Bleck | Schultz | Conen | Frerk | Henke | Leiber | Fuchs

\title{
Selbstbestimmt teilhaben in Altenpflegeeinrichtungen
}

Empirische Analysen zu fördernden

und hemmenden Faktoren

\section{Nomos}


Christian Bleck | Laura Schultz | Ina Conen Timm Frerk | Stefanie Henke | Simone Leiber Harry Fuchs

\section{Selbstbestimmt teilhaben in Altenpflegeeinrichtungen}

Empirische Analysen zu fördernden und hemmenden Faktoren

In Zusammenarbeit mit Helene Maqua, Henry Kieschnick

\section{Nomos}


Die Finanzierung der vorliegenden Publikation erfolgte im Rahmen der Förderung der Projekts „Selbstbestimmt teilhaben in Altenpflegeeinrichtungen. Ein Musterrahmenkonzept" durch die Stiftung Wohlfahrtspflege NRW.



Die Deutsche Nationalbibliothek verzeichnet diese Publikation in der Deutschen Nationalbibliografie; detaillierte bibliografische Daten sind im Internet über http://dnb.d-nb.de abrufbar.

\section{Auflage 2020}

(c) Christian Bleck, Laura Schultz, Ina Conen, Timm Frerk, Stefanie Henke, Simone Leiber, Harry Fuchs

In Zusammenarbeit mit Helene Maqua, Henry Kieschnick

Publiziert von

Nomos Verlagsgesellschaft mbH \& Co. KG

Waldseestraße 3-5 | 76530 Baden-Baden

www.nomos.de

Gesamtherstellung:

Nomos Verlagsgesellschaft mbH \& Co. KG

Waldseestraße 3-5| 76530 Baden-Baden

ISBN (Print): 978-3-8487-6709-0

ISBN (ePDF): 978-3-7489-0766-4

DOI: https://doi.org/10.5771/9783748907664

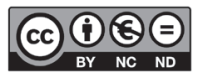

Dieses Werk ist lizenziert unter einer

Creative Commons Namensnennung - Nicht kommerziell Keine Bearbeitungen 4.0 International Lizenz.

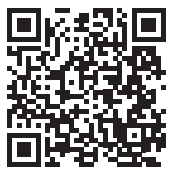

Onlineversion Nomos elibrary 


\section{Geleitwort \\ zum Abschlussbericht des Projektes „Selbstbestimmt teilhaben in Altenpflegeeinrichtungen" (STAP)}

Wenn bestmögliche Selbstbestimmung und Teilhabe das Leben von Bewohnerinnen und Bewohnern in Altenheimen prägen, dann wird sich ein „Ich will auf keinen Fall ins Heim“ in ein „Ich gehe gern ins Altenheim“ wandeln können.

Die pflegerische Versorgung ist in den katholischen Altenpflegeeinrichtungen des Erzbistums Köln auf einem hohen Stand, das bestätigen verschiedene Prüfinstanzen, Pflegebedürftige und ihre Angehörigen immer wieder. Zugleich besteht die Sorge, dass mit Einzug in eine Altenhilfeeinrichtung die Selbstbestimmung und Teilhabe quasi „an der Pforte“ abgegeben werden.

Im Zuge der intensiven Befassung u. a. von Prof. Dr. Harry Fuchs mit der Frage, wie sich selbstbestimmte Teilhabe am gesellschaftlichen Leben für die Bewohnerinnen und Bewohner von Altenpflegeeinrichtungen besser verwirklichen lässt, wurde vor drei Jahren das nun abgeschlossene Projekt aufgelegt.

Dabei geht es für die Seniorinnen und Senioren um ganz konkrete Alltagsfragen: Kann ich noch zu einem Fußballspiel oder ins Theater fahren, obwohl ich hilfebedürftig bin? Kann ich weiter mit meinen Enkeln skypen? Finde ich Möglichkeiten der Beschäftigung, auch wenn ich nur noch ganz schlecht höre oder sehe? Wie viel Einfluss habe ich eigentlich auf meine Pflege? Solchen Fragen ging die nun vorliegende Untersuchung zur aktuellen Teilhabewirklichkeit in den stationären Altenpflegeeinrichtungen nach und formuliert Anforderungen dafür, dass Teilhabe gelingen kann.

Hilfreich und interessiert machten sich vier Mitgliedseinrichtungen über drei Jahre engagiert auf den Weg. Sie ließen dafür die wissenschaftlichen Mitarbeiterinnen der Hochschule Düsseldorf bei sich hospitieren, beobachten und Interviews führen. Sie gewährten Einblick in die hausinternen Abläufe bei Pflege und Betreuung. Auch die Bewohnerinnen und Bewohner bzw. ihre Bevollmächtigten gaben ihr Einverständnis. Zudem fand sich bereits eine weitere Einrichtung zur Erprobung des Musterrahmenkonzeptes.

Dieses Musterrahmenkonzept, dessen Erstellung das Projektziel war, liegt nun vor. Damit ist erstmalig eine Untersuchung zu selbstbestimmter 
Teilhabe in stationären Einrichtungen der Altenhilfe gelungen. In dem Konzept sind Faktoren für gelingende Teilhabe in den Einrichtungen herausgearbeitet, geordnet nach Struktur, Prozess und Ergebnis. Die Erkenntnisse des Projektes sind sehr hilfreich auf dem Weg in eine moderne Pflege, Versorgung und Betreuung in Altenpflegeeinrichtungen. Diese Weiterentwicklung voranzubringen, treibt uns an. Das Konzept gilt es nun in der Praxis umzusetzen - das wird für die Einrichtungen der Altenhilfe eine wichtige Herausforderung der kommenden Jahre sein.

Der Diözesan-Caritasverband für das Erzbistum Köln dankt der Stiftung Wohlfahrtspflege NRW, dass sie das Projekt finanziell entscheidend unterstützt hat. Herrn Prof. Dr. Harry Fuchs, Frau Prof. Dr. Simone Leiber und Herrn Prof. Dr. Christian Bleck und dem gesamten STAP-Team der Hochschule Düsseldorf gilt unser Dank für den Ideenreichtum und die vertrauensvolle Zusammenarbeit während der gesamten Projektphase. Und der Dank gilt den Einrichtungen der stationären Altenhilfe im Erzbistum Köln, die sich zum Wohle heutiger und künftiger Bewohnerinnen und Bewohner beteiligt haben.

Dr. Frank Joh. Hensel

Diözesan-Caritasdirektor 


\section{Inhaltsverzeichnis}

$\begin{array}{ll}\text { Abbildungsverzeichnis } & 11\end{array}$

$\begin{array}{ll}\text { Tabellenverzeichnis } & 13\end{array}$

$\begin{array}{ll}\text { Abkürzungsverzeichnis } & 15\end{array}$

1. Einleitung 17

1.1. Projekthintergründe 19

1.2. Untersuchungsleitende Fragen und Projektziel 22

1.3. Aufbau der Publikation 23

2. Forschungsstand, rechtlicher und konzeptioneller Rahmen 24

2.1. Systematische Literaturrecherche 24

2.2. Forschungsstand 25

2.3. Selbstbestimmte Teilhabe: rechtlicher und konzeptioneller Rahmen 35

2.3.1. Rechtliche Verankerung von selbstbestimmter Teilhabe $\quad 36$

2.3.2. Konzeption selbstbestimmter Teilhabe in STAP 39

3. Forschungsdesign 45

3.1. Aufbau: Sequenzieller Mixed-Methods-Ansatz 45

3.2. Qualitative Analyse 48

3.2.1. Erhebungen in vier ausgewählten Einrichtungen 49

3.2.1.1. Samplingstrategie 49

3.2.1.2. Merkmale der Einrichtungen $\quad 50$

3.2.1.3. Erhebungsmethoden 65

3.2.1.4. Auswertungsmethoden 75

3.2.2. Fokusgruppendiskussion $\quad 79$

3.2.2.1. Samplingstrategie $\quad 79$

3.2.2.2. Erhebungsmethode $\quad 80$

3.2.2.3. Auswertungsmethode $\quad 81$ 
3.2.3. „Good-Practice“-Analyse als projektergänzender Zugang $\quad 82$

3.3. Quantitative Analyse 85

3.3.1. Stichprobenzugänge und Stichprobe $\quad 86$

3.3.2. Erhebungsmethode $\quad 89$

3.3.3. Auswertungsmethode 91

4. Ergebnisse der qualitativen Analyse 92

4.1. Wunschäußerung und -erfassung als Ausgangspunkt der

Teilhabeförderung

4.1.1. Zum Begriff Wunsch

4.1.2. Phasen der Wunschäußerung, -erfassung, -berücksichtigung und -verwirklichung 94

4.1.3. Wunschäußerung 95

4.1.4. Wunscherfassung 100

4.1.4.1. Erfassung formeller Art 100

4.1.4.2. Erfassung informeller Art 103 4.1.5. Wunschberücksichtigung 104

4.2. Alltags- und Angebotsgestaltung als Kontexte und Elemente der Teilhabeförderung

106

4.2.1. Alltags- und Angebotsgestaltung innerhalb und außerhalb der Einrichtung

4.2.1.1. Alltags- und Angebotsgestaltung innerhalb der Einrichtung 5

(10)

4.2.1.2. Alltags- und Angebotsgestaltung außerhalb der Einrichtung

4.2.2. Alltagsstrukturierende Schlüsselprozesse aus dem Blickwinkel der Beobachtung

4.2.2.1. Alltags- und Angebotsgestaltung 117

4.2.2.2. Mahlzeiten

4.2.2.3. Pflegeprozesse

4.2.2.4. Informelle Gespräche mit Bewohner*innen im Kontext der Beobachtung

4.3. Fördernde und hemmende Faktoren der Teilhabeförderung auf Ebene der Bewohner*innen, Mitarbeiter"innen sowie der Einrichtung und Organisation $\quad 128$

4.3.1. Bewohner*innen $\quad 128$

4.3.1.1. Physische und psychische Voraussetzungen 
4.3.1.2. Weitere Differenzmerkmale der Bewohner*innenschaft

4.3.2. Mitarbeiter*innen

4.3.2.1. Kompetenzen 135

4.3.2.2. Arbeitskultur und Arbeitsengagement 140

4.3.3. Einrichtung und organisatorische Abläufe $\quad 145$

4.3.3.1. Organisationskultur 145

4.3.3.2. Formelle Verankerung in Konzepten $\quad 150$

4.3.3.3. Schnittstellen und Zusammenarbeit innerhalb der Arbeitsprozesse

4.3.3.4. Handlungsspielräume innerhalb von Arbeitsprozessen

4.4. Besondere und zusätzliche Ressourcen 163

4.4.1. Zusätzliche Betreuungskräfte 164

4.4.2. Ehrenamtler*innen 167

4.4.3. Angehörige 169

4.4.4. Zusätzliche finanzielle Mittel 173

4.4.5. Mobilitätshilfen und -unterstützung 175

4.4.6. Räume 178

4.4.7. Vernetzung mit dem Quartier 179

4.5. Zwischenfazit zur qualitativen Analyse 182

5. Ergebnisse der quantitativen Analyse 189

5.1. Beschreibung der Einrichtungen 190

5.2. Verankerung von Teilhabe 193

5.3. Zusammenarbeit und Aufgaben der Arbeitsbereiche 196

5.4. Handlungsspielräume und Ressourcen 202

5.5. Arbeitskulturelle Orientierungen 206

5.6. Wichtige Faktoren und dringende Veränderungen 207

5.7. Zwischenfazit zur quantitativen Analyse 210

6. Entwicklung und Erprobung des Musterrahmenkonzeptes 212

6.1. Entwicklung und Inhalte des Musterrahmenkonzeptes 212

6.2. Auswahl und Merkmale der Erprobungseinrichtung 214

6.3. Durchführung der Erprobung 216

6.3.1. Vorstellung und Einführung des MRK 218 
6.3.2. Selbsteinschätzung und Kriterienauswahl

6.3.3. Einrichtungsinterne Maßnahmenentwicklung und initiale Implementation

6.4. Implementationsanalyse

6.4.1. Vorarbeiten und Entwicklung der Implementationsanalyse

6.4.2. Analyseleitende Fragen und Forschungsdesign

6.4.2.1. Fokusgruppeninterview

6.4.2.2. Kurzinterviews

6.5. Ergebnisse der Implementationsanalyse

6.5.1. Ergebnisse der Erhebung auf der Leitungsebene

(Fokusgruppeninterviews)

6.5.1.1. Akzeptanz

6.5.1.2. Angemessenheit

6.5.2. Ergebnisse der Erhebung auf der Mitarbeiter*innenebene (Kurzinterviews)

6.6. Zwischenfazit zur Erprobung

7. Schlussbetrachtung und Ausblick

7.1. Fördernde Faktoren selbstbestimmter Teilhabe in Altenpflegeeinrichtungen

7.2. Ausblick zum Musterrahmenkonzept 265

7.3. Empfehlungen an die Politik 266

7.4. Weiterer Forschungs- und Entwicklungsbedarf 272

$\begin{array}{ll}\text { Literatur } & 275\end{array}$

Anhang: Auszüge aus dem Musterrahmenkonzept 287 


\section{Abbildungsverzeichnis}

Abbildung 1: Überblick über den Aufbau der Studie 46

$\begin{array}{lll}\text { Abbildung 2: } & \text { Zeitlicher Ablaufplan } & 47\end{array}$

Abbildung 3: Prototypische Abfolge von Wunschäußerung, -erfassung, -berücksichtigung und -verwirklichung 95

Abbildung 4: Teilnehmende an der Umfrage nach Regierungsbezirk 191

$\begin{array}{lll}\text { Abbildung 5: Verankerung von Teilhabe (Ist-Zustand) } & 194\end{array}$

Abbildung 6: Verankerung von Teilhabe (Bewertung der Relevanz) 195

Abbildung 7: Zusammenarbeit zwischen den Arbeitsbereichen

(Ist-Zustand und Bewertung der Relevanz) 197

Abbildung 8: Beitrag zur Umsetzung von Teilhabewünschen nach Arbeitsbereichen 198

Abbildung 9: Planung der Teilhabemaßnahmen und Fallbesprechungen (Ist-Zustand und Bewertung der Relevanz)

Abbildung 10: Arbeitsbereiche, die in der Regel an Fallbesprechungen teilnehmen

Abbildung 11: Handlungsspielräume (Ist-Zustand und Relevanz der Bewertung)

Abbildung 12: Kenntnisse über Handlungsspielräume im Rahmen von Vorschriften und Bestimmungen (Ist-Zustand)

Abbildung 13: Personelle Ressourcen innerhalb und außerhalb der Einrichtung (Ist-Zustand)

Abbildung 14: Arbeitskulturelle Orientierung

Abbildung 15: Dringendste Veränderung für Teilhabe (Score-Werte nach Rangfolge) 
Abbildung 16: Wichtige Faktoren für die Teilhabeförderung (Score-Werte nach Rangfolge)

Abbildung 17: Bewertung des neuen Wochenplans

Abbildung 18: Kernprozess sowie Faktoren zur Förderung selbstbestimmter Teilhabe

Abbildung 19: Formelle und informelle Erfassungsformen von Wünschen und Bedürfnissen in Altenpflegeeinrichtungen 


\section{Tabellenverzeichnis}

Tabelle 1: Suchbegriffe für die Schlagwortsuche nach Kategorien

Tabelle 2: $\quad$ Einflussfaktoren auf selbstbestimmte Teilhabe in Altenpflegeeinrichtungen

Tabelle 3: $\quad$ Trägerschaft und Lage der Einrichtungen

Tabelle 4: Anzahl der Plätze, Wohnbereiche und Arten der Zimmer

Tabelle 5: Ambulante und stationäre Angebote der Einrichtungen

Tabelle 6: Soziodemografische Merkmale der Bewohner*innen

Tabelle 7: $\quad$ Einschränkungen und Hilfebedarfe der Bewohner*innen

Tabelle 8: Rechtliche Vertretung der Bewohner*innen

Tabelle 9: $\quad$ Aufenthaltsdauer, Einzugsalter und vorherige Wohnorte der Bewohner*innen

Tabelle 10: Anzahl der Mitarbeiter*innen nach Einrichtungs- und Aufgabenbereichen (in Vollzeitäquivalenten)

Tabelle 11: Beispiel für einen Beobachtungsplan der Einrichtung C

Tabelle 12: Kategoriensystem der Interviews

Tabelle 13: Einrichtungen der „Good-Practice“-Analyse

Tabelle 14: Vier Bewohner*innengruppen auf Basis der Interviewaussagen

Tabelle 15: Angaben zu den Einrichtungen, die teilgenommen haben

Tabelle 16: Gliederung des MRK

Tabelle 17: Ablauf und eingesetzte Ressourcen für die Erprobung des Musterrahmenkonzeptes 


\section{Abkürzungsverzeichnis}

$\begin{array}{ll}\text { ANG } & \text { Angehörige } \\ \text { BW } & \text { Bewohner*innen } \\ \text { EL } & \text { Einrichtungsleitung } \\ \text { GP } & \text { „Good-Practice“-Analyse } \\ \text { HT } & \text { Haustechnik } \\ \text { HW } & \text { Hauswirtschaft } \\ \text { Int. } & \text { Interview } \\ \text { ÖPNV } & \text { offentlicher Personennahverkehr } \\ \text { PDL } & \text { Pflegedienstleitung } \\ \text { PFK } & \text { Pflegefachkraft } \\ \text { SD } & \text { Sozialer Dienst (Leitung und Mitarbeitende) } \\ \text { SIS } & \text { Strukturierte Informationssammlung } \\ \text { STAP } & \text { Projekt „Selbstbestimmt teilhaben in Altenpflegeeinrichtun- } \\ & \text { gen“ } \\ \text { UN-BRK } & \text { UN-Behindertenrechtskonvention } \\ \text { WHO } & \text { Weltgesundheitsorganisation (World Health Organisation) } \\ \text { WTG NRW } & \text { Wohn- und Teilhabegesetz des Landes Nordrhein-Westfalen } \\ \text { ZBK } & \text { Zusätzliche Betreuungskräfte }\end{array}$




\section{Einleitung}

Angesichts des demografischen Wandels ist die Organisation "guter" Sorge und Pflege für ältere und alte Menschen eine der zentralen gesellschaftlichen Zukunftsaufgaben. Das Projekt „Selbstbestimmt teilhaben in Altenpflegeeinrichtungen - STAP" fokussiert auf die stationäre Altenhilfe und Fragen der selbstbestimmten Teilhabe von Bewohner"innen in Pflegeeinrichtungen. Alte Menschen mit Hilfe- und Pflegebedarf sollen nicht nur qualitativ hochwertig medizinisch und pflegerisch versorgt werden, sondern auch ein würdiges und selbstbestimmtes Leben führen können, das eine Teilhabe am gesellschaftlichen Leben weiterhin ermöglicht. Dazu verpflichten nicht nur die Vorgaben der UN-Behindertenrechtskonvention (UN-BRK), sondern auch die Heimgesetze einiger Bundesländer. Auch in Nordrhein-Westfalen (NRW) ist dieses Anliegen bereits gesetzlich verankert. Hintergrund des Projektes sind somit auch die Anforderungen des $\$ 5$ Wohn- und Teilhabegesetz Nordrhein-Westfalen (WTG NRW).

Teilhabe verstehen wir in Anlehnung an Fuchs (2009, S. 20f) als gleichberechtigten Zugang zu und die Mitbestimmung über soziale Umweltbeziehungen nach den Wünschen einer Person zur persönlichen Daseinsentfaltung. Selbstbestimmung wird dabei als ein zentrales Prinzip von Teilhabe verstanden, nach dem diese auszugestalten ist (ausführlich zur Konzeption von selbstbestimmter Teilhabe im STAP-Projekt vgl. Kapitel 2.3).

Auf Seiten der Einrichtungsträger in der stationären Altenhilfe gibt es längere Traditionen und vielfältige Angebote der sogenannten sozialen Betreuung, die von Ausflügen über Kreativ-, Spiele- und Fitnessangebote bis hin zu Selbsthilfekreisen reichen. Auf diese Angebote, die v. a. von Fachkräften des Sozialen Dienstes sowie - zumeist in Einzel- oder Kleingruppenarbeit - von zusätzlichen Betreuungskräften nach 43b SGB XI (früher $\$ 87$ b SBG XI) durchgeführt werden, baut das Projektvorhaben STAP prinzipiell auf. Das Vorhandensein bestimmter - unter Umständen eher paternalistisch geprägter - Angebotsstrukturen in Bezug auf gesellschaftliche Teilhabe bedeutet jedoch nicht, dass auch eine Kultur der Unterstützung und Routinen der Berücksichtigung von Selbstbestimmung und Teilhabe der Bewohner*innen im Selbstverständnis und Alltagshandeln der Einrichtungen verankert sind. Zudem machen Veränderungen in der Bewohner*innenstruktur stationärer Altenpflegeeinrichtungen (Zunahme des Grades an Pflegebedürftigkeit und der Demenzerkrankungen, sinkende 


\section{Einleitung}

Verweildauern) diesen Anspruch besonders voraussetzungsvoll. In der Praxis steht eine Umsetzung gesellschaftlicher Teilhabe im Rahmen stationärer Einrichtungen, die nach dem Prinzip der Selbstbestimmung auch die individuellen Wünsche und Bedarfe der Bewohner*innen berücksichtigt, vor großen Herausforderungen:

„Ein Wechsel in eine stationäre Pflegeeinrichtung wird von den Betroffenen [...] als Autonomieverlust und als Manifestation von Gebrechlichkeit und fehlender Lebensqualität gedeutet" (BMFSFJ, 2005, S. 7). Dieser ist daher selten positiv besetzt, erst recht nicht mit Assoziationen von Selbstbestimmung und Teilhabe. Der Anspruch an eine fortgesetzte, an den individuellen Wünschen und Bedürfnissen ausgerichtete gesellschaftliche Teilhabe wird daher von den Bewohner*innen und ihrem Umfeld häufig gar nicht aufrechterhalten und somit auch nicht eingefordert. Dies macht es besonders schwer, die notwendige Anpassung der Strukturen und Prozesse hin zu einer teilhabeorientierten Ausrichtung von stationären Einrichtungen voranzutreiben. Auch werden hierfür fehlende Ressourcen im Arbeitsalltag der stationären Altenhilfe angeführt, wenngleich vielfach bereits ein „teilhabeorientierter Blick“ der Mitarbeitenden - aus der Pflege ebenso wie der Sozialen Betreuung und Hauswirtschaft - und eine entsprechende Ausrichtung in der Konzeption und Struktur der Einrichtung zu einer verbesserten selbstbestimmten Teilhabesituation der Bewohner*innen führen könnte.

Teilhabe und Selbstbestimmung sind zwar viel genutzte Schlagwörter, aber ihre alltagspraktische Bedeutung in der stationären Altenhilfe bleibt bislang diffus. Das macht auch die Überprüfung der Ergebnisqualität hinsichtlich Teilhabe und Selbstbestimmung bisher nahezu unmöglich, nochmals verschärft durch eingeschränkte kognitive Fähigkeiten der Bewohner*innenschaft. Bewohner*innen von Altenpflegeeinrichtungen werden ihre Selbstbestimmungs- und Teilhaberechte aber nur dann verwirklichen können, wenn sowohl (bereits vorhandene) Angebotsstrukturen und prozessuale Rahmenbedingungen sowie auch das Selbstverständnis der Einrichtung und der darin Beschäftigten konsequent auf Teilhabe und Selbstbestimmung ausgerichtet werden. Zur Umsetzung einer selbstbestimmten gesellschaftlichen Teilhabe fehlt es an praxistauglichen Orientierungen für Einrichtungen der Altenhilfe. Ein Orientierungsrahmen dafür, wie dies in den Einrichtungen bewerkstelligt und nachgehalten werden kann, ist in diesem Projekt entwickelt und erprobt worden. 


\subsection{Projekthintergründe}

Während des Gesetzgebungsverfahrens zum Wohn- und Teilhabegesetz Nordrhein-Westfalen (WTG NRW) wurde die Frage, ob, in welchem Umfang und in welcher Ausführung man Anforderungen der UN-Behindertenrechtskonvention (UN-BRK) umsetzt, sehr ausführlich - teilweise auch kontrovers - diskutiert. ${ }^{1}$

Im Ergebnis hat sich der Gesetzgeber dafür entschieden, bereits die Zweckbestimmung des Gesetzes auf die Gewährleistung des selbstbestimmten Lebens auszurichten ( $\$ 1$ Abs. 1 WTG NRW). Dabei soll die Leistungserbringung ausdrücklich auch auf die Förderung der Teilhabemöglichkeiten ausgerichtet werden ( $\$ 1$ Abs. 3 WTG NRW). Bewohner*innen von Einrichtungen der Alten- und Behindertenhilfe sollen ein möglichst selbstbestimmtes Leben führen können und in der Wahrnehmung ihrer Selbstverantwortung unterstützt werden ( $\$ 1$ Abs. 4 WTG NRW).

Im Rahmen der Begriffsbestimmung ( $\int 3$ WTG NRW) stellt der Gesetzgeber klar, dass „soziale Betreuung“ Tätigkeiten umfasst, die Menschen in einer selbstbestimmten Lebensführung unterstützen sowie der Förderung einer unabhängigen Lebensführung und der vollen Teilhabe am Leben in der Gesellschaft dienen.

Nach $\int 5$ WTG NRW (Teilhabe am Leben in der Gesellschaft) haben Leistungsanbieter*innen im Rahmen ihrer jeweiligen Leistungsvereinbarung die gleichberechtigte Teilhabe der Nutzer*innen am Leben in der Gesellschaft zu unterstützen und zu fördern. Der Gesetzgeber ordnet damit die Gewährleistung der Zweckbestimmung mit der Leistung „Soziale Betreuung" den Leistungserbringer"innen zu.

Bereits die Diskussion während des Gesetzgebungsverfahrens zum WTG NRW enthielt jedoch deutliche Hinweise, dass das Wissen bei den am Verfahren Beteiligten über die UN-BRK und die sich aus ihren Anforderungen ergebenden Folgen, insbesondere für die Lebenswirklichkeit in Einrichtungen der Alten- und Behindertenhilfe, sehr heterogen war und entsprechende anwendungsbezogene Analysen fehlten. So wurde insbesondere der Bedarf an Orientierungen dazu deutlich, wie eine Förderung von Selbstbestimmung und Teilhabe in der Altenhilfe praktisch umgesetzt werden kann und welche konkreten Faktoren in Bezug auf die Strukturen und Prozesse in Pflegeeinrichtungen zu berücksichtigen sind. Vor diesen

1 Siehe hierzu beispielsweise das Protokoll über die öffentliche Anhörung des Ausschusses für Arbeit Gesundheit und Soziales zum GEPA NRW vom 2.10.2014 - 28. Sitzung des Ausschusses am 12.9.2013 - APr 16/319 vom 12.9.2013. 
Hintergründen entstand die Überlegung, in einem wissenschaftlichen Projekt einerseits den derzeitigen Entwicklungsstand der auf die selbstbestimmte Teilhabe orientierenden Rahmenbedingungen, Aktivitäten und Maßnahmen in stationären Altenpflegeeinrichtungen zu untersuchen, andererseits eine praxisbezogene Orientierungshilfe für die Organisation und Förderung von selbstbestimmter Teilhabe in den Einrichtungen zu entwickeln.

Zur Verwirklichung dieses Projektes, das durch die Stiftung Wohlfahrtspflege NRW über einen dreijährigen Zeitraum vom 1.1.2017 bis zum 31.12.2019 gefördert wurde, konnte Prof. Dr. Harry Fuchs den DiözesanCaritasverband für das Erzbistum Köln e. V. in Kooperation mit der Hochschule Düsseldorf gewinnen. Die Trägerschaft und Projektleitung von STAP lag beim Diözesan-Caritasverband für das Erzbistum Köln e. V., während die wissenschaftliche Leitung durch die Hochschule Düsseldorf erfolgte. Personell standen auf Seiten des Diözesan-Caritasverbandes für das Erzbistum Köln e. V. eine halbe Stelle für einen Projektmitarbeiter und auf Seiten der Hochschule Düsseldorf, aufgrund des hohen Forschungsanteils in STAP, drei halbe Stellen für wissenschaftliche Mitarbeiter*innen zur Verfügung. ${ }^{2}$ Zum Projektteam zugehörig waren ferner zwei Berater*innen, die an der Projektplanung und -steuerung kontinuierlich, u. a. über regelmäßige Teamsitzungen, beteiligt waren. ${ }^{3}$

Der Projektverlauf wurde darüber hinaus durch zwei Gremien, die einerseits aus dem Wissenschaftlichen Beirat ${ }^{4}$ und andererseits aus dem Pro-

2 Auf Seiten des Diözesan-Caritasverbandes für das Erzbistum Köln e. V. lag die Projektleitleitung bei Helene Maqua; Henry Kieschnick war als Projektmitarbeiter und Ingeborg Heinrich als Projektassistenz tätig. Die wissenschaftliche Durchführung der STAP-Studie erfolgte auf Seiten der Hochschule Düsseldorf unter der Leitung von Prof. Dr. Christian Bleck (bis August 2017 gemeinsam mit Prof. Dr. Simone Leiber) durch die wissenschaftlichen Mitarbeiter*innen Laura Schultz, Ina Conen, Timm Frerk (bis April 2019) und Stefanie Henke (ab Juli 2019) sowie unter Mitwirkung der studentischen Mitarbeitenden Jennifer van Berk und Patrick Stumpf.

3 Fortlaufend beraten wurde das Projekt im Bereich „Teilhabe“ durch Prof. Dr. Harry Fuchs (freier Berater; nebenamtlich Honorarprofessor an der Hochschule Düsseldorf) und ab September 2017 im Bereich „Pflege/Pflegepolitik“ durch Prof. Dr. Simone Leiber (nach ihrem Wechsel von der Hochschule Düsseldorf an die Universität Duisburg-Essen).

4 Mitglieder des Wissenschaftlichen Beirats waren in alphabetischer Reihenfolge: Prof. i. R. Dr. Gerhard Bäcker (Universität Duisburg-Essen), Stephan Enzweiler (AWO Bezirksverband Mittelrhein e. V.), Stefan Juchems (Stiftung Wohlfahrtspflege NRW), Prof. i. R. Dr. Ernst von Kardorff (Humboldt-Universität zu Berlin), 
jektrat ${ }^{5}$ bestanden, begleitet und begutachtet. Damit war gewährleistet, dass STAP sowohl aus wissenschafts- als auch aus praxisorientierter Sicht regulär Rückmeldungen und Einschätzungen zu dessen Projektverlauf und -ergebnissen erhielt.

Das STAP-Team dankt allen Kooperationspartner*innen, welche in unterschiedlichen Zusammenhängen die Forschungs- und Entwicklungsarbeiten von STAP unterstützt haben. Insbesondere danken wir der Stiftung Wohlfahrtspflege für die maßgebliche Finanzierung des Projektes sowie den Leitungskräften, Mitarbeitenden, Bewohner"innen und Angehörigen aus den beteiligten Altenpflegeeinrichtungen für ihre Unterstützung im Rahmen der Interviews, teilnehmenden Beobachtungen und weiteren Projektaktivitäten von STAP. Ferner danken wir den Mitgliedern des Wissenschaftlichen Beirats für die hilfreichen Hinweise im Projektverlauf.

An dieser Stelle ist darüber hinaus festzuhalten, dass die Veröffentlichung der vorliegenden STAP-Studie in die Zeit der - seit Anfang 2020 zunehmend auch in Deutschland wahrnehmbaren - Corona-Pandemie fiel, während deren Durchführung davon noch in Gänze unberührt war. Die Verbreitung sowie die Maßnahmen zur Eindämmung von Covid-19 hatten und haben zum Zeitpunkt der Veröffentlichung für die Bewohner*innen, Mitarbeiter*innen und Angehörigen von Altenpflegeeinrichtungen besonders belastende und teils dramatische Folgen. Auch wenn sich damit während dieser Corona-Krise zugleich die Rahmenbedingungen in Altenpflegeeinrichtungen für eine Teilhabeförderung und -verwirklichung wesentlich verändert haben (z. B. in Bezug auf Abstandsregeln, Besuchsverbote bzw. -einschränkungen), gehen die Autor*innen ausdrücklich davon aus, dass die hier dargestellten Studienergebnisse nach Abschluss der Hochphase der Corona-Pandemie grundsätzlich ihre Gültigkeit behalten. Ferner ist zu betonen, dass die Ausgangsfrage von STAP - die Frage nach der Verwirklichung des Rechts auf selbstbestimmte Teilhabe von Bewohner*innen stationärer Altenpflegeeinrichtungen - ebenso in Zeiten einer langwierigen Pandemie zu beantworten ist. Unter Berücksichtigung angemessener Schutz- und Hygienemaßnahmen gilt es auch in dieser Situation, Zugänge, Wege und Lösungen zu finden, wie die selbstbestimmte Teilhabe der Bewohner*innen unterstützt und gewährleistet werden kann. Da

Prof. Dr. Mathilde Niehaus (Universität Köln), Dr. Thomas Otten (Diözesanbeauftragter für Ethik im Gesundheitswesen), Dr. Martin Theisohn (Landesseniorenvertretung NRW), Frank Wübbold (Der Paritätische Nordrhein-Westfalen).

5 Der Projektrat war zusammengesetzt aus den vier Projekteinrichtungen des Caritasverbandes - siehe hierzu die näheren Angaben in Kapitel 3.2.1. 


\section{Einleitung}

die Ergebnisse der STAP-Studie vor allem grundlegende Faktoren der Ermittlung, Organisation und Durchführung von adäquaten Teilhabeaktivitäten in Altenpflegeeinrichtungen aufzeigen, können diese an vielen Stellen auch Anregungen für die Teilhabeförderung unter den einschränkenden Bedingungen der Corona-Pandemie bieten.

\subsection{Untersuchungsleitende Fragen und Projektziel}

Ziel des Projekts war es, auf empirischer Basis fördernde und hemmende Faktoren zur praktischen Unterstützung und Verwirklichung der selbstbestimmten Teilhabe von Bewohner*innen stationärer Altenpflegeeinrichtungen zu identifizieren und auf dieser Grundlage ein Musterrahmenkonzept zu entwickeln. Mit dem Musterrahmenkonzept soll Leitungen und Mitarbeitenden von Altenpflegeeinrichtungen ein praxisorientierter Handlungsrahmen für die Förderung der Teilhabe von Bewohner*innen am Leben in der Gesellschaft auf Basis des Selbstbestimmungsprinzips zur Verfügung gestellt werden. Die Projektdurchführung beinhaltete daher im ersten Schritt eine umfassende Phase der Erforschung von fördernden und hemmenden Faktoren zur Unterstützung und Verwirklichung von selbstbestimmter Teilhabe in Altenpflegeeinrichtungen. Die zweite Phase widmete sich dem Musterrahmenkonzept, das auf Basis der empirischen Erkenntnisse entwickelt und in einer Einrichtung - im Rahmen einer Implementationsanalyse - erprobt und bewertet wurde. Zeitlich bereits parallel dazu sowie über die Projektlaufzeit hinaus erfolgte die Transferphase zur Präsentation von (Zwischen-)Ergebnissen des STAP-Projektes aus der Empirie und zum Musterrahmenkonzept in verschiedenen Kontexten von Wissenschaft, Politik und Praxis.

Vor diesen Hintergründen lagen der - in diesem Bericht im Vordergrund stehenden - empirischen Studie von STAP folgende untersuchungsleitende Forschungsfragen zugrunde:

1) Wie können Wünsche und Bedürfnisse von Bewohner*innen stationärer Pflegeeinrichtungen in Bezug auf gesellschaftliche Teilhabe innerhalb und außerhalb der Einrichtung festgestellt und berücksichtigt werden?

2) Wie kann auf dieser Basis das Recht auf selbstbestimmte Teilhabe dieser Bewohner*innen umgesetzt und überprüft werden? 


\subsection{Aufbau der Publikation}

Die vorliegende Publikation dokumentiert die theoretische Konzeptionierung, das Forschungsdesign und die empirischen Ergebnisse der wissenschaftlichen Studie im Rahmen des STAP-Projektes. Begonnen wird im nachfolgenden Kapitel mit der Darstellung des Forschungstandes im Themenfeld selbstbestimmter Teilhabe im Kontext stationärer Altenhilfe sowie der Erläuterung der rechtlichen und begrifflich-konzeptionellen Grundlagen der STAP-Studie. Im dritten Kapitel wird das Mixed-MethodsDesign der Studie vorgestellt, indem die genutzten qualitativen und quantitativen Forschungszugänge in Bezug auf ihre Stichproben bzw. Samples, ihre Erhebungs- und Auswertungsmethoden methodologisch sowie methodisch gerahmt und begründet werden. Das Kapitel vier stellt die Ergebnisse der qualitativen Analysen vor und den umfassendsten empirischen Bestandteil der Studie dar. Im fünften Kapitel werden die Ergebnisse der quantitativen Analyse erläutert, die auf den Ergebnissen der qualitativen Erhebungen aufbaut. Das sechste Kapitel bezieht sich auf die zweite Projektphase und demnach auf die Entwicklung des Musterrahmenkonzeptes sowie vor allem auf dessen praktische Erprobung und dessen empirische Bewertung im Rahmen einer Implementationsanalyse. Das siebte und letzte Kapitel schließt mit einem Gesamtfazit und Ausblick, indem zentrale empirische Ergebnisse resümiert und diskutiert, ein Ausblick zur Nutzung und zum Transfer des Musterrahmenkonzepts vermittelt und Empfehlungen an die Politik formuliert werden. 


\section{Forschungsstand, rechtlicher und konzeptioneller Rahmen}

Im Folgenden werden einerseits der Forschungsstand zur Förderung von Selbstbestimmung und Teilhabe im Alter sowie in Altenpflegeeinrichtungen vorgestellt, der dem STAP-Projekt dazu diente, Forschungslücken zu identifizieren. Andererseits werden der rechtliche und konzeptionelle Rahmen für die vorliegende STAP-Studie erläutert.

\subsection{Systematische Literaturrecherche}

Das Ziel der systematischen Literaturrecherche und -analyse war die Aufarbeitung des Forschungstandes im Themenfeld selbstbestimmter Teilhabe und ihrer Förderung im Kontext stationärer Altenhilfe. In der ersten Phase wurde über die Schlagwortsuche nach Teilhabe, Selbstbestimmung oder Autonomie und verwandten Konzepten (Tabelle 1, Punkt 1) in Kombination mit Pflegeeinrichtungen, Pflege oder alte Menschen und verwandten Perspektiven (Tabelle 1, Punkt 2) gesucht. Anschließend wurde die Suche nach Teilhabe in Verbindung mit weiteren ausgewählten Konzepten und Perspektiven (u. a. Sozialraum, Empowerment) und um Forschungsliteratur mit Bezug zu Teilgruppen (Menschen mit Demenz) ergänzt (Tabelle 1, Punkt 3). Abschließend wurde die Literatur in das Literaturverwaltungsprogramm Zotero eingepflegt. Die Suche erfolgte in deutscher und englischer Sprache in den Datenbanken Pro Quest, PubMed, MEDLINE, ciando, wiso, SocINDEX, SSG Sozialwissenschaft, EBSCO sowie über Google Scholar. In der zweiten Phase wurde die recherchierte Literatur mittels Schneeballsystem um weitere relevante Forschungsliteratur und Praxisbeispiele erweitert. 
Tabelle 1: Suchbegriffe für die Schlagwortsuche nach Kategorien

\begin{tabular}{|l|l|l|}
\hline & Deutsch & Englisch \\
\hline 1 & $\begin{array}{l}\text { Teilhabe, Partizipation, Selbstbe- } \\
\text { stimmung, Autonomie, selbstbe- } \\
\text { stimme Teilhabe, Lebensführung }\end{array}$ & $\begin{array}{l}\text { Participation, autonomy, self-de- } \\
\text { termination }\end{array}$ \\
\hline 2 & $\begin{array}{l}\text { Pflege, Altenhilfe, Altersheim, Al- } \\
\text { tenheim, Altenhilfeeinrichtun- } \\
\text { gen, Pflegeheim, Pflegeeinrich- } \\
\text { tung, stationär, alte Menschen, äl- } \\
\text { tere Menschen, pflegebedürftig }\end{array}$ & $\begin{array}{l}\text { Care, elder care, nursing home, } \\
\text { home for the elderly, institutional } \\
\text { care, retirement home, old peo- } \\
\text { ples home, ltc, residential care }\end{array}$ \\
\hline 3 & $\begin{array}{l}\text { Empowerment, Demenz, Quar- } \\
\text { tier, Sozialraum, Lebensqualität, } \\
\text { gute Praktiken, Menschenrechte, } \\
\text { Emotionsarbeit }\end{array}$ & $\begin{array}{l}\text { Empowerment, dementia, social } \\
\text { environment, quality of life, dis- } \\
\text { ability, best practise, human } \\
\text { rights }\end{array}$ \\
\hline
\end{tabular}

\subsection{Forschungsstand}

Teilhabe hat sich als wissenschaftliches Konzept und als politisches Schlagwort in den letzten Jahren stark verbreitet. Die Förderung von Teilhabe und Selbstbestimmung ist als übergeordnetes Ziel mit dem SGB IX (2001) in Deutschland eingeführt worden. Mit der Unterzeichnung der UN-Konvention über die Rechte von Menschen mit Behinderungen (UN-BRK) und dem darauf aufbauenden Bundesteilhabegesetz (2016) wurden weitere rechtliche Grundlagen geschaffen, die allen Menschen das Recht auf Teilhabe ermöglichen sollen (u. a. Degener \& Diehl, 2015). Teilhabe hat sich seitdem als Leitprinzip politisch und rechtlich etabliert und erstreckt sich neben der Sozialpolitik auf weitere Politikfelder wie die Arbeitsmarkt-, Bildungs- und Gesundheitspolitik (Brütt et al., 2016; Nullmeier, 2015). Mit der Internationalen Klassifikation der Funktionsfähigkeit, Behinderung und Gesundheit (ICF) der Weltgesundheitsorganisation (WHO) steht seit 2001 zudem ein globales Referenzsystem zur Verfügung. In dem biopsychologischen Modell werden die Folgen von Krankheiten in Bezug auf Körperfunktionen bzw. Körperstrukturen, Aktivitäten und Partizipation (Teilhabe) unter Berücksichtigung der Kontextfaktoren eines Menschen klassifiziert. Die Klassifikation der WHO dient als zentraler Bezugsrahmen für die interdisziplinäre Teilhabeforschung, die darauf abzielt, in allen gesellschaftlichen Bereichen Möglichkeiten und Risiken von Teilhabe zu ergründen (Brütt et al., 2016; Farin, 2012). Disziplinär sind neben der Reha- 
bilitationswissenschaft u. a. auch die Fächer Pflegewissenschaft, Soziale Arbeit, Pädagogik, Soziologie, Psychologie, Medizin und Recht beteiligt (Farin, 2012). Die Mehrzahl der Arbeiten fokussieren auf Menschen mit Behinderung, wenngleich auch speziell Möglichkeiten und Grenzen der Teilhabe von Menschen im hohen Lebensalter untersucht werden. Empirische Forschung zu Teilhabe und Selbstbestimmung im Kontext von stationärer Altenhilfe, das hat die systematische Literaturanalyse ergeben, liegt im nationalen als auch im internationalen Kontext bisher nur wenig vor.

Ein praxistaugliches Modellkonzept zur tatsächlichen Umsetzung von Teilhabe in stationären Einrichtungen, wie es in diesem Projekt geplant war, ist bislang nicht vorhanden, wenngleich unlängst verschiedene Praxisforschungen zur stationären Altenhilfe vorliegen. Beispielsweise befasste sich ein Praxisprojekt des AWO-Bundesverbandes von 2005 bis 2007 mit der verwandten Frage, wie Lebensqualität und Teilhabe von Menschen mit Demenz in der Praxis der stationären Pflege realisiert werden kann. Die Forschenden entwickelten das Rahmenkonzept „Lebensqualität für Menschen mit Demenz" (Dittrich, 2009), welches in unserer Studie, deren Zielgruppe über Demenzerkrankte hinausgeht, berücksichtigt wurde. Im „Modellprojekt zur Messung der Lebensqualität und subjektiven Wohlbefindens im Pflegeheim (MoMeL)“ (2011 bis 2014) wurde beleuchtet, was die Lebensqualität kennzeichnet, und ein Instrument entwickelt, das die Lebensqualität erfassen soll. Selbstbestimmung wurde als eine zentrale Dimension für das Wohlbefinden von Bewohner"innen herausgearbeitet und in dem Instrument entsprechend verankert (Baas et al., 2017). Ein weiteres Projekt, „JointConImprove“, zielt auf die Verbesserung der sozialen Teilhabe von Pflegeheimbewohner"innen mit Gelenkkontrakturen ab (Saal et al., 2018). ${ }^{6}$ Dabei stehen aber insbesondere medizinisch-pflegerische Aspekte der Teilhabeförderung im Vordergrund (siehe auch Bartoszek et al., 2015). Doch drei Forschungsstränge können das Projektvorhaben prinzipiell informieren: (1) Forschung zu den Themenkomplexen Teilhabe, Selbstbestimmung und Autonomie; (2) Forschung zu Teilhabe aus einer Quartiersperspektive; (3) teilhaberelevante Aspekte der Forschung zu Qualitätsmessung und Lebensqualität.

6 Das Projekt befindet sich aktuell in der dritten Phase (Laufzeit September 2017 bis August 2020), in der die Wirksamkeit der entwickelten Intervention evaluiert werden soll. 
Teilhabe, Selbstbestimmung und Autonomie

Das Projekt STAP geht von der Prämisse aus, dass sich Teilhabeförderung in der stationären Altenhilfe an den Wünschen, Bedürfnissen und Ressourcen der Bewohner*innen orientiert. Daher sind präferierte Vorstellungen, die sich in Teilhabewünschen ausdrücken, sowie Äußerungen oder Signale des beispielsweise Rückzugs- oder Ruhebedürfnisses zu erkennen und so weit wie möglich anzunehmen. Zudem ist es für die Frage der selbstbestimmten gesellschaftlichen Teilhabe innerhalb und außerhalb von Einrichtungen von Belang, welche relevanten Wahlmöglichkeiten („choice“) zwischen sozialen Angeboten bestehen, inwiefern individuelle Wünsche und Bedürfnisse der Bewohner*innen überhaupt formalisiert - im Sinne von Mitbestimmung - einbezogen und geäußert werden („voice“) ${ }^{7}$ sowie welche Unterstützungsressourcen für deren Realisierung - insbesondere für Menschen mit kognitiven oder starken physischen Beeinträchtigungen - zur Verfügung gestellt werden können. Damit stehen Teilhabeperspektiven in engem Zusammenhang mit Fragen der Autonomie, Selbstbestimmung und Selbstständigkeit von Bewohner*innen in Einrichtungen stationärer Altenhilfe.

Empirische Forschungen, die sich mit verschiedenen Schwerpunktsetzungen auf Selbständigkeit, Selbstbestimmung und Autonomie in Altenpflegeeinrichtungen beziehen, sind durchaus umfangreich vorhanden (Davies et al., 1997; exemplarisch Kuhlmey \& Tesch-Römer, 2013; Kümpers \& Heusinger, 2012; Schopp et al., 2001). ${ }^{8}$ Gleichwohl werden in den Studien nur vereinzelt und am Rande Aspekte von sozialer Teilhabe und ihrer Förderung thematisiert (Ausnahmen sind Kardorff \& Meschnig, 2009c; sowie aus Menschenrechtsperspektive Aronson \& Mahler, 2016; Fix \& KurzkeMaasmeier, 2009).

Dennoch lassen sich einige Grundsatzfragen und Zielkonflikte aus diesen Studien auf Fragen der Teilhabeförderung übertragen. Autonomie, wörtlich „sich selbst Gesetz gebend“, ist laut Definition der WHO „die Fähigkeit, die für das Alltagsleben notwendigen persönlichen Entscheidungen zu treffen, sie zu kontrollieren und mit ihnen umzugehen, und zwar

7 So sehen Kardoff \& Meschnig (2009a, S. 36) das Vorhandensein von Mitbestimmung („voice“) und die Existenz subjektiv bedeutsamer Wahlmöglichkeiten („choice“) als zentrale Grundvoraussetzungen für Selbstbestimmung an.

8 Auch eine Reihe von internationalen Studien, v. a. aus Schweden und Großbritannien, bezieht sich insbesondere auf ethische Dilemmata in Bezug auf den Umgang mit Patient*innenautonomie in der stationären Pflege (exemplarisch Elander, Drechsler \& Persson, 1993; Mattiasson \& Andersson, 1995). 
im Rahmen der persönlichen Bedürfnisse und Präferenzen“ (WHO, 2002, S. 13). ${ }^{9}$ Divergierende Sichtweisen bestehen hinsichtlich der Voraussetzungen für Autonomie bzw. Teilhabe. Als wesentliche Voraussetzung werden die kognitive Funktionsfähigkeit, die Unabhängigkeit von kontrollierenden Einflüssen sowie die Fähigkeit zu beabsichtigenden Handlungen genannt (Bobbert, 2002). Dieser Ansicht widersprechen - insbesondere mit Blick auf Bewohner*innen von Pflegeheimen - die Autor*innen Wulff et al. (2010), die davon ausgehen, dass eine Graduierung der Selbstbestimmungsmöglichkeiten vorliegt, wie Wunder (2008) es bei Demenzerkrankten beschreibt. Angelehnt an Collopy (1988) wird zwischen Entscheidungs- und Durchführungsautonomie unterschieden. So können zwar Entscheidungen autonom getroffen werden, zur Umsetzung aber die Unterstützung von Dritten notwendig werden.

Auch Garms-Homolová und Theiss (2009) gehen - in ihren Analysen, die dezidiert Teilhabe auch auf alte Menschen mit Pflegebedarf beziehen davon aus, dass individuelle Voraussetzungen den Grad der Teilhabe bestimmen: Kognitive, soziale, physische und sensorische Kompetenzen beeinflussen, in welcher Weise Teilhabe in Anspruch genommen und durchgeführt werden kann. So führen die beiden Autorinnen beispielsweise aus, dass für die Entscheidungsfähigkeit kognitive Kompetenzen, die Wahrnehmung von Spielräumen und die Kenntnisse eigener Fähigkeiten relevant und für die Artikulation von Wünschen Fähigkeiten im Bereich Hören, Sehen und Sprachkenntnisse erforderlich sind. Sie betonen aber, dass Teilkompetenzen eruiert werden können und eine Aktivierung der latenten Potenziale möglich sei, um Einschränkungen zu kompensieren (GarmsHomolová \& Theiss, 2009b, 2009c). Als weitere Voraussetzung wird ein soziales Netz gesehen, das durch Kontakte, Transaktionen und Unterstützung sichert. Zudem können bauliche Veränderungen dazu beitragen, die Umgebung an die Bedürfnisse der Zielgruppen anzupassen (Garms-Homolová \& Theiss, 2009c).

Der formulierte Bedarf an Unterstützung betont die Rolle der Pflegekräfte im besonderen Maße: Pflegende müssen demzufolge abwägen, wie sie die Autonomie der Pflegebedürftigen durch unterstützende Maßnahmen und Berücksichtigung der Wünsche und Bedürfnisse wahren können. Huber et al. (2005) führen Fähigkeiten und Kompetenzen von Pflegekräften auf, die für eine autonomiefördernde Pflege notwendig sind. Unter den Sach- und Methodenkompetenzen werden die Wahrnehmung und Be-

9 Ausführlich zur Begriffsgenese und unterschiedlichen Verständnissen im pflegerischen Kontext z. B. Kümpers \& Zander (2012). 
urteilung von Wünschen sowie die Fähigkeit und Bereitschaft, Verantwortung zu übernehmen, genannt. Unter sozial-kommunikativen und personalen Kompetenzen werden die Kommunikations-, Reflexions- und Konfliktfähigkeit angeführt (Huber et al., 2005, S. 75). Die Herausforderung in der Wahrnehmung von Wünschen und Bedürfnissen zeigt sich in der Studie bei Kardorff und Meschnig (2009c), die in Abgrenzung zu Menschen mit Behinderung keine aktive Artikulation eigener Bedürfnisse der Pflegeheimbewohner*innen festgestellt haben. Vielmehr zeigt sich eine grundsätzliche Dankbarkeit und das Bestreben, sich mit der Situation im Pflegeheim zu arrangieren, die generationsspezifisch, alterstypisch oder aus dem Abhängigkeitsverhältnis dazu führt, dass Wünsche und Bedürfnisse nicht formuliert oder eingefordert werden (Kardorff \& Meschnig, 2009c, S. 266ff.). Dagegen hat die Metastudie von Andresen et al. gezeigt, dass Pflegeheimbewohner*innen ohne kognitive Einschränkungen die Bedeutsamkeit der Entscheidungsautonomie und die Kontrolle über den Alltag hervorheben. Es wird betont, dass es von den Bewohner"innen als besonders wichtig empfunden wird, zu entscheiden, wann, wie und mit wem Aufgaben der täglichen Routine sowie Aktivitäten beruhend auf den intrinsischen Interessen erbracht werden (Andresen et al., o. J., zit. nach Andresen, 2009). Mit dem „Hertz Perceived Enactment of Autonomy Scale“ besteht ferner ein wissenschaftliches Instrumentarium, um das selbst wahrgenommene Autonomieerleben von Pflegeheimbewohner*innen zu erheben (Wulff et al., 2012).

Autonomie entsteht im Zusammenspiel von Selbstbestimmung einerseits und Handlungsfähigkeit und Selbständigkeit andererseits. Sie ist relational zu betrachten und dabei von individuellen wie umwelt- bzw. kontextbezogenen Faktoren abhängig. Methoden zur Verbesserung oder Stabilisierung der Selbstständigkeit in Alltagsaktivitäten haben das Ziel der Rehabilitation pflegebedürftiger Menschen. Ergebnisse und Konzepte der rehabilitativ aktivierenden Pflege liegen im breiten Umfang vor (Ackermann \& Oswald, 2006). In Gerontologie, Pflegewissenschaften und Sozialer Arbeit ist vor allem das Verhältnis von Autonomie, Selbstbestimmung und Selbstständigkeit umstritten: So problematisieren etwa Behrens und Zimmermann (2006) eine zu enge Verkoppelung von Autonomie und Selbstständigkeit. Sie kritisieren die einseitige Ausrichtung pflegewissenschaftlicher Aktivierungskonzepte auf Selbständigkeit. Selbstständigkeit sei demnach nur eine von mehreren potenziellen Ressourcen für Autonomie neben Wissen und Informiertheit, sozialer Kompetenz oder früheren Erfahrungen beispielsweise. Auch könnten kontext- und umweltbezogene Autonomieressourcen fehlende individuelle Ressourcen kompensieren. Auto- 
nomie ist nach diesem Verständnis nicht mit Selbständigkeit gleichzusetzen. Kammerer, Falk und Kümpers (2012) beschreiben, dass Selbständigkeit auch klar von Selbstbestimmung abgegrenzt werden muss. In der Fokusgruppenstudie von Kardorff und Meschnig (2009, S. 278) wird deutlich, dass auch der professionelle Blick von Pflegeleitungskräften und Pflegekräften eher auf Selbstständigkeit gerichtet ist, als auf Selbstbestimmung. Autonomie kann aber eben auch bedeuten, selbstbestimmt darüber zu entscheiden, bestimmte Dinge nicht eigenständig zu übernehmen, etwa weil dies zeitlich zu aufwändig oder mit Schmerzen verbunden wäre (vgl. auch Kümpers \& Zander, 2012). ${ }^{10}$ Selinger et al. (2010) betonen, dass Selbstständigkeit nur ein Weg von mehreren zur selbstbestimmten Teilhabe ist. „Vielmehr stellt sie sich im Falle von Behinderung und Pflegebedürftigkeit häufiger als Rückgewinnung und Wahrung von gewünschter und individuell gefärbter Selbstbestimmung im Sinne von Entscheidungsspielräumen trotz Unterstützungsbedarfen dar" (Selinger et al., 2010, S. 72).

Klie und Krahmer (2009) arbeiten Aspekte heraus, die für eine an Selbstbestimmung und Selbständigkeit orientierte Pflege charakteristisch sind (vgl. auch den zusammenfassenden Literaturüberblick zum Thema Selbstbestimmung in Hasseler et al., 2016, S. 76). Einige dieser Punkte erscheinen uns auch in Bezug auf die Förderung von selbstbestimmter Teilhabe relevant. Dazu zählen: ein Verzicht auf erzieherische Maßnahmen gegenüber Pflegebedürftigen; Berücksichtigung individueller Lebensgewohnheiten, Beachtung lebensweltlicher und kultureller Besonderheiten, insbesondere bei Menschen mit Migrationshintergrund; Planung der Pflege gemeinsam mit den Pflegebedürftigen oder deren Vertrauenspersonen; strenge Beobachtung der gesetzlichen Selbstbestimmungs- und Freiheitsrechte der Pflegebedürftigen; Berücksichtigung von Selbstbestimmung und Selbständigkeit als wesentliche Dimensionen von Ergebnisqualität.

Ein weiteres wichtiges Spannungsfeld, das in der pflegewissenschaftlichen Forschung thematisiert wird, stellt das Verhältnis von Autonomie und Schutz dar. Die Pflegekräfte sind neben dem Prinzip der Selbstbestimmung auch der Vermeidung von Selbst- und Fremdgefährdung verpflichtet (Giesbers, 2016; Kotsch \& Hitzler, 2013). Prozesse der Professionalisierung der Pflege, insbesondere, wenn diese einseitig auf standardisierter Qualitätssicherung und externer Evidenz fußten, gingen mit Gefahren einer

10 Ressourcen und Barrieren für Autonomie trotzen Schmerzen von multimorbiden Pflegeheimbewohner*innen wurden im Forschungsprojekt PAIN untersucht (Dräger et al., 2013). 
Einschränkung von Selbstbestimmungsmöglichkeiten einher (Behrens \& Zimmermann, 2006). In Bezug auf medizinisch-pflegerische Versorgungssituationen wird herausgearbeitet, welche Zielkonflikte ${ }^{11}$ zwischen der Berücksichtigung von Autonomie bzw. Selbstbestimmung und der Sicherstellung der Versorgung entstehen können (Geisler, 2004; Kümpers \& Zander, 2012). Dies gilt insbesondere auch in Bezug auf Bewohner*innen mit demenziellen Erkrankungen (z.B. Fossey et al., 2002; Marshall, 1997; Werner, 2013). Kotsch und Hitzler (2013) heben in ihrer Studie hervor, dass es sich bei den Interaktionen zwischen Pflegekräften und Bewohner*innen weniger um absichtsvolle Bevormundung handelt als um Dilemmata im Umgang mit vermeintlich unselbstständigen Personen, insbesondere wenn die Willensbekundungen uneindeutig werden oder scheinbar ausbleiben.

Als einflussreich hervorzuheben sind an dieser Stelle zudem die sogenannten MUG-Studien (MUG - Möglichkeiten und Grenzen selbstständiger Lebensführung in Einrichtungen), wovon hier nur die letzte Studie (MUG IV) aufgegriffen werden soll. So wurden auch im Rahmen von MUG IV auf repräsentativer Stichprobenbasis etwa Hilfe- und Pflegebedarfe sowie die Wohn-, Lebens- und Versorgungssituation der Bewohner*innen im Heimkontext erfasst. Festgehalten wurde dabei u.a., dass durchaus „beispielhafte Lösungen für die vielfältigen Anforderungen an eine menschenwürdige Gestaltung des Alltags in Pflegeeinrichtungen" identifiziert werden konnten. Gleichermaßen ist aber zu konstatieren, dass Schwierigkeiten und Defizite „weniger in der körperlichen Versorgung als im Bereich der psychosozialen Betreuung und den individuellen Partizipationsmöglichkeiten der Bewohnerinnen und Bewohner sowie den Gestaltungsmöglichkeiten für die Pflegekräfte liegen" (Heusinger \& Knoch, 2007, S. 325). Ein Blick in die internationale Forschung zeigt mit der Studie von Andresen et al. (2009) Effekte von individuell zugeschnittenen Programmen (umfasst physische und soziale Aktivitäten) auf das Autonomieerleben in dänischen Pflegeheimen auf. Zum Vergleich wurde eine Kontrollgruppe gebildet, die an den bestehenden Aktivitäten teilgenommen haben. Es konnte nach 12 bzw. 24 Wochen jedoch kein signifikanter Unterschied im Autonomieerleben zwischen den beiden Gruppen festgestellt werden.

11 Ein Beispiel für einen solchen Zielkonflikt ist die Frage nach einem Recht auf Verwahrlosung für die Bewohner*innen der Einrichtungen. 
Teilhabe aus einer Quartiersperspektive

Im Rahmen von STAP werden Ziele für die Teilhabeförderung in den Blick genommen und definiert, die sich sowohl auf Maßnahmen und Ressourcen innerhalb als auch außerhalb bzw. das Umfeld der Altenpflegeeinrichtungen beziehen. Mit letzterer Perspektive ist somit von Interesse, inwieweit Forschungen zu Ansätzen der Quartiers- bzw. Sozialraumorientierung in der stationären Altenhilfe existieren. Dabei ist zunächst hervorzuheben, dass in der Altenhilfe auf der Ebene von Praxisentwicklung, Konzeption und Strategiepapieren in den letzten Jahren zunehmend Perspektiven des Sozialraums/Quartiers aufgegriffen werden (Bogert, 2013; Vries, 2009; z. B. Deutscher Evangelischer Verband für Altenarbeit und Pflege e. V., 2008; Falk \& Wolter, 2018; Schönberg, 2011). Insbesondere ist hierbei aber auf den durch das Kuratorium Deutscher Altershilfe entwickelten Ansatz der „KDA-Quartiershäuser“ hinzuweisen, der für eine Neuausrichtung von Alten- und Pflegeheimen steht (Michell-Auli \& Sowinski, 2012). Hierbei bleibt das Normalitätsprinzip mit dem Prinzip „Leben in der Gemeinschaft" weiterhin grundlegender Bestandteil, der auch für Ansätze der Teilhabeförderung innerhalb der Einrichtung von zentraler Bedeutung ist. Neben dem weiteren Prinzip „Leben in Privatheit" ist nun quartiersbezogene Teilhabe im Zusammenhang mit dem neuen Prinzip „Leben in der Öffentlichkeit" von Interesse. Hier wird Teilhabe gefördert, indem sich die Pflegeeinrichtungen einerseits stärker - z. B. mit offenen Veranstaltungen - dem „Quartier öffnen“ und andererseits Bewohner*innen gezielter Aktivitäten im Quartier wahrnehmen (Bleck, Rießen, Knopp \& Schlee, 2018; vgl. z. B. Michell-Auli \& Sowinski, 2012, S. 64ff.). ${ }^{12}$

Auch wenn die zunehmende Relevanz von Quartiersperspektiven in Altenpflegeeinrichtungen konzeptionell und programmatisch hinlänglich begründet ist, liegen bislang nur singulär empirische Studien zu quartiersbzw. sozialraumbezogenen Fragestellungen in der stationären Altenhilfe vor. So hat Hämel (2012) anhand qualitativer Fallstudien zivilgesellschaftliche und gemeinschaftliche Ressourcen mit Blick auf Potenziale einer „Heimöffnung“ untersucht. Ferner wurden jüngst durch Bleck et al. 2018, auf Basis von qualitativen und quantitativen Forschungszugängen, in Düsseldorfer Einrichtungen aktuelle Sozialraumbezüge in der stationären Altenhilfe analysiert. Dabei wurden insbesondere Kooperationen im Stadtteil sowie Nutzungen der Einrichtungen durch Stadtteilbewohner*innen so-

12 In der Praxis finden sich Beispiel für eine quartiersbezogene Ausrichtung der stationären Pflegeeinrichtungen (z. B. Benninghoven, 2013). 
wie Nutzungen des Stadtteiles durch Einrichtungsbewohner*innen in den Blick genommen (Bleck, et al., 2018; Bleck, Rießen \& Schlee, 2018). Die konkrete Förderung von selbstbestimmter Teilhabe in Altenhilfeeinrichtungen unter Berücksichtigung des Kontextes Quartier wurde aber bislang nicht empirisch untersucht.

Teilhabe, Qualitätsmessung und Lebensqualität

Dimensionen sozialer Teilhabe sind ebenfalls im Kontext von Ansätzen und Instrumenten zur (Ergebnis-)Qualitätserfassung in der stationären Altenhilfe wiederzufinden (z. B. Bräutigam, 2008; Oswald et al., 2014; Wingenfeld et al., 2011) sowie in verschiedenen Studien, die sich mit Lebensqualität oder (sozialem) Wohlbefinden in stationären Pflegeeinrichtungen befassen (z. B. Bernsteiner \& Boggatz, 2016; Gerritsen et al. 2010; Kane, Pratt \& Schoeneman, 2004; Netten et al., 2010). ${ }^{13}$ Fuchs (2016) stellt allerdings auch fest, dass die Forschung bisher weit überwiegend an die sozialrechtlichen Vorgaben für die Qualitätssicherung der Pflege ( $\$ 115$ SGB XI) anknüpfe, „die bisher als - zu gewährleistendes und zu überprüfendes Kriterium ,Lebensqualität', nicht jedoch die selbstbestimmte Teilhabe vorgibt. Lebensqualität ist auf dem Hintergrund der UN-BRK jedoch nur ein Aspekt der selbstbestimmten Teilhabe am Leben in der Gesellschaft.“

Hasseler et al. (2016) zielen in ihrer Studie auf die Entwicklung eines theoretisch-konzeptionellen Qualitätsverständnisses für die Pflege- und Lebensqualität ab. Dafür wurden eine systematische Literaturrecherche und eine Beschreibung unterschiedlicher Kennzeichen für die Qualität in der pflegerischen Versorgung vorgenommen. Berücksichtigt wurde auch das Merkmal der Teilhabe. So kommen Hasseler et al. (2016, S. 131) zur Einschätzung, dass eine qualitativ hochwertige Teilhabe in Einrichtungen der Altenhilfe und Langzeitpflege insgesamt auf folgenden Punkten beruht:

- „Berücksichtigung des Normalitätsprinzips,

- Verhinderung von Scheinalternativen,

- Herstellung einer symmetrischen Beziehung zwischen den Beteiligten,

- Entwicklung eines individuellen Maßnahmenpaketes (Hilfeplan) auf der Basis der Einschätzung,

- individuelle Einschätzung (Assessment) der Teilhabebedarfe, Ziele,

- individuelles Maßnahmenpaket (Hilfeplan),

13 Für einen ausführlichen Überblick über die vielfältigen Forschungsströmungen zur „Lebensqualität im Alter“ siehe zum Beispiel Weidekamp-Maicher, 2016. 
- aktive Sozialraumgestaltung mit der Möglichkeit der Betroffenen, sich nach eigenen Wünschen und Bedürfnissen am Leben der Gemeinde/des Sozialraumes zu beteiligen,

- Erfüllung alltagspraktischer Bedürfnisse nach individuellen Vorstellungen wie Kommunikation, Mobilität, Wohnen, Bildung, kulturelles Erleben, Kontakte,

- Gewährung von Kontrolle über eigene Entscheidungen,

- Möglichkeiten der Teilnahme an Aktivitäten und Maßnahmen gemäß individueller Vorstellungen und Ressourcen und

- Abbau von Barrieren (bauliche Barrieren, Barrieren im Bereich des Wohnens, der Vorstellungen von Fachkräften etc.).“

Um den Bedarf zu ermitteln wird vorgeschlagen, die Bedarfsermittlung beruhend auf einem Assessment der Teilhabebeeinträchtigung durchzuführen, um auf dieser Grundlage eine Planung der Teilhabeförderung zu entwickeln, die eine Ressourcenorientierung berücksichtigt und die Möglichkeiten zur Selbsthilfe unterstützt (Hasseler, Stemmer, Macsenaere, Arnold \& Weidekamp-Maicher, 2016; Metzler, 2010). Auch der Arbeitskreis „Teilhabeorientierte Pflege“ regt an, dass es eines verbindlichen Einschätzungsverfahrens bedarf, um die individuellen Teilhabe-, Hilfe- und Pflegebedarfe festzustellen (Arbeitskreis Teilhabeorientierte Pflege, o. J.).

Auch Praxisansätze zur Verbesserung von Transparenz und Vergleichbarkeit der Qualität in Altenhilfeeinrichtungen, wie der "Grüne Haken“ (Heimverzeichnis gGmbH, 2017), enthalten ansatzweise Teilhabedimensionen. In diesen Ansätzen steht die Situation der einzelnen Bewohner*innen in der Regel stärker im Vordergrund und es werden etwa Indikatoren zur gesundheits- und versorgungsbezogenen Lage sowie zu würdevollem Umgang, Selbständigkeit, Selbstbestimmung, Tagesgestaltung und sozialen Beziehungen von Bewohner*innen stationärer Altenhilfe aufgegriffen. Bezüge zu Teilhabeperspektiven sind etwa in Bezug auf Indikatoren zur bedürfnisgerechten Beschäftigung und Teilnahme an Aktivitäten von Bewohner*innen (Wingenfeld et al., 2011, S. 186ff. und 194), zur sozialen und gesellschaftlichen Teilhabe (Bräutigam, 2008, S. 16), zu sozialen Kontakten und Beziehungen (Oswald et al., 2014, S. 64ff.) sowie zu „Relationships, Enjoyment, Spiritual well-being, and Meaningful activity“ (Kane et al., 2004, Kapitel 3) vorhanden. Die sogenannte HILDE-Studie (Heidelberger Instrument zur Erfassung der Lebensqualität Demenzkranker) hat ebenfalls ein Instrument zur Erfassung von Lebensqualität entwickelt, dass insbesondere auf demenzerkrankte Heimbewohner*innen ausgerichtet ist (Kruse \& Schröder, 2006). Das Projekt PELI-D will das auf Selbsteinschätzung beruhende Befragungsinstrument PELI den Präferenzen des tägli- 
chen Lebens von alten Menschen für den deutschen Raum erproben und nutzbar machen. ${ }^{14}$ Durch diese Studien liegen somit Indikatoren und Instrumente vor, die standardisiert auch Teilhabeaspekte im Rahmen von Ergebnis- oder Lebensqualität in Altenpflegeinrichtungen erfassen. $\mathrm{Zu}$ den Ressourcen und konkreten Faktoren, die eine Förderung von Teilhabe in der stationären Pflege und Betreuung ermöglichen, treffen sie aber keine Aussagen. ${ }^{15}$ Im Forschungsprojekt kann sehr gut auch an jene Erfahrungen angeschlossen werden, die zurzeit in dem Projekt EQiSA (Ergebnisqualität in der stationären Altenhilfe) durch das Institut für Pflegewissenschaft an der Universität Bielefeld (im Anschluss an die sogenannte „Wingenfeld-Studie“; Wingenfeld et al., 2011) näher erprobt werden.

Die Literaturanalyse hat gezeigt, dass konzeptionelle und empirische Anknüpfungspunkte für die STAP-Studie bestehen, die eine wichtige Grundlage für Teilaspekte bieten. Unter anderem werden Schwierigkeiten bei der Erfassung von Wünschen und Bedarfen benannt, die Kompetenzen und Fähigkeiten von Pflegekräften zur Verwirklichung selbstbestimmter Teilhabe herausarbeiten und die Herausforderungen von Bewohner*innen mit eingeschränkten kognitiven Fähigkeiten thematisiert sowie für Konflikte im Spannungsfeld von Autonomie und Fürsorge sensibilisiert. Bestehende Instrumente, die bestimmte Perspektiven und Teilgruppen in den Blick nehmen, können als Referenz in der Entwicklung des Musterrahmenkonzeptes berücksichtigt werden. Es liegen aber keine empirischen Befunde vor, die umfassende Erkenntnisse über die praktische Verwirklichung von selbstbestimmter Teilhabe in stationären Einrichtungen der Altenhilfe liefern.

\subsection{Selbstbestimmte Teilhabe: rechtlicher und konzeptioneller Rahmen}

Nach dem Überblick zum Forschungsstand und der Beschreibung der Forschungslücke, die im Projekt STAP adressiert wird, werden in diesem Ab-

14 Siehe hierzu die Beschreibung der zum Zeitpunkt der vorliegenden Berichtslegung noch laufenden Studie auf den Internetseiten des DZNE: www.dzne.de/fors chung/studien/projekte-der-versorgungsforschung/peli-d/ (Zugriff 20.12.2019).

15 In einigen internationalen Studien wird „meaningful activity“, also soziale Aktivitäten, die von Bewohner*innen als bedeutsam, interessant und nicht langweilig empfunden werden, stattdessen als unabhängige Variable zur Erklärung der Verringerung von Depressionen Pflegebedürftiger in stationären Einreichungen herangezogen (z. B. Meeks et al., 2007; Meeks \& Looney, 2011). 
schnitt die rechtlichen und begrifflich-konzeptionellen Grundlagen der vorliegenden Studie ausgeführt.

\subsubsection{Rechtliche Verankerung von selbstbestimmter Teilhabe}

Leistungsanbieter*innen sind nach den Bestimmungen der UN-Behindertenrechtskonvention (zentral für die selbstbestimmte Teilhabe sind insbesondere Art. 3. Buchst. a-c ${ }^{16}$, Art. 19 sowie Art. 26 UN-BRK; ausführlich z. B. Degener, 2009; Degener \& Diehl, 2015), dem Alten- und Pflegesetz Nordrhein-Westfalen (APG), dem Wohn- und Teilhabegesetz NordrheinWestfalen (WTG) sowie der Durchführungsverordnung dazu verpflichtet, die Teilhabe der Bewohner*innen von Altenpflegeeinrichtungen am Leben in der Gesellschaft innerhalb und außerhalb der Einrichtungen zu fördern. Dazu gehört Folgendes:

- Bei Planung, Gestaltung und Betrieb bzw. der Ausführung von Angeboten ist darauf hinzuwirken, dass die Bestimmungen des Gesetzes zu dem Übereinkommen der Vereinten Nationen vom 13. Dezember 2006 über die Rechte von Menschen mit Behinderungen (UN-Behindertenrechtskonvention, UN-BRK) berücksichtigt werden ( $\$ 2$ Abs. 2 APG NRW). Schon bei der Planung von Angeboten sind übergreifende Aspekte der Teilhabe zur Sicherung eines würdevollen, inklusiven und selbstbestimmten Lebens einzubeziehen ( $\$ 7$ Abs. 3 Satz 3 APG NRW).

- Die UN-Behindertenrechtskonvention erwartet danach von den Leistungsanbieter*innen geeignete Maßnahmen, um die volle Einbeziehung aller Bewohner*innen von Altenhilfeeinrichtungen in die Teilhabe an der Gesellschaft zu erleichtern (Art. 19 Satz 1 UN-BRK). Sie sollen durch wirksame und geeignete Maßnahmen in die Lage versetzt werden, ein Höchstmaß an Unabhängigkeit, umfassende körperliche, geistige und soziale Fähigkeiten sowie die volle Einbeziehung in alle Aspekte des Lebens und die volle Teilhabe an allen Aspekten des Lebens zu erreichen und zu bewahren (Art. 26 Abs. 1 Satz 1 UN-BRK). Zudem sollen auch bei älteren Menschen weitere Behinderungen

16 Dieser lautet: „Die Grundsätze dieses Übereinkommens sind: a. die Achtung der dem Menschen innewohnenden Würde, seiner individuellen Autonomie, einschließlich der Freiheit, eigene Entscheidungen zu treffen, sowie seiner Unabhängigkeit; b. die Nichtdiskriminierung; c. die volle und wirksame Teilhabe an der Gesellschaft und Einbeziehung in die Gesellschaft.“ 
durch entsprechende Leistungen möglichst gering gehalten oder vermieden werden (Art. 25 Buchst. b UN-BRK).

- Die Leistungsanbieter"innen haben ihre Leistungserbringung auf die Förderung der Teilhabemöglichkeiten auszurichten. Sie sollen den Menschen, die Angebote nach diesem Gesetz nutzen, eine angemessene und individuelle Lebensgestaltung, insbesondere durch die gleichberechtigte Teilhabe am gemeinschaftlichen kulturellen Leben in der Gesellschaft, ermöglichen ( $\$ 1$ Abs. 3 WTG NRW).

- Vom WTG erfasste Wohnangebote sollen in räumlicher Anbindung an Wohnsiedlungen errichtet werden und so gelegen sein, dass den Nutzerinnen und Nutzern eine Teilhabe am Leben in der örtlichen Gemeinschaft möglich ist ( $\$ 4$ Abs. 6 WTG NRW).

- Die Leistungsanbieter*innen haben im Rahmen ihrer jeweiligen Leistungsvereinbarungen die gleichberechtigte Teilhabe der Nutzerinnen und Nutzer am Leben in der Gesellschaft zu unterstützen und zu fördern ( $\$ 5$ WTG NRW). Der Sicherung der Selbstbestimmung und gleichberechtigten Teilhabe der Nutzerinnen und Nutzer am Leben in der Gesellschaft dient nach $\$ 5$ WTG NRW unter anderem

- die Einbeziehung von Angehörigen und anderen Bezugspersonen sowie bürgerschaftlich Engagierten in das von dem jeweiligen Angebot unterstützte Alltagsleben sowie die Öffnung der Angebote für Kooperationen und Veranstaltungen mit externen Institutionen, Vereinen und anderen Stellen;

- zielgruppenbezogene Betätigungen anzubieten, die die Fertigkeiten der Nutzerinnen und Nutzer in alltagsnahen und gewohnten Handlungen zur Geltung bringen;

- in Zusammenarbeit mit geeigneten Einrichtungen und Organisationen regelmäßig über Veranstaltungen und Aktivitäten im Quartier $\mathrm{zu}$ informieren und die Teilnahme daran zu unterstützen und zu fördern und

- die Wahrnehmung auswärtiger Termine zu unterstützen und zu fördern.

- Die Leistungsanbieter*innen müssen ein Qualitätsmanagement betreiben, das u. a. eine Beschreibung der Kernprozesse des Betriebes umfasst ( $\$ 4$ Abs. 3 Nr. 5 WTG NRW). Zu diesen Kernprozessen zählt mit Blick auf die sich aus der UN-Behindertenrechtskonvention, insbesondere

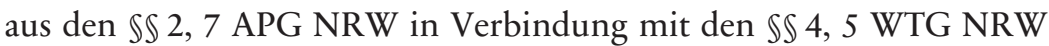
ergebenden Pflichten die Förderung der Selbstbestimmung und gleichberechtigten Teilhabe am Leben in der Gesellschaft. 
Die Bedeutung dieser Vorgaben wird zudem durch die Verpflichtung der Leistungsanbieter*innen zur Fortbildung der Beschäftigten über die Grundsätze der UN-Behindertenrechtskonvention ( $\$ 3$ Abs. 2 WTG DVO) wie auch durch die Beteiligungs- ( $\$ 10 \mathrm{Nr}$.7 WTG DVO) und Mitwirkungsrechte ( $\$ 12$ Abs. 1 Nr. 8 WTG DVO) des Einrichtungsbeirates bei den Maßnahmen zur sozialen Betreuung und Teilhabe am Leben in der Gemeinschaft verstärkt. Allein die Anzahl der zu beachtenden Regelungen und die Zahl der darin verankerten konkreten Förderaspekte belegt das Erfordernis eines übersichtlichen, am Alltag in den Einrichtungen und den verfügbaren Ressourcen orientierten Musterhandlungsrahmens, welcher die Umsetzung von Teilhabe in der Praxis erleichtert.

Im Sozialrecht wird der Begriff Teilhabe v. a. mit dem Sozialgesetzbuch (SGB) IX verbunden, das mit dem Titel „Rehabilitation und Teilhabe von Menschen mit Behinderungen " überschrieben ist. Teilhabe ist ein Element des modernen Behinderungsbegriffs der ICF, in welchem Behinderung als ein Zustand charakterisiert wird, der durch ein Defizit an Teilhabe am Leben in der Gesellschaft geprägt ist (Welti, 2005, S. 537, 753). Die Bedeutung von Teilhabe wird im SGB IX somit mittelbar über den Bezug zur ICF deutlich:

„Der Begriff Teilhabe ist über die Internationale Klassifikation der Funktionsfähigkeit, Behinderung und Gesundheit (ICF) der Weltgesundheitsorganisation als internationaler Standard des gesundheitswissenschaftlichen Behinderungsbegriffs und über die Ausformung des von den Grundrechten geprägten Sozialstaats im 9. Sozialgesetzbuch definiert" (Fuchs, 2009, S. 21).

Dabei stehen neun Bereiche der Teilhabe im Zentrum der ICF: Lernen und Wissensanwendung; allgemeine Aufgaben und Anforderungen; Kommunikation; Mobilität; Selbstversorgung; häusliches Leben; interpersonelle Interaktion und Beziehungen; bedeutende Lebensbereiche sowie Gemeinschafts-, soziales und staatsbürgerliches Leben. Deutschland orientiert mit dem SGB IX sein nationales Sozialrecht an der ICF, in deren Rahmen Teilhabe die Übersetzung von „participation“ darstellt, welche wiederum als ,involvement in a live situation“, also die Gesamtheit der sozialen Umweltbeziehungen von Menschen, verstanden wird (ebd.). 
Im SGB XI, welches die soziale Pflegeversicherung regelt, wird der Teilhabebegriff nicht expliziert. ${ }^{17}$ In $\$ 2$ SGB XI wird allerdings ein Anspruch der Leistungsberechtigten auf Selbstbestimmung geregelt, wenngleich dieser nicht den Anforderungen der UN-BRK entspricht. Dort heißt es:

„(1) Die Leistungen der Pflegeversicherung sollen den Pflegebedürftigen helfen, trotz ihres Hilfebedarfs ein möglichst selbständiges und selbstbestimmtes Leben zu führen, das der Würde des Menschen entspricht. Die Hilfen sind darauf auszurichten, die körperlichen, geistigen und seelischen Kräfte der Pflegebedürftigen, auch in Form der aktivierenden Pflege, wiederzugewinnen oder zu erhalten.

(2) Die Pflegebedürftigen können zwischen Einrichtungen und Diensten verschiedener Träger wählen. Ihren Wünschen zur Gestaltung der Hilfe soll, soweit sie angemessen sind, im Rahmen des Leistungsrechts entsprochen werden. Wünsche der Pflegebedürftigen nach gleichgeschlechtlicher Pflege haben nach Möglichkeit Berücksichtigung zu finden.

(3) Auf die religiösen Bedürfnisse der Pflegebedürftigen ist Rücksicht zu nehmen. Auf ihren Wunsch hin sollen sie stationäre Leistungen in einer Einrichtung erhalten, in der sie durch Geistliche ihres Bekenntnisses betreut werden können.

(4) Die Pflegebedürftigen sind auf die Rechte nach den Absätzen 2 und 3 hinzuweisen.“

Die Begriffe Selbstbestimmung und Selbständigkeit werden dabei zwar unterschieden, aber nicht näher umrissen, was das in Abschnitt 2.2. bereits angesprochene Problem verstärkt, dass die Begriffe in der Literatur zum Teil synonym verwendet werden (Hasseler, 2016, S. 74).

\subsubsection{Konzeption selbstbestimmter Teilhabe in STAP}

Während sich aus den beschriebenen Rechtsgrundlagen vergleichsweise klare Anforderungen ergeben, dass selbstbestimmte Teilhabe für die Bewohner*innen von Altenpflegeeinrichtungen zu ermöglichen ist, ist die Frage, was darunter genau zu verstehen ist, rechtlich nicht eindeutig ge-

17 Dies macht die Sichtweise umso bedeutsamer, dass pflegebedürftige Menschen zugleich als behinderte Menschen im Sinne des SGB IX mit dem Recht auf Zugang zu Teilhabeleistungen zu betrachten sind (ausführlich Fuchs 2009, Kapitel 1.6). 
klärt. Auch in der wissenschaftlichen Diskussion sind die Konzeptionen von Teilhabe und Selbstbestimmung, sogar innerhalb der einzelnen in Abschnitt 2.2. bereits vorgestellten disziplinären Zugänge, höchst vielfältig und teils umstritten (für ausführliche Überblicksdarstellungen vgl. Kardoff \& Meschnig, 2009b; Garms-Homolová \& Theiss, 2009; Hasseler, Stemmer, Macsenaere, Arnold \& Weidekamp-Maicher, 2016, Kapitel 7 und 13).

Im Rahmen von STAP legen wir ein menschenrechtsbasiertes Verständnis von Teilhabe zugrunde, wie es auch in der UN-BRK zum Ausdruck kommt (vgl. z. B. auch Degener, 2009). Aufbauend auf das Verständnis von „participation" der UN-BRK sowie Fuchs (2009, S. 20f) verstehen wir Teilhabe als den gleichberechtigten Zugang zu und Mitbestimmung über soziale Umweltbeziehungen nach den Wünschen einer Person zur persönlichen Daseinsentfaltung. Soziale Umweltbeziehungen fassen wir dabei bewusst weit, so dass sie die neun ICF-Bereiche, die oben bereits beschrieben wurden, umfassen und sich ebenfalls auf verschiedene Stufen der sozialen Teilhabe alter Menschen mit Hilfe- und Pflegebedarf beziehen können (z. B. Kümpers \& Wolter, 2015, S. 137). Die Bandbreite möglicher teilhaberelevanter Aspekte in stationären Einrichtungen ist in unserem Verständnis von selbstbestimmter Teilhabe also weit gefächert und kann unterschiedliche Aktionsradien umfassen. Sie kann etwa von der selbstbestimmten Handlungswahl bei teilhaberelevanten Aspekten der Lebensführung im Nahraum der Einrichtung oder des eigenen Zimmers (z. B. Öffnen und Schließen von Türen, Zeit und Ort der Mahlzeiteneinnahme) bis hin zur regelmäßigen Wahrnehmung subjektiv bedeutsamer Wahlangebote und Aktivitäten außerhalb der Einrichtung (z. B. Ausflüge oder die Teilnahme an einem kommunalen Gremium) reichen. ${ }^{18}$

In Bezug auf die Übersetzung des Begriffs „participation“ mit Teilhabe wird häufig kritisiert, dass „Teilhabe“ auf eine bloße „Teilnahme“ verkürzt zu werden drohe und der Aspekt der Mitbestimmung verloren gehe (ausführlich zum Begriff der Partizipation vgl. Kardoff, 2014). In unserem Verständnis betonen wir daher ausdrücklich den Mitbestimmungsaspekt.

18 Mit Bezug auf die landesrechtlichen Grundlagen in Nordrhein-Westfalen wurde in der vorliegenden Studie in den untersuchungsleitenden Forschungsfragen der im Wohn- und Teilhabegesetz NRW verwendete Begriff der gesellschaftlichen Teilhabe genutzt. Entsprechend den hier vorgenommenen Ausführungen gehen wir in STAP grundsätzlich von einem weiten Teilhabeverständnis aus, bei dem etwa soziale und gesellschaftliche Teilhabe nicht differenziert, sondern synonym in unserem Sinne der Definition von selbstbestimmter Teilhabe betrachtet werden. 
Selbstbestimmung betrachten wir als ein untrennbar mit dem Grundsatz der Teilhabe in Verbindung stehendes Prinzip, nach dem die Teilhabe gestaltet wird. Selbstbestimmung ist „die reale Möglichkeit, mit eigener Stimme $\mathrm{zu}$ sprechen, Freiheiten auszuüben und Entscheidungen $\mathrm{zu}$ treffen" (Fuchs, 2009, S. 20, im Original ohne Hervorhebung), denn gerade für pflegebedürftige Menschen ist nicht nur die rechtliche Freiheit im Sinne negativer Freiheitsrechte und der „Abwesenheit institutionalisierter Zwänge und bevormundender Fachlichkeit" (Steiner, 1999, zit. in Kardoff \& Meschnig, 2009b, S. 75) zentral, sondern es sind insbesondere die tatsächlichen Voraussetzungen zur Wahrnehmung von Freiheiten im Sinne positiver Freiheitsrechte von Belang. Selbstbestimmung ist mit Heusinger und Falk (2011, S. 624) von der Selbständigkeit klar abzugrenzen: „Wenn ich etwas nicht mehr selbständig tun kann, kann ich doch selbstbestimmt darüber entscheiden, wer dies wann und wie für mich tut" und beinhaltet insbesondere auch das Recht zur Unselbständigkeit sowie zur Nichtteilhabe.

Nach Kardorff und Meschnig (2009b, S. 75) sind zwei Voraussetzungen zentral für die Selbstbestimmung im Kontext pflege- und hilfebedürftiger Menschen: (1) dass wirkliche, für die betroffenen Individuen auch bedeutungsvolle Alternativen vorhanden sind; und (2) dass diese auch die kognitive Kompetenz mitbringen, zwischen Alternativen entscheiden und dies kommunizieren zu können - „egal auf welche Weise“. Der Nachsatz der Autoren weist bereits auf das Spannungsfeld hin, dass kognitive Beeinträchtigungen in der stationären Altenhilfe zunehmen, was die Ermöglichung von Selbstbestimmung erschwert. Wir argumentieren jedoch auf Basis von Forschungen zur Kommunikation mit demenzerkrankten Menschen, dass Einrichtungen, die den Grundsatz selbstbestimmter Teilhabe umsetzen wollen, Willens- und Befindlichkeitsäußerungen auch kognitiv beeinträchtigter Menschen ernst nehmen können und dabei gefordert sind, besonders gute Rahmenbedingungen für die Kommunikation und das wechselseitige Verständnis zwischen Bewohner*in und Einrichtungspersonal zu schaffen.

Aus dem menschenrechtsbasierten Verständnis von Teilhabe wird häufig auch abgeleitet, dass das Normalisierungsprinzip auf alle Lebensbereiche von Menschen mit Behinderung und/oder Pflegebedarf angewandt werden soll. Zurückgehend auf Ideen des Dänen Niels Erik Bank-Mikkelsen aus den 1950er und 1970er Jahren in Bezug auf das Leben von Menschen mit geistiger Behinderung versteht man unter Normalisierung, dass Menschen mit Behinderungen oder Pflegebedarf ein Leben führen, das „so normal wie möglich" gestaltet ist und somit weitgehend dem von nicht be- 
einträchtigten Menschen entspricht (ausführlich zu Historie und auch Kritik des Ansatzes Thimm, 2005).

Für unser Verständnis von Teilhabe ist jedoch insbesondere das Normalitätsprinzip, verstanden als Möglichkeit zur Gestaltung des Lebens nach den in der eigenen Biografie angelegten, gewohnten Normen und Routinen, zentral. Anders als beim Normalisierungsprinzip ist der Maßstab für die Normalität hier weniger eine abstrakte gesellschaftliche Norm vermeintlich nicht beeinträchtigter Menschen, die auch die Gefahr der Stigmatisierung einer Gruppe als abweichend mit sich bringt, als vielmehr die Gewohnheiten und Wünschen, die aus der individuellen biografischen Erfahrung vor dem Heimeintritt stammen.

Im Rahmen des psychobiografischen Pflegemodells prägte Erwin Böhm (1999) den Begriff Normalitätsprinzip. Böhm geht davon aus, dass jeder Mensch auf Grund seiner Sozialisation, Kultur und Erfahrungen eine persönliche Lebensform entwickelt, aus der sich sein Verständnis von einem „normalen“ Handeln ergibt. Menschen mit Demenz greifen auf diese früheren Handlungsweisen zurück - dafür will Böhm sensibilisieren. Auch unabhängig von einer Demenzerkrankung kann die im Lebensverlauf entwickelte „Normalität“ vor dem Heimeintritt ein wichtiger Referenzpunkt für die Verwirklichung selbstbestimmter Teilhabe in der stationären Altenhilfe sein. Die Möglichkeit zur Aufrechterhaltung von Gewohnheiten im Tagesablauf (z. B. spätes Aufstehen, abendliche Aktivitäten) oder in sozialen Beziehungen (z. B. Fortsetzung gewohnter Besuche von Menschen oder Orte/Veranstaltungen außerhalb der Einrichtung), auch wenn sie nicht im Einklang mit den Einrichtungsroutinen steht, stellt hierbei eine wichtige Norm dar. Bei beiden Perspektiven, dem Normalisierungs- und dem Normalitätsprinzip, stehen nicht die Bedarfe der Institution, sondern die der alten, hilfe- und pflegebedürftigen Menschen im Vordergrund. Die Orientierung an dem Normalitätsprinzip kann als Maßstab für den Bedarf an Förderung der selbstbestimmten Teilhabe dienen und auch auf die Differenz hinweisen, zwischen dem, was ein nicht pflegebedürftiger Mensch tun möchte und kann, im Verhältnis zu dem, was ein pflegebedürftiger Mensch tun möchte und (nur) noch tun kann.

Die ICF sensibilisiert uns dafür, dass der Grad der Behinderung von Menschen - und damit auch der Grad ihrer Teilhabe am gesellschaftlichen Leben - vom Zusammenspiel personenbezogener und umweltbezogener Faktoren abhängt. Mit Garms-Homolová und Theiss (2009b, S. 121ff.) lassen sich vier Ebenen von selbstbestimmter Teilhabe unterscheiden: (1) die Ebene des Individuums, (2) die Ebene der Mikroumwelt, wie etwa Angehörige oder andere soziale Beziehungen und Netzwerke im Nahraum, (3) 
die Ebene des Versorgungssettings der Einrichtung sowie (4) die Ebene der Systeme der sozialen Sicherung und der Gesamtgesellschaft. Basierend auf den vorhandenen Erkenntnissen in der Literatur (vgl. Abschnitt 2.2) sowie auf eigenen Überlegungen gehen wir davon aus, dass entlang dieser vier Ebenen die folgenden in Tabelle 2 zusammengestellten Faktoren für die Verwirklichung selbstbestimmter Teilhabe ausschlaggebend sein können. Dieses Modell ist im Sinne eines „sensibilisierende[n] Konzeptes“ (Blumer, 1954; Denzin, 1970) zu verstehen, das dazu genutzt wurde, die qualitative Analyse anzuregen, ohne die für den qualitativen Forschungsprozess zwingend notwendige Offenheit für das empirische Material zu verlieren (ausführlich zum methodischen Vorgehen vgl. auch Kapitel 3). Wichtig ist dabei, dass dies nicht als starres, sondern dynamisches Modell zu betrachten ist, bei dem Wechselwirkungen zwischen den Bereichen sowie Lern- und Anpassungsprozesse der Bewohner*innen über Zeit zu beachten sind.

Tabelle 2: Einflussfaktoren auf selbstbestimmte Teilhabe in Altenpflegeeinrichtungen

\begin{tabular}{|c|c|}
\hline $\begin{array}{l}\text { Individuelle Ebene der } \\
\text { Bewohner*innen }\end{array}$ & Ebene der Mikroumwelt \\
\hline $\begin{array}{l}\text { Personenbezogene Faktoren, insbe- } \\
\text { sondere } \\
\text { Gesundheit, Behinderung, Pflege- } \\
\text { bedürftigkeit } \\
\text { Resilienz } \\
\text { Persönlichkeit und Überzeugungen } \\
\text { Biografie (v. a. Bildung und Beruf) } \\
\text { Milieu, Lebensformen, Lebensstile, } \\
\text { Erwartungen } \\
\text { Einkommen, finanzieller Status } \\
\text { Geschlecht } \\
\text { Ethnizität } \\
\text { Generation } \\
\text { Individuelle Wünsche und deren } \\
\text { Einforderung }\end{array}$ & $\begin{array}{l}\text { Familiäre Beziehungen } \\
\text { Angehörige } \\
\text { Familiäre Konflikte } \\
\text { Soziale Netzwerkeinbindung, z. B. } \\
\text { Freunde } \\
\text { Bekannte } \\
\text { andere Bewohner*innen } \\
\text { ehrenamtliche Hilfe }\end{array}$ \\
\hline
\end{tabular}




\begin{tabular}{|c|c|}
\hline $\begin{array}{l}\text { Ebene der Dienstleistung und } \\
\text { Versorgung }\end{array}$ & $\begin{array}{l}\text { Ebene der gesellschaftlichen } \\
\text { Umwelt und der Systeme sozialer } \\
\text { Sicherung }\end{array}$ \\
\hline $\begin{array}{l}\text { Personenbezogene Faktoren auf der } \\
\text { Ebene der Mitarbeiter*innen, z. B. } \\
\text { Haltung } \\
\text { Empathie } \\
\text { Qualifikation } \\
\text { Quartierswissen } \\
\text { Biografiewissen } \\
\text { Institutionelle Faktoren auf der } \\
\text { Ebene der Einrichtung, z. B. } \\
\text { Ressourcen, insbesondere Personal- } \\
\text { situation und Räume } \\
\text { Konzept } \\
\text { Leitung } \\
\text { Kooperation } \\
\text { Planung und Dokumentation } \\
\text { Einrichtungskultur } \\
\text { Organisatorische Abläufe } \\
\text { Partizipationsmöglichkeiten }\end{array}$ & $\begin{array}{l}\text { Gegebenheiten außerhalb der Ein- } \\
\text { richtung/ des Quartiers } \\
\text { Rechte und Ressourcen des sozialen } \\
\text { Sicherungssystems } \\
\text { Arbeitskreise und Verbände } \\
\text { Gesellschaftliche Normen und Dis- } \\
\text { kurse }\end{array}$ \\
\hline
\end{tabular}

Quelle: eigene Darstellung nach Garms-Homolová \& Theiss (2009b, S. 121ff.) und Hasseler et al. (2016, S. 126) 


\section{Forschungsdesign}

In diesem Kapitel wird das Forschungsdesign der STAP-Studie erläutert, die durch einen sequenziellen Mixed-Methods-Ansatz gekennzeichnet ist. Entsprechend der Chronologie der durchgeführten Forschungssequenzen wird zunächst das methodische Vorgehen der qualitativen und dann dasjenige der darauf aufbauenden quantitativen Analyse vorgestellt, indem jeweils auf die Sampling- bzw. Stichprobenstrategie sowie die Erhebungsund Auswertungsmethodik eingegangen wird.

\subsection{Aufbau: Sequenzieller Mixed-Methods-Ansatz}

Ziel des Projekts war die Erarbeitung eines musterhaften Rahmenkonzepts zur Förderung einer selbstbestimmten, gleichberechtigten gesellschaftlichen Teilhabe pflegebedürftiger Bewohner*innen von Altenpflegeeinrichtungen auf empirischer Basis. Die Erhebungs- und Auswertungsstrategie folgte daher dem Ziel, möglichst eng an der sozialen Wirklichkeit einer Altenpflegeeinrichtung förderliche und hemmende Faktoren selbstbestimmter Teilhabe sowohl qualitativ zu entdecken als auch quantitativ zu verallgemeinern und bewerten.

Ein solches Vorhaben bedingt die Auswahl eines deskriptiven Ansatzes, als Zustands- und Prozessanalyse mit starkem Gegenwartsbezug zum Ablauf von aktuellen Geschehnissen. Daher liegt der Fokus auf Prozessen und die Organisation der selbstbestimmten Teilhabe sowie deren Einordnung durch die Befragten in den Strukturen der Altenpflegeeinrichtungen.

Als geeignete Methoden der Datengewinnung standen hierfür $(A)$ Interviews und ergänzend Beobachtungen sowie Gruppendiskussionen im Rahmen der qualitativen Analyse im Vordergrund, um damit explorativ, offen und möglichst praxisnah die Wissensinhalte und Deutungen der interviewten Personen identifizieren bzw. die Handlungspraxis in den Altenpflegeeinrichtungen erfassen zu können. Im zweiten Schritt diente eine quantitative Folgestudie $(B)$ dazu, zentrale Ergebnisse der qualitativen Analyse anhand einer umfangreicheren Stichprobe mit einer standardisierten Onlinebefragung zu verallgemeinern und bewerten. Somit wurden die Daten auf Basis eines sequenziell aufgebauten Mixed-Methods-Forschungsprozesses erho- 


\section{Forschungsdesign}

ben und ausgewertet, wie aus dem folgenden Schema hervorgeht und anschließend näher erläutert wird.

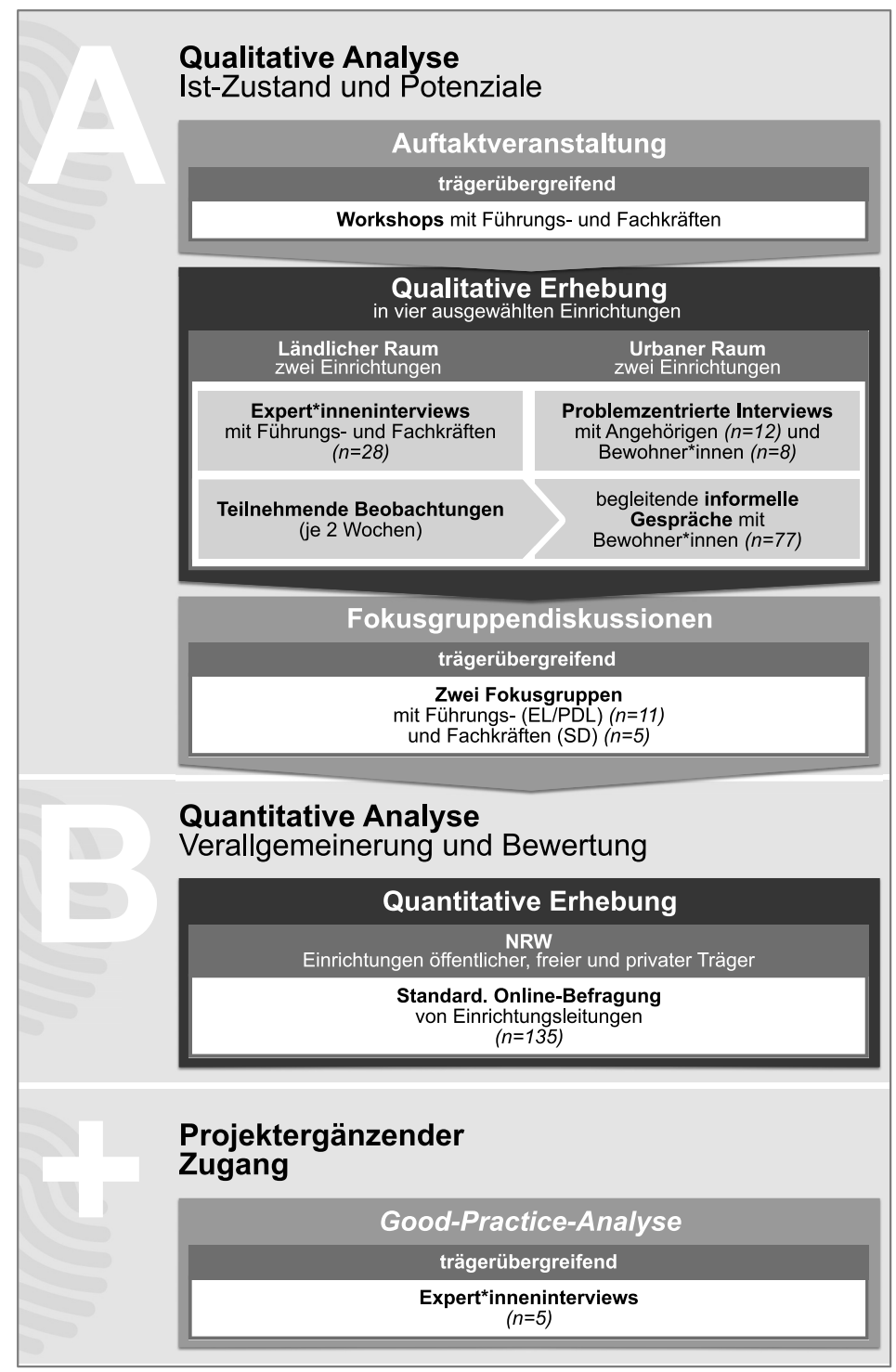

Abbildung 1: Überblick über den Aufbau der Studie 
Abbildung 1 zeigt also im Überblick die einzelnen Erhebungsmethoden sowie die jeweils berücksichtigte Personengruppe und -anzahl im Rahmen der gestuft und aufeinander aufbauenden qualitativen und quantitativen Analysezugänge. Im Sinne des Verallgemeinerungsmodells nach Mayring wurden so ausgewählte Ergebnisse der qualitativen Studie - hier zum IstStand und den Potenzialen zur Organisation selbstbestimmter Teilhabe hinsichtlich ihrer Übertragbarkeit auf andere Einrichtungen analysiert sowie um eine Bewertung von identifizierten Faktoren ergänzt.

Innerhalb der qualitativen Erhebungsphase wurden die Methoden Einzelinterview, Beobachtung und Gruppendiskussion zudem informierend zur gegenseitigen Ergänzung genutzt. Dabei sollte die Varianz der Feldzugänge (Auswahl über „Gatekeeper“, eigene Ansprache, „Convenience“), Methoden (individuell-kollektiv, unterschiedliche Standardisierungsgrade) und Datensorten der Erhebung (visuell und kommunikativ) dazu führen, dass die gewonnenen Ergebnisse miteinander konvergieren und sich anreichernd ergänzen. Die Ergebnisse aus den Interviews, der Gruppendiskussion und Beobachtung wurden aber explizit nicht in ein kongruentes Verhältnis gesetzt. Vielmehr näherten sie sich an, da beispielsweise die Analyse von beobachten Phänomenen ebenfalls in den Interviews verbalisiert und kodiert wurden. Sie werden aber wegen der unterschiedlichen Datenquellen nicht als deckungsgleich bewertet.

Im Folgenden wird der zeitliche Ablauf der wissenschaftlichen Analysen in STAP dargestellt. Die realisierte Durchführung der einzelnen Forschungsphasen wich von der in der Antragstellung anvisierten Zeitplanung nur in Bezug auf den Beginn der quantitativen Erhebung leicht ab.

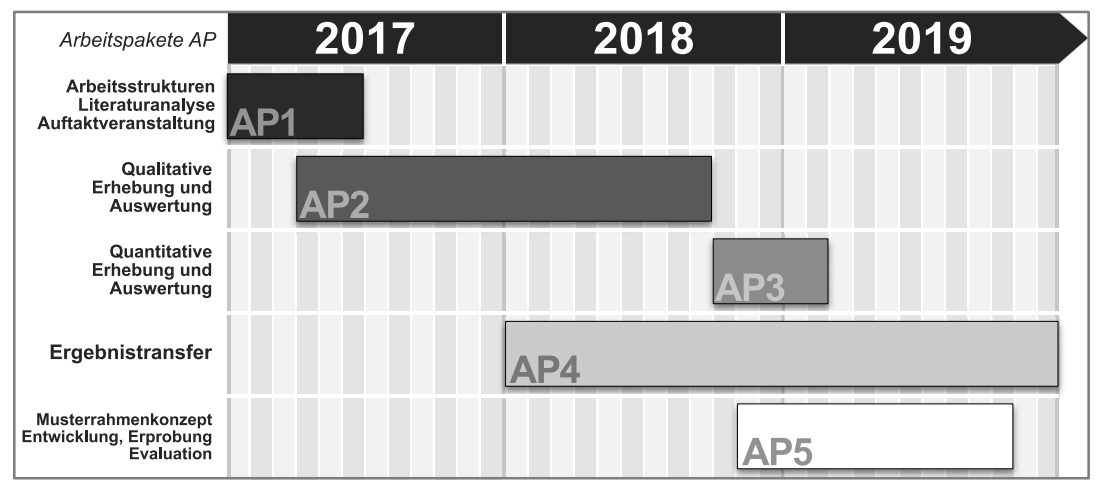

Abbildung 2: Zeitlicher Ablaufplan 


\section{Forschungsdesign}

Aufgrund der ausdrücklich anwendungsorientierten Zielsetzung von STAP, ein Musterrahmenkonzept zu entwickeln, sollte der Forschungsprozess möglichst eng mit den Akteur*innen im Feld verbunden erfolgen. So wurden nicht nur im Feld aktuelle Expertise und Einschätzungen über die Interviewpersonenzugänge zu Mitarbeiter*innen, Angehörigen und Bewohner*innen eingeholt, sondern auch verschiedene Gremien und Veranstaltungen dazu genutzt, die Akzeptanz für ein zukünftiges Musterrahmenkonzept und dessen empirischen Fundierung über die hier vorgestellte STAP-Studie zu prüfen und abzustimmen. Dies wurde über verschiedene Austauschprozesse im Rahmen von folgenden Gremien und Veranstaltungen erreicht:

- Trägerübergreifende Workshops in der Auftaktveranstaltung: Vorstellung und offene Diskussion von Projektfragen in moderierten Untergruppen

- Projektrat der an der Studie beteiligen Einrichtungen: Vorstellung und Abstimmung von Fragen und Vorgehensweisen im Projekt sowie Forschungsergebnissen

- Interdisziplinär zusammengesetzter Wissenschaftlicher Beirat: Vorstellung und Abstimmung von methodischen Vorgehensweisen und Forschungsergebnissen

- Lernworkshops in beteiligten Einrichtungen: strukturierte Erläuterung, Diskussion und Validierung von Forschungsergebnissen

- Institutionsübergreifende und überregionale Bewohner"innenbeiratstreffen: Vorstellung und Diskussion von Forschungsergebnissen

- Trägerübergreifende Fokusgruppen: strukturierte Diskussion von Projektfragen und Impulsen aus Forschungsergebnissen

- Teilnabme an Fachkonferenzen: Vorstellung und Diskussion von methodischen Vorgehensweisen und Forschungsergebnissen

\subsection{Qualitative Analyse}

Im folgenden Kapitel werden die empirischen Zugänge und das methodische Vorgehen im Rahmen der qualitativen Analyse der STAP-Studie vorgestellt. Hier wird zunächst auf die umfassenden Erhebungen in vier Projekteinrichtungen des Diözesan-Caritasverbandes für das Erzbistum Köln e. V. eingegangen, die den Schwerpunkt der qualitativen Analyse darstellen. Danach wird der chronologisch daran anschließende Zugang der trägerübergreifenden Fokusgruppendiskussionen erläutert. Abschließend wird auf eine projektergänzende „Good-Practice“-Analyse eingegangen, 
die außerhalb der regulären Projektstruktur stattgefunden hat, aber durch STAP angeregt und von STAP als ergänzende Datenquelle genutzt wurde.

\subsubsection{Erhebungen in vier ausgewählten Einrichtungen}

Im Zentrum der qualitativen Analyse standen Erhebungen in vier ausgewählten stationären Altenpflegeeinrichtungen des Caritasverbandes. Bei diesen handelt es sich um das Altenzentrum St. Martin Düsseldorf, das Altenzentrum Helenenstift Hennef, das Franziskusheim Wipperfürth und das St. Josefshaus Refrath. Zum Schutze der zugesicherten Anonymität von Mitarbeiter*innen, Bewohner*innen und Angehörigen werden die weiteren Aussagen, die sich auf die Einrichtungen beziehen, nicht mehr diesen Einrichtungen namentlich zugeordnet. ${ }^{19}$

Im Folgenden werden nun die Auswahl und Merkmale dieser vier Projekteinrichtungen ebenso wie die Erhebungs- und Auswertungsmethoden innerhalb dieses Parts der qualitativen Analyse detaillierter vorgestellt.

\subsubsection{Samplingstrategie}

Als Projekt des Diözesan-Caritasverbandes für das Erzbistum Köln e. V. bezog sich die Auswahl der Einrichtungen zunächst - im Schwerpunkt der qualitativen Analyse - auf verbandsinterne Einrichtungen. Durch die gewählten Feldzugänge und Erhebungsmethoden, die explizit im Alltag der Einrichtungen verankert und wahrnehmbar sind, musste eine Bereitschaft der Einrichtungsleitung zur intensiven Kooperation gegeben sein, die somit die Zugangsmöglichkeit als Voraussetzung für die Einrichtungsauswahl setzte und hier folglich auch an einer Strategie des „Convenience-Sampling“" (Flick, 2014) anknüpfte.

Innerhalb der Einrichtungen, die sich prinzipiell für eine Teilnahme an der Studie bereit erklärten, erfolgte aber eine kriteriengeleitete Auswahl. Die im qualitativen Stichprobenplan enthaltenen Merkmale des Samplings basierten auf folgenden Vorannahmen, die auch von dem Forschungsstand abgeleitet wurden:

19 Wie in Tabelle 3 dargestellt, werden die Einrichtungen im Folgenden mit einem Buchstaben bezeichnet, die Reihenfolge entspricht nicht der oben genannten Aufzählung. 


\section{Forschungsdesign}

- Teilhabe wird in den Abläufen einer spezifischen Organisation hergestellt (daher: Einbeziehung der gesamten Organisation).

- Teilhabe wird durch infrastrukturelle Bedingungen der Umwelt beeinflusst (daher: Unterschiede räumliche Lage in Stadt, Land).

- Teilhabe kommt überpersonell unter den Ressourcenbedingungen zustande, die sich in großen und kleinen Einrichtungen unterscheiden (daher: Unterschiede in Einrichtungsgröße).

- Die Anzahl der Einrichtungen muss genügend Varianz erlauben, ist aber innerhalb eines qualitativen Designs noch in der gebotenen Tiefe bearbeitbar, daher vier Organisationen (je zweimal Großstadt und Mittelstadt; je zweimal große und kleine Einrichtung). Diese Anzahl bietet genügend Typik hinsichtlich der Merkmale Lage und Ressourcenausstattung, aber in der Summe auch genügend Varianz.

Damit ergaben sich zwei zentrale Samplingkriterien:

a) Größe der Einrichtung unterschieden nach Plätzen (nach Projektdefinition entsprechen Einrichtungen mit über 100 Plätzen einer großen, unter 100 Plätze einer kleinen bis mittelgroßen Einrichtung).

b) Räumliche Lage als „Stadt-Land Differenz“ im Rahmen vorhandener Varianz in Nordrhein-Westfalen (kleine Mittelstadt: 20.000 bis 50.000 Einwohner*innen; kleine Großstadt: 100.000 bis 500.000 Einwohner*innen; große Großstadt: ab 500.000 Einwohner*innen - vgl. hierzu die Klassifikation des Bundesamtes für Bauwesen und Raumordnung, 2018). Um ein möglichst breites Verständnis zu erlangen, sollten diese Kriterien bzw. Merkmale der Einrichtungen in ihrer Ausprägung - im Sinne eines „heterogeneous sampling“ (Patton, 2002) - differieren, so dass von den Einrichtungen mit einem bekundeten Interesse an der Studienteilnahme die vier oben benannten Projekteinrichtungen ausgewählt wurden, deren Merkmale im Folgenden näher beschrieben werden.

\subsubsection{Merkmale der Einrichtungen}

In diesem Kapitel werden die in die Studie einbezogenen Einrichtungen hinsichtlich ihrer Struktur in der Organisation, des Personals und der Bewohner*innenschaft differenziert beschrieben. Denn damit soll einerseits die ausgewählte Teilmenge stationärer Altenpflegeeinrichtungen als Sample für diesen wesentlichen Part der qualitativen Analyse transparent gemacht werden. Andererseits ermöglicht die Darstellung von Merkmalen der Einrichtungen exemplarisch Einblicke in strukturelle Rahmenbedingungen für die Förderung selbstbestimmter Teilhabe in der stationären Al- 
tenhilfe, die auch für die Einordnung der empirischen Ergebnisse von Interesse sind. Die dazu herangezogene Datenbasis beruht auf den Angaben der Einrichtungen, die in standardisierten Erhebungsbögen erfasst wurden. Die Erhebungsbögen wurden von den STAP-Mitarbeiter*innen in Abstimmung mit dem Projektrat entwickelt und beinhalten für die Darstellung des Sampling relevante Angaben zur Organisations-, Personalund Bewohner*innenstruktur. Zusätzlich erfolgt eine kurze Beschreibung des Wohnumfeldes der Einrichtungen hinsichtlich der räumlichen Lage, aber auch teilhaberelevanter Faktoren des Wohnquartiers (wie z. B. in Bezug auf die Infrastruktur). Diese Angaben beruhen auf einer teilstandardisierten Erhebung im Rahmen einer Begehung des Wohnumfeldes, die in Abstimmung mit dem STAP-Team von einer Praktikantin von STAP durchgeführt wurde.

\section{Organisationsstruktur}

Wie Tabelle $3 \mathrm{zu}$ entnehmen ist, weisen die vier Einrichtungen unterschiedliche Trägerschaften - innerhalb ihrer übergeordneten Zuordnung zum Diözesan-Caritasverband für das Erzbistum Köln e. V. - auf. Im Falle der Einrichtung A ist der Träger eine Stiftung, die noch andere soziale Einrichtungen und Dienste (auch außerhalb der Altenhilfe) vorhält. Einrichtung B und D sind Einrichtungen des Caritasverbandes einer Stadt bzw. einer Region, die Verbände sind jeweils eingetragene Vereine. Währenddessen wird die Einrichtung $\mathrm{C}$ in der Trägerschaft einer gemeinnützigen Gesellschaft mit beschränkter Haftung $(\mathrm{gGmbH})$ geführt, die noch weitere drei Altenpflegeeinrichtungen innehat.

Die vier Einrichtungen variieren hinsichtlich ihrer Lage in Gemeinden mit einer unterschiedlichen siedlungsstrukturellen Prägung und Bevölkerungsdichte zwischen der in einer kleinen Mittelstadt gelegenen Einrichtung A mit 181,6 Einwohner*innen je Quadratkilometer bis zu Einrichtung B mit 2815,8 Einwohner*innen je Quadratkilometer als große Großstadt. Dies begründet sich dadurch, dass unter den Einrichtungen des Diözesan-Caritasverbandes für das Erzbistum Köln e. V., die ein Interesse an der Studienteilnahme bekundeten, keine Einrichtung die Lage in einer kleinen Kleinstadt oder einer Landgemeinde aufwies. Dabei ist allerdings zu berücksichtigen, dass der Diözesan-Caritasverband für das Erzbistum Köln e. V. generell mit seiner Lage im Ballungsraum Nordrhein-Westfalens wenig Auswahl an Einrichtungen in einer Gemeinde unter 10.000 Einwohner*innen bietet. 
Tabelle 3: Trägerschaft und Lage der Einrichtungen

\begin{tabular}{|l|l|l|l|}
\hline Name & Träger & $\begin{array}{l}\text { Gemeindetyp, }{ }^{20} \\
\text { siedlungs- } \\
\text { strukturelle } \\
\text { Prägung21 }\end{array}$ & $\begin{array}{l}\text { Bevölke- } \\
\text { rungsdichte } \\
\text { (Einwoh- } \\
\text { ner*innen je } \\
\left.\mathrm{km}^{2}\right)^{22}\end{array}$ \\
\hline Einrichtung A & $\begin{array}{l}\text { Stiftung Kirchenge- } \\
\text { meinde, mehrere } \\
\text { soziale Einrichtun- } \\
\text { gen und Dienste }\end{array}$ & $\begin{array}{l}\text { kleine Mittelstadt, } \\
\text { teilweise städtisch } \\
\text { geprägt }\end{array}$ & 181,6 \\
\hline Einrichtung B & $\begin{array}{l}\text { Caritasverband } \\
\text { Region, e. V. }\end{array}$ & $\begin{array}{l}\text { große Großstadt, } \\
\text { städtisch geprägt }\end{array}$ & 2815,8 \\
\hline Einrichtung C & $\begin{array}{l}\text { gGmbH von 4 } \\
\text { Altenpflegeeinrich- } \\
\text { tungen }\end{array}$ & $\begin{array}{l}\text { kleine Großstadt, } \\
\text { überwiegend städ- } \\
\text { tisch geprägt }\end{array}$ & 1340,3 \\
\hline Einrichtung D & $\begin{array}{l}\text { Caritasverband } \\
\text { Region, e. V. }\end{array}$ & $\begin{array}{l}\text { kleine Mittelstadt, } \\
\text { überwiegend städ- } \\
\text { tisch geprägt }\end{array}$ & 444,2 \\
\hline
\end{tabular}

20 Stadt- und Gemeindetypen gemäß der Klassifikation des Bundesamtes für Bauwesen und Raumordnung.

21 Gemäß der Definition des Bundesinstituts für Bau-, Stadt-, Raumforschung zu Raumtypen. Definition der siedlungsstrukturellen Ausprägung: (1) Überwiegend städtisch: Gemeinden/Gemeindeverbände mit mindestens $50 \%$ Flächenanteil städtisch geprägter Umgebung; das bedeutet, dass diejenigen Gemeinden/ Gemeindeverbände, deren Fläche überwiegend in städtischer Umgebung liegt, insgesamt als überwiegend städtisch geprägt eingestuft werden. (2) Teilweise städtisch: Gemeinden/Gemeindeverbände mit einem Flächenanteil von mindestens $23 \%$ städtisch geprägter Umgebung (Bundeswert) oder mit einer Fläche städtisch geprägter Umgebung von mindestens $15 \mathrm{~km}^{2}$, das heißt, dass alle Gemeinden/Gemeindeverbände mit einem höheren Flächenanteil städtisch geprägter Umgebung als das gesamte Bundesgebiet $(23 \%)$ als teilweise städtisch geprägt gelten. (3a) Ebenfalls unter diesen Typ fallen Gemeinden/Gemeindeverbände mit einer absoluten Fläche in städtischer Umgebung in der Größenordnung der halben Durchschnittsgröße einer Gemeinde (rund $15 \mathrm{~km}^{2}$ ). Das letzte, absolute Kriterium trägt insbesondere den in jüngster Zeit durch Gemeindegebietsreformen gewachsenen Flächengrößen Rechnung. (3) Ländlich: gemeindefreie Gebiete und alle anderen Gemeinden/Gemeindeverbände.

22 Gemäß IT.NRW (2017): Kommunalprofil [der jeweiligen Stadt]: Bevölkerungsstand, Bevölkerungsdichte, Anteil der Bevölkerung über 65 Jahren, verfügbares Einkommen. 
Die Größe der Einrichtung, gemessen an der Platzzahl, variiert deutlich zwischen einer Einrichtung kleinerer bis mittlerer Größe mit 64 Bewohner*innenplätzen und einer großen Einrichtung mit 184 Bewohner*innenplätzen in den ausgewählten Einrichtungen. Wie sich das Bild hinsichtlich der Bewohner*innenplätze und dem Anteil der Einzelzimmer in den Einrichtungen zeigt, ist in Tabelle 4 dargestellt.

Tabelle 4: Anzabl der Plätze, Wohnbereiche und Arten der Zimmer

\begin{tabular}{|l|l|l|l|l|l|}
\hline Einrichtung & A & B & C & D & Gesamt \\
\hline $\begin{array}{l}\text { Bewohner*innen- } \\
\text { plätze }\end{array}$ & $88^{23}$ & 64 & $184^{24}$ & $119^{25}$ & 455 \\
\hline $\begin{array}{l}\text { Art der Zimmer } \\
\text { Einzelzimmer } \\
\text { Doppelzimmer }\end{array}$ & 56 & 50 & $\begin{array}{l}152 \\
16\end{array}$ & $\begin{array}{l}111 \\
4\end{array}$ & $\begin{array}{l}369 \\
43\end{array}$ \\
\hline Wohnbereiche & 3 & 3 & 5 & 4 & 15 \\
\hline
\end{tabular}

Obwohl die Anzahl der Plätze zwischen 64 und 184 liegt, ist die Differenz der Wohnbereiche zwischen den Einrichtungen nicht sehr hoch. Die kleinste Einrichtung B (64 Plätze) verfügt über drei Wohnbereiche, genauso wie Einrichtung A mit 88 Plätzen. Ebenso fällt die Differenz der Wohnbereiche zwischen der Einrichtung $\mathrm{C}$ und D verglichen mit ihrer Platzanzahl von 184 und 119 Plätzen nicht so hoch aus: Einrichtung C umfasst fünf, Einrichtung D vier Wohnbereiche. Drei der Einrichtungen bieten Kurzpflege an, die unterschiedlich organisiert ist. Die fünf eingestreuten Plätze der Kurzpflege in Einrichtung A bilden keinen eigenen Einrichtungsbereich. In der Einrichtung $\mathrm{C}$ werden 15 der Plätze als eingestreute Plätze für die Kurzzeitpflege räumlich und konzeptionell einem eigenen Einrichtungsbereich (der Kurzzeitpflege) zugeordnet. Einrichtung D bietet fünf Kurzpflegeplätze, integriert in den Wohnbereich, an. Diese Bewohner*innen haben einen eigenen Versorgungsvertrag und sind demnach nicht in die 119 Bewohner*innenplätze integriert.

23 Fünf Kurzzeitpflegeplätze sind eingestreut und daher in den vollstationären Bereich eingerechnet.

24 Fünfzehn Kurzzeitpflegeplätze sind eingestreut und daher in den vollstationären Bereich eingerechnet.

25 Fünf Kurzzeitpflegeplätze sind nicht in den vollstationären Bereich eingerechnet. 


\section{Forschungsdesign}

Die Einrichtungen haben den seit August 2018 gesetzlich vorgeschriebenen Anteil von $80 \%$ Einzelzimmern ${ }^{26}$ zum Erhebungszeitpunkt (Juli 2017) noch unterschiedlich realisiert: In der Einrichtung D sind 93,3\% der Zimmer Einzelzimmer, in der Einrichtung A ist dies im Falle von 63,6\% gegeben. Dazwischen liegt die Einrichtung B, in der 78,1 \% der Zimmer Einzelbettenzimmer sind. Im Fall C liegt die Einzelzimmerquote bei 82,6\%.

Die Einrichtungen verfügen über unterschiedliche ambulante und stationäre Angebote und Strukturen, wie in Tabelle 5 ersichtlich wird.

Tabelle 5: Ambulante und stationäre Angebote der Einrichtungen

\begin{tabular}{|l|l|l|l|l|}
\hline Einrichtung & A & B & C & D \\
\hline Kurzzeitpflege & $\mathrm{x}$ & - & $\mathrm{x}$ & $\mathrm{x}$ \\
\hline Tagespflege & - & - & $\mathrm{x}$ & \\
\hline $\begin{array}{l}\text { Wohnbereich, der für } \\
\text { spezifische } \\
\text { Bewohner*innen } \\
\text { konzeptioniert ist }\end{array}$ & - & - & $\begin{array}{l}\mathrm{x} \\
\text { zwei } \\
\text { Wohnbe- } \\
\text { reiche für } \\
\text { demenziell } \\
\text { veränderte } \\
\text { Menschen }\end{array}$ & - \\
\hline Senior*innenwohnen & - & & $\begin{array}{l}\text { x } \\
\text { Wohnen } \\
\text { mit Service }\end{array}$ & $\begin{array}{l}\text { x } \\
\text { Senior*in- } \\
\text { nenwoh- } \\
\text { nungen }\end{array}$ \\
\hline
\end{tabular}

Insbesondere die Kurzzeitpflege (Verhinderungspflege) stellt ein Angebot dar, das in drei der vier Einrichtungen vorhanden ist. Einrichtung C, als große Einrichtung, unterhält zudem eine Tagespflege und hat sich räumlich und konzeptionell in zwei von fünf Wohnbereichen auf die Pflege und Betreuung demenziell veränderter Bewohner*innen spezialisiert. In der vergleichsweise kleinen Einrichtung B bestehen keine weiteren ambulanten oder teilstationären Angebote.

Mit Senior*innenwohnungen in einem separaten Wohnbereich (Einrichtung D) oder Wohnen mit Service innerhalb eines Einrichtungsbereiches (Einrichtung C) halten zwei Einrichtungen weitere Angebote vor. Die Mieter*innen leben hier selbstständig, nutzen aber teilweise vertraglich ge-

$26 \rrbracket 20$ Abs. 3 Satz 2 WTG Fassung, 2.10.2014. 
regelt ausgewählte Leistungen der Einrichtung, wie beispielsweise das Mahlzeitenangebot.

In allen vier Einrichtungen können zusätzliche Leistungen in Anspruch genommen werden. So stehen den Bewohner*innen beispielsweise Friseurleistungen zur Verfügung, die im Rahmen einer mehrtägigen Öffnung eines Friseursalons innerhalb der Einrichtung oder durch eine Friseurleistung im Wohnbereichsbad angeboten werden. Bewohner*innen können außerdem einzelne medizinische Leistungen, die nicht von Pflegekräften erbracht werden, durch ärztliche Verordnung über die Einrichtung organisieren lassen. Dazu gehören die Ergo-, Physio- und Sprachtherapie. Die Einrichtung organisiert in dem benannten Fällen den Kontakt zu den Dienstleister*innen, stellt gegebenenfalls Räume zur Verfügung und unterstützt bei zuzahlungspflichtigen Leistungen möglicherweise mit der Abwicklung der Zahlung bzw. Beantragung der Kostenübernahme bei Kostenträger*innen.

Bewohner*innenstruktur

Die Zusammensetzung der Bewohner*innenschaft zum Stichtag nach soziodemografischen Merkmalen zeigt sich wie folgt:

Tabelle 6: Soziodemografische Merkmale der Bewohner"innen

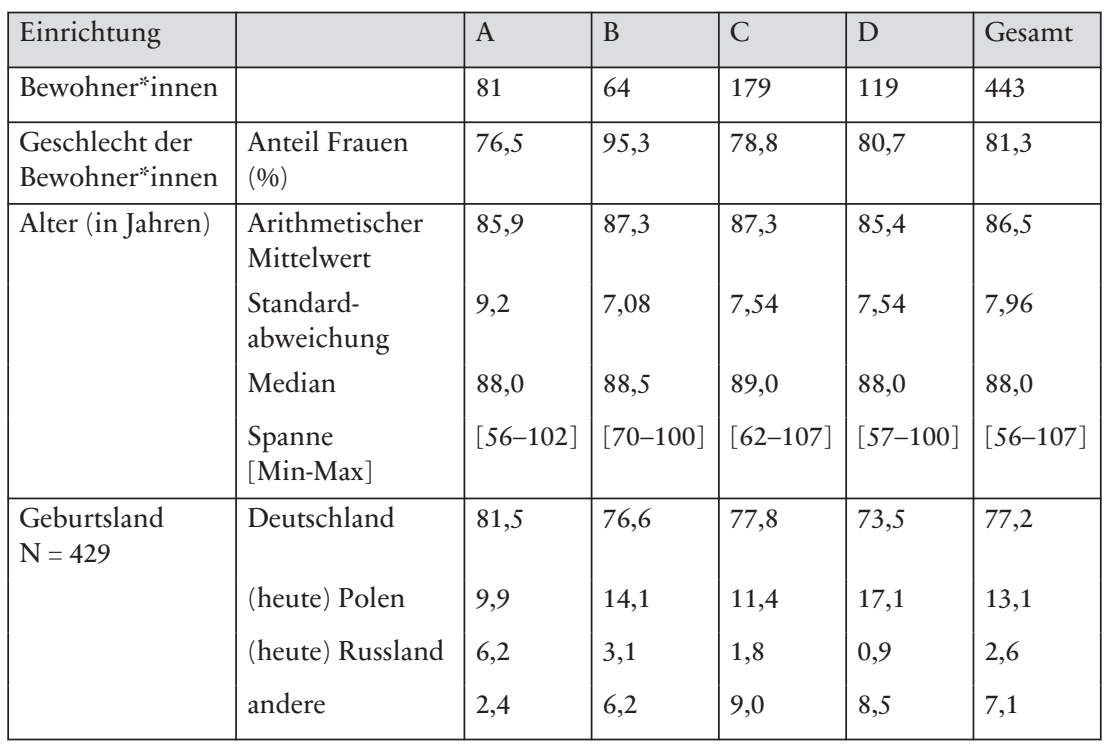




\begin{tabular}{|l|l|l|l|l|l|l|}
\hline Einrichtung & & A & B & C & D & Gesamt \\
\hline $\begin{array}{l}\text { Sozialhilfeemp- } \\
\text { fänger*in (in \%) }\end{array}$ & & 33,3 & 40,6 & 29,6 & 49,6 & 37,25 \\
\hline
\end{tabular}

Die Auswertungen der vorliegenden Studie beruhen auf 443 Bewohner*innendaten. Diese Zahl weicht von den tatsächlich in allen Einrichtungen zur Verfügung stehenden 455 Plätzen ab, da zum Erhebungsstichtag nicht in allen vier Einrichtungen alle Plätze belegt waren und nicht für alle Bewohner*innen die Erlaubnis zum Einbezug ihrer Daten vorlag.

Innerhalb der vier Einrichtungen verteilten sich die Männer und Frauen zum Zeitpunkt der Datenerhebung so, dass Frauen mindestens drei Viertel der Bewohner*innenschaft stellten. Insbesondere in der Einrichtung B war der Anteil der Männer mit 4,7\% gering, während in der Einrichtung A 24,5 \% Männer lebten.

Das durchschnittliche Alter der Bewohner*innen der Einrichtungen wich währenddessen recht gering von dem gesamten arithmetischen Mittelwert aller Bewohner*innen der vier Einrichtungen von 86,5 Jahren ab gleiches gilt für den hier robusteren Kennwert des Medians. Bis auf die Einrichtung $\mathrm{C}$ lebten in jeder der vier Einrichtungen auch pflegebedürftige Menschen unterhalb des gesetzlichen Rentenalters, wenngleich der Anteil hochaltriger Menschen, gemeint sind Bewohner*innen mit mindestens 80 Jahren bzw. - je nach Definition von Hochaltrigkeit - 85 Jahren, ${ }^{27}$ deutlich im Vordergrund steht, wie die mittleren Werte bereits erkennen lassen. In der Einrichtung A war die Altersspanne am stärksten ausgeprägt und der durchschnittliche Alterswert streute etwas weiter zum Mittelwert als in den anderen Einrichtungen.

Mit durchschnittlich 77,2\% sind die meisten der Bewohner*innen in Deutschland geboren oder sind deutschstämmiger. Nur durchschnittlich 7,1 \% aller Bewohner*innen der vier Einrichtungen besitzen einen Migrationshintergrund, dieser Personenkreis ist in der Einrichtung $\mathrm{C}$ mit 9,0\% am stärksten und in der Einrichtung A mit 2,4\% am geringsten vertreten ${ }^{29}$.

27 „Der Vierte Altenbericht definiert pragmatisch den Altersabschnitt von 80 bis 85 Jahren als den Beginn des hohen Alters" (Bundesministerium Familie, Soziales, Frauen und Jugend, 2002, S. 45).

28 Die Deutschstämmigkeit entsteht dadurch, dass ihr Geburtsort in einem Gebiet Deutschlands in den Grenzen vor 1948 lag.

29 Kohls (2012) gibt bezogen auf Daten aus dem Jahr 2010 an: „In vollstationären Pflegeeinrichtungen haben $53 \%$ der befragten Heimleitungen angegeben, dass der Anteil der Versorgten mit Migrationshintergrund zwischen $0 \%$ und $9 \%$ be- 
Blickt man auf den Anteil der Bewohner*innen, die zur Bestreitung ihres Lebensunterhaltes auf Leistungen des SGB XII (Grundsicherung im Alter und bei Erwerbsminderung ${ }^{30}$ ) angewiesen sind, so sind durchschnittlich 37,3\% aller Bewohner*innen der vier Einrichtungen auf staatliche Transferleistungen angewiesen. In der Einrichtung D bezieht fast jede/ $r$ zweite Bewohner"in Sozialhilfe, in der Einrichtung C liegt die Bezugsquote hingegen bei 29,6\%.31 Dies ist verglichen mit der Anzahl der Empfänger*innen der Grundsicherung im Alter - hier allerdings an der gesamten Alterskohorte oberhalb der Regelaltersgrenze von 65+ Jahren gemessen im Jahr 2017 mit 3,1\% (Institut für Qualifikation und Arbeit, 2017) hoch. 32

Die Zusammensetzung der Bewohner*innenschaft hinsichtlich der Pflegegrade und der Alltagskompetenz sowie die Anteile von Bewohner"innen, bei denen eine vollständige Immobilität (gemessen an den Qualitätsprüfungsrichtlinien bzw. der sogenannten QPR-Liste) gegeben ist und die Hilfsmittel der Mobilität und Kommunikation nutzen, werden im Folgenden aufgeführt.

trägt [...] In $7 \%$ der Einrichtungen liegt der Anteil zwischen $10 \%$ und 19\%, während in $27 \%$ der Einrichtungen keine Personen mit Migrationshintergrund wohnen“ (ebd., S. 4).

30 Der Leistungsbezug ist daran gekoppelt, wenn „aus Altersgründen nicht mehr erwartet werden kann, dass die materielle Notlage einer Person durch Ausübung einer Erwerbstätigkeit überwunden wird, oder die Ausübung einer Erwerbstätigkeit aus gesundheitlichen Gründen dauerhaft nicht möglich ist" (Bundesministerium für Arbeit und Soziales, 2018).

31 Die Einrichtung liegt in dem Gebiet, bei dem die Einwohner*innen - verglichen mit den Gebieten der anderen Einrichtung - das höchste Pro-Kopf-Einkommen besitzen. Die Bewohner*innen dieser Einrichtung wohnten vor dem Einzug zu 93,4\% in dem Einzugsgebiet der Einrichtung (bis $25 \mathrm{~km}$ ).

32 Berechnung des Instituts für Qualifikation und Arbeit auf Basis der Daten des Statistischen Bundesamtes (www.sozialpolitik-aktuell.de/tl_files/sozialpolitik-akt uell/_Politikfelder/Sozialstaat/Datensammlung/PDF-Dateien/abbIII51.pdf, Zugriff 1.4.2019). Mit dem Einzug in eine stationäre Altenpflegeeinrichtung sind Kosten, aber auch Unterstützung bei der Beantragung von sozialrechtlichen Leistungen durch den Sozialen Dienst verbunden. Es besteht ein zudem lebenslagenbedingtes Risiko, im Pflegefall Transferleistungen beziehen zu müssen. Der Anteil der in einem Pflegeheim wohnenden Bezieher*innen von Hilfe zur Pflege lag 2016 - verglichen zu Nichtbewohner*innen - bei $72 \%$ (www.sozialpolitik-aktuel 1.de/tl_files/sozialpolitik-aktuell/_Politikfelder/Sozialstaat/Datensammlung/PDFDateien/abbIII55.pdf, Zugriff 1.4.2019). 
Tabelle 7: Einschränkungen und Hilfebedarfe der Bewohner*innen

\begin{tabular}{|l|l|r|r|r|r|r|}
\hline Einrichtung & & A & B & C & D & Gesamt \\
\hline Pflegegrad (in \%) & ohne & 0,0 & 0,0 & 0,0 & 2,5 & 0,7 \\
& 1 & 0,0 & 0,0 & 0,0 & 0,8 & 0,2 \\
& 2 & 30,8 & 26,6 & 18,4 & 25,2 & 23,7 \\
& 3 & 29,6 & 35,9 & 24,6 & 28,6 & 28,2 \\
& 4 & 22,2 & 23,4 & 39,1 & 30,3 & 31,4 \\
& 5 & 17,3 & 14,1 & 17,9 & 12,6 & 15,8 \\
\hline Eingeschränkte Alltags- & & 64,2 & 40,6 & 78,2 & 57,1 & 64,6 \\
kompetenz (in \%) & & & & & & \\
\hline Vollständige Immobilität & ja & 3,7 & 3,1 & 4,5 & 2,5 & 3,6 \\
\hline Kommunikationshilfe & Brille & 67,9 & 57,8 & 35,2 & 54,6 & 49,7 \\
& Hörgerät & 21,0 & 26,6 & 14,0 & 20,2 & 18,7 \\
\hline Mobilitätshilfen (in \%) & Rollstuhl & 45,7 & 48,4 & 51,4 & 45,4 & 48,3 \\
& Rollator & 51,9 & 45,3 & 45,8 & 54,6 & 49,2 \\
& Andere & 13,6 & 0,0 & 5,6 & 5,0 & 6,1 \\
\hline Personen ohne Mobili- & & 11,1 & 6,2 & 19,6 & 16,0 & 15,1 \\
tätshilfe (Anteil in \%) & & & & & & \\
\hline
\end{tabular}

Es zeigt sich hier deutlich, dass die Bewohner*innenstruktur durch das Vorliegen höherer Pflegegerade gekennzeichnet ist: Während kaum Bewohner*innen ohne einen Pflegegrad oder mit Pflegegrad 1 vertreten sind, besteht grob eine Dreiteilung bei den Pflegegraden 2, 3 und 4. Hinsichtlich des Pflegegrades 5 zeigt sich ein Bild, bei dem sich 12 bis $17 \%$ der Bewohner*innen durch eine hohe Pflegebedürftigkeit auszeichnen. Durch die Spezialisierung der Einrichtung C in Form eines "Demenzhauses“ zeichnet sich ein anderes Bild hinsichtlich der eingeschränkten Alltagskompetenz. Bei 78,2\% der Bewohner*innen wurde eine eingeschränkte Alltagskompetenz angegeben. Diese Quote übersteigt die der Einrichtung B fast um die Hälfte, während sie zwischen den Einrichtungen A $(64,2 \%)$ und D $(57,1 \%)$ nicht so stark abweicht.

Nur ein geringer und in den vier Einrichtungen relativ nah beieinanderliegender Anteil der Bewohner*innen ist vollständig immobil (im Mittel 3,6\%). Auf die Kommunikationshilfe Brille greifen rund die Hälfte und auf Hörgeräte circa ein Fünftel aller Bewohner*innen zurück, während Mobilitätshilfen von durchschnittlich $85 \%$ der Bewohner*innen genutzt werden. Die Bewohner*innen (gesamt) greifen dabei hälftig auf den Rollstuhl und hälftig auf den Rollator als Mobilitätshilfe zurück. Bei den Zahlen ist allerdings zu beachten, dass einerseits Mehrfacherfassungen enthalten sind (z. B. besitzen manche Bewohner*innen einen Rollator für kurze Wege und einen Rollstuhl für längere Wege oder die Mobilität außerhalb der Einrichtung), andererseits wurden unter Umständen Rollstühle ange- 
geben, die zwar vorhanden sind, aber derzeit nicht genutzt werden (können).

In der Einrichtung $\mathrm{C}$, mit einem hohen Anteil von Menschen mit eingeschränkter Alltagskompetenz, spielen im Vergleich der vier Einrichtungen Kommunikationshilfen eine geringere Rolle, dies ist aber nicht der Fall bei den Mobilitätshilfen.

Die Zusammensetzung der Bewohner"innenschaft hinsichtlich ihrer rechtlichen Vertretung ist in folgender Tabelle 8 dargestellt.

Tabelle 8: Rechtliche Vertretung der Bewohner*innen

\begin{tabular}{|l|l|r|r|r|r|r|}
\hline Einrichtung & & $A$ & $B$ & $C$ & $D$ & Gesamt \\
\hline Gesetzliche Betreuung & Angehörige & 7,4 & 3,1 & 7,8 & 4,2 & 6,1 \\
durch (in \%) & Berufsbetreuer*innen & 11,1 & 12,5 & 11,2 & 12,4 & 12,0 \\
& Keine & 81,5 & 84,4 & 79,9 & 82,4 & 81,5 \\
\hline Vollmacht (in \%) & $\mathrm{Ja}$ & 46,9 & 81,3 & 75,4 & 75,6 & 71,1 \\
\hline
\end{tabular}

Hinsichtlich des Anteils an Bewohner*innen mit einer gesetzlichen Betreuung unterscheiden sich die Einrichtungen nur gering, die meisten, also durchschnittlich 81,5\% der Bewohner*innen, werden nicht durch eine gesetzliche Betreuung vertreten. Besteht eine gesetzliche Betreuung, so übernehmen diese Vertretungen doppelt so häufig Berufsbetreuer"innen verglichen mit dem Anteil der Angehörigen.

Angaben zur Aufenthalts- bzw. Wohndauer, zum Einzugsalter sowie zu vorherigen Wohnorten der Bewohner*innen werden in der folgenden Tabelle 9 aufgeführt.

Tabelle 9: Aufenthaltsdauer, Einzugsalter und vorherige Wohnorte der Bewohner*innen

\begin{tabular}{|l|l|r|r|r|r|r|}
\hline & $\mathrm{A}$ & $\mathrm{B}$ & $\mathrm{C}$ & $\mathrm{D}$ & Gesamt \\
\hline $\begin{array}{l}\text { Aufenthalts- } \\
\text { dauer seit } \\
\text { Einzug (in } \\
\text { Jahren) }\end{array}$ & $\begin{array}{l}\text { Arithmeti- } \\
\text { scher Mit- } \\
\text { telwert, }\end{array}$ & 4,02 & 2,23 & 3,58 & 3,04 & 3,32 \\
& $\begin{array}{l}\text { Standardab- } \\
\text { weichung, } \\
\text { Median } \\
\text { Spanne } \\
\text { [Min.- } \\
\text { Max.] }\end{array}$ & 3,88 & 1,7 & 3,04 & 3,0 & 3,1 \\
& 3,03 & 1,79 & 2,89 & 2,37 & 2,5 \\
{$[0,13-17,54]$} & {$[0,15-6,93]$} & {$[0,13-21,34]$} & {$[0,11-16,62]$} & {$[0,11-21,34]$} \\
\hline
\end{tabular}




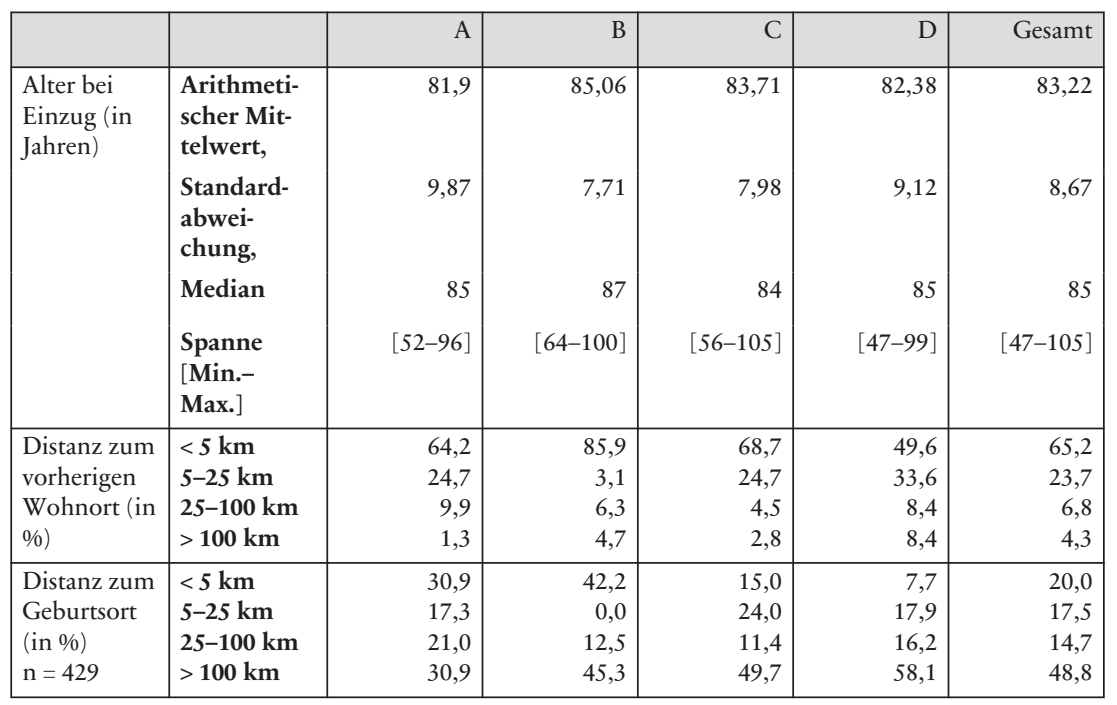

Die in die Studie einbezogenen Bewohner*innen leben zum Zeitpunkt der Abfrage durchschnittlich seit 3,32 Jahren in der Einrichtung und sind durchschnittlich als hochaltrige Menschen, also als über 80-jährige Menschen, eingezogen. Hier liegen die Werte der vier Einrichtungen nah beieinander. Innerhalb der jeweiligen Einrichtungen variieren die Angaben zur Wohndauer allerdings beträchtlich. So leben einzelne Bewohner*innen bis zu 21 Jahren in der Einrichtung, was mit einem geringen Einzugsalter zu erklären ist. Dennoch gehört eine kurze Aufenthaltsdauer nach dem Einzug mehrheitlich zur Realität in allen vier Einrichtungen, wie der Median zwischen 1,79 und 3,03 Jahren zu erkennen gibt.

Betrachtet man nun den Aspekt, inwiefern Bewohner*rinnen nach dem Umzug in die Einrichtung innerhalb ihres vorherigen Sozialraumes bzw. ihres Wohnortes verblieben sind, so ist feststellbar, dass 88,9\% der Bewohner"innen zuvor in einem Umkreis von bis zu 25 Kilometern lebten. Nur $11,1 \%$ der Bewohner*innen haben räumlich und sozial das gewohnte Umfeld mit dem Einrichtungseinzug verlassen. Das gewohnte räumliche und soziale Umfeld entspricht bei 37,5\% der Bewohner*innen sogar dem Geburtsort und verweist damit auf eine hohe Kontinuität. Bei nahezu der Hälfte der Bewohner*innen besteht jedoch eine hohe räumliche Distanz von über 100 Kilometern zu ihrem Geburtsort. 


\section{Räumliches und soziales Wohnumfeld}

Die folgenden Ausführungen beziehen sich auf ein Teilprojekt im Rahmen einer Studienarbeit ${ }^{33}$ im Wintersemester 2017/2018.

In Anlehnung an die Checkliste „Generationsgerechtes Wohnumfeld“34 des Kuratoriums Deutsche Altershilfe (Mehnert \& Kremer-Preiß, 2014) wurde eine für das vorliegende Forschungsprojekt angepasste Checkliste erstellt, die sowohl ausgewählte "physische und bauliche Strukturen“ (ebd., S. 6) erfasst als auch „sozial-infrastrukturelle Potenziale“ (ebd.) aufzeigen soll. ${ }^{35}$ Diese Checkliste ermöglichte eine detaillierte Erfassung von möglichen teilhaberelevanten Aspekten im direktem Umfeld ${ }^{36}$ der jeweiligen Einrichtung.

Alle vier Einrichtungen befinden sich in einem Wohnumfeld mit mittlerer Bebauung und direkter Nachbarschaft. Insgesamt lässt sich feststellen, dass alle Einrichtungen im Umkreis von 250 Metern über eine Infrastruktur verfügen, die den täglichen und mittelfristigen Bedarf des täglichen Lebens deckt. ${ }^{37}$ Ebenfalls findet sich in jedem Wohnumfeld im 250-MeterUmkreis mindestens ein gastronomisches Angebot, eine Anbindung an den öffentlichen Personennahverkehr (ÖPNV), mit zwei bis vier Haltestellen in der Nähe, und mindestens eine Sitzbank.

In drei der Einrichtungen ( $\mathrm{A}, \mathrm{C}$ und $\mathrm{D}$ ) gehört zum einrichtungseigenen Gelände ein eigener, parkähnlicher Garten, der auch öffentlich zugänglich ist. ${ }^{38}$ Die Einrichtung $\mathrm{C}$ öffnet ihre Grünflächen zeitweilig für eine benachbarte Einrichtung der Kindertagespflege.

33 Jennifer van Berk verfasste diese Arbeit im Rahmen einer Prüfungsleistung ihres Praxissemesters an der Hochschule Düsseldorf.

34 Sie ist enthalten in einer Handreichung im Rahmen des Förderbausteins 3.1.1 „Projekte mit Ansatz zur Quartiersentwicklung“ des Deutschen Hilfswerks, die vom Kuratorium Deutsche Altershilfe herausgegeben wurde.

35 In der Begehung wurden erfasst: Straßenbedingungen, öffentlicher Nahverkehr, Grünfläche, Ruhebänke, Dienstleistungen/Nahversorgung, Gastronomie, soziale Begegnungsräume und sonstige Eindrücke.

36 Das fußläufig erreichbare Nahumfeld wurde auf einen Radius von 250 Metern um die jeweilige Einrichtung festgelegt.

37 Darunter fallen beispielsweise bei der Einrichtung A folgende Geschäfte: (1) täglicher Bedarf: Lebensmitteldiscounter, Bäcker, Kiosk; (2) mittelfristiger Bedarf: Kleidungsgeschäfte, Geschenkeläden, Schmuckladen, Blumenladen.

38 In den Beobachtungen in allen drei Einrichtungen zeigte sich aber, dass hier eine geringe, eigenmotivierte bzw. selbstständige Nutzung der Bewohner*innen zu verzeichnen ist. Ebenso zeigten sich dort in den Beobachtungszeiträumen keine externen Besucher*innen. 


\section{Forschungsdesign}

Auffällig ist, dass in drei von vier Einrichtungen keine öffentlich nutzbaren Grünflächen in fußläufiger Entfernung liegen. Die Einrichtung B verfügt als einzige der vier Einrichtungen über einen fußläufigen Zugang zu einem Park, der allerdings von Hügeln durchzogen ist. Ebenso haben drei Einrichtungen keine sozialen Begegnungsräume in ihrer direkten Umgebung, dies ist nur im Umkreis von 250 Metern bei der Einrichtung C mit einer katholischen Kirche inklusive Pfarrbüro und Bücherei gegeben.

Bei den Straßenbedingungen ist ein sehr gemischtes Bild zu verzeichnen. Es gibt überall abgesenkte Bordsteine, aber nur in zwei Einrichtungen sind alle Gehsteige im Umkreis von 250 Metern mindestens 150 Zentimeter breit. In zwei der vier Einrichtungen sind die Gehwege teilweise uneben. Eine besondere Barriere stellen die teilweise sehr kurzen Grünphasen an den Ampeln in der Nähe der Einrichtungen dar. Viele Ampeln haben eine Grünphase von 30 Sekunden und weniger, besonders kurz war die Grünphase einer Ampel in der Nähe der Einrichtung A mit 9 Sekunden. In dieser Einrichtung fällt zudem die topografische Lage der Einrichtung auf: Sie befindet sich auf einem Hügel, daher führen die umgebenden Straßen teilweise stark bergab und bergauf.

Das Nahumfeld der Einrichtung D ist teilweise geprägt durch Kopfsteinpflaster und sehr enge Gehwege. Vor der Einrichtung verläuft eine stark befahrene Straße ohne eine Ampelanlage, so dass Bewohner*innen diese ungesichert überqueren müssen, um beispielsweise zu den Einkaufsmöglichkeiten zu gelangen.

\section{Personalstruktur}

Die hier aufgezeigte Personalsituation in den einzelnen Einrichtungen beruht auf Angaben zu den Vollzeitstellenäquivalenten (VÄ) pro Einrichtung. Dies ist zu unterscheiden von der Anzahl der Mitarbeitenden („Köpfe“), da Mitarbeitende (MA) beispielweise in Teilzeit oder auch regelmäßig in zwei oder mehreren Einrichtungen arbeiten. So wechseln sich beispielsweise die Einrichtungsleitung und stellvertretende Einrichtungsleitung der Einrichtung D als Leitung in jeweils zwei Häusern ab und bilden so ein Vollzeitäquivalent. Zum besseren Verständnis wird aber auch die Anzahl der Mitarbeiter*innen mit aufgeführt (Tabelle 10). 
Tabelle 10: Anzabl der Mitarbeiter*innen nach Einrichtungs- und Aufgabenbereichen (in Vollzeitäquivalenten)

\begin{tabular}{|l|l|l|l|l|l|l|l|l|}
\hline & \multicolumn{2}{l}{ A } & \multicolumn{2}{l}{ B } & \multicolumn{2}{l|}{ C } & D \\
\hline & MA & VÄ & MA & VÄ & MA & VÄ & MA & VÄ \\
\hline $\begin{array}{l}\text { Einrichtungs- } \\
\text { leitung }\end{array}$ & 1 & 1 & 1 & 1 & 1 & 1 & 2 & $1^{39}$ \\
\hline Verwaltung & 2 & $1^{40}$ & 1 & 0,75 & 14 & 8,69 & 6 & $2,83^{41}$ \\
\hline Pflege & 55 & 33,5 & 31 & 25,25 & 92 & 70,26 & 59 & $41,32^{42}$ \\
\hline Sozialer Dienst & $4^{43}$ & 2 & 2 & 1,75 & 5 & 3,8 & 5 & $3,95^{44}$ \\
\hline $\begin{array}{l}\text { Zusätzliche Be- } \\
\text { treuungskräfte }\end{array}$ & 8 & 4,4 & 4 & $3,2^{45}$ & 13 & 8,8 & 8 & 6,75 \\
\hline Hauswirtschaft & 32 & $13,1^{46}$ & 7 & $5,5^{47}$ & 68 & 34,76 & 35 & $19,51^{48}$ \\
\hline Haustechnik & 3 & 1,3 & 1 & 0,75 & 8 & 3,99 & 3 & $1,5^{49}$ \\
\hline
\end{tabular}

Während der Stellenschlüssel von Leitungen (Einrichtungs- und Pflegedienstleitung) sowie von Pflegekräften gesetzlich normiert ist, so besteht bei dem Personalschlüssel der anderen Einrichtungsbereiche ein Spielraum. Unterschiede in der Personalausstattung zeigen sich insbesondere in der Organisation der Hauswirtschaft sowie in der Verwaltung, bei der einzelne Dienstleistungen bzw. Aufgabenbereiche ein- oder ausgelagert sind. So arbeiten in der Hauswirtschaft der Einrichtung B, als eine kleine Einrichtung ohne eigene Küche und Wäscherei, 5,5 Mitarbeiter*innen in Voll-

39 Einrichtungsleitung und stellvertretende Einrichtungsleitung zählen jeweils 0,5 Stellenanteile.

40 Personalabteilung und Finanzbuchhaltung sind ausgelagert (1,2 VÄ externe Verwaltung).

41 1,83 Stellen sind reine Rezeptionsmitarbeiter"innen.

42 Bewohnernahe Haushaltstätigkeiten werden von Hauswirtschaftsmitarbeiter"innen übernommen.

43 In den Interviews zählen Leitung und Stellvertretung auch zu den Mitarbeitenden des Sozialen Dienstes, sie werden hier als „Köpfe“ angeführt, aber nicht in den Stundenkontingenten mitberechnet.

44 Davon 1,25 keine Fachkräfte.

45 Betreuungsassistenten plus Stelle für ein Freiwilliges Soziales Jahr.

46 Flachwäsche in Form von Mietwäsche ausgelagert.

47 Essenslieferung aus einem anderen Haus, Wäsche extern.

48 Davon 15 Personen auf den Wohnbereichen (VÄ 4,91), bei 2 VÄ handelt es sich um Auszubildende in der Hauswirtschaft. Wäscheversorgung und Reinigung ist ausgelagert.

49 Wobei alle Mitarbeiter*innen stundenweise andere Objekte mitbetreuen. 


\section{Forschungsdesign}

zeitstundenäquivalenten umgerechnet, während dies in der großen Einrichtung $\mathrm{C}$ - mit einer eigenen Küche - 34,76 Stellenanteile von Mitarbeitenden sind. Innerhalb der Verwaltung der Einrichtung A sind die Personalabteilung sowie die Finanzbuchhaltung ausgelagert, so dass dort eine Verwaltungsmitarbeitende in einem Vollzeitstundenäquivalent zu finden ist. Dies stellt sich bei den Einrichtungen C und D unterschiedlich dar. Die große Einrichtung C, bei der auch eine Tagespflege, eine Kurzzeitpflege sowie Seniorenwohnungen angebunden sind, verfügt über 8,69 Mitarbeiter*innen in Vollzeitstundenäquivalenten. Innerhalb der Einrichtung D sind dies 2,83 Mitarbeiter*innen in Vollzeitstundenäquivalenten, bei denen 1,83 Stellenanteile für Rezeptionstätigkeiten vorbehalten sind. Die Finanzbuchhaltung und die Personalabteilung sind in der Einrichtung D ausgelagert.

Für die vorliegende Studie interessiert auch die Anzahl der Mitarbeitenden, die als Fachkräfte im Sozialen Dienst und als zusätzliche Betreuungskräfte tätig sind. Durch die gesetzlichen Vorgaben ist die Relation der Mitarbeiter*innenanzahl - ausgehend von Vollzeitäquivalenten im Verhältnis zu Bewohner*innenplätzen - relativ ähnlich.

Für die Rahmenbedingungen der Teilhabeförderung ist zusätzlich zu der Anzahl des beschäftigten Personals interessant, wo die Stellen des Sozialen Dienstes und die der zusätzlichen Betreuungskräfte organisatorisch in der Einrichtung integriert sind. Es bestehen zwei unterscheidbare Modelle, die aber nicht in einer Reinform existieren. Innerhalb des ersten Modells werden die Mitarbeiter*innen des Sozialen Dienstes und die zusätzlichen Betreuungskräfte einem Wohnbereich so zugeordnet, dass diese unmittelbar in die Planung, Kommunikation und den Ablauf im Wohnbereich eingebunden sind. Innerhalb des zweiten Modells besteht keine feste Zuordnung der Mitarbeiter*innen und der zusätzlichen Betreuungskräfte zu einem Wohnbereich, die Zusammenarbeit erfolgt primär über oder in Bezug auf die jeweiligen Gruppenangebote und Einzelbetreuung des Sozialen Dienstes bzw. der zusätzlichen Betreuungskräfte.

Außerdem bestehen Unterschiede in Bezug auf eine Leitungsfunktion bzw. die Vorgesetztenregelung beim Sozialen Dienst. Während es in zwei Einrichtungen eine Leitung des Sozialen Dienstes gibt, ist in den anderen beiden Einrichtungen der Soziale Dienst direkt der Einrichtungs- oder Pflegedienstleitung unterstellt. 


\subsubsection{Erhebungsmethoden}

A) Interviews

Um die Forschungsfragen im Rahmen der qualitativen Analyse in den vier ausgewählten und oben vorgestellten Einrichtungen multiperspektivisch beleuchten zu können, sollte das Sampling der Interviewpersonen die in einer Einrichtung vorrätigen, unterschiedlichen Wissensinhalte als Expert*innenwissen (der Leitungen und Mitarbeiter*innen verschiedener Einrichtungsbereiche) und alltagsgebundenes Laienwissen (der Bewohner*innen und Angehörigen) berücksichtigen (Schütz, 1972). Angeregt von der Unterscheidung in „knowing that“ und „knowing how“, bei der Wissen und Können als differente Formen des Handelns betrachtet werden (Ryle, 1945) steht hier das Wissen - als „knowing that“ - der Interviewten zu den Prozessen und der Organisation selbstbestimmter Teilhabe in stationären Altenpflegeeinrichtungen im Zentrum. ${ }^{50}$ So wird das Expert*innen- und Laienwissen innerhalb der folgenden neun Akteur*innengruppen in den ausgewählten Einrichtungen wie folgt berücksichtigt:

Leitungen und Mitarbeiter*innen $(n=28)$

1. Einrichtungsleitung $(n=4)$

2. Leitung bzw. Mitarbeitende Sozialer Dienst $(n=4)$

3. Hauswirtschaftsleitung $(\mathrm{n}=4)$

4. Leitung Haustechnik $(\mathrm{n}=4)$

5. Pflegedienstleitung $(\mathrm{n}=4)$

6. Pflegefachkraft $(\mathrm{n}=4)$

7. Zusätzliche Betreuungskraft $(n=4)$

50 Die Erkundung des „knowing how“ findet demgegenüber vornehmlich in den teilnehmenden Beobachtungen und ihren Analysen statt. Das Projekt folgt hier dem praxeologischen Verständnis von Reckwitz (2010): „Die Kritik dieser im weitesten Sinne ,praxeologischen' Analytiken richtet sich gegen die rationalistischen Voraussetzungen eines Homo oeconomicus und eines Homo sociologicus gleichermaßen und damit gegen das klassische Konzept einer Handlungstheorie, die vom zweckrationalen oder regelorientierten Handeln ausgeht. Stattdessen treten die wissensabhängigen rekursiven Praktiken in den Blick, wobei, Wissen' sich in dieser Perspektive von einem Aussagesystem primär zu einem körperlich-leiblich verankerten know how-Wissen transformiert" (Reckwitz, 2010, S. 185f). 


\section{Forschungsdesign}

Bewohner*innen und Angehörige $(n=20)$

8. Bewohner*in $(\mathrm{n}=8)$

9. Angehörige $(\mathrm{n}=12)$

\section{Zugang zu den Interviewpersonen}

Bei der Auswabl der konkreten Interviewpersonen innerhalb der Akteur*innengruppen spielten die Bereitschaft und Zugänglichkeit eine große Rolle, da Bereitschaft zu einem intensiven Gespräch aber auch praktische Erwägungen, wie beispielsweise die Verfügbarkeit für ein Interview während der Arbeitszeit, berücksichtigt werden mussten. Die Auswahl innerhalb der Befragungsgruppen erfolgte durch Gatekeeper, in der Regel durch die Einrichtungs- oder Pflegedienstleitungen und Mitarbeitende des Sozialen Dienstes. Das Auswahlkriterium lag auf der Bereitschaft zur Teilnahme an dem Interview. Dieses Argument wog schwerer als das Argument, dass dadurch möglicherweise aus Einrichtungssicht „problematische“ Interviewpersonen, die kritische Inhalte äußern könnten, nicht ausgewählt werden. Bei den Angehörigen wurde mit Bezug zu den Forschungsfragen versucht, solche Personen zu finden, die sich zu unterschiedlichen Bewohner*innentypen - unterschieden nach „Orientierungsgrad“ und „Mobilität“ - äußern konnten. Innerhalb der Bewohner*inneninterviews wurde in der Auswahl zudem versucht, die in der Literatur bekannten unterschiedlichen Teilhabewünsche von Männern und Frauen ${ }^{51}$ abzubilden. Außerdem wurden für diese umfassenden, leitfadengestützten Bewohner"inneninterviews bewusst keine Bewohner*innen mit einer diagnostizierten Demenzerkrankung ausgewählt (siehe dazu im Folgenden die Berücksichtigung dieser Bewohner*innengruppen im Rahmen der teilnehmenden Beobachtung und informellen Kurzkontakte).

Die Befragungsgruppen bilden somit multiperspektivisch die verschiedenen in einer Einrichtung agierenden Gruppen nach Lebenswelten bzw. professionellen Perspektiven ab: Teilhabe als berufliche Aufgabe von Mitarbeitenden, Teilhabe als eigenes Recht und Wunsch von Bewohner*innen

51 Die Autor*innen des Landesförderplanes Alter und Pflege betonen die Bedeutung der Berücksichtigung von folgenden Aspekten bei der Gestaltung selbstbestimmter Teilhabe: „Zu den wesentlichsten Aspekten zählen insbesondere Migration, Religion, Gender und geschlechtliche Identität" (Ministerium für Gesundheit, Emanzipation, Pflege und Alter des Landes Nordrhein-Westfalen, 2017, S. 10). 
der Einrichtung sowie Teilhabe als Recht und Wunsch von Angehörigen. Erhoben wurde einerseits das praxisgesättigte Betriebs- und Kontextwissen, welches als Expert"innenwissen der Mitarbeitenden und Leitungskräfte (Meuser \& Nagel, 1991) vorliegt. In den Interviews mit Bewohner*innen und Angehörigen wurden andererseits vornehmlich die subjektive Wahrnehmung und Verarbeitungsweise der hier im Fokus stehenden Problemstellung (Witzel, 2000) als betroffene „Laien“ eruiert.

\section{Aufbau des Interviewleitfadens}

Die Interviews verliefen leitfadengestützt unter Berücksichtigung von narrativen Elementen, um durch die Gesprächslenkung das breite Spektrum von Inhalten sowohl zur Organisation als auch die Einschätzung und Relevanzsetzung der Interviewperson zum Thema selbstbestimmte Teilhabe allgemein und zum Stand der selbstbestimmten Teilhabe in der Einrichtung zu sichern. Dies ist strukturell insbesondere bei dem Typus des problemzentrierten Interviews (Witzel, 2010) durch die Verknüpfung von Offenheit und Gegenstandsbezug gegeben, dessen Prinzipien sowohl in den Interviews mit den Bewohner*innen und Angehörigen als auch bei den Expert"inneninterviews mit den Mitarbeitenden berücksichtigt wurden. ${ }^{52}$ Offenheit entsteht durch den im Interview vorgesehenen Einstieg. Der erste offene Erzählstimulus bietet Gelegenheit zur narrativen Entfaltung der eigenen Relevanzsetzung und der subjektiv (berufs-)biografischen Einordnung von Ereignissen. Gegenstandsbezug entsteht im Anschluss durch das Wechselspiel von Fragen und Antworten zum Interviewthema, bei der die Interviewperson themenbezogen das eigene Begründungswissen als Expert"in expliziert.

Der Leitfaden orientierte sich an Fragen mit thematischem Bezug zu Inhalten, die in einem einrichtungsumfassenden Musterrahmenkonzept interessieren können und wurde auf Basis von theoriegeleiteten Überlegungen im Projektteam entwickelt sowie im Wissenschaftlichen Beirat und im Projektrat in Bezug auf die Struktur und Themenkomplexe vorgestellt und

52 Auch wenn bei Expert*inneninterviews üblicherweise andere Wissenszugänge und -formen als in problemzentrierten Interviews fokussiert werden und diesbezüglich auch bei STAP - wie beschrieben - die Expert*innen in Bezug auf ihr Betriebs- und Kontextwissen in und zu den Einrichtungen von Interesse waren, so wurden aber strukturelle Prinzipien des problemzentrierten Interviews zur Leitfadengestaltung ebenfalls bei den Expert*inneninterviews berücksichtigt. 


\section{Forschungsdesign}

abgestimmt. Davon ausgehend wurden vier Leitfäden unterschieden (nach Leitungskräften, Mitarbeitenden, Bewohner*innen und Angehörigen), die in den Fragestellungen und im Umfang den unterschiedlichen Befragungsgruppen angepasst wurden. Im Folgenden wird exemplarisch der thematische Aufbau des Interviewleitfadens für Mitarbeitende aufgeführt:

Teil 1: Zur Person (z. B. Tätigkeitsdauer und Aufgaben)

Teil 2: Eruierung Relevanzrahmen (Beschreibung Arbeitsalltag)

Teil 3: Handlungsleitende Deutungsmuster (z. B. Verständnis von Teilhabe)

Teil 4: Erkundung Praxis (z. B. Teilhabeziele, Teilhabekultur, Zielgruppenberücksichtigung, Angebotsplanung)

Teil 5: Prozess- und Ergebnisqualität (z. B. Ergebnissicherung und Dokumentation der Teilhabeförderung)

Teil 6: Potenziale und Grenzen (z. B. Bedingungen teilhabeförderlicher Arbeit, Mitarbeiter*innenpotenziale)

Teil 7: Möglichkeit zu einer Schlussbemerkung (als Einstellung, Meinung oder als Verbesserungsvorschlag).

Innerhalb von Teil 4 ist als Besonderheit zu erwähnen, dass den Interviewpersonen eine Ressourcenliste vorgelegt wurde, die sowohl finanzielle, personale als auch sachlich-räumliche Ausstattung als Ressourcen umfasste. Damit wurde ein weites Begriffsverständnis zugrunde gelegt.

Zudem ist darauf hinzuweisen, dass im Rahmen des sechsten Teils des Interviews anhand von drei Dilemmasituationen, die widersprüchliche Anforderungen zwischen Sicherheit und Ressourcen einerseits sowie Selbstbestimmung und Teilhabe andererseits beinhalteten, Begründungen der Interviewpersonen provoziert wurden.

\section{Pretest und Anpassung}

Alle Interviewleitfäden wurden jeweils innerhalb eines Interviews in einer Einrichtung des Caritasverbandes, die nicht zu den vier Projekteinrichtungen zählte, von beiden Interviewerinnen getestet. Die jeweils andere anwesende Interviewerin sowie ein weiterer Projektmitarbeiter beobachteten und führten neben der Audioaufzeichnung ein Protokoll. Insbesondere die Interviewleitfäden der Interviewpersonen mit vergleichsweise geringerer Qualifikation sowie mit Laienwissen wurde verändert. 
Interviewdurchführung und -dauer

Die Durchführung der Interviews erfolgte innerhalb der jeweiligen Einrichtung, entsprechend den gegebenen Bedingungen in verfügbaren Räumen, in der Regel im Besprechungsraum.

Die Dauer der Interviews variierte deutlich und umfasste bei den Leitungen und Mitarbeitenden zwischen 47 und 153 Minuten. Bei den Interviews mit den Bewohner*innen variierte die Dauer zwischen 29 und 68 Minuten sowie bei den Angehörigeninterviews zwischen 30 und 64 Minuten.

B) Teilnehmende Beobachtung und informelle Gespräche

Neben den Interviews waren teilstrukturierte, teilnehmende Beobachtungen in Verbindung mit nichtstrukturierten, informellen Kurzgesprächen der zweite wesentliche Bestandteil der qualitativen Analysen in den Projekteinrichtungen. Intention war dabei, insbesondere die Alltags- und Angebotsgestaltung in den Einrichtungen und damit wesentliche Kontexte und Elemente der Teilhabeförderung unmittelbar und situativ erfahren zu können, um damit ergänzende Einsichten generieren zu können, die nicht oder weniger über Interviews zugänglich sind. Angenommen wird hier, dass der Ort des impliziten Wissens zu selbstbestimmter Teilhabe die soziale Praxis selbst ist (Reckwitz, 2010), insbesondere deswegen, da Mitarbeiter*innen bislang nicht auf ausgearbeitete Konzepte, Handlungsempfehlungen oder Dokumentationsinstrumente zur Teilhabeförderung in den Einrichtungen zurückgreifen können.

Das implizite, praktische Wissen und dessen Performativität - das Können im Sinne eines „knowing how" der Mitarbeitenden und Bewohner“innen - erschließt sich somit erst in der Beobachtung der Praxis von Teilhabe. So ist ein direkter Zugang zum praktischen Handeln der Bewohner*innen und Mitarbeitenden herzustellen, um Informationen über die Herstellung von Teilhabe in der konkreten sozialen Situation zu generieren. Untersuchungsgegenstand ist somit die Handlungspraxis der beforschten Personen innerhalb „natürlicher“ Gesprächs- und Praxissituationen (Bohnsack et al., 2007; Streblow, 2005). Mit der Methodik der offenen, teilnehmenden Beobachtung (Döring \& Bortz, 2016) in der natürlichen Umgebung besteht die Möglichkeit, dass die Forschenden in den Handlungssituationen in Interaktionen und Kommunikation einbezogen sind oder dies verständnisgenerierend initiieren. Im Rahmen der teilnehmenden Beobach- 


\section{Forschungsdesign}

tungen wurden zudem informelle Kurzgespräche mit Bewohner*innen geführt, die offen und spontan in den Beobachtungssituationen erfolgten.

Die teilnehmenden Beobachtungen wurden in STAP auch als Strategie zum Einbezug von kognitiv eingeschränkten Bewohner*innen - etwa Menschen mit einer Demenz - genutzt. Gerade durch die informellen Kurzgespräche und Interaktionen innerhalb von Handlungssituationen mit Bewohner*innen können diese so einbezogen werden, dass sie ihren subjektiven sozialen Sinn der Gesprächs- und Praxissituationen verdeutlichen können. Durch den konkret erfahrbaren Handlungsgegenstand (die gerade erlebte Praxissituation) und die Kürze des Gesprächs konnten also auch Äußerungen von Bewohner*innen mit einem eingeschränkten Kurzzeitgedächtnis berücksichtigt und kontextbezogen nachvollzogen werden. Im Gesprächskontakt mit kognitiv eingeschränkten bzw. psychisch veränderten Bewohner*innen wurde sich an vorher im STAP-Team dokumentierten Regeln zur Selbstverpflichtung in der Beobachtung und bei Gesprächen mit vulnerablen Personen gehalten, die an Konzepte des „informed consent“ (z. B. Klie, Vollmann \& Pantel, 2014) und das auf Menschen mit Demenz bezogene Konzept des „process (ongoing) consent“ (Dewing, 2007) angelehnt waren. Mit Blick auf die Akteur*innengruppe (die Bewohner*innen) ist zur Methodenwahl der Beobachtung im Rahmen von STAP abschließend hervorzuheben, dass die routinierten Abläufe der eigenen Lebensführung oder die der Teilhabe in einer stationären Einrichtung so habitualisiert sein können, dass dies den Bewohner*innen eine Reflexion sowie eine Versprachlichung erschwert. Kommen noch krankheitsbedingte Einschränkungen bei den Explikationen des Erlebten hinzu, erweist sich eine Beobachtung als ein vergleichsweise geeigneter Erhebungszugang, um das Erleben und Aussagen der Bewohner*innen kontextnah zu erfassen.

\section{Feldvorbereitung}

Im Rahmen der Feldvorbereitung besuchten die Beobachterinnen ${ }^{53}$ einführend einen projektinternen Workshop mit einer Forscherin im Bereich „Medical Anthropology“, die bereits über umfassende Erfahrungen in der Durchführung von teilnehmenden Beobachtungen im Kontext medizi-

53 Die Beobachtungen wurden von weiblichen Mitgliedern des Forschungsteams durchgeführt. 
nisch-pflegerischer Versorgung verfügt. ${ }^{54}$ Hierbei wurden insbesondere Fragen der praktischen Umsetzung im Feld thematisiert, wie etwa das Führen des Feldtagebuches. Aufgrund der geplanten Zeit von circa 10 Tagen im Feld sowie mit Blick auf Forschungsthema und -setting wurde dem STAP-Team geraten, das Ziel einer qualitativen, teilstrukturierten Beobachtung beizubehalten und dabei aber (a) vorher Beobachtungssituationen und ihre Gegenstände zu fokussieren und (b) die Settings auch nach Beobachtungspfaden, v. a. ausgehend vom Sozialen Dienst, auszuwählen.

Zur Vorbereitung der Feldaufenthalte war zudem von zentraler Bedeutung, dass nicht nur die Mitarbeiter*innen, sondern auch die Bewohner*innen, ihre nahen Angehörigen oder gegebenenfalls die gesetzlichen Betreuer*innen sowohl schriftlich als auch im Rahmen von Gremien und Veranstaltungen über STAP informiert und dass für die Bewohner*innen Einwilligungserklärungen eingeholt wurden. Für jede/n Bewohner*in der vier Projekteinrichtungen wurde eine differenzierte Einwilligungs- bzw. Nichteinwilligungserklärung zu den beiden Erhebungsmethoden der Beobachtung und Kurzgespräche ausgegeben.

Sampling und Zugang zu den Beobachtungssituationen

In allen vier Einrichtungen der Studie wurde zunächst ein Tag vereinbart, an dem die Beobachterinnen sich mit der Leitung oder Mitarbeitenden des Sozialen Dienstes in der Einrichtung bewegten und beispielsweise den Wohnbereichsleitungen vorgestellt wurden. Dies war in einer Einrichtung nicht der Fall, so dass die Beobachterin im Anschluss an einen gemeinsamen Termin das Haus selbst erkundete und Verantwortliche ansprach, um Vereinbarungen zu treffen und das Projekt vorzustellen. Innerhalb dieses ersten Tages wurden Notizen von den Beobachterinnen zu möglichen Beobachtungsanlässen, den räumlichen Gegebenheiten und Hinweise auf teilhaberelevante Initiativen einzelner Personen angefertigt.

Als Beobachtungssituationen wurden zunächst die folgenden vier Teilhabesettings (in Anlehnung an Garms-Homolová \& Theiss, 2009) theoriegeleitet entwickelt, die sich wesentlich nach Formalität und Sozialität unterscheiden:

54 Die Referentin und Beraterin im Rahmen des Workshops war Dr. Julia QuarzTopp (Forschungsstudien u. a. QUASER, SituCare). 
1. Angebote innerhalb und außerhalb der Einrichtung

Aktivität: kommunizierte, formalisierte Angebote innerhalb und außerhalb der Einrichtung $\rightarrow$ räumlich (z. B. Aufenthaltsraum, Saal, „Funktionsräume“ wie Kegelbahn, Malatelier usw. sowie Büro des Sozialen Dienstes, Ausflugsziel)

2. Regelmäßige informelle Treffen

Aktivität: z. B. Kartenspielen, Rauchen $\rightarrow$ räumlich: Wohnbereich, Treffpunkte, Bewohner*innenzimmer

3. Informelle Begegnungen

Aktivität: Pflege von Freundschaften und Kontakten im Alltag $\rightarrow$ räumlich: Wohnbereich, Bewohner*innenzimmer

4. Alleinsein

Aktivität: (angenommene) Nichtnutzung, Rückzug und Passivität $\rightarrow$ räumlich: Bewohner*innenzimmer, in der Nähe eines Angebotes

Orientiert an den theoretisch vordefinierten Teilhabekontexten wurden dann die konkreten Beobachtungssettings und -pfade in der einzelnen Einrichtung mit dem oder der Projektverantwortlichen der Einrichtung abgestimmt und anschließend in einem Beobachtungsplan (Flick, 2006, S. 207) festgehalten. Der vorher festgelegte Beobachtungsplan (siehe Tabelle 11) war aus den folgenden drei Gründen notwendig und hilfreich: Erstens lag nicht für alle Bewohner*innen eine Einwilligungserklärung zu einer Beobachtung vor. Fehlte sie oder wurde sie abgelehnt, so mussten diese Bewohner*innen explizit in der Absprache mit der jeweiligen vorab informierten Wohnbereichsleitung ausgeschlossen oder ein anderer Beobachtungsbereich gewählt werden. Zweitens war eine Vorbereitung der Mitarbeiter*innen sinnvoll, um die Intention der Studie und die Rolle der Beobachterin zu verdeutlichen. Dies erwies sich auch deswegen als förderlich, weil die Beobachtungsituationen von den Mitarbeiter*innen ansonsten eventuell als die gewohnten Qualitätsprüfungen interpretiert worden wären und sie sich dementsprechend verhalten hätten. Drittens wurde planerisch in der zweiwöchigen Beobachtungsphase versucht, Selbstbestimmung und Teilhabe in Routinen, in unorganisierten und organisierten Settings zu verschiedenen Tageszeiten von unterschiedlichen Bewohner"innentypen abzudecken. Dazu erwiesen sich die Rückmeldungen und Anregungen der Mitarbeiter*innen und Bewohner*innen innerhalb des Beobachtungsprozesses als sehr hilfreich. So w0urde der Beobachtungsplan mehrfach verändert und in Absprache mit der Einrichtung angepasst. Tabelle 11 zeigt exemplarisch den Beobachtungsplan in einer Einrichtung. 
Tabelle 11: Beispiel für einen Beobachtungsplan der Einrichtung C

\begin{tabular}{|c|c|c|}
\hline Termin & Beobachtungsvorhaben & Ort \\
\hline $\begin{array}{l}\text { 3.7.2017, } \\
\text { 9:00-16:45 Uhr }\end{array}$ & $\begin{array}{l}\text { Begleitung des Sozialen Dienstes, Kennenler- } \\
\text { nen der Wohnbereiche, Verabredungen; Beob- } \\
\text { achtung der Leitungsrunde der Einrichtung } \\
\text { und einer Einzelbetreuung im Zimmer }\end{array}$ & $\begin{array}{l}\text { Haus, Büro, } \\
\text { Bewohner*innen- } \\
\text { zimmer }\end{array}$ \\
\hline $\begin{array}{l}\text { 9.7.2017, } \\
\text { 9:30-12:00 Uhr }\end{array}$ & $\begin{array}{l}\text { Besuch des Gottesdienstes mit vorheriger An- } \\
\text { sprache, wer interessiert ist; Weg in die Kapelle, } \\
\text { Gottesdienstteilnahme, Weg zurück. }\end{array}$ & $\begin{array}{l}\text { Wohnbereich 1, } \\
\text { Kapelle }\end{array}$ \\
\hline $\begin{array}{l}\text { 10.7.2017, } \\
\text { 13:30-ca. 16:15 Uhr }\end{array}$ & $\begin{array}{l}\text { Selbstorganisierte Spielrunde: Aufbau, erste } \\
\text { Runde mit zwei Frauen, Spielerunde zu viert, } \\
\text { Abbau }\end{array}$ & $\begin{array}{l}\text { Konferenzraum, } \\
\text { unten }\end{array}$ \\
\hline $\begin{array}{l}\text { 11.7.2017, } \\
\text { 6:30-12:30 Uhr }\end{array}$ & $\begin{array}{l}\text { Wohnbereich 1: Beginn Frühschicht, Übergabe } \\
\text { des Nachtdienstes, morgendliche Gestaltung, } \\
\text { insbesondere Wecken, Aufstehen, Frühstücken; } \\
\text { Angebot Vorlesen, Übergang zum Mittagessen }\end{array}$ & Wohnbereich 1 \\
\hline $\begin{array}{l}\text { 12.7.2017, } \\
\text { 14:00-17:00 Uhr }\end{array}$ & $\begin{array}{l}\text { Nachmittagsgestaltung: ggf. Spaziergänge, Nut- } \\
\text { zung des Umfelds, Begleitung der Betreuungs- } \\
\text { assistenz }\end{array}$ & Wohnbereich 3 \\
\hline $\begin{array}{l}\text { 17.7.2017, } \\
\text { 16:30 Uhr }\end{array}$ & Ausflug Männerrunde & $\begin{array}{l}\text { Gaststätte, } \\
\text { Biergarten }\end{array}$ \\
\hline $\begin{array}{l}\text { 19.7.2017, } \\
\text { 9:00-14:30 Uhr }\end{array}$ & $\begin{array}{l}\text { Vormittagsgestaltung Wohnbereich 2: Bedeu- } \\
\text { tung von Medien/Zeitung. Anschließend Be- } \\
\text { such Beiratssitzung, Interview Bewohner }\end{array}$ & $\begin{array}{l}\text { Wohnbereich } 2 \text {, } \\
\text { Konferenzraum }\end{array}$ \\
\hline $\begin{array}{l}\text { 25.6.2017, } \\
\text { 10:00-12:00 Uhr }\end{array}$ & $\begin{array}{l}\text { Interview Bewohnerin (9:15 Uhr), Angebot } \\
\text { Musik }\end{array}$ & $\begin{array}{l}\text { Wohnbereich } 1 \text {, } \\
\text { Konferenzraum }\end{array}$ \\
\hline $\begin{array}{l}\text { 26.7.2017, } \\
\text { 7:30-ca. 11:30 Uhr, } \\
\text { Übergabe, } \\
\text { 17:00-21:00 Uhr }\end{array}$ & $\begin{array}{l}\text { Wohnbereich 3: morgendliche Gestaltung, ins- } \\
\text { besondere Wecken, Aufstehen, Frühstücken; } \\
\text { Bedeutung von Medien, insbesondere Fernse- } \\
\text { hen. Wohnbereich 2: Abendgestaltung (Abend- } \\
\text { essen, Zubettbegleiten/Abendpflege, informelle } \\
\text { Treffs, ggf. am Empfang oder im Speisesaal un- } \\
\text { ten) }\end{array}$ & $\begin{array}{l}\text { Wohnbereich } 3 \text {, } \\
\text { Wohnbereich } 2 \text {, } \\
\text { Haus }\end{array}$ \\
\hline
\end{tabular}

\section{Beobachtungsprotokoll}

Die teilstrukturierte Protokollierung der Beobachtung erfolgte anhand von Feldnotizen innerhalb und im Anschluss an die Situation (Lamnek, 2005). Hierbei wurden vorher festgelegte Kriterien berücksichtigt, die sich auf Kontexte (z. B. Uhrzeit, Ort, Personengruppen) und Dimensionen der Beobachtung (s. u.) bezogen haben. Konnten in der Situation nur Stichworte mitgeschrieben werden, so wurden diese möglichst zeitnah, noch teilweise 


\section{Forschungsdesign}

in der Einrichtung vervollständigt. Für sehr umfassende Beobachtungen wurde auch das Aufnehmen von diktierten Eindrücken per Aufnahmegerät genutzt, die anschließend in schriftliche Notizen überführt wurden.

Die Beobachtungsdimensionen waren:

- Zugang oder Nichtzugang zu Situationen, Prozessen und Angeboten der Teilhabe

- Herstellen oder Nichtherstellen von Teilhabe in der Interaktion

- Wird - individuell - das Prinzip der Selbstbestimmung im Rahmen von Teilhabe ermöglicht, wenn ja wie? Oder inwieweit wird es verhindert?

- Ressourcen (Art) und Ressourceneinsatz (Weise) bei Teilhabe oder Nichtteilhabe

Unter den einzelnen Kategorien subsumiertes Material wurde hinsichtlich zweier Merkmale geordnet und gewichtet. Diese Merkmale waren an der Normalität $^{55}$ und an die für die Teilhabeförderung entstehenden Effekte ${ }^{56}$ orientiert.

\section{Informelle Kurzgespräche}

Um einen Zugang zu dem in der Situation subjektiven Sinn der Teilhabe zu erfahren, wurden einzelne Bewohner*innen innerhalb oder im Anschluss an die Situation auf einzelne Aspekte angesprochen. Dazu zählten die Beobachtungssituation kontextualisierende Fragen an die Bewohner"innen, wie beispielsweise danach, was ihnen an dem Angebot gefallen und nicht gefallen hat, was davon für sie wichtig und bedeutsam ist, ob sie auch schon früher vor ihrem Einzug ähnliche Aktivitäten ausgeübt haben.

Die informellen Gespräche ergaben sich zufällig in der Situation durch den aktiven Einbezug der Beobachterinnen oder wurden von diesen im Anschluss an eine Beobachtungssituation spontan initiiert. Sie verliefen daher offen und orientierten sich an den aufgebrachten Themen der Bewohner*innen. So wurde auch die Suche nach geeigneten Interviewpersonen (über dieses Verfahren wurden zwei Personen gewonnen) unterstützt,

55 Wie bereits in Kapitel 2.3.2 benannt, wird das Normalitätsprinzip in STAP als die Möglichkeit zur Gestaltung des Lebens nach den in der eigenen Biografie angelegten gewohnten Normen und Routinen verstanden.

56 Prozessgestaltung orientierte sich a) an der Aufrechterhaltung von Normalität oder orientierte sich b) nicht an der Aufrechterhaltung von Normalität und wurde somit im Effekt als teilhabefördernd oder hemmend bewertet. 
die selbstbestimmte Teilhabe detailreicher darstellten und somit für die Teilnahme an leitfadengestützten Interviews zugänglich waren. Die informellen Kurzgespräche wurden ebenso wie die Beobachtungen im Rahmen der Feldnotizen dokumentiert.

\subsubsection{Auswertungsmethoden}

Mit den Interviews und den teilnehmenden Beobachtungen sowie den damit verbundenen informellen Gesprächen lagen verschiedene Datenquellen vor, deren Verhältnis zueinander nicht nur in der Durchführung, sondern auch in der Auswertung zu klären war. Zeitlich war die Beobachtungsphase der Interviewphase nachgeordnet oder lediglich teilweise überlappend, so dass eine Fülle von einrichtungsinternen Informationen, aber auch Begegnungen in der Einrichtung vor der Beobachtung vorlagen. Insgesamt lag der Schwerpunkt der qualitativen Analyse auf der Auswertung der Interviews, so dass die teilnehmende Beobachtung eine ergänzende Stellung in der Analyse einnahm (vgl. z. B. Farrokhzad \& Mäder, 2014, S. 50). Angewendet auf das Forschungsdesign des vorliegenden Forschungsprojektes bedeutete dies:

- Interviewpersonen schilderten regelmäßig vorkommende Ereignisse, die anschließend beobachtet werden konnten,

- vorangegangene Beobachtungen konnten in anschließend stattfindenden Interviews herangezogen werden,

- Beobachtungen konnten als Kontext in die Interviewanalyse einbezogen werden, entweder ergänzend oder als Kontrapunkt.

Auswertungsmethode der Interviews und Beobachtungen

Sowohl mit Bezug auf die Zielsetzung, einen möglichst breiten Überblick über fördernde und hemmende Faktoren zur Unterstützung und Verwirklichung von selbstbestimmter Teilhabe zu gewinnen, als auch mit Blick auf den Umfang des erhobenen Materials über verschiedene Gruppen von Interviewpersonen und Einrichtungen (allein die Transkription der 48 Interviews hatte einen Gesamtumfang von rund 2.100 Textseiten), fiel die Wahl auf ein inhaltsanalytisches Verfahren (Mayring, 2010) - hier dann speziell im Sinne der inhaltlich strukturierenden Analyse nach Kuckartz (2016). Qualitativ inhaltsanalytische Verfahren können als „Forschungsmethode zur Systematisierung und Interpretation von manifesten und laten- 


\section{Forschungsdesign}

ten Kommunikationsinhalten unter Verwendung eines Kategoriensystems" (Stamann et al., 2016, Abschnitt 24) verstanden werden. Ziel der inhaltlich-strukturierenden Inhaltsanalyse bei STAP war es, das Material im Hinblick auf einzelne Aspekte - hier ausgewählte Themen mit Bezug zu einem möglichen teilhabeförderlichen Musterrahmenkonzept - systematisch kategorisierend zu ordnen. Die Themen wurden deduktiv festgelegt und im Verlauf der Analyse induktiv ausdifferenziert. Diese bildeten zugleich die Struktur des Kategoriensystems (vgl. Mayring, 2015; Schreier, 2014).

Auch die Daten aus den Beobachtungen und Kurzgesprächen wurden inhaltsanalytisch ausgewertet. Inhaltsanalytische Verfahren können ebenfalls auf Beobachtungsinhalte angewendet werden, wenn folgendes Methodenverständnis vorliegt: Beobachtungsinhalte sind Kommunikationsinhalte und anhand eines Sender-Empfänger-Modells intersubjektiv für die Beobachterin nachvollziehbar. Der/die mitweltliche Beobachter*in (Merkens, 1992) hat ein fachlich begründetes Vorverständnis und versteht prinzipiell den Sinn der beobachteten Praktiken aus ihrer Perspektive, die vor und nach der Beobachtung unter fachlich definierbaren Kategorien subsumierbar sind.

Transkription der Interviews

Die vollständige Übertragung der Audiodaten erfolgte mit einem einfachen Transkriptionssystem (Flick, 2016), welches sich auf lautsprachlichen Äußerungen bezieht. Im Sinne der Fragestellung wurde weder eine linguistische noch eine auf die Konversation bezogene Analyse intendiert. Anschließend wurde das verschriftlichte Material mit einer EDV-gestützten Auswertung über das Programm MAXQDA (Rädiker \& Kuckartz, 2019) bearbeitet.

Kategoriensystem Interviews

Das Kategoriensystem orientierte sich deduktiv sowohl an den Untersuchungsfragen als auch an dem Interviewleitfaden sowie an Dimensionen der Aufbau- und Ablauforganisation von Altenpflegeeinrichtungen. Im zweiten Schritt erfolgte die Bildung induktiver Kategorien aus dem Material. Eine Übersicht zu den deduktiv gebildeten Hauptkategorien des Kategoriensystems bietet die folgende Tabelle. 
Tabelle 12: Kategoriensystem der Interviews

\begin{tabular}{|l|l|}
\hline Code & Definition \\
\hline Wünsche & $\begin{array}{l}\text { Äußerungen, die sich auf das Erbringen und Er- } \\
\text { fassen von BW- Bedürfnissen und Vorstellungen } \\
\text { beziehen. Auch negativ: Kritik als ein Ände- } \\
\text { rungswunsch. }\end{array}$ \\
\hline $\begin{array}{l}\text { Praxis der Selbst- } \\
\text { bestimmung }\end{array}$ & $\begin{array}{l}\text { Aussagen, die sich auf die Herstellung und Um- } \\
\text { setzung des Alltagslebens nach BW-Relevanzen } \\
\text { beziehen. }\end{array}$ \\
\hline Praxis der Teilhabe & $\begin{array}{l}\text { Aussagen, die sich auf die Herstellung und Um- } \\
\text { setzung des Alltagslebens, insbesondere des so- } \\
\text { zialen Lebens von BW innerhalb und außerhalb } \\
\text { der Einrichtung beziehen. }\end{array}$ \\
\hline $\begin{array}{l}\text { Ergebniskontrolle der } \\
\text { Teilhabe }\end{array}$ & $\begin{array}{l}\text { Aussagen, die Maßnahmen und/oder Instru- } \\
\text { mente der Überprüfung von Teilhabe beinhal- } \\
\text { ten und/oder deren Verankerung in Einrich- } \\
\text { tungsdokumenten thematisieren. }\end{array}$ \\
\hline Bewohner*innen & $\begin{array}{l}\text { Aussagen, in denen Eigenschaften, Situationen } \\
\text { oder Lebenslagen von BW beschrieben werden. }\end{array}$ \\
\hline Mitarbeiter*innen & $\begin{array}{l}\text { Mitarbeiter*innenbezogene Aussagen, die sich } \\
\text { auf arbeitsbezogene Einstellungen, Fähigkeiten } \\
\text { und Eigenschaften beziehen. }\end{array}$ \\
\hline Kultur & $\begin{array}{l}\text { Aussagen, die sich auf das den Handlungen hin- } \\
\text { terlegte Verständnis von Werten und Normen } \\
\text { beziehen, als Gruppe (Team, Kolleg*innen) } \\
\text { oder des eigenen Berufsstandes. }\end{array}$ \\
\hline $\begin{array}{l}\text { Ressourcen/Rahmen- } \\
\text { bedingungen } \\
\text { organisation }\end{array}$ & $\begin{array}{l}\text { Aussagen, die einen faktischen oder potenziel- } \\
\text { len Beitrag oder Einfluss auf Teilhabeunterstüt- } \\
\text { zung umfassen. }\end{array}$ \\
\hline Planung/Ablauf- \\
organisation & $\begin{array}{l}\text { Aussagen, die sich auf die strukturellen Grund- } \\
\text { lagen der Einrichtung beziehen. }\end{array}$ \\
\hline $\begin{array}{l}\text { Aussagen, die sich auf Einrichtungsprozesse zur } \\
\text { Erbringung von Dienstleistungen und deren } \\
\text { Gestaltung sowie Kontrolle beziehen. }\end{array}$ \\
\hline $\begin{array}{l}\text { Umgang mit Zeit und/oder Zeit als strukturie- } \\
\text { render Faktor. }\end{array}$ \\
\hline andbau-
\end{tabular}




\section{Forschungsdesign}

Nach der ersten Sichtung des Materials konnten nur wenige Spezifika gefunden werden, die auf grundlegend differierende Themen und Aussagen zwischen den einzelnen Interviewpersonengruppen hinweisen. Ebenso ließ sich keine gewichtige Differenz hinsichtlich von Themen und Aussagen zwischen den Einrichtungen finden, so dass die Interpretation nicht akteur*innengruppen- oder einrichtungsbezogen, sondern queranalytisch über den gesamten Textkorpus erfolgte. Allerdings wurde bei der späteren Interpretation von Interviewergebnissen und -aussagen die Akteur*innengruppenquelle berücksichtigt.

Insgesamt wurde - auch angesichts des anwendungsorientierten Charakters der Forschungsstudie - eine deskriptive, aussagenorientierte Analyse angestrebt, bei der die zu einem Code vorgefundenen Aussagen in ihrer Breite dargestellt wurden. Vorbereitend wurden die Textpassagen (Codes) der Haupt- sowie Subkategorien paraphrasiert und anschließend nach Themen zusammengefasst (Mayring, 2010).

Darüber hinaus wurden die Aussagen pro Thema, möglichst eng am Material, hinsichtlich förderlicher und hemmender Orientierungen und Bedingungen, differenziert. Unterschieden wurde zwischen einer Orientierung an der Normalität (förderlich) - im Sinne des Normalitätsprinzips und einem für die Teilhabeförderung entstehenden Effekt und einer Nichtorientierung an der Normalität (hemmend) und keinem oder geringem für die Teilhabeförderung entstehenden Effekt.

\section{Kategoriensystem Beobachtungen}

Ziel der Auswertung der teilnehmenden Beobachtung war, entsprechend den Beobachtungsdimensionen kontextualisierende Informationen über die Aspekte der Herstellung von Teilhabe zu gewinnen. Diese konnten in der Beobachtung - ergänzend zu den Interviews - insbesondere in Bezug auf Gesprächs- und Handlungssituationen gewonnen werden, die den Alltag der Bewohner*innen strukturieren. Dazu wurde ein Kodierungsschema entwickelt, das nach Sichtung des Beobachtungsmaterials auf den Bewohner"innenalltag strukturierende Schlüsselprozesse im Material fokussierte. ${ }^{57}$

57 Die Definition „den Bewohner*innalltag strukturierenden Schlüsselprozesse“ lautete wie folgt:

Alle überindividuellen, faktisch den Tagesablauf der Bewohner*innen zeitlich und inhaltlich in seinen Üblichkeiten strukturierenden Sequenzen, die von Sei- 
Klassifikation der einzelnen Beobachtungen nach Schlüsselprozessen und Tageszeit:

- Morgendliche und abendliche Routinen (v. a. Wecken, Aufstehen, Grundpflege sowie Ins-Bett-Bringen, Gestaltung des Tagesendes)

- Mahlzeiten: Frühstück, Mittagessen, Nachmittagskaffee, Abendessen

- Vormittags/nachmittags/evtl. abends: Nutzung/Gestaltung von Angeboten (innerhalb, außerhalb, auch Nutzung/Gestaltung Raum- und Materialangebote wie Garten und Lesematerial)

- Vormittags/nachmittags: Nutzung/Gestaltung von Einzelbetreuung

- Vormittags/nachmittags: Nutzung/Gestaltung von Dienstleistungen inhouse (z. B. medizinisch-therapeutische Dienstleistungen, Frisör, Wäscherei, Haustechnik)

- Ganztägig: freie Zeit (individuell verbrachte Zeit, kollektive Wartezeit)

\subsubsection{Fokusgruppendiskussion}

Die Fokusgruppendiskussionen (nach Kühn \& Koschel, 2011) dienten zum Abschluss der in den vier Projekteinrichtungen durchgeführten qualitativen Datenerhebungen - dazu, die in den Einrichtungen des Caritasverbandes gewonnenen Ergebnisse trägerübergreifend zu analysieren und damit auch die Fragebogenentwicklung für die Onlinebefragung im quantitativen Analysepart vorzubereiten. So sollten in den leitfadengestützten Fokusgruppen abschließend kollektiv die Einstellungen zu selbstbestimmter Teilhabe und deren Organisation durch Leitungskräfte und Mitarbeitende der stationären Altenhilfe mit Blick auf ihre Erfahrungen in Einrichtungen unterschiedlicher Träger erfasst werden.

\subsubsection{Samplingstrategie}

Der Feldzugang zu Interviewpersonen für die Fokusgruppen erfolgte über Hinweise auf den Termin und die vorgesehenen Akteur*innengruppen in Gremien auf Landesebene (NRW) und dann im Schneeballsystem über die Gremienteilnehmer*innen (Vertreter*innen auf Leitungsebene freier, pri-

ten der Organisation auch als Arbeitsprozesse definiert sind und im hohen Maße dazu beitragen, dass diese Sequenz entsteht. Im Vordergrund steht der Alltag der Bewohner*innen und nicht der Arbeitsalltag der Mitarbeitenden (also z. B. keine Unterstützungsprozesse und Managementprozesse). 


\section{Forschungsdesign}

vater und öffentlicher Träger), so dass hier wiederum kombiniert Anteile des „Typical Case“-Sampling und „Convenience“-Sampling vorlagen. Abweichend zu den im Forschungsprozess geplanten ${ }^{58}$ drei Fokusgruppen bestehend aus (1) Einrichtungsleitungen, (2) Pflegedienstleitungen und (3) Sozialem Dienst kam es bedingt durch die Anzahl der Rückmeldungen zur Bildung von zwei Gruppen mit insgesamt 16 Personen. So bestanden die Gruppen aus (1) Fachbereichsleitungen, Einrichtungsleitungen und Pflegedienstleitungen $(\mathrm{n}=11)$ sowie (2) Leitung und Mitarbeitende des Sozialen Dienstes $(\mathrm{n}=5)$. Die Gruppenteilnehmer*innen der ersten Gruppe erwiesen sich hinsichtlich ihrer übergeordneten leitenden Funktion als relativ homogen in Bezug auf ihre Sicht auf den Gegenstand. Das Spezifikum der zweiten Gruppe bestand darin, innerhalb des Sozialen Dienstes zu arbeiten, womit der intendierten Homogenität innerhalb einer Akteur*innengruppe ebenfalls entsprochen wurde. Bezogen auf den Träger ihrer Einrichtungen waren die Gruppendiskussionsteilnehmer*innen aber - der Zielsetzung für die Fokusgruppen entsprechend - heterogen und wiesen Zugehörigkeiten zu verschiedenen freigemeinnützigen Trägern (Deutsches Rotes Kreuz, Diakonie, Caritas, Arbeiterwohlfahrt) sowie einem öffentlichen und zwei privat-gewerblichen Trägern auf.

\subsubsection{Erhebungsmethode}

Die Verwendung von Fokusgruppendiskussionen lässt sich mit verschiedenen Zielsetzungen verbinden und wird dabei häufig in Bezug auf andere Erhebungsmethoden im Rahmen von Methodenkombinationen begrün$\operatorname{det}$ (z. B. Kühn \& Koschel, 2011, S. 18ff). Im Rahmen von STAP wurden die Fokusgruppen eingesetzt, um die bisherigen Befunde aus den Einzelinterviews und den Beobachtungen trägerübergreifend validieren sowie die standardisierte Befragung dementsprechend überprüft vorbereiten zu können (siehe hierzu die Verwendungskontexte der „Methodenkombination“ und „Vorstudie“ bei Kühn \& Koschel, 2011, S. 19ff).

Lamnek \& Krell (2016, S. 388) oder auch Kruse (2014, S. 195) stellen heraus, dass es sich um ein kommunikativ ermittelndes Verfahren handelt, welches thematisch strukturiert mittels von Impulsfragen aufgebaut ist (ebd., S. 195). Innerhalb des Forschungsdesigns von STAP diente die Me-

58 Im Projektantrag war ursprünglich nur eine Fokusgruppe vorgesehen, im Forschungsprozess haben wir uns aber für eine Differenzierung in ausgewählte Akteur*innengruppen entschieden. 
thodenkombination der Gruppendiskussion mit den anderen Methoden einer komplementären Strategie (Kühn \& Koschel, 2011, S. 21; Lamnek \& Krell, 2016, S. 389), welche der angesprochenen trägerübergreifenden Validation diente.

Die Diskussionen in den Fokusgruppen zielte daher einerseits auf den Vergleich mit einzelnen Ergebnissen der qualitativen Erhebung und rückten aber auch gering berücksichtigte Aspekte in den Blick. In den Fokusgruppen wurden daher einzelne Fragen, angelehnt an die Interviewleitfäden, strukturierend eingebracht. Durch diese Impulsfragen wurde ein Vergleich mit den Interviews möglich (Schulz et al., 2012, S. 27); zugleich ermöglichte die offene Fragestellung, dass explorativ neue Aspekte herausgearbeitet werden konnten (Flick, 2006, S. 190f).

Andererseits wurden aus den qualitativen Interviews relevante Ergebnisdimensionen und zentrale Aussagen als Diskussionsanreiz (z. B. als Interviewzitat) in die Fokusgruppe eingebracht. So konnten Einschätzungen zu den bisher herausgearbeiteten Erkenntnissen gewonnen werden. Sichergestellt wurde dies durch einer an einem Leitfaden orientierten Moderation, die von einer zweiten Person durch Visualisierungsmethoden unterstützt wurde.

Durch die Länge der leitfadengestützten Diskussion von rund zwei Stunden bot sich eine Audioaufzeichnung an (Schulz et al., 2012), zusätzlich wurde ein diskussionsbegleitendes Protokoll verfasst. Die vollständig transkribierten Aufzeichnungen wurden mithilfe der Protokolle ergänzend angereichert.

\subsubsection{Auswertungsmethode}

Die Auswertung der Fokusgruppendiskussionen erfolgte wiederum orientiert an der inhaltlich-strukturierenden Inhaltsanalyse. Jedoch wurde bereits in der Durchführung der Gruppendiskussion deutlich, dass die bisherigen Ergebnisse in Bezug auf die bis dato induktiv entwickelten Subkategorien bzw. -themen sowie die dabei identifizierten fördernden und hemmenden Faktoren selbstbestimmter Teilhabe in Altenpflegeeinrichtungen von den Diskussionsteilnehmer*innen bestätigt wurden. Daher fokussierte die Auswertung primär auf Aussagen, welche die bisherigen Ergebnisse in besonderer Weise inhaltlich ergänzten. 


\subsection{3. „Good-Practice“-Analyse als projektergänzender Zugang}

Die "Good-Practice“-Analyse wurde im Rahmen eines Forschungssemesters von Prof. Dr. Christian Bleck im Jahr 2018 mit Nähe zur STAP-Studie durchgeführt. Ziel war es, näher zu beleuchten, auf welche Weise und mit welchen Ressourcen es in bundesweit ausgewählten Altenpflegeeinrichtungen - mit besonderen Erfahrungen im Themengebiet - gelingt, die selbstbestimmte Teilhabe der dort lebenden Bewohner*innen zu fördern.

Auf den Erfahrungen und Erkenntnissen des zu diesem Zeitpunkt seit 1,5 Jahren laufenden Projektes STAP aufbauend, sollte die „Good-Practice"-Analyse nun in spezifischerer Weise besondere Ansätze zur Förderung von Selbstbestimmung und Teilhabe untersuchen, die sich in Einrichtungen mit innovativen und teils prämierten Konzepten bereits bewährt haben. Somit stellt die "Good-Practice“-Analyse eine ergänzende Studie zu STAP dar, die nun nicht mehr grundlegend fördernde und hemmende Faktoren im Überblick, sondern primär beispielhaft besondere Vorgehensweisen und Ressourcen im Rahmen von Ansätzen guter Praxis identifizieren und die Erfahrungen damit in den ausgewählten Einrichtungen beleuchten möchte.

Der Begriff "good practice“ wurde in der Analyse mit erfolgreichen oder besonderen bzw. innovativen Ansätzen der Praxis verbunden. Der eng verwandte Begriff der „best practice“ wurde daher bewusst nicht verwendet, weil auf die Auswahl einer einzigen „richtigen Lösung“ ebenso wie auf die Idee des Benchmarkings mit einer vergleichenden Analyse verzichtet wird (Krems, 2011).

\section{Forschungsfragen}

Folgende untersuchungsleitende Forschungsfragen wurden für die „GoodPractice“-Analyse formuliert:

- In welcher Weise werden in den ausgewählten stationären Altenpflegeeinrichtungen Selbstbestimmung und Teilhabe ihrer Bewohner*innen gefördert?

- Mit welchen Ressourcen werden in den ausgewählten stationären Altenpflegeeinrichtungen Selbstbestimmung und Teilhabe ihrer Bewohner*innen gefördert? 
Recherche und Auswahl von „Good-Practice“-Einrichtungen

In einem ersten Schritt wurden Einrichtungen der stationären Altenhilfe bundesweit recherchiert, die besondere Ansätze oder innovative konzeptionelle Schwerpunkte zur Förderung von Selbstbestimmung und Teilhabe vorhalten. Die Recherche erfolgte sowohl über deutschsprachige Fachdatenbanken („Gerolit“, „wiso Sozialwissenschaften“, „GESIS-Suche“) als auch über das Internet, wobei die Resultate der Internetanalyse bei diesem Recherchegegenstand ertragreicher waren. Im Ergebnis der Recherche wurden 15 Altenpflegeeinrichtungen identifiziert, wovon wiederum acht Einrichtungen ausgewählt wurden, weil für diese Auszeichnungen oder Zeitschriftenartikel vorlagen, die ihre Arbeit in besonderer Weise würdigten, was als bestätigendes Kriterium für die „good practice“ in diesen Einrichtungen betrachtet werden kann. Letztendlich haben sich für ein Interview vier der acht angefragten Einrichtungen bereit erklärt (siehe Tabelle 13).

Tabelle 13: Einrichtungen der „Good-Practice“-Analyse

\begin{tabular}{|l|l|l|l|l|}
\hline Einrichtung & $\begin{array}{l}1 \\
\text { St. Marienstift }\end{array}$ & $\begin{array}{l}2 \\
\text { CBT-Wohnhaus } \\
\text { Upladin }\end{array}$ & $\begin{array}{l}3 \\
\text { Haus 2 des } \\
\text { Städtischen Se- } \\
\text { niorenzentrums } \\
\text { Köln-Riehl }\end{array}$ & $\begin{array}{l}\text { Maria-Martha- } \\
\text { Stift }\end{array}$ \\
\hline Träger & Caritas & $\begin{array}{l}\text { CBT - Caritas- } \\
\text { Betriebsfüh- } \\
\text { rungs- und Trä- } \\
\text { gergesellschaft } \\
\text { mbH }\end{array}$ & $\begin{array}{l}\text { Sozial-Betriebe- } \\
\text { Köln gemein- } \\
\text { nützige GmbH }\end{array}$ & $\begin{array}{l}\text { Diakonie-Lin- } \\
\text { dau }\end{array}$ \\
\hline Ort & Bochum & $\begin{array}{l}\text { Leverkusen } \\
\text { Opladen }\end{array}$ & Köln & Lindau \\
\hline $\begin{array}{l}\text { Für die Analyse } \\
\text { relevante kon- } \\
\text { zeptionelle Be- } \\
\text { sonderheiten }\end{array}$ & $\begin{array}{l}\text { Autonomie, } \\
\text { Teilhabe und }\end{array}$ & Quartiershaus & Mitmachhaus & $\begin{array}{l}\text { Konzept nach } \\
\text { EDEN; Lebens- } \\
\text { stilkonzept }\end{array}$ \\
\hline $\begin{array}{l}\text { Für die Recher- } \\
\text { che relevante } \\
\text { Quellen }\end{array}$ & $\begin{array}{l}\text { Altenheim-Zu- } \\
\text { kunftspreis 2015 }\end{array}$ & $\begin{array}{l}\text { Artikel und Vor- } \\
\text { träge }\end{array}$ & Artikel & $\begin{array}{l}\text { Altenpflege- } \\
\text { Preis 2014 }\end{array}$ \\
\hline
\end{tabular}




\section{Forschungsdesign}

Erhebungsmethode

Die Interviews mit ausgewählten Vertreter*innen dieser Einrichtungen wurden im Verständnis von Expert"inneninterviews nach Meuser und Nagel (2002) durchgeführt. So waren Interviews mit den Einrichtungsleitungen oder unter Umständen mit den Pflegedienstleitungen zur Eruierung des Kontextwissens sowie Interviews mit Mitarbeiter"innen des Sozialen Dienstes zur Beleuchtung der operativen Ebene im Sinne des Betriebswissens vorgesehen.

Insgesamt wurden in den vier angegebenen Einrichtungen fünf Interviews mit acht Interviewpersonen mit einer Länge zwischen einer und rund eineinhalb Stunden geführt. Die Gestaltung des Interviewsettings erfolgte aufgrund von feldspezifischen Anforderungen, forschungsökonomischen Beschränkungen und forschungspraktischen Überlegungen jedoch flexibel. So wurde den Einrichtungen etwa zur Begrenzung ihres zeitlichen Aufwandes auch ein Interview durch den Interviewenden mit zwei Interviewpersonen ermöglicht.

\section{Aufbau des Interviewleitfadens}

Ziel des Interviews war es, anknüpfend an die Zwischenergebnisse der STAP-Studie, besondere Ansätze und Ressourcen der Selbstbestimmungsund Teilhabeförderung in den ausgewählten Einrichtungen zu identifizieren. Daher beinhaltete der teilstrukturierte Interviewleitfaden einerseits die Themenkomplexe (A) Ansätze der Förderung von Selbstbestimmung und (B) Ressourcen der Förderung von Selbstbestimmung sowie andererseits (C) Ansätze der Förderung von Teilhabe und (D) Ressourcen der Förderung von Teilhabe. Innerhalb dieser Themenkomplexe wurden offene Hauptfragen formuliert, die danach fragen, in welcher Weise die Selbstbestimmung bzw. Teilhabe und mit welchen Ressourcen Selbstbestimmung bzw. Teilhabe gefördert werden sowie was dabei fördernde Faktoren sind. $\mathrm{Zu}$ den Hauptfragestellungen wurden ergänzend Nachfragen formuliert, die optional gestellt wurden, sofern deren Inhalte nicht selbst von den Interviewten thematisiert wurden. Die letzten Themenkomplexe beziehen sich auf einen (E) Vergleich und Ausblick, bei dem danach gefragt wurde, was die jeweilige Einrichtung möglicherweise von anderen Einrichtungen unterscheidet und was sich ändern müsste, um Selbstbestimmungs- und Teilhabemöglichkeiten von Bewohner*innen in Zukunft zu verbessern. Hier hat sich die Frage nach dem Vergleich mit anderen Einrichtungen als 
schwieriger zu beantworten erwiesen, weil den Interviewten der „Vergleichsmaßstab“ fehlte. Der abschließende Themenkomplex (G) beinhaltet die übliche Frage danach, ob zu dem Interviewthema noch etwas zu erwähnen wäre, was in dem Interview noch nicht angesprochen wurde.

\section{Auswertungsmethode}

Die vollständig transkribierten Interviews wurden inhaltsanalytisch ausgewertet. Dabei erfolgte die Auswertung im Sinne der qualitativen Inhaltsanalyse nach Mayring (2010) und hier konkret nach der strukturierenden Inhaltsanalyse, wobei die Hauptfragen des Interviewleitfadens deduktiv als Auswertungskategorien genutzt und innerhalb dieser induktiv Subthemen identifiziert wurden.

Dateneinbezug in der Auswertung des qualitativen Teils der STAP-Studie

Ausgewählte Ergebnisse der „Good-Practice“-Analyse wurden innerhalb der Auswertung des qualitativen Teils der STAP-Studie herangezogen, wenn sie als Gelingensfaktoren der teilhabefördernden Arbeit benannt wurden und thematisch die empirischen Befunde der STAP-Studie mit besonderen Inhalten oder Beispielen ergänzten. Zur Kennzeichnung von Zitaten aus den „Good-Practice“-Interviews wird hier in Kapitel 4 das Kürzel „GP Int." mit Nummerierung verwendet, wobei die Nummerierung nicht mit der tabellarischen Reihenfolge übereinstimmt und somit keine Rückführung auf die einzelne Einrichtung möglich ist.

\subsection{Quantitative Analyse}

Entsprechend dem sequenziellen Mixed-Methods-Design ergänzt die nachgeordnete quantitative Analyse die qualitative Studie, indem ausgewählte Ergebnisse verallgemeinert wurden (vgl. Mayring, 2001). Eine Auswahl der Ergebnisse war notwendig, da die qualitative Analyse, entsprechend dem explorativen Vorgehen, eine Vielzahl an Ergebnissen generierte, die den Umfang einer durchführbaren quantitativen Befragung übertrafen. Die Auswahl bedeutete eine Fokussierung auf Ergebnisse, die als besonders relevant eingeschätzt wurden, die Veränderungspotenzial beinhalteten (z. B. in der Handlungspraxis der Mitarbeiter*innen) oder Ergebnisse, die auf 


\section{Forschungsdesign}

eine Lücke im Forschungsstand hinwiesen bzw. konträr zum Forschungsstand waren. Dieser empirische Baustein war damit nicht darauf ausgerichtet, neue hemmende und fördernde Faktoren zu herauszuarbeiten, sondern vielmehr die bereits identifizierten zu analysieren. Dabei strukturierten drei Leitfragen sowohl die Entwicklung des Fragebogens als auch die Analyse:

1. Liegen die als relevant identifizierten Rahmenbedingungen in anderen Einrichtungen vor? (Ist-Zustand)

2. Wie bewerten Einrichtungsleitungen die Relevanz der identifizierten fördernden und hemmenden Faktoren? (Bewertung der Relevanz)

3. Unterscheiden sich Einrichtungen hinsichtlich des Ist-Zustandes und der Bewertung der Relevanz aufgrund von Kontextfaktoren? (Kontext)

Die erste Frage stellte sicher, dass bzw. ob und inwieweit als relevant markierte Bedingungen oder Ressourcen keinen Einzelfall darstellen, sondern auch in anderen Einrichtungen vorliegen (z. B.: Werden Wünsche der Bewohner*innen in der Übergabe thematisiert?). Die zweite Frage sollte die Bewertung und Gewichtung der qualitativ identifizierten Faktoren ergänzen, indem Einrichtungsleitungen die Relevanz der Faktoren einschätzten (z. B.: Ist es nach der Einschätzung der Einrichtungsleitung förderlich, wenn die Wünsche der Bewohner"innen in der Übergabe thematisiert werden?). Die dritte Frage sollte dem Umstand Rechnung tragen, dass in der qualitativen Studie die Vielfalt an Einrichtungen nicht abgebildet werden konnte. Daher sollte ein Einfluss des Kontextes auf die Leitfragen 1 und 2 analysiert werden (z. B.: Unterscheiden sich Einrichtungen abhängig von der Trägerschaft, (a) ob Wünsche in der Übergabe thematisiert werden und (b) unterscheiden sie sich in der Bewertung der Relevanz?).

Ausgehend von diesen drei Leitfragen sollen im Folgenden die Zielgruppe und der Zugang erläutert (Kapitel 3.4.1), die Entwicklung des Erhebungsinstruments dargestellt (Kapitel 3.4.2) und die Auswertungsmethoden vorgestellt werden (Kapitel 3.4.3).

\subsubsection{Stichprobenzugänge und Stichprobe}

Die Umfrage richtete sich an Einrichtungsleitungen von Pflegeeinrichtungen mit vollstationärer Dauerpflege in NRW (Untersuchungseinheit). Anders als im qualitativen Ansatz wurde in der quantitativen Datenerhebung nur die Perspektive der Einrichtungsleitungen erfasst. Einrichtungsleitungen wurden als geeignete Zielgruppe ausgewählt, da davon ausgegangen wird, dass sie Fragen zu allen Arbeitsbereichen der Einrichtungen beantworten kön- 
nen, Einsichten in zentrale Strukturen und Prozesse sowie Zugang zu ausgewählten Kennzahlen der Einrichtungen besitzen. Es kann aber nicht ausgeschlossen werden, dass Mitarbeiter*innen aus anderen Arbeitsbereichen derselben Einrichtung andere Aussagen treffen würden, daher ist die Monoperspektive bei der Interpretation der Daten stets zu berücksichtigen. Im Vorfeld wurde durchaus auch die Aufnahme von Mitarbeiter*innen anderer Einrichtungsbereiche für die Befragung in Erwägung gezogen, was aber schließlich auch aus forschungspraktischen Gründen - in Bezug auf Restriktionen in den hier möglichen Stichprobenzugängen für die Onlinebefragung - nicht realisiert wurde.

Die genaue Anzahl an Einrichtungen in Nordrhein-Westfalen zum Zeitpunkt der Befragung war nicht bekannt; die Daten der Pflegestatistik dienten als Referenz und wiesen 2190 Einrichtungen aus (Stand 15.12.2017, IT.NRW, 2019). Angestrebt wurde, alle Einrichtungsleitungen zur Teilnahme an der Umfrage einzuladen.

Die Einladung an die Einrichtungsleitungen erfolgte per E-Mail und wurde über zwei Schritte hergestellt. Im ersten Schritt wurde über Vorabkontakte für die Teilnabme an der Befragung geworben, da für Onlineumfragen gezeigt werden konnte, dass unter anderem Vorabkontakte (Cook et al., 2000) und vorherige Informationen (Fan \& Yan, 2010) die Teilnahmebereitschaft erhöhen. Dazu wurde in einem Gremium, an dem die Leitungsebenen der Wohlfahrtsverbände in NRW teilnehmen, das Projekt vorgestellt und darum gebeten, eine E-Mail an die Einrichtungsleitungen weiterzuleiten (Zugang 1.1). Um auch Einrichtungen in privater und öffentlicher Trägerschaft zu erreichen, wurden zudem die Leitungsebene des Bundesverbands privater Anbieter sozialer Dienste e. V., des Verbandes der kommunalen Senioren- und Behinderteneinrichtungen in NRW e. V. (VKSB) sowie der Verband Deutscher Alten und Behindertenhilfe e. V. (VDAB) kontaktiert (Zugang 1.2). Der Vorteil dieses Vorgehens bestand darin, dass die Unverbindlichkeit durch eine direkte E-Mail-Einladung durch die Hochschule Düsseldorf reduziert wurde. Der Nachteil bestand darin, dass nicht nachvollzogen werden konnte, wie viele Einrichtungsleitungen auf diesem Weg tatsächlich erreicht wurden.

Im zweiten Schritt wurden aus öffentlichen Quellen (Deutsches Seniorenportal und Der Pflegekompass) 1746 E-Mail-Adressen recherchiert und die Einrichtungsleitungen direkt per Mail eingeladen (Zugang 2), um den über den ersten Zugang erreichten Rücklauf steigern zu können. Auch wenn es über diesen Weg nicht möglich war, eine vollständige Liste aller Einrichtungen mit vollstationärer Pflege zu erstellen (als Richtwert wurde von 2190 ausgegangen (IT.NRW, 2017) und damit alle Einrichtungen ein- 


\section{Forschungsdesign}

zuladen, so konnte damit eine äußerst umfassende Adressliste gewonnen werden, für die eine angemessene Verteilung (im Abgleich mit der Pflegestatistik) der recherchierten Einrichtungen auf die fünf Regierungsbezirke in NRW geprüft wurde und vorlag. Des Weiteren konnte über diesen $\mathrm{Zu}-$ gang nicht nachvollzogen werden, in welchem Umfang die Einladungen die Einrichtungsleitungen tatsächlich erreicht haben (z. B. durch veraltete E-Mail-Adressen, Spam-Ordner, Firewall) und nicht ausgeschlossen werden, dass die Teilnahmebereitschaft der Einrichtungsleitungen systematisch unterschiedlich ist. Allerdings kann wiederum festgehalten werden, dass auch die erzielte Stichprobe der in die Befragung einbezogenen Einrichtungen angemessene Verteilungen im Vergleich mit der Grundgesamtheit in NRW aufwies. Geht man davon aus, dass die Lage der Einrichtungen und die Trägerschaft das Antwortverhalten determinieren, so zeigt sich, dass die Einrichtungen sich prozentual mit nur geringen Abweichungen (maximal 2,1\%) auf die Regierungsbezirke verteilen, wie dies aus der Pflegestatistik zu erwarten wäre. Mit etwas größeren Abweichungen (maximal 12,5\%) trifft dies auch auf die Trägerschaft zu (siehe Ergebniskapitel $5)$.

Die per E-Mail versendete Einladung enthielt neben dem Link zur Umfrage eine Kurzbeschreibung des Projektes, einen Link zu weiterführenden Informationen sowie Kontaktdaten für weitere Nachfragen. Es wurde darüber informiert, dass die Befragung vollständig anonym erfolgt und die Teilnahme freiwillig ist. Es wurden keine Daten erhoben, die einen Rückschluss auf die Personen oder die Einrichtungen zulassen. Die gespeicherten Antworten der Teilnehmer*innen wurden auf dem Server der Hochschule Düsseldorf gespeichert.

Die Versendung der Einladungen per E-Mail erfolgte im Zeitraum von November 2018 bis Januar 2019. Im November wurden die Zugänge 1.1 und 1.2 (Vorabkontakte) gewählt, im Dezember erfolgte eine Erinnerung an die Vorabkontakte. Im Januar wurden der Zugang 2 genutzt und die Einrichtungsleitungen direkt per E-Mail zur Teilnahme eingeladen; nach einer Woche wurde per E-Mail an die Umfrage erinnert. Die adressierten Einrichtungsleitungen verteilten sich wie folgt auf die Regierungsbezirke in NRW: 368 in Düsseldorf, 342 in Köln, 441 in Arnsberg, 296 in Detmold und 299 in Münster.

An der Umfrage haben 135 Einrichtungsleitungen komplett bzw. bis zum Ende teilgenommen. Ausgeschlossen wurden alle Datensätze von Teilnehmer*innen, die die Umfrage nicht beendet haben (97) und Teilnehmer"innen, die keine Items beantwortet haben, aber bis zum Ende teilgenommen haben (3). Die exakte Rücklaufquote lässt sich aufgrund der 
Zugänge nicht berechnen, da die tatsächliche Anzahl der erreichten Einrichtungen unbekannt ist (vgl. Wagner \& Hering, 2014, S. 667). Legt man als Divisor 2190 Einrichtungen zugrunde, liegt die Rücklaufquote bei 6,2\%. Berechnet man die Rücklaufquote anhand der Anzahl der E-Mails, die versendet wurden, liegt die Quote bei 7,7\%. Es ist anzunehmen, dass die tatsächliche Anzahl der erreichten Einrichtungsleitungen geringer ausfällt (siehe oben) und somit die tatsächliche Rücklaufquote höher liegt.

\subsubsection{Erhebungsmethode}

Ausgehend von den qualitativen Ergebnissen wurde ein standardisierter Fragebogen für die Onlinebefragung konstruiert, der sich an Einrichtungsleitungen richtet und in Anlehnung an die Hinweise von Porst (2014, S. 146f) zur Dramaturgie eines Fragebogens strukturiert wurde. Items zum gleichen Thema wurden in Frageblocks zusammengefasst und durch Überschriften voneinander abgegrenzt sowie darüber hinaus in eine übergeordnete Struktur integriert. Die übergeordnete Struktur orientierte sich an den drei Leitfragen: Im ersten Abschnitt wurde der Ist-Zustand zu den vier Themenblöcken mit Blick auf die eigene Einrichtung erhoben (Leitfrage 1). Durch einen kurzen Text wurde in den zweiten Abschnitt übergeleitet. Es wurde eine Einschätzung von hemmenden und fördernden Faktoren erfragt (Leitfrage 2). Im letzten Abschnitt wurden Angaben zur Einrichtung abgefragt, die den Kontext der Einrichtung beschreiben (Leitfrage 3). Die Items der ersten beiden Abschnitte wurden analog unter folgenden Überschriften gruppiert: (1) Verankerung von Teilhabe, (2) Zusammenarbeit und Aufgaben der Arbeitsbereiche, (3) Handlungsspielräume und Ressourcen sowie (4) Arbeitskulturelle Orientierung. Der Befragung wurde eine Definition von Teilhabe vorangestellt, um sicherzustellen, dass alle Befragungspersonen den Begriff in der gleichen Weise verstehen.

In den ersten beiden analog aufgebauten Abschnitten (Leitfragen 1 und 2) zielte der erste inhaltliche Themenkomplex auf Ansatzpunkte ab, in denen Teilhabe in den Einrichtungen verankert sein könnte. Dies umfasste sowohl die formellen Fundierungen in Leitbildern und Konzepten als auch die Thematisierung von Teilhabe durch Leitungskräfte oder in Gremien. Ebenso wurde nach der Implementation von Teilhabeaspekten in der täglichen Routine gefragt, die sich durch die Integration in Instrumenten oder durch gezielte Maßnahmen ausdrückt. Der zweite Themenkomplex nahm die Zusammenarbeit und die Aufgaben der Arbeitsbereiche in den Blick. Neben dem Verständnis von Teilhabe als bereichsüber- 


\section{Forschungsdesign}

greifende Aufgabe ergab die qualitative Untersuchung, dass insbesondere die Arbeitsweise zwischen den Arbeitsbereichen eine relevante Schnittstelle für die Teilhabeförderung darstellt. Im Fragebogen bezogen sich die Fragen insbesondere auf die Weitergabe (z. B. Übergaben), die Dokumentation (z. B. im Rahmen des Pflegeprozesses) oder die Besprechung (z. B. Fallbesprechungen) von teilhaberelevanten Informationen. Der dritte Themenkomplex umfasste die Handlungsspielräume und Ressourcen der Mitarbeiter*innen. Anknüpfend an die qualitativen Erkenntnisse umfasste dieser Themenkomplex Items zum flexiblen und situativen Umgang mit Aufgaben und Zeiten sowie zur Legitimation durch Vorgesetzte und Akzeptanz von Mitarbeiter*innen. Bezugnehmend auf den dritten Themenkomplex wurde im vierten nach der handlungsleitenden Arbeitskultur gefragt und zwischen den individuellen Bedürfnissen der Bewohner*innen und den festgelegten Abläufen der Einrichtung unterschieden. Im dritten Abschnitt wurden die Einrichtungen um Angaben zu den Einrichtungen und der Bewohnerstruktur gebeten. Im dritten Abschnitt (Leitfrage 3) wurden neben der Trägerschaft sowie Angaben zur Lage und Größe der Einrichtung auch der Anteil an Menschen mit Demenz sowie Sozialhilfeempfänger*innen unter den Bewohner*innen sowie an derzeit ehrenamtlich tätigen Personen in der Einrichtung erfragt.

Insgesamt umfasste der Fragebogen 62 Items. Darunter bezogen sich 36 Items auf den Ist-Zustand und 20 Items auf die Bewertung der Relevanz, jeweils in Hinsicht auf die inhaltlichen Themenkomplexe. Abschließend bezogen sich sieben Items auf die Kontextfaktoren. Es wurde angestrebt, dass die Beantwortung der Fragen nicht länger als 15 bis 20 Minuten beträgt. Dies hat sich empirisch als realistische Einschätzung erwiesen (Median: 14,4 Minuten, arithmetisches Mittel: 18,9 Minuten).

Um die Qualität und Durchführbarkeit des Fragebogens ex ante zu optimieren, wurde ein Pretest durchgeführt (siehe auch Weichbold, 2014). Dabei wurde das Frageverständnis, auf Basis des vierstufigen Modells des Antwortprozesses (Faulbaum et al., 2009, S. 40), evaluiert. Der Fragebogen wurde anschließend in LimeSurvey implementiert.

Auch wenn eine sorgfältige Prüfung des Fragebogens erfolgte, kann nicht ausgeschlossen werden, dass die Teilnehmer*innen trotz anonymer Antwortabgabe ihre Einrichtung positiv darstellten (soziale Erwünschtheit). Dass die Teilnahmebereitschaft der Einrichtungsleitungen möglicherweise nicht zufällig unterschiedlich war (z. B. Einrichtungsleitungen, deren Einrichtungen bereits Maßnahmen zur Verbesserung der Teilhabe ergriffen haben, nahmen möglicherweise eher teil), kann die Stichprobe verzerren (positive Selektion). Bei der Interpretation in Kapitel 5 ist weiter 
zu berücksichtigen, dass die Items nur aus der Perspektive der Einrichtungsleitungen beantwortet wurden und dass anhand der Items nur beschrieben werden konnte, ob Faktoren vorliegen und ob sie als relevant für die Teilhabeförderung bewertet werden, es kann aber keine Aussage über die konkrete Umsetzung gemacht werden.

\subsubsection{Auswertungsmethode}

Die gewonnenen Daten wurden mit dem Statistikprogramm $R$ aufbereitet und ausgewertet. Neben der deskriptiven Auswertung der Ergebnisse zur Beurteilung der ersten und zweiten Leitfrage wurden zur Beurteilung der dritten Leitfrage Signifikanztests zur Analyse von Gruppenunterschieden durchgeführt. Für die Kontextfaktoren (Trägerschaft und Gemeindetyp) wurden ungerichtete Unterschiedshypothesen angenommen: Es gibt einen Unterschied in Bezug auf den Ist-Zustand und der Bewertung der Relevanz (nach Angaben der Einrichtungsleitungen) von der Trägerschaft oder des Gemeindetypus. Analog dazu wurde für die Anzahl der Bewohner*innenplätze in einer Einrichtung eine ungerichtete Zusammenhangshypothese formuliert: Der Ist-Zustand und die Bewertung der Relevanz sind von der Anzahl an Bewohner*innenplätzen einer Einrichtung abhängig. Entsprechend dem Skalenniveau und den Annahmevoraussetzungen wurden folgende Tests eingesetzt: der Chi-Quadrat-Test auf Unabhängigkeit und der Mann-Whitney-U-Test sowie für Vergleiche mit mehr als zwei Gruppen der Kruskal-Wallis-Test (Eid et al., 2017). Hierbei besitzen die nonparametrischen Testverfahren den Vorteil, dass sie auch bei kleinen Stichproben robuste Ergebnisse liefern. Der Einsatz von parametrischen Verfahren, die eine höhere Teststärke aufweisen würden, war hier aufgrund der Verletzung der Annahmevoraussetzungen nicht sinnvoll (u. a. Eid et al., 2017; Rasch, Friese, Hofmann, \& Naumann, 2014). Ebenso wurden Rangkorrelationsanalysen nach Spearman berechnet, um Zusammenhangshypothesen zu analysieren, und die Effektstärke nach Cohen bestimmt. Sofern zwei metrische Variablen vorlagen, erfolgte eine Korrelationsanalyse nach Pearson, nachdem die Voraussetzungen geprüft wurden (z. B. durch den Shapiro-Wilk-Test auf Normalverteilung). Bei der Beurteilung der Ergebnisse wurden die Einschränkungen (soziale Erwünschtheit, Stichprobenverzerrung, Monoperspektive der Einrichtungsleitungen) berücksichtigt. 


\section{Ergebnisse der qualitativen Analyse}

In diesem Kapitel werden die Ergebnisse der qualitativen Erhebungen beschrieben. Hierzu zählen die Ergebnisse aus den im Rahmen der STAPStudie durchgeführten Interviews mit Bewohner*innen, Angehörigen sowie Leitungs- und Fachkräften sowie den teilnehmenden Beobachtungen in den vier Projekteinrichtungen und Ergebnisse aus den trägerübergreifenden Fokusgruppendiskussionen. Hinzu kommen ausgewählte Ergebnisse aus der "Good-Practice"-Analyse, die projektergänzend im Rahmen eines Forschungsfreisemesters der wissenschaftlichen Leitung durchgeführt wurde.

Im Zentrum der qualitativen Analyse standen die beiden Forschungsfragen:

1. Wie können Wünsche und Bedürfnisse von Bewohner*innen stationärer Pflegeeinrichtungen in Bezug auf gesellschaftliche Teilhabe innerhalb und außerhalb der Einrichtung festgestellt und berücksichtigt werden?

2. Wie kann auf dieser Basis das Recht auf selbstbestimmte Teilhabe dieser Bewohner*innen praktisch verwirklicht und dessen Umsetzung überprüft werden?

Von diesen untersuchungsleitenden Fragen ausgehend sowie unter Berücksichtigung der theoretischen Vorüberlegungen wurden in einem iterativen Prozess die relevanten Themenkomplexe in dem qualitativen Datenmaterial ausgewählt. Als relevant wurden Themen identifiziert, die in den Interviews und Fokusgruppen multiperspektivisch bekräftigt und mehrfach benannt, in den Beobachtungen bestätigt und im Rahmen von Projekttreffen durch die Projekteinrichtungen (z. B. Projektrat, Lernworkshops) als gültig und relevant erachtet sowie im Rahmen der „Good-Practice"-Analyse ergänzend gestützt oder vertieft wurden.

Im Folgenden werden die Ergebnisse der qualitativen Analyse zunächst in Bezug auf den Themenkomplex der 1.) Wunschäußerung und -erfassung vorgestellt, der in STAP als Ausgangspunkt der Teilhabeförderung betrachtet wird. Anschließend werden 2.) Ergebnisse in den Blick genommen, welche die Angebots- und Alltagsgestaltung als Kontexte der Teilhabeförderung näher beschreiben. Vor diesem Hintergrund werden dann 3.) fördernde und hemmende Faktoren der Teilhabeförderung erläutert, die auf Ebene der Bewohner"innen, den Mitarbeitenden und der Einrichtung als Organisation identifi- 
ziert werden konnten. Abschließend werden 4.) zentrale Ressourcen der Teilhabeförderung beschrieben.

\subsection{Wunschäußerung und-erfassung als Ausgangspunkt der Teilhabeförderung}

Die individuellen Wünsche zur Teilhabe von Bewohner*innen in Altenpflegeeinrichtungen werden im Rahmen von STAP als Ausgangspunkt und daher die Wunschäußerung und -erfassung als die wesentlichen Ausgangsprozesse zur Förderung der selbstbestimmten Teilhabe erachtet. Wie in Kapitel 1.2 erläutert, fokussiert die erste untersuchungsleitende Fragestellung dementsprechend darauf, wie Wünsche von Bewohner*innen stationärer Pflegeeinrichtungen in Bezug auf gesellschaftliche Teilhabe innerhalb und außerhalb der Einrichtung festgestellt und berücksichtigt werden können. In diesem Kapitel werden daher die wesentlichen Ergebnisse der qualitativen Erhebungen dargestellt, die Antworten auf diese erste Forschungsfrage geben.

\subsubsection{Zum Begriff Wunsch}

Im Rahmen der empirischen Erhebungen sowie der vorliegenden Berichtslegung wurde der Begriff des Wunsches - in Bezug auf Teilhabe - aus Gründen der Anschlussfähigkeit und des Verständnisses bei verschiedenen Akteur*innengruppen in Altenpflegeeinrichtungen gewählt, da dieser Begriff in der beruflichen Praxis und im Alltag der Einrichtungen maßgeblich verwendet wird und sowohl für Mitarbeitende als auch für Bewohner"innen und Angehörige als Terminus nachvollziehbar bzw. zugänglich ist. Allerdings gehen wir im STAP-Projekt ausdrücklich von einem weit gefassten Verständnis des Wunschbegriffs aus. So werden darunter selbstbestimmtes Wollen ebenso wie selbstbestimmte Bedürfnisse (die in der Untersuchungsfrage zunächst separat formuliert wurden), Interessen und Gewohnheiten von Bewohner*innen verstanden. Direkt und indirekt geäußerte präferierte Vorstellungen von Teilhabe gehören daher wesentlich dazu.

Jedoch bleibt die Wunschperspektive im Rahmen von STAP immer auf Teilhabe gerichtet, deren Förderung erst dann nach dem Prinzip der Selbstbestimmung funktioniert, wenn die so verstandenen Wünsche der Bewohner*innen eruiert werden. Im Rahmen der Teilhabeförderung liegt der Fokus auf der dem Menschen zuerkannten Würde und dem damit einhergehenden Menschenrecht. In der Argumentation von Fuchs (2009, 
S. 19ff), entspricht die Menschenwürde einer Norm, die sich in Grundrechten und Freiheitsrechten der Menschenrechte verankert findet. Die Norm der Würde begründet zum einen systematisch den Abbau von Ungleichheit durch Teilhaberechte, die durch die Lebenssituation - hier Pflegebedürftigkeit und das Leben in einer stationären Altenhilfeeinrichtung entsteht (Fuchs, 2009, S. 20). Zum anderen findet sie sich in der Gewährung von Wunsch- und Wahlrechten der Anspruchsberechtigten bei der Inanspruchnahme von Leistungen des Sozialgesetzbuches wieder. Ein im beruflichen Alltag der stationären Altenhilfe ins Zentrum gesetzter Wunschbegriff mit einem in dieser Weise gefassten Verständnis kann für relevante Kontexte und Prozesse der Äußerung, Erfassung, Berücksichtigung und Verwirklichung von Wünschen der einzelnen Bewohner*innen sensibilisieren. Vor diesem Hintergrund verwenden wir daher den Begriff des Wunsches prioritär in der folgenden Darstellung der Ergebnisse.

\subsubsection{Phasen der Wunschäußerung, -erfassung, -berücksichtigung und -verwirklichung}

Damit Wünsche zur Teilhabe von Bewohner*innen in Altenpflegeeinrichtungen verwirklicht werden können, müssen diese zunächst von ihnen geäußert werden. Dies kann in unterschiedlicher Art und Weise sowie in verschiedenen Kontexten im Alltag einer Altenpflegeeinrichtung geschehen, wie später Beispiele aus der Empirie zeigen. Hier kann etwa auch eine „Weckung" von Wünschen seitens der Mitarbeitenden eine Rolle spielen, ohne dass damit das Prinzip der Selbstbestimmung unterlaufen würde. Geäußerte Wünsche von Bewohner*innen sollten anschließend erfasst und unter den Mitarbeiter*innen kommuniziert werden, damit die Wünsche daraufhin in der Planung und Organisation berücksichtigt und praktisch verwirklicht werden (können).

Hierbei handelt es sich also um eine prototypische Abfolge von drei Prozessphasen -1 . Wunschäußerung, 2. Wunscherfassung, 3 . Wunschberücksichtigung und -verwirklichung -, die in der beruflichen Praxis jedoch nicht immer trennscharf und als Aufeinanderfolge wiederzufinden sind. So kann es etwa auch sein, dass der zweite Prozessschritt (Erfassung) in bestimmten Fällen (beispielsweise bei kleinen, alltagsnahen Wünschen) nicht stattfindet, weil der Bewohner*innenwunsch direkt verwirklicht wird. 


\section{Wunsch- äußerung

Abbildung 3: Prototypische Abfolge von Wunschäußerung, -erfassung, -berücksichtigung und-verwirklichung

Quelle: eigene Darstellung

\subsubsection{Wunschäußerung}

Im Rahmen der Interviews wurden verschiedene Themenbereiche der Wunschäußerung von Bewohner*innen, Mitarbeitenden und Angehörigen - teilweise eher indirekt - benannt, die in teilhaberelevante Wünsche von Bewohner"innen innerhalb und außerhalb der Einrichtung unterschieden werden können. So gehören hierzu zum einen kleinere, alltagsnahe Wünsche innerhalb der Einrichtung (z. B. Wünsche in Bezug auf die Mahlzeiten, die Pflege und Versorgung, die Kleidung, das Zimmer), die sich oft auf die unmittelbare Alltagsgestaltung oder auf Voraussetzungen für Teilhabe im Alltag der Einrichtung beziehen. Spezifische Wünsche in Bezug auf Teilhabeangebote innerhalb der Einrichtung werden hier im Kontext der Wunschäußerung nicht direkt benannt oder hervorgehoben. Zum anderen werden aber Wünsche von Bewohner*innen zur Teilhabe genannt, die außerhalb der Einrichtung stattfindet (z. B. Einkaufen, Stadtbesuche, Ausflüge, Ferien).

Darüber hinaus wird aber auch der selbstbestimmte Wunsch der Nichtteilnabme an Teilhabeangeboten ausdrücklich zum Thema der Wunschäußerung erwähnt: „Manchmal hat sie auch keine Lust, [...] dann sitzt sie in ihrem Sessel. Also das ist eben so, [...] das ist ja jetzt ihr Wunsch, und da gehen die auch drauf ein, ne? Das gehört so vielleicht jetzt dazu, ne?" (ANG).

Ferner wird die Nichtäußerung von Wünschen im Rahmen der Interviews mehrfach - sowohl von Mitarbeitenden als auch von Angehörigen - beschrieben und hervorgehoben. Dazu wird benannt, dass häufig gar keine Wunschäußerungen von Seiten der Bewohner*innen erfolgen bzw. keine konkreten Teilhabewünsche genannt werden, dass eine gewisse Zurückhaltung und Bescheidenheit bei manchen Bewohner*innen existiert sowie 
dass aufgrund physischer oder psychischer Einschränkungen Voraussetzungen zur Äußerung fehlen.

Auch von den interviewten Bewohner"innen werden auf die Frage nach ihren Wünschen oder Ideen dazu, was sie gerne machen möchten, entweder keine Wünsche („Ich bin mit dem zufrieden, was man mir anbietet hier", BW) oder eher kleine alltagsnahe Wünsche benannt, wie auch von den Mitarbeitenden beschrieben: „Oder wenn was ist [...] den Wunsch können sie äußern, ne? Können sie sagen - oder wenn sie es mit dem Magen haben oder abends kommen sie auch fragen, ob man Brei haben will, ne?" (BW). Eine andere Bewohnerin beschreibt, dass sie „kleine bescheidene Wünsche“ (BW) äußere, aber gar nicht sagen könne, um welche es sich handele. Zum Ausdruck kommt dies auch in der folgenden Gesprächssituation mit einer Bewohnerin, als nach einem Interview über ihre Schwierigkeit, Wünsche zu äußern, gesprochen wurde, da sie vorher sagte, dass sie „wunschlos glücklich ist“ (BW). Auf die spontane Nachfrage der Interviewerin „Ich schlage vor, es gäbe eine gute Fee, und sie hätte drei Wünsche frei“, äußerte die Bewohnerin, dass "Gesundheit das Wichtigste" sei und „ein Tisch für ihr Zimmer" und nach einer Weile des Überlegens noch „eigenes frisches Obst“ (BW).

Es lassen sich insgesamt verschiedene fördernde und hemmende Faktoren im Rahmen der Interviews identifizieren, welche sich auf die Äußerung von Wünschen beziehen.

\section{Fördernd:}

- Eine vertrauensvolle Beziehung zwischen Bewohner*innen und Mitarbeiter"innen erleichtert die Wunschäußerung.

- In allen vier Einrichtungen werden aber auch konkrete Anlässe und Settings zur Wunschäußerung geschaffen, die Wunschäußerungen unterstützen (können); hierzu gehören etwa die Mahlzeitenplanung sowie die Jahresangebotsplanung. Sie erfolgen etwa im Rahmen von unterschiedlichen Mitbestimmungsgremien, zum Teil im Bewohner*innenbeirat ${ }^{59}$ (wenngleich ebenfalls geschildert wird, dass die Partizipation von Be-

59 Die Aufgabe des Bewohner*innenbeirats wird im Rahmen des Wohn- und Teilhabegesetzes (WTG) NRW wie folgt beschrieben: „Der Beirat vertritt die Interessen der Nutzerinnen und Nutzer ohne Unterscheidung nach sexueller Orientierung und geschlechtlicher Identität, Herkunft, religiöser Weltanschauung oder anderen persönlichen Merkmalen. Seiner Mitwirkung unterliegen Fragen der Unterkunft, Betreuung und der Aufenthaltsbedingungen. Der Mitbestimmung unterliegen die Grundsätze der Verpflegungsplanung, die Freizeitgestaltung und die Hausordnung in der Einrichtung" (WTG NRW $\$ 22$ Abs.2). Das heißt, es 
wohner*innen im Rahmen des Bewohner*innenbeirates in der Praxis aufgrund der veränderten Bewohner*innenstruktur zunehmend eine Herausforderung darstellt) oder in Form von niedrigschwelligen Angeboten, die für alle Bewohner*innen zugänglich sind (beispielsweise die sogenannte „Wunschrunde“, ${ }^{60}$ Kapitel 4.1.4).

- Aufgrund der Erfahrung, dass Bewohner*innen eher selten konkrete Wünsche in Bezug auf Teilhabeangebote äußern, haben die Einrichtungen auch ein „Angebote-machen-System“ entwickelt. Das heißt, damit Angebote entstehen, werden Vorschläge gemacht, die mit den Bewohner"innen besprochen und abgestimmt werden: „Meistens gehe ich in Vorleistung, ne, das heißt, ich biete an und bitte darum, ja oder nein" (SD).

- Durch das „Trial-and-Error"-Verfahren (Versuch und Irrtum) werden zudem Bewohner*innen in Einzel- oder Gruppenangebote einbezogen, die sich nicht mehr äußern können, denen aber Teilhabe ermöglicht werden soll. Dabei sind jedoch Beobachtungskompetenz sowie das Lesen und Interpretieren der jeweiligen Reaktionen von besonderer Bedeutung: „Die keine Wünsche äußern, die werden dann mit hinzugenommen und dann wird beobachtet, einfach an der Gestik und Mimik, ob demjenigen das gefällt oder nicht" (PDL). Zündel (2012) beschreibt hierzu, dass die nichtsprachliche Kommunikation zu einer wichtigen Interaktion gehört, die situativ zustande kommt. Dabei sei es signifikant, dass das Gegenüber (Pflegekräfte und andere Mitarbeitende) auf das reagiert, „was der Interaktionspartner [Bewohner*in] ihm gezeigt hat und dieser wiederum darauf eingeht" (Zündel, 2012, S. 183). Für die Mitarbeitenden in einer Altenpflegeeinrichtung bedeutet dies also, dass sie sich selbst und die Situation reflektieren müssen, um die Zeichen der Bewohner*innen wahrzunehmen und interaktiv darauf zu reagieren (ebd., S. 184).

- Ein häufig genanntes Thema (im Rahmen der Interviews und der trägerübergreifenden Fokusgruppendiskussion) ist das Wissen um die und die Orientierung an der Biografie der Bewohner"innen. Biografisches Wissen bietet in diesem Zusammenhang auch Ansatzpunkte für ein „Trialand-Error"-Vorgehen und unterstützt dabei, Wunschäußerungen we-

handelt sich um ein gewähltes Gremium, welches die Wünsche und Bedürfnisse der Bewohner*innen vertritt (Kempchen, 2017, S. 78).

60 Die „Wunschrunde“ ist ein niedrigschwelliges Angebot in einer der vier Projekteinrichtungen: Einmal im Quartal lädt der Soziale Dienst alle Bewohner*innen der Altenpflegeeinrichtung zur „Wunschrunde“ ein. Im Rahmen dessen können die Bewohner*innen ihre Wünsche, Anliegen und Verbesserungsvorschläge einbringen. 
cken oder angemessen interpretieren zu können. Allerdings konnten hierzu unterschiedliche, teils in Konflikt stehende Perspektiven identifiziert werden:

Zunächst ist festzuhalten, dass mehrfach die Bedeutung der Biografieorientierung hervorgehoben wird, um eine Begleitung und Förderung der Bewohner*innen anbieten zu können, die sich an den individuellen Interessen, Vorlieben und Fähigkeiten orientiert. Als Voraussetzung - und bei Bewohner*innen mit einer Demenz auch als Herausforderung - wird hierbei aber auch beschrieben, die Informationen der individuellen Biografie zu erhalten bzw. zu kennen, die gegenwärtig für die Bewohner*innen von Relevanz sind. Dazu wird in den Interviews häufiger erwähnt, dass insbesondere (nahen) Angehörigen die Orientierung an der Biografie von ihren, oft lange bekannten Angehörigen in der Einrichtung sehr wichtig ist, um ihre Persönlichkeit und Identität zu würdigen. So äußert etwa eine Angehörige deutlich dahingehend Kritik, dass ihrer Mutter Kleidung angezogen wird, die sie früher, vor ihrer Erkrankung, nicht getragen hätte, wenngleich sie dies auch mit Personalmangel begründet („die [Mitarbeiter] sind ja auch begrenzt“, ANG). Allerdings wird demgegenüber auch von Mitarbeitenden benannt, dass sich Wünsche der Bewohner*innen ändern können und daher wichtig sei, die aktuellen Wünsche, Interessen und Vorlieben zu erkennen und zu berücksichtigen (siehe auch das folgende Kapitel). Dennoch kann an dieser Stelle festgehalten werden, dass ein guter Kontakt zwischen Mitarbeitenden und den Angehörigen förderlich sein kann, um relevante Aspekte aus der Biografie der Bewohner*innen zu erhalten, die zur Weckung und Interpretation von Wunschäußerungen genutzt werden können (Kapitel 4.4.3).

\section{Hemmend:}

- Als hemmender Faktor bei der Wunschäußerung wird von der Mitarbeiter"innen-Seite häufig das Thema der „Dankbarkeit" (EL) von Bewohner*innen beschrieben. So konnten Fachkräfte etwa beobachten, dass Bewohner*innen zu höflich sind, ein Freizeitangebot abzulehnen, da sich die Einrichtung so viel „Mühe“ (PDL) gibt.

- Noch weiter mit Blick auf das Verhältnis der Bewohner*innen zur Institution und zu ihren Mitarbeitenden gehen in den Interviews Äußerungen, die auf ein gewisses „Abhängigkeitsverbältnis“ (EL) der Bewohner*innen zu den Mitarbeitenden hinweisen. So wollen die Bewohner“innen die Abläufe nicht „stören“ (EL) und sind daher bei der Wunschäußerung gehemmt. Auch in der Literatur wird beschrieben, dass 
Bewohner*innen in einem Abhängigkeitsverhältnis (insbesondere in Kontexten der Pflege) stehen und sich die Betroffenen daher aus „Angst vor Repressalien nicht trauen, z. B. Beschwerden vorzutragen“ (Kempchen, 2017, S. 78) oder Kritik zu äußern (vgl. Jenull-Schiefer, 2005).

- Es gibt ferner zurückhaltende oder bescheidene Bewohner"innen, welche sich aufgrund ihrer Persönlichkeit oder relativierenden Lebenserfahrungen bezüglich ihrer Wünsche nicht äußern, weil sie keine Ansprüche stellen (möchten): „[...] ist deshalb nicht unzufrieden, weil sie keine Ansprüche stellt in dieser Weise" (ANG). Gemutmaßt wird, dass es sich bei den jetzigen Bewohner*innen auch um eine zurückhaltende „Kriegsgeneration“ handelt, die aufgrund des erlebten Mangels in ihrem Leben selten(er) Ansprüche stellt („[eine] Kriegsgeneration [..., die sich] mit vielem [...] bescheiden geben“, SD). Hier besteht die Gefahr, dass diese Personengruppe mit ihren Wünschen übergangen bzw. übersehen wird, denn diese zurückhaltenden Bewohner*innen werden von den Mitarbeiter"innen als besonders positiv wahrgenommen, da von ihnen keine Anforderungen gestellt werden und somit kein zu bearbeitender Mehraufwand entsteht. Allerdings wird auch beschrieben, dass der Anteil der Kriegsgeneration in den kommenden Jahren abnehmen werde und dann „anspruchsvollere“ Bewohner"innen in die Einrichtungen einziehen würden.

- Ferner wird auch ein verändertes Verhalten von Bewohner"innen im Laufe des Eingewöhnungsprozesses in eine Altenpflegeeinrichtung beobachtet und benannt. Demnach passen sich Bewohner*innen an die Gegebenheiten und Tagesabläufe in der Einrichtung an: „Das ist - das ist einfach - diese Eingewöhnungsphase ist eigentlich für mich ein Aufgeben dessen, wofür man sein Leben lang gearbeitet hat, wo man sich sein Leben lang drum bemüht hat, wo man sich versucht hat, es so schön wie möglich zu machen. Und das ist alles weg. Und das wird auch nicht mehr wiederkommen. Also irgendwie ist das eher ein Aufgeben und Sich-Anpassen auch an diese bestimmten Abläufe, die einfach sind, ne, die Essenszeiten sind einfach dann gegeben" (SD). Auch im Rahmen der Fokusgruppendiskussion werden das Anpassungsverhalten und die Nichtäußerung von Wünschen konkret miteinander in Verbindung gesetzt: „Ja aber der Bewohner ordnet sich da unseren Strukturen schon so unter, dass er diesen Wunsch gar nicht äußert" (EL/PDL). Dies scheint ebenfalls (indirekt) in den Bewohner*inneninterviews zum Ausdruck zu kommen: „Ich bin mit dem zufrieden, was man mir hier anbietet" (Bewohner*in). Gleichermaßen wird bereits in der Literatur beschrieben, dass sich die Mehrheit der in einer Altenpflegeeinrichtung 
lebenden Personen in kurzer Zeit an das Leben in einer Einrichtung anpasst. Eine für das Individuum gelingende Anpassung sei jedoch von vielen Faktoren abhängig, so Hahn (2011): die Erwartungshaltung, die eigene Lebensgeschichte, Zufriedenheit mit der neuen Situation und verschiedene Persönlichkeitsvariablen wie soziale Kontakte, frühere Aktivitäten und der Gesundheitszustand (Hahn, 2011, S. 112).

- Als Grund für die Nichtäußerung von Wünschen werden des Weiteren mehrfach gesundheitliche Einschränkungen von und über Bewohner*innen genannt. So können sich manche Bewohner*innen aufgrund von physischen oder psychischen Einschränkungen nicht mehr äußern, was die verbale und nonverbale Kommunikation zwischen Mitarbeitenden und Bewohner"innen beeinträchtigt, denn es gebe „viele, die [...] ihre Wünsche so gar nicht mehr artikulieren können" (ZBK). Andere Bewohner*innen nennen einzelne Wünsche bewusst nicht, da sie aufgrund ihrer jeweiligen körperlichen Verfassung nicht in der Lage sind bzw. sich nicht mehr in der Lage fühlen, früheren Interessen und Hobbys nachzugehen: „Ich möchte das nicht mehr [..., ist mir zu anstrengend" (BW). Wenngleich es sich hier bei Letzterem um eine selbstbestimmte Entscheidung der Bewohner"innen der Nichtteilhabe handelt, ist damit auch eine Restriktion der Wunschäußerung verbunden, so dass dieser Aspekt auch als hemmender Faktor an dieser Stelle angeführt wird.

\subsubsection{Wunscherfassung}

In den Einzel- und Gruppeninterviews werden - direkt oder indirekt - verschiedene Formen der Wunscherfassung benannt, die sich in formelle und informelle Erfassungsformen unterscheiden lassen. Mit formellen Formen der Wunscherfassung sind hier Vorgehensweisen gemeint, die mit spezifischen Vorgaben, Regelungen oder Konzepten verbunden sind, welche demgegenüber bei informellen Erfassungsformen fehlen. Beide werden im Folgenden - wiederum mit Bezug auf fördernde und hemmende Faktoren - erläutert.

\subsubsection{Erfassung formeller Art}

Eine Erfassung von Wünschen formeller Art geschieht einerseits im Rahmen von individuellen Gesprächen und der dazugehörigen Dokumentati- 
on. Mehrfach ausdrücklich benannt wurden hier die Gespräche in der Einzugs- und Eingewöhnungsphase zwischen Bewohner*innen und Mitarbeitenden („Eingewöhnungsgespräche oder Einzugsgespräche und [...] Aussagen, was war da mal? Wie ist das Leben so abgelaufen?" eine Informationsweitergabe durch Angehörige an Mitarbeitende, wenn sich der/die Bewohner*in nicht mehr äußern kann („Also da halt wirklich eher über Angehörige“, EL). Im Rahmen der Einzugsphase wird häufig auch eine biografische Datenaufnahme bzw. ein Biografiegespräch zwischen den Mitarbeitenden des Sozialen Dienstes und Bewohner*innen/ Angehörigen geführt (Kapitel 4.4.3). Eine Leitungskraft beschreibt in der trägerübergreifenden Fokusgruppendiskussion hierzu ergänzend und als besonderes Beispiel, dass es in ihrer Einrichtung Reflexionsgespräche im Rahmen der Einzugsphase von Bewohner*innen gebe, die von einer externen Beraterin durchgeführt werden. Diese Beraterin komme "feinfühlig“ ins Gespräch mit Bewohner*innen, Angehörigen und Mitarbeitenden der Einrichtung, um „dann wirklich in allen Bereichen mit uns aufzuarbeiten, wo wir noch nachjustieren müssen" (EL/PDL).

Auch die Dokumentation im Rahmen der Pflegeprozessplanung, einschließlich des Pflegeberichtes, gehört hier weitergehend zur formellen Form der Wunscherfassung, auch wenn dieses Instrument im Rahmen der Interviews eher eine indirekte Erwähnung findet. Das heißt, sie wurde auf die Frage nach der Wunscherfassung nicht offensiv erwähnt und als reguläres Instrument für die Wunscherfassung konkret benannt, sondern eher in dem Sinne erwähnt, dass Teilhabewünsche dort auch einfließen könnten oder sollten („Also die Dokumentation lässt oft zu wünschen übrig. Und wichtige - wichtige Aussagen der Bewohner gehen oft so im Alltag dann auch verloren, wo wir das dann auch in der Tat oft nicht mitbekommen, wenn Wünsche und Bedürfnisse dann doch mal artikuliert werden“, EL).

Zum anderen erfolgt eine formelle Erfassung von Wünschen durch Gremien und Angebote der Mitbestimmung und des Verbesserungsmanagements, die überindividuell existieren und allen Bewohner*innen eine Äußerung von Wünschen und Beschwerden in Bezug auf die Einrichtung ermöglichen. Hierzu zählen zuvorderst der Bewohner*innenbeirat, der als Mitwirkungsgremien originär den Auftrag hat, die Interessen der Bewohner*innen zu vertreten. Eine Wunscherfassung findet hier etwa im Rahmen von Sprechstunden des Bewohner*innenbeirats statt. Teils auch vom Bewohner*innenbeirat (mit)geführt, teils davon unabhängig gehören auch Verfahren des Verbesserungs- bzw. Beschwerdemanagements zu formellen Erfassungsformen: „Wir haben unten so einen Kummerkasten im Eingangsbereich“ (SD). Weitere Angebote der Mitbestimmung werden in Bezug auf 
die Mahlzeiten genannt, indem etwa regelmäßig „Menübesprechungen“ $(\mathrm{HW})$ von Seiten der Hauswirtschaft durchgeführt werden. Darüber hinaus wird in einer Einrichtung explizit auf die bereits erwähnte „Wunschrunde“ (SD) hingewiesen (Kapitel 4.1.3), bei der Wünsche in Bezug auf Angebote des Sozialen Dienstes erfasst werden. Sie wird auch von einer Bewohnerin auf die Nachfrage, wie Angebote entstehen und ob dazu Wünsche geäußert werden können, erwähnt: „Ja, dafür gibt es ja die Wunschrunde, ne? Die ist alle drei Monate. Und dann werden - ich glaube, da können so Wünsche geäußert werden und dann wird das notiert und wird dann in den Reihen der Sozialarbeiter diskutiert und wenn das machbar ist, wird das gemacht, ne" (BW).

Es lassen sich wiederum verschiedene fördernde und hemmende Faktoren identifizieren, welche sich auf die formelle Erfassung von Wünschen beziehen.

\section{Fördernd:}

- Als förderlich wurde benannt, wenn Leitungskräfte ihre Mitarbeiter*innen für den Nutzen der (auch) für die Wunscherfassung vorgesehenen und hilfreichen Instrumente, Verfahren und Methoden sensibilisieren: „Und die Mitarbeiter immer wieder auch zu sensibilisieren, wie wichtig das ist, dass Wünsche und Bedürfnisse, die artikuliert werden, Ressourcen, die vorhanden sind, dass sie auch mit in der Pflegeprozessplanung, in den sogenannten AEDLs verschriftet werden" (SD).

- So wird die Wunscherfassung dann auch durch eine systematische Nutzung der formell vorhandenen Verfahren und Instrumente unterstützt.

- Als ein zentraler methodischer Ansatz, der die - formelle wie informelle - Erfassung von Wünschen unterstützt, wird auch in diesem Zusammenhang die Biografieorientierung bzw. -arbeit mehrmals herausgestellt.

- Um Wünsche von Bewohner*innen zu erfassen, die sich nicht mehr äußern können, wird auch im Kontext von formellen Erfassungsformen der Kontakt zu Angehörigen wahrgenommen. So können - z. B. im Rahmen von Einzugsgesprächen - über die Angehörigen zumindest potenzielle Wünsche dieser Bewohner*innen erfasst und später anhand der Reaktionen von Bewohner*innen überprüft werden.

\section{Hemmend:}

- Es wurde benannt, dass feblende oder veraltete biografische Angaben und alleinige Informationen von Angehörigen hemmend für eine gegenwärtig adäquate Wunscherfassung sein können. So werden beispielsweise "Gewohnheiten beschrieben [...], die schon 20 Jahre alt sind" 
(SD). Die Fähigkeiten, Interessen und Vorlieben von Bewohner*innen können sich aber im Laufe der Zeit verändern, so dass frühere Aspekte aus der Biografie in der Gegenwart an Bedeutung verlieren (Kapitel 4.4.3).

- Eine mangelnde Weiterleitung und eine fehlende Dokumentation von Wünschen wurden hier als ein wesentlicher hemmender Faktor genannt. Eine Einrichtungsleitung beschreibt: „In den meisten Fällen werden sie [die Wünsche] nicht dokumentiert. Das passiert so im alltäglichen Gespräch“ (EL).

- Aber auch eine mangelnde Kooperation zwischen Pflege und Sozialem Dienst kann die Weitergabe und Erfassung von Informationen erschweren. Einzelne Mitarbeitende äußerten auch die Sorge, dass in der Strukturierten Informationssammlung (SIS) die Biografie weniger "präsent“ ist, zumindest dann, wenn separat kein „Biografieblatt“ mehr existiert.

\subsubsection{Erfassung informeller Art}

Eine Wunscherfassung informeller Art geschieht ohne spezifische Regelungen und ergibt sich häufig im Rahmen alltäglicher Gespräche oder Beobachtungen in der jeweiligen Pflege-, Versorgungs- oder Betreuungssituation („Wichtige Quelle ist natürlich der Bewohner selber, der dann vielleicht auch stolz von Dingen erzählt", SD). Wenn daran eine Weitergabe der erfassten Wünsche anschließt, erfolgt diese - in informeller Art - etwa über eine anschließende mündliche Weitergabe an einzelne Kolleg*innen oder - in formeller(er) Art - in Gremien (z. B. Übergabeteam, Leitungsrunde). Der informelle Charakter bleibt bei der Weitergabe aber insbesondere dann bestehen, wenn hierzu keine weitergehende Dokumentation geschieht: „Die dokumentieren das nicht. Höchstens, dass sie mal das sagen, ne? Hör mal, die möchte gerne - und so" (ZBK). Allerdings ist es auch möglich, dass eine informelle Wunscherfassung nicht formell dokumentiert wird, wenn eine Realisierung des Wunsches unmittelbar erfolgt: „Also oftmals ist es ja auch so, man kriegt was mit und kann ja dann direkt reagieren, ne, ohne dass man das jetzt formell festhält“ (SD). So lassen sich wiederum fördernde und hemmende Faktoren bezogen auf die informelle Erfassung von Wünschen identifizieren.

\section{Fördernd:}

- Fördernd ist es, wenn die Wunscherfassung als ein Teil der wahrgenommenen Aufgabe der Mitarbeiter*innen verstanden wird und die Mitarbei- 
ter*innen auch spontan in Pflege-, Versorgungs- und Betreuungssituationen Wünsche wahrnehmen und erfassen.

- Es findet eine Überfübrung in eine Formalisierung statt, indem die informell erfassten Wünsche dokumentiert und in die vorhandenen Instrumente (z. B. Pflegeprozessplanung und -berichte) übertragen werden oder andere Verfahren existieren, die erfassten Wünsche zu dokumentieren (als Beispiel wurde ein Verfahren an der Rezeption genannt, mit dem, Bewohner*innenwünsche festgehalten werden).

- Die enge bzw. regelmäßige Abstimmung zwischen den Mitarbeitenden verschiedener Schichten und Einrichtungsbereiche ist förderlich. Insbesondere der Informationsfluss zwischen der Pflege und dem Sozialen Dienst wird als relevant markiert (siehe hierzu auch das Kapitel „Schnittstellen der Arbeitsbereiche").

- Förderlich ist dementsprechend auch, wenn der Aspekt der Wunscherfassung bzw. die vorhandenen Wünsche der Bewohner*innen integral zum Besprechungswesen gehören (z. B. in Übergaben, Fallbesprechungen).

\section{Hemmend:}

- Was an Teilhabewünschen erfasst und weitergegeben wird, wird oft als "personenabhängig" (EL) beschrieben, weil es unterschiedlich gehandhabt wird, im Ermessen der Mitarbeitenden liegt oder auch vom individuellen Arbeitsengagement abhängig ist. So kann es eine hemmende Auswirkung haben, wenn es keine Vorgaben gibt, wie das zu erfolgen hat: „Und da kannst du dich ja drum kümmern“ (EL).

- Teilhabewünsche sind zudem nicht prioritärer Zweck der Aufgabenerfüllung oder eben nicht integraler Teil der Besprechung. Sie kommen on top und unsystematisch dazu, müssen nicht miterfasst und umgesetzt werden.

- Zudem führt das kollektive Besprechen von Wünschen bzw. der informelle Austausch darüber oft nicht zu einer mit personeller Verantwortung versehenen Berücksichtigung und Verwirklichung.

\subsubsection{Wunschberücksichtigung}

Aussagen, die in den Interviews die Wunschberücksichtigung und -verwirklichung thematisieren, beziehen sich inhaltlich einerseits auf die Angebotsgestaltung innerhalb und außerhalb der Einrichtung (z. B. Angebotsinhalte, Ausflugsziele) und andererseits auf die individuelle Alltagsgestaltung und 
Tagestrukturierung (z. B. Aufstehzeiten, Mahlzeiten innerhalb eines Rahmens), auf Essenswünsche (innerhalb einer Essensauswahl) sowie die Zimmergestaltung (z. B. Bilder, Mobiliar). Des Weiteren beziehen sich die thematisierten Bereiche auf die Privatheit und Intimität der jeweiligen Bewohner*innen (z. B. geschlossene Zimmertüre, Alleinsein mit Partner*in). Die fördernden und hemmenden Faktoren werden hier zunächst - im unmittelbaren Anschluss an die Dimensionen der Wunschäußerung und -erfassung - auf die Wunschberücksichtigung bezogen. Während die Berücksichtigung der Wünsche an dieser Stelle bedeutet, dass die erfassten Wünsche in der Planung und Vorbereitung von Teilhabeangeboten beachtet werden, bezieht sich die Verwirklichung auf die Realisierung des gewünschten Teilhabeangebotes in der Alltags- und Angebotsgestaltung, worauf später eingegangen wird.

\section{Fördernd:}

- Als förderlich wurde benannt, wenn die Wunschberücksichtigung (und -verwirklichung) in der jeweiligen Situation der Wunscherfassung mit dem/der angesprochenen Mitarbeiter*in und den situativ vorhandenen Ressourcen möglich ist. Insbesondere Wünsche, die an die Versorgung geknüpft sind (vor allem an Mahlzeiten), können gut berücksichtigt werden: „Individuelle Wünsche, die sowieso in den Alltagstätigkeiten drinnen sind, das ist das geringste Problem“ (EL).

- Förderlich für die Wunschberücksichtigung scheint aus Sicht der Mitarbeitenden auch zu sein, wenn Teilhabewünsche von Bewohner*innen eher klein und alltagsnah sowie auf individueller Ebene verortet sind. Diese wären im Einrichtungsalltag mit einem geringeren Aufwand platzierbar, präsent und gut an die bzw. unter den Mitarbeiter"innen weiterzugeben und umzusetzen (z. B. Einkauf im nahegelegenen Supermarkt).

- Ein Wunsch wird darüber hinaus als besonders legitim und berücksichtigenswert beurteilt, so dass sich der/die Mitarbeiter*in dafür engagiert, bei - einem für den/die Mitarbeiter/in nachvollziehbaren Wunsch: „Also ein Buch, ne, etwas vom höheren Niveau zum Beispiel, ne? Dass diese Wünsche, finde ich ganz wichtig, würde ich auch sofort versuchen umzu[setzen]" (SD).

- einer seltenen Wunschäußerung der jeweiligen Person, die dann als positiv wahrgenommen wird: „Wenn das jetzt irgendwas Besonderes wäre, wo man wüsste, okay, das ist jetzt nicht jede Woche, [...], dann versuchen wir das natürlich auch möglich zu machen, ne?“ (SD). 
- Im Rahmen der Interviews wurde zudem mehrfach das Arbeitsengagement der einzelnen Mitarbeitenden (siehe Kapitel 4.3.2) als Faktor benannt, der zu einer Wunschberücksichtigung beiträgt.

\section{Hemmend:}

- Hemmend, bezogen auf die Mitarbeiter*innenebene, ist an dieser Stelle im Wesentlichen der prognostizierte Aufwand, wenn ein Wunsch nicht in der Situation mit dem/der angesprochenen Mitarbeiter*in und den vorhandenen Ressourcen unmittelbar berücksichtigt werden kann.

\subsection{Alltags- und Angebotsgestaltung als Kontexte und Elemente der Teilhabeförderung}

Im Sinne des $₫ 5$ WTG NRW sind die stationären Einrichtungen dazu aufgefordert, „die gleichberechtigte Teilhabe der Nutzerinnen und Nutzer am Leben in der Gesellschaft zu unterstützen und zu fördern" und hierfür Angebote - in Zusammenarbeit mit u. a. Angehörigen, bürgerschaftlich Engagierten und Institutionen der Gemeinde - zu gestalten. Um die „Selbstbestimmung und gleichberechtigte Teilhabe der Nutzerinnen und Nutzer am Leben in der Gesellschaft" zu gewährleisten, sollen etwa zielgruppenbezogene Betätigungen in alltagsnahen und gewohnten Handlungskontexten angeboten werden, die die Fertigkeiten der Nutzer*innen fördern. Gemeinsam mit anderen Einrichtungen oder Organisationen soll zudem regelmäßig über Veranstaltungen oder Aktivitäten im Quartier informiert werden und eine Teilnahme der Bewohner*innen daran unterstützt werden. Zudem soll die Wahrnehmung auswärtiger Termine der Bewohner*innen unterstützt und gefördert werden.

Angesichts dieser landesrechtlichen Bestimmungen in $₫ 5$ WTG ist die Förderung und Verwirklichung der gewünschten Teilhabe von Bewohner*innen im Rahmen der Alltags- und Angebotsgestaltung innerhalb und außerhalb der Einrichtung nicht als besondere Dienstleistung, sondern als reguläre Aufgabe einer Altenpflegeeinrichtung und als Rechtsanspruch der Bewohner"innen zu betrachten. Daher soll in diesem Kapitel näher und exemplarisch auf die Formen und Inhalte der Alltags- und Angebotsgestaltung eingegangen werden, die in den untersuchten Einrichtungen benannt oder beobachtet wurden. Damit werden wesentliche Kontexte beschrieben, in denen Teilhabeförderung stattfindet und die zur Teilhabeförderung beitragen. Zugleich sind damit auch wesentliche Elemente der ak- 
tuell gelebten Praxis von Teilhabeverwirklichung in stationären Altenpflegeeinrichtungen beschrieben.

Um die zweite Forschungsfrage (Wie kann auf dieser Basis das Recht auf selbstbestimmte Teilhabe dieser Bewohner*innen praktisch verwirklicht und dessen Umsetzung überprüft werden?) beantworten zu können, werden im Folgenden zunächst die Ergebnisse zur Alltags- und Angebotsgestaltung innerhalb und außerhalb der Einrichtung auf Grundlage der Einzel- und Gruppeninterviews - wie bisher - wiedergegeben. Im Anschluss daran werden in diesem Kapitel ausdrücklich auch die Ergebnisse der teilnehmenden Beobachtungen aufgenommen, da diese auf die Beobachtung der Alltags- und Angebotsgestaltung fokussieren und hier die Perspektiven aus den Interviews ergänzen.

\subsubsection{Alltags- und Angebotsgestaltung innerhalb und außerhalb der Einrichtung}

Die Trennung zwischen der Alltags- und Angebotsgestaltung innerhalb und außerhalb der Einrichtung bezieht sich zunächst auf den Ort der Teilhabeförderung. Bezogen auf die Sozialform der Alltags- und Angebotsgestaltung ist ferner zwischen Gruppen- und Einzelangeboten zu unterscheiden, die ebenfalls innerhalb und außerhalb der Einrichtung stattfinden können. Darüber hinaus lässt sich eine Unterteilung in Bezug auf den Grad der Planung vornehmen und zwischen geplanten und zeitlich terminierten sowie ungeplanten und spontanen Angeboten unterscheiden.

\subsubsection{Alltags- und Angebotsgestaltung innerhalb der Einrichtung}

Im Rahmen der Interviews werden verschiedenste geplante Gruppen- und Einzelangebote von Seiten der Interviewpartner*innen beschrieben, für die der Umfang und das Spektrum des Angebotes mehrfach positiv hervorgehoben werden. Diese geplante Alltags- und Angebotsgestaltung findet vorwiegend während der Zeiten zwischen den Mahlzeiten - zwischen Frühstück und Mittagessen sowie zwischen Nachmittagskaffee und Abendessen - statt und scheint auch der Alltagsgestaltung zwischen den Prozessen der Pflege und Versorgung zu dienen: „Hauptaufgaben sind natürlich die Organisation des Alltages rund um Pflege und Essen mit - ja, Beschäftigungsangeboten, also im breitesten Sinne Großgruppenangebote, von denen wir 
in der Regel zwei am Tag haben" (SD). Folgende Inhalte von Gruppenund Einzelangeboten können hier exemplarisch aufgeführt werden:

- Die wohnbereichsübergreifend offerierten Gruppenangebote sind breit gefächert und lassen sich inhaltlich grob in sport- und gesundheitsbezogene Angebote (z. B. Gymnastik, Sitztanz, Qigong, Rollatortraining, Kegeln), Musikangebote (z. B. Singen, Tanztee), Kreativ- und Werkangebote (z. B. Basteln, Werken), hauswirtschaftliche Angebote (z. B. Koch- und Backangebote), Gesellschaftsspiele (z. B. Bingo, „Mensch ärger Dich nicht"), Informations- und Bildungsangebote (z. B. Zeitungsrunde, Vorträge), Entertainmentangebote (Filmnachmittage, Angebote über Spielkonsolen wie Bowling) sowie religiöse Angebote (z. B. evangelische und katholische Gottesdienste, Rosenkranzgebet) differenzieren.

„Bewährte“ Gruppenangebote wurden zum Teil von den jeweiligen Vorgänger*innen des Sozialen Dienstes übernommen („Also ich habe sie geerbt", SD) und aufgrund der Zufriedenheit von Bewohner*innen beibehalten. Gruppenangebote finden unter der Woche mehrmals täglich und bislang noch seltener am Wochenende statt.

Neben den wöchentlich oder monatlich wiederkehrenden Gruppenangeboten werden auch Highlight- $b z w$. Sonderveranstaltungen benannt, die als etwas Besonderes wahrgenommen werden, weil sie selten und oft in einem größeren Rahmen stattfinden. Dazu gehören v. a. jahresbezogene Feierlichkeiten (z. B. Karnevals-, Sommer- und Herbstfeste, Osterund Weihnachtsfeiern, Erntedankfest), aber auch themenbezogene Sonderveranstaltungen (z. B. Konzerte).

Darüber hinaus kann bei den Gruppenangeboten innerhalb der Einrichtung zwischen Angeboten entschieden werden, die in der Regel für eine größere Gruppe in zentral gelegenen Mehrzweckräumen stattfinden und Angeboten, die eher in kleineren Gruppen in den Wohnbereichen stattfinden, die sich auch auf die oben genannten Inhalte beziehen können. Hierzu wird benannt, dass in den letzten Jahren - auch durch eine veränderte Bewohner*innenschaft (Kapitel 4.3.1) und die Unterstützung der zusätzlichen Betreuungskräfte (Kapitel 4.4.1) - eine Verlagerung von Angeboten auf die Wohnbereiche stattfinde: „Aber wir gehen immer mehr zum Beispiel von - von der Organisation her mehr in - in die Wohnbereiche selber, bieten da etwas an" (SD).

- Unter Einzelangeboten werden Aktivitäten verstanden, die Bewohner*innen individuell angeboten und vorwiegend von zusätzlichen Betreuungskräften oder Ehrenamtler*innen zu bestimmten bzw. vorab abgestimmten Zeiten durchgeführt werden (Kapitel 4.4.1 und 4.4.2). In 
den Interviews werden inhaltlich etwa Einzelangebote der Beschäftigung, der (basalen) Stimulation oder des Gesprächs benannt: „Der Einzelkontakt kann auch sehr schön sein. Und ich denke, da sind unsere Betreuungsassistenten durch unsere Ergotherapeutin auch sehr gut angeleitet, haben da auch viele basale stimulierende Angebote. Wir haben auch eine ehrenamtliche Mitarbeiterin, die ein Klangschalenangebot macht. Da sind auch einige Mitarbeiter vom Sozialdienst geschult worden" (PDL). Eine andere Pflegedienstleitung bemängelt demgegenüber, dass Einzelangebote zu Lasten von Gruppenangeboten gehen und in diesem Moment nur einzelne Bewohner*innen ansprechen, während andere Bewohner*innen zur gleichen Zeit nicht betreut werden können: „[Das ist] für den Einzelnen sehr schön, aber dann fällt die Gruppe [...] aus. Und insofern - ja, ist einfach die personelle Ressource das Problem für Einzelangebote“ (PDL). Hervorgehoben wird aber auch die Ressource der Ehrenamtler*innen für Einzelangebote: „Und was nicht zu unterschätzen ist, sind diese - diese Einzelkontakte. Also wenn Ehrenamtlerinnen Bewohner, die nicht mehr so mobil sind, im Haus aufsuchen, das sagte ich eben, auch wenn Menschen reinkommen ins Haus und dann erzählen von der Stadt, was passiert ist“ (PDL). Neben den geplanten Gruppen- und Einzelangeboten werden ungeplante bzw. spontane Aktivitäten und Angebote innerhalb der Einrichtung erwähnt, bei denen der Alltag insbesondere in individuellen Kontakten mit folgenden Personengruppen gestaltet wird:

- Mitarbeiter"innen: Hier werden etwa Kurzkontakte und -gespräche mit Mitarbeitenden genannt, wie hier mit einer Pflegekraft: „, [der] Nachtschwester [...] dann werden mal so zwei, drei Worte gequatscht und dann gehe ich dann schlafen" (BW). Hinzu kommen spontane Einzelbegleitungen, die insbesondere durch zusätzliche Betreuungskräfte erfolgen, wenn etwa akuter Bedarf der Begleitung besteht: „Gerade wenn unruhige Bewohner da sind, ist das sehr belastend für die Mitarbeiter der Pflege und das ist dann schön, eine Unterstützung durch die Alltagsbegleitung" (PDL). Da häufig vom Wetter abhängig, kann hier die Begleitung von Bewohner*innen durch Mitarbeitende in den Garten der Einrichtung auch als spontane Aktivität hinzugezählt werden: „Betreuungsassistenzen mehr zuständig $[\ldots]$ in Sommerzeit rausgefahren in Garten" (HT).

- Andere Bewohner"innen: Im Rahmen von einzelnen Interviews wird beschrieben, wie die Bewohner*innen Kontakte zueinander pflegen und etwa miteinander Gespräche führen, gemeinsam fernsehen oder in Spielerunden miteinander spielen: „Ja, wir spielen Rummy Cup [...] 
Das machen wir jeden Tag“ (BW). Auch alte Freundschaften haben sich, nach Aussagen einzelner Interviewpartner*innen, in den Einrichtungen wiedergefunden. Benannt werden auch Begegnungsorte innerhalb der Einrichtung, an denen die Bewohner*innen spontan in Kontakt kommen können, wie z. B. in der Cafeteria.

- Angehörige und Bekannte: Die Besuche von Angehörigen oder auch Bekannten können teils auch zu ungeplanten bzw. für die Bewohner*innen spontanen und in der Regel aber zu den individuellen Kontakten und Aktivtäten in der Einrichtung gezählt werden. Neben der Gesprächsführung mit den Angehörigen und dem gemeinsamen Kaffeetrinken in der Einrichtung oder im Zimmer werden auch alte bzw. gewohnte Hobbys und Interessen der Bewohner*innen unterstützt. Die Inhalte der Aktivitäten sind auch bei den Angehörigen oft spontan vor dem Hintergrund, dass die Tagesform der Bewohner*innen zu berücksichtigen ist: „Ja, ich setze mich dann ans Bett und gucke. Also wenn ich - wenn sie wach ist, rede ich mit ihr, aber vielfach lese ich auch" (ANG). Ein Angehöriger beschreibt etwa, dass er für seine Mutter einen digitalen Bilderrahmen mit Fotos aus der Vergangenheit und Gegenwart zur Verfügung gestellt hat und dann damit das Gespräch mit seiner Mutter spontan anregt: „Dass sie sich [...] aus der Erinnerung raus vielleicht an irgendeine oder die andere Sache erinnert" (ANG).

Der Alltag innerhalb der Einrichtung wird darüber hinaus auch durch die Bewohner"innen selbst (für sich) gestaltet. So wird im Rahmen der Interviews von Bewohner*innen benannt, dass sie zwar gerne an den Angeboten des Sozialen Dienstes teilnehmen, aber ebenso gerne alleine Zeit verbringen, wobei vorwiegend Aktivitäten im eigenen Zimmer benannt werden.

- Im Zimmer: Verschiedenste Aktivitäten im Zimmer werden benannt, wie etwa fernsehen, lesen, Musik und Hörbücher hören oder Handarbeit: „Ich mache ja auch viel Handarbeit. Auch für andere. Und da habe ich immer einige Aufträge, [so] dass ich immer beschäftigt bin. Ich kenne keine Langeweile in dem Sinn" (BW). Eine Bewohnerin, die aufgrund ihrer körperlichen Einschränkungen nicht mehr so aktiv an Angeboten teilnehmen kann, beschreibt etwa, dass sie gerne am Fenster sitzt und ihr Umfeld beobachtet. Einzelne setzen auch alte Rituale oder Gewohnheiten fort und trinken z. B. ihr allabendliches Bier: „Sie hat zu Hause immer abends eine kleine Flasche Bier getrunken [...] auch jetzt“(ANG). 


\subsubsection{Alltags- und Angebotsgestaltung außerhalb der Einrichtung}

Im Rahmen der Interviews wurden ferner Gruppenangebote außerhalb der Einrichtung beschrieben, welche im Folgenden zudem in Ausflüge innerhalb und außerhalb des Ortes der Einrichtung unterschieden werden können. Der Grad der Planung ist hier unterschiedlich.

Während Ausflüge außerhalb des Ortes eine längere Planung benötigen, können Ausflüge innerhalb des Ortes sowohl zuvor festgelegt als auch in selteneren Fällen spontan stattfinden, z. B. bei gutem Wetter und dann vorhandenen personalen Ressourcen: „ [... Sie] gehen mal hinten zum Markt, mit ein paar Leuten, aber das ist natürlich auch je nachdem, wie das Personal vorhanden ist, abhängig davon“ (HT). Folgende Angebotsinhalte von Ausflügen in der Gruppe lassen sich exemplarisch für Ausflüge innerhalb und außerhalb des Ortes nennen:

- Ausflüge innerhalb des Ortes: Im Rahmen der Interviews werden etwa der Besuch von besonderen Highlight-Veranstaltungen vor Ort benannt, die an bestimmte Termine gebunden sind, wie der Besuch von Schützenfesten, Karnevalszügen, Weihnachtsmärkten und die Beteiligung am Seniorentag. Ferner werden Ausflüge in den Ort beschrieben, die alltäglicher und weniger an bestimmte Termine im Jahr gebunden sind, wie der Besuch von Eiscafés, Wochenmärkten, Geschäften oder einem Einkaufszentrum. Der Ausflug in ein nahegelegenes Einkaufszentrum findet in einer Einrichtung in einem Turnus von drei Monaten statt. In einem anderen Interview werden bewusst Ausflüge in „Lokalitäten im Umkreis“ (PDL) erwähnt, um die Kontakte im aktuellen und früheren Wohnumfeld zu fördern: „[Dorthin,] wo die Menschen auch herkommen, da mal zum Kaffeetrinken, und wenn man dann den Wirt kennt oder so" (PDL).

- Ausflüge außerhalb des Ortes: Hierzu zählen Ausflüge, die über einen ganzen oder halben Tag gehen und einen anderen Ort zum Ziel haben. Inhaltlich wurden etwa Ausflüge in umliegende Orte mit Kaffeetrinken ebenso wie Tierpark- und Zoobesuche, Besuche von Konzerten, Kinovorstellungen und Museen sowie Schifffahrten genannt. Die Ausflüge finden in einzelnen Einrichtungen einmal im Monat statt, in anderen bewusst ausschließlich während der wärmeren Jahreszeit: „Donnerstags haben wir zum Beispiel nachmittags immer jetzt in der schönen Jahreszeit, so von März bis Oktober, unsere Ausflüge“ (SD).

Damit Ausflüge stattfinden können, müssen zum Teil lange Anfahrtswege in Kauf genommen werden. Gerade eine „ländlicher“ gelegene Einrichtung beklagt die langen Fahrten, die aber weiter in Kauf genom- 
men werden, da es positive Rückmeldungen von den Bewohner*innen zu diesen Ausflügen gäbe. Ausflüge außerhalb des Ortes werden vorher in Bezug auf die Teilnehmer*innenplätze normiert und einerseits für eine bestimmte Anzahl an Bewohner*innen organisiert, damit mehr Bewohner*innen „was davon haben“, und andererseits entsprechend den Platzkapazitäten in Bezug auf das Transportmittel (z. B. Bus) und personelle Ressourcen für die Begleitung begrenzt.

- Urlaubsreisen: Als weiteres, besonderes und selteneres Gruppenangebot außerhalb der Einrichtung werden Urlaubsfahrten beschrieben, die zumindest in einzelnen Einrichtungen mit einer Auswahl an Bewohner*innen in größeren Abständen vorgenommen werden: „[...] dass hier welche gemeinschaftlich in den Urlaub fahren" (ANG). Diese Urlaubsreisen werden teils durch Spenden finanziert, wenn die Kosten einer solchen Reise die regulären finanziellen Mittel der Bewohner*innen und der Einrichtung übersteigen: „Dann ist der - der Förderverein fördert zum Beispiel auch unsere Urlaubsfahrt, -fahrten, die wir mehrfach gemacht haben" (EL).

Wiederum werden in den Interviews auch geplante wie ungeplante Einzelangebote und -begleitungen außerhalb der Einrichtung benannt, die von folgenden Personengruppen durchgeführt werden:

- Mitarbeiter"innen und Ehrenamtler"innen: Benannt werden vor allem Ausflüge oder Erledigungen in der näheren Umgebung, die oft von zusätzlichen Betreuungskräften und teils auch von Ehrenamtler*innen begleitet werden: „Wie wir zum REWE gelaufen sind, oder - ja, oder eben das Eis auch mal essen können, was man nicht alleine kann dann" (ZBK); „,Wir] hatten [...] eine Bewohnerin, die ist regelmäßig nach Gummersbach mit einer Ehrenamtlerin gefahren. Im Moment haben wir eben eine, die regelmäßig zum Friedhof fährt und zum - zum Aldi einkaufen“ (PDL). Hinzu kommt häufiger auch die Begleitung von einzelnen Bewohner*innen bei Arztbesuchen, die von unterschiedlichen Mitarbeiter*innengruppen durchgeführt werden kann (z. B. Pflegekräfte, Betreuungskräfte oder aber auch Kräfte, die einen Freiwilligendienst oder eine Beschäftigungsförderungsmaßnahme absolvieren, je nach Voraussetzungen, die die Bewohner*innen mitbringen). In einer Einrichtung werden diese Begleitungen vom Sozialen Dienst koordiniert (Kapitel 4.4.5).

- Angehörige und Bekannte: Manche Bewohner*innen gehen mit ihren Angehörigen oder Bekannten im Ort z. B. etwas einkaufen, zur Bank oder ein Eis essen. Eine Bewohnerin berichtet auch davon, wie sie ihren Geburtstag im nahegelegenen Restaurant mit einer Freundin ver- 
bracht hat: „[Da] hatte ich Geburtstag [...] habe ich meine Freundin zum Essen eingeladen, sind wir hier vorne an der Ecke [...] zum Essen gewesen" (BW).

Darüber hinaus werden auch individuelle und selbstständige Alltagsgestaltungen außerhalb der Einrichtung benannt, die sich vor allem auf die nähere Umgebung beziehen, oft mit Mobilitätshilfen erfolgen und nur noch von einer kleineren Minderheit der Bewohner*innen alleine wahrgenommen werden können:

- Selbstständige Ausflüge in der Umgebung: So wird im Rahmen der Interviews berichtet, dass einzelne orientierte Bewohner*innen durchaus ihren Alltag außerhalb der Einrichtung gestalten und zwar mithilfe von verschiedensten Hilfsmitteln. Eine Bewohnerin geht etwa - unterstützt durch ihren Rollator - einkaufen: „[Sie ist eine] Bewohnerin, die noch - noch mit Rollator eigentlich gut mobil zu Fuß ist, [sie| geht einkaufen hier schräg gegenüber" (PDL). Andere Bewohner“innen gehen selbstständig spazieren oder fahren auch mit öffentlichen Verkehrsmitteln alleine in einen nahegelegenen Ort. Ein Bewohner erzählt, wie er mithilfe seines Elektrorollstuhls in der Umgebung durchaus längere Entfernungen wahrnehmen kann: „Ja, und dann wird das Ding wieder oben in die Ecke gestellt und aufgeladen. Ne, ungefähr 10 Kilometer kann ich damit fahren" (BW). Zu einem anderen Bewohner wird berichtet, wie er einem allabendlichen Ritual in der Umgebung nachgeht: „Der geht jeden Abend in die Kneipe und trinkt da sein Klärchen, also seinen - seinen klaren Schnaps und kommt dann abends dann nach Hause“ (PDL).

Im Folgenden werden die fördernden und hemmenden Faktoren beschrieben, welche sich auf die Alltags- und Angebotsgestaltung innerhalb und außerhalb der Einrichtung beziehen.

\section{Fördernd:}

- Es existiert eine Vielfalt an Gruppen- und Einzelangeboten in den verschiedenen Einrichtungen, um die Teilhabe von den Bewohner"innen zu unterstützen. Insbesondere montags bis freitags finden täglich mehrere Gruppenangebote statt, um den Alltag der Bewohner*innen zu gestalten und zu strukturieren, wobei laut den Interviews eine Stärkung von Angeboten der Alltags- und Angebotsgestaltungen an den Wochenenden gewünscht wird.

- Unterstützung bei der Durchführung der Angebote erhält der Soziale Dienst (bestehend aus Mitarbeiter"innen unterschiedlicher Professionen, z. B. Sozialarbeiter*innen/-pädagog*innen, Pädagog*innen, Ergo- 
therapeut*innen und zusätzlichen Betreuungskräften) von Ehrenamtler"innen, vereinzelt auch von Angehörigen, die mit an Angeboten teilnehmen.

- Je nach Rückmeldung nach den Angeboten, den jeweiligen Teilnehmer*innenzahlen und dem von Bewohner*innen mitgeteilten thematischen Interesse finden Angebote häufiger statt oder werden reduziert. Wichtig ist, dass die Akzeptanz der Angebote regelmäßig reflektiert bzw. evaluiert und der jeweiligen Bewohner*innenschaft angepasst wird. $\mathrm{Ob}$ und inwiefern Bewohner"innen zufrieden waren und ob sie erneut an einem Angebot teilnehmen möchten, wird in einer Einrichtung explizit in den Berichtsblättern dokumentiert: „,[Das] ist unterschiedlich. Also sagen wir mal so, eigentlich, wenn was fest eingeplant ist, zum Beispiel Bingo,[...]. Es sei denn, sie [eine Bewohnerin; Anm. d. Verf.] wollte da mal an dem Tag nicht. Aber was Neues, oder wenn da was neu zugekommen oder was auffällt, dann schreibe ich dann. Manchmal ein-, zweimal in der Woche und manchmal auch alle zwei - alle zwei Wochen, dass ich dann mal [...]wirklich individuell“ (ZBK).

- In den Einrichtungen wird unterschiedlich damit verfahren, wie die Informationsweitergabe bzgl. der zukünftig stattfindenden Angebote erfolgt. In zwei der Einrichtungen wird explizit genannt, dass es Wochenpläne für Freizeitangebote gibt, die in ihrem Angebot pro Woche mal mehr, mal weniger variieren. Dies steigert die Übersichtlichkeit für die „orientierten“ Bewohner"innen, sie können selbstständig den Wochenplan einsehen und im Vorfeld selbst bestimmen und entscheiden, an welchen Angeboten sie teilnehmen möchten. Benannt wird auch eine monatliche Hauszeitung, in der die jeweiligen wöchentlichen Aktivitäten dargestellt werden. Hinzukommen aushängende Ankündigungen für spezielle Angebote, die außerhalb des Hauses stattfinden und für die man sich anmelden muss.

- Mitarbeitende sprechen Bewohner*innen gezielt an, um diese auf eine Aktivität hinzuweisen und sie zu motivieren, an dieser teilzunehmen, respektieren aber auch Signale oder Aussagen in Bezug auf Wünsche der Nichtteilhabe (Kapitel 4.1.3).

- In Bezug auf die Organisation der Angebote ist die Information der Mitarbeitenden über die Angebote sowie die diesbezügliche Kommunikation und Abstimmung unter den Mitarbeitenden von großer Bedeutung (Kapitel 4.3.3.3). Es wird jedoch auch beschrieben, dass es personenabhängig sei, inwieweit Mitarbeitende das im Blick haben: „Meistens ist das ja mitarbeiterabhängig. Der eine hat das auf dem Schirm, der andere nicht. Und dann gehe ich einfach noch mal hin und sage: Bitte, 
denkt dran, die ist bei der Gruppe dabei, das ist das einzige Angebot, was sie bei mir hat. Ist das nicht möglich, dass die später zum Friseur kommt, ne?“ (SD). Förderlich wäre demnach, wenn es weniger von den einzelnen Mitarbeiter*innen abhängig ist und die Information über die Angebote nicht nur für alle Mitarbeitende transparent, sondern auch die Informationsverwertung geregelt wäre.

- Wie bereits beschrieben, werden Angebotsinteressen auch von Seiten der Mitarbeitenden erfragt (Kapitel 4.1.4). Das heißt, sie gehen etwa in „Vorleistung“, entwickeln Ideen und stellen diese den Bewohner"innen vor, so dass die Teilhabe der Bewohner*innen trotz allem ermöglicht werden kann. Gerade der Bewohner*innenbeirat wird bei der Angebotsentwicklung mit einbezogen. Das heißt, dass durch die eigene Interessenvertretung Teilhabe potenziell ermöglicht wird. Andere Angebote wiederum kommen durch das Wissen um die jeweilige Biografie der Bewohner*innen zustande.

- In jede Einrichtung kommen regelmäßig oder zu bestimmten Anlässen Externe. Dabei stehen mögliche Angebotsplanungen und -inhalte im Zusammenhang mit den Kontakten, die eine Einrichtung nach außen pflegt (z. B. Karnevalsvereine, Kirchengemeinde, Kindergärten, Schulen; Kapitel 4.4.7). Auch Verbindungen zum Einzelhandel spielen eine Rolle, so wird beschrieben, dass beispielsweise Modegeschäfte in die Einrichtung kommen, ein Dorfladen und ein Schuhgeschäft. Friseur*innen, Podolog*innen und Physiotherapeut*innen kommen ebenfalls in die Einrichtungen und können auch zur Teilhabe im Alltag innerhalb der Einrichtung beitragen.

\section{Hemmend:}

- In den Einrichtungen gibt es bisher nur eingeschränkt Angebote an den Wochenenden oder in der Abendzeit. Zurückzuführen ist dies auf die knappere personelle Besetzung an den Wochenenden und entsprechende Restriktionen in der Dienstplangestaltung. Dass eingeschränkte personelle Ressourcen im Abend- und Wochenendbereich auch Einschränkungen der Teilhabeförderung mit sich bringen können, wird auch bereits in der Literatur thematisiert, wie im Folgenden von Schumacher (2018) beispielhaft für die Abendzeit festgestellt wird: „Interne Regelsysteme wie die Dienstplanorganisation machen abendlichen Ausgang oder andere Aktivitäten unmöglich, und Standardversorgungsleistungen (Leistungstypen) erfüllen nur teilweise den individuellen Bedarf [...]. Dies führt dazu, dass grundlegende Kompetenzen nicht mehr ausgeübt werden können und Zugänge zu selbstgewählten Aktivitäten 
nicht ermöglicht werden [...]. Probleme werden nur insoweit gesehen und bearbeitet, als in der Organisation Lösungen zur Verfügung stehen oder generiert werden können" (Schuhmacher, 2018, S. 99).

- Die Angebots-Zufriedenheit der Bewohner*innen wird teilweise anhand der Teilnehmer"innenanzabl gemessen. Wenn sich die Angebotsplanung und -gestaltung primär an hohen Teilnahmequoten orientiert, kann dies für individuelle Teilhabeperspektiven und -wünsche einschränkend sein und sollte - nicht nur - in Perspektive selbstbestimmter Teilhabe als Erfolgsindikator kritisch betrachtet werden.

- Angebotsdifferenzierung nach bestimmten Bewohner"innengruppen - z. B. zwischen Menschen mit und ohne Demenz (Kapitel 4.3.1) - können in der Angebotsgestaltung unterstützend sein, aber auch exkludierend für einzelne Bewohner*innen wirken und zu Verfestigungen von Ungleichheiten unter den Bewohner*innen in der Einrichtung führen.

- Im Rahmen der Interviews werden verschiedene Ressourcen als fehlend oder begrenzt hervorgehoben, die bereits von Bedeutung für die Angebotsplanung sind: Neben fehlenden personellen Ressourcen, insbesondere für Aktivitäten außerhalb der Einrichtung, sind dies v. a. auch begrenzte Räumlichkeiten (Kapitel 4.4.6) und Budgets, welche dem Sozialen Dienst für Angebote zur Verfügung stehen (Kapitel 4.4.4).

\subsubsection{Alltagsstrukturierende Schlüsselprozesse aus dem Blickwinkel der Beobachtung}

Dieses Kapitel ist den Ergebnissen der teilnehmenden Beobachtung (und den in diesem Kontext geführten Kurzgesprächen) gewidmet. Da im Zentrum dieser Erhebungsmethode die Beobachtung der sozialen Praxis der Alltags- und Angebotsgestaltung in den Einrichtungen lag, werden die Ergebnisse ausschließlich in dieses Kapitel integriert.

Die Ziele und methodische Vorgehensweise im Rahmen der teilnehmenden Beobachtung wurden bereits erläutert (Kapitel 3). An dieser Stelle soll nur wiederholend erwähnt werden, dass für die Auswertung ein Kodierungsschema entwickelt wurde, das nach Sichtung des Beobachtungsmaterials auf den Bewohner*innenalltag strukturierende Schlüsselprozesse im Material fokussierte. Unter strukturierenden Schlüsselprozessen im Bewohner*innenalltag werden alle überindividuellen, den Tagesablauf der Bewohner*innen zeitlich und inhaltlich in seinen Üblichkeiten strukturierenden Sequenzen verstanden, die von Seiten der Organisation auch als Arbeitsprozesse definiert sind. Im Vordergrund steht jedoch der Alltag der 
Bewohner*innen und nicht der Arbeitsalltag der Mitarbeitenden. In den Blick genommen wurde im Rahmen der Auswertung daher auch die Aufrechterhaltung der Normalität im Rahmen dieser Schlüsselprozesse im Bewohner*innenalltag.

\subsubsection{Alltags- und Angebotsgestaltung}

Im Zuge der Beobachtungen wurden verschiedene (Gruppen- und Einzel-)Angebote betrachtet. Diese finden in der Regel im Vormittags- und Nachmittagsbereich unter der Woche statt. Auch die individuelle Alltagsgestaltung der Bewohner*innen wurde zu verschiedenen Tageszeiten, insbesondere in den Wohnbereichen, beobachtet. Die Beobachtungen fanden in (Allzweck-)Räumen und den Wohnbereichen, auf den Bewohner*innenzimmern sowie außerhalb der Einrichtung statt. In diesem Zusammenhang wurden ferner verschiedene Dienstleistungsangebote in die Beobachtung integriert (z. B. Frisör, Schuhverkauf in der Einrichtung).

\section{Fördernd: ${ }^{61}$}

- Die Gruppenangebote innerhalb der Einrichtungen wurden von einer gröBeren Anzahl von Bewohner*innen besucht, wobei in dieser Gruppensituation eine sebr positive Grundstimmung herrschte - es wurde miteinander gelacht, geredet und sehr aktiv an den Inhalten des Angebotes teilgenommen.

- Bei den meisten beobachteten Angeboten war der zeitliche Rabmen stets angemessen, so dass dieser zu keiner Erschöpfung oder gegebenenfalls Überlastung auf Seiten der Bewohner"innen führte.

- In zwei Einrichtungen wurden Handlungserfolge der Bewohner"innen durch schnelles Erfassen der Aufgaben und Mitmachen beobachtet (z. B. Tanztee, Kegeln).

- Die Bewohner*innen konnten ohne An-/Einleitung teilnehmen, egal, welche körperlichen oder kognitiven Einschränkungen sie hatten. Das heißt, dass einzelne Angebote in besonderer Weise barrierefrei und inklusiv ausgelegt waren (Kapitel 4.2.1.2). So wurden etwa Freizeitangebote für Menschen mit und ohne physische und/oder psychische Einschränkungen angeboten. Damit auch Personen mit größeren Ein-

61 Beobachtungen, die auf die Aufrechterhaltung von Normalität, eine Orientierung an Lebensgewohnheiten und Interessen im Rahmen der Alltags- und Angebotsgestaltung hinweisen. 
schränkungen unterstützt werden konnten, halfen zusätzliche Betreuungskräfte im Rahmen der Angebote mit.

- In den Einrichtungen gestalten auch externe Personen verschiedenste (Dienstleistungs-)Angebote für die Bewohner*innen. Im Rahmen der Beobachtung kamen etwa eine Puppenspielerin, ein Hundebesuchsdienst und ein Schuhgeschäft (der sogenannte Pfennigmarkt) in die verschiedenen Einrichtungen, die den Alltag „belebten“. So brachte etwa der Hundebesuchsdienst positive Erinnerungen an früher hervor, wie einzelne Reaktionen der Bewohner*innen zeigten. Durch den Besuch des Schuhgeschäftes wird innerhalb der Einrichtung auch ein Kauf von Konsumgütern ermöglicht, wodurch Situationen entstanden, in denen Bewohner*innen - etwa durch die Auswahl von Produkten auch ein Kauferlebnis hatten und ein Gefühl, aktive Kund"innen zu sein. Des Weiteren wurde auch der Besuch eines Friseurs beobachtet, bei dem ebenfalls das die Selbstbestimmung fördernde Gefühl, Kund"in zu sein, beobachtet werden konnte, wenn die Vorlieben der Bewohner"innen erfragt wurden.

- Die Einzelbetreunng, mit der vor allem auch die Teilhabeförderung der stärker eingeschränkten Bewohner*innen gesichert wird, findet sowohl in den Zimmern der jeweiligen Bewohner*innen als auch außerhalb der Einrichtung statt (Kapitel 4.2.1.2). Es handelte sich hierbei hauptsächlich um Bewohner*innen, die bettlägerig oder schwer zu mobilisieren sind. Es wird etwa basale Stimulation durchgeführt oder, bei „fitteren" Bewohner*innen, die sich noch äußern können, etwa Gedächtnistraining.

- Das „Trial-and-Error"-Verfahren wird bei Bewohner"innen angewendet, die sich nicht mehr äußern können. Hier wird sehr auf die Mimik und Gestik der Bewohner*innen geachtet, um Vorlieben und Abneigungen erkennen zu können.

- In zwei Einrichtungen gibt es aktuell regelmäßig stattfindende Gesellschaftsspielrunden, die auf eigene Initiative von Bewohner*innen zustande gekommen sind.

- Der Wohnbereich, das Foyer (zumindest in einzelnen Einrichtungen) und das eigene Zimmer werden von den Bewohner*innen rege genutzt und stellen beliebte Aufenthaltsorte zur individuellen Alltagsgestaltung dar. Die Raucher"innenecken/-Terrassen werden in beinahe jeder Einrichtung von den Bewohner*innen genutzt - auch von Personen, die nicht rauchen. Es handelt sich anscheinend um einen geselligen Ort, an dem sich einige Bewohner*innen gerne aufhalten, um andere zu treffen. 
- In zwei Einrichtungen versammelten sich im Beobachtungszeitraum am Abend Bewohner*innen im Wohnbereich, um gemeinsam fernzusehen (Nachrichten, Fernsehsendungen). Während sich die Runde in einer der Einrichtungen schnell nach den Nachrichten auflöste, hatte die Fernsehrunde in der anderen Einrichtung mehr Ausdauer und entwickelte eine Dynamik, so dass zur "Stammrunde“ (drei bis fünf Personen) auch zwischenzeitig andere Bewohner*innen hinzukamen.

- Im Rahmen eines Gruppenangebotes außerhalb der Einrichtung (Ausflug) wurde mit Unterstützung von Mitarbeitenden der Besuch in einer Gaststätte ermöglicht. Es konnten auch mehrfach eingeschränkte Bewohner*innen daran teilnehmen. Unabdingbar waren ein Kleinbus mit der Möglichkeit zum Transport von Rollstühlen sowie die entsprechende Fahrerlaubnis von Seiten der Mitarbeitenden.

- Durch die individuelle Betreuung über Ehrenamtler"innen, zusätzliche Betreuungskräfte oder Angehörige im Rahmen von Einzelangeboten außerhalb der Einrichtung wurde es Bewohner*innen ermöglicht, in das Wohnumfeld (z. B. zum Supermarkt) begleitet zu werden, um alte Routinen aufrechterhalten zu können (z. B. Kauf von Zeitschriften oder Schokolade). Gerade in einer Einrichtung wurden Bewohner*innen beobachtet, die das direkte Quartier mit nahegelegenen Infrastrukturen (z. B. Einkaufsmöglichkeiten und Restaurants) mit Begleitung von zusätzlichen Betreuungskräften, Angehörigen oder auch selbstständig nutzten.

\section{Hemmend:62}

- Gruppenangebote, die nur für bestimmte Bewohner*innengruppen vorgesehen sind, können von den Bewohner*innen als exkludierend wahrgenommen werden. So konnten vereinzelt Menschen mit Demenz beispielsweise nicht an Ausflügen teilnehmen, die für Menschen ohne Demenz geplant und vorbereitet wurden - umgekehrt galt das gleiche. Dies kann zum Gefühl der Ungleichbehandlung führen, was im Kontext der teilnehmenden Beobachtung von einer Mitarbeiterin des Sozialen Dienstes erwähnt wurde. Dass Angebotsdifferenzierungen für den Prozess der Angebotsteilnahme jedoch auch von Vorteil und etwa entlastend für die Bewohner*innen sein können, zeigt auch die kontroverse Diskussion zu den Vor- und Nachteilen integrativer und segregativer

62 Beobachtungen, die auf eine geringe Aufrechterhaltung von Normalität und Orientierung an Lebensgewohnheiten und Interessen im Rahmen der Alltags- und Angebotsgestaltung hinweisen. 
Betreuungsformen für Menschen mit Demenz (z. B. Schuhmacher, 2017, S. 101).

- Ferner konnte auch beobachtet werden, dass vereinzelt nicht genügend Betreuungspersonen im Rahmen von Angeboten vorgehalten werden konnten, um den verschiedenen Bewohner*innenvoraussetzungen und -ansprüchen gerecht zu werden.

- Als hemmender Faktor konnte vereinzelt auch die nichtoptimale methodisch-didaktische Umsetzung von Gruppenangeboten identifiziert werden, die möglicherweise mit fehlenden Grundlagen im Bereich des Fach- und Methodenwissen oder auch unterschiedlichen Voraussetzungen in der Sozialkompetenz der Angebotsdurchführenden verbunden sein kann. So entstand beispielsweise im Rahmen eines Angebots ein sehr schulischer Charakter, indem keine Seitengespräche zugelassen wurden und ausschließlich frontal gesprochen wurde. In einem anderen Fall wurde am festen Repertoire von Inhalten konsequent festgehalten, ohne Abweichungen zuzulassen - auch bei Unruhe. Reaktionsund Teilnahmslosigkeit von Bewohner*innen führten in einer anderen Beobachtungssituation in einem Gruppenangebot dazu, dass die zusätzlichen Betreuungskräfte sich miteinander unterhielten und nicht versuchten, das Angebot zu ändern, um die Bewohner*innen zu aktivieren. So kam es auch vor, dass über den Kopf einer Bewohnerin hinweg entschieden wurde - ohne sie nach ihrer Meinung zu fragen.

- Zeiten, in denen keine Ansprache oder Angebote erfolgten - gerade vor und nach den Mahlzeiten- wurden als Wartezeiten erlebt und stellten daher für viele Bewohner*innen (insbesondere für jene mit kognitiven Einschränkungen) eine große Herausforderung dar. Typische, längere und für die Bewohner*innen problematische Wartesituationen waren einerseits das Warten nach dem Frühstück, wo manche Bewohner*innen - teils ohne Anregung, Ansprache oder Angebote der Selbstbeschäftigung - an ihren Tischen verblieben. Andererseits beziehen sich Wartesituationen auf das Warten nach dem Abendessen auf die Begleitung in das eigene Zimmer, wo Bewohner*innen auch Unmut über das Warten und Orientierungsfragen äußerten (z. B. wann und von wem sie abgeholt würden).

- Am Abend (teils bereits nach 19:00 Uhr und teils erst nach 20:00 Uhr) waren sowohl in den Wohnbereichen als auch in den wohnbereichsübergreifenden Räumen selten Bewohner"innen aufzufinden (eine Ausnahme siehe oben) und Gruppen- oder Einzelangebote fanden dort zu dieser Zeit - zumindest im Beobachtungszeitraum - nicht statt. Hier stellt sich jedoch die Frage, inwieweit diese Situation auch auf Wunsch 
der Bewohner*innen (z. B. physische Erschöpfung, Rückzug in das eigene Zimmer) oder aufgrund von Routinen und fehlenden Ressourcen in den Einrichtungen (z. B. Arbeitszeiten und -aufkommen im Spätdienst) zustande kommt.

- Die Auswabl der Leistung innerhalb einer beobachteten Friseurdienstleistung bemaß sich nur an der vorhandenen Kundenkarte. So wurde nicht abgefragt, ob es etwas grundsätzlich anderes als das übliche Angebot geben solle. Es war zudem nicht möglich, Trinkgeld zu geben, so dass durch das bargeldlose Zahlen (Betrag wurde über Bewohner*innenkonto abgerechnet) am Ende des Friseurbesuchs ein entscheidender Moment der Kundensouveränität fehlte.

- Spezifische Konsumangebote innerhalb der Einrichtungen, wie ein (anderer) an bestimmten Terminen stattfindender Schuhverkauf und ein durchgängig verfügbarer Einrichtungskiosk, wurden in dem Beobachtungszeitraum nicht genutzt, wozu der hemmende Faktor jedoch nur interpretiert werden kann. Es könnte etwa daran liegen, dass die Preise über denen von günstigeren Angeboten außerhalb der Einrichtung gelegen haben.

- Teils wurden Gärten nicht aufgesucht, obwohl das Wetter während der Beobachtungen schön war. Eine Erklärung könnte beispielsweise die schlechte Erreichbarkeit innerhalb einer Einrichtung sein, da man das Gebäude durch den Haupteingang verlassen musste, um den Garten durch ein Tor betreten zu können. Dies könnte ein Hemmnis für Bewohner*innen (mit einer Gehbehinderung) darstellen.

\subsubsection{Mahlzeiten}

Die Beobachtungen fanden zu verschiedenen Tageszeiten und an unterschiedlichen Orten in den Einrichtungen statt. So wurden auch das Frühstück, Mittagessen, Kaffeetrinken und Abendessen beobachtet - sowohl in einzelnen Wohnbereichen als auch in der Cafeteria. Da die Mahlzeiten damit nicht nur viermal täglich, sondern auch zu festgelegten Uhrzeiten bzw. in bestimmten Zeitkorridoren stattfinden, gehören sie eindeutig zu zentralen alltagsstrukturierenden Schlüsselprozessen für die Bewohner*innen, die ihnen aber auch Teilhabe ermöglichen. 
Fördernd: ${ }^{63}$

- Unter „orientierten“ Bewohner*innen war in einzelnen Einrichtungen eine selbstständige Tischkommunikation zu beobachten, die auch während der Mahlzeit fortgesetzt wurde. Die „orientierten“ Bewohner“innen wurden während der Mahlzeiten in der Cafeteria von Mitarbeitenden nicht begleitet. Sie nahmen die Mahlzeiten selbstständig ein und wurden nicht „aktiviert“. Lediglich die hauswirtschaftlichen Mitarbeiter*innen nahmen währenddessen Kontakt zu den Bewohner*innen auf. Dabei verhielten sie sich freundlich und den Bewohner*innen zugewandt.

- Kognitiv eingeschränkte Bewohner*innen wurden während der Mahlzeiten in den Wohnbereichen von zusätzlichen Betreuungskräften oder Pflegekräften begleitet und unterstützt. Dabei gestalteten oder förderten (in zwei Einrichtungen) die zusätzlichen Betreuungskräfte die Kommunikation - hierbei boten sie den Bewohner*innen eine gute Orientierung, stellten eine gesellige und dennoch ruhige Tischsituation her und motivierten die Bewohner*innen, das Essen zu sich zu nehmen.

- In einer Einrichtung wurde im Beobachtungszeitraum zum Frühstück und Abendessen von Tablett- auf Buffetform umgestellt. Das spontane Zusammenstellen von Mahlzeiten wurde von den Bewohner*innen und den Mitarbeiter"innen als positiv wahrgenommen. So ermöglicht das Buffet insofern eine Orientierung an der Normalität und Selbstbestimmung der Bewohner*innen, indem die Bewohner*innen zum Zeitpunkt der Mahlzeit wählen können, was sie essen möchten. In einer anderen Einrichtung gab es ein sogenanntes rollendes Buffet in der Cafeteria. Mitarbeiter*innen hielten mit einem Servierwagen an den jeweiligen Tischgruppen und die Bewohner*innen konnten sich auf ihrem Platz sitzend aussuchen, was sie zum Frühstück oder Abendessen zu sich nehmen wollten.

- Auch wenn die Mahlzeiten zu bestimmten Zeiten eingenommen wurden, herrschte dabei zumindest für die Bewohner*innen kein Zeitdruck. Jedenfalls konnte innerhalb der Beobachtungszeiträume in keiner Einrichtung beobachtet werden, dass zeitlicher Druck auf die Bewohner*innen ausgeübt wurde, die Mahlzeiten schnell einzunehmen oder frühzeitig zu beenden.

63 Beobachtungen, die auf die Aufrechterhaltung von Normalität, eine Orientierung an Lebensgewohnheiten und Interessen im Rahmen der Mahlzeiten hinweisen. 


\section{Hemmend: 64}

- Insbesondere in der Cafeteria gibt es feste Sitzplätze, die - aufgrund „gewachsener" bzw. vorhandener Sitzplatzstrukturen - nicht von den Bewohner*innen gewählt werden können, sondern von den Mitarbeitenden festgelegt werden. Dies kann dazu führen, dass Bewohner*innen ohne Bezug zueinander zusammensitzen, zumindest konnten Tischformationen beobachtet werden, bei denen keinerlei Beziehung bzw. Gesprächskontakt zwischen Bewohner*innen zu erkennen war.

- Im Rahmen einer sogenannten Verteilerküche ist es oft notwendig, dass Bewohner*innen mehrere Tage im Voraus ihre Mahlzeiten festlegen müssen. Das heißt im konkreten Fall, dass Bewohner*innen mitunter eine Woche im Voraus ihren Mahlzeitenwunsch angeben mussten und es nicht möglich war, sich spontan für ein anderes Essen zu entscheiden, da die Mengen für die jeweilige Mahlzeit (durch die Bestellung und Lieferung) jeweils festgelegt waren.

\subsubsection{Pflegeprozesse}

Des Weiteren wurden verschiedene Beobachtungen im Rahmen der morgendlichen, nachmittäglichen und abendlichen Pflege durchgeführt, deren Ergebnisse im Folgenden vorgestellt werden. Hier ist $\mathrm{zu}$ betonen, dass nicht die Pflegequalität, sondern viel mehr die Pflegeprozesse in ihrer Relevanz für Fragen der Selbstbestimmung und Teilhabe in den Blick genommen wurden.

\section{Fördernd:65}

- Die aktivierende Pflege wurde im Rahmen der Beobachtungen in allen Einrichtungen angewendet. Die Bewohner"innen wurden dabei motiviert, die Tätigkeitsschritte, die sie selbst noch ausführen können, selbstständig umzusetzen. Dabei kommunizierten die jeweiligen Pflegefachkräfte mit den Bewohner*innen und stellten so eine angenehme Pflegesituation her. Die Gesprächsthemen beinhalteten die jeweiligen

64 Beobachtungen, die auf eine geringe Aufrechterhaltung von Normalität und Orientierung an Lebensgewohnheiten und Interessen im Rahmen der Mahlzeiten hinweisen.

65 Beobachtungen, die auf die Aufrechterhaltung von Normalität, eine Orientierung an Lebensgewohnheiten und Interessen im Rahmen von Pflege bzw. Pflegeroutinen hinweisen. 
Tagespläne der Bewohner*innen, ihre körperlichen Beschwerden und in einem Fall auch einen speziellen Wunsch. Kleine alltagsnahe Wünsche (z. B. selbstständig die Vorhänge aufziehen) konnten im Rahmen der Pflege direkt umgesetzt werden.

- In einer Einrichtung wurde eine sogenannte „Tourenplanung“ angewendet: Bei dem Rundgang der Pflegefachkraft erklärt diese, es gäbe Frühaufsteher und Langschläfer und sie würde das in ihrer Tourenplanung mit berücksichtigen. So gehe sie in die Zimmer, in denen teilweise schon Türen geöffnet sind oder Bewohner*innen auf dem Gang zu sehen sind. Das Wissen über Vorlieben von Bewohner*innen findet demnach Berücksichtigung. Somit entsteht eine geschickte Zusammenführung von Prozessen und Synergien.

- Eine andere Pflegekraft richtete sich im Rahmen der morgendlichen Pflege nach den Belangen der Bewohner"innen und orientierte sich daran, wer bereits wach war. So entstanden sehr spontane Pflegesituationen, welche positiv von den Bewohner*innen wahrgenommen wurden.

\section{Hemmend: 66}

- Da ein begrenzter Zeitkorridor für das Frübstück existiert, wurden Bewohner*innen teilweise auch geweckt - obwohl sie unter Umständen gerne länger geschlafen hätten. Dies führte manchmal zu Unmut auf Seiten der Bewohner*innen, auch wenn gleichermaßen Verständnis für die eingeschränkten personellen Ressourcen vorhanden ist.

- Bewohner*innen wurden am frühen Abend (19:00 oder 20:00 Uhr) „bettfertig“ gemacht. Eine Vermutung ist hierzu, dass auch daher von den Bewohner*innen als Aufenthaltsort das eigene Zimmer bevorzugt wird, weil sie sich ungern im Pyjama/Nachthemd außerhalb ihres Zimmers aufhalten möchten. Pfleger*innen versuchten im Rahmen der Beobachtung auch nicht, Bewohner*innen dazu zu motivieren, im Aufenthaltsraum zu verbleiben. Sie versuchten vielmehr, verbliebene Bewohner*innen für die abendliche Pflege in ihre Zimmer zu bewegen.

- Ein Wunsch, der außerhalb der geplanten Routine der Pflegemitarbeitenden lag, konnte nicht umgesetzt werden. Der Bewohner musste auf einen späteren Zeitpunkt vertröstet werden: „Über die Anlage wird um 6:30 Uhr ein Anruf aus einem Bewohnerzimmer zugestellt. Der Bewoh-

66 Beobachtungen, die auf eine geringe Aufrechterhaltung von Normalität und Orientierung an Lebensgewohnheiten und Interessen im Rahmen der Pflege bzw. Pflegeroutinen hinweisen. 
ner aus der 1. Etage möchte jetzt Unterstützung beim Anziehen bekommen: ,Kann jemand kommen? Ich kann das nicht alleine.' Mitarbeiterin: ,Herr F., es ist noch zu früh. Wir kommen um 7:00 Uhr zu ihnen. ' Herr F. antwortet, er könne das nicht alleine. Mitarbeiterin: ,Wir wissen das. Wir kommen um 7:00 Uhr und helfen Ihnen dabei.' Herr F. klingt beruhigt und sagt, dann sei es ja gut" (Beobachtung in einer Einrichtung).

\subsubsection{Informelle Gespräche mit Bewohner*innen im Kontext der Beobachtung}

Die informellen Gespräche dienten als ergänzender Zugang zu den Interviews und der teilnehmenden Beobachtung dazu, den subjektiv erfahrenen Sinn der Alltags- und Angebotsgestaltung aus Sicht der Bewohner"innen im Beobachtungkontext situativ zu eruieren. Im Rahmen dieser Kurzgespräche konnten auch Bewohner*innen mit Demenz berücksichtigt werden, wobei sich die Forscherinnen stets an der von STAP verfassten „Selbstverpflichtung - Beobachtung und Gespräche mit vulnerablen Personen" (Kapitel 3) orientierten.

Im Vordergrund stand der Alltag der Bewohner*innen (Aussagen zur individuellen Alltagsgestaltung wie Hobbys, Freundschaften zwischen Bewohner*innen und Kontakt zu Angehörigen; geplante Alltagsgestaltung durch Angebote der Einrichtung; Mahlzeiten; Zimmer). Zentral waren aber auch hier Aussagen zur (Nicht-)Aufrechterhaltung der subjektiv bedeutsamen Normalität, so dass etwa geäußerte (Un-)Zufriedenheit und Wünsche zur teilhabebezogenen Alltags- und Angebotsgestaltung in den Blick genommen werden konnten. Insgesamt ist aber festzuhalten, dass sich die Bewohner*innen primär positiv über die Einrichtungen geäußert und kaum kritische Aspekte benannt haben. Nicht auszuschließen sind hierbei Effekte sozialer Erwünschtheit, indem die Bewohner*innen, als sie im jeweiligen Beobachtungssetting angesprochen wurden, die Einrichtung positiv darstellen wollten. Menschen, die in Einrichtungen leben, neigen dazu, ,in der Beurteilung von Dienstleistungsangeboten sozial erwünschte Antworten zu geben“ (Jenull-Schiefer, 2005, S. 149). Gerade dann, wenn es um „heikle“ Themen geht oder die Bewohner"innen einen Zusammenhang zwischen der Institution und dem/der Interviewer*in wahrnehmen, steigt die Tendenz der sozialen Erwünschtheit (ebd.). 
Fördernd: ${ }^{67}$

- Einzelne Bewohner*innen berichteten im Rahmen der informellen Gespräche, dass sie frühere Hobbys in der Einrichtung fortführen (z. B. Kegeln, Sammeln von Puppen), aber auch neue Freizeitbeschäftigungen gefunden haben (z. B. Malen, Singen). Der Soziale Dienst bzw. die Einrichtung, so zwei Bewohnerinnen, unterstütze sie bei der Besorgung von Materialien, die sie zum Ausleben ihrer Hobbys benötigten.

- Verschiedene Bewohner*innen äußerten, dass sie sehr zufrieden mit den Freizeitangeboten der Einrichtungen seien und diese auch gerne nutzten (innerhalb und außerhalb der Einrichtung, z.B. Gottesdienste).

- Es sei immer eine selbstbestimmte Entscheidung, ob man an Angeboten der Einrichtung teilnehmen möchte oder nicht.

- Bewohner*innen erhielten durch zusätzliche Betreuungskräfte und/oder Angehörige spezifische Möglichkeiten und Unterstützung, selbstbestimmt teilzuhaben. So konnten sie dadurch alte Gewohnheiten auch außerhalb der Einrichtung weiter fortführen (z. B. der wöchentliche Einkauf von Zeitschriften, Geld abheben am nahegelegenen Geldautomaten, essen gehen im Restaurant etc.).

- Eine Bewohnerin bezog sich auch auf die nahegelegene Infrastruktur und betonte, dass sie selbstständig in den Ort gehen und einkaufen könne.

- Gerade bevor Freizeitangebote oder Mahlzeiten in den Einrichtungen stattfanden, wurden die öffentlichen Räume und Aufenthaltsbereiche (z. B. Foyer, Wohnbereiche) von den Bewohner"innen genutzt. So beschrieb eine Bewohnerin, dass sie vor den Mahlzeiten immer mit ihren Freundinnen zusammensitze und sich mit ihnen unterhalte. Eine andere Bewohnerin hob hervor, dass sie die Interaktion, das Beisammensein vor den Angeboten genieße.

- Im Foyer entwickelten sich Gespräche mit Bewohner*innen, die Zeitungen lasen. Sie fanden es wichtig, sich lokal auf dem Laufenden zu halten, um zu erfahren, was aktuell im Ort passiere oder wer gestorben sei.

- Aber auch der Rückzug, die Privatheit des eigenen Zimmers, wurde von den befragten Bewohner*innen als wichtig erachtet. Hier zogen sie sich gerne zum Fernsehen oder Lesen zurück (Kapitel 4.2.1).

- Die Mablzeiten wurden mehrfach allgemein für "gut“ befunden, im Rahmen der informellen Gespräche gab es keine Beschwerden darüber.

67 Aussagen im Rahmen von informellen Gesprächen, die auf die Aufrechterhaltung von Normalität, eine Orientierung an Lebensgewohnheiten und Interessen von Bewohner*innen hinweisen. 


\section{Hemmend: 68}

- Im Rahmen der informellen Gespräche wurde von verschiedenen Bewohner*innen berichtet, dass sie einzelne ehemalige Hobbys aufgegeben haben (z. B. „shoppen“, malen, lesen, Besuch des Schützenvereins), aber nicht, weil die Einrichtung sie daran hindern würde, sondern vielmehr aufgrund von persönlichen, zum Teil aus gesundheitlichen Gründen („Traurigkeit“ und Antriebslosigkeit oder bei einer Bewohnerin aufgrund ihrer Demenz).

- Eine blinde Bewohnerin merkte in einem Gespräch an, dass es keine adäquaten Angebote für sie gebe. Sie höre gerne Hörbücher, leider stelle die Einrichtung aber keine für sie bereit. Dies werde alles privat durch ihre Familie organisiert.

- Einige Bewohner*innen berichteten, dass ihnen der Einzug in die Einrichtung schwergefallen und es ein großer Schritt gewesen sei, sich mit dem neuen Leben dort abzufinden. Gerade die Einzugsphase ist eine sehr emotionale Phase, in denen die neuzugezogenen Bewohner"innen von der Einrichtung sehr eng begleitet werden (sollten) (Kapitel 4.1).

- Im Zuge der informellen Gespräche wurde auch über die Räumlichkeiten der jeweiligen Einrichtung gesprochen. In einer Einrichtung wurde von einem Bewohner betont, dass es keine Ausweichmöglichkeiten in andere Räume gebe, da nur ein großer Mehrzweckraum für Angebote existiere. Dies wurde auch von Mitarbeitenden im Rahmen der Interviews bestätigt und bei der Beobachtung wahrgenommen. In einer anderen Einrichtung wurde berichtet, dass der Wohnbereich zum freizeitlichen Aufenthalt nicht genutzt werde und sich die Bewohner*innen lieber auf ihren jeweiligen Zimmern aufhalten würden. Woran das lag, wurde nicht weiter benannt.

- Die Sprechstunde, ein regelmäßig stattfindendes Angebot des Bewohner*innenbeirates, werde zum Bedauern eines Mitglieds des Bewohner*innenbeirates von den anderen Bewohner*innen nicht angenommen. Selten erscheine jemand mit einem bestimmten Anliegen. Dies stimmt mit den Aussagen der Mitarbeitenden im Rahmen der Interviews überein. Hier wurde als Grund genannt, dass es sich bei der jetzigen Bewohner*innenschaft um die „dankbare“ (EL) Kriegsgeneration handle, die selten Anliegen oder Wünsche äußere (Kapitel 4.1).

68 Aussagen im Rahmen von informellen Gesprächen, die auf eine geringe Aufrechterhaltung von Normalität und Orientierung an Lebensgewohnheiten und Interessen von Bewohner*innen hinweisen. 


\subsection{Fördernde und hemmende Faktoren der Teilhabeförderung auf Ebene der Bewohner*innen, Mitarbeiter*innen sowie der Einrichtung und Organisation}

Nachdem in den vorausgegangenen Kapiteln mit den Themen Wunschäußerung und -erfassung zentrale Ausgangsprozesse sowie mit dem Thema Alltags- und Angebotsgestaltung wesentliche Kontexte und Elemente zur Förderung und Verwirklichung selbstbestimmter Teilhabe in stationären Altenpflegeeinrichtungen angesprochen wurden, sollen im Folgenden fördernde und hemmende Faktoren der Teilhabeförderung wiederum auf Basis der verschiedenen qualitativen Interviewzugänge beschrieben werden, die nun noch einmal spezifisch auf die Ebenen der Bewohner*innen, der Mitarbeiter"innen sowie der Einrichtung und Organisation fokussieren. Dies gestaltet sich insofern nicht trennscharf von den Inhalten der vorausgegangenen Kapitel, da auch dort bereits verschiedene fördernde und hemmende Faktoren in Bezug auf die Bewohner*innen, Mitarbeiter*innen, Einrichtung und Organisation benannt wurden. Hier werden sie jedoch nochmals ausgehend von den Aussagen beleuchtet, die speziell in Bezug auf die Bewohner*innen, Mitarbeiter*innen und Einrichtung sowie deren Organisationsprozesse als fördernd und hemmend benannt wurden, denn die Bewohner*innen und Mitarbeiter*innen sind zunächst die beiden zentralen Akteur*innengruppen der Teilhabeförderung, deren Handeln im Kontext von Rahmenbedingungen und Prozessen in einer Einrichtung stattfindet, so dass auf diesen Ebenen jeweils die in den Interviews benannten fördernden und hemmenden Faktoren der Teilhabeförderung von Interesse sind.

\subsubsection{Bewohner*innen}

Die Voraussetzungen zur selbstbestimmten Teilhabe und für ihre Förderung durch die Mitarbeitenden sind auf Seiten der Bewohner*innen äuBerst heterogen, wie die folgenden in den Interviews identifizierbaren Differenzierungen der Bewohner*innenschaft verdeutlichen. Besonders deutlich kommen Differenzsetzungen in Bezug auf unterschiedliche physische und psychische Voraussetzungen der Bewohner*innen zum Ausdruck. Darüber hinaus finden sich auch mehrfach Hinweise auf weitere für die Teilhabeförderung relevante Differenzmerkmale wie das Geschlecht, die finanzielle Lage und die Herkunft. 
Betrachtet man theoriegeleitete Differenzierungen zu Bewohner*innenmerkmalen in der Literatur, so wird auch dort eine vergleichbare Unterteilung zwischen der "Gesundheit" der Bewohner*innen vorgenommen (die in STAP auf Basis des Interviewmaterials noch ausdifferenziert wurde) sowie auf weitere Unterscheidungskriterien, wie die „soziale und finanzielle Situation“, "Geschlecht" und „ethnische Differenzen“ der Bewohner"innen (vgl. Bode, 2014), die sich empirisch auch in der STAP-Studie herausgebildet haben, eingegangen.

\subsubsection{Physische und psychische Voraussetzungen}

Die physischen und psychischen Voraussetzungen der einzelnen Bewohner*innen beeinflussen sowohl die Möglichkeiten der Wunschäußerung und -weckung als auch die Wahrnehmung bzw. Nutzung von teilhabebezogenen Alltagsaktivitäten und Angeboten.

In den Interviews erfolgten dabei direkte oder indirekte Differenzierungen von Bewohner*innen in verschiedene Gruppen - sowohl durch Mitarbeitende als auch durch Bewohner*innen. Auf Seiten der Mitarbeitenden scheint hier eine Unterteilung pragmatisch zu erfolgen, um entweder Unterteilungen in Fördergruppen vorzunehmen oder aber aus Sicht der vorhandenen Angebote zu prüfen, wer an welchen Angeboten teilnehmen kann oder nicht. Eine grobe Unterscheidung bezieht sich hierbei auf Bewohner*innen, die an großen Gruppenangeboten des Sozialen Dienstes teilnehmen können und „die, die es nicht mehr können“ (BW), wobei hier v. a. zwischen Bewohner*innen mit und ohne einer (fortgeschrittenen) Demenz unterschieden wird (siehe auch Alltags- und Angebotsgestaltung): „,[Wir] strukturieren das allerdings, [...] gerade was die kognitiven Fähigkeiten angeht, auch in Angebote für Bewohner mit und ohne Demenz" (EL). Betrachtet man jedoch die Gesamtheit der bewohner"innenbezogenen Aussagen, lässt sich darüber hinaus die folgende differenziertere Einteilung in vier Bewohner*innengruppen erkennen. 
Tabelle 14: Vier Bewohner*innengruppen auf Basis der Interviewaussagen

\begin{tabular}{|l|l|}
\hline & Bewohner*innengruppen \\
\hline 1. & $\begin{array}{l}\text { „Orientierte“ Bewohner*innen (Unterteilung in zwei Untergruppen): } \\
-\quad \text { aktive Bewohner*innen } \\
-\quad \text { sich zurückziehende Bewohner*innen }\end{array}$ \\
\hline 2. & Kognitiv eingeschränkte und psychisch veränderte Bewohner*innen \\
\hline 3. & Physisch stark eingeschränkte Bewohner*innen \\
\hline 4. & Bewohner*innen in der Sterbephase \\
\hline
\end{tabular}

1. „Orientierte“ Bewohner*innen: Eine Bewohner"innengruppe wird häufig mit der Umschreibung „orientierte“ Bewohner"innen versehen. Damit werden Bewohner*innen verbunden, bei denen keine oder nur geringe kognitive Einschränkungen und psychische Erkrankungen vorliegen, welche die Orientierung beeinflussen. Diese Bewohner*innenschaft kann sich außerdem „frei“ bewegen (zu Fuß oder mithilfe eines Rollators oder Elektrorollstuhls) und selbstständig den Tagesablauf bestimmen und gestalten (z. B. auch Besuche in der Stadt, Einkäufe, Treffen mit Freund"innen innerhalb und außerhalb der Einrichtung). Für diese Gruppe wird allerdings auch ausgesagt, dass ihr Anteil innerhalb der Bewohner*innenschaft relativ klein ist und in den letzten Jahren abgenommen hat. Innerhalb dieser Gruppe lassen sich in den Aussagen zudem zwei Untergruppen identifizieren: Es gibt zum einen Bewohner*innen, die als „orientiert“ oder „fit“ bezeichnet werden und sich aktiv an dem Geschehen in der Einrichtung beteiligen, und zum anderen „orientierte“ Bewohner*innen, die sich mehrheitlich zurückziehen:

a) Aktive Bewohner"innen: Sie nehmen sowohl aktiv an Angeboten in der Einrichtung als auch außerhalb der Einrichtung teil. Wobei dazu nochmals betont wird, dass es sich hierbei um eine kleine Teilgruppe von Bewohner*innen handelt: „Das ist dann aber auch halt eben ein ganz spezieller kleiner Kreis, ne, die da total begeistert sind und zuhören und das auch wirklich noch umsetzen können" (ZBK).

b) Sich zurückziehende Bewohner*innen: Hierbei handelt es sich um eine Personengruppe, die zwar „orientiert“ ist, sich aber zurückzieht und es bevorzugt, alleine zu bleiben. Das heißt, sie nehmen nicht gerne an Einrichtungsangeboten teil, weder innerhalb noch außerhalb des Hauses und lehnen dann auch entsprechende Anfragen und Motivation zur Teilnahme an Gruppenangeboten ab: „Er möchte in Ruhe gelassen werden, dann muss man es vielleicht auch akzeptieren“ (PFK). 
2. Kognitiv eingeschränkte und psychisch veränderte Bewohner*innen: Hierzu zählen insbesondere Bewohner*innen mit demenziellen Erkrankungen, die als große Gruppe und oft im fortgeschrittenen Stadium beschrieben werden. Für einen Teil dieser Bewohner*innengruppe werden auch Verhaltensweisen benannt, die herausfordernd und mit einem besonderen Unterstützungsbedarf im Einrichtungsalltag verbunden sind (z. B. Rufen, Aggressivität, Hinlauftendenz). In einzelnen Interviews werden zudem Bewohner*innen mit psychischen Veränderungen und Erkrankungen (z. B. Depression) erwähnt, die nicht auf einer Demenz basieren. Diese Bewohner*innen werden ebenfalls dieser Gruppe zugeordnet, weil hier vergleichbare Herausforderungen für die Mitarbeitenden, auch in Bezug auf Möglichkeiten und Grenzen der Teilhabeförderung, beschrieben werden, wobei bei dieser Gruppe häufig Grenzen benannt werden, wie es z. B. in der folgenden Aussage zum Ausdruck kommt: „[Wir] versuchen, ein bisschen $\mathrm{zu}$ aktivieren. Also das ist schwierig, wenn Bewohner, insbesondere wenn sie ein psychisches Krankheitsbild entwickeln, wenn eine Depression im Vordergrund steht, sie zu erreichen und sie auch zu motivieren, an der Gesellschaft, an der Gemeinschaft teilzunehmen“ (EL).

3. Physisch stark eingeschränkte Bewohner*innen: $\mathrm{Zu}$ dieser Gruppe zählen vor allem Bewohner*innen, die in ihrer Mobilität stark eingeschränkt sind, wozu dann auch Bewohner*innen gehören, die von den Mitarbeitenden oft als „bettlägerig“ bezeichnet werden. Die in den Interviews benannten physisch bedingten Einschränkungen beziehen sich aber nicht nur auf die Mobilität, sondern auch auf Sinneseinschränkungen und Einschränkungen in der Kommunikation. Hier ist keineswegs eine Trennschärfe zur zweiten Gruppe „kognitiv eingeschränkte und psychisch veränderte Bewohner"innen" gegeben, weil auch etwa bei Menschen mit Demenz physische Einschränkungen im Krankheitsverlauf zunehmen und das späte bzw. letzte Stadium oft durch eine „Bettlägerigkeit“ gekennzeichnet ist. Die Trennung dieser beiden Bewohner"innengruppen erfolgt hier aber ausdrücklich mit der Perspektive, dass die hier beschriebenen Merkmale jeweils im Vordergrund der Betrachtungen durch die Interviewpersonen standen und zu einer Differenzierung in diese Bewohner*innengruppen führen, die in der Berufspraxis offenbar als Differenzsetzung von Relevanz ist.

4. Bewohner"innen in der Sterbephase: $\mathrm{Zu}$ einer vierten, spezifisch benannten Gruppe zählen in den Interviews Bewohner*innen in der Sterbephase. Die Anzahl dieser Bewohner*innen hat nach Aussagen der Interviewpersonen in den letzten Jahren zugenommen, womit auch verbunden ist, dass die Bewohner*innen teilweise mit einer vergleichsweise kürzeren Lebens- 
zeit in den Einrichtungen wohnen und bei einer palliativen Begleitung auch vorwiegend in ihren Zimmern leben: „[Wir haben] viel[e] palliativ versorgte Bewohner. Also die absehbar, sagen wir mal, auf keinen Fall länger als drei oder sechs Monate noch bei uns sind“ (PFK).

\subsubsection{Weitere Differenzmerkmale der Bewohner*innenschaft}

Quer liegend zu den oben erläuterten Differenzierungen in Bezug auf physische und psychische Voraussetzungen werden weitere Differenzmerkmale benannt, die sich in den Interviews primär auf das Geschlecht und die finanzielle Lage der Bewohner*innen beziehen. Ferner ist die Herkunft als weiteres Differenzmerkmal erkennbar, das aber nur in der trägerübergreifenden Gruppendiskussion und nicht in den Interviews in den vier Projekteinrichtungen angesprochen wurde (der Anteil von Menschen mit $\mathrm{Zu}$ wanderungsgeschichte war in den untersuchten Einrichtungen - im Erhebungszeitraum - gering).

- Geschlecht: Die Aussagen, die Differenzierungen in Bezug auf das Geschlecht vornehmen, beziehen sich vorwiegend auf männliche Bewohner und dabei teils auch auf entsprechend geschlechterdifferenzierte Angebote mit unterscheidbaren Teilhabeinteressen.

- Finanzielle Lage: Es wird auf eine große Heterogenität unter den Bewohner*innen hinsichtlich der finanziellen Lage und auf die für viele Bewohner*innen hohen Heimkosten hingewiesen sowie darauf, dass einige Bewohner"innen wenig Geld zur freien Verfügung haben.

- Herkunft: Es wird benannt, dass es nicht immer einfach ist, auf die kulturellen Unterschiede verschiedener Bewohner*innen einzugehen. Gerade unterschiedliche sprachliche Voraussetzungen auf Seiten der Bewohner*innen und Mitarbeitenden könnten eine Herausforderung in der Teilhabeförderung darstellen.

Insgesamt werden in Bezug auf die Bewohner*innenschaft zwei Entwicklungen festgestellt bzw. für die Zukunft prognostiziert. Wie bereits erwähnt, wird einerseits mehrfach hervorgehoben, dass der Anteil der Bewohner"innen mit gravierenden physischen und/oder psychischen Einschränkungen in den letzten Jahren deutlich zugenommen habe (exemplarisch für die einbezogenen Einrichtungen siehe Kapitel 3.2.1.2). Es wird erwartet, dass sich diese Veränderungstendenz in Zukunft fortsetzen bzw. noch verstärken wird, da es die Konsequenz der zunehmend verwirklichten altenpolitischen Leitlinie „ambulant vor stationär" sei. Dabei wird im Zuge der Interviews etwa beschrieben, dass die Bewohner*innen in Zukunft zunehmend im hohen 
Alter, mit multimorbiden Krankheitsbildern und schwerer Pflegebedürftigkeit einziehen werden, sich ihre Aufenthaltszeit weiter verkürzt und selbstbestimmte Teilhabe dann schwieriger umzusetzen sein wird: „Aber die Altersgrenze wird immer höher. Und immer immobiler. Und da kann es natürlich auch sein, dass in 20 Jahren nur noch die Leute ins Altenheim gehen, die wirklich kaum noch in der Lage sind, auch hier, was Selbstbestimmung angeht, da sich überhaupt zu äußern. Aber das bleibt ja“ (PDL).

Andererseits wird von Leitungskräften im Rahmen der trägerübergreifenden Fokusgruppendiskussion beschrieben, dass die Kriegsgeneration „aussterbe“ und anspruchsvollere Bewohner"innen in die Einrichtungen einziehen würden: „Die Bewohner, die jetzt einfach kommen, die haben viel mehr Ansprüche und ganz andere Ansprüche, da müssen wir sowieso verändern und anpassen. Da wird jetzt nach WLAN gefragt und nicht nach irgendwelchen Bingogruppen“" (EL/PDL).

Im Folgenden werden fördernde und hemmende Faktoren der Teilhabeförderung in Bezug auf verschiedene Bewohner*innengruppen aufgeführt.

\section{Fördernd:}

- Manche Bewohner*innengruppen können besser oder ausschließlich mit Einzelangeboten bzw. -betreuung erreicht werden, die inzwischen vor allem durch zusätzliche Betreuungskräfte durchgeführt werden. Dazu zählen etwa Bewohner*innen, die dazu neigen, sich zurückzuziehen, sowie Bewohner*innen, die physisch oder psychisch in besonderem Maße eingeschränkt sind. Diese Angebote sind individuell teilhabeförderlich und können unter Umständen Prinzipien der Selbstbestimmung und Normalität besser entsprechen als Gruppenangebote.

- Andere Bewohner*innen erhalten nicht nur Einzelbetreuung, sondern nehmen auch an Gruppenangeboten des Sozialen Dienstes teil. Individuelle Differenzierungen der Bewohner"innen in Bezug auf die Teilnahme an Einzel- und/oder Gruppenangeboten können jeweils unterschiedlichen Wünschen nach Zugängen zu Umweltbeziehungen gerechter werden.

- Das Beobachten des Bewohner"innenverhaltens in Verbindung mit einem sensibel eingesetzten „Trial-and-Error“-Verfahren wird als eine bewährte Vorgehensweise betrachtet, um auch Bewohner*innen, die sich nicht mehr äußern können (ob aus physischen oder psychischen Gründen), in ihrer Teilhabe fördern zu können (Kapitel 4.1).

\section{Hemmend:}

- Differenzierungen nach spezifischen Bewohner*innengruppen können Stigmatisierungen und defizitorientierte Betrachtungen von Bewohner"innen 
zur Folge haben, was etwa deutlich in der oben erwähnten Unterteilung zwischen Bewohner*innen, die noch an Angeboten teilnehmen können, und jenen, die es nicht mehr können, zum Ausdruck kommt.

- Die Veränderung der Bewohner*innenschaft, bei der die Gruppe der „fitten" und „orientierten" Bewohner"innen in Relation zu den Bewohner*innen mit gravierenden physischen und psychischen Einschränkungen deutlich kleiner geworden ist, führt dazu, dass die Herausforderungen, selbstbestimmte Teilhabe zu fördern und zu gestalten, zunehmen (z. B. in Bezug auf Wunschäußerung und -erfassung, Gestaltung von Gruppenangeboten).

- In einzelnen Interviews wird demgegenüber auch beschrieben, dass manche Bewohner"innen von anderen Bewohner"innen als störend wahrgenommen werden, so dass sie in bestimmten Teilhabekontexten nicht oder nur schwer in eine Gruppe integriert werden können, was insbesondere auf Menschen mit einer fortgeschrittenen Demenz bezogen wird. In diesen Situationen stellt es für die Mitarbeiter"innen eine besondere Herausforderung dar, beiden Bewohner*innengruppen "gerecht" zu werden. Schuhmacher (2018) bestätigt diese Beschreibungen und spricht von einem erhöhten Exklusionsrisiko, indem sich diese Personengruppe nicht an die „Spielregeln“ halten könne und somit das geregelte Zusammenleben verhindere, "gelten sie auch innerhalb der Heimbewohnerschaft als Außenseiter" (Pleschberger, 2005, S. 132, zit. in Schuhmacher, 2017, S. 99).

- Die Bewohner*innen werden von den Mitarbeitenden in mehreren Interviews sowie im Rahmen der trägerübergreifenden Fokusgruppendiskussion als „dankbar" und „zufrieden" (EL) mit dem Vorhandenen beschrieben, so dass sie gegebenenfalls ihre Wünsche zurückhalten (Kapitel 4.1). Das betrifft vor allem die Gruppe der „orientierten“ Bewohner*innen.

In mehreren Einrichtungen werden Differenzierungen für Menschen mit und ohne (fortgeschrittene) Demenz auch strukturell in Hinsicht auf spezielle Wohnbereiche und Angebote für Menschen mit Demenz vorgehalten, die sowohl mit fördernden als auch mit hemmenden Faktoren der Teilhabeförderung verbunden werden können. So ist etwa die Rede von einem „Demenzhaus" (SD; wohl im Sinne eines segregativen Einrichtungs- oder Wohnbereichs) und einer „Demenzgruppe“ (SD; im Sinne eines teilsegregativen Angebots). Wie bereits benannt, werden solche Angebotsdifferenzierungen kontrovers diskutiert und jeweils mit Vor- und Nachteilen verbunden. So erklärt etwa Schuhmacher (2018), dass spezifische Wohnbereiche für Menschen mit Demenz zwar deutlich besser auf die Zielgruppe 
eingehen können (durch die regelmäßige Erhebung von biografischen Daten), die Bewohner*innen öfter gerontopsychiatrisch behandelt werden und daher Antidementiva erhalten. „Demgegenüber ist in der integrierten Betreuung, also in Wohnbereichen, in denen demenziell erkrankte Menschen mit kognitiv orientierten pflegebedürftigen Menschen zusammenleben, aber die Besuchshäufigkeit höher, die Einbindung von Angehörigen stärker, und die Bewohnerinnen und Bewohner nehmen häufiger an kompetenzfördernden Aktivitäten teil“" (Schuhmacher, 2018, S. 101). Insgesamt, so Schuhmacher, gebe es bisher aber keine einheitliche Bewertung der Wirkungen von integrativer und segregativer Betreuung von Menschen mit Demenz (ebd.).

\subsubsection{Mitarbeiter*innen}

Sowohl Angehörige, Bewohner*innen als auch Mitarbeitende (verschiedener Bereiche und Ebenen) teilen in den Interviews die Überzeugung bzw. Vorstellung, dass Umfang und Qualität der Teilhabeförderung maßgeblich von individuellen Kompetenzen und persönlichen Eigenschaften der jeweiligen Mitarbeiter*innen abhängig sind, was auch in der trägerübergreifenden Gruppendiskussion deutlich bestätigt wurde. Die Aussagen in den Interviews zu personenbezogenen Eigenschaften werden hier einerseits verschiedenen Kompetenzdimensionen sowie andererseits nochmals separat und speziell den Bereichen der Arbeitseinstellung und des Arbeitsengagements zugeordnet.

\subsubsection{Kompetenzen}

In den Einzel- und Gruppeninterviews bezog sich eine Vielzahl von Aussagen auf verschiedene Kompetenzen, die Mitarbeiter*innen für die Förderung selbstbestimmter Teilhabe benötigen oder haben (sollten). In der Auswertung wurde auf die häufig verwendete Unterscheidung zwischen Fach-, Methoden-, Sozial- und Selbstkompetenz (vgl. z. B. Enggruber \& Bleck, 2005) zurückgegriffen. 
Fachkompetenz

Hierunter wurde in der Auswertung das Wissen in Bezug auf fachliche Inhalte verstanden, wie etwa das Fachwissen über medizinisch-pflegerische Grundlagen (z. B. Krankheiten oder Medikamente). Diese und andere hierzu benannte Themen werden im Folgenden als fördernde und hemmende Faktoren der - aus Sicht der Interviewpersonen für die Teilhabeförderung erforderlichen - Fachkompetenz erläutert.

\section{Fördernd:}

- Ein angemessenes medizinisches Fachwissen über Krankheitsbilder (z. B. über Demenz bzw. ein demenzielles Syndrom) sowie über medizinische Versorgung (z. B. Wirkungen einer Medikation) ist wichtig, um Voraussetzungen und Verhaltensweisen von Bewohner*innen in Teilhabekontexten angemessen berücksichtigen und einschätzen zu können.

- Auch das - unter Umständen physiotherapeutische - Wissen zur Förderung der Mobilisation wird benannt, ebenso wie jenes zur Unterstützung der Körperwahrnehmung der Bewohner*innen. Beide Aspekte beziehen sich auf die Berücksichtigung von physischen Voraussetzungen sowie die entsprechenden Möglichkeiten und Grenzen in der Teilhabeförderung.

- Das Fachwissen um unterschiedliche Voraussetzungen der Wunschäußerung auf Seiten der Bewohner"innen ist ebenso von Bedeutung wie das Wissen um Wahrnehmungstechniken, mit denen Signale der Wunschäußerung sensibel erkannt und interpretiert werden können.

- Als fachliches Wissen im weiteren Sinne lässt sich auch das Wissen um Rahmenbedingungen fassen, damit sich Bewohner*innen in der Einrichtung und in ihrem Zimmer wohl und „zuhause“ (BW) fühlen können. Benannt werden dabei etwa die Achtung der Privatsphäre oder die Unterstützung der Angehörigen bei einer biografienahen Zimmergestaltung der Umgebung bzw. des Zimmers (z. B. durch Gegenstände aus der früheren Wohnung) und die Rücksicht, den Einzug der Bewohner*innen den jeweiligen Voraussetzungen der Bewohner*innen angemessen und sensibel zu gestalten.

\section{Hemmend:}

- Die geringe fachliche Qualifikation der zusätzlichen Betreuungskräfte wird als hemmend angesehen, sofern diese über keine notwendige Verbindung zwischen fachlichen und sozialen Kompetenzen verfügen: „160Stunden-Ausbildung [...] das kann nur klappen, wenn diese Menschen 
sowieso von - von Haus aus eine hohe soziale Kompetenz haben“ (PDL; Kapitel 4.4.1).

- Unwissenheit oder Unvorsichtigkeit beim Umgang mit unterschiedlichen Erkrankungen der Bewohner*innen kann die Teilhabe einschränken. Auch Pflegepersonal fehle es manchmal an Wissen darum, so eine Einrichtungsleitung: „Und auch, ich erschrecke mich manchmal, wie gering die Vorstellung von Krankheiten bei Pflegefachkräften tatsächlich ist" (EL)

\section{Methodische Kompetenz}

Unter der methodischen Kompetenz wurden insbesondere die Fähigkeiten im Umgang mit Vorgehensweisen, Techniken und Instrumenten des beruflichen Handelns verstanden, wie beispielsweise aktivierende Vorgehensweisen in der Pflege oder Kommunikation. Im Folgenden werden die fördernden und hemmenden Faktoren im Bereich der Methodenkompetenz benannt.

\section{Fördernd:}

- Hier ist noch einmal die Anwendung des „Trial-and-Error“-Verfahren zu nennen, die hier als eine wichtige methodische Kompetenz in der Arbeit mit Menschen zu sehen ist, die sich nicht mehr äußern können (Kapitel 4.1). So ist es hier Fähigkeit, kreativ und sensibel sowie ressourcenorientiert Neues zu entdecken, aufzuzeigen, um mit den Bewohner*innen auszuprobieren, um verschiedene und - davon ausgewählt geeignete Zugänge zur Teilhabe zu ermöglichen. Hierbei ist auch die Fähigkeit der Beobachtung und Interpretation von Reaktionen der Bewohner*innen von Relevanz (z. B. Mimik, Gestik, Körperhaltung): „[Es passiert] immer wieder, dass Wünsche von - von Bewohnern, ja, die er nicht selber äußern kann, der Bewohner, unterschiedlich interpretiert oder wahrgenommen werden" (SD). Mitarbeiter"innen müssen auch im Rahmen von Angeboten in der Lage sein, spontan auf die Reaktionen von Bewohner*innen einzugehen.

- Aktivierende Vorgehensweisen werden als förderlich beschrieben, wozu etwa Varianten der motivierenden Gesprächsführung und aktivierenden Pflege gehören. So wird versucht, Bewohner*innen zur Mitwirkung in der Pflege und Teilnahme an Angeboten zu motivieren. Auch hierzu wird betont, dass Signale der Ablehnung und Grenzen auf Seiten der Bewohner*innen wahrzunehmen und zu berücksichtigen sind. 
So ist es relevant, sich Zeit für den/die Einzelne*n zu nehmen und auch mit möglichen Konsequenzen einer anschließenden Mehrarbeit zu leben: „Einen nach dem anderen. So, und das gibt ihnen die Routine und die Erfahrung, zu sagen, so, dem und dem kann ich das zutrauen. Oder - und wenn ich dann später dieses ganze Badezimmer wischen muss, aber derjenige hat sich doch selber seine Zähne gemacht“ (PFK).

\section{Hemmend:}

- Betont wird etwa, dass fehlende Beobachtungskompetenz und Sensibilität dazu führen können, dass aktuelle Wünsche und Veränderungen in den Wünschen übersehen werden, was sich teilhabehemmend auswirken könnte: „Und ich denke, da müsste man noch mal hinschauen, auch noch mal sensibilisieren, dass man da das auch aufnimmt, wenn sich das verändert. Wenn jemand immer gerne Fisch gegessen hat und es eben, weil er sich vielleicht auch nicht mehr äußern kann, aber über den Tellerrand spuckt, dann sollte man das schon da auch verändern" (HW).

\section{Sozialkompetenz}

Die Sozialkompetenz beschreibt hier die Fähigkeiten, Beziehungen und Interaktionen zu gestalten. Zum Beispiel ist hier ein ausgeprägtes Einfühlungsvermögen von Vorteil, um Wünsche von Bewohner*innen zu erkennen und richtig einzuschätzen. Diese und weitere fördernde und hemmenden Faktoren werden untenstehend weitergehend erläutert.

\section{Fördernd:}

- Der Kontakt zwischen Bewohner*innen und Mitarbeiter*innen wird auch als Teilhabesituation beschrieben und dazu als förderliche Sozialkompetenz benannt, Kontakt zu den Bewohner*innen aufbauen zu können (z. B. durch kurze Gespräche, sich Zeit nehmen, mit Namen ansprechen, mit freundlichem Umgangston).

- Einfühlungsvermögen bzw. Empathie der Mitarbeitenden gegenüber Bewohner*innen ist eine zentrale Voraussetzung, um die Teilhabewünsche erkennen und in Teilhabesituationen angemessen fördern zu können.

- Die jeweilige Sozialkompetenz wird oft mit dem persönlichen Arbeitsengagement in Verbindung gebracht: „Wenn man die Angst nicht zeigt, sondern wirklich mit - mit Herzen da - da dran geht und mit Liebe und 
sagt: ,Ach, das haben Sie so toll gemacht', und dieses - dieses noch dieses Gutes zuzusprechen, dann sind sie stolz, dann - dann - dann blüht das Leben wieder in denen auf.“ (PFK)

\section{Hemmend:}

- Im Zusammenhang mit einer motivierenden Gesprächsführung kommt allerdings auch in besonderer Weise die Herausforderung zum Ausdruck, Teilhabeförderung tatsächlich nach dem Prinzip der Selbstbestimmung vorzunehmen und ein Nein der Bewohner*innen zu akzeptieren.

\section{Subjektkompetenz}

Der Begriff Subjektkompetenz - auch personale Kompetenz - wurde in der Auswertung als die Fähigkeit der Selbstregulation in Wahrnehmung und Handlung definiert. Hierzu zählt beispielsweise die Flexibilität in Bezug auf (Pflege-)Handlungen oder das Zulassen und die Akzeptanz der Mitarbeitenden bezüglich der selbstbestimmten Entscheidung der Bewohner*innen. Fördernde und hemmende Faktoren werden im Folgenden erläutert.

\section{Fördernd:}

- Mehrfach wird in den Interviews die Reflexion der Beziehung und der jeweiligen Kontaktqualität zwischen Bewohner*innen und Mitarbeitenden als förderlich benannt (z. B. bezogen auf Konflikte und Frustrationserfahrungen, „Helfer“innenfalle“, Beziehungsaufbau mit Pflegebedürftigen), um persönliche und professionelle Möglichkeiten und Grenzen der Teilhabeförderung zu erkennen und zu berücksichtigen.

- Auch die Flexibilität in Arbeitshandlungen und -abläufen sowie das situative Erkennen und Nutzen von Handlungsspielräumen weisen Interviewpersonen den persönlichen Eigenschaften der Mitarbeitenden zu und können hier als wichtiges Kriterium der Selbstkompetenz verstanden werden (Kapitel 4.3.3.4).

- Ferner werden bewohner*innenbezogen das Zulassen von Abweichungen (z. B. Umgang von Mitarbeitenden mit herausfordernden Verhaltens- 
weisen ${ }^{69}$ ) sowie die Akzeptanz der Entscheidungen von Bewohner*innen (z. B. Wunsch der Nichtteilhabe) als wichtige Aspekte der Selbstkompetenz in Teilhabekontexten erachtet.

\section{Hemmend:}

- Als Problematik wird beschrieben, dass sich Mitarbeiter*innen zurückhalten müssen, aufgrund der (vermeintlich) bekannten Vorlieben und Abneigungen vorschnell Entscheidungen für Bewohner*innen zu übernehmen. Sofern Entscheidungen übernommen werden, widerspricht dies dem Grundsatz der Selbstbestimmung.

- Als hemmend wird auch ausdrücklich eine geringe Flexibilität im Umgang mit der Zeit (und möglicherweise der gewählten Reihenfolge von Handlungen) benannt.

- Wenn die Hektik und Arbeitsdichte im Arbeitsalltag als Belastung wahrgenommen und davon nicht die individuelle Situation der Bewohner*innen getrennt betrachtet wird, kann es teilhabehemmende Auswirkungen haben: „Also die meisten erleben es als total stressig, den Pflegealltag. Und nehmen sich leider Gottes auch nicht mehr so die Zeit, wie ich mir das oft wünschen würde, da mal mehr hinzuspüren und hinzuhören" (EL).

- Die „Fürsorgepflicht“ der Mitarbeitenden und die Selbstbestimmung der Bewohner*innen können miteinander „kollidieren" und somit teilhabehemmend sein: „Und da ist immer so dieser Schutz und Selbstbestimmung. Und das kollidiert komischerweise“ (EL).

\subsubsection{Arbeitskultur und Arbeitsengagement}

In den Interviews wurde vielfach angesprochen und betont, dass die individuellen Motivationen, Orientierungen und Selbstverständnisse im beruflichen Handeln der Mitarbeitenden von großer Bedeutung dafür sind, inwieweit die einzelne Person teilhabeorientiert arbeitet und sich entsprechend einsetzt. Diese Interviewaussagen wurden den Bereichen der Ar-

69 Der Begriff „herausforderndes Verhalten“ wird hier in dem Sinne verstanden, dass er „Verhaltensweisen kennzeichnet, die die Umgebung herausfordern, die also auch bestimmte Anforderungen an das Verhalten der Pflegenden stellen" (Bartholomeyczik et al., 2006). Damit enthält der Begriff eine psychosoziale Perspektive, die von intrinsischen und extrinsischen Faktoren als Ursache des Verhaltens ausgeht und es besteht hierbei die Auffassung, dass das Verhalten für diejenigen, die sich verhalten, immer einen Sinn hat (ebd.). 
beitskultur und des Arbeitsengagements zugeordnet. Auch wenn diese alternativ verschiedenen oben bereits aufgeführten Kompetenzdimensionen zugeordnet werden könnten (z. B. Selbstkompetenz), werden sie hier aufgrund der besonderen Bedeutungszuschreibung in den Interviews separat aufgeführt.

\section{Arbeitskultur}

Der Begriff der Arbeitskultur steht im engen Zusammenhang mit der später auf Einrichtungsebene beschriebenen Organisationskultur: Während sich die Organisations- bzw. Unternehmenskultur auf in der Organisation geteilte Annahmen und Überzeugungen sowie organisationale Werte und Normen bezieht, fokussiert demgegenüber die Arbeitskultur auf das Arbeitshandeln bzw. die Arbeitsergebnisse und deren Bedeutung für das Individuum, das Unternehmen wie auch für die Gesellschaft und damit auf einen übergreifenden, nicht nur unternehmensspezifischen Entwicklungsprozess der Arbeit (Widuckel, 2015, S. 30). Unter dem Begriff der Arbeitskultur werden hier „die Arbeit und das Arbeitshandeln als individuelle und gesellschaftliche Tätigkeit prägenden Werte und Normen sowie die ihr unterliegenden sozialen Beziehungen verstanden“ (ebd., S. 30).

In der arbeitskulturellen Perspektive wurden in den Interviewaussagen also die individuellen Annahmen, Überzeugungen und Werte sowie das individuelle Arbeitshandeln in den Blick genommen. Hierzu zählten zum Beispiel Leitorientierungen und Ziele in der eigenen Arbeit, der Umgang mit Fehlern und Abweichungen oder auch ein gewisses „Säulendenken“ in Bezug auf „wir“ und „die“ innerhalb der einzelnen Einrichtungsbereiche. Im Rahmen der Interviews wurden folgende förderliche und hemmende Faktoren benannt.

\section{Fördernd:}

- Als förderliche arbeitskulturelle Orientierung wird betrachtet, dass die Bewohner"innen den Ausgangspunkt im beruflichen Handeln bilden und ihre Bedürfnisse im Mittelpunkt stehen. Als Beispiele für ein an Bewohner*innen orientiertes Handeln wurden genannt:

- Übernahme von Aufgaben, die dem/der Bewohner"in aktuell dienen, auch wenn sie nicht in der formellen Zuständigkeit liegen (das Mitnehmen im Rollstuhl innerhalb der Einrichtung, für jeden „zuständig zu sein"), 
- Nichtbeharren auf Pflegeroutinen (z. B. bei der „Ganzkörperwäsche"),

- kleine Dinge des Alltags aufwerten.

Hierbei steht das Einbinden von Bedürfnissen der Bewohner*innen im Vordergrund. Die Einbindung ist verbunden mit einem individualisierten und flexiblen Vorgehen.

- Ferner ist als Wertvorstellung für ein teilhabeorientiertes Handeln förderlich, wenn auch alte, pflegebedürftige Menschen, die in einer Altenpflegeeinrichtung leben, als entwicklungsfähig betrachtet werden: „Aber jeder Mensch ist ja individuell für sich. Und das ist wirklich diese Entdeckungsreise, zu wissen, so, wo will dieser Bewohner hin? Wo sind unsere Ziele noch? Was können wir therapeutisch noch erreichen? Weil es ist ja - heißt ja nicht, ein Altenheim ist Endstation, wir lassen die Leute in Ruhe, sondern wir wollen da noch irgendetwas rausholen. Und denen noch was zeigen" (PFK).

- Höflichkeit und Zugewandtheit werden als ein wichtiger Bestandteil der "guten Betreuung“ angesehen und werden insbesondere von den Angehörigen als erkennbares Kriterium einer positiven Arbeitseinstellung gesehen. Aber auch von den Mitarbeitenden wird in den Interviews betont, dass Vorgesetzte - insbesondere bei der Einarbeitung - vermitteln sollten, dass Höflichkeit und Respekt im Auftreten wesentlich sind: "[...] und dass es ganz wichtig ist, den Leuten eigentlich mitzuteilen, dass das - ja, ich will jetzt nicht sagen, dass es jetzt das neue Zuhause ist, aber zumindest so Leute ein bisschen darauf - ja, man muss - die Mitarbeiter muss man da ein bisschen einstellen, also, dass sie nicht direkt so ohne zu klopfen ins Zimmer rein, also man muss - den Respekt muss man schon vor dem Bewohner haben" (HT).

- Ein positives Verständnis von Kollegialität wird im Zusammenhang mit Unterstützungsleistungen und gegenseitiger Hilfe beschrieben. So wird das kooperative Handeln unter Kolleg*innen als notwendig für die Unterstützung der Teilhabe von Bewohner*innen erachtet, aber auch grundlegend dafür, das Arbeitspensum erfüllen zu können: „Da, wo Not am Mann ist, wird miteinander gearbeitet und untereinander ausgeholfen, ne? Und auch, wenn ich hier fertig bin, dann gehe ich rüber und helfe da weiter" (PDL). Häufig wird im Zuge dieser Sichtweise auch betont, dass die Leitung diese Arbeitsweise unterstützen muss, damit auch kooperativ gehandelt wird (Kapitel 4.3.3.1). 


\section{Hemmend:}

- Einzelne Mitarbeitende orientieren sich primär an Strukturen, Regeln und Routinen, von denen sie nicht abweichen wollen - dies bietet ihnen Orientierung und Sicherheit. Gerade in der Versorgung von pflegebedürftigen Menschen wird ein vorgegebener Rahmen gebraucht, so die Annahme einzelner Interviewpartner"innen, aber dieser ist auch hemmend für die individuelle, situative und kreative Förderung von Teilhabe: „[Teilhabe] ist, denke ich, immer mehr Thema in der letzten Zeit, aber sicherlich noch nicht so wirklich verinnerlicht. Schon mal gar nicht, sage ich mal, im täglichen Tun der Pflegenden, die ja doch sehr eingebunden sind in ihre Tätigkeiten, ja, mit einem hohen Arbeitsanfall, der bewältigt werden muss. Und wo sicherlich so routiniertes Arbeiten erst mal im Vordergrund steht und dann auch nicht immer so im täglichen Tun die Zeit da ist, Dinge zu reflektieren oder sich mit neuen Dingen auseinanderzusetzen“ (PDL).

- Hemmend ist auch eine grundsätzliche Orientierung an der Sicherheit der Bewohner*innen, die mit der Angst vor negativen, persönlichen Konsequenzen verbunden ist, die mit der Verantwortungsübernahme einhergeht: „Aber ich sage mal, auf der Seite, das ist, glaube ich, das Schlimmste. Die haben immer Angst, es passiert was [...] Und die sind verantwortlich und kriegen dann nachher Stress“ (PDL).

\section{Arbeitsengagement}

Das Arbeitsengagement wird auch auf der Ebene der/des individuellen Mitarbeiter"in betrachtet und knüpft an die Arbeitskultur an. Darunter versteht das Projekt STAP die jeweilige Einstellung zum Arbeiten (Arbeitsorientierung, Stellenwert der Tätigkeit) und die damit verbundenen Erwartungen an die Arbeit, wie beispielsweise Wachstumsbedürfnisse (Sinn vs. Erwerb). Aber auch die Arbeitsorientierung (instrumentell vs. inhaltlich) und die Arbeitsbedingungen (z. B. Arbeitszeit und Entlohnung) spielen hier mit eine Rolle (angelehnt an Heeg, 1991, S. 58). Hierzu konnte in den Interviews mehrfach die Vorstellung identifiziert werden, dass Teilhabeförderung in Altenpflegeeinrichtungen nur durch ein überdurchschnittliches Arbeitsengagement unter Beteiligung persönlicher Ressourcen (z. B. Überstunden, private Einkäufe von Material) hinreichend umgesetzt werden kann. So wird das jeweilige Engagement der Mitarbeiter"innen auch als personenabhängig eingestuft: „Aber ich glaube, dass es da - dass es da Mitarbeiter gibt, die gucken, von wegen, was ist das für ein Mensch, und 
was kann ich dem denn mal Gutes tun?“ (EL). Auch im Rahmen der trägerübergreifenden Fokusgruppendiskussion wurde die Personenabhängigkeit betont: „Also ich glaub, dass man gar nichts verallgemeinern kann, weil es abhängig ist von Charakteren und Persönlichkeiten“ (SD).

\section{Fördernd:}

- In Bezug auf verschiedene Motivationen in der und Einstellungen zur Arbeit wird mehrfach die Unterscheidung zwischen 1.) der Freude im Umgang mit Menschen und 2.) der Sachorientierung bzw. dem Geldverdienen aufgestellt: „Auch in der - in der Pflege, das merkt man. Kommt da einer, weil ihm das Spaß macht, in der Pflege zu arbeiten, oder kommt da einer, weil er nur Geld verdienen will? Und das merkt man am Umgang“ (HW). Insbesondere von Angehörigen werden für die Arbeit mit pflegebedürftigen Menschen nichtfachliche persönliche Eigenschaften als besonders bedeutsam erachtet, die sich als besondere Befähigungen für den Beruf sowie den Umgang mit Menschen („Berufung“, „Herzblut“) und dazu passenden, stabilen Leitorientierungen und Prinzipien im beruflichen Handeln (oft als „Haltung“ thematisiert) zusammenfassen lassen und hier die Nähe zur Arbeitskultur zeigen.

- Als förderlich wurde erachtet, wenn Mitarbeitende sich auch außerhalb ihrer Regelaufgaben für Bewohner*innen einsetzen - häufig werden eben diese Mitarbeiter*innen als besonders engagiert wahrgenommen (z. B. Einrichtungsleitung begleitet Bewohner*innen zu Ausflügen).

- Gerade der überdurchschnittliche Einsatz, der über das Arbeitsverhältnis hinausgeht, wird auch von den Mitarbeitenden - untereinander - als besonders positiv wahrgenommen. Einzelne Mitarbeiter*innen beginnen beispielsweise früher mit ihrer Arbeit, damit Bewohner*innen zu der gewünschten Zeit Kaffee zu sich nehmen können, andere finanzieren ihre Fortbildungen selber oder schaffen Material aus eigenen finanziellen Mitteln an. Auch wenn ein solches Engagement als teilhabeförderlich beschrieben wurde, sei an dieser Stelle allerdings ausdrücklich anzumerken, dass dies gleichzeitig - durch die Überschreitung der Arbeitszeit und den deutlichen Rückgriff auf private Ressourcen - nicht als Maßstab für gute, teilhabefördernde Arbeit genommen werden kann.

\section{Hemmend:}

- Der sogenannte „Dienst nach Vorschrift“ (ANG) wird von den Interviewpersonen als teilhabehemmend wahrgenommen - gerade auch, wenn 
Bewohner*innen, Angehörige oder Kolleg*innen ein anderes Engagement in der gleichen Einrichtung und Funktion zum Vergleich haben, z. B. durch einen Wechsel von Mitarbeiter*innen.

- Ferner wird als hemmend betrachtet, wenn in der Altenpflege Menschen tätig sind, die diesen Beruf nur als „Job" bzw. „Broterwerb“ betrachten: ,[...] Dann ist ja immer das Problem, wer möchte den Job noch machen? Da kommt ja - und wenn mal welche Leute kommen, dann denkst du, hm [...] passt nicht so, ne? Weil die kommen dann nur zum Arbeiten oder nur, um Geld zu verdienen. Und ich sage mal, in einem Altenheim zu arbeiten, ist nicht nur Geld verdienen. Man muss das auch - man muss das mögen" (HW).

- Für Mitarbeitende kann es eine Herausforderung darstellen, alle Bewohner*innen gleich(berechtigt) zu behandeln und mit dem gleichen Arbeitsengagement zu begegnen, denn Sympathie und Antipathie gegenüber Bewohner*innen können das Engagement und die Teilhabeförderung beeinflussen. Eine Einrichtungsleitung beschreibt, dass eine „professionelle Distanz" (EL) bei der Arbeit in einer Altenpflegeeinrichtung besonders wichtig sei.

\subsubsection{Einrichtung und organisatorische Abläufe}

Die Rahmenbedingungen und Strukturen sowie organisatorischen Abläufe in der Einrichtung spielen eine wesentliche Rolle für den Blick auf und die Berücksichtigung sowie Gestaltung von selbstbestimmter Teilhabe in Altenpflegeeinrichtungen. Als bedeutsam hervorgehoben werden im Folgenden einerseits - mit den Themen der Organisationskultur und Verankerung in Konzepten - Rahmenbedingungen, die zur Bewusstseinsbildung bei den Mitarbeitenden beitragen. Andererseits werden verschiedene organisationale Strukturen und (Arbeits-)Abläufe beschrieben, die sich in den Interviews insbesondere auf die Schnittstellen und Zusammenarbeit zwischen verschiedenen Einrichtungsbereichen sowie auf Handlungsspielräume innerhalb von Arbeitsprozessen bezogen haben.

\subsubsection{Organisationskultur}

Der bereits im Zusammenhang mit der Arbeitskultur erwähnte Begriff der Organisationskultur kann hier mit Schmitt (2015, S. 75) noch konkreter verstanden werden als die von den Mitgliedern einer Organisation bzw. 
Einrichtung geteilten Annahmen und Überzeugungen; die geteilten expliziten und impliziten Werte; die Mittel der Organisation, Entscheidungen und Handlungen ihrer Mitglieder im Sinne dieser Annahmen, Überzeugungen und Werte zu prägen; sowie schließlich auch die für Außenstehende erkennbaren, kulturbedingten Merkmale.

Die Bedeutsamkeit der Organisationskultur zur Förderung von selbstbestimmter Teilhabe kam insbesondere im Rahmen der "Good-Practice“Analyse sehr deutlich zum Ausdruck, wenn von den Interviewpersonen rückblickend überlegt wurde, was dazu geführt hat, dass die Förderung von Selbstbestimmung und Teilhabe in ihrer Einrichtung erfolgreich „umgesetzt" wird. Hinweise dazu finden sich aber ebenfalls in den Interviews in den vier Projekteinrichtungen von STAP sowie in den trägerübergreifenden Fokusgruppen. Darüber hinaus wurde die Relevanz der Organisationskultur im Sinne geteilter Überzeugungen und Werte in den Rückmeldungen des Projektrates und im Rahmen der Lernworkshops ${ }^{70}$ vielfach deutlich bestätigt.

\section{Fördernd:}

- So wurde mehrfach betont, dass vor allem die Einrichtungsleitung und mit ihr das Leitungsteam aus den verschiedenen Einrichtungsbereichen wesentlich dafür verantwortlich sind, dass in der Einrichtung bei den hauptamtlichen Mitarbeiter*innen und ehrenamtlichen Kräften eine entsprechende Organisationskultur, aber auch bei Bewohner*innen und Angehörigen das (Selbst-)Verständnis aufgebaut und gefördert wird, dass die Gewährleistung und Förderung von Selbstbestimmung und Teilhabe der Bewohner*innen von zentraler Bedeutung im Handeln aller Beteiligten ist. Die Leitungskräfte erfüllen hier wesentlich die Funktion, grundlegend und stetig für die Themen Selbstbestimmung und Teilhabe zu sensibilisieren und zu motivieren sowie die Bedeutung dieser Themen in ihrem Verhalten und ihrer Führung vorzuleben, wobei hierzu als Voraussetzung gesehen wird, dass das Leitungsteam ein gemeinsames Verständnis hat und gemeinsame Ziele verfolgt. Im Rahmen der trägerübergreifenden Fokusgruppendiskussion wurde die Einstellung und Vorbildfunktion der Leitungskräfte ebenfalls thematisiert und als relevant erachtet: „Wenn man neue Mitarbeiter schult, neue

70 Die Lernworkshops wurden auf Nachfrage des Projektrates initiiert. Hier wurden vorläufige Ergebnisse des Projektes STAP in den vier Projekteinrichtungen vorgestellt und mit den Einrichtungsleitungen, den Pflegedienstleitungen und Vertreter*innen der Sozialen Dienste diskutiert. 
Mitarbeiter hat. Wenn man mit den Kollegen ins Gespräch geht. Das ist mir total wichtig. Ich will nicht, dass die Kollegen das Gefühl haben, die machen es nicht gut. Nein, sondern dass die wissen, was ist unsere Kultur, unser Ziel hier im Haus? Was braucht der Mensch? So, also, dass wir Situationen erkennen und sie gemeinsam lösen. Weil die Menschen, die das tun - ich bin davon entfernt, früher habe ich immer gedacht: ,Sind die denn bescheuert? ${ }^{\text {- }}$, also ich bin davon entfernt, also ich kann mit dieser Haltung meinen Leuten ja nicht begegnen. Ich hab' was, was die nicht können, und die, also die sind ja nicht alle grundsätzlich so, aber es schleicht sich manchmal so was ein. Und da sind wir gefordert" (SD).

- Von den "Good-Practice“-Einrichtungen wurden ferner Mitarbeiter"innenfortbildungen oder Coachings bzw. Beratungen der Leitungsteams als bedeutsamer Faktor benannt, um das Wissen zu relevanten Inhalten fundieren sowie die Arbeits- und Unternehmenskultur fördern zu können. Die benannten Fortbildungsinhalte waren durchaus heterogen (z. B. mit Bezug auf „Personzentrierung“, den „Umgang mit Menschen mit Demenz“, „Sozialraumorientierung“, „Lebensstilkonzept“, „Sterbebegleitung“), wurden aber jeweils als bedeutsam beschrieben, um eine „Initialzündung“ zu geben, das Wissen der Mitarbeitenden - teils auch bereichsübergreifend - zu fundieren und die Arbeits- und Unternehmenskultur entsprechend zu fördern.

- Betont wurde in den Interviews einerseits, dass Fortbildungen einen Einstieg und ersten Ausgangspunkt zur Bewusstseinsbildung bieten, und andererseits, dass es danach die große Herausforderung sei, die „Theorie“ in die „Praxis“ zu überführen. So wird die stete im Arbeitsalltag verankerte Förderung der Organisationskultur als eine zentrale Gelingensbedingung in allen „Good-Practice“-Einrichtungen beschrieben. Es sei ein langfristiger und fortlaufender Prozess, der immer wieder neues Engagement für die Sache erfordere: „Es gehört jeden Tag erkämpft und man steckt sehr viel Energie rein [...] Man muss am Ball bleiben“ (GP Int. 4). Wenn die gewünschte Organisationkultur hinreichend spürbar ist, kann es dazu führen, dass neue Mitarbeitende sich dieser automatisch anpassen: „Neue Mitarbeiter [..., die sich dann halt dem Team anpassen müssen [..., die beginnen dann halt auch diesen Gedanken zu leben, aber das passiert erst dann, wenn das tatsächlich integriert ist und gepflegt wird“ (GP Int. 3). Dennoch gibt es keinen finalen Zeitpunkt, an dem die Organisationskultur immerwährend verankert ist: „Also man kann nicht sagen, so jetzt haben wir es geschafft, jetzt ist gut, jetzt denken alle so" (GP Int. 3). 
- Als zentrale Orientierungen und geteilte Werte im Rahmen der Organisationskultur können in den Interviews einerseits die Orientierung an dem Prinzip der Selbstbestimmung sowie andererseits an dem Normalitätsprinzip wiedergefunden werden:

Das Selbstbestimmungsprinzip wird in den Interviews mehrfach als grundlegende Orientierung hervorgehoben, die den Umgang mit den Bewohner*innen sowie die Alltagsgestaltung bestimmen sollte. Als Erfahrung wird dazu dann auch geschildert, dass eine den Bewohner*innen gegenüber offen kommunizierte Orientierung an dem Selbstbestimmungsprinzip dazu führe, dass sich die Bewohner*innen selbst aktiver und offener in Hinsicht auf eigene Wünsche zur Alltagsgestaltung und sozialen Teilhabe, aber auch zur Ablehnung von Teilhabeangeboten äußern würden.

Auch zum Normalitätsprinzip als wichtige Leitorientierung finden sich viele Hinweise im Rahmen der Interviews in den „Good-Practice“-Einrichtungen ebenso wie in den Projekteinrichtungen und in den Fokusgruppen. So wird die Relevanz des Normalitätsprinzips mit vielen Beispielen und unterschiedlichen Bezügen verdeutlicht: zum Beispiel in Bezug auf Normalität in den alltäglichen Gewohnheiten, wie die Reihenfolge und Dauer von Alltagsaktivitäten, Gewährleistung von Privatsphäre, ein „normaler“ Umgang mit Bewohner“innen, eine der individuellen Normalität entsprechende Möblierung und Gestaltung des Zimmers.

\section{Hemmend:}

- In den Interviews mit den Leitungskräften der „Good-Practice“-Einrichtungen werden allerdings auch Grenzen der Orientierung an Prinzipien der Selbstbestimmung und Normalität benannt, die sich auf verschiedene Umsetzungsebenen und -bereiche im Alltag beziehen, wie etwa in Bezug auf Abstimmungsbedarfe in einer Gemeinschaft („Natürlich hat man immer, wenn man in Gruppen oder in Nachbarschaften oder sehr wohl auch in Abhängigkeiten zusammen ist, hat man da Abklärungsbedürfnisse, ja“, GP Int. 4) oder bei Gesundheitsgefahren („Also man muss dann eben schon gucken, wie weit geht jetzt Selbstbestimmung, also wenn es jetzt in Richtung gesundheitsschädigend geht, dann muss man eben intervenieren“, GP Int. 5).

- Auch gibt es häufig das explizite Verständnis, dass Umfang und Qualität der Teilhabeförderung personenabhängig bzw. mitarbeiter*innenabhängig sind. Das individuelle Arbeitsengagement sei demnach nur begrenzt oder wenig von einer geteilten Organisationskultur beeinflusst. 
Teilhabe werde besonders von den Mitarbeitenden umgesetzt, die dazu eine gewisse verinnerlichte Haltung und Befähigung haben: „Sicherlich ist das auch personenabhängig, ja? Also es kann nicht jeder, der eine Betreuungskraft ist, das gleich so machen wie - wie - wie der andere. Also wie auch ein Pflegemitarbeiter nicht - nicht einer ist wie der andere, ja? Und jeder hat da einen eigenen - eigenen Ansatz und ein anderes Verständnis. Ja?“" (PDL). Selbst wenn ein/e Mitarbeiter“in eine gute Qualifikation mitbringt, heißt dies nicht, dass sie dieses Teilhabeverständnis tatsächlich innehat: „Also personelle Größen sind unheimlich wichtig, gutes Personal zu haben, engagiertes, motiviertes Personal zu haben. Das heißt nicht, dass jeder Examinierte und gut Bezahlte auch gleichzeitig eine qualitativ hochwertige Arbeit leistet“ (EL).

- So wurden im Rahmen der Interviews auch Kritik an einem gewissen "Versorgungsprimat" geäußert, womit gemeint ist, dass die Versorgung der Bewohner*innen - insbesondere im Sinne medizinisch-pflegerischen Versorgung - im Vordergrund des Einrichtungsalltags steht und vorrangig gegenüber anderen Zielen und Leistungen betrachtet wird: „Also eine Pflegeeinrichtung heißt ja nicht umsonst Pflege. Und Pflege ist der wichtigste Punkt, und alles andere ist untergeordnet" (EL). Das Selbstverständnis einer Altenpflegeeinrichtung sei demnach auf die Pflege konzentriert, so dass ein Teilhabeverständnis eher nebensächlich ist: „Also Pflege ist gerne pflegelastig. Ich glaube nicht, dass die sich um Teilhabe jetzt so die großen Gedanken machen. Vielleicht wird das dann auch eher als Anstrengung empfunden“ (PDL). „Bei den Mitarbeitern der Pflege ist Teilhabe glaube ich kein Thema“ (PDL). Gleichermaßen wird als Ideal geäußert, dass bereichsübergreifend viel deutlicher eine ganzheitliche und subjektorientierte Betrachtung der Bewohner*innen als Orientierung dienen sollte (mit dem kritischen Blick auf die oft vorhandene Existenz unterschiedlicher Orientierungen: Primat der medizinisch-pflegerischen Versorgung „in der Pflege“ versus Primat der sozialen Betreuung „im Sozialen Dienst“), ohne dass dabei die jeweiligen Kompetenzschwerpunkte negiert werden (Kapitel 4.3.3.3): „Aber es gibt ja Bewohner, die können das nicht so äußern. Und da guckt man dann. Und dann gibt es auch da Rangeleien, sage ich jetzt mal, zwischen Mitarbeitern und auch zwischen Arbeitsbereichen. Dann weiß die Pflege das besser als der Soziale Dienst“ (EL).

- Eine Überzeugung, die zu Einschränkungen der Selbstbestimmung und individuellen Normalität im Rahmen der Teilhabeförderung führen kann, bezieht sich auf Normierungen und einheitliche Handhabungen, z. B. im Kontext von Gruppenangeboten. Diese dienen aus Sicht man- 
cher Mitarbeitenden der Fairness und scheinen für eine Gemeinschaftsunterkunft angemessen. Auch werden sie zum Teil auf den Personalmangel zurückgeführt: „Aber so muss man einfach gucken, also der hat immer so - versucht schon mit neuen Ideen, aber wie lässt sich das eben mit der Gleichberechtigung, wie lässt sich das vereinbaren? Das ist ja gar nicht so leicht“ (SD). So werden etwa auch neue Ideen, die möglicherweise nur für einzelne Bewohner*innen von Bedeutung sein könnten, nicht umgesetzt, weil Teilhabeangebote möglichst viele erreichen sollen. Dadurch erfolgen implizit, aber zwangsläufig Normierungen in der Teilhabeförderung, die individuellen Teilhabewünschen entgegenstehen können.

- Ferner gibt es - an den vorangegangenen Aspekt anschließend - auch Hinweise auf Effizienzorientierungen, in dem Sinne, dass Interessen und Ressourcen zu bündeln sind, um ressourcensparend Angebote umzusetzen: „Und da kann ich natürlich schlecht sagen, auch wenn - auch wenn das super ist, wenn - wenn der Mensch dann überglücklich ist, aber ich kann halt nicht irgendwie nur sieben Leute glücklich machen und die anderen 64 - das, ne, kann ich mal punktuell, klar. Ich werde nie immer alle erreichen" (EL).

\subsubsection{Formelle Verankerung in Konzepten}

Die Frage danach, wo Teilhabeziele im Rahmen von Konzepten formell verankert bzw. konkret festgehalten sind, fällt vielen Interviewpersonen schwer zu beantworten. Die meisten Befragten deuten eher vage auf „das Konzept" hin - es wird nicht näher bezeichnet, welches damit gemeint ist; zu vermuten ist, dass es sich um das Leitbild oder Einrichtungskonzept handeln könnte. Einzelne Mitarbeitende nennen aber als Orte der Verankerung konkret das (Rahmen-)Konzept des Sozialen Dienstes, das Pflegeleitbild bzw. Pflegekonzept und das Leitbild und Einrichtungskonzept. Eine Einrichtungsleitung bringt hier auch das „Einzugskonzept“ (EL) ein. Es wird aber in mehreren Interviews auch offen gesagt, dass sie davon ausgehen, dass in diesen Konzepten etwas dazu festgehalten ist, ohne es genauer zu wissen.

Ferner geben einzelne Interviewpersonen ausdrücklich an, dass es kein konkretes „Teilhabekonzept“ gebe, auch wenn Bezüge auf den Teilhabebegriff direkt und indirekt in anderen Konzepten enthalten seien: „Nein, bis jetzt sage ich ja nicht. Müssen wir ja machen. Hatten wir schon mal kurz drüber gesprochen. Außer in den - in den - in den anderen Konzepten, 
die vielleicht in die Richtung gehen, ja? Ein - ein Einzugskonzept, was wir haben, wo auch die Integrationsgespräche drin sind, das ist natürlich - hat was mit - mit - mit - mit Teilhabe zu tun. Ich glaube aber, dass das Wort, vielleicht steht es sogar noch ein-, zweimal da drin, aber es steht definitiv nicht oben drüber Teilhabekonzept. Das Einzugskonzept soll aber die Teilhabe fördern. Und das - die Gewaltprävention soll Teilhabe fördern und die freiheitsent-, die Vermeidung von freiheitsentziehenden Maßnahmen soll auch Teilhabe fördern. Und unser Sozialdienstkonzept soll auch Teilhabe fördern" (EL).

Vor diesem Hintergrund werden zu diesem Themenbereich keine fördernden oder hemmenden Faktoren aufgeführt, wenngleich in STAP die Annahme besteht, dass eine konzeptionelle Verankerung förderlich ist, damit die Förderung von Selbstbestimmung und Teilhabe als Organisationsziel festgehalten wird und eine gemeinsame Orientierung daran erfolgt. Bestätigt wurde dies durch die „Good-Practice“-Analyse, in deren Interviews mehrfach hervorgehoben wurde, dass es grundsätzlich wichtig sei, die Unterstützung von Selbstbestimmung und Teilhabe als Ziele der Einrichtung auch konzeptionell zu verankern: „Ich glaube das Konzept ist ja das, überhaupt das Wesentliche“ (GP Int. 5). Vor diesem Hintergrund und ebenso angesichts dessen, dass die Interviewpersonen der Projekteinrichtungen mehrheitlich eben keine konkreten Aussagen über Bezugnahmen auf selbstbestimmte Teilhabe in den Konzepten tätigen konnten, wurde das Thema näher aufgegriffen. So war es auch im Hinblick auf die Erstellung des Musterrahmenkonzeptes im Rahmen von STAP von Interesse, ob und inwieweit bislang Bezüge zu Selbstbestimmung und Teilhabe in den Konzepten der Projekteinrichtungen als Anknüpfungspunkte vorhanden sind. Daher erfolgte eine ergänzende Analyse der Konzepte, die an dieser Stelle als „methodischer Exkurs“ integriert wird.

Der nächste Abschnitt bezieht sich auf diese Analyse zur Verankerung von Teilhabe in den Konzepten der Einrichtungen. Dazu stellten alle Projekteinrichtungen ihre Konzepte zur Verfügung. Die Datenbasis umfasste folgende Konzepte:

- das Einrichtungsleitbild,

- das Einrichtungskonzept,

- das Konzept des Sozialen Dienstes,

- das Pflegekonzept,

- das Konzept der zusätzlichen Betreuung,

- das Konzept der Gewaltprävention, 
- das Konzept der freiheitsentziehenden/freiheitseinschränkenden Maßnahmen,

- das Inhaltsverzeichnis des Qualitätsmanagementhandbuches.

Die betrachteten Einrichtungen besitzen unterschiedliche Systeme und Formen von Konzepten. Das Spektrum reicht von (a) einem gesamten, einrichtungsumfassenden Konzept über (b) die Fusion von zwei größeren Einrichtungsbereichen in einem Konzept nebst Teilkonzepten bis hin zu (c) mehreren größeren Teilkonzepten.

Nicht alle Einrichtungen nutzen ein gesondertes Pflegekonzept oder ein gesondertes Konzept für den Sozialen Dienst, da sie Inhalte innerhalb eines gesamten Konzeptes für die Einrichtung oder innerhalb des Einrichtungskonzeptes ${ }^{71}$ integriert haben. Auch ein gesondertes Konzept der zusätzlichen Betreuung findet sich bei zwei Einrichtungen nicht. Bei einer Einrichtung ist dies Bestandteil des Konzeptes Sozialer Dienst, bei der anderen besteht ein Konzept, welches sich spezifisch auf die Nachmittagsbetreuung durch zusätzliche Betreuungskräfte bezieht. Im Folgenden werden Bezugnahmen auf die Begriffe und Themen der Teilhabe und Selbstbestimmung in den Konzepten der Projekteinrichtungen erläutert und exemplarisch zitiert.

Teilhabe

Allgemein variieren die Formulierungen der Teilhabeziele und Teilhabedimensionen zwischen den Konzepten vor allem hinsichtlich ihrer Konkretheit und ihrer Gegenstandsbenennung. So finden sich als allgemeine Formulierungen „Teilhabe am gesellschaftlichen Leben innerhalb und außerhalb der Einrichtung“ oder als „Teilhabe am gemeinschaftlichen Leben innerhalb und außerhalb der Einrichtung“. Innerhalb eines Konzeptes bezieht sich Teilhabe auf die Betreuung mit dem Ziel der „Teilnahme am gemeinschaftlichen Leben“. Im Kontext von „Teilhabe am gesellschaftlichen Leben" werden zur Umsetzung vor allem die Förderung des sozialen Austausches von Bewohner*innen durch Einzel- und Gruppenangebote mit Freizeitcharakter genannt.

Blickt man nun konkret auf das explizit formulierte Vorkommen von „Teilhabe am gesellschaftlichen Leben“ in den übergeordneten Konzepten, also den Einrichtungskonzepten und innerhalb des Einrichtungsleitbildes, so

71 Das Einrichtungskonzept ist nicht sinngleich mit einem umfassenden Konzept, was alle Einrichtungsbereiche und Themen einbezieht. 
zeigt sich, dass ein solcher Bezug selten existiert: So wird Teilhabe einmal innerhalb eines Einrichtungskonzeptes und einmal innerhalb eines Einrichtungsleitbildes aufgeführt.

Teilhabe findet sich begrifflich und als Ziel jedoch in beiden Konzepten der zusätzlichen Betreuung, etwa wenn im Rahmen der Einzel- und Gruppenangebote „Teilhabe am gemeinschaftlichen Leben“ erfahrbar ist. Der Teilhabebegriff findet sich darüber hinaus aber nicht als direkt formulierte Zieldimension in den Konzepten des Sozialen Dienstes wieder, sondern es sind eher indirekte Bezüge zu finden, wenn zum Beispiel als Ziel, „Geselligkeit und Teilnahme am sozialen Gruppengeschehen“ formuliert wird. Weist man dem Sozialen Dienst eine besondere Rolle in der Förderung und Verwirklichung des Rechts auf Teilhabe zu, so ist dabei zum Zeitpunkt der Erhebung aber auch zu berücksichtigen, dass in der Hälfte der untersuchten Einrichtungen das Konzept des Sozialen Dienstes aktuell oder zeitnah überarbeitet wird und bei der anderen Hälfte kein Einzelkonzept des Sozialen Dienstes existiert.

\section{Selbstbestimmung}

Im Vergleich zum Aspekt der Teilhabe werden in den verschiedenen Konzepttypen (z. B. Einrichtungsleitbild, Pflegekonzept und Konzept Sozialer Dienst) häufiger und vielfältig Aussagen getroffen, die sich auf die Förderung von Selbstbestimmung und auf die Orientierung an individuellen Bedürfnissen beziehen sowie - auf der Ebene von Aufgaben - auf die Durchführung von Einzel- und Gruppenangeboten. So findet sich der Begriff Selbstbestimmung vielfach konkret sowie in verschiedenen alternativen Formulierungen und oft - wie bei STAP - im Verständnis eines Arbeitsprinzips wieder. Dabei geht es etwa um

- das Bestehen auf ein Recht der Selbstbestimmung, der Anerkennung von Einzigartigkeit der Bewohner*innenschaft,

- den Erhalt oder die Stärkung von Eigenständigkeit, Eigen- oder Selbstverantwortung und Individualität,

- den Respekt vor und die Berücksichtigung von persönlichen Bedürfnissen und Mitsprache.

Insbesondere wird Selbstbestimmung als Maßstab erwähnt, an dem sich Maßnahmenplanung, Betreuung und Pflege orientieren. Der Grad der Konkretheit von Aussagen variiert auch hier zwischen den Konzepten der Einrichtung. In den Einrichtungskonzepten, in denen Selbstbestimmung überwiegend benannt wird, finden sich allgemeinere Aussagen mit und 
ohne konkrete Zuordnungen auf Mitarbeiter*innen- oder Bewohner*innengruppen. Die Aussagen zur Selbstbestimmung als Zieldimension, bei der Mitarbeiter*innen- oder Bewohner*innengruppen adressiert werden, zeichnen sich durch die Nennung einer Arbeitsmethodik aus (Biografiearbeit, Integration in Alltagsverrichtungen, Einbezug von Wünschen aus der Gremienarbeit, Gemeinwesensarbeit). Währenddessen stellt das Führen eines möglichst selbstbestimmten und selbstständigen Lebens in der Einrichtung eine allgemeine, übergeordnete Zieldimension dar.

Innerhalb der Konzepte für die zusätzliche Betreuung findet Selbstbestimmung einmal als ein bei Bewohner*innen zu förderndes Ziel neben dem der Individualität Erwähnung. Liegt ein gesondertes oder einrichtungsübergreifendes Pflegekonzept vor, so wird Selbstbestimmung auch dort explizit aufgeführt und bezieht sich etwa auf die Unterstützung für ein „möglichst selbstbestimmtes und selbstständiges Leben“.

Angeführt werden können zudem indirekte und direkte Aussagen zur Selbstbestimmung innerhalb verschiedener Konzepttypen, die einen spezifischen normativen Gesichtspunkt hervorheben. Beispielsweise dann, wenn Bewohner*innenbedürfnisse „im Mittelpunkt stehen“, bis hin zur umsetzungsorientierten Nennung von Instrumenten, etwa „der Pflege- und Betreuungsplan berücksichtigt das Selbstbestimmungsrecht".

Betrachtet man nun die zugeschriebene Wirkung des Einbezugs von Teilhabe in Leitbildern und Konzepten hinsichtlich der Teilhabeförderung aus Sicht der Leitungen und Mitarbeitenden, so finden sich dazu - wie bereits erwähnt - vor allem Aussagen in den Interviews der "Good-Practice“Analyse. So wurde es im Rahmen der „Good-Practice“-Analyse als wichtig erachtet, dass die Zielsetzungen der Förderung von Selbstbestimmung und Teilhabe konkret und zentral in der Konzeption und dabei insbesondere im Leitbild der Einrichtung verankert sind: „Und ich glaube zunächst mal, um überhaupt Selbstbestimmung und Teilhabe zu generieren, ist es zunächst erstmal Aufgabe des Trägers, dies auch in einem Leitbild zu verankern. Das ist also von meiner Seite aus eine ganz klare Sache, die top-down vom Management vorgegeben sein muss, dass Selbstbestimmung eigentlich im Grunde das höchste Gut und der Anspruch einer solchen Einrichtung sein muss" (GP Int. 2).

Eine andere Interviewperson betont die Orientierungsfunktion von Konzepten, die erkennbare Prioritäten setzen: „Die Autonomie als solche absolute Priorität in unserem Leitbild, im Unternehmensleitbild. Das ist das Erste, was genannt wird, und wir hatten immer den Anspruch und gepaart mit der Normalität, also zu sagen, ne [...] eben nicht institutionell zu 
generieren, sondern einfach sich an der Häuslichkeit zu orientieren" (GP Int. 3).

\subsubsection{Schnittstellen und Zusammenarbeit innerhalb der Arbeitsprozesse}

Im Rahmen einer Vielzahl von Arbeitsprozessen in einer Altenpflegeeinrichtung existieren Schnittstellen zwischen unterschiedlichen Einrichtungsbereichen. Betont wird in den Interviews, dass es für die Versorgung, Betreuung und Begleitung der Bewohner*innen unabdingbar sei, dass verschiedene Funktions-, Aufgaben- und damit auch Dienstleistungsbereiche vorhanden sind und die jeweiligen Mitarbeitenden miteinander kooperieren. Allein dadurch bestehe eine Qualität der Zusammenarbeit, in der sich verschiedene Berufsgruppen mit ibren Qualifikationen und Kompetenzschwerpunkten einbringen: „Also wir machen reine Pflege, das macht der Sozialdienst nicht. Der macht keine Wunden, dies, jenes. Aber ansonsten, die Alltagsbegleiter, die - die Pfarren, die Ehrenamtlichen, der Sozialdienst, wir müssen alle zusammen - Physiotherapie, der Logopäde, der Ohrenarzt, gehört alles dazu" (PFK). Auch Bode (2014) nimmt eine Unterteilung der verschiedenen Mitarbeitendengruppen vor (Pflege, Betreuung, Sozialer Dienst), konzentriert sich hier aber weniger auf die Schnittstellenproblematik als vielmehr auf die jeweiligen Aufgabenbereiche, die klar voneinander getrennt sind. Er spricht von einer Akteurslandschaft, die er als „außerordentlich komplex" (Bode, 2014, S. 111) beschreibt. Für die Akteur"innen gilt es allerdings, zusammenzuarbeiten, um ein teilhabeförderliches Vorgehen der verschiedenen Berufsgruppen zu erzielen.

Konkrete Schnittstellen in der Zusammenarbeit werden von den Interviewpersonen aus unterschiedlichen Einrichtungsbereichen, aber vor allem immer wieder zu folgenden drei Themenkomplexen beschrieben:

1. Der erste Themenkomplex bezieht sich auf die Zusammenarbeit bei gröBeren Veranstaltungen, Gruppenangeboten und Ausflügen, die über das „alltägliche“ Regelangebot hinausgehen. So sind geplante, zentral gesteuerte Schnittstellen im Rahmen von großen Veranstaltungen (z. B. Festen) über die verschiedenen Funktionsbereiche wiederkehrend gegeben: „Wenn wir zum Beispiel eine große hauseigene Veranstaltung jetzt haben wir bald unser Sommerfest, dann ist da die Hauswirtschaft, der Soziale Dienst, die Haustechnik, der Pflegedienst und - und ich bin halt dabei“ (EL). Diese Schnittstellen zeichnen sich durch eine große Routine aus. Die Größe der Veranstaltung und damit verbundene bereichsübergreifende Aufgabenstellungen bedingen, dass Veranstaltun- 
gen von mehreren Schnittstellen und einem im Voraus geplanten $\mathrm{Zu}$ sammenwirken geprägt sind, das sich eingespielt zu haben scheint: „Auch die Haustechniker müssen uns diesen Grill aufbauen und wieder - also wie auch hier mit Musik, also das machen alles Haustechniker. Oder stellen die Tische schon mal auf, also das ist Hand-in-HandArbeiten. Also das ist wirklich dann schon - ja, mhm, alles organisiert. Also das klappt wunderbar" (PFK). Eine Zusammenarbeit über verschiedene Funktionsbereiche ergibt sich auch bei größeren besonderen Gruppenangeboten, so dass sich zwischen den beteiligten Mitarbeiter*innen ein Team für die Durchführung des Angebots bildet und entsprechend Personal aus den unterschiedlichen Einrichtungsbereichen eingeplant wird: „[Da] gibt es Tage, wo man weiß, da muss ich mehr Personal haben, weil da das Tanzcafé stattfindet, ja? Okay, obwohl das über Betreuungsassistenten, Sozialdienstmitarbeiter, es wird über die alles organisiert in Zusammenarbeit mit der Pflege, das ist bei uns hier gut geregelt" (SD). Auch bei Ausflügen ergibt sich diese Zusammenarbeit, etwa in der Form, dass die beteiligten Bewohner*innen rechtzeitig von Seiten der Pflege „vorbereitet“ werden oder dass Mitarbeitende aus der Pflege den Ausflug begleiten: „Und zu den großen [Ausflügen] wie Zoo oder hier die Ausflüge, mal kommt $[\ldots]$ ein Kollege $[\ldots]$ mit $[\ldots]^{\text {“ }}$ (ZBK).

2. Alltäglich und damit regelmäßig entstehen aber vor allem Schnittstellen im Zusammenhang mit den verschiedenen, „regulären" Gruppen- und Einzelangeboten der sozialen Betreuung, die hier als zweiter, vielfach benannter Themenbereich aufgeführt werden sollen und insbesondere in Bezug auf die Zusammenarbeit zwischen Pflege und Sozialer Dienst angesprochen wurden.

Hierzu wird einerseits auf die Notwendigkeit der (Ein-)Planung von Angeboten der sozialen Betreuung im Rahmen der von der Pflege geplanten Termine von Bewohner*innen (z. B. Frisör- und Arzttermine) hingewiesen und darauf, dass hierfür die Relevanz der sozialen Angebote für die Bewohner*innen vom Pflegeteam nachvollzogen werden müsse.

Andererseits ergibt sich mehrmals täglich die Zusammenarbeit im unmittelbaren Vorfeld oder nach Abschluss von Einzel- und Gruppenangeboten der sozialen Betreuung. Hier entsteht als besondere Herausforderung, dass Arbeitsprozesse inhaltlich und zeitlich unmittelbar aufeinanderfolgen, etwa wenn Bewohner*innen im Anschluss an eine pflegerische Versorgung an einem Angebot teilnehmen. Die Pflege muss also beendet sein, damit eine Teilnahme möglich ist: „Weil wir - wir brauchen den und den und der muss dann und dann aus dem Bett sein, weil der 
möchte da gerne dran teilnehmen. Da kann man ja schon ein bisschen unangenehm werden, in der Pflegerealität ist das ja manchmal nicht ganz einfach. Das hat sich aber jetzt auch gerade, weil wir selber so viele Helfer haben und wir dadurch auch Entlastung bieten können, also gerade in diesem Frühstücks- und Mittagessen und auch Abendsituation deutlich verbessert" (SD). Dabei ist eine pflegerische Aufgabe und Unterstützungsleistung häufig die Voraussetzung dafür, dass Bewohner*innen an einem Einzel- oder Gruppenangebot teilnehmen können, was auch von den Teilnehmenden der trägerübergreifenden Fokusgruppendiskussion ausdrücklich bestätigt wird. Typischerweise gehören hierzu Unterstützungen bei Toilettengängen, der Mobilisation bzw. dem Transfer und dem Anziehen. Auch das Hinbringen zu oder Abholen von den Angeboten wird hierbei als wichtiger Prozess der $\mathrm{Zu}$ sammenarbeit angesprochen, die unterschiedlich organisiert sein können: „Ja, kommen auch ab und zu zu uns. Und also die holen auch dann die Bewohner nach unten, wenn jetzt unten was stattfindet hier in der Beschäftigungstherapie, holen sie die auch ab und - oder es - es findet unten eine Zeitungsrunde an bestimmten Tagen statt, dann kommen auch die Bewohner. Also das ist so - quasi so eine Organisatja, wie soll ich das jetzt sagen? [...] Und dann sind auch die Ehrenamtler dabei und entweder werden die durch die hier runtergebracht oder unten betreut und wir bringen sie runter" (PFK).

Umgekehrt nutzen Pflegemitarbeiter*innen ebenfalls die Expertise des Sozialen Dienstes, um die Betreuungsmöglichkeiten für ihre Bewohner*innen des Wohnbereiches individuell auszuloten. Die gegenseitige Unterstützung kann hier auch ungeplant zu Situationen des Zusammenwirkens führen, indem Kolleg*innen spontan fachlich konsultiert werden: „Oder dann auch mal meine Kollegin anrufen. ,Du, kannst du mal gucken? Vielleicht kannst du ihn locken oder so, ne?‘ Da muss man zusammenarbeiten. Und auch mal - ja, wie will ich es sagen? Aber es ist im Leben ja auch so, dass man so schon mal lavieren muss, ne?"“ (SD). Neben diesen Aussagen zu Schnittstellen, die sich unmittelbar auf die Zusammenarbeit in Bezug auf Einzel- und Gruppenangebote des Sozialen Dienstes beziehen, wurde mehrfach grundsätzlich auf die entlastende Arbeit der zusätzlichen Betreuungskräfte hingewiesen (Kapitel 4.4.1).

3. Der dritte, in den Interviews identifizierbare Themenkomplex bezieht sich auf die Zusammenarbeit innerhalb von bewohner*innenbezogenen Besprechungen. Eine Zusammenarbeit entsteht etwa, wenn aus den Bereichen Pflege und Soziale Betreuung unterschiedliche, auf Einzelfallebene 
relevante Informationen zusammengetragen werden sollen, um zum Beispiel anlassbezogen einen gezielten Informationsaustausch (z. B. im Rahmen einer Fallbesprechung) vorzunehmen: „Die Zusammenarbeit mit den Pflegekräften und auch Hauswirtschaft hat sich eigentlich auch ganz gut entwickelt. Wir sind jetzt im Moment noch mal dabei, unsere Fallbesprechungsstrategie neu aufzulegen" (SD). Im Zuge der "Good-Practice“-Analyse wurde bestätigt, dass Bewohner*innen- und Fallbesprechungen wichtig sind, um die individuelle Perspektive der Bewohner*innen (z. B. in Bezug auf aktuelle Wünsche, Bedarfe und Ressourcen) auch einrichtungsbereichsübergreifend abzustimmen, damit in deren Rahmen auch Fragen der Selbstbestimmung und Teilhabe auf Ebene der einzelnen Bewohner*innen besprochen und abgestimmt werden: „Es gibt regelmäßige Besprechungen, wo Fallbesprechungen gemacht werden zwischen Sozialer Dienst, gesetzlicher Betreuung und Pflege. Da werden genau diese Dinge thematisiert, was tut dem Bewohner gut, was braucht er, was müssen wir bei ihm fördern, welche Angebote machen wir ihm?" (GP Int. 4).

Im Folgenden sollen wiederum fördernde und hemmende Faktoren, bezogen auf die Schnittstellen innerhalb von für die Teilhabeförderung relevanten Arbeitsprozessen beschrieben werden.

Fördernd:

- Prinzipiell als förderlich wird betrachtet, wenn bereichsübergreifend gemeinsame Ziele und Leitorientierungen in Bezug auf die Bewohner*innen existieren (Kapitel 4.3.3.1).

- Auch wird das gegenseitige Verständnis für die jeweiligen Aufgaben und die Relevanz der Einrichtungsbereiche als wichtig angesehen.

- Wenn Schnittstellen zwischen den Mitarbeitenden verschiedener Einrichtungsbereiche gut funktionieren und eingespielt sind, kann Teilhabe auch gut umgesetzt werden. Eine routinierte Kooperation besteht insbesondere bei den zentral geplanten Großveranstaltungen zwischen den verschiedenen Arbeitsbereichen in den Einrichtungen.

- So funktioniert die Zusammenarbeit an relevanten Schnittstellen auch besser, wenn diese grundsätzlich (ein)geplant, organisiert und gut kommuniziert ist.

- Förderlich scheint auch zu sein, wenn die Wirkungen der Unterstützung aus anderen Einrichtungsbereichen sichtbar und spürbar sind. So äußern sich Mitarbeitende aus Pflege und Sozialem Dienst hier auch mehrfach wertschätzend hinsichtlich der Unterstützung durch die Einrichtungsbereiche Hauswirtschaft und Haustechnik für das eigene Arbeiten so- 
wie über die im Vergleich zu früher zusätzlichen personellen Ressourcen nach Einführung der zusätzlichen Betreuungskräfte (Kapitel 4.4.1).

\section{Hemmend:}

- In Bezug auf das gegenseitige Verständnis der Einrichtungsbereiche insbesondere zwischen Pflege und Sozialem Dienst - und ihrer jeweiligen Zuständigkeitsbereiche (hier auch die "Sicht" des Wohnbereiches als Einheit gegenüber dem Sozialen Dienst als wohnbereichsübergreifende Organisationseinheit) wird ein „Säulendenken" als hemmender Faktor beschrieben (auch Kapitel 4.3.3.3). Damit ist gemeint, dass etwa 1.) $z u$ wenig Kenntnis in Bezug auf die jeweiligen Abläufe besteht („Aber wieder so, dass wir wieder nicht wissen, wie genau das abläuft, ne? Die sind für sich. Wir sind für uns“, PFK), dass 2.) unterschiedliche Ziele und Leitorientierungen existieren und zu wenig die Funktionen der anderen Bereiche in der eigenen Planung berücksichtigt werden: „Wir haben schon eine ganz andere Vorstellung, ganz andere Ziele bezüglich des - also Teilhabe am persönlichen - also jetzt nicht pflegerisch, sondern wie geht es dem, was mag er, was hat er für Vorstellungen, Geschmäcker und all so was. Da wird manchmal von einigen Schwestern gar nicht - kein Wert drauf gelegt. Satt, sauber und gut gepflegt, kein Dekubitus und so was, ne?" (ZBK) und dass 3.) eine Hierarchie der Aufgaben und Konflikte zwischen den Einrichtungsbereichen in Bezug auf die Relevanz der jeweiligen Aufgaben wahrgenommen wird: „Wir waren so ein bisschen die lästigen Bespaßer“ (SD); „Der typische Konflikt zwischen Pflege und Betreuung [...:] die reden und die machen schönen Sachen und die anderen arbeiten“ (GP Int. 3); „Aber wie gesagt, man muss sehr, sehr dickes Fell haben. Man muss selbstbewusst sein, um dann auch wirklich zu bestehen gegenüber der Pflege, ne? [...] Was will der schon wieder? Der - der nervt mich jetzt nur" (ZBK).

- In Bezug auf die Informationsweitergabe und Zusammenarbeit zwischen den Arbeitsbereichen wird als hemmend benannt, wenn Mitarbeitende des Sozialen Dienstes nicht an der Pflegeprozessplanung bzw. an der Dokumentation im Rahmen des Pflegeprozesses beteiligt werden. Auch ein fehlender Zugriff auf Instrumente der Pflegeanamnese und -planung (hier in Bezug auf die SIS) durch den Sozialen Dienst wird vereinzelt als Problem gesehen.

- Die Weitergabe von Informationen im Besprechungssystem der Pflege (im Wesentlichen in Bezug auf die täglichen Übergaben zwischen den „Schichten“) orientiert sich primär an der medizinisch-pflegerischen Versorgung. Teilhabebezogenen Wünschen und Maßnahmen kommt da- 
bei - nach Aussagen der Interviewpersonen - eine geringere Bedeutung zu. Eine Beteiligung und Teilnahme von Mitarbeitenden des Sozialen Dienstes an den Übergaben erfolgt in den Einrichtungen unterschiedlich, aber nicht regulär.

- Multiprofessionell zusammengesetzte Gruppen finden innerhalb des Besprechungswesens selten statt. Dazu gehören etwa Teambesprechungen der Wohnbereiche (z. B. einmal im Monat oder Quartal) oder anlassbezogene Fallbesprechungen (etwa veranlasst durch die den Pflegeprozess steuernde Pflegefachkraft) oder auch kurze Tagesrunden von den Leitungen der jeweiligen Arbeitsbereiche (in einer Einrichtung einmal am Tag, morgens). Nach Aussagen einiger Interviewpersonen besteht Bedarf nach mehr bzw. strukturierterem Austausch zwischen den Arbeitsbereichen, so dass Teilhabewünsche besser weitergegeben werden können.

- Es besteht selten eine zugeordnete, personelle Verantwortung für den Gesamtprozess der Wunscherfassung, Berücksichtigung und Kontrolle über Bereiche und Professionsgruppen der Beteiligten hinweg. Daher komme es vor, dass Wünsche von Bewohner*innen nicht weitergegeben werden oder ihre Umsetzung im Alltag „untergeht“.

- Wie oben bereits skizziert, ist ein wesentlicher hemmender Faktor auch, wenn die unmittelbare Zusammenarbeit im Kontext von Gruppen- und Einzelangeboten an der Schnittstelle zwischen Pflege und Sozialem Dienst nicht funktioniert, insbesondere wenn es an gegenseitiger Information mangelt (z. B. zu Angeboten des Sozialen Dienstes und pflegerelevanten Terminen) und rechtzeitige Unterstützung fehlt (z. B. bei Toilettengängen).

\subsubsection{Handlungsspielräume innerhalb von Arbeitsprozessen}

Handlungsspielräume innerhalb von Arbeitsprozessen wurden in den Interviews als ein relevantes Thema markiert, da sich nur über grundsätzlich vorhandene Handlungsspielräume - auch situativ entstehende - individuelle Wünsche und Bedürfnisse von Bewohner*innen berücksichtigen lassen. Das Erkennen und Nutzen von Handlungsspielräumen ist einerseits auf der Ebene der individuellen Mitarbeiter*innen als Subjektkompetenz einzuordnen und wurde dort im Zusammenhang mit Flexibilität bereits benannt. Andererseits wurden in den Interviews Handlungsspielräume aber auch mit strukturellen und organisationskulturellen Rahmenbedingungen auf Ebene der Einrichtung verbunden. So ist hier zu fragen, inwieweit Struk- 
turen der Einrichtung sowie vorhandene Werte und Normen der Vorgesetzten und Kolleg*innen Handlungsspielräume ermöglichen und tolerieren. Im Rahmen der Interviews wurden verschiedene fördernde und hemmende Faktoren genannt, welche sich auf Handlungsspielräume innerhalb von Arbeitsprozessen beziehen:

\section{Fördernd:}

- Es besteht offenbar bereichsübergreifend das Bewusstsein, dass in der Arbeit mit Menschen nicht alle Tätigkeiten (z. B. in Bezug auf Zeiten und Abläufe) festgelegt bzw. standardisiert werden können: „Also da kann man sich nicht so festlegen, also das - das wäre dann unmenschlich“ (PFK). Daher dienen Vorgaben für Arbeitsaufgaben und -abläufe als Rahmenplan der Orientierung, haben aber nicht die oberste Priorität: „Und also diesen Rahmenplan, sagen wir mal, der - den hat - haben wir relativ großzügig, ne? Also ich versuche, wenn ich da fünf Leute mit Einzelbetreuung morgens auf dem Plan stehen habe, dann versuche ich die fünf Leute also auch zu betreuen. Aber nicht so nach dem Zeitplan, ich bin jetzt um halb zehn bei dem und um viertel nach zehn bei dem, das mache ich nicht“ (ZBK); „Also so, dass man irgendwie einen Plan aufstellt und nach diesem Plan hat man dann da zu arbeiten. Ich finde, das funktioniert einfach nicht so“ (ZBK).

- Besondere, temporär vorhandene Bedarfe von Bewohner*innen werden berücksichtigt, auch wenn sie (noch) nicht den Zeitvorgaben entsprechen: „Wo wir wissen, dass er dann nachts sehr unruhig ist, dann gehen wir auch öfters hin, unabhängig davon, dass man diese festen Zeiten hat, wie man sagt, dieses drei Mal, dann geht man trotzdem hin, guckt dann leise um die Ecke, ob dann alles in Ordnung ist“" (PFK). Auch für die Mahlzeiten werden die Zeitkorridore großzügig gehalten, wenn es den Wünschen der Bewohner"innen entspricht: „Also letztlich bestimmt der Bewohner, wann er aufsteht oder wann er schlafen möchte. Letztlich bestimmt er wann er und wo er hier ist, weil es gibt verschiedenste Orte. Natürlich haben auch wir bestimmte Zeitkorridore für die verschiedenen Mahlzeiten, die aus dem Haus angeboten werden, aber wenn sie die zusammen, wenn sie zusammenpacken, dann können sie Tag und Nacht essen" (GP Int. 4).

- Vorgesetzte legitimieren und unterstützen Handlungsspielräume bei den Mitarbeitenden und auch die Mitarbeitenden untereinander tolerieren $\mathrm{Ab}-$ weichungen von Routinen und Vorgaben (z. B. zwischen den Schichten), wenn diese situativ erforderlich sind. Abweichungen werden nach Aussagen der Interviewpersonen dann akzeptiert, wenn Veränderun- 
gen etwa im Ablauf, im Zeitpunkt oder im Umfang noch innerhalb eines grundsätzlichen Rahmens erfolgen und das Arbeitsergebnis „stimmt": „Und es gibt halt eben auch Mitarbeiter, die hier noch ein Schwätzchen, und da wissen sie, der hat das noch gern, und gehen so auf persönliche Sachen [ein]. [...] Sie schaffen auch vieles dann eben anders" (HW). Allerdings kann es auch sein, dass besondere Situationen größere „Abweichungen“ verlangen, die auch akzeptiert werden sollten.

- In der Personaleinsatzplanung wird in arbeitsintensiven Zeiten mehr Personal eingesetzt: „Also ich habe im Schnitt von sieben bis elf das meiste, also von 7 Uhr morgens bis 11 Uhr vormittags habe ich im Schnitt das allermeiste Personal im Haus“ (EL).

\section{Hemmend:}

- Das Handeln der Mitarbeitenden wird - insbesondere in der Pflege mit unzureichenden Rahmenbedingungen in Bezug auf personelle Ressourcen verbunden, die den Arbeitsalltag oft zu dominieren scheinen. So werden immer wieder Personalmangel und Arbeitsdichte („Wegen dem Personalmangel, ja, das ist ja überall, ne? Aber das sagen sie dann. Dann sagen sie, ach ja, wir haben wieder - es ist viel zu tun und wir müssen jetzt - ja den anderen helfen“, ANG) sowie gestiegene Anforderungen durch veränderte Bewohner*innenvoraussetzungen als wesentliche Faktoren innerhalb der Arbeitsprozesse benannt, die Handlungsspielräume einschränken.

- Auch das Denken und Handeln innerbalb von "Schichten“" (Früh-, Spät-, Nachtdienst) kann die Spielräume im Handeln der Mitarbeitenden wesentlich einschränken, wenn primär die Arbeiten und Aufgaben aus der jeweiligen Schicht und nicht die aktuellen Bedarfe der Bewohner*innen im Vordergrund stehen: „[Dass in] einer Dienstschicht alle Bewohner ordnungsgemäß versorgt sind und am besten so, dass der Nachtdienst [...] halt alle Leute gut übergeben bekommt" (EL).

- Im Rahmen der trägerübergreifenden Fokusgruppendiskussion wird das "Schichtdenken“ ebenfalls thematisiert und zudem mit der individuellen Arbeitskultur und -einstellung sowie der Organisationskultur der Einrichtung in Verbindung gebracht: „Das hat aber auch wirklich was zu tun mit, ich sag das mal ganz brutal, ich muss meine Bewohner fertig machen. Das ist die Haltung, die dahintersteht. Wenn ich gehe und die Schicht nach mir kommt. ,Wieso muss ich den jetzt noch waschen - hast du das heute morgen nicht gepackt?‘ Dann muss ich da auch noch Arbeit wegmachen. Das ist eine Frage der Kultur, aber da setzt für 
mich auch die Frage der Führung ein, Führung und Leitung“ (EL/ PDL).

- Auch die Orientierung an Sicherheit ist ein besonderer Aspekt, der die Auswahl, aber auch die Durchführung von Handlungen bestimmt und Handlungsspielräume für die Teilhabeförderung nach dem Prinzip der Selbstbestimmung einschränken kann. Dies kann selbst dann Einfluss nehmen, wenn die eigentliche fachliche Leitorientierung „Hilfe zur Selbsthilfe“ lautet: „Eigentlich ist ja auch gerade jetzt bei Sozialpädagogik so ein Ansatz Hilfe zur Selbsthilfe“ (SD); „Wie ein Bewohner jetzt der will sich nicht waschen lassen, ne? Ja, das ist ein Recht auf Selbstbestimmung [...] Und irgendwann, dann schwindet das, ne, mit dem Verständnis dafür, ja? Und dann sieht man das anders, ne? Ich würde sagen, wenn da so [lachend:] drei oder vier Wochen ins Land gehen, ne, dann sieht man das dann schon - schon anders" (PDL). Der Konflikt zwischen Freiheit und Sicherheit - hier im Sinne des Konfliktes von Selbstbestimmung und Selbstgefährdung - kann also auch Handlungsspielräume auf Seiten der Mitarbeitenden einschränken.

- Als Einschränkung der Handlungsspielräume werden in dieser Hinsicht dann auch spezifische rechtliche Vorgaben und Vorschriften (z. B. Brandschutz, Hygienevorschriften) wahrgenommen: „Und wir müssen bei so was wie Backen, wie Waffeln und so [...] müssen wir extra aus dem Keller Hütchen nehmen und die Brand-, diesen Brandschutz abdecken" (ZBK); "Sobald das Eis in der Verpackung zu ist, ist es kein Thema, ne? Aber alles andere, [damit] müssen die hier scheinbar sehr vorsichtig sein. Oder mit Eiern, ne, da wird also kein frisches Ei genommen, sondern zum Backen, da muss das irgend so was - gibt es was anderes" (ANG).

\subsection{Besondere und zusätzliche Ressourcen}

In diesem Kapitel werden Ressourcen in stationären Altenpflegeeinrichtungen beschrieben, die eine Teilhabeförderung nach dem Prinzip der Selbstbestimmung besonders unterstützen und von den Interviewpersonen als wichtige Ressourcen hervorgehoben wurden. Auffällig ist, dass die meisten der benannten Ressourcen - direkt oder indirekt - personalen Ressourcen zuzuordnen sind. Insgesamt wurden zum Beispiel direkt zusätzliche Betreuungskräfte, Ehrenamtler*innen und Angehörige sowie indirekt zusätzliche finanzielle Mittel, Mobilitätsunterstützung und Verbindungen zum Quartier als Ressource benannt. Allein Räumlichkeiten und 
(technische) Mobilitätshilfen kommen hier primär als sachliche Ressource vor.

\subsubsection{Zusätzliche Betreuungskräfte}

Zusätzliche Betreuungskräfte (im Folgenden ZBK) nach $₫ 43$ b SGB XI stellen keine zusätzliche Ressource dar, da sie reguläre Mitarbeitende der Einrichtung sind (für die einbezogenen Einrichtungen siehe exemplarisch Kapitel 3.2.1.2). ZBK werden aber insgesamt mehrfach als unterstützend sowie entlastend und damit als besondere Ressource wahrgenommen - sowohl von Angehörigen als auch von Leitungskräften und Mitarbeiter*innen (und hierbei von der Pflege wie auch vom Sozialen Dienst). Sie werden aktuell auch deswegen als besondere Ressourcen wahrgenommen, weil einige Mitarbeitende noch die Zeit vor Einführung der ZBK kennen: „,Sie bedeuteten] auch eine Entlastung für uns, weil man natürlich die ganzen vielen Jahre vorher, wo wir alleine waren, auch immer gesehen hat, wo die Grenzen sind, ne? Wo - wo man weiß, jetzt hättest du eigentlich da noch mal hingehen müssen, aber irgendwann willst du auch mal nach Hause“ (SD). Auch Bode (2014) verbindet die ZBK mit hinzugekommenen Ressourcen, indem der Einsatz von ZBK einerseits „Zeit für die eigentliche Pflegearbeit freigesetzt" (Bode, 2014, S. 98) habe. Andererseits erhielten Bewohner*innen durch die ZBK vermehrte Zugänge zu Teilhabe, durch Teilnahme an Gruppenaktivitäten oder durch individuell geführte Gespräche (ebd.).

Grundsätzlich fällt in Bezug auf die ZBK auf, dass ihre Aufgabenbereiche mehrfach in Verbindung mit den gesetzlichen Vorgaben thematisiert und in Abgrenzung zu den Aufgaben der Fachkräfte des Sozialen Dienstes (zumeist mit einem Studienabschluss) betrachtet werden: „Aber alle Angebote, die sie unterbreiten, stehen unter dem Fokus oder dem - der Vorgabe, dass es niederschwellige Angebote sind. Und keinen oder kaum therapeutischen Charakter hat. So sieht es der Gesetzgeber. Mehr darf auch nicht sein, weil letztendlich der Rahmen ihrer Qualifikation zu knapp gehalten ist mit 160 Stunden" (EL). An anderer Stelle wird demgegenüber beklagt, dass die Vorgaben zu eng seien und es weitere Möglichkeiten der Teilhabeförderung gäbe, die von den Betreuungskräften aber nicht wahrgenommen werden dürfen: „Bei den Betreuungskräften: Die könnten - die könnten Teilhabe noch an einer Stelle viel mehr unterstützen, dürfen sie aber aus - aus der Politik heraus nicht. Sie wissen ja, da gibt es ja diese ganz klare Regelung, von wegen, was die machen dürfen, was die nicht - 
und sie dürfen auf keinen Fall in pflegerische - [...] Handlungen eingebunden werden" (EL).

Der primäre Aufgabenbereich der ZBK in den Einrichtungen ist die Einzelbetreuung. Hierzu zählen beispielsweise Einkaufsbegleitungen und Spaziergänge, die auf Anfrage und je nach zeitlichen Ressourcen von den ZBK übernommen werden ebenso wie etwa individuelle Angebote der Gesprächsführung, des Gedächtnistrainings, der basalen Stimulation, des Vorlesens etc. Des Weiteren erfolgt eine individuelle Betreuung von bettlägerigen Menschen durch die ZBK: „Ja, natürlich auch Einzelbetreuung, weil ja auch viele bettlägerig sind" (BW).

Ein weiterer wichtiger Aufgabenbereich der ZBK ist die Begleitung und Durchfübrung von Gruppenangeboten. Hierzu zählen zunächst die Ansprache und Motivation zu Angeboten, das Abholen zu und Zurückbringen von wohnbereichsübergreifenden Angeboten des Sozialen Dienstes. Ferner wirken sie auch als zusätzliche Begleitung in diesen Gruppenangeboten des Sozialen Dienstes mit, als Beispiel wurde hier die Assistenz bei einzelnen Bewohner*innen im Rahmen von Bingo genannt. Daneben führen die ZBK aber auch selbst Gruppenangebote durch, wobei es sich hierbei gemäß den gesetzlichen Vorgaben nur um kleinere Gruppen und „,niederschwellige Angebote“ (EL) handeln sollte. Zu den genannten Gruppen gehören dann aber ganz unterschiedliche Angebote wie beispielsweise Kegeln, Kochen und Backen sowie Spiel- und Bewegungsangebote etc. Im Rahmen dieser Angebote werden mehrere Personen gleichzeitig erreicht, was gerade auch von den ZBK hervorgehoben wird. Hier findet aus Zeitgründen inzwischen auch bei den ZBK eine „Bündelung“ von einzelnen Betreuungen statt.

ZBK werden auch bei der Begleitung von Mablzeiten eingesetzt (in Form einer Assistenz oder eines Trainings). Hierbei unterstützen sie besonders Mitarbeitende aus der Pflege und Hauswirtschaft. Es wird berichtet, dass durch ihre Unterstützung an den jeweiligen Tischen mehr Ruhe während der Mahlzeiten entsteht: „Ich glaube schon, dass viele aufgeatmet haben, weil die Betreuungsassistenten ja schon viel auch in den Mahlzeiten vorhanden sind, ne, und das auch entspannen, durch ein Mit-am-Tisch-Sitzen" (HW).

ZBK übernehmen zudem Begleitungen von Bewohner*innen außerhalb der Einrichtung und auch Fahrdienste und Kurierfahrten. So wird etwa beschrieben, dass sie Bewohner*innen bei Arztbesuchen begleiten. Im Zuge der trägerübergreifenden Fokusgruppendiskussion wird dies bestätigt und gerade auch die Organisation und Begleitung von Arztbesuchen als Zeitfresser in den Einrichtungen identifiziert. 
Die Vorgesetztenregelung und die Anbindung der zusätzlichen Betreuungskräfte an Wohnbereiche und/oder den Sozialen Dienst sind unterschiedlich geregelt und offerieren teilweise Klärungsbedarf. Für zwei Einrichtungen wird konkret benannt, dass ZBK explizit auf den Wohnbereichen bzw. -etagen eingesetzt werden: „Oder wir haben auch die Betreuungsassistenz hier im Haus auf jeder Etage. Wir sind ja dann eher für die Dementen $[\mathrm{da}]$ “ (PFK).

Mehrfach wird aber benannt, dass die ZBK-Stellen organisatorisch an den Sozialen Dienst angebunden sind bzw. durch die Leitung des Sozialen Dienstes koordiniert werden (sollten). Neben der organisatorischen Steuerung wird dabei in einzelnen Interviews aber auch die Notwendigkeit einer fachlichen Leitung und Kontrolle durch Fachkräfte des Sozialen Dienstes zum Ausdruck gebracht, auch wenn die Vorgesetztenfunktion rechtlich durch die Pflege(dienstleitung) erfolgt.

Um Bewohner*innen ausreichend im Alltag begleiten zu können, stellen Ehrenamtler"innen und Praktikant"innen wiederum eine wichtige Unterstützung für die ZBK dar, da ihr Tätigkeitspektrum bzw. -umfang offenbar mit der Zeit immer weiter gewachsen ist. So wird von den ZBK selbst beschrieben, dass sie auch unter Zeitmangel leiden: „Ja, auf jeden Fall der Begleitdienst durch Ehrenamtliche, weil wir es halt einfach auch nicht mehr stemmen können, ne?“ (ZBK).

Besonders eine Pflegedienstleitung betont ausdrucksstark, dass ein weiterer Ausbau von $Z B K$ die Pflege noch mehr entlasten und die Teilhabeförderung der Bewohner*innen verbessern könnte, wenn diese ausdrücklich und kontinuierlich im Wohnbereich für die soziale Betreuung der Bewohner*innen präsent wären und ausschließlich hierfür mehr Zeit zur Verfügung stünde.

Faktoren, die darüber hinaus als teilhabeförderlich und -hemmend zu den ZBK als besondere Ressource benannt wurden, werden im Folgenden beschrieben.

\section{Fördernd:}

- Einzelbetreuung kann in dem derzeit bestehenden Umfang nur durch das Vorhandensein von ZBK angeboten werden. Dabei können sie für die Umsetzung von individuellen Wünschen eingesetzt werden, was als förderlich angesehen wird, da dadurch auch bettlägerige Menschen erreicht werden.

- Durch die ZBK kann gerade auch zu Randzeiten ein Einzelangebot innerhalb oder außerhalb der Einrichtung stattfinden, was in diesen sonst weniger durch Angebote belegten Zeiten teilhabeförderlich sein kann. 
- Die Anwesenheit von ZBK bei Mablzeiten ist unterschiedlich geregelt, wird aber vielfach als förderlich für den Teilhabeaspekt der Mahlzeiteneinnahme (soziale Situation des Essens) wahrgenommen.

- Es wird beschrieben, dass die ZBK eine besondere Nähe zu den Bewohner*innen haben, gerade auch wenn sie in den Wohnbereichen vor Ort präsent sind bzw. eingesetzt werden und so mit einem größeren Verständnis auf individuelle Wünsche der Bewohner“innen eingehen können.

\section{Hemmend:}

- Die Möglichkeiten zur Teilhabeförderung durch die ZBK werden im besonderen Maße als personenabhängig beschrieben, auch weil ihre Qualifikation im Rahmen ihrer Ausbildung als zu gering erachtet wird.

- Um individuelle Teilhabe in Zukunft noch besser gestalten zu können, wäre es wünschenswert, wenn über einen weiteren Ausbau der ZBKStellen nachgedacht wird.

- ZBK übernehmen Tätigkeiten, die nicht in ibren Aufgabenbereich fallen (sollten). Dadurch fehlt Zeit für die einzelnen Bewohner*innen und ihre Teilhabeförderung.

\subsubsection{Ehrenamtler*innen}

Ehrenamtler*innen werden neben den Professionellen und den Familienangehörigen als die sogenannte dritte Säule in der Betreuung von älteren pflegebedürftigen Menschen gesehen. „Diese Hilfsleistungen sind unverzichtbar, wenn es um die Betreuung jener Zielgruppe von Hilfsbedürftigen und dort besonders von demenzkranken Menschen geht" (Fischbach \& Veer, 2008, S. 235). Durch die drei Säulen soll, so das Bundesministerium für Familien, Senioren, Frauen und Jugend (2005), die Betreuung für Menschen mit Hilfebedarf verbessert und ihre Lebensqualität gesteigert werden (BMFSFJ, 2005, S.349). Auch in $\$ 5$ Abs. 1 WTG NRW wird die Einbeziehung von bürgerschaftlich Engagierten ausdrücklich genannt, um die Teilhabe der Nutzer*innen am Leben in der Gesellschaft zu sichern. Die Relevanz von ehrenamtlichem Engagement in der Altenhilfe scheint unbestritten, gleichwohl ist aus professionspolitischer Perspektive stets auch kritisch zu hinterfragen, wo die Grenzen zwischen hauptamtlichen und ehrenamtlichen Aufgaben und Tätigkeiten liegen, was in den Interviews ebenfalls zum Ausdruck kommt. 
So finden sich in den Interviews einige Hinweise auf die große Bedeutung von Ehrenamtler*innen für die - insbesondere individuelle - Teilhabeförderung, aber auch auf die Notwendigkeit ihrer Anbindung an die hauptamtlichen Kräfte. Die Koordination der Ehrenamtler"innen erfolgt in den Altenpflegeeinrichtungen über den Sozialen Dienst. Es wird zwischen den „aktiven“ (EL) oder „regelmäßigen“ (EL) Ehrenamtler"innen und denen, die nur „monatlich“ (EL) oder bei Festen und Feierlichkeiten anwesend sind, unterschieden: „Und das haben wir aufgebaut. Wir haben insgesamt zurzeit um die 30, die wirklich ganz aktiv täglich, wöchentlich oder monatlich ein Ehrenamt bekleiden [...]“ (EL).

Es wird beschrieben, dass das Ehrenamt nicht ausgenutzt werden sollte und Ehrenamtler"innen nicht als kostenlose Mitarbeiter"innen betrachtet werden dürften. Eine Gefahr sei es, dass durch Ehrenamtliche die Stellen von Hauptamtlichen eingespart werden könnten und es daher wichtig sei, Ehrenamtler*innen stets als zusätzliche Ressource zu betrachten: „Immer mit der Zielsetzung, niemals irgendeinem Mitarbeiter eine Stelle wegzunehmen, die man darüber einsparen kann. Es gibt ja auch Häuser, die geben sogar einem Ehrenamtler eine Gruppenleitung für ein Beschäftigungsangebot, das kann und darf nicht Sinn und Zweck von Ehrenamt sein. Ehrenamt soll das Haus bereichern, soll das - soll den Bewohner unterstützen, soll das Haus beleben, soll dem Bewohner Teilhabe ermöglichen. Und wenn der Ehrenamtler dann dafür sorgt zum Beispiel, auch regelmäßig Fahrten zum Friedhof zu ermöglichen, den versicherungstechnischen und finanziellen Rahmen aber von uns bekommt, dann ist das eine tolle Sache, die wir so eigentlich nicht leisten könnten. Die auch nicht refinanziert werden" (EL). Auch in der Literatur wird das Verhältnis von Haupt- und Ehrenamt durchaus kritisch beleuchtet und davor gewarnt, dass das freiwillige Engagement auch als Kostensenkung herangezogen wird, um hauptamtliche Mitarbeitende zu ersetzen. Darüber hinaus werden, gerade in Bezug auf die Betreuung von Menschen mit Demenz, Gefahren der Unfachlichkeit (falsche Balance zwischen Nähe und Distanz) durch Ehrenamtler*innen kritisch angeführt (Fischbach \& Veer, 2008, S. 236).

Ehrenamtler"innen werden nach den Schilderungen der Interviewpersonen in den Projekteinrichtungen aber primär für die individuelle Betreuung und Unterstützung von Bewohner*innen eingesetzt. Darüber hinaus werden sie als ergänzende Unterstützung in Gruppenangeboten innerhalb und außerhalb der Einrichtung (z. B. bei Ausflügen) genutzt.

Die meisten Ehrenamtler"innen haben selbst bereits das Rentenalter erreicht und sind mehrheitlich weiblich. Das Ehrenamt wird teilweise auch von Angehörigen mit übernommen, manchmal wird dieses nach dem Tod 
der Eltern weiter fortgesetzt (Kapitel 4.4.3). Fischbach und Veer (2008) sprechen bei diesen Ehrenamtlichen von den „dankbaren früheren Angehörigen". Diese engagieren sich aus einer gewissen Verbundenheit mit der Einrichtung aufgrund der guten Pflege der Familienangehörigen und „der Begleitung der eigenen Person“ (Fischbach \& Veer, 2008, S. 247).

Im Folgenden werden die als förderlich und hemmend beschriebenen Faktoren in Bezug auf das Ehrenamt wiedergegeben.

\section{Fördernd:}

- Ehrenamtler*innen werden als Unterstützung angesehen - sie stützen die „dünne Personaldecke“ (ANG) und ermöglichen die Teilhabe für Bewohner*innen: „Ja, wie gesagt, wir haben ja viele Ehrenamtliche auch, die - die sind auch immer parat, also das finde ich faszinierend. Wenn irgendwas ist, sind sie alle da und das ist einfach toll, ne?“ (ZBK).

- Ehrenamtler*innen unterstützen die Hauptamtler*innen bei verschiedenen teilhabefördernde Gruppenangeboten und Veranstaltungen innerhalb und außerhalb der Einrichtung.

- Sie werden oft eingesetzt, um individuelle Wünsche von Bewohner*innen zu erfüllen und werden hierbei gerade in Bezug auf die Einzelbetreuungen als „Joker“ wahrgenommen.

\section{Hemmend:}

- Die Rekrutierung von Ehrenamtler*innen fällt den Einrichtungen schwer und teils wird eine Abnahme des Interesses am Ehrenamt benannt, so dass die individuelle Betreuung durch Ehrenamtliche nicht immer sichergestellt werden kann.

- Ehrenamtler"innen sind selbst im Rentenalter sowie meist weiblich und können daher körperlich anstrengende Tätigkeiten (z. B. Rollstuhlschieben) oft nur eingeschränkt leisten.

\subsubsection{Angehörige}

Angehörige $^{72}$ der Bewohner*innen spielen im Alltag von Altenpflegeeinrichtungen eine wichtige Rolle und sind eine besondere Ressource für die Förderung und Ermöglichung von Teilhabe, denn einerseits haben sie einen biografischen Bezug zu den ihnen nahestehenden Bewohner*innen und verfügen daher über spezifische persönliche Kenntnisse und emotio-

72 Angehörige sind hier auch Zugehörige oder entfernte Verwandte. 
nale Zugänge zu den Bewohner*innen. Andererseits unterstützen sie die Arbeit der Mitarbeitenden in verschiedenen Aufgaben, die sie mit den oder für die Bewohner*innen übernehmen. Auch im WTG NRW $(\mathbb{} 5$ Abs. 1) werden Angehörige als wichtige Ressource zur Ermöglichung der selbstbestimmten Teilhabe genannt. In den Interviews finden sich sowohl Hinweise auf teilhabeförderliche als auch teilhabehemmende Faktoren.

\section{Fördernd:}

- Mit Angehörigen werden von den Mitarbeitenden der Pflege und des Sozialen Dienstes Gespräche geführt, um Informationen über die Biografie der Bewohner*innen (und somit teilhaberelevante Informationen) zu erhalten - gerade dann, wenn sich die Bewohner*innen nicht mehr äußern können, welche Interessen und Vorlieben sie haben (z. B. im Rahmen von Einzugs- und Biografiegesprächen). Das Bewohner*innenwissen, das Angehörige haben, ist damit ein wichtiger ergänzender Zugang zu biografischen Hintergründen der Bewohner*innen: „Wir brauchen die Angehörigen. Und die Angehörigen wissen Dinge, die wir nicht wissen" (PFK).

- Angehörige haben darüber hinaus oft eine intensivere emotionale Bindung zu den verwandten Bewohner*innen, die einerseits an sich das Erleben einer besonderen sozialen Beziehung ausmacht und andererseits in eventuell schwierigen Situationen vermittelnd oder motivierend wirken kann: „Wir kommen nicht an den Angehörigen vorbei. Die haben eine ganz andere Beziehung zu denen. Die gehen auch über - von ihren - von ihren Emotionen ganz anders an die Bewohner ran" (PFK). „Die brauchte wirklich den letzten Kick von ihrer Tochter, die gesagt hat: ,Mama, du hast doch früher immer gemalt.' Und sie [hatte] sich, glaube ich, am Anfang gar nicht zu[ge]traut[e], dass sie wieder malen kann $[\ldots]^{\text {“ }}(\mathrm{PFK})$.

- Angehörige übernehmen spezifische Aufgaben in der individuellen Begleitung und Unterstützung der Bewohner*innen, indem sie beispielsweise Taxifahrten planen, Arzttermine begleiten oder auch Friseurbesuche organisieren: „Das regeln wir dann so, ja. Ansonsten hole ich sie - ja, die Arztbesuche mache ich mit ihr. Da hole ich sie ab. Und was ihr wichtig ist, $[. .$.$] zum Friseur zu fahren" (ANG).$

- Darüber hinaus unternehmen Angehörige mit den Bewohner*innen oftmals freizeitbezogene Ausflüge außerhalb der Einrichtung, wobei das Engagement von Angehörigen unterschiedlich intensiv ist: „Ne? Also wir haben ja eine Gruppe von - von Bewohnern, die auch von Angehörigen noch abgeholt wird, ne? Die also so mobil sind, dass sich das An- 
gehörige zutrauen und das auch machen und dann mit ihren Eltern etwas unternehmen. Da ist allerdings das Engagement von Kindern ja auch unterschiedlich, aus welchen Gründen auch immer, ne?“ (PDL).

- In den Begleitungen ihrer verwandten Bewohner*innen integrieren manche Angehörige auch weitere Bewohner"innen und fördern ihre Teilhabe, indem sie sich etwa mit mehreren Bewohner*innen unterhalten, etwas Vorlesen oder auch einen Spaziergang unternehmen: „Wir haben sehr viele Angehörige, die sich sehr um ihre eigenen Angehörigen kümmern, die aber auch andere mitnehmen." (HW)

- Viele Angehörige unternehmen nicht nur Einzelbegleitungen mit ihren Verwandten, sondern unterstützen auch den Sozialen Dienst bei Gruppenangeboten und -aktivitäten innerhalb und außerhalb des Hauses: „Ressourcen über Angehörige, zum Teil. Wie gesagt, ich denke jetzt gerade an diese Singrunde, wo dann die Angehörigen da sich um diese Mappen gekümmert haben, die auch ganz toll gemacht" (SD). „Hatte mich auch angeboten, wie gesagt, bei Ausflügen mitzufahren“ (ANG).

- Es gibt Hinweise über verschiedene Arten von Spenden, die von Angehörigen übernommen werden. So wird etwa von monetären Spenden und von Sachspenden aus dem Kreis der Angehörigen berichtet. Mit monetären Spenden könnten beispielsweise Ausflüge oder besondere Veranstaltungen finanziert werden - dies trägt somit auch unmittelbar zur Finanzierung von Angeboten der Teilhabeförderung bei. In einzelnen Einrichtungen sind auch Sachspenden in Zusammenhang mit besonderen Veranstaltungen und Aktivitäten möglich; es ist etwa üblich, dass zu Feierlichkeiten Kuchen und Salate gespendet werden.

- Verschiedene Mitarbeiter"innen beschreiben, dass sich gerade Angehörige als ehrenamtliche Kräfte beteiligen - auch, wenn ihre Verwandten bereits verstorben sind, bleiben sie in der Einrichtung aktiv: „Ehrenamt sind aber ganz oft Angehörige, das muss ich auch sagen. Also Angehörige spielen ganz, ganz oft eine große Rolle“ (EL). Durch das ehrenamtliche Engagement kann Teilhabe unterstützt werden (Kapitel 4.4.2).

- Angehörige stellen schließlich auch dann eine Ressource dar, wenn sie als Gäste an Veranstaltungen im Haus teilnehmen, wenn sie Interesse am Leben in der Einrichtung zeigen und auch einfach als Besucher"innen daran teilhaben: „Also für mich ist eine Ressource auch, wenn ich zum Beispiel ein Konzert mache zum Mitmachen, Mitmachkonzert, und sie bitte zu kommen, weil ich weiß, die Bewohner finden das ganz toll, ne, und die helfen dann auch mit. Manche wollen auch nur genießen. Aber es ist auch eine Art Ressource, wenn man die Ressource mal so 
ein bisschen weiter sieht. Ne? Die alle ins Boot zu nehmen, das ist schon auch eine Ressource“ (SD).

\section{Hemmend:}

- Hemmend für die Teilhabeförderung kann sein, wenn Angehörige zwar Informationen über Vorlieben und Interessen der mit ihnen verwandten Bewohner*innen weitergeben, diese aber nicht mehr aktuell sind: „Da ist auch ein bisschen halt [die] Biografie überbewertet, ne? Ich mache jetzt mal ein ganz krasses Beispiel. Wenn jemand früher unheimlich gerne Motorrad gefahren ist, ja, und ganz, ganz viel - das ist er vielleicht in den letzten zehn Jahren zu Hause, wo er in Rente ist, wo vielleicht auch die - eigentlich Augenlicht und so was nachgelassen hat, da gar nicht mehr so gerne gemacht, ja? Dafür hat er vielleicht eine andere Kompensation gefunden" (EL).

- Spezifische Ansichten und Ansprüche von Angehörigen in Bezug auf das Leben ihrer verwandten Bewohner*innen, die sich von jenen der Bewohner*innen und Mitarbeitenden unterscheiden, werden aber auch als teilhabehemmend bzw. Einschränkungen der Teilhabeförderung durch die Mitarbeitenden beschrieben: „Die sich in allem einmischen, immer was zu kritisieren haben. Da stehen also erst mal die Angehörigen. Wieso läuft meine Mutter alleine zu Edeka rüber?“ (PDL); „Der Wunsch der Angehörigen ist, dass die Frau in ihrem Zimmer alleine isst, wobei die Frau oft sagt: ,Warum muss ich denn auf mein Zimmer? Warum darf ich denn nicht hier mit essen?““ (EL).

- Hemmend für die Teilhabeförderung kann dann auch sein, wenn weniger die Wünsche der Bewohner*innen als die Wünsche der Angehörigen im Mittelpunkt stehen: „Ich finde, als Einrichtungsleitung ist es eher, dass man die Angehörigen zufrieden stellt als die Bewohner“ (EL).

- Angehörige werden im Einzelfall nicht als Ressource wahrgenommen, weil sie sich nicht engagieren bzw. zu wenig präsent sind. Als Gründe nennen die Mitarbeitenden vor allem das Alter, die Wohnortdistanz und das berufliche Eingebundensein: „Ich sage jetzt mal, die faulen Angehörigen. Aber auf der anderen Seite, Angehörige sind vielleicht dann auch schon 70 Jahre oder um den Dreh, oder drüber, haben ihre eigenen Gebrechen oder [stehen] so wie ich am Ende ihrer Berufstätigkeit. Da guckst du auch schon hier, wo kannst du mal eine kleine Pause einlegen? Da sind die auch satt, ne? Die sind einfach schon selbst sehr erschöpft. Und denken, okay, jetzt haben wir das im Pflegeheim [...] entschieden, wir waren die letzten zehn Jahre schon zu Hause da, ne? Wir haben uns dafür jetzt entschieden, ich bringe dir ein Blümchen zu 
Muttertag und guten Tag und guten Weg, ne?“ (PFK). Aber auch aus der Angehörigensicht wird eingebracht bzw. bedauert, dass man beruflich zu sehr eingespannt sei und daher leider keine Zeit finde, sich in der Einrichtung zu engagieren: „Weil ich beruflich zu sehr eingespannt und eingebunden bin [...] und deshalb habe ich da gar nicht die Zeit für" (ANG).

\subsubsection{Zusätzliche finanzielle Mittel}

Auch zusätzliche finanzielle Mittel der Einrichtungen, welche die Regelfinanzierung ergänzen, fanden im Rahmen der Interviews als relevante Ressourcen Erwähnung. Zum einen werden finanzielle Ressourcen benannt, die die Einrichtung betreffen und sich auf zusätzliche Mittel für Personal (z. B. Projektstellen) und - noch häufiger - auf Mittel für besondere Aktivitäten und Sachmittel beziehen. Zum anderen werden finanzielle Ressourcen erwähnt, die individuell für Bewohner*innen eingesetzt werden, wenn ihnen finanzielle Mittel für Teilhabeangebote fehlen. Es lassen sich einzelne fördernde und hemmende Faktoren im Rahmen der Interviews identifizieren, welche sich auf zusätzliche finanzielle Ressourcen beziehen.

\section{Fördernd:}

- Aus allen vier Projekteinrichtungen gibt es Hinweise, dass Spenden, etwa über Sponsoring, Stiftungen oder (eigene) Fördervereine („Wir haben einen eigenen Förderverein, der schon sehr aktiv ist. Und der uns schon einfach Sachen ermöglicht.", EL), vorhanden sind und genutzt werden, um besondere Teilhabeangebote oder hierfür erforderliche Voraussetzungen zu finanzieren.

- Über Spenden - ausgewählt oder ergänzend -werden auf Einrichtungsebene zum Beispiel finanziert:

- Gebrauchsgegenstände und Ausstattung: „Wir haben oben zum Beispiel eine fahrbare Küche, die man also auch durch die Zimmer fahren kann, um da mal was zu machen und ja, die ist über - auch über einen Fonds irgendwie gestiftet worden“ (ZBK); „Viele Busse, ich sage mal so, verteilt worden an - an Altenheime als Spende“ (PDL); „Ja, wir haben jetzt vor kurzem eine [Nintendo] Wii bekommen als Spende“ (HT).

- Ausflüge: „und an Spenden ist auch manchmal - jetzt wie wir wieder - wo Anfang des Jahres wieder so viel Spenden, da können wir 
die Ausflüge auch finanzieren. Das kostet ja letztendlich auch. So haben sie das dann finanziert" (ZBK).

- Urlaubsfabrten: „Thema Urlaub, Urlaubsreise [...] Und es ist auch eine sehr kostenintensive Angelegenheit. Da müssen wir noch einen Sponsor finden" (EL).

- Spenden werden darüber hinaus auch auf Bewohner"innenebene genutzt, um einzelnen Bewohner"innen, die nicht über die finanziellen Mittel verfügen, die Teilnahme an besonderen Aktivitäten zu ermöglichen (z. B. über die Finanzierung von Eintrittsgeldern): „Aber die Eintrittskarten und so was" (BW).

- Im Rahmen der Interviews mit den „Good-Practice"-Einrichtungen wurden zudem Projektmittel benannt, die dann auch für zusätzliches Personal in befristeten Projekten genutzt werden können: „Auch immer wieder neue Projektanträge zu bekommen und dadurch auch personelle Ressourcen refinanziert zu bekommen und auch immer weiter innovativ zu denken und das hört auch nicht auf“ (GP Int. 1); „[auf] zwei Jahre, das war eine Projektstelle“ (GP Int. 3).

- Betont wird, dass das gesamte zur Verfügung stehende Budget ausgenutzt werde, um Teilhabe für die Bewohner*innen zu ermöglichen („Wir schöpfen unsere finanziellen Ressourcen komplett aus“, EL). Dazu sei aber eine Jahresplanung notwendig, um alle Kosten im Vorfeld absehen zu können. Diese Jahresplanung wird von den Sozialen Diensten als koordinierende Stelle für die sozialen Angebote übernommen, auch wenn die Einrichtungsleitungen ein „Letztentscheidungsrecht" über die Finanzen haben: „Genau, also diese Organisation, diese Vorabplanung, ein Jahr vorher oder im Herbst des Jahres vorher, plane ich mit diesen ganzen auch Honorarkräften, die dahinter stehen teilweise. Mit der Berücksichtigung des Budgets, natürlich hat da die Hausleitung den Hauptblick drauf, aber so grob weiß ich ja auch, was wer bekommt" (SD).

- Als förderlich wird zudem benannt, wenn eine Personalstelle - etwa auf Ebene des Trägers und nicht nur auf Ebene der Einrichtung - für die Akquise von Spenden und Projektmitteln zuständig ist: „Es gibt auch eine Stelle, $[\ldots]$ wer sich um - um Spendengelder kümmert“ (PFK).

\section{Hemmend:}

- Prinzipiell wird mehrfach erwähnt, dass die finanzielle Situation in Altenpflegeeinrichtungen - insbesondere mit Bezug auf die personellen Ressourcen - grundsätzlich unzureichend ist: „Finanzielle Mittel, finanzi- 
ell ist immer alles zu wenig im Altenheim. Das ganze Gesundheitswesen ist aus meiner Sicht unterbezahlt" (PDL).

- Als spezifischer hemmender Faktor in Bezug auf zusätzliche finanzielle Mittel wird konkret aber nur der zusätzliche administrative Aufwand für ihre Akquise und Verwaltung benannt: „Trotzdem ist da auch irgendwie sehr viel Sachbearbeitung mit verbunden, und Mails schreiben und dergleichen" (EL).

\subsubsection{Mobilitätshilfen und -unterstützung}

Mobilitätshilfen und eine personelle Unterstützung der Mobilität von Menschen mit Handicaps stellen zentrale Ressourcen für die Ermöglichung von selbstbestimmter Teilhabe dar, was nicht nur für Bewohner*innen in Altenpflegeeinrichtungen gilt: „Lediglich in eingeschränktem Maße mobil sein zu können oder gänzlich auf Mobilität verzichten zu müssen, grenzt daher nicht nur die allgemeine Partizipations-, sondern auch die Möglichkeiten gesteigerter Lebensqualität im Alter merklich ein" (Böhmer, 2015, S. 132). Die Mobilität in den Altenpflegeeinrichtungen wird nach Aussagen der Interviewpersonen - vor allem über personelle Ressourcen hergestellt, die in Begleitungen durch Mitarbeitende (v. a. Pfleger*innen, Sozialdienstmitarbeiter*innen und ZBK) sowie Helfer"innen (Ehrenamtler*innen, Praktikant"innen) liegen. Darüber hinaus werden auch sachliche Ressourcen benannt, die als Mobilitätshilfen für die Ermöglichung von Teilhabe eingesetzt werden (z. B. Fahrzeuge).

\section{Mobilität innerhalb der Einrichtung}

Als Anlässe zur individuellen Mobilität innerhalb der Einrichtung für Bewohner*innen, die auf Mobilitätsunterstützung angewiesen sind, werden die Mablzeiten, Veranstaltungen und Angebote im Haus benannt. Die Mobilitätsunterstützung bezieht sich hier auf die Bewohner*innen, die sich aufgrund von physischen und/oder psychischen Einschränkungen nicht selbstständig zu diesen Aktivitäten bewegen können. Diese personelle Unterstützung ist innerhalb der Einrichtung täglich mit einem nicht unerheblichen Aufwand verbunden, aber inzwischen vor allem auch durch Mitwirkung der zusätzlichen Betreuungskräfte regulär gewährleistet. Bei den Angeboten des Sozialen Dienstes ist die Schnittstelle zwischen Pflege und Sozialem Dienst von Bedeutung und mit hier wesentlichen fördernden sowie 
hemmenden Faktoren verbunden (Kapitel 4.3.3.3), damit - nach Prozessen der Pflege (z. B. Toilettengang) - eine rechtzeitige Mobilitätsunterstützung vor den Angeboten bzw. ein rechtzeitiger Transfer zu den Angeboten gewährleistet werden kann. Für die anderen Bewohner*innen, die sich selbstständig bewegen, sind in diesem Zusammenhang vor allem Mobilitätshilfen wie Gehstöcke, Rollatoren und Rollstühle von Bedeutung.

\section{Mobilität außerhalb der Einrichtung}

Einzelne Bewohner*innen, die mobil und „orientiert“ sind, bewegen sich auch selbstständig außerhalb der Einrichtung (Kapitel 4.2.1 und 4.3.1). Hier dienen wiederum Hilfsmittel wie Rollatoren und Rollstühle der Mobilitätsunterstützung, wobei hier auch Elektrorollstühle eine besondere Rolle einnehmen: „Elektrorollstuhl: Spielt hier aber dann wieder eine ganz große Rolle. Das gibt es schon Bewohner, die sich dann eigenständig bewegen“ (EL). Auch nutzen einzelne Bewohner*innen den öffentlichen Nahverkehr, sofern sie selbstständig in die Umgebung fahren möchten (und sich die dadurch entstehenden finanziellen Kosten leisten können).

Für Einzelausflüge von Bewohner*innen werden auch Taxifahrten genutzt, die insbesondere bei Kosten für ein behindertengerecht ausgestattetes Taxi als hoch eingeschätzt werden: „Und Taxifahrten für Behinderte sind sehr teuer, also wenn man jetzt so ein Behindertentaxi bestellt" (PDL). Aber auch insgesamt ist zu erkennen, dass Einzelausflüge, die nicht durch ein Angebot der Einrichtung berücksichtigt werden, oft nur über die Eigenmittel von Bewohner*innen finanzierbar sind: „Vielfach wird das ja dann hier mit dem Taxi geregelt. Die, die sich das finanziell dann leisten können" (HW).

Drei der vier Projekteinrichtungen verfügen über ein eigenes Fabrzeug (z. B. Kleinbus), um damit auch die Mobilität der Bewohner*innen, etwa im Rahmen von Ausflügen, zu gewährleisten. Die Anschaffung der einrichtungseigenen Fahrzeuge wird oft über Spenden (mit)finanziert. Für besondere Ausflüge wird auch schon mal ein Reisebus gemietet: „[...] einen Reisebus, in den tatsächlich alle Rollstühle [der Personen], die dann mitmöchten, auch rein passen. Weil Ausflüge mit Rollstuhlfahrern [sind] immer unheimlich schwierig“ (EL).

Die Unterstützung der Mobilität außerhalb der Einrichtung ist in den Einrichtungen unterschiedlich organisiert. Während Fahrzeuge in beinahe jeder Einrichtung vorhanden sind, so variiert die Organisation der Begleitdienste, gleichwohl Begleitdienste als bedeutsamer teilhabefördernder Fak- 
tor beschrieben werden: „Also oberstes Thema [sind] eigentlich Begleitdienste, um überhaupt mal außerhalb des Altenheims am Leben teilnehmen zu können" (ZBK). Hol- und Bringdienste werden selten zentral gesteuert, sondern scheinen dem jeweiligen Arbeitsprozess zugehörig: „Es gibt aber keinen Hol- und Bringdienst, wo wir Termine machen, die muss jetzt da in die Kirche, da musst du die abholen, du musst die abholen, so organisiert ist es nicht. Aber wenn im Pflegeplan steht, Bewohner Frau XY geht sonntags in die Kirche, muss hingebracht werden, dann reicht das. Dann ist das der Auftrag an - an - an die Pflege, ja?“ (PDL).

\section{Fördernd:}

- Als förderlich (hinsichtlich der Teilhabe außerhalb der Einrichtung) wird gesehen, wenn der Einsatz von Fahr-, Hol- und Bringdiensten zur Begleitung von Bewohner*innen außerhalb der Einrichtung von einer Funktionsstelle (hier z. B. dem Sozialen Dienst) koordiniert wird.

- Gleichermaßen ist fördernd, wenn überhaupt hinreichend personale Ressourcen zur Begleitung in der Mobilität außerhalb des Hauses vorhanden sind - Erwähnung finden hier insbesondere zusätzliche Betreuungskräfte, Ehrenamtler*innen und Maßnahmeteilnehmer*innen.

- Vereinzelt wird davon berichtet, dass die jeweilige Kommune die Finanzierung von Taxifahrten in einem begrenzten Umfang übernimmt: „Scheinbar ist da der Kreis recht großzügig. Die können dann so zwei, drei, manchmal auch vier Taxifahrten im Monat kostenlos haben" (PDL); „Das gibt es ja, glaube ich, in anderen Städten nicht, (hm-hm) dass diese Fahrmarken ermöglichen, dass man wirklich auch teilhaben kann an Sachen außerhalb“ (SD).

\section{Hemmend:}

- Regulär installierte Fahr-, Hol- und Bringdienste für die Unterstützung der Mobilität von Bewohnern*innen außerhalb der Einrichtung fehlen. Zwar ist dies nach SGB IX und nach dem Rahmenvertrag nach $\$ 75$ SGB XI bezogen auf Fahrdienste außerhalb der Einrichtung nicht geregelt, d. h., dies ist keine Aufgabe der Einrichtung. Dennoch könnten regulär installierte Fahrdienste die selbstbestimmte Teilhabe unterstützen und wären daher aus der Teilhabeperspektive förderlich.

- Ausflüge von Bewohner*innen, die nicht im Rahmen eines Angebotes der Einrichtung erfolgen, können nur über die Eigenmittel der Bewohner*innen finanziert werden. Die finanziellen Ressourcen von vielen Bewohner*innen sind aber aufgrund der hohen Kosten in einem Altenpflegeheim bereits gering. Die zusätzliche finanzielle Belastung kann 
dazu führen, dass individuelle Einzelausflüge aus diesem Grund nicht stattfinden.

- Die Anzabl der Rollstüble, die in einrichtungseigenen Fahrzeugen transportiert werden können, ist häufig geringer, als die Nachfrage bei Ausflügen.

- Die Nutzung der Einrichtungsfahrzeuge, so eine Interviewperson, ist gekoppelt an einen Personenbeförderungsschein, wenn Fahrten geschäftsmäßig ${ }^{73}$ durchgeführt werden. Wenn dieser nicht vorliegt, darf das Fahrzeug in diesen Fällen nicht von Personen gefahren werden, die auch individuelle Begleitungen ermöglichen könnten (z. B. von Ehrenamtlichen, freiwilligen Diensten, Praktikant*innen).

\subsubsection{Räume}

Räume sind insofern wichtige Ressourcen für die Teilhabeförderung, als dass sie das Setting der Alltags- und Angebotsgestaltung innerhalb der Einrichtung ausmachen und mitbestimmen. In den Interviews haben sich die Aussagen zu Räumen sowohl auf Einzel- als auch auf Gruppenangeboten bezogen.

Einzelangebote innerhalb einer Einrichtung können an unterschiedlichen Orten in einer Einrichtung stattfinden (z. B. im Bewohner*innenzimmer, Aufenthaltsraum, in Multifunktionsräumen). Hierzu wird oft der Wunsch beschrieben, einen Ort zu haben, an dem man mit den Bewohner*innen auch ungestört sein kann. Gruppenangebote finden oft in Aufenthaltsräumen in den Wohnbereichen oder in zentralen Räumlichkeiten statt, die für größere Gruppen ausgelegt sind. Bei Letzteren handelt es sich oft um Multifunktionsräume, die beispielsweise mit mobilen Möbeln und Trennwenden vielseitig genutzt werden. Zwei Einrichtungen verfügen über sogenannte Gymnastikräume, welche aber auch für andere Angebote genutzt werden.

\section{Fördernd:}

- Trotz räumlichen Mangels wird versucht, kreativ mit der Situation umzugehen. Es wurden mobile Möbel und Trennwände angeschafft, um An-

73 Geschäftsmäßig bedeutet in diesem Zusammenhang nicht unbedingt entgeltlich, aber regelmäßig und auf die Aufgaben der Einrichtung bezogen. Wenn jedoch ein/e Ehrenamtler*in eine/n Bewohner*in in seinem PKW mitnimmt, braucht er dafür keinen Personenbeförderungsschein. 
gebote vielseitig gestalten zu können. Somit wird der Raummangel kompensiert.

\section{Hemmend:}

- Die Mehrzahl der Mitarbeitenden gibt im Rahmen der Interviews an, dass die räumlichen Ressourcen zu gering sind. Gerade durch die - mit den zusätzlichen Betreuungskräften - gewachsene Anzahl der Mitarbeitenden in der sozialen Betreuung sei es eine Herausforderung, für die breite Anzahl von Einzel- oder Gruppenangeboten angemessene Räumlichkeiten zu finden. Die Raumbelegung ist dadurch teils schwer zu gestalten. Eins-zu-eins-Betreuungen werden v. a. in den jeweiligen Bewohner*innenzimmern durchgeführt, obwohl hier häufig der Wunsch genannt wird, Einzelbetreuungen in anderen Räumen anbieten zu können: „Wäre schön, wenn da noch einer da wäre, wo sich vielleicht auch ein Betreuungsassistent mal mit jemandem einzeln zurückziehen kann" (HW).

- In einer Einrichtung wird beklagt, dass zu viele kleine Räumlichkeiten vorhanden sind, so dass größere Gruppenangebote schlecht realisiert werden können: „Weil also das, was uns manchmal mangelt an geschlossenen Räumen für Großgruppen, haben wir ja ganz, ganz viel für kleine [Gruppen]. Überall steht ein Tisch mit ein paar Stühlchen“ (EL).

- Da mehrheitlich Multifunktionsräume genutzt werden müssen, besteht ein hoher zeitlicher Aufwand durch das anschließende Aufräumen und Umgestalten der Räume: „Also zum einen ist es natürlich - viel Arbeit entsteht dadurch, dass wir die Räume immer für verschiedene Anlässe wieder umgestalten müssen. Dieser Pavillon, den man abtrennen kann, für einen Filmnachmittag,[da] muss ich die ganzen Tische wegräumen, nachher wieder alle hinräumen" (SD). Diese Zeit, die in das Auf- und Umräumen investiert wird, könnte auch in die Ermöglichung von Teilhabeangeboten investiert werden.

- In einem Haus wird bemängelt, dass die Räumlichkeiten aufgrund des „Krankenhauscharakters" nicht zeitgemäß und wenig ansprechend gestaltet sind.

\subsubsection{Vernetzung mit dem Quartier}

Das Quartier als Wohnumfeld der Einrichtungen in ihrer Kommune wird von den Interviewten nicht nur als Ort der Teilhabe (siehe Kapitel 4.2.1), sondern auch als besondere Ressource für die Teilhabeförderung betrachtet, 
wenn die Einrichtung mit diesem hinreichend vernetzt ist. Insbesondere im Rahmen der „Good-Practice“-Analyse wurden Prinzipien und Vorgehensweisen der Quartiers- bzw. Sozialraumorientierung benannt und bei der Frage nach „Ansätzen der Teilhabeförderung“ ausdrücklich hervorgehoben oder sogar primär aufgeführt. Aber auch in den Interviews in den Projekteinrichtungen sowie im Rahmen der trägerübergreifenden Gruppendiskussion finden sich einige Hinweise auf Verbindungen mit dem Quartier und deren Notwendigkeit für die Teilhabeförderung. Insgesamt wird einerseits die Relevanz der Vernetzung und Kooperation mit anderen Institutionen im Quartier und andererseits die Notwendigkeit von Öffnungen der stationären Altenhilfe für und in das Quartier benannt.

Die Kooperation und Vernetzung mit anderen Institutionen und Gruppen aus unterschiedlichen professionellen und bürgerschaftlichen Sektoren im Quartier wird als wichtige Voraussetzung oder sogar als das „A und O“ (GP Int. 2) für eine quartiersorientierte Arbeit betrachtet, welche damit auch erst Öffnungen der Einrichtungen für und in das Quartier ermöglicht.

Eine Öffnung für das Quartier impliziert prinzipiell eine erweiterte Nutzung von Angeboten und Räumen einer Pflegeeinrichtung durch Bürger"innen oder Netzwerke - etwa Vereine und Gremien - aus dem Quartier. Die Teilhabe der Bewohner*innen der Einrichtung kann hier gefördert werden, weil dadurch das Quartier quasi ,ins Haus kommt“ und Kontakte entstehen können. Eine Öffnung für das Quartier kann aber auch implizieren, dass Bürger*innen oder Institutionen aus dem Quartier in die Einrichtung bewusst eingeladen werden, um Angebote für und mit den Bewohner"innen durchzuführen (z. B. die Kita oder der Karnevalsverein). Weitergehend kann eine Öffnung für das Quartier auch auf eine Ausdifferenzierung des bisherigen Angebotsspektrums sowie auf veränderte Angebotsstrukturen der stationären Altenhilfe bezogen werden, indem sich etwa ambulante Hilfen mit stationären Angeboten bedarfsgerecht ergänzen (Bleck et al., 2018, S. 5). Beide hierzu zählenden Öffnungsperspektiven für das Quartier werden von den Einrichtungen geschildert - hier zum Beispiel das Stadtteilfrühstück, der geöffnete Gottesdienst, das Sommerfest, der „REHA-Sport“, die Kita und der Musiklehrer in der Einrichtung sowie ein differenziertes Angebotsspektrum, das neben der vollstationären Pflege auch etwa eine Beratung, Begegnungsstätte und Tagespflege beinhaltet.

Für die Verwirklichung der Teilhabe von Bewohner*innen am Leben in der Gesellschaft außerhalb der Einrichtung sind Öffnungen in das Quartier zentral. Diese beziehen sich dann auf Nutzungen des Quartiers durch die Bewohner*innen der Pflegeeinrichtungen und soll ihre soziale Teilhabe 
vor Ort im Wohnumfeld fördern (Bleck et al., 2018, S. 5), Auch diese Öffnungsperspektive wurde in den Interviews mehrfach aufgegriffen- hier etwa das Generationenkonzert oder der traditionelle Kirmesbesuch.

Das Quartier nimmt eine relevante Stellung in der Ermöglichung von Teilhabe am Leben in der Gesellschaft ein. Dazu sollen im Folgenden die förderlichen und hemmenden Faktoren dargestellt werden.

\section{Fördernd:}

- Mehrere Interviewpartner*innen geben im Rahmen der durchgeführten Interviews an, dass es Kooperationen und Vernetzungen mit (evangelischen und katholischen) Kirchengemeinden, Kitas, Schulen und Vereinen (beispielsweise Schützen-, Hospiz-, Gesangs-, Musik- und Hundeverein sowie Altenhilfeverein) gibt. Eine Einrichtung erwähnt auch die Beteiligung an einem „kommunalen Aktionsbündnis Senioren“ (PDL). Über diese Verbindungen entstehen zur Nutzung von Teilhabeangeboten für die Bewohner*innen innerhalb und außerhalb der Einrichtungen Zugänge, die über politische Mitwirkung verbessert werden können.

- Es gibt verschiedene Aktivitäten, die außerhalb von der Einrichtung geplant und gezielt im Quartier stattfinden (Theater, Aktivitäten in einer Veranstaltungshalle, Quartiersfeste; Kapitel 4.2.1.2). Die Teilhabe der Bewohner*innen vor Ort - als Bürger*innen des Quartiers - wird so von Einrichtungsseite aktiv unterstützt: „Ja, wir verknüpfen ja im Moment mit der Teilhabe die Teilhabe am Gemeinwesen, das heißt, teilnehmen an den Festen und Angeboten der Stadt" (PDL).

- Des Weiteren wird von verschiedenen Einrichtungen beschrieben, dass es auch Veranstaltungen gibt, die innerhalb des Hauses stattfinden - indem das Quartier "ins Haus geholt" wird (Modenschau/Modegeschäft, Supermarkt; Kapitel 4.2.1.1). Diese Kontakte zum Einzelhandel stellen gerade für ländlich gelegene Einrichtungen eine relevante teilhabeförderliche Methode dar, um einen „normalen“ Alltag durch ein „Kund"innengefühl“ aufrechtzuerhalten.

- Fördernd für die Vernetzung ist eine im Quartier wahrnehmbare Öffentlichkeitsarbeit von Seiten der Einrichtungen.

- Einrichtungen, die städtisch liegen, haben kurze Wege zu Einrichtungen des täglichen Bedarfs. Dies wird von den meisten Befragten als sehr vorteilhaft angesehen, um Zugänge zur Teilhabe an Infrastrukturen zu haben. 


\section{Hemmend:}

- Kritisch beleuchtet wird, inwieweit Bewohner*innen mit zunehmenden Beeinträchtigungen von Öffnungen der Altenpflegeeinrichtungen für und zum Quartier adäquat profitieren können. Im Zuge der „GoodPractice“-Analyse wurde dies bestätigt: „Die demenziell veränderten Menschen sind, was die klassische Quartiersarbeit im engeren Sinne angeht, nicht so integriert. Das ist sehr, sehr schwierig bei der Angebotsstruktur [...] es gibt möglicherweise Ansätze, wie man das noch verbessern kann" (GP Int. 2).

- Als hemmender Faktor wird auch eine ungünstige geografische Lage von Einrichtungen erachtet. Eine Projekteinrichtung liegt etwa auf einer Anhöhe. Diese Lage wird als teilhabehemmend für die Bewohner"innen angesehen, da die in ihrer Mobilität eingeschränkten Bewohner"innen die Einrichtung nicht ohne Hilfe verlassen können, um beispielsweise einen Supermarkt aufzusuchen oder in die Stadt zu gehen. Auch für Helfer*innen stellt dies ein Hindernis dar, da es viel Kraft erfordere, um eine im Rollstuhl sitzende Person hinaufzuschieben. Um diese Hemmnis abzubauen, gibt es einen Bus, der von der Einrichtung zur Verfügung gestellt wird. Darüber hinaus kann auch ein Bürger*innenbus genutzt werden, welcher direkt vor der Einrichtung hält.

\subsection{Zwischenfazit zur qualitativen Analyse}

In Anbetracht der in diesem Kapitel vorgestellten Untersuchungsergebnisse ist zunächst als Zwischenfazit zur qualitativen Analyse festzuhalten, dass die praktische Verwirklichung des Rechts auf selbstbestimmte Teilhabe von Bewohner*innen in Altenpflegeeinrichtungen von einer Vielzahl von Faktoren beeinflusst ist. Damit schließen die Ergebnisse der qualitativen Analyse an die in den Kapiteln 2.2 und 2.3 erläuterten Erkenntnisse und Vorüberlegungen aus dem Forschungsstand und der konzeptionellen Rahmung an, bestätigen diese aber nicht nur, sondern differenzieren sie auf empirischer Grundlage maßgeblich aus, indem ein umfassendes Raster zentraler Themenkategorien mit fördernden und hemmenden Faktoren auf unterschiedlichen Analyseebenen herausgearbeitet werden konnte. So hat sich in den Einzel- und Gruppeninterviews im Rahmen von STAP ebenso wie in der projektergänzenden „Good-Practice“-Analyse deutlich bestätigt, dass die Förderung von selbstbestimmter Teilhabe kein monolinearer, auf wenige Faktoren begrenzter Prozess ist, sondern dass diese stets eine „Wechselwirkung von mehreren Komponenten“ (Garms-Homolová \& 
Theiss, 2009a, S. 190) darstellt, deren Bedeutung somit auch je Bewohner*in und Situation variieren kann. Angeregt durch die konzeptionellen Vorüberlegungen, orientiert an den Forschungsfragen sowie dann insbesondere geprägt durch das empirische Material im Rahmen von STAP wurden die in der qualitativen Analyse identifizierten fördernden und hemmenden Faktoren auf den Ebenen der Bewohner*innen, der Mitarbeitenden und der Einrichtung unterschieden. Davon getrennt wurden die Wunschäußerung und -erfassung als zentraler Ausgangsprozess der Teilhabeförderung festgehalten, auf den die Berücksichtigung und Verwirklichung gewünschter Teilhabe im Kontext der Alltags- und Angebotsgestaltung innerhalb und außerhalb von Altenpflegeeinrichtungen folgt bzw. folgen sollte. Darüber hinaus wurden besondere Ressourcen für die Teilhabeförderung identifiziert und erläutert.

Aus der Vielfalt der vorgestellten fördernden und hemmenden Faktoren sollen im Folgenden noch einmal wesentliche Ergebnisbereiche resümierend hervorgehoben werden, die in besonderer Weise in ihrer Relevanz bestätigt wurden: durch die vielfache Betonung aus unterschiedlichen Perspektiven (z. B. durch Mitarbeiter*innen verschiedener Ebenen und Einrichtungsbereiche, Angehörige und Bewohner*innen), in unterschiedlichen Themenbereichen (z. B. Faktoren, die auf verschiedenen Ebenen wiederholend vorkommen) und verschiedenen Erhebungszugängen (Einzelinterviews, Beobachtungen, Gruppeninterviews, „Good-Practice“-Analyse) sowie Validierungszugängen (z. B. Lernworkshops mit den Projekteinrichtungen, Projektratssitzungen, Tagung der Bewohner*innenbeiräte).

Der Themenkomplex „Wünsche der Bewohner"innen" bzw. der Umgang mit ihren Wünschen wurde in STAP in der ersten der beiden übergeordneten Untersuchungsfragen berücksichtigt und somit bereits im Vorfeld der empirischen Erhebungen als zentral erachtet. Im Rahmen der qualitativen Analysezugänge - insbesondere der Expert*inneninterviews und Fokusgruppen - wurde der Themenkomplex „Wünsche“ - und damit die Annahme, dass Teilhabeförderung entsprechend den individuellen Wünschen der Bewohner*innen differenziert erfolgen muss - nicht nur vielfach thematisiert, sondern auch in seiner Relevanz als Ausgangsprozess zur Förderung und Realisierung von selbstbestimmter Teilhabe deutlich bestätigt. Zur Feststellung und Berücksichtigung von Wünschen sind die Voraussetzungen zur Wunschäußerung bei den Bewohner*innen sehr heterogen. Daher sind die Einbeziehungsformen durch Mitarbeitende individuell an den oder die Bewohner*in anzupassen, aber auch Wünsche der Nichtteilhabe einzelner Bewohner*innen sensibel zu berücksichtigen und zu akzeptieren. Gerade institutionellen Reglementierungen sowie dem Anpassungsver- 
halten von Bewohner*innen an empfundene oder existierende institutionelle Vorgaben oder Abläufe sollte von Seiten der Mitarbeitenden bewusst entgegengewirkt werden. Vielmehr sollten die Bewohner*innen bereits im Rahmen der Einzugsphase motiviert werden, ihre Wünsche zu äußern, damit hier kein Anpassungs- und Rückzugsprozess seitens der Bewohner*innen erfolgt.

Festzuhalten ist auch, dass bereits ausreichend formelle und informelle Erfassungs- und Kommunikationsformen existieren, bei denen aber teilhabebezogene Wünsche grundsätzlich mehr Gewicht erhalten sollten. Doch nicht nur in der für das Einleben der Bewohner*innen wichtigen Einzugsphase sollten Wunscherfassungen im Rahmen der vorhandenen Instrumente (wie Einzugsgespräch, Pflegeanamnese) stattfinden, sondern auch im weiteren Verlauf, nach der Eingewöhnungsphase, sollten Wünsche in regelmäßigen Abständen erfragt oder geweckt und erfasst werden (z. B. im Rahmen der weiteren Pflegeprozessplanung). Um die Wünsche von Bewohner*innen für die Angebotsgestaltung zu berücksichtigen, hat beispielsweise eine Einrichtung eine sogenannte „Wunschrunde“ implementiert, die als ein niedrigschwelliges Angebot für alle Bewohner“innen entwickelt wurde. Neben dem Bewohner*innenbeirat, der auch verschiedene Zugänge der Wunscherfassung anbietet (z. B. über Sprechstunden), bietet dieses Format eine Möglichkeit, sich individuell zu beteiligen und Wünsche gegenüber dem Sozialen Dienst in Bezug auf die Angebote zu äußern. Zu benennen ist aber auch, dass aus Sicht der Bewohner*innen nur wenige konkrete Teilhabewünsche genannt wurden, was möglicherweise mit der Interviewsituation zusammenhängt, aber wohl auch darauf zurückzuführen ist, dass die jetzige Bewohner*innenschaft, die der (Nach-)Kriegsgeneration angehört, seltener Anforderungen stellt. Gleichwohl konnten in den anderen Interviewzugängen Teilhabewünsche sowie fördernde und hemmende Faktoren zu ihrer Berücksichtigung identifiziert werden. Die Erfassung und Berücksichtigung von Wünschen der Teilhabe sollte professionsbzw. bereichsübergreifend im Selbst- und Aufgabenverständnis intensiver verankert werden. Dabei sind auch Zugänge zu und die Zusammenarbeit im Rahmen von bestehenden Kommunikations- und Dokumentationsformen (z. B. Übergaben, Instrumente des Pflegeprozesses) stärker für das „gesamte Team" zu berücksichtigen. Wenn danach gefragt wird, wird Teilhabeförderung zwar ausdrücklich als bereichsübergreifende Aufgabe verstanden, bislang wirkt aber immer noch ein gewisses „Säulendenken“ zwischen den verschiedenen Bereichen ebenso hemmend wie nicht hinreichend transparente oder gestaltete Schnittstellen zwischen den Einrichtungsbereichen. Auch wenn bisher typischerweise und im Wesentlichen 
der Soziale Dienst eine koordinierende Funktion in der Förderung selbstbestimmter Teilhabe übernimmt, so ist dieser abhängig von der Kooperation mit den anderen Bereichen. Zudem ist die Förderung selbstbestimmter Teilhabe eben ausdrücklich nicht nur Aufgabe des Sozialen Dienstes, sondern eine bereichsübergreifende Aufgabe, die sowohl im gemeinsamen, kooperativen Handeln unterschiedlicher Bereiche zum Ausdruck kommen sollte als auch unmittelbar in Alltagssituationen der jeweiligen Bereiche stattfinden kann - etwa im Rahmen der pflegerischen Versorgung ebenso wie bei den Mahlzeiten oder in der Zimmergestaltung.

Neben der unmittelbaren Berücksichtigung von Wünschen der Teilhabe in verschiedenen Alltagssituationen findet Teilhabeförderung über die Planung und Gestaltung von Einzel- und Gruppenangeboten innerhalb und außerhalb der Einrichtungen statt. Hier sollte leitend sein, die Teilhabeförderung und -verwirklichung in den verschiedenen Kontexten der Alltags- und Angebotsgestaltung stets an den Prinzipien der Selbstbestimmung und Normalität auszurichten. Um angesichts - auch im Sozialen Dienst - begrenzter personeller Ressourcen möglichst vielen Bewohner*innen zeitgleich Teilhabe zu ermöglichen, sind Angebote innerhalb und außerhalb der Einrichtung in Gruppenform ein wesentliches Setting der Teilhabeförderung. Daneben finden Kleingruppenangebote und Einzelbetreuungen - seit Einführung der zusätzlichen Betreuungskräfte in einem größeren Umfang als davor - statt. Gerade die von zusätzlichen Betreuungskräften und teils auch Ehrenamtler*innen durchgeführte Einzelbetreuung ermöglicht die Verwirklichung individueller Wünsche der Teilhabe innerhalb sowie auBerhalb der Einrichtung und dabei etwa auch die individuelle Betreuung von bettlägerigen Bewohner*innen, die selten an Gruppenangeboten teilnehmen. Demgegenüber bieten Gruppenangebote aber eben auch den $\mathrm{Zu}$ gang zu einer Gemeinschaft in der Gruppe des Angebotes. Allerdings ist zu beachten, dass in der Planung und Gestaltung von Gruppenangeboten keine „Normierungen“ vorgenommen werden, die der Teilhabe nach dem Prinzip der Selbstbestimmung widersprechen. Normierungstendenzen entwachsen - nach Darstellungen in den Interviews - etwa aus den Gedanken, keine/n im Sinne der Gemeinschaft zu bevorzugen, oder der Effizienz, mit einer/m Mitarbeitenden möglichst viele Bewohner*innen zu erreichen. So werden Angebote bevorzugt für mehrere Personen bereitgestellt und möglicherweise vor allem dann als erfolgreich erachtet, wenn viele Bewohner*innen daran teilnehmen - der aus Sicht von STAP primäre Gedanke einer Teilhabeförderung, die auch den individuellen Wünschen entspricht, gerät dadurch aber unter Umständen in den Hintergrund. 
Ferner wurden in der qualitativen Analyse (weitere) fördernde und hemmende Faktoren zur selbstbestimmten Teilhabe auf Ebene der Bewohner*innen, Mitarbeiter*innen und Einrichtung ermittelt. So konnte zunächst auf Ebene der Bewohner*innen ausdifferenziert werden, dass die Voraussetzungen der Bewohner*innen zur selbstbestimmten Teilhabe und ihrer Förderung äußerst heterogen sind. Auf Basis der Einzel- und Gruppeninterviews wurden verschiedene Bewohner"innengruppen identifiziert, die sich vor allem auf physische und psychische Voraussetzungen bezogen haben: (1) „Orientierte“ Bewohner*innen (unterteilt in „aktive“ und „sich zurückziehende" Bewohner*innen), (2) kognitiv eingeschränkte und psychisch veränderte Bewohner*innen, (3) physisch stark eingeschränkte Bewohner*innen sowie (4) Bewohner*innen in der Sterbephase. Darüber hinaus wurden weitere Differenzmerkmale benannt und festgehalten, die sich querliegend zu den oben benannten Unterscheidungen auf das Geschlecht, die Herkunft und die finanzielle Situation der Bewohner*innen beziehen. Die Unterteilung der Bewohner*innenschaft zeigt nicht nur auf, dass die Voraussetzungen zur Teilhabeförderung der Bewohner*innen heterogen sind, sondern sie verweist auch auf mögliche Unterscheidungen in Teilgruppen innerhalb der Bewohner*innenschaft, die teilweise auch unter den Mitarbeitenden pragmatisch einer Zuordnung von in Frage kommenden Bewohner*innen für spezifische Gruppenangebote oder Einzelbetreuungen dient. Hierbei wird offenbar im Vorfeld überlegt, wer an welchem Angebot teilnehmen kann.

In Bezug auf Voraussetzungen auf Seiten der Mitarbeiter*innen war die Annahme verbreitet, dass die persönlichen „Eigenschaften“ und Kompetenzen der Mitarbeitenden (oft wurde ausgesagt, dies sei „personenabhängig“) maßgeblich für die Qualität und Intensität der Teilhabeförderung von Bewohner*innen verantwortlich seien. Sofern diese flexibel in ihrer Arbeitsweise sind und mit hohem Arbeitsengagement agieren sowie hinreichend über soziale Kompetenzen verfügen, gilt dies für Mitarbeitende als teilhabeorientiert bzw. -förderlich. Darüber hinaus konnten aber auch weitere Inhalte in anderen Kompetenzdimensionen erfasst werden, die auf Seiten der Mitarbeiter*innen Zugänge zur Teilhabeförderung unterstützen und teilweise auch über Fortbildungen vermittelbar wären (z. B. in Bezug auf spezifische Fach- und Methodenkompetenzen).

Damit die Teilhabeförderung aber nicht abhängig von persönlichen Eigenschaften der einzelnen Mitarbeitenden bleibt, ist von zentraler Bedeutung, dass das Thema der selbstbestimmten Teilhabe von Bewohner*innen auf Ebene der Organisation bzw. Einrichtung verankert und gefördert wird. So hat sich in der qualitativen Analyse eine entsprechende Organisati- 
onskultur als wesentlicher Faktor zur Unterstützung der selbstbestimmten Teilhabe in stationären Altenpflegeeinrichtungen herausgestellt. Um eine solche Organisationskultur zu entwickeln und dauerhaft zu begünstigen, ist es von Bedeutung, dass das Leitungsteam aus unterschiedlichen Bereichen einer Einrichtung ein gemeinsames Verständnis - mit geteilten Orientierungen und Werten - der Förderung von selbstbestimmter Teilhabe in dieser Einrichtung hat und dass dieses Verständnis immer wieder im Arbeitsalltag geplant oder spontan situativ thematisiert wird. Einem „Säulendenken“ zwischen den Bereichen und Tendenzen zu einem gewissen „Versorgungsprimat" (Primat der "medizinisch-pflegerischen Versorgung" gegenüber der „sozialen Betreuung“ der Bewohner"innen) sind hier zugunsten einer gemeinsamen, bereichsübergreifenden Idee und Praxis selbstbestimmter Teilhabe entgegenzuwirken.

Um gemeinsame Bezugspunkte und Grundlagen zur Förderung selbstbestimmter Teilhabe zu haben, scheint auch dessen Verankerung in Konzepten der Einrichtung hilfreich. Bislang finden sich zwar einzelne Bezugnahmen auf die Begriffe Selbstbestimmung und/oder Teilhabe in einzelnen Konzepten der Einrichtungen (z. B. Einrichtungskonzept, Konzept Sozialer Dienst, Pflegekonzept), es liegen aber weder eigene "Teilhabekonzepte“ vor noch wird die Förderung der Teilhabe dort differenzierter aufgegriffen bzw. erläutert.

Spezifische organisatorische Abläufe - und Rahmenbedingungen für diese - haben sich in der qualitativen Analyse ebenfalls als relevant für eine gelingende Teilhabeförderung herausgestellt, wovon die Wunscherfassung bereits separat als zentraler Ausgangsprozess benannt wurde. Aber auch ein gewisser von Leitungsseite legitimierter Handlungsspielraum innerhalb von Arbeitsprozessen, beispielsweise beim Verteilen der Aufgaben über verschiedene Schichten, ist ein wichtiger teilhabeförderlicher Aspekt, da damit durch Mitarbeitende flexibler auf situativ vorhandene Wünsche reagiert werden kann. Wie bereits angesprochen, sind auch die Kooperation und Schnittstellen zwischen den Bereichen - insbesondere diejenigen zwischen Pflege und Sozialem Dienst - von besonderer Bedeutung für die Teilhabeförderung. Der direkte und regelmäßige Austausch zwischen den Einrichtungsbereichen wird zwar als relevant erachtet, findet aber eher seltener bereichsübergreifend und mit konkretem Fokus auf Teilhabe in dem vorhandenen Besprechungswesen statt. Absprachen zu aktuellen Teilhabewünschen erfolgen oft eher „zwischen Tür und Angel“. Dies birgt die Gefahr, dass teilhaberelevante Informationen über Bewohner*innen nicht weitergegeben werden. 
Die Förderung der Teilhabe benötigt insbesondere personale Ressourcen, die in den Interviews immer wieder als fehlend benannt wurden. Gerade die zusätzlichen Betreuungskräfte ${ }^{74}$ und auch die ehrenamtlichen Helfer*innen werden als besondere Ressourcen für die Teilhabeförderung benannt und bilden - neben den Fachkräften des Sozialen Dienstes - eine wesentliche personale Basis, um Teilhabe zu verwirklichen. Zusätzliche personelle Ressourcen können Auszubildende und Praktikant"innen oder auch Projektstellen über ergänzende finanzielle Mittel im Rahmen von beantragten Projekten sein.

Ein wichtiger Ressourcenbereich bezieht sich hier auf die Unterstützung der Mobilität der Bewohner*innen innerhalb und außerhalb der Einrichtung, die mehrheitlich über die zusätzlichen Betreuungskräfte und Ehrenamtler*innen sowie darüber hinaus die Angehörigen erfolgt. Als förderlich hat sich hier herausgestellt, wenn die Kräfte, Termine und Aufgaben der Mobilitätsunterstützung koordiniert werden, so wie es teilweise in den Einrichtungen über den Sozialen Dienst erfolgt.

Eine gute Vernetzung im Quartier stellt ebenfalls eine zentrale Ressource dar, da sie dem Aufrechterhalten oder der Weckung von unterschiedlichen Teilhabezugängen innerhalb und außerhalb der Einrichtung dienen kann. Ressource ist die Vernetzung mit Akteur*innen im Quartier, etwa einerseits für Angebote, die von Externen in der Einrichtung offeriert werden und somit besondere Angebote für die Bewohner“innen darstellen, deren Teilhabepotenzial noch erweitert werden kann, wenn auch Bürger"innen des Quartiers daran teilnehmen können (im Sinne einer Öffnung für das Quartier). Andererseits bieten Kooperationen mit Institutionen, Geschäften, Unternehmen und Schlüsselpersonen aus dem Quartier auch Ressourcen, damit die Bewohner*innen Angebote im Quartier und damit die Teilhabe am gesellschaftlichen Leben außerhalb der Einrichtung stärker - mit oder ohne Begleitung - wahrnehmen können, etwa indem Gruppenausflüge ermöglicht oder individuelle Teilnahmen der Bewohner*innen an spezifischen Veranstaltungen oder regelmäßigen Aktivitäten vermittelt werden können (im Sinne einer Öffnung in das Quartier).

74 Im engeren Sinne stellen zusätzliche Betreuungskräfte keine „Ressource“ dar, da sie als reguläre Mitarbeitende der Einrichtung angestellt sind. Dennoch wurden sie in den Interviews als besondere personelle Ressource gedeutet und in diesem Sinne so häufig benannt, so dass sie hier aufgeführt wurden. 


\section{Ergebnisse der quantitativen Analyse}

In diesem Kapitel werden die Ergebnisse der Onlinebefragung präsentiert. Die Umfrage adressierte die Einrichtungsleitungen von Altenpflegeeinrichtungen in NRW mit vollstationären Pflegeplätzen. Die quantitative Analyse wurde als Folgeuntersuchung konzipiert und zielte darauf ab, ausgewählte Ergebnisse der qualitativen Analyse (siehe Kapitel 4) hinsichtlich dreier Leitfragen zu untersuchen: (1) Ist-Zustand: Liegen die als relevant identifizierten Rahmenbedingungen in anderen Einrichtungen vor? (2) Bewertung der Relevanz: Wie bewerten Einrichtungsleitungen die Relevanz der identifizierten fördernden und hemmenden Faktoren? (3) Kontext: Unterscheiden sich Einrichtungen hinsichtlich des Ist-Zustandes und der Bewertung der Relevanz aufgrund von Kontextfaktoren?

Die Analyse erfolgte entlang der inhaltlichen Abschnitte (1) Verankerung von Teilhabe, (2) Zusammenarbeit und Aufgaben der Arbeitsbereiche, (3) Handlungsspielräume und Ressourcen sowie (4) arbeitskulturelle Orientierung (siehe Kapitel 3.3.2). Dabei wurde die Analyse durch die drei oben genannten Leitfragen strukturiert: Der Ist-Zustand der identifizierten Rahmenbedingungen sowie die Einschätzung der Einrichtungsleitungen, ob diese Faktoren hemmend oder fördernd sind, werden im Folgenden jedoch zusammenfassend beschrieben. Dabei kann die überwiegende Anzahl an Items zum Ist-Zustand direkt in Bezug zur Einschätzung der Relevanz gesetzt werden. Ausnahmen bilden einzelne Items, zu denen, aufgrund des begrenzten Umfangs, nur der Ist-Zustand oder ausschließlich eine Bewertung der Relevanz erhoben wurde. Um abzusichern, dass die qualitativen Ergebnisse nicht nur für Einrichtungen mit bestimmten Kontextfaktoren gültig sind, sondern verallgemeinert werden können, wurden Items, die sich in ihrer zentralen Tendenz unterscheiden und für die Voraussetzungen für den Test erfüllt sind (Kapitel 3.3.3), auf einen Einfluss von Kontextfaktoren analysiert. Es wird eine ungerichtete Unterschiedshypothese zugrunde gelegt: Es gibt einen Unterschied in Bezug auf den IstZustand und der Bewertung der Relevanz (nach Angaben der Einrichtungsleitungen) von der Trägerschaft oder dem Gemeindetypus. Analog dazu wird für die Anzahl der Bewohner*innenplätze in einer Einrichtung eine ungerichtete Zusammenhangshypothese formuliert: Der Ist-Zustand und die Bewertung der Relevanz, nach Angaben der Einrichtungsleitungen, sind von der Anzahl an Bewohner*innenplätzen einer Einrichtung ab- 
hängig. Wenn die Bewertung der Relevanz eine Varianz aufweist und der Ist-Zustand in Bezug dazu erfasst wurde, dann wird untersucht, ob der IstZustand die Bewertung der Relevanz beeinflusst. Die dargestellten Angaben beziehen sich auf die gültigen Antworten; Abweichungen zur Anzahl aller Einrichtungen $(n=135)$ werden berichtet.

Eine Beschreibung der Einrichtungen (Kapitel 5.1) auf Basis der Angaben der teilgenommenen Einrichtungsleitungen wird der Darstellung der inhaltlichen Ergebnisse (Kapitel 5.2 - 5.5) vorangestellt. Eine abschließende Rangfolge zu den wichtigsten Faktoren und zu den dringendsten Änderungsansätzen für die Förderung von Teilhabe (Kapitel 5.6) ermöglicht eine Gewichtung der Ergebnisse. Abschließend werden die Ergebnisse zusammengefasst (Kapitel 5.7).

\subsection{Beschreibung der Einrichtungen}

Im Folgenden werden die Einrichtungen anhand der Angaben zu den Kontextfaktoren der 135 Einrichtungsleitungen, die an der Umfrage teilgenommen haben, beschrieben. Die Angaben beziehen sich zum einen auf die Einrichtungsstruktur und zum anderen auf die Bewohner*innenstruktur. Die Daten zu den Einrichtungen ermöglichen eine Einschätzung, welche Einrichtungen durch die gewählten Zugänge erreicht wurden. Angestrebt wurde, Einrichtungen unterschiedlicher Regionen und Trägerschaften für die Teilnahme zu gewinnen. Die regionale Verortung wurde sowohl aus der Lage in NRW (Regierungsbezirke) bestimmt, als auch aus dem Gemeindetyp, in dem die Einrichtung liegt (Großstadt, Mittelstadt, Kleinstadt, kleiner Ort/Dorf). In Abbildung 4 sind die absoluten Teilnehmenden je Regierungsbezirk, der Anteil an den gesamten teilnehmenden Einrichtungen und die Abweichung zu den Angaben der Pflegestatistik dargestellt. 


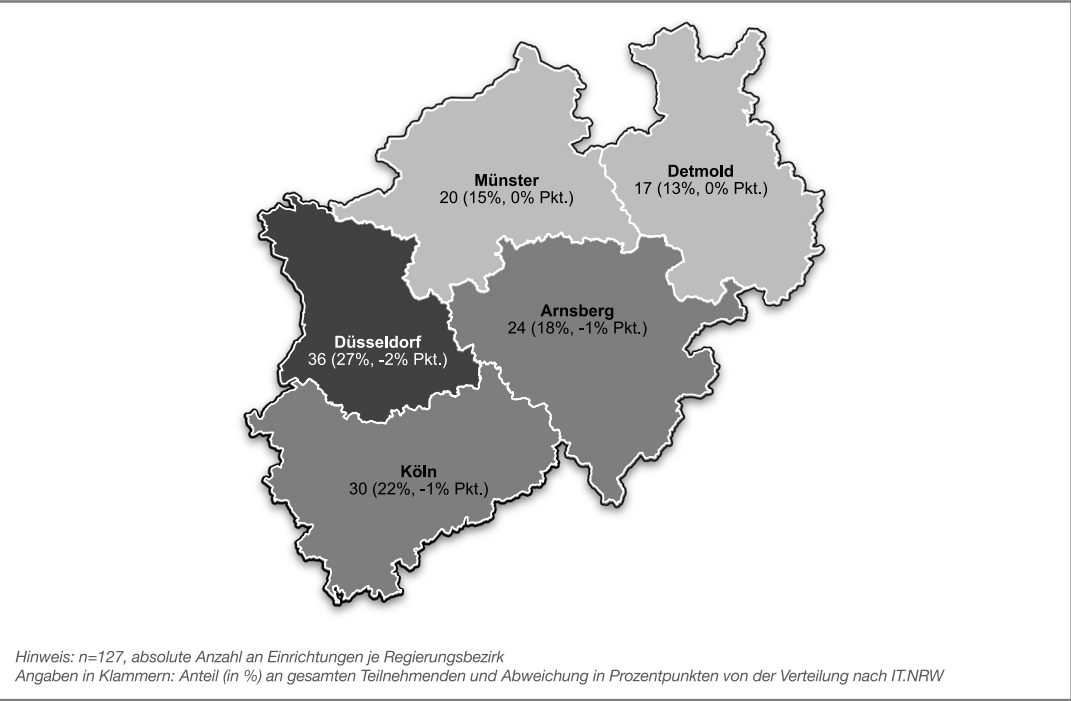

Abbildung 4: Teilnehmende an der Umfrage nach Regierungsbezirk

Legt man die Pflegestatistik zugrunde (IT.NRW, 2017), dann entspricht die Verteilung auf die Regierungsbezirke annähernd der Verteilung der teilgenommenen Einrichtungen nach Regierungsbezirken (maximale Abweichung 2 Prozentpunkte).

Tabelle 15: Angaben zu den Einrichtungen, die teilgenommen haben

\begin{tabular}{|l|l|l|}
\hline \multirow{5}{*}{ Gemeindetyp } & Großstadt & $41(30,4 \%)$ \\
\cline { 2 - 3 } & Mittelstadt & $23(17,0 \%)$ \\
\cline { 2 - 3 } & Kleinstadt & $45(33,3 \%)$ \\
\cline { 2 - 3 } & kleiner Ort/Dorf & $26(19,3 \%)$ \\
\hline \multirow{2}{*}{$\begin{array}{l}\text { Trägerschaft }(\mathbf{n}= \\
\text { freigemeinnützig }\end{array}$} & $88(67,2 \%)$ \\
\cline { 2 - 3 } & privat-gewerblich & $27(20,6 \%)$ \\
\cline { 2 - 3 } $\begin{array}{l}\text { Bewohner*innen- } \\
\text { plätze (n=133) }\end{array}$ & öffentlich & $16(12,2 \%)$ \\
\hline $\begin{array}{l}\text { Ehrenamtliche } \\
\text { (n= 132) }\end{array}$ & $\begin{array}{l}\text { Arithmetisches Mittel = 91,3 } \\
(\mathrm{SD}=38,3) ; \text { Median }=87\end{array}$ \\
\hline
\end{tabular}




\begin{tabular}{|l|l|l|}
\hline $\begin{array}{l}\text { Menschen mit } \\
\text { Demenz }(\mathbf{n}=\mathbf{1 3 2})\end{array}$ & $\begin{array}{l}\text { in \% an Bewoh- } \\
\text { ner*innen }\end{array}$ & $\begin{array}{l}\text { Arithmetisches Mittel = 62,6 } \\
(\mathrm{SD}=18,8) ; \text { Median }=65\end{array}$ \\
\hline Sozialhilfe $(\mathbf{n}=\mathbf{1 2 9})$ & $\begin{array}{l}\text { in \% an Bewoh- } \\
\text { ner*innen }\end{array}$ & $\begin{array}{l}\text { Arithmetisches Mittel = 47,3 } \\
(\mathrm{SD}=23,5) ; \text { Median }=45\end{array}$ \\
\hline
\end{tabular}

In Tabelle 15 sind zusätzlich Angaben zum Gemeindetyp, zur Trägerschaft und zu den verfügbaren Plätzen sowie Angaben zur Bewohner*innenstruktur aufgeführt. Für den Gemeindetyp liegt kein Referenzwert vor, es zeigt sich aber, dass Einrichtungen aus den vier Gemeindetypen vertreten sind, wenngleich mehr Einrichtungsleitungen aus Klein- und Großstädten (>30\%) und weniger aus Mittelstädten und kleinen Orten $(<20 \%)$ teilgenommen haben. Die Angaben zur Trägerschaft zeigen, dass etwa zwei Drittel der Einrichtungen in freigemeinnütziger, etwa $21 \%$ in privat-gewerblicher und $12 \%$ in öffentlicher Trägerschaft sind. Vergleicht man diese Verteilung mit den Angaben der Pflegestatistik (IT.NRW 2017), sind Einrichtungen in freigemeinnütziger $(+4,8 \%)$ und in öffentlicher Trägerschaft $(+7,7 \%)$ leicht überpräsentiert und private Einrichtungen $(-2,5 \%)$ in der Befragung unterrepräsentiert.

Die weiteren Angaben der Einrichtungsleitungen lassen sich wie folgt zusammenfassen: Im Durchschnitt haben die Altenpflegeeinrichtungen etwa 91 vollstationäre Plätze $(S D=38,3$, Median $=87)$, inklusive eingestreuter Plätze für Kurzzeitpflege. Dabei haben $25 \%$ der Einrichtungen weniger als 70 (1. Quantil) und 25\% mehr als 109 Bewohner*innenplätze. Basierend auf den Angaben der Einrichtungsleitungen kommen im Mittel etwa 13 Ehrenamtliche in die Einrichtungen ( $S D=13,4$, Median =7), die im Durchschnitt mehr als eine Stunde in der Woche vor Ort sind (1. Quantil: 3, 3.Quantil: 20). Dabei besteht ein - erwartbar - signifikanter, aber moderater positiver Zusammenhang zwischen der Einrichtungsgröße und der Anzahl der Ehrenamtlichen ( $r$ o $=0.49, \mathrm{p}<0.01$ ).

Die Einrichtungsleitungen haben angegeben, dass etwa $63 \%$ (SD $=18,8$, Median $=65$ ) ihrer Bewohner*innen Menschen mit Demenz sind. Die Auswertung hat ergeben, dass der Anteil der Bewohner*innen mit Demenz in $25 \%$ der Einrichtungen weniger als $52 \%$ (1. Quantil) beträgt und in $25 \%$ der Einrichtungen der Anteil über 75,25\% liegt. Etwa die Hälfte der Bewohner*innen $(47,3 \%, S D=23,5$, Median $=45)$ empfangen Sozialhilfe, dabei liegt das 1. Quantil bei $30 \%$ und das 3. Quantil bei $64 \%$.

Zusammenfassend zeigen die Angaben, dass die betreffenden Einrichtungen annährend in der zu erwartenden Verteilung (IT.NRW, 2017) in den fünf Regierungsbezirken liegen. Weiter haben Einrichtungen aus allen Gemeindetypen und aus unterschiedlicher Trägerschaft (hier vergleichs- 
weise größere Abweichungen zur erwarteten Verteilung) an der Befragung teilgenommen. Damit lassen sich die qualitativen Ergebnisse an einer hinreichend heterogenen Gruppe absichern.

\subsection{Verankerung von Teilhabe}

Im Abschnitt Verankerung von Teilhabe wurde sowohl nach formellen Fundierungen zur Teilhabeförderung (z. B. in Konzepten) gefragt als auch deren Thematisierung durch Leitungskräfte oder in Gremien behandelt. Zudem zielten Items darauf ab, zu erfragen, ob Teilhabewünsche in Form von Instrumenten implementiert oder Maßnahmen erfasst oder dokumentiert werden. Ein sehr hoher Anteil an Einrichtungsleitungen hat die dichotomen Fragen (ja/nein) zum Ist-Zustand in ihrer Einrichtung bejaht, wobei hiermit nur das Vorhandensein der ausgewählten Aspekte, nicht aber deren Intensität erfragt wurde. Zudem kann nicht ausgeschlossen werden, dass soziale Erwünschtheit, dass Teilhabe bereits in Altenpflegeeinrichtungen verankert ist, die hohen Werte begünstigt hat. Ferner kann eine positive Selektion der Stichprobe dahingehend nicht ausgeschlossen werden, dass sich Einrichtungsleitungen, die einen besonderen Wert auf Teilhabe legen, eventuell zu größeren Anteilen an der Umfrage beteiligt haben. Ebenso haben die Items, in denen die Relevanz bewertet werden sollte, hohe Zustimmungsraten erhalten. Die Einschätzung zur Relevanz wurde anhand einer vierstufigen Skala (Stimme voll zu/Stimme eher zu/ Stimme eher nicht zu/ Stimme nicht zu) bewertet; die Einrichtungsleitungen haben zu einem hohen Anteil voll oder eher zugestimmt, dass die meisten Faktoren für die Teilhabeförderung in ihrer Einrichtung notwendig sind oder wären.

Im Folgenden werden die Angaben zum Ist-Zustand und zur Bewertung der Relevanz vorgestellt und der Einfluss von Kontextfaktoren berichtet. Die Ratingskalen werden hinsichtlich ihrer Verteilung deskriptiv dargestellt, auf die Angabe einer zentralen Tendenz wurde verzichtet, um dem ordinalen Skalenniveau Rechnung zu tragen. 


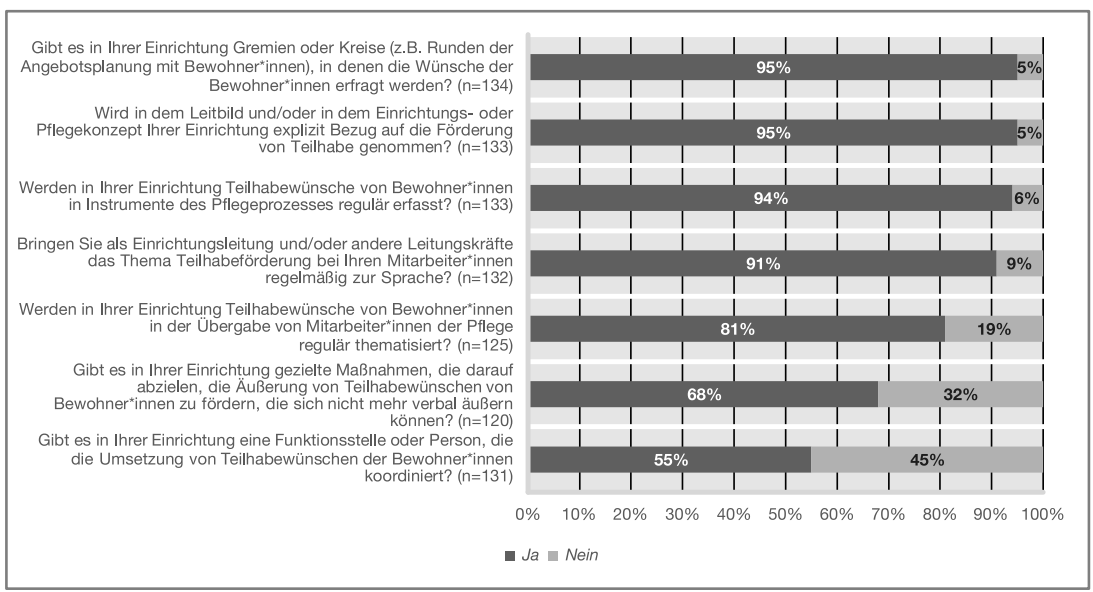

\section{Abbildung 5: Verankerung von Teilhabe (Ist-Zustand)}

In Abbildung 5 sind die Fragen und Angaben der Einrichtungsleitungen zum Ist-Zustand dargestellt. Fast alle Einrichtungsleitungen haben angegeben, dass in ihrer Einrichtung die Förderung von Teilhabe im Leitbild oder im Einrichtungs- oder Pflegekonzept verankert ist (95\%). Ebenso thematisieren sie oder andere Leitungskräfte das Thema Teilhabeförderung regelmäßig gegenüber ihren Mitarbeiter*innen (91\%). Die weiteren Fragen beziehen sich auf die Teilhabewünsche der Bewohner*innen. Die Erfassung von Teilhabewünschen der Bewohner*innen in Gremien oder Kreisen (95\%) findet in fast allen Einrichtungen statt. Für Bewohner*innen, die sich verbal nicht mehr äußern können, gibt es hingegen nur in $68 \%$ der Einrichtungen gezielte Maßnahmen, um die Äußerung von Teilhabewünschen für die Bewohner*innengruppe zu fördern. Die reguläre Erfassung von Teilhabewünschen in Instrumenten des Pflegeprozesses erfolgt in fast allen befragten Einrichtungen (94\%). Teilhabewünsche werden in den Übergaben der Mitarbeiter*innen der Pflege in vielen Einrichtungen regulär thematisiert $(81 \%)$, wenngleich dies kein einrichtungsübergreifender verbindlicher Bestandteil in Übergaben zu sein scheint. Die Umsetzung von Teilhabewünschen wird nur in etwas mehr als der Hälfte der Einrichtungen durch eine Funktionsstelle oder Person zentral koordiniert (55\%).

Die aufgeführten Item-Inhalte zur Verankerung von Teilhabe wurden von den Einrichtungsleitungen auch überwiegend als notwendig zur Förderung der Teilhabe bewertet, nur wenige Befragte haben eher nicht oder nicht zugestimmt. Die Bewertung der Relevanz umfasste fünf Items, die 
sich direkt auf ausgewählte Fragen zum Ist-Zustand beziehen und in Abbildung 6 dargestellt sind.

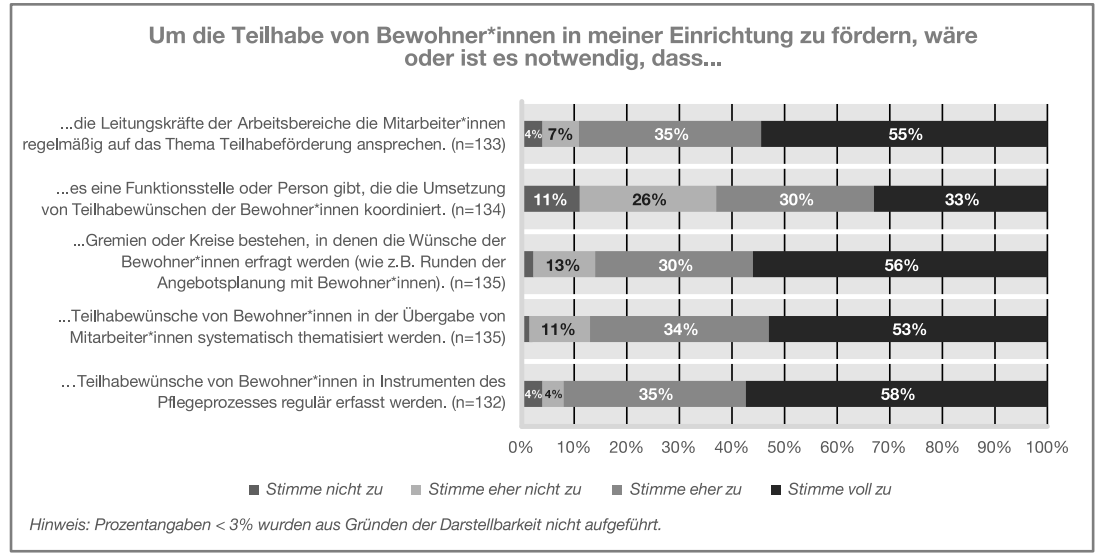

Abbildung 6: Verankerung von Teilhabe (Bewertung der Relevanz)

Die Einrichtungsleitungen haben voll (55\%) bzw. eher (35\%) zugestimmt, dass die regelmäßige Thematisierung der Teilhabeförderung gegenüber den Mitarbeiter*innen für die Teilhabeförderung in ihren Einrichtungen notwendig ist. Teilhabewünsche in Gremien oder Kreisen zu erfragen, wird ebenfalls als notwendig angesehen (stimme voll $\mathrm{zu} 56 \%$, stimme eher zu $30 \%$ ). Die reguläre Erfassung in Instrumenten des Pflegeprozesses (stimme voll zu $58 \%$, stimme eher zu $35 \%$ ) wie auch die systematische Thematisierung in der Übergabe von Mitarbeiter*innen werden zu großen Anteilen voll oder eher zugestimmt (stimme voll zu 53 \%, stimme eher zu $34 \%$ ). Eine Mehrheit der befragten Einrichtungsleitungen stimmt zu, dass eine Funktionsstelle oder Person, die die Umsetzung von Teilhabewünschen der Bewohner*innen koordiniert, notwendig ist oder wäre (stimme voll zu 33 \%, stimme eher zu $30 \%$ ). Der Anteil der Einrichtungsleitungen, die zu einer anderen Einschätzung kommen, weicht von dem bisherigen Antwortverhalten ab (26\% stimmen eher nicht zu, $11 \%$ stimmen nicht zu). Dabei bewerten Einrichtungen, die bereits eine Koordinationsstelle oder eine zuständige Person haben, diese als förderlicher im Vergleich zu Einrichtungen, in denen die Teilhabewünsche nicht zentral koordiniert werden (Mann-Whitney-U-Test, $\mathrm{U}=2812, \mathrm{p}<.01$ ). Insgesamt stimmen 83 Einrichtungsleitungen der Aussage voll (33\%) oder eher zu (30\%), dass eine Funktionsstelle für die Förderung von Teilhabe notwen- 
dig wäre. Auf die Frage, wo diese Koordinationsstelle angesiedelt werden sollte (Wenn Sie an Ihre Einrichtung denken, in welchem Arbeitsbereich sollte eine Funktionsstelle oder Person angesiedelt sein, die die Umsetzung der Wünsche der Bewohner*innen koordiniert?), geben $83 \%$ den Sozialen Dienst und $16 \%$ die Pflege an.

Unterschiede, die auf Kontextfaktoren (Trägerschaft, Einrichtungsplätze, Gemeindetyp) zurückzuführen sind, wurden für die Funktionsstellen zur Koordinierung untersucht (Ist-Zustand und Bewertung der Relevanz). Die Analyse der Kontextfaktoren soll dem Umstand Rechnung tragen, dass in der qualitativen Studie die Vielfalt an Einrichtungen nicht abgebildet werden kann. Daher wurden der Einfluss der Trägerschaft, die Größe einer Einrichtung und die räumliche Lage analysiert. Es konnte nicht nachgewiesen werden, dass Kontextfaktoren einen Einfluss besitzen.

Aus den Ergebnissen der quantitativen Umfrage lässt sich in Bezug auf die qualitativen Ergebnisse ableiten, dass viele als relevant identifizierten Faktoren bereits in Einrichtungen bestehen bzw. angewendet werden (> $90 \%$ ), wobei hier nur deren Existenz (ja/nein), nicht aber die Intensität ihrer Umsetzung erfragt wurde. Jedoch wurden diese Faktoren auch überwiegend als notwendig angesehen, um die Teilhabe der Bewohner*innen zu fördern (> $50 \%$ stimmen voll zu, > $30 \%$ stimmen eher zu). Ausnahmen - mit vergleichsweise geringeren Werten - bilden gezielte Maßnahmen, um die Teilhabewünsche zu erfassen (Ist-Zustand), und eine zentrale Stelle oder Person, die die Umsetzung der Teilhabewünsche koordiniert (Ist-Zustand und Bewertung der Relevanz).

\subsection{Zusammenarbeit und Aufgaben der Arbeitsbereiche}

Die qualitativen Ergebnisse haben gezeigt, dass die Arbeitsweise zwischen den Arbeitsbereichen eine relevante Schnittstelle in der Planung und Verwirklichung von Teilhabeangeboten darstellt sowie dass es förderlich ist, wenn Teilhabeförderung als bereichsübergreifende Aufgabe verstanden wird. Neben der Weitergabe von Informationen, der Besprechung von teilhaberelevanten Themen und der Dokumentation von Teilhabewünschen wurde in diesem inhaltlichen Abschnitt auch nach der Verantwortlichkeit und den Schnittstellen zwischen den Arbeitsbereichen gefragt. 


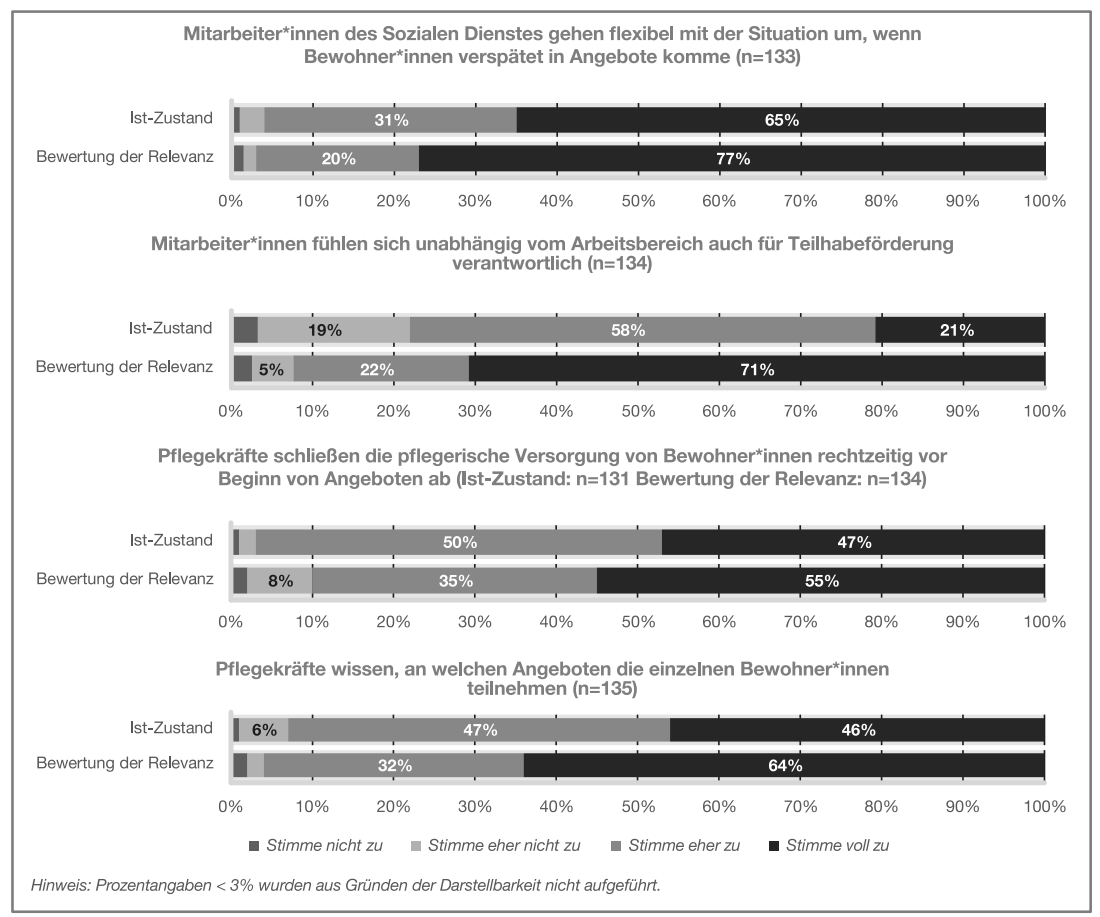

\section{Abbildung 7: Zusammenarbeit zwischen den Arbeitsbereichen (Ist-Zustand und Bewertung der Relevanz)}

In Abbildung 7 sind Items zur Zusammenarbeit zwischen den Arbeitsbereichen dargestellt, zu denen die Einrichtungsleitungen sowohl den Ist-Zustand als auch die Bewertung der Relevanz auf einer vierstufigen Skala bewertet haben. Vergleichbar mit den Angaben zur Verankerung von Teilhabe zeigt das Gesamtbild ebenfalls eine allgemein hohe Zustimmung beim Ist-Zustand (stimme voll bzw. stimme eher zu). Die Relevanz wurde bei allen Items noch höher bewertet als der Ist-Zustand. Dies zeigt sich insbesondere bei der Frage nach der Verantwortung für Teilhabeförderung als bereichsübergreifende Aufgabe. Die Einrichtungsleitungen stimmten voll (21\%) und mehrheitlich eher zu (58\%), dass die Mitarbeiter*innen sich unabhängig von ihrem Arbeitsbereich für die Teilhabeförderung verantwortlich fühlen. Die Relevanz für die Teilhabeförderung wird von den Einrichtungsleitungen aber noch höher bewertet. Der Aussage, dass sich alle Mitarbeiter*innen für die Teilhabeförderung verantwortlich fühlen sollten, um die Teilhabe der Bewohner*innen zu fördern, stimmen über 
zwei Drittel der Einrichtungsleitungen voll (71\%) oder eher (22\%) zu. Dieser Unterschied, auf einem insgesamt hohen Zustimmungsniveau, zwischen Ist-Zustand und der Bewertung der Relevanz verweist auf ein Potenzial, aus der sich ein Ansatzpunkt für die Entwicklung des Musterrahmenkonzeptes ableiten lässt. Die Analyse der Kontextfaktoren hat hier ergeben, dass die Einschätzung zum Ist-Zustand positiv mit der Anzahl der Plätze einer Einrichtung korreliert ( $r$ ho $=0.18, \mathrm{p}<.05)$. Dabei handelt es sich nach Cohen (1992) allerdings um einen schwachen Effekt.

Doch auch zu den weiteren Item-Inhalten sind hohe Zustimmungswerte in Abbildung 7 erkennbar. So weisen die Angaben der Einrichtungsleitungen auch darauf hin, dass die Pflegekräfte wissen, an welchen Angeboten die Bewohner*innen teilnehmen (46\% stimmen voll zu, $47 \%$ stimmen eher $\mathrm{zu})$ und dies auch als wichtig erachten $(64 \%$ stimmen voll zu, $32 \%$ stimmen eher zu). Sie geben an, dass die Mitarbeiter*innen anstreben, die Pflege rechtzeitig vor Beginn von Angeboten abzuschließen $(47 \%$ stimmen voll zu, 50\% stimmen eher zu) und beschreiben dies auch als notwendig ( $55 \%$ stimmen voll zu, $35 \%$ stimmen eher zu). Ebenso scheint es auch nur ein geringes oder gar kein Problem zu sein, wenn Bewohner*innen verspätet zu Angeboten des Sozialen Dienstes kommen. Die Einrichtungsleitungen haben voll $(65 \%)$ oder eher $(31 \%)$ zugestimmt, dass die Mitarbeiter*innen flexibel mit verspäteter Teilnahme in Angeboten umgehen und diese Flexibilität auch als notwendig bewerten $(77 \%$ stimmen voll zu, $20 \%$ stimmen eher zu).

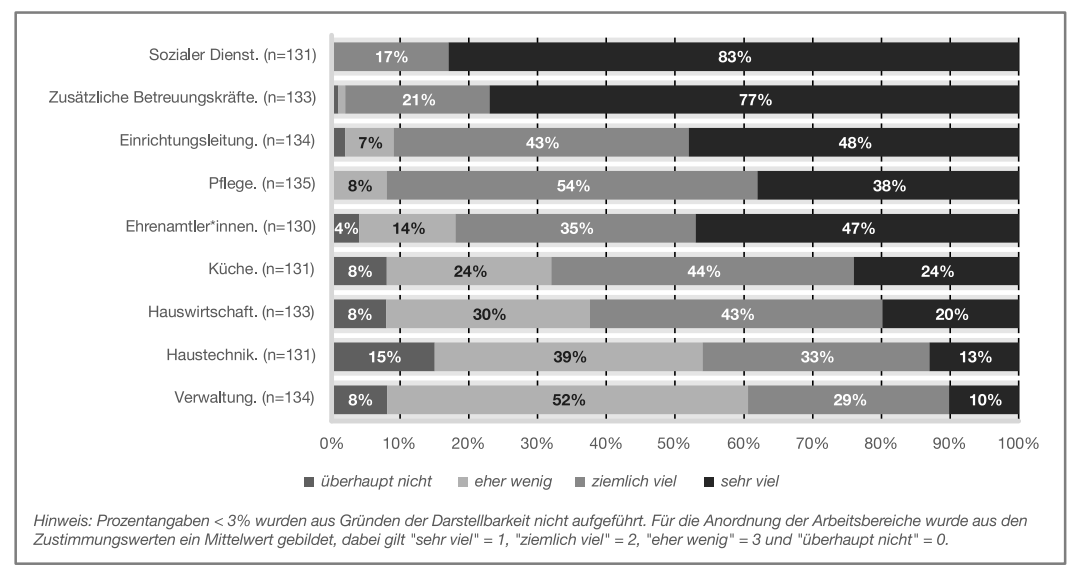

Abbildung 8: Beitrag zur Umsetzung von Teilhabewünschen nach Arbeitsbereichen 
In Abbildung 8 ist nun der jeweilige Anteil dargestellt, den die einzelnen Arbeitsbereiche nach Einschätzung der Einrichtungsleitungen zur Umsetzung von Teilhabewünschen der Bewohner*innen beitragen. Auf einer vierstufigen Skala (sehr viel, ziemlich viel, eher wenig, überhaupt nicht) wurde der Beitrag des Sozialen Dienstes und der zusätzlichen Betreuungskräfte als besonders hoch eingeschätzt. Auch die Einrichtungsleitung, Pflege und Ehrenamtler*innen tragen viel zur Umsetzung von Teilhabewünschen bei, während die Küche, Hauswirtschaft, Haustechnik und Verwaltung einen vergleichsweise geringeren Beitrag leisten. Auch wenn aufgrund der Aufgabenzuschreibungen ein unterschiedlicher Beitrag nicht überraschend erscheint, so zeigen die Daten einerseits aus Sicht der Befragten deutlich, von welchen Bereichen zur Umsetzung von Teilhabewünschen in vergleichsweise höherem Ausmaß beigetragen wird, andererseits bieten die Daten Anlass zur Reflexion, inwieweit Entwicklungspotenzial besteht, Teilhabeförderung als bereichsübergreifende Aufgabe zu verstehen.

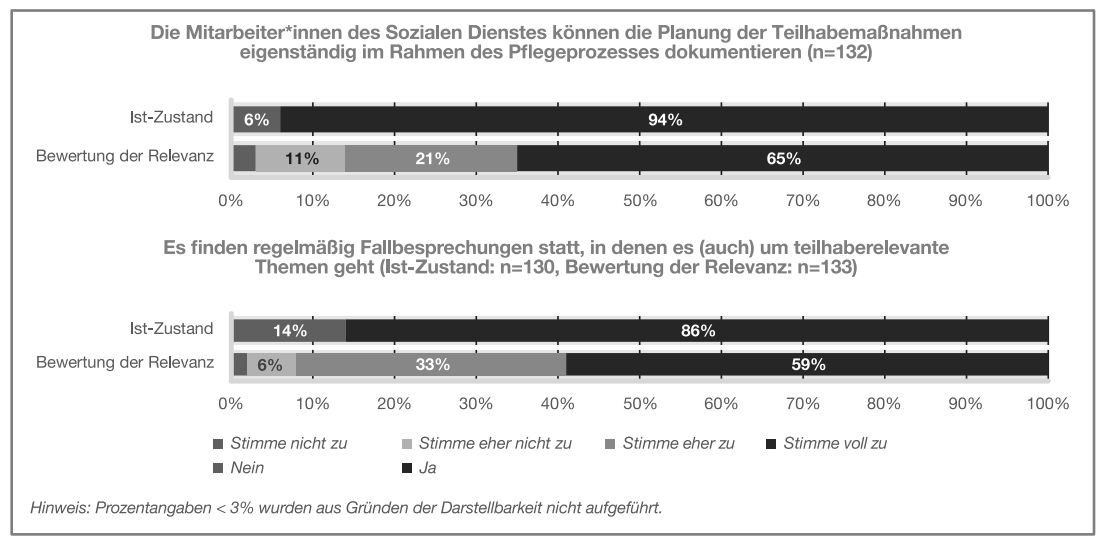

$\begin{aligned} \text { Abbildung 9: } & \text { Planung der Teilhabemaßnahmen und Fallbesprechungen (Ist- } \\ & \text { Zustand und Bewertung der Relevanz) }\end{aligned}$

Die nächsten Items in Abbildung 9 thematisieren die Planung von Teilhabemaßnahmen sowie die Existenz regelmäßiger Fallbesprechungen mit teilhaberelevanten Themen. In Bezug auf ihre Einrichtungen haben $94 \%$ der Einrichtungsleitungen angegeben, dass die Mitarbeiter"innen des Sozialen Dienstes Teilhabemaßnahmen der Bewohner"innen eigenständig im Rahmen des Pflegeprozesses dokumentieren können. Bei der Bewertung der Relevanz zeigt sich ebenfalls eine hohe Zustimmung: So halten die Einrichtungsleitungen es wiederum mehrheitlich für notwendig, dass 
die Mitarbeitenden des Sozialen Dienstes, die Teilhabemaßnahmen dokumentieren können, um die Teilhabe zu fördern (63\% stimmen voll zu, $21 \%$ stimmen eher zu). Dass die Mitarbeiter*innen des Sozialen Dienstes in Abstimmung mit der zuständigen Pflegefachkraft Teilhabemaßnahmen im Rahmen des Pflegeprozesses planen, wird aus Sicht der Einrichtungsleitungen ebenfalls als wichtig bewertet $72 \%$ stimmen voll zu, $22 \%$ stimmen eher zu; nicht in der Abbildung dargestellt).

Eine Möglichkeit, in der die Teilhabesituation von einzelnen Bewohner*innen besprochen werden kann, stellen Fallbesprechungen dar. Wie auch in Abbildung 9 aufgeführt, finden Fallbesprechungen, in denen es (auch) um teilhaberelevante Themen geht, nach Angaben der Einrichtungsleitungen in $86 \%$ der Einrichtungen statt und werden als wichtig eingeschätzt ( $59 \%$ stimmen voll zu, $33 \%$ stimmen eher zu). Dass die Häufigkeit und die Teilnehmer*innengruppen nach Arbeitsbereichen aber variieren, zeigt die Auswertung der folgenden Filterfragen: In der Mehrheit der Einrichtungen, deren Einrichtungsleitungen die Existenz von regelmäBigen Fallbesprechungen bejaht haben, finden die Fallbesprechungen mindestens einmal im Monat statt (44\%) bzw. einmal in 2 bis 3 Monaten (36\%), einmal in 4 bis 6 Monaten ( $7 \%$ ) bzw. einmal in 7 bis 12 Monaten ( $5 \%$; Wie häufig finden Fallbesprechungen statt, in denen es - auch - um teilhaberelevante Themen geht?; hier ohne Abbildung). Weitere Einrichtungsleitungen haben unter der offenen Kategorie „Andere Häufigkeit“ etwa angegeben, dass in ihrer Einrichtung die Fallbesprechungen nach Bedarf stattfinden oder in der Eingewöhnungsphase nach dem Einzug (8\%).

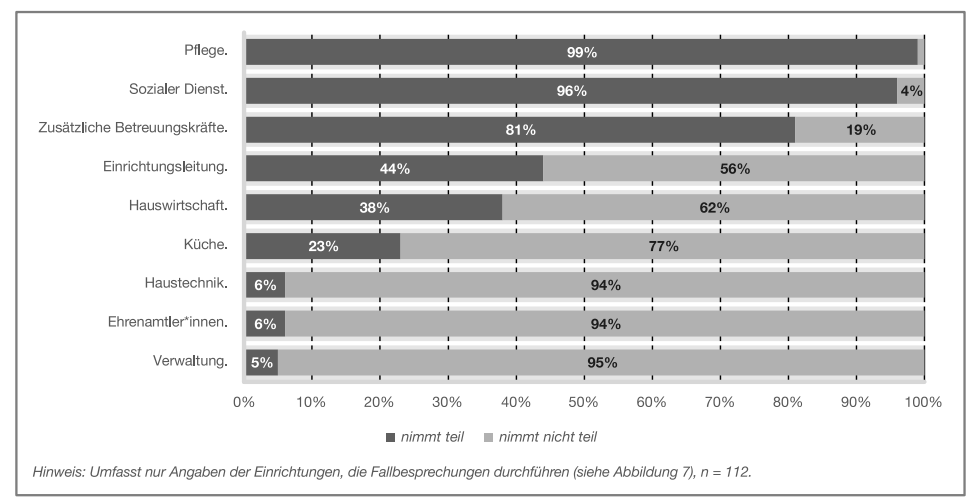

Abbildung 10: Arbeitsbereiche, die in der Regel an Fallbesprechungen teilnehmen 
In Abbildung 10 sind die Angaben der Einrichtungsleitungen zu den Arbeitsbereichen dargestellt, die in der Regel an den Fallbesprechungen teilnehmen (Bitte geben Sie in Bezug auf Ihre Einrichtung an, welche Arbeitsbereiche in der Regel an Fallbesprechungen teilnehmen, in denen es um teilhaberelevante Themen geht). In fast allen Einrichtungen nehmen die Mitarbeiter*innen der Pflege (99\%) und des Sozialen Dienstes (96\%) teil, auch die zusätzlichen Betreuungskräfte (81\%) sind in der überwiegenden Anzahl der teilnehmenden Einrichtungen in die Besprechungen eingebunden. In weniger als der Hälfte der Einrichtungen nehmen die Einrichtungsleitungen (44\%), die Mitarbeiter*innen der Hauswirtschaft (38\%) und der Küche (23\%) an den Fallbesprechungen teil. Nur in seltenen Fällen sind Mitarbeiter*innen der Haustechnik (6\%), Ehrenamtler*innen $(6 \%)$ oder Verwaltungskräfte $(5 \%)$ Teilnehmer*innen von Fallbesprechungen, in denen es auch um teilhaberelevante Themen geht. Diese Angaben verweisen darauf, dass der Austausch über Fallbesprechungen zu teilhaberelevanten Themen - erwartungsgemäß - nicht alle Arbeitsbereiche gleichermaßen erreicht und drei Bereiche dabei im Vordergrund zu stehen scheinen.

Die Ergebnisse der quantitativen Erhebung deuten darauf hin, dass die aus der qualitativen Studie gewonnenen Erkenntnisse um zentrale Schnittstellen in den meisten Einrichtungen bereits im Sinne der Teilhabeförderung gestaltet werden. Die Einrichtungsleitungen stimmen voll oder eher zu, dass die Zusammenarbeit durch Flexibilität im Umgang mit Verspätungen und dem Wissen um anschließende Angebote der Bewohner*innen gekennzeichnet ist und die Dokumentation von Teilhabemaßnahmen auch durch Mitarbeiter*innen des Sozialen Dienstes erfolgen kann. Diese Schnittstellen werden auch als entsprechend relevant für die Teilhabeförderung eingeschätzt. Dass sich alle Mitarbeitenden, unabhängig vom Arbeitsbereich, für die Teilhabeförderung verantwortlich fühlen, wird als notwendig bewertet, scheint aber nicht in allen Einrichtungen der Ist-Zustand zu sein. Hier hat die Analyse der Kontextfaktoren gezeigt, dass dies in Einrichtungen mit mehr Plätzen eher der Fall ist als in kleineren. Insbesondere die Arbeitsbereiche Verwaltung, Hauswirtschaft und Haustechnik tragen nach Angaben der Einrichtungsleitung wenig zur Umsetzung von Teilhabewünschen bei. Der differenzierte Blick auf Fallbesprechungen verdeutlicht, dass in den meisten Einrichtungen Fallbesprechungen zu teilhaberelevanten Themen stattfinden, die unterschiedlich oft durchgeführt werden und selten alle Arbeitsbereiche einschließen. Für das Musterrahmenkonzept könnten Fallbesprechungen oder andere Besprechungen, in denen teilhaberelevante Themen diskutiert bzw. bearbeitet werden, ein 
Mechanismus darstellen, um das Ziel der Teilhabeförderung als bereichsübergreifende Aufgabe zu fördern.

\subsection{Handlungsspielräume und Ressourcen}

Im Abschnitt Handlungsspielräume und Ressourcen wird der Umgang von Mitarbeiter*innen mit Aufgaben und Zeiten sowie die Legitimation durch Vorgesetzte und die Akzeptanz durch Kolleg*innen, wenn von routinierten Abläufen abgewichen wird, in den Blick genommen. Die qualitativen Ergebnisse haben gezeigt, dass es fördernd ist, wenn Mitarbeitende Vorgaben flexibel und situativ (Zeiten/Aufgaben) den aktuellen Bedingungen und Bedürfnissen der Bewohner*innen anpassen können. Diese Arbeitsweise wird erleichtert, wenn das Denken und Handeln nicht an das „Schichtprinzip“ geknüpft ist, sondern Abweichungen akzeptiert und legitimiert sind. Der Umgang mit diesen Arbeitsanforderungen wird dabei maßgeblich den persönlichen Eigenschaften und Kompetenzen - der Fähigkeit zum flexiblen Handeln - zugeschrieben. Als erschwerend für die Teilhabeförderung werden Personalmangel und fehlende zeitliche Ressourcen beschrieben.

In der quantitativen Befragung wurde der Ist-Zustand hierzu fast ausnahmslos positiv (Ich stimme voll zu bzw. stimme eher zu) beschrieben. Ein heterogenes Bild zeigt sich hingegen bei den Angaben zur Bewertung der Relevanz: Die Einrichtungsleitungen haben hier weitaus weniger häufig zugestimmt, dass die aufgeführten Faktoren ein Hemmnis darstellen, wenn die Faktoren nicht vorliegen. Zu erwähnen und berücksichtigen ist hierzu aber: Um einer inhaltsunabhängigen Zustimmungstendenz entgegenzuwirken (Porst, 2014), wurde in den Items zur Bewertung der Relevanz diesmal gefragt, ob das „Nichtvorliegen“ ein „Hemmnis“ für die Teilhabeförderung darstellt. Dass diese Veränderung in der Frageformulierung einen Effekt auf das Antwortverhalten hatte, kann nicht ausgeschlossen werden, da die Zustimmungswerte hier vergleichsweise gering ausfallen. Dem entgegen und damit passend zu den geringen Zustimmungswerten steht, dass bei der Bewertung der dringendsten Veränderungen (Abschnitt 5.6) die Handlungsspielräume aus Sicht der Einrichtungsleitungen am wenigsten dringend verändert werden müssten. 


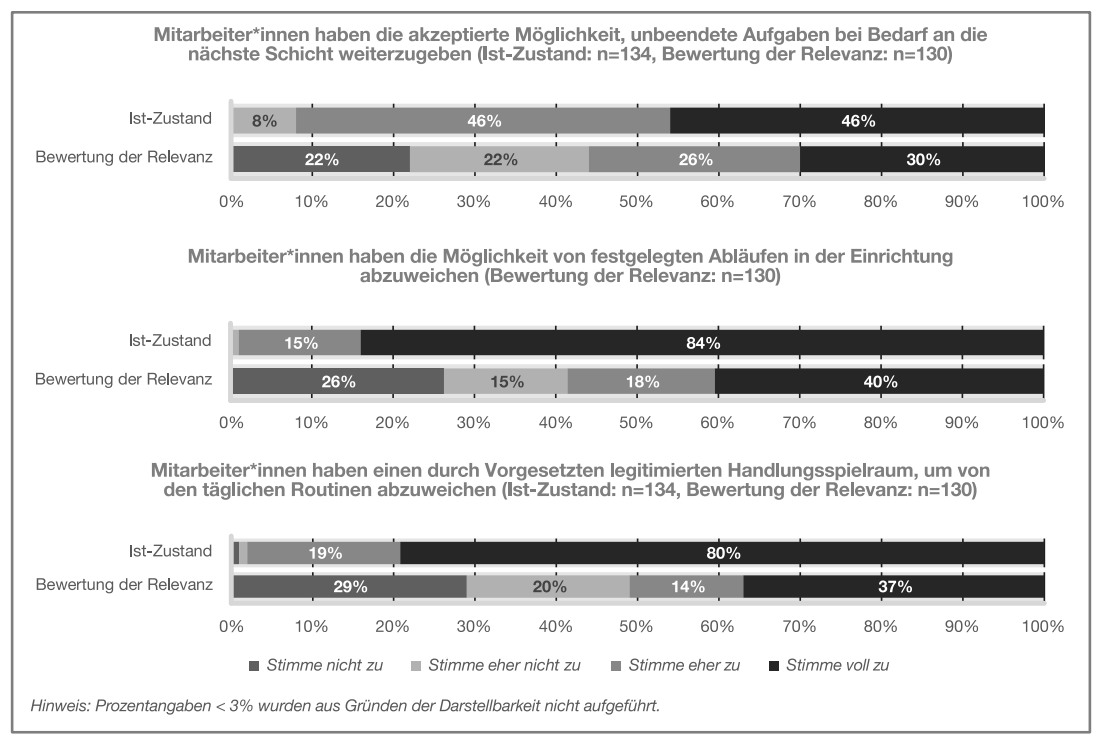

\section{Abbildung 11: Handlungsspielräume (Ist-Zustand und Relevanz der Bewertung)}

Abbildung 11 können also wieder Einschätzungen zum Ist-Zustand und zur Relevanz entnommen werden: Die Möglichkeit, dass Mitarbeiter*innen unbeendete Aufgaben an die nächste Schicht weitergeben, ist nach Angaben der Einrichtungsleitungen verbreitet $(46 \%$ stimmen voll zu, $46 \%$ stimmen eher zu). Etwas mehr als die Hälfte der Einrichtungen bewerten es als ein Hemmnis, wenn die Möglichkeit nicht bestehen würde (30\% stimmen voll zu, $26 \%$ stimmen eher zu). Dass Mitarbeiter*innen die Möglichkeit haben, von festgelegten Abläufen in der Einrichtung abzuweichen, ist nach Angaben der Einrichtungsleitungen fast immer möglich (84\% stimmen voll zu, $15 \%$ stimmen eher zu). Wenn Mitarbeiter"innen diese Flexibilität nicht besitzen würden, dann bewerten die Einrichtungsleitungen dies mehrheitlich als Hemmnis (40\% stimmen voll zu, $18 \%$ stimmen eher zu). Dass die Mitarbeiter"innen einen durch Vorgesetzten legitimierten Handlungsspielraum haben, ist nach Angaben der Einrichtungsleitungen fast immer gegeben $(80 \%$ stimmen voll zu, 19\% stimmen eher zu). Wenn kein legitimierter Handlungsspielraum besteht, dann wäre dies für etwa die Hälfte der Einrichtungsleitungen ein Hemmnis (37\% stimmen voll zu, $14 \%$ stimmen eher zu). 


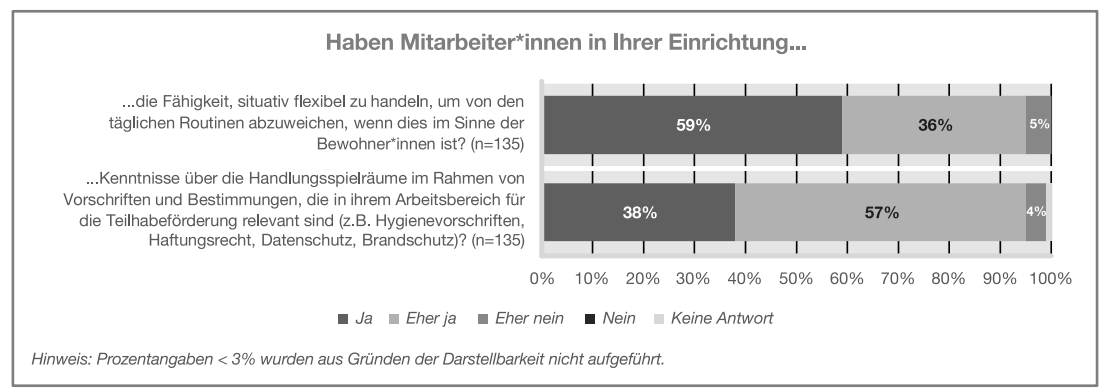

\section{Abbildung 12: Kenntnisse über Handlungsspielräume im Rabmen von Vorschriften und Bestimmungen (Ist-Zustand)}

Nach Angaben der Einrichtungsleitungen verfügen die Mitarbeitenden auch über die Fähigkeit, situativ und flexibel zu handeln, um von den täglichen Routinen abzuweichen, wenn dies im Sinne der Bewohner*innen ist (Abbildung 12). Auf einer vierstufigen Skala (ja/eher ja/eher nein/nein) bejahten fast alle Einrichtungsleitungen diese Frage (59\% ja, $36 \%$ eher ja). Wenn diese Fähigkeit nicht bestehen würde (Wenn Sie an Ihre Einrichtung denken, was ist oder wäre ein Hemmnis, um die Teilhabe der Bewohner*innen zu fördern? Die Mitarbeiter*innen haben nicht die Fähigkeit, situativ flexibel zu handeln, um von den täglichen Routinen abzuweichen), dann würde dies für mehr als die Hälfte der Einrichtungen ein Hemmnis für die Teilhabeförderung darstellen (37\% stimmen voll zu, $24 \%$ stimmen eher zu).

Neben der Fähigkeit wurden Einrichtungsleitungen gefragt, ob die Mitarbeiter*innen Kenntnisse über die Handlungsspielräume im Rahmen von Vorschriften und Bestimmungen, die in ihrem Arbeitsbereich für die Teilhabeförderung relevant sind (z. B. Hygienevorschriften, Haftungsrecht, Datenschutz, Brandschutz), besitzen (Abbildung 12). Die Einrichtungsleitungen gaben an, dass dies meistens zutrifft ( $39 \%$ ja) bzw. mehrheitlich eher zutrifft ( $57 \%$ eher ja). Fehlende Kenntnisse würden mehr als die Hälfte der Einrichtungsleitungen als Hemmnis beschreiben (29\% stimmen voll zu, $26 \%$ stimmen eher zu). 


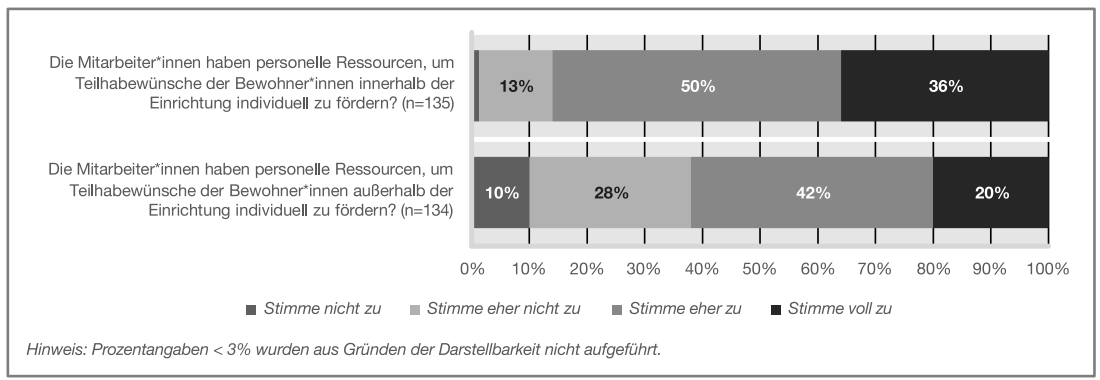

Abbildung 13: Personelle Ressourcen innerhalb und außerhalb der Einrichtung (Ist-Zustand)

Die Personalsituation wird abweichend zu den qualitativen Ergebnissen aus Sicht der Einrichtungsleitungen eher positiv beschrieben (Abbildung 13), wenn es sich um die individuelle Förderung von Teilhabewünschen innerhalb der Einrichtungen handelt (Die Mitarbeiter*innen meiner Einrichtung haben personelle Ressourcen, um Teilhabewünsche der Bewohner*innen innerhalb der Einrichtung individuell zu fördern). Die Mehrheit der Einrichtungen stimmt zu (36\% stimmen voll zu, $50 \%$ stimmen eher $\mathrm{zu}$ ), dass personelle Ressourcen vorhanden sind. Geringere Zustimmungswerte gaben die Einrichtungsleitungen an, wenn es um die personellen Ressourcen für die Förderung von individuellen Teilhabewünschen außerhalb der Einrichtung geht (Die Mitarbeiter*innen meiner Einrichtung haben personelle Ressourcen, um Teilhabewünsche der Bewohner*innen außerhalb der Einrichtung individuell zu fördern). Aber auch hier geben deutlich mehr als die Hälfte an, dass auch für die Förderung auBerhalb der Einrichtung personelle Ressourcen vorhanden sind (20\% stimmen voll zu, $42 \%$ stimmen eher zu). Dabei konnte für die Items zu den personellen Ressourcen kein signifikanter Effekt der Trägerschaft, des Gemeindetyps oder der Größe der Einrichtung nachgewiesen werden.

Die Ergebnisse weisen auch in Bezug auf den Handlungsspielraum darauf hin, dass die in der qualitativen Studie identifizierten fördernden Faktoren in den meisten Einrichtungen vorliegen: Mitarbeitende haben - in der Bewertung durch die Einrichtungsleitungen - den Spielraum und die Fähigkeit, um von den festgelegten Abläufen abzuweichen, dieser situativ flexibler Umgang ist in den meisten Einrichtungen durch die Vorgesetzten legitimiert und von den Kolleg*innen akzeptiert. In der qualitativen Studie wurde die Personalsituation als einschränkender Faktor für die Teilhabeförderung benannt, dies wird von den Einrichtungsleitungen, die an der quantitativen Umfrage teilgenommen haben, anders angegeben, wenn- 
gleich die Zustimmungswerte hier vergleichsweise geringer als bei anderen Items waren. Diese Diskrepanz kann unter Umständen insofern aus der Monoperspektive resultieren, als dass sich die Perspektive der Einrichtungsleitung von der Ansicht der Mitarbeiter*innen aus anderen Arbeitsbereichen unterscheidet. Die Bewertung der Relevanz ist, wie zu Beginn des Abschnitts beschrieben, möglicherweise durch die veränderte Frageformulierung verzerrt. Es konnte nicht gezeigt werden, dass die Kontextfaktoren einen Einfluss auf die Bewertung der Handlungsspielräume und Ressourcen besitzen.

\subsection{Arbeitskulturelle Orientierungen}

Die Arbeitskultur bezieht sich auf Werte und Normen der Mitarbeitenden, die das individuelle Handeln und die Beziehung zwischen Bewohner*innen und Mitarbeitenden prägen. Dabei wurde hier zwischen Handlungen, die sich (1) an den individuellen Bedürfnissen der Bewohner*innen ausrichten und Handlungen, die sich (2) am Aufrechterhalten der Versorgung und den Strukturen der Einrichtung orientieren, unterschieden, da die qualitativen Ergebnisse darauf hinweisen, dass die erste Dimension sich förderlich und die zweite sich hemmend auf die selbstbestimmte Teilhabe der Bewohner*innen auswirkt. In der quantitativen Studie wurden beide Dimensionen erfragt. Da sich beide Handlungsorientierungen nicht zwingend gegenseitig ausschließen, wurden diese nicht als Endpunkte einer Dimension verstanden, sondern als zwei getrennte Dimensionen erfasst.

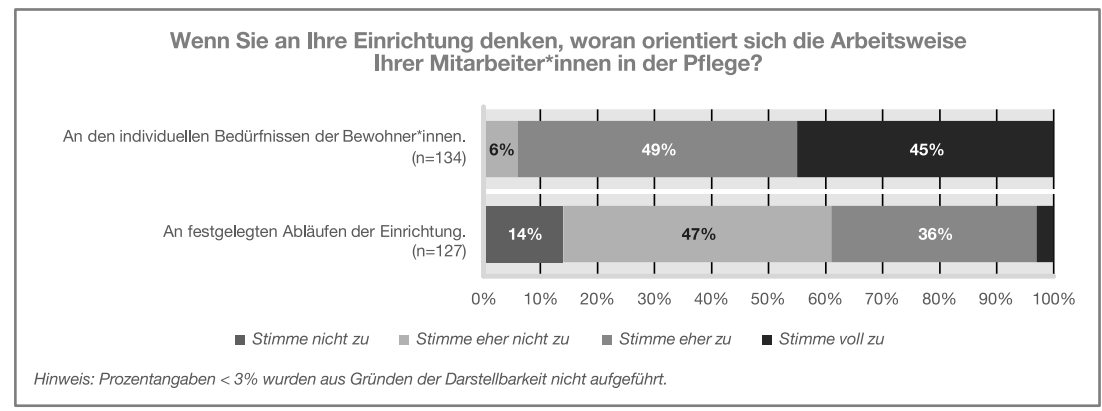

Abbildung 14: Arbeitskulturelle Orientierung

In Abbildung 14 sind die Angaben der Einrichtungsleitungen dargestellt. Demnach orientiert sich die Arbeitsweise der Mitarbeiter*innen nach Ein- 
schätzung der Einrichtungsleitungen zu sehr hohen Anteilen an den individuellen Bedürfnissen der Bewohner*innen (45\% stimmen voll zu, $49 \%$ stimmen eher zu). Die festgelegten Abläufe der Einrichtung werden deutlich weniger als Handlungsorientierung angegeben $(2 \%$ stimmen voll zu, $36 \%$ stimmen eher zu) und spielen damit aus der Perspektive der Einrichtungsleitungen eine untergeordnete Rolle für die Arbeitsweise der Mitarbeiter*innen (14\% stimmen nicht zu, $47 \%$ stimmen eher nicht zu).

\subsection{Wichtige Faktoren und dringende Veränderungen}

Die qualitativen Ergebnisse haben eine Vielzahl von Bedingungen identifiziert, die für die Teilhabe der Bewohner*innen wichtig sind, sowie zahlreiche Ansatzpunkte abgeleitet, die zur Förderung der Teilhabe beitragen können. In der in diesem Kapitel dargestellten quantitativen Folgestudie haben die Einrichtungsleitungen sowohl den Ist-Zustand als auch die Relevanz von ausgewählten Ergebnissen bewertet. Um zudem im Vergleich besonders wichtige Faktoren und dringende Änderungsbedarfe identifizieren zu können, sollten die Einrichtungsleitungen abschließend in Bezug auf die inhaltlichen Abschnitte zwei Rangfolgen bilden.

Die Einrichtungsleitungen wurden gebeten, vorgegebene Antwortmöglichkeiten in eine Reihenfolge zu bringen, um anzugeben, (a) was in ihrer Einrichtung am wichtigsten ist, damit die Teilhabe von Bewohner*innen gefördert wird, und (b) was am dringendsten verändert werden müsste, um die Teilhabe zu fördern.

Um die Antwortoptionen über die Ränge vergleichen zu können, wurde für jede Antwortoption ein Score-Wert ermittelt, der das durchschnittliche Ranking angibt. Für jede Antwortoption $\mathrm{j}$ wurde der Score wie folgt berechnet:

$$
\text { Score }_{j}=\frac{\sum_{i=1}^{n}\left(x_{i} * w_{i}\right)}{x_{0}+\sum x_{i}} * \frac{100}{n}
$$

Dabei stellt $x$ die Beantwortungsanzahl für jeden Rang $i, \ldots, n$ dar und $w$ gibt die Gewichtung an. Dabei gilt $w_{i}=(n+1)-i$, so dass die Option auf dem ersten Rang das höchste Gewicht und auf dem letzten Rang ein Gewicht von 1 erhält. Berücksichtigt sind nur Teilnehmende, die mindestens einen Faktor bewertet haben. Antworten, die nicht als relevant erachtet wurden (siehe Anweisung zur Beantwortung), werden als $\mathrm{x}_{0}$ in der Berechnung der Gesamtanzahl an Beobachtungen berücksichtigt und haben damit ein Gewicht von 0. Der Score-Wert ist damit auf 0 bis 100 normiert. 
Dabei gilt ein Wert von 100, wenn alle Befragten diesem Faktor als am wichtigsten bewertet haben und ein Wert von 0 , wenn kein/e Teilnehmer*in diesen Faktor als relevant bewertet hat. Die Angaben können nur relational zu den anderen Antwortmöglichkeiten interpretiert werden.

Damit (a) die Teilhabe von Bewohner*innen gefördert wird, sollten die Einrichtungsleitungen eine Reihenfolge erstellen, geordnet nach dem wichtigsten Faktor, und angeben, ob es von den Bewohner*innen, von der/dem einzelnen Mitarbeiter*in, von den Ressourcen der Einrichtung oder von der Zusammenarbeit zwischen den Mitarbeiter*innen abhängt (Wovon hängt es in Ihrer Einrichtung ab, ob die Teilhabe von Bewohner*innen gefördert wird? Bitte bringen Sie die Antwortmöglichkeiten in eine Reihenfolge, die oberste Antwort ist dabei am wichtigsten. Wenn Antworten nicht relevant sind, bitte diese nicht einordnen).

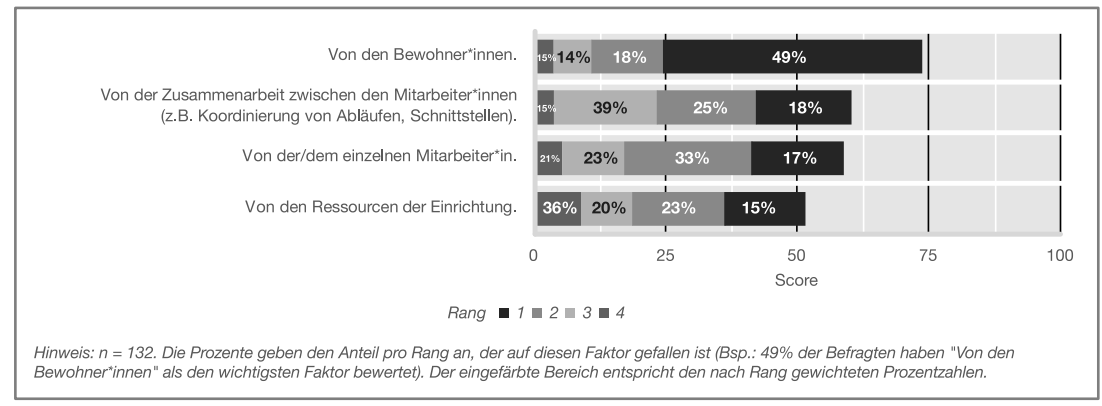

\section{Abbildung 15: Dringendste Veränderung für Teilhabe (Score-Werte nach Rangfolge)}

In Abbildung 15 sind die Score-Werte abgetragen und die Prozentzahlen angegeben, die sich auf die einzelnen Ränge beziehen. Wenn sich alle Antworten zu gleichen Anteilen auf die Ränge verteilen, dann hätte jede Antwort einen Score-Wert von 62,5. Die Einrichtungsleitungen haben durchschnittlich angegeben, dass die Teilhabeförderung an erster Stelle abhängig ist von den Bewohner*innen (Score-Wert: 74), gefolgt von der Zusammenarbeit zwischen den Mitarbeiter*innen (60), der/dem einzelnen Mitarbeiter"in (59) und relational am wenigsten von den Ressourcen der Einrichtung (52). Die Zuordnung, dass Teilhabeförderung vorrangig von den Bewohner*innen selber abhängig ist und weniger von den Ressourcen einer Einrichtung, lässt Interpretationsspielraum, kann aber einen Hinweis darauf geben, dass - aus Sicht der Einrichtungsleitungen - möglicherweise weniger die Umsetzungsressourcen die Teilhabeförderung beeinflussen 
bzw. beschränken, sondern vielmehr die (heterogenen) Voraussetzungen der Bewohner*innen über die Möglichkeiten zur Teilhabe und ihrer Förderung entscheiden.

Neben den wichtigsten Faktoren, die sich auf die aktuelle Situation in den Einrichtungen bezieht, wurde (b) nach den dringendsten Veränderungen gefragt (Um die Teilhabe von Bewohner*innen zu fördern, gibt es viele Ansatzpunkte. Was müsste in Ihrer Einrichtung am dringendsten verändert werden, um die Teilhabe von Bewohner*innen zu fördern? Bitte bringen Sie die Antwortmöglichkeiten in eine Reihenfolge, die oberste Antwort ist dabei am wichtigsten. Wenn Antworten nicht relevant sind, bitte diese nicht einordnen). Die Einrichtungsleitungen sollten in Bezug auf ihre Einrichtung folgende Antwortmöglichkeiten in eine Rangfolge bringen: Die Mitarbeiter*innen brauchen die fachliche Voraussetzung zur Teilhabeförderung; die Mitarbeiter*innen brauchen mehr Handlungsspielraum, um Wünsche umsetzen zu können; es muss eine verbindliche Weitergabe von Wünschen stattfinden; es werden mehr personelle Ressourcen benötigt; und Teilhabe muss als bereichsübergreifende Aufgabe verstanden werden. Wenn alle Antworten zu gleichen Teilen den Rängen zugeordnet wären, dann beträgt der Score-Wert 60.

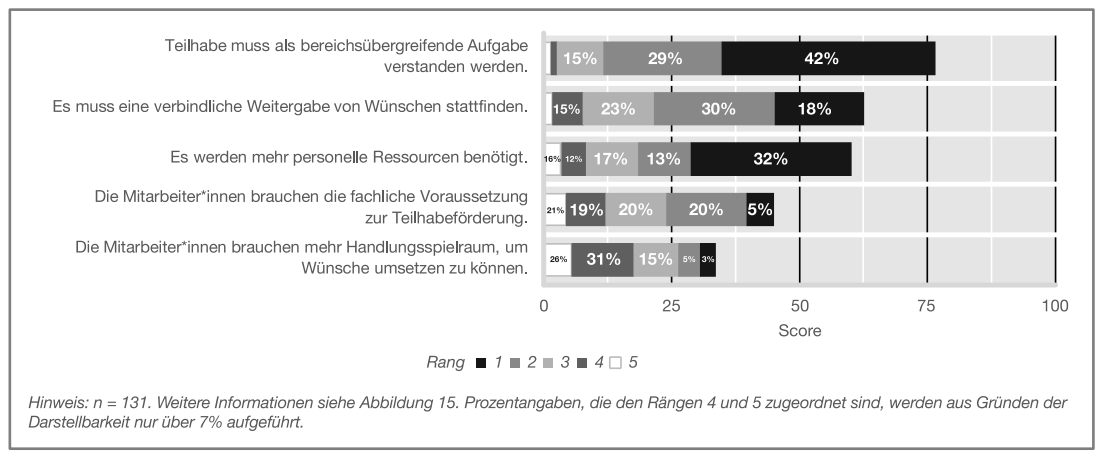

Abbildung 16: Wichtige Faktoren für die Teilhabeförderung (Score-Werte nach Rangfolge)

In Abbildung 16 sind die Score-Werte dargestellt sowie die Prozente für die Nennungen pro Rang. Die dringendste Veränderung aus Sicht der Einrichtungsleitungen besteht darin, dass Teilhabe als eine bereichsübergreifende Aufgabe verstanden werden sollte (Score-Wert: 77). Nachfolgend werden die verbindliche Weitergabe von Wünschen (63), mehr personelle Ressourcen (61), die fachliche Voraussetzung der Mitarbeiter*innen zur 
Teilhabeförderung (45) und mehr Handlungsspielraum zur Umsetzung der Wünsche (33) als Ansatzpunkte für Veränderungen aufgeführt. Analog zu den Angaben in Abschnitt 5.3 (Zusammenarbeit und Aufgaben der Arbeitsbereiche), in dem der Unterschied zwischen dem Ist-Zustand und der Bewertung der Relevanz bezogen auf die bereichsübergreifende Verantwortung für Teilhabeförderung vergleichsweise groß war, markiert dieser Aspekt einen zentralen Ansatz für Veränderungen. Mit einem sehr geringen Score-Wert haben die Einrichtungsleitungen angegeben, dass die Handlungsspielräume der Mitarbeiter“innen vergrößert werden müssten, was damit den Angaben in Abschnitt 5.4 (Handlungsspielräume und Ressourcen) entspricht. Der Handlungsspielraum ist nach den Angaben der Einrichtungsleitungen zu einem hohen Anteil gegeben und die Relevanz für die Teilhabeförderung wird im Verhältnis zu den anderen Bereichen als gering bewertet. Die personellen Ressourcen werden von fast einem Drittel der Teilnehmenden auf den ersten Rang, aber von vergleichsweise wenigen Einrichtungsleitungen auf die nachfolgenden Plätze gesetzt und von etwa $10 \%$ als nicht relevant bewertet, so dass in der Verrechnung der Ränge die verbindliche Weitergabe von Wünschen einen höheren ScoreWert erreicht.

\subsection{Zwischenfazit zur quantitativen Analyse}

Die quantitative Folgestudie hatte das Ziel, ausgewählte qualitative Ergebnisse hinsichtlich der drei Leitfragen zu untersuchen. (1) Ist-Zustand: Liegen die als relevant identifizierten Rahmenbedingungen in anderen Einrichtungen vor? Die Einrichtungsleitungen haben für die meisten Faktoren zu einem sehr hohen Anteil angegeben, dass sie in ihrer Einrichtung vorliegen. Es ist nicht auszuschließen, dass soziale Erwünschtheit und eine Stichprobenverzerrung (Einrichtungsleitungen, die einen besonderen Wert auf Teilhabe legen) diese Angaben verzerrt haben. Abgesehen von wenigen Ausnahmen lässt sich für die qualitativen Ergebnisse aber schlussfolgern, dass es offenbar weniger um die Implementierung von neuen Faktoren als vielmehr um die Ausgestaltung dessen geht, was bereits vorliegt. Die Einrichtungsleitungen sagen jedoch auch, dass es von den Bewohner*innen abhängt, ob die Teilhabe gefördert wird bzw. werden kann. (2) Bewertung der Relevanz: Wie bewerten Einrichtungsleitungen die Relevanz der identifizierten fördernden und hemmenden Faktoren? Die befragten Einrichtungsleitungen bewerteten die identifizierten fördernden und hemmenden Faktoren fast ausschließlich in derselben Richtung, wie die 
qualitativen Ergebnisse es erwarten ließen. Abstufungen ergaben sich fast nur auf einem sehr hohen Zustimmungsniveau. Eine Ausnahme bildeten die Items zu den Handlungsspielräumen und den Ressourcen, die im Vergleich mit den anderen Faktoren von weniger Einrichtungsleitungen als relevant eingeschätzt wurden, wobei hier auch die besondere Perspektive der Einrichtungsleitung zu berücksichtigen ist. Die Ergebnisse unterstützen damit weitestgehend (mit Ausnahme der beiden Faktorenbereiche) die Annahme, dass die in der qualitativen Analyse identifizierten und für die Onlinebefragung ausgewählten sowie operationalisierten Faktoren relevant für die Förderung der Teilhabe der Bewohner*innen sind. Als dringendste relationale Veränderung wurde angegeben, dass Teilhabe als bereichsübergreifende Aufgabe verstanden wird. (3) Kontext: Unterscheiden sich Einrichtungen hinsichtlich des Ist-Zustandes und der Bewertung der Relevanz aufgrund von Kontextfaktoren? Es konnte nur in einem Fall ein Hinweis darauf gefunden werden (mit einer schwachen Effektstärke), dass der Ist-Zustand oder die Bewertung der Relevanz von Kontextfaktoren abhängig ist. Es ist daher davon auszugehen, dass die qualitativen Ergebnisse nicht für einzelne Träger, Einrichtungsgrößen oder bestimmte Umgebungen förderlich oder hemmend sind bzw. als solche bewertet werden und somit potenziell auch auf andere Einrichtungen übertragen werden können.

Die quantitative Analyse weist daher darauf hin, dass die fördernden Faktoren in vielen Einrichtungen - aus Sicht der Einrichtungsleitungen prinzipiell bereits etabliert sind, wenngleich nur wenige Informationen zur Art und Intensität der Umsetzung vorliegen, die fördernden Faktoren fast ausnahmslos als relevant erachtet werden und die untersuchten Kontextfaktoren keine selektive Relevanz erwarten lassen. 


\section{Entwicklung und Erprobung des Musterrahmenkonzeptes}

Nachfolgend werden einerseits die Entwicklung des Musterrahmenkonzeptes und andererseits dessen Erprobung in einer hierfür ausgewählten Einrichtung sowie die in diesem Rahmen durchgeführte Implementationsanalyse näher erläutert.

\subsection{Entwicklung und Inhalte des Musterrabmenkonzeptes}

Das Musterrahmenkonzept (MRK) wurde auf Grundlage der empirischen Befunde entwickelt und als ein Instrument der Reflexion, Selbsteinschätzung und Weiterentwicklung der Umsetzung von selbstbestimmter Teilhabe in stationären Altenpflegeeinrichtungen konzipiert. Inhaltlich wurden die Themen der empirischen Ergebnisse ausgewählt, die mehrfach in ihrer Relevanz bestätigt wurden und die über die Situationsbeschreibung hinausgehend Hinweise auf veränderungssensible fördernde Faktoren für das Handeln der Leitungskräfte und Mitarbeitenden in den Einrichtungen geben. Da die einzelnen Kriterien in dem Musterrahmenkonzept nachvollziehbar beschrieben und - gegebenenfalls mit Beispielen - erläutert werden sollten, wurde hierfür insbesondere auf das Datenmaterial und die Ergebnisse der tiefer in die Praxisbedingungen gehenden und kontextualisierenden qualitativen Analyse zurückgegriffen. Weil die empirischen Ergebnisse aber im Rahmen des explorativ-deskriptiven Forschungsdesigns keinen Anspruch auf Vollständigkeit haben können, erfolgten darüber hinaus Ergänzungen einzelner Kriterien und Erläuterungen sowie die Nennung von Beispielen bei einzelnen Kriterien, die nicht unmittelbar aus den empirischen Material entnommen wurden, sondern auf anderen Anregungen beruhen - durch Rückmeldungen auf Projektveranstaltungen (z. B. Projektrat, Wissenschaftlicher Beirat, Tagungen), durch vorhandene sowie aktuelle Rahmenvorgaben (z. B. WTG NRW) sowie durch Erfahrungen der Projektleitung und Projektmitarbeitenden auf Seiten des Caritasverbandes.

Wie im folgenden Kapitel näher erläutert wird, wurde das auf diesem Wege im Mai 2019 in seiner ersten Version vollständig erarbeitete MRK abschließend in einer Altenpflegeeinrichtung im Rahmen der Erprobungsphase und der damit verbundenen Implementationsanalyse u. a. in Bezug auf das Verständnis und die Handhabung des Gesamtkonzeptes überprüft. 
Darüber hinaus haben die vier Projekteinrichtungen das MRK zur näheren Überprüfung des Verständnisses, der Realitätsnähe der formulierten Kriterien sowie der von ihnen erwarteten Praxistauglichkeit des Gesamtkonzeptes erhalten und ihre Rückmeldung dazu auf einer hierfür vorgesehenen Sitzung des Projektrats - auf Basis der bereits im Rahmen der Erprobungsphase überarbeiteten zweiten MRK-Version - gegeben.

Das MRK ist in seiner finalen Fassung in Auszügen im Anhang abgebildet. Die folgende Tabelle zeigt die Übersicht der darin enthaltenen Kapitel. Dass die Inhalte des MRK auf den Ergebnissen der hier dokumentierten STAP-Studie beruhen, ist an den Kapitelbezeichnungen bereits erkennbar. Um die Zugänglichkeit und Übertragbarkeit für die Praxis zu vereinfachen, wurden die Kriterien im Musterrahmenkonzept den in Altenpflegeeinrichtungen bekannten Ebenen von Struktur, Prozess und Ergebnis zugeordnet und die Kapitel entsprechend strukturiert.

\section{Tabelle 16: Gliederung des MRK}

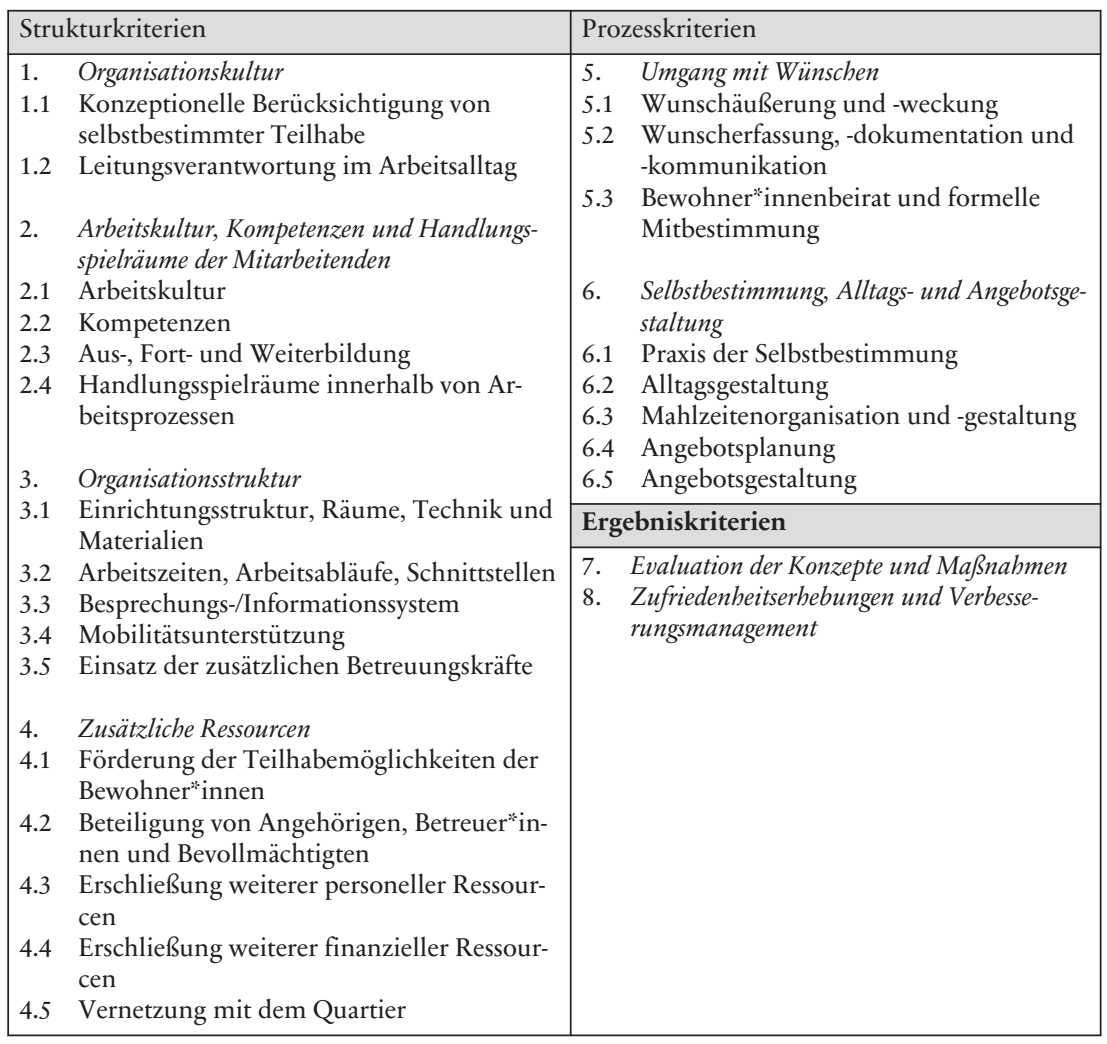


Innerhalb dieser Kapitel sind jeweils Kriterien aufgeführt, die aus Sicht von STAP wesentliche Anforderungen zur Umsetzung der selbstbestimmten Teilhabe von Bewohner*innen in stationären Altenpflegeeinrichtungen darstellen. Ergänzend wurden zu jedem Kriterium Erläuterungen und/ oder Beispiele benannt, damit Nutzer*innen des MRK das jeweilige Kriterium besser nachvollziehen können und Ideen für dessen Umsetzung erhalten. In der Bearbeitungsversion ${ }^{75}$ besteht zusätzlich die Möglichkeit, den Ist-Zustand zu den einzelnen Anforderungen für die eigene Einrichtung einzuschätzen, indem auf einer vierstufigen Ratingskala der Handlungsbedarf bewertet wird. Außerdem können - basierend auf der Selbsteinschätzung des Ist-Standes - Ideen oder Vorschläge für Verbesserungsmaßnahmen abgeleitet und in einer dafür vorgesehenen Spalte näher beschrieben werden. Damit kann das MRK potenziell auch als Instrument der Weiterentwicklung und etwa als Grundlage für die Erstellung und Priorisierung von zukünftigen Maßnahmen zur Förderung und Verwirklichung der selbstbestimmten Teilhabe in der eigenen Einrichtung genutzt werden. Einführend enthält das MRK zudem Hinweise zu dessen Nutzung, mit denen u.a. Ansatz und Ziele, zentrale Begriffe sowie die einzelnen Spalten und Funktionen des MRK erläutert werden.

\subsection{Auswabl und Merkmale der Erprobungseinrichtung}

Die Auswahl der Erprobungseinrichtung erfolgte innerhalb von Einrichtungen des Diözesan-Caritasverbandes für das Erzbistum Köln. Entgegen anfänglicher Überlegungen wurde im Projektverlauf entschieden, dass die Erprobungseinrichtung nicht aus dem Kreis der vier Projekteinrichtungen stammen sollte, da diese sich über die Projektbeteiligung (z. B. im Rahmen der Erhebungen, der Projektsitzungen, der Lernworkshops und der davon einrichtungsintern abgeleiteten Maßnahmen) bereits zu tiefgehend mit dem Teilhabethema beschäftigt hatten, um eine unvoreingenommene Erprobung des Musterrahmenkonzeptes vornehmen zu können. Für die Auswahl einer Erprobungseinrichtung war dann zu berücksichtigen, dass die Teilnahme an der Erprobung für die betreffende Einrichtung mit einem nicht unerheblichen Aufwand über den geplanten Zeitraum von sechs Monaten verbunden war. So verpflichtete sich die Einrichtung, ne-

75 Neben der hier beschriebenen Bearbeitungsversion existiert auch eine Leseversion, die einen schnelleren Überblick über die Inhalte des MRK ermöglichen soll und daher keine Bearbeitungselemente enthält. 
ben der konkreten, intensiven Auseinandersetzung mit dem Musterrahmenkonzept auch dazu, für die Erhebungen im Rahmen der Implementationsanalyse zur Verfügung zu stehen. Deshalb wurde auf der Grundlage einer Samplingstrategie, die wiederum am ehesten dem Vorgehen eines "Convenience“- oder vielmehr „Availability"-Samplings entsprach (vgl. z. B. Patton 1990, S. 180f), einerseits eine Vorauswahl von Einrichtungen getroffen, die bereits an dem STAP-Projekt Interesse gezeigt hatten und anderseits Freiwilligkeit und Kooperationsbereitschaft auf Seiten der Einrichtung als Auswahlkriterium vorausgesetzt.

Konkret wurden alle Einrichtungen des Caritasverbandes angeschrieben, die ursprünglich auf der STAP-Auftaktveranstaltung im Mai 2017 grundsätzlich ihr Interesse an einer Projektbeteiligung signalisiert hatten, und gefragt, inwieweit sie mit welchen Ressourcen für die Erprobung des Musterrahmenkonzeptes zur Verfügung stehen würden. Auf diesem Wege konnte verbandsintern als Erprobungseinrichtung schließlich das CBTWohnhaus An Sankt Georg in Köln ausgewählt werden.

\section{Die Erprobungseinrichtung}

Das CBT-Wohnhaus An Sankt Georg in Köln befindet sich in der Trägerschaft der Caritas-Betriebsführungs- und Trägergesellschaft (CBT). Mit 75 Wohnplätzen verteilt auf zwei Wohnbereiche ist das Haus eher den Altenpflegeeinrichtungen kleinerer bis mittlerer Größe zuzurechnen. Von den Beschäftigten der Einrichtung waren zum Zeitpunkt der Erprobung 35 Mitarbeiter*innen in der Pflege tätig. Darüber hinaus absolvierten sechs Personen eine Pflegeausbildung. Der Bereich der sozialen Betreuung verfügte zum Erprobungszeitpunkt über drei Mitarbeiter"innen im Sozialen Dienst und sieben zusätzliche Betreuungskräfte.

Die Einrichtung befindet sich im Zentrum eines belebten Innenstadtviertels. Entsprechend ausgeprägt ist die infrastrukturelle Anbindung im Nahumfeld im Vergleich zu Altenpflegeeinrichtungen im ländlich geprägten Raum. So sind zwei ÖPNV-Haltestellen im Umkreis von 250 Metern über barrierefreie Zugänge erreichbar. Eine hochfrequentierte Geschäftsstraße verläuft in unmittelbarer Nähe des Hauses, so dass zum Beispiel in einem Bäckerei-Café und einem Lebensmittelhandel Waren des täglichen Bedarfs verfügbar sind. Ein Kiosk in der Nachbarschaft der Einrichtung beliefert zudem die Bewohner*innen auf Wunsch. Zur medizinischen Versorgung können verschiedene Arztpraxen in den umliegenden Straßen genutzt werden. Mehrere Sitzbänke befinden sich in unmittelbarer Nähe des 
Gebäudes. In der grundstücksangrenzenden Kirchengemeinde können von den Bewohner*innen verschiedene Angebote genutzt werden. Die Einrichtung selbst verfügt über einen Garten mit Terrasse, auf der die Bewohner*innen prinzipiell auch ihre Mahlzeiten einnehmen können.

\subsection{Durchfübrung der Erprobung}

Die Erprobung des Musterrahmenkonzeptes erfolgte in einem vergleichsweise kurzen Zeitraum von knapp sechs Monaten im letzten Projektjahr und fokussierte zwei Organisationsebenen: 1.) Das Leitungsteam, das sich unmittelbar mit dem Musterrahmenkonzept im Gesamten auseinandersetzte, sowie 2.) die Mitarbeiter"innen, welche eine aus ausgewählten MRKKriterien abgeleitete Maßnahme in ihre Arbeitsroutinen integrieren sollten. Im ersten Schritt - nach einer Einführung durch STAP ${ }^{76}$ - wurde das MRK seitens der Leitungsebene der Erprobungseinrichtung bearbeitet, um anhand der Kriterien des MRK über eine Selbsteinschätzung den Ist-Zustand in Bezug auf selbstbestimmte Teilhabe in der eigenen Einrichtung zu erheben. Abgeschlossen wurde diese Phase durch die Identifizierung von MRK-Kriterien mit Handlungsbedarf, um eine konkrete Problemstellung zu bearbeiten. Daraufhin erfolgte im zweiten Schritt die Entwicklung und Umsetzung einer Maßnahme, die den ausgewählten Kriterien mit Handlungsbedarf sowie den Rahmenbedingungen der Einrichtung entsprach. Nachfolgend werden der Ablauf und die Aktivitäten der Erprobung zunächst im Überblick tabellarisch vorgestellt und anschließend im Detail erläutert.

$76 \mathrm{Zu}$ diesem Zeitpunkt lagen in der ersten Version des MRK noch nicht die Hinweise zur Nutzung des MRK vor, so dass eine Einführung durch STAP sowohl hierfür als auch für die Vorstellung und Abstimmung der Erprobung und Implementationsanalyse notwendig war. 
Tabelle 17: Ablauf und eingesetzte Ressourcen für die Erprobung des Musterrabmenkonzeptes

\begin{tabular}{|c|c|c|c|}
\hline \multicolumn{2}{|c|}{ Aktivitäten } & \multirow{2}{*}{$\begin{array}{l}\text { Beteiligte } \\
\text { Leiterin der Einrichtung, Projekt- } \\
\text { mitarbeitende STAP }\end{array}$} & \multirow{2}{*}{\begin{tabular}{|l|} 
Dauer \\
$\begin{array}{l}\text { 1 Arbeits- } \\
\text { stunde }\end{array}$ \\
\end{tabular}} \\
\hline a) & $\begin{array}{l}\text { Vorstellung und Einführung des MRK } \\
\text { - Gespräch zur Vorstellung des } \\
\text { Projekts und des MRK im } \\
\text { Überblick }\end{array}$ & & \\
\hline & $\begin{array}{l}\text { Erster Workshop - Einführung in } \\
\text { das MRK und die Erprobung: } \\
-\quad \text { Vorstellung des MRK } \\
-\quad \text { Ablauf und Abstimmung } \\
\\
\text { der Erprobung des MRK } \\
-\quad \text { Abstimmung der „Hausauf- } \\
\\
\text { gabe“: Selbsteinschätzung } \\
\text { mittels MRK }\end{array}$ & $\begin{array}{l}\text { Lenkungsgruppe der Einrichtung } \\
\text { (Einrichtungsleitung, Sozialer } \\
\text { Dienst, Pflegedienstleitung, Wohn- } \\
\text { und Pflegeberatung sowie Leitung } \\
\text { des zentralen Qualitätsmanage- } \\
\text { ments des Trägers), Projektmitar- } \\
\text { beitende STAP }\end{array}$ & $\begin{array}{l}3 \text { Arbeits- } \\
\text { stunden }\end{array}$ \\
\hline b) & $\begin{array}{l}\text { Selbsteinschätzung und Kriterienaus- } \\
\text { wabl } \\
\text { - Einrichtungsinterne Selbstein- } \\
\text { schätzung des Ist-Standes an- } \\
\text { hand des MRK durch die Er- } \\
\text { probungseinrichtung }\end{array}$ & $\begin{array}{l}\text { Lenkungsgruppe der Einrichtung } \\
\text { (Einrichtungsleitung, Sozialer } \\
\text { Dienst, Pflegedienstleitung, Wohn- } \\
\text { und Pflegeberatung in Abstim- } \\
\text { mung mit der Leitung des zentra- } \\
\text { len Qualitätsmanagements des Trä- } \\
\text { gers) }\end{array}$ & $\begin{array}{l}\text { ca. } 30 \\
\text { Arbeitsstun- } \\
\text { den über } \\
\text { einen Zeit- } \\
\text { raum von } 6 \\
\text { Wochen }\end{array}$ \\
\hline & $\begin{array}{cl}\text { Z Zweiter Workshop-Auswertung } \\
\text { der Arbeit mit dem MRK: } \\
-\quad \text { Reflexion der Selbstein- } \\
\text { schätzung zum Ist-Stand } \\
\text { selbstbestimmter Teilhabe } \\
-\quad \text { Identifizierung einer Maß- } \\
\text { nahme zur Teilhabeförde- } \\
\text { rung mittels MRK-Kriteri- } \\
\text { en mit Handlungsbedarf } \\
-\quad \text { Feedback zum MRK für die } \\
\text { Implementationsanalyse }\end{array}$ & $\begin{array}{l}\text { Lenkungsgruppe der Einrichtung } \\
\text { (Einrichtungsleitung, Sozialer } \\
\text { Dienst, Pflegedienstleitung, Wohn- } \\
\text { und Pflegeberatung sowie Leitung } \\
\text { des zentralen Qualitätsmanage- } \\
\text { ments des Trägers), Projektmitar- } \\
\text { beitende STAP }\end{array}$ & $\begin{array}{l}8 \text { Arbeits- } \\
\text { stunden }\end{array}$ \\
\hline c) & $\begin{array}{l}\text { Einrichtungsinterne Maßnabmenent- } \\
\text { wicklung und initiale Implementation } \\
-\quad \text { Vorstellung des STAP-Projektes } \\
\text { in einer Mitarbeiter*innenver- } \\
\text { sammlung } \\
-\quad \text { Entwicklung und Abstimmung } \\
\text { einer konkreten teilhabeför- } \\
\quad \text { dernden Maßnahme } \\
-\quad \text { Initialer Einsatz der Maßnahme }\end{array}$ & $\begin{array}{l}\text { Einrichtungsleitung, Pflegedienst- } \\
\text { leitung, Sozialer Dienst in Abstim- } \\
\text { mung mit den Wohnbereichslei- } \\
\text { tungen sowie weiteren Mitarbei- } \\
\text { tenden der Pflege und zusätzlichen } \\
\text { Betreuungskräften }\end{array}$ & $\begin{array}{l}\text { ca. } 10 \\
\text { Arbeitsstun- } \\
\text { den über } \\
\text { einen Zeit- } \\
\text { raum von } \\
\text { 3,5 Mona- } \\
\text { ten }\end{array}$ \\
\hline
\end{tabular}




\subsubsection{Vorstellung und Einführung des MRK}

In einem ersten Vorgespräch führte ein Projektmitarbeiter die Leiterin der Erprobungseinrichtung in das Projektvorhaben, die Anforderungen an die Erprobungseinrichtung sowie das Musterrahmenkonzept selbst ein. Im nächsten Schritt wurde ein von der Einrichtungsleiterin zusammengestelltes Lenkungsteam (Einrichtungsleitung, Pflegedienstleitung, Leitung Sozialer Dienst sowie Wohn- und Pflegeberatung der Einrichtung und zudem die Leitung des zentralen Qualitätsmanagements des Trägers) in einem Einfübrungsworkshop einbezogen, in dem die Themen- und Kriterienbereiche des Musterrahmenkonzeptes von Seiten des STAP-Teams näher vorgestellt sowie der Ablauf der Konzepterprobung - Selbsteinschätzung und Maßnahmenumsetzung - und Implementationsanalyse thematisiert und abgestimmt wurden.

\subsubsection{Selbsteinschätzung und Kriterienauswahl}

Im Anschluss an den Workshop bestand die „Hausaufgabe“ für das Lenkungsteam darin, das Musterrahmenkonzept bezogen auf ihre Einrichtung zu bearbeiten, d. h., den Ist-Stand der Teilhabeförderung mittels der entwickelten Konzeptkriterien einzuschätzen.

Für die Ausgestaltung der konkreten Bearbeitung des Musterrahmenkonzepts wurde seitens der Erprobungseinrichtung zunächst die Entscheidung getroffen, das Erprobungsvorgehen (Organisation der Bearbeitung des MRK, Kommunikationsstrategie, Maßnahmenentwicklung und initiale Implementation) in die Steuerung der Einrichtungsleitung zu legen. So war gewährleistet, dass die Vorgehensschritte auf der Grundlage aktuell vorhandener Ressourcen (Personal, Zeit, Finanzen) in die Arbeitsabläufe der Einrichtung integrierbar waren und sich nach realen praktischen Erfordernissen ausrichteten. Für das von der Einrichtung schließlich realisierte Vorgehen war dann aber vor allem prägend, dass entschieden wurde, die Leitungsebene bereichsübergreifend als Lenkungsgruppe für den eigentlichen Bearbeitungsprozess des MRK einzubeziehen, um das Thema der selbstbestimmten Teilhabe in der Einrichtung aus verschiedenen relevanten fachlichen Perspektiven beleuchten zu können.

Ein erstes Treffen dieser Lenkungsgruppe diente dazu, den weiteren organisatorischen Rahmen für die Bearbeitung des Konzeptes festzusetzen, wobei u. a. die aktuellen Arbeits- und Urlaubszeiten in der Terminierung weiterer Treffen berücksichtigt wurden. Daran anschließend wurde das MRK 
in verschiedenen Konstellationen der Lenkungsgruppe, aber stets in einem Team bestehend aus mindestens zwei Mitarbeiter*innen, bearbeitet und beurteilt. So fanden einrichtungsintern insgesamt fünf Treffen innerhalb des Leitungsteams mit einer Dauer von ein bis drei Stunden statt. Gearbeitet wurde in wechselnden Teams, so dass die Vertreter*innen der betreffenden Fachbereiche am Gesamtprozess durchgängig beteiligt waren, aber nicht immer alle anwesend sein mussten. So war die Einrichtungsleitung mit sechs Stunden, die Leitung des Sozialen Dienstes mit fünf Stunden, die Pflegedienstleitung mit drei Stunden und die Leitung der Wohn- und Pflegeberatung mit sieben Stunden in den Bearbeitungs- und Beurteilungsprozess involviert. Es ergab sich für das Leitungsteam ein Aufwand von $21 \mathrm{Ar}-$ beitsstunden. Darüber hinaus war die Leitung des zentralen Qualitätsmanagements des Trägers mit neun Arbeitsstunden in die Organisation, Abstimmung und Bearbeitung involviert. In Summe wurden demnach vom Haus und der Trägerorganisation 30 Arbeitsstunden für die Bearbeitung des Musterrahmenkonzeptes aufgewendet, worunter im Fall der Erprobungseinrichtung aber nicht nur die Einschätzung des Ist-Standes und die Beurteilung des Handlungsbedarfes in der Einrichtung, sondern - als Bestandteil der Implementationsanalyse - auch die Beurteilung des Musterrahmenkonzeptes fallen (siehe hierzu das Kapitel 6.4).

Im Rahmen der Ermittlung des Ist-Zustandes mittels MRK identifizierte das Lenkungsteam verschiedene MRK-Kriterien mit Handlungsbedarf, wovon folgende Kriterien - in Abstimmung mit dem STAP-Team auf dem zweiten Workshop - als prioritär für die Maßnahmenumsetzung ausgewählt wurden und in der Zeit der Erprobung bearbeitet werden sollten: ${ }^{77}$

- 2.2.3: Die Abläufe von Pflege und SD/ZBK sind so aufeinander abgestimmt, dass die Bewohner*innen zur geplanten Zeit an den Beschäftigungsangeboten teilnehmen und Dienstleistungen in Anspruch nehmen können.

- 2.2.5: Die Pflegemitarbeiter*innen sind darüber informiert, zu welchen Zeiten an welchen Beschäftigungsangeboten die Bewohner*innen ihres Wohnbereichs/ihrer Organisationseinheit teilnehmen.

77 Von den MRK-Kriterien, bei denen das Leitungsteam noch Handlungsbedarf in der Einrichtung identifiziert hat, wurde wiederum eine Auswahl an Kriterien getroffen, die im Rahmen der Erprobungsphase verfolgt werden sollten und konnten. 
- 4.6.1: Die Begleitung von Bewohner*innen mit Hilfebedarf zu den Angeboten und zurück ist bewohnerorientiert organisiert, sowohl im Wohnbereich/der Organisationseinheit als auch außerhalb. ${ }^{78}$

Idee und Ziel des Lenkungsteams war es, hinsichtlich dieser ausgewählten Kriterien ein Instrument zu entwickeln, das den Mitarbeiter*innen der Pflege transparent macht, welche Bewohner*innen wöchentlich aktuell an welchen Angeboten des Sozialen Dienstes teilnehmen wollen, um den Bewohner*innen durchgängig die Teilhabeoptionen innerhalb der Einrichtung zu ermöglichen, die diese sich wünschen, indem etwa Pflegemaßnahmen entsprechend terminiert werden.

\subsubsection{Einrichtungsinterne Maßnahmenentwicklung und initiale Implementation}

Nachdem im zweiten Workshop Kriterien zur Bearbeitung ausgewählt worden waren, wurden von der Lenkungsgruppe wiederum einrichtungsintern weitere Mitarbeiter"innen in die Planung einer entsprechenden Maßnahme einbezogen, indem zunächst die beiden Wohnbereichsleitungen über das Erprobungsvorhaben und die ausgewählten Kriterien informiert wurden. Anschließend fand eine Mitarbeiter"innenversammlung statt, in der alle interessierten Mitarbeiter*innen über STAP informiert und für das Teilhabethema sensibilisiert wurden. Danach waren alle Mitarbeiter*innen, die von der Maßnahme unmittelbarer betroffen sind, aufgefordert, an der Ideenfindung und Maßnahmenentwicklung mitzuarbeiten. Das heißt, die Kommunikation wurde im Sinne eines symbiotischen Vorgehens, das sowohl Top-down- als auch Bottom-up-Elemente enthielt, gestaltet.

Um für die Mitarbeiter*innen der Pflege und die zusätzlichen Betreuungskräfte transparent zu machen, welche Angebote wöchentlich aktuell vom Sozialen Dienst angeboten werden und welche Bewohner*innen jeweils den Wunsch haben, daran teilzunehmen, wurde die angebotsbezogene Wochenübersicht durch einen weiteren Wochenplan mit bewohner"innenbezogenen Angaben ergänzt. Dieser - maßgeblich vom Sozialen Dienst erarbeitete - Wochenplan ist jeweils wohngruppenspezifisch farblich unterlegt, so dass alle betroffenen Mitarbeiter*innen Einblick in eine aktuelle Übersicht haben, aus der hervorgeht, wann welche Bewohner*innen an

78 Die Nummern der Kriterien entsprechen der ersten und nicht der finalen Version des MRK. 
welchem Angebot teilnehmen möchten, so dass pflegerische Maßnahmen dementsprechend terminiert werden können.

Die Erprobung des Musterrahmenkonzepts selbst, die daraus abgeleitete Maßnahme sowie deren Reüssieren in der Arbeitspraxis wurden auf Basis einer Implementationsanalyse reflektiert und ausgewertet.

\subsection{Implementationsanalyse}

Die Erprobung des Musterrahmenkonzeptes wurde von der Hochschule Düsseldorf mit einer Implementationsanalyse begleitet, die im Schwerpunkt am Ende der Erprobungsphase mit qualitativen und teilstrukturierten Erhebungsmethoden erfolgte und im Folgenden näher begründet und erläutert wird. Die Analyse intendierte und implizierte, das Musterrahmenkonzept bis zum Ende der Projektlaufzeit zusätzlich anhand spezifischer praktischer Fragestellungen und Erfahrungen zu erproben, reflektieren und verbessern. Zudem sollten für die Implementation des MRK sowie teilhabebegünstigender Maßnahmen förderliche wie hemmende Faktoren identifiziert werden, um dieses Wissen für künftige Anwendungen des MRK bereitstellen und nutzen zu können.

Implementationsforschung ist allgemein definiert als die „Beschreibung und Analyse von Prozessen bei der Umsetzung von Konzepten oder Programmen" (Petermann, 2014, S. 122) und hat in den letzten beiden Jahrzehnten auch in der Gerontologie, Pflege und Sozialen Arbeit insbesondere in Verbindung mit und im Anschluss an Wirkungs- bzw. Wirksamkeitsstudien bzw. im Kontext der Evidenzbasierung an Bedeutung gewonnen (z. B. Hoben, 2016, S. 118ff; Wahl \& Diehl, 2016, S. 138ff), obwohl im internationalen Vergleich innerhalb des deutschsprachigen Raums bislang noch deutlich weniger Erfahrungen vorliegen (für die Pflegewissenschaften konstatiert dies z. B. Hoben, 2016, S. 115). Vor allem in den Vereinigten Staaten hat sich mit den „Implementational Sciences“ ein eigenes Forschungsgebiet etabliert. Implementational Sciences beanspruchen für sich, die Brücke zwischen theoretischen Konzepten und deren praktischer Umsetzung schließen zu wollen (Fixsen \& Blase, 2009). Insofern können Implementationen als Schlüsselmomente von Modellprojekten interpretiert werden, da sich in diesem Prozessschritt entscheidet, ob das entwickelte Konzept von den jeweiligen Adressat"innen akzeptiert und im Arbeitsalltag tatsächlich angewendet wird (vgl. Euler \& Sloane, 1998; ReinmannRothmeier \& Mandl, 1998). 
Die Durchführungsziele und -weisen von Implementationsanalysen sind sehr heterogen und schwer systematisch zu überblicken, auch wenn verschiedene Sortierungsversuche mit unterschiedlichen Ausgangspunkten existieren (z. B. Petermann, 2014; Beelmann \& Karing, 2014). Dabei wird auf unterschiedliche konzeptionelle Ansätze in der Implementationsforschung hingewiesen (z. B. Voigt-Radloff et al., 2013, S. 30), welche eine Vielzahl von Faktoren beschreiben, die eine Implementation von neuen Interventionen beeinflussen können (vgl. z. B. die Metaanalyse von Fixsen et al., 2009). Entsprechend werden in der Literatur auch mehrere Ebenen von Implementationsfaktoren und -prozessen aufgeführt (z. B. Beelmann \& Karing, 2014), indem etwa zwischen individueller, institutioneller sowie politisch-administrativer und gesellschaftlicher Ebene unterschieden wird. Ferner wird analytisch in spezifische Zielsetzungen von Implementationsanalysen unterschieden (z. B. Petermann, 2014), die aber nicht trennscharf abgrenzbar und in der Praxis der Implementationsforschung noch nicht durchgängig wiederzufinden sind. Insbesondere im deutschsprachigen $\mathrm{Pu}$ blikationsraum scheint der Rahmen für Implementationsanalysen damit noch denkbar offen, nicht nur, weil Implementationsstudien sehr heterogen methodologisch begründet und methodisch durchgeführt werden können, sondern auch, weil ihre Zielsetzungen - auch begrifflich - uneinheitlich bestimmt werden.

Unter dem Begriff der Implementation soll hier zunächst grundsätzlich der soziale Prozess verstanden werden, „in dem aus der formalen Entscheidung, eine neue Verfahrensweise einzuführen, ein dauerhaft geändertes Verhalten der Anwender wird" (Voigt-Radloff et al., 2013, S. 54). Das heißt, Implementation zielt somit nicht allein auf die Einführungssituation einer Maßnahme, sondern über diesen Zeitpunkt hinaus. Dies spiegelt sich in verschiedenen Phasenmodellen zu Implementationsprozessen wider. Beispielsweise differenzieren Fixsen et al. (2007, S. 5) folgende sechs Stadien einer Implementation: „Exploration“, „Installation“, „Initial“, „Implementation“, „Innovation“ und „Sustainability“. Greenberg et al. (2005) hingegen interpretieren Implementationsprozesse als in drei Phasen segmentiert: „Pre-adoption“, „Delivery“ und „Post-adoption“. Setzen wir diese Phasen in Relation zu den gegebenen Ressourcen und Zielstellungen des Projektes STAP, wird schnell deutlich, dass eine Analyse hier die „Post-Implementations-“ bzw. „Nachhaltigkeitsphase“ nicht umfassen kann. Vielmehr fokussierte die Analyse erste Erfahrungen in Bezug auf die Akzeptanz und Angemessenheit des Musterrahmenkonzeptes sowie dabei fördernde und hemmende Faktoren bis zur initialen Maßnahmenimplementation. 


\subsubsection{Vorarbeiten und Entwicklung der Implementationsanalyse}

Das Vorgehen für die Erprobung des MRK und die Implementationsanalyse wurde unter der Maßgabe entwickelt, sowohl praktische als auch fachwissenschaftliche Erfahrungen und Wissensbestände zu integrieren. Deshalb war auch die gesamte Entwicklungsphase des Konzeptes durch den Projektrat wie den Wissenschaftlichen Beirat qualitätssichernd flankiert. Entsprechend wurden Konzeptkriterien und -strukturen in regelmäßigen Feedbackschleifen aus den verschiedenen Perspektiven entwickelt, diskutiert sowie gegebenenfalls modifiziert.

Im Rahmen des zweiten Erprobungsworkshops im CBT-Wohnhaus An Sankt Georg wurde zudem der erste praktische Einsatz des Konzeptes reflektiert. Beispielsweise wurde rekapituliert, wie das Leitungsteam der Einrichtung zur Bearbeitung des Konzeptes vorgegangen war und wie sich im Sinne der Selbsteinschätzung der aktuelle Ist-Stand in Bezug auf Teilhabeförderung darstellte. Darüber hinaus war es Gegenstand des Workshops, eine erste Beurteilung des MRK aus arbeitspraktischer Perspektive vorzunehmen. So wurden etwa die Struktur des MRK sowie alle Einzelkriterien auf ihre Verständlichkeit und ihren Teilhabebezug hinterfragt. Wenngleich die Ergebnisse der Diskussionen im Projektrat, im Wissenschaftlichen Beirat wie auch im Erprobungsworkshop noch nicht Bestandteil der methodisch kontrollierten Implementationsanalyse waren, wurden sie dennoch jeweils gesichert und mündeten bei Bedarf in entsprechenden Konzeptadaptionen.

\subsubsection{Analyseleitende Fragen und Forschungsdesign}

Die methodisch-kontrollierte Analysephase setzte schließlich nach der Bearbeitung des Musterrahmenkonzeptes durch das Leitungsteam der Erprobungseinrichtung ein. Für die Entwicklung des Untersuchungsdesigns wurde ein pragmatischer Zugang gewählt, wie es in der Evaluations- und Implementationsforschung nicht unüblich ist. So wurde, um Analyseziele und -fragen für die Implementationsanalyse des Projektes zu identifizieren, folgender Orientierungsrahmen einbezogen:

1. Implementationswissenschaftliche Konzepte und Modelle,

2. die Forschungsfragen des Projektes STAP sowie

3. der Projektrahmen und die Projektressourcen.

Als konzeptionelle Grundlage für die Implementationsanalyse diente schließlich die von Michie et al. (2005) entwickelte und von Petermann 
(2014) leicht modifizierte und für den deutschen Sprachraum angepasste Taxonomie. Diese umfasst als Implementationsoutcomes die Faktoren Akzeptanz, Übernahme, Angemessenheit, Machbarkeit, Wiedergabetreue, Implementationskosten sowie Durchdringung. Gespiegelt an den Projektzielen und -ressourcen zeigte sich, dass sich in erster Linie die Implementationskomponenten Akzeptanz, Angemessenheit und Machbarkeit als wesentlich für die Beurteilung der initialen praktischen Bewährung des Musterrahmenkonzeptes eigneten.

Zum Verständnis der einzelnen Analysedimensionen wurden folgende Definitionen zugrunde gelegt:

- Akzeptanz impliziert den „Bezug auf eine Person oder Gruppe als Akzeptanzsubjekte [...], an die ein gegenstandsbezogenes Akzeptanzobjekt gebunden ist. Das Verhältnis von Akzeptanzobjekt und Akzeptanzsubjekt wird weiterhin immer im Rahmen eines Akzeptanzkontextes betrachtet" (Wirth \& Husmann, 2013, S. 3), indem der Zeitpunkt, die Gründe und der Zusammenhang der Akzeptanz genauer angeschaut werden. Auf der Ebene des Akzeptanzsubjektes kann zwischen Einstellungs- und Verhaltensakzeptanz unterschieden werden.

- Unter der Einstellungsakzeptanz werden affektive Komponenten (Gefühlsfaktoren), kognitive (Vorstellungen, Ideen und Glaubensüberzeugungen, Kosten- und Nutzen-Abwägung) und konative Komponenten (Verhaltensbereitschaft gegenüber dem Akzeptanzobjekt) verstanden.

- Verhaltensakzeptanz beschreibt das tatsächliche Verhalten einer Person, indem das Akzeptanzobjekt durch Nutzung aktiv angenommen wird.

Demnach kann man Akzeptanz einerseits als einen Ausdruck einer grundlegenden positiven Einstellung der Mitarbeitenden und Leitungskräfte gegenüber dem MRK definieren, die sich andererseits durch die konkrete Anwendung und Nutzung des MRK oder Elementen davon äußert (Olbrecht, 2010). Im Projekt STAP werden dabei vor allem die Einstellungsakzeptanz zum MRK und die Verhaltensakzeptanz in Bezug auf ein ausgewähltes Element sowie die ersten Schritte dazu in den Blick genommen.

- Angemessenheit meint die wahrgenommene Kompatibilität einer Intervention für ein bestimmtes Setting. Das Konzept der Angemessenheit hat dabei auch „eine wichtige Funktion zur Einschätzung des Implementationsaufwandes" (Petermann, 2014, S. 124). Der Aufwand könnte beispielsweise als zu groß wahrgenommen werden, indem die Einrichtung beispielsweise das MRK als eine weitere Belastung wahr- 
nimmt oder auch wenn die Intervention nicht den Erwartungen der Mitarbeitenden und Leitungskräfte entsprechen würde (ebd.).

- Machbarkeit bedeutet "das Ausmaß, in dem ein neues Behandlungsangebot erfolgreich in einem definierten Setting eingesetzt werden kann“ (Petermann, 2014, S. 124) und bezieht sich in STAP primär auf die ersten ausgewählten Elemente bzw. Kriterien des MRK und deren Bearbeitung. Hier geht es im Vergleich zur Angemessenheit weniger um die wahrgenommene Kompatibilität als vielmehr darum, inwieweit die ausgewählten Maßnahmen auf Basis des MRK tatsächlich in der Praxis umgesetzt werden können.

Insgesamt vollzogen sich - wie beschrieben - die Erprobung und damit auch Implementation des Musterrahmenkonzeptes in der Erprobungseinrichtung auf zwei Ebenen: Auf der Leitungsebene des Lenkungsteams erfolgte die konkrete Auseinandersetzung mit dem MRK, die der Ermittlung des Ist-Zustandes und des Handlungsbedarfes in Bezug auf selbstbestimmte Teilhabe diente. Die zweite Ebene war die Entwicklung einer konkreten Maßnahme zur Teilhabeförderung und deren Einsatz durch die Mitarbeiter*innen innerhalb ihrer alltäglichen Arbeitsroutinen. Durch die Einbeziehung dieser Ebenen werden auch die beiden entscheidenden, sensiblen Bereiche einer Maßnahmenimplementation erfasst: das Verständnis und die Interpretation eines Konzeptes vor dem Hintergrund eines konkreten organisationalen Rahmens sowie die Haltungen und Handlungsweisen der betroffenen Mitarbeiter*innen in Bezug auf davon abgeleitete Maßnahmen. Mit Bezug auf die oben genannten Analysedimensionen wurden für diese Ebenen schließlich folgende analyseleitende Fragen abgeleitet.

Für die Erhebung auf der Leitungsebene

Im Sinne der Akzeptanz:

- Verständlichkeit: Sind die Kriterien im MRK für die „Anwender*innen“ (EL, PDL, SD etc.) verständlich? Sind eventuell weitere Erläuterungen oder Beispiele notwendig?

- Motivation: Sind die Kriterien für die Anwender*innen ansprechend und motivierend formuliert?

Im Sinne der Angemessenheit:

- Vollständigkeit: Sind die wichtigsten teilhaberelevanten Aspekte aus Sicht der beteiligten „Anwender“innen“ enthalten? 
- Passung: Kann man mit dem MRK den Ist-Zustand zur Teilhabe einschätzen? Kann man aus dieser Selbsteinschätzung bzw. aus der Anwendung des MRK geeignete Maßnahmen ableiten?

- Aufwand: Inwieweit erleben die Leitungskräfte die Selbsteinschätzung als Mehraufwand oder gar Stressor?

Im Sinne der Machbarkeit:

- Aufwand: Können wesentliche Anforderungen des MRK in eigene Konzepte, Verfahren, Instrumente etc. überführt werden? Welche Anforderungsbereiche werden in ihrer Umsetzung als aufwändig eingeschätzt, welche als mit geringem Aufwand im Rahmen vorhandener Ressourcen realisierbar?

- Umsetzungsfaktoren und -barrieren: Welches sind die wichtigsten fördernden Faktoren und die stärksten Barrieren für die Implementation unter Routinebedingungen? Welche spezifischen Strategien reduzieren die identifizierten Barrieren und stärken die wichtigsten fördernden Faktoren?

Für die Erhebung auf der Mitarbeiter*innenebene

Im Sinne der Akzeptanz:

- Verständlichkeit: Haben die Mitarbeiter*innen die Zielsetzung des entwickelten Instrumentes zur Teilhabeförderung verstanden? Welche Relevanzen schreiben sie diesem zu?

- Motivation: Inwiefern artikulieren die Mitarbeiter*innen Zustimmung oder Ablehnung in Bezug auf die entwickelte und implementierte Maßnahme?

Im Sinne der Angemessenheit:

- Passung: Inwiefern fügt sich die entwickelte Maßnahme in die gegebenen Arbeitsroutinen ein? Wo sehen die Mitarbeiter*innen gegebenenfalls Anpassungsbedarf?

- Aufwand: Inwiefern empfinden sie die Maßnahme als entlastend? Inwiefern erleben die Mitarbeiter*innen die implementierte Maßnahme als Mehraufwand oder gar Stressor?

Die beiden Frageebenen wurden in zwei entsprechenden Forschungszugängen - 1. Fokusgruppeninterview auf der Leitungsebene und 2. Kurzinterviews auf der Mitarbeiter*innenebene - umgesetzt. Insgesamt wurde für diese Analyse ein primär qualitativer Forschungszugang gewählt, da dieser ermöglichte, Relevanz- und Bedeutungskonstruktionen innerhalb ihres or- 
ganisationalen Rahmens nachzuvollziehen und die ihnen zugrunde liegenden sozialen Prozesse einzubinden.

\subsubsection{Fokusgruppeninterview}

\section{Samplingstrategie}

Für das Fokusgruppeninterview, das auf der Leitungsebene des Lenkungsteams ansetzte, wurde auf ein Sampling verzichtet, da eine Vollerhebung durchgeführt werden konnte, d. h., ausnahmslos alle Personen des Lenkungsteams, die an der Bearbeitung des Musterrahmenkonzeptes beteiligt waren, konnten in die Erhebung im Sinne einer natürlichen Gruppe einbezogen werden. Dementsprechend waren in der Erhebungssituation die identischen fachlichen Perspektiven vertreten, die auch bei den Einschätzungen mittels MRK mitgewirkt hatten.

\section{Erhebungsmethode}

Der Erfolg oder Misserfolg von Maßnahmenimplementationen, hier eines neuen Konzepts, hängt nicht nur von den einzelnen Mitarbeiter*innen in ihrem Handeln ab, sondern wird zum Beispiel auch ganz wesentlich durch die Kommunikations- und Konfliktkultur in einer Einrichtung bedingt. Das kommunikative Setting eines Gruppeninterviews (vgl. Kühn \& Koschel, 2018) erlaubte es, diese Spezifika mit zu explorieren und entsprechende Sinnkonstruktionen in ihrem interaktiven Charakter zu erfassen. So wurde für die Analyse auf der Leitungsebene konkret ein leitfadenstrukturiertes Fokusgruppeninterview (vgl. Mayerhofer, 2009, S. 477-490) als Erhebungsmethode ausgewählt, da diese Methode in besonderer Weise geeignet ist, Einstellungen und Meinungen in natürlichen Gruppen, wie etwa Arbeitskollegien, innerhalb ihres Konstruktionsgefüges zu erheben (Lamnek \& Krell, 2016, S. 407). Aus diesem Grund wurden in das Fokusgruppeninterview auch alle am Bearbeitungsprozess beteiligten Personen einbezogen, um innerhalb dieses etablierten Kontextes die Diskussionen und Entscheidungen des Prozesses rekonstruieren zu können. 
Aufbau des Fokusgruppeninterviews

Die Themenkomplexe des Leitfadens orientierten sich inhaltlich an den vorab identifizierten Analysedimensionen der Akzeptanz, der Angemessenheit und der Machbarkeit (Teil 2). Darüber hinaus wurden zum einem das organisatorische Vorgehen zur Bearbeitung des MRK abgefragt und beurteilt (Teil 1) und zum anderen Nachhaltigkeitsbedingungen des MRK-Einsatzes exploriert (Teil 3), so dass sich schließlich folgende Leitfadenstruktur ergab:

Teil 1: Organisatorisches Vorgehen zur Bearbeitung des MRK

Teil 2: Bewertungen des MRK - Akzeptanz, Angemessenheit, Machbarkeit

Teil 3: Nachhaltigkeitsbedingungen des MRK

Teil 4: Schlussbemerkungen

Auswertung

Ausgewertet wurde das Transkript des Fokusgruppeninterviews kategoriengeleitet mittels inhaltlich-strukturierender Inhaltsanalyse (gemäß Kuckartz, 2018, S. 97-122), um einen unmittelbaren Bezug zu den Befunden der vorausgegangenen empirischen Erhebungen und den Forschungsfragen der Implementationsanalyse herzustellen. Die Auswertung fokussierte insbesondere die für die Analyse als leitend eingestuften Bereiche der Akzeptanz, Angemessenheit und Machbarkeit sowie die übergeordneten Fragen nach Hemmnissen und förderlichen Faktoren für den Einsatz des MRK. Die erhobenen Daten wurden zunächst vor diesem Kategoriensystem interpretiert und die Kategorien schließlich im Analyseprozess weiter ausdifferenziert (vgl. ebd., S. 47).

\subsubsection{Kurzinterviews}

Samplingstrategie

Die Auswahl der Mitarbeiter"innen für die zweite Analyseebene ergab sich über die Frage, inwiefern sie in ihren Arbeitsroutinen von der Erprobungsmaßnahme betroffen waren. Dazu diente ein Sampling, orientiert an einer 
Perspektive im Sinne der Expert*innenauffassung für Expert"inneninterviews: „Der Experte steht für eine Problemperspektive, die typisch ist für den institutionellen Kontext, in dem er sein Wissen erworben hat und in dem er handelt"“ (Meuser \& Nagel, 2009, S. 469). In der Fallauswahl wurde darauf geachtet, multiperspektivisch alle von der teilhabefördernden Maßnahme im Praxishandeln betroffenen Bereiche in die Erhebung zu integrieren. Für die Zusammenstellung des Sample diente die Einrichtungsleitung - unter Abstimmung mit den Wohnbereichsleitungen, der Leitung des Sozialen Dienstes sowie der Pflegedienstleitung - als Gatekeeper -, da sie, anders als das STAP-Team, beurteilen konnte, welche Mitarbeiter"innen in der Erledigung ihrer Arbeitsaufgaben tatsächlich vom Einsatz der Maßnahme bzw. des neuen Instrumentes betroffen sind. Interviewt wurden schließlich zwei Mitarbeiter"innen des Sozialen Dienstes, zwei Bewohnerassistent"innen bzw. zusätzliche Betreuungskräfte, ${ }^{79}$ zwei Pflegefachkräfte, eine Pflegeassistentin und eine Wohnbereichsleitung. Darüber hinaus wurde eine maximale Varianz in den Kriterien Berufserfahrung und Alter der Interviewten umgesetzt. So verfügten die Befragten über 0,5 bis 35 Jahre Beschäftigungsdauer in der Einrichtung und waren zwischen 32 und 62 Jahre alt. Die Interviews dauerten zwischen zehn und fünfzehn Minuten und fanden in einem separaten Raum im CBT-Wohnhaus selbst statt.

\section{Erhebungsmethode}

Übergeordnetes Ziel der zweiten Analyseebene der Implementationsanalyse war es, hemmende wie fördernde Faktoren für den Einsatz der entwickelten Maßnahme zu identifizieren. Um dabei sowohl den subjektiven Erfahrungs- und Deutungsrahmen allgemein erfassen als auch Einschätzungen zur Maßnahmenplanung und -realisierung reflektieren zu können, sollten zur Gestaltung der Kurzinterviews zwei grundlegende Ebenen zum Tragen kommen: einerseits der subjektive Arbeitskontext und dessen Bezüge zur Teilhabe der Mitarbeiter"innen sowie andererseits deren Einschätzung der neuen Maßnahme.

Deshalb wurde im Rahmen der Implementationsanalyse ein Kurzinterview entwickelt, das narrative Sequenzen im Sinne eines teilstandardisierten Interviews in der Erhebungssituation an einen quantitativen Kurzfragebogen koppelte, der in erster Linie aus einer skalierten Itembatterie be-

79 In der Erprobungseinrichtung wurde diese Mitarbeitendengruppe als Betreuungsassistent*innen bezeichnet. 
stand. Mittels der teilstandardisierten Items wurde zunächst der subjektive Erfahrungs- und Deutungsrahmen erfasst. Anschließend wurden durch verschiedene Ratingskalen (zusammenfassend vgl. z. B. Franzen, 2014, S. 701-712) Einschätzungen, bezogen auf die Akzeptanz, die Angemessenheit sowie die Machbarkeit, erhoben, um diese unmittelbar quantifizierbar und in ihren Entwicklungen abbildbar zu gestalten.

Aufbau der Kurzinterviews

Teil 1: Zur Person

Teil 2: Kontext und Bezüge zur Teilhabe

Teil 3: Sozialstatistische und berufsbiografische Angaben

Teil 4: Bewertung der Maßnahme

Teil 5: Schlussbemerkungen

\section{Auswertung}

Die Auswertung der transkribierten narrativen Sequenzen der Kurzinterviews erfolgte analog zur Auswertung des Fokusgruppeninterviews im Sinne einer inhaltlich-strukturierenden qualitativen Inhaltsanalyse (Kuckartz, 2018, S. 97-122). Die quantitativen Items im Kurzfragebogen wurden hingegen mittels univariater Maßzahlen und bivariater Analyseverfahren der deskriptiven Statistik (vgl. z. B. Weins, 2010, S. 65-89) dargestellt bzw. ausgewertet.

\subsection{Ergebnisse der Implementationsanalyse}

Für die nachfolgend dargelegten Ergebnisse der Implementationsanalyse und ihre Interpretation ist nochmals hervorzuheben, dass die Erprobungseinrichtung nicht nur vor der Aufgabe stand, das MRK für sich als Instrument der Selbsteinschätzung einzusetzen, sondern dieses auch für die Implementationsanalyse bewerten sollte und damit für die Erprobungseinrichtung ein höherer Aufwand, aber eventuell auch ein anderer Motivationseffekt vorhanden war. Die inhaltliche Auswertung erfolgte dann anhand der vorab differenzierten Analysedimensionen der Akzeptanz, Angemessenheit und Machbarkeit. 
6.5.1. Ergebnisse der Erhebung auf der Leitungsebene (Fokusgruppeninterviews)

\subsubsection{Akzeptanz}

Die Auseinandersetzung mit dem MRK und dem Teilhabethema allgemein trafen in der Erprobungseinrichtung auf eine explizit positiv artikulierte Einstellungsakzeptanz. So wurde deutlich geäußert, dass die Berücksichtigung von Teilhabe wesentlich für die Qualität einer Einrichtung und die eigene Arbeit sei und für die Einordnung der eigenen Arbeitsleistung sogar durchaus einen geradezu sinnstiftenden Charakter annehmen könne: „Also ich kann da nur dahinterstehen, wenn ich davon überzeugt bin. Wenn ich von dem Wohnhaus und der Arbeit, die hier geleistet wird, wirklich überzeugt bin. [...] Wenn ich wüsste, hier ist alles Mist, könnte ich nicht den Angehörigen und den zukünftigen Bewohnern sagen, was wir machen und wie das hier abläuft, könnte ich nicht mit mir vereinbaren, dann würd' ich hier aufhören und sagen: ,Nee““.80 In diesem Zusammenhang wurden das Thema Teilhabe und das MRK weniger als zusätzliche Belastung eingeordnet, sondern als notwendiges, bedeutsames Thema und Instrument. Dieser Einstellung entsprechend erfolgte während der Erprobungsphase eine sehr intensive Auseinandersetzung mit dem Thema, eine Erweiterung der vorab festgelegten Problemstellung auf weitere teilhaberelevante Fragestellungen sowie mitunter positiv relativierende Aussagen bezüglich des Zeitaufwandes, der mit der Erprobung verbunden war. Auch in Bezug auf die - auf dieser Ebene ursprünglich nicht adressierte Verhaltensakzeptanz war eine sich bereits verstetigende Teilhabeorientierung in der Einrichtung auszumachen. Deutlich wurde das, weil bereits ein Zeitraum für die erneute Bearbeitung des Konzeptes im kommenden Jahr festgesetzt wurde. Zudem wurden das MRK konsequent mit den vorhandenen Konzepten der Einrichtung abgeglichen und entsprechende teilhabeorientierte Konzeptmodifizierungen vorgenommen.

80 In diesem Kapitel werden zugunsten der Anonymisierung keine Funktionszuordnungen der Interviewzitate vorgenommen. 
a) Verständlichkeit: Sind die Kriterien im MRK für die „Anwender“innen“ (EL, PDL, SD etc.) verständlich? Sind eventuell weitere Erläuterungen oder Beispiele notwendig?

Die Einzelkriterien wurden von den Anwender*innen ausdrücklich als mehrheitlich verständlich beurteilt. Das Lenkungsteam betonte, dass insbesondere die Beispiele und Erläuterungen für sie entscheidend zum Verständnis der Kriterien beigetragen hätten, insbesondere um diese trennscharf voneinander abzugrenzen. Weitere Erläuterungen oder Beispiele seien aber nicht notwendig.

Demgegenüber ergaben sich Verständnisprobleme nicht in Bezug auf einzelne Kriterien, sondern eher im Sinne einer gewissen Unübersichtlichkeit aufgrund der Vielzahl der Kriterien. Entsprechend beschrieben die Mitglieder des Lenkungsteams, dass es für sie bei der ersten Bearbeitung nicht immer ganz klar war, ob es sich bei einigen Kriterien auf verschiedenen inhaltlichen Gliederungsebenen eventuell um Dopplungen handeln könnte.

Unabhängig davon, wie verständlich die Kriterien formuliert sind, wurde ein für die Konzeptbearbeitung weiterer gewichtiger Aspekt deutlich. So wurde ersichtlich, dass die Einschätzung des Handlungsbedarfs eine subjektive Interpretationsleistung bleibt und daher als sinnvoll erachtet wurde, eine endgültige Entscheidung für die Bewertung des jeweiligen Handlungsbedarfs zu den Kriterien erst nach einem Austausch aus verschiedenen Fachbereichsperspektiven vorzunehmen.

b) Motivation: Ist die Auseinandersetzung mit dem MRK für die Anwender*innen motivierend?

Die Interviewten des Lenkungsteams beschrieben die Arbeit mit dem MRK insgesamt als sehr motivierend. Motivierend an der Arbeit mit dem MRK sei, dass es ermögliche, alle Facetten von Teilhabeförderung in den Blick zu nehmen und, wie bereits während des Erprobungsworkshops geäußert wurde, „über den Tellerrand zu schauen [... D]ieses Instrument finde ich persönlich sehr sinnvoll, sehr sinnvoll“. Insbesondere die aufgeführten Beispiele ermöglichten es, diese aufzugreifen oder, durch diese inspiriert, neue teilhabefördernde Ideen zu entwickeln. So wurde die Beispielspalte des Konzeptes immer wieder positiv hervorgehoben, da diese nicht nur zum Verständnis der Einzelkriterien beitrüge, sondern vor allem auch Anregungen für die Praxis enthielte, die zum Weiterdenken und -gestalten der eigenen Teilhabeorientierung einladen würden. Den Beispielen kann 
vor diesem Verständnis durchaus auch die Funktion eines Ideenpools für teilhabefördernde Maßnahmen zugeschrieben werden.

Das MRK wurde zudem insofern als motivierend erlebt, als es erlaubt, den Stand der eigenen Einrichtung in Bezug auf Teilhabe abzubilden und sich so auch zu vergewissern, welche Leistungen bereits erbracht werden. Der mithilfe des MRK ermittelte Ist-Zustand ermögliche es, sich der Qualität der eigenen Arbeit bewusst zu werden. In diesem Zusammenhang scheint die Motivation zur Auseinandersetzung mit dem MRK nicht allein durch dieses selbst bedingt zu sein, sondern auch davon abzuhängen, ob das Thema Teilhabe bzw. Teilhabeorientierung auf Leitungsebene als bedeutsam eingeschätzt wird. Die festgestellte(n) Motivation(seffekte) könnte(n) hier also auch durch die in der Erprobungseinrichtung bereits vorhandene Organisationskultur geprägt sein. So vermittelte die Einrichtung den Forscher*innen den Eindruck einer ausgeprägten Qualitäts- und vor allem Bewohner*innenorientierung, was sich auch in der intensiven Auseinandersetzung mit dem MRK niederschlug.

\subsubsection{Angemessenheit}

Zusammenfassend lässt sich zu dieser Dimension festhalten, dass bei der Beurteilung der Angemessenheit des MRK zwei Grundpositionen nebeneinander existierten. Einerseits wurde die Vollständigkeit im Sinne einer guten Übersicht relevanter Kriterien und die Passung in Bezug auf die Möglichkeiten zur Selbsteinschätzung des Ist-Zustandes ausdrücklich positiv betrachtet. Andererseits wurde in Bezug auf den Aufwand der Umfang des MRK kritisch beurteilt. Auch wenn die Erprobungseinrichtung dies im Projektrahmen nicht als Stressor empfunden hat, so sah sie darin aber - bei der Vielfalt der in Pflegeeinrichtungen zu erfüllenden Aufgaben - eine eventuell abschreckende Wirkung für andere Einrichtungen: „Im Grunde genommen, beide Seiten würde ich verstehen, wenn es so bliebe. Wir sind auch der Meinung, das geht nun mal im Detail, aber schließlich und endlich, besonders, wenn es um den Bereich der Pflege geht, die haben so viel, wo man diese Qualitätsprüfung, zweimal wieder erfassen muss. Das ist so viel, dass man schon sagen muss, das ist irgendwie manchmal zu viel. Und die Bewohner sind auch noch da und die müssen auch noch versorgt werden. Und die Angehörigen auch, so, und deshalb denke ich mir, muss kurz, praxisbezogen sein, intensiv natürlich, aber so, dass man damit gut arbeiten kann, aber nicht, manchmal kann man nicht alles abfragen.[...] und entmutigend, weil, das ist sehr umfassend und wenn man so ein 
Stückchen Papier sieht, da denkt man: Da habe ich keine Zeit." Diese zweipolige Positionierung - einerseits die Betonung der Chancen der anregungsreichen Multiperspektivität des MRK und andererseits dessen großer Umfang - wurde von allen Diskutant*innen geteilt.

Seitens der Einrichtung und der QM-Verantwortlichen wurde aber vorgeschlagen, diesen Widerspruch mittels einer Digitalisierung des Konzeptes aufzulösen. So könnten eine Verschlagwortung und eine Suchfunktion eine raschere Orientierung gewährleisten und gleichzeitig die Kriterienzahl beibehalten werden. Darüber hinaus wurde eine Modularisierung nach Gliederungsebenen als sinnvoll erachtet, so dass es für Altenpflegeeinrichtungen leichter möglich wäre, einzelne Abschnitte nach einiger Zeit nochmals gezielter zu analysieren.

a) Vollständigkeit: Sind die wichtigsten teilhaberelevanten Aspekte aus Sicht der beteiligten „Anwender“innen“ enthalten?

Das MRK wurde von den Anwender*innen einhellig als sehr vollständig beurteilt. Dass das Konzept damit inhaltlich sehr perspektivenreich und aussagekräftig, aber eben auch sehr ausführlich ist, wurde in diesem Zusammenhang wiederum als ambivalent bewertet - diesmal mit Blick auf unterschiedliche Ausgangssituationen in den Einrichtungen. Einerseits wurde betont, dass das MRK - auch in seiner Breite - bestens für Altenpflegeeinrichtungen geeignet sei, die sich bislang noch weniger differenziert mit Teilhabeorientierung und -förderung in Altenpflegeeinrichtungen auseinandergesetzt haben. Andererseits wurde hinterfragt, ob das MRK für Einrichtungen, die schon relativ gut für das Teilhabethema sensibilisiert sind, in der Breite erforderlich sei.

b) Passung: Kann man mit dem MRK den Ist-Zustand zur Teilhabe einschätzen? Kann man aus dieser Selbsteinschätzung bzw. aus der Anwendung des MRK geeignete Maßnahmen ableiten?

Mehrfach wurde innerhalb der Gruppendiskussion und auch schon vorab im Erprobungsworkshop die Einschätzung vorgenommen, dass mittels des MRK sehr gut eine Selbsteinschätzung des Ist-Zustandes in Bezug auf Teilhabe in Altenpflegeeinrichtungen vorgenommen werden könne. So wurde etwa geäußert: „Man kann sehen, wo man selbst steht, was man schon bietet und wo vielleicht, ist ja auch so drin, Handlungsbedarf besteht. Dafür ist 
das [das MRK, Anm. d. Verf.] sehr wichtig." Oder eine andere Person der Fokusgruppe betonte: „Ich finde, die Tabelle ist sehr gut, weil man auch Notizen machen kann. Für sich. Auf jeden Fall finde ich die gut. Man kann sie jedes Jahr durchgehen und die Notizen machen: Wo sind wir gerade? Was hat man verändert? [...]. So in Form der Tabelle und dass wirklich Notizen da sind, das finde ich ganz wichtig. [...], aber man kann sagen, hat man, hat man und ergänzen“. Darüber hinaus zeigte sich, wie sehr sich der Blick auf Teilhabe und die Vielzahl teilhaberelevanter Arbeitssituationen, Alltagssituationen weitet und differenziert, in dem nicht nur die Diagnostik des Ist-Zustandes mittels dem MRK möglich war, sondern auch eine allgemeine bzw. vertiefende Sensibilisierung für das Thema in all seinen Facetten erfolgte. Anhand der ausgewählten Kriterien sei es dann auch möglich, entsprechende Maßnahmen abzuleiten.

Seitens der Erprobungseinrichtung wurde es als sinnvoll erachtet, zur Bearbeitung des MRK für interessierte Einrichtungen eine einführende Schulung anzubieten: „Dieses Instrument finde ich persönlich sehr sinnvoll, sehr sinnvoll, dass man mindestens eine Einführung hat und diese Einführung sollte sein, zum Beispiel dass man alle in Einrichtungen $\mathrm{Zu}$ ständigen einlädt, die für das Projekt verantwortlich [das MRK, Anm. d. Verf.] sind, kommen dahin, [...], da muss man wirklich einen Tag planen, [sich] damit auseinandersetzen, vorstellen, was ist damit gemeint, damit derjenige, der als Multiplikator, der dafür verantwortlich ist, Bescheid weiß.“

c) Aufwand: Inwieweit erleben die Leitungskräfte die Selbsteinschätzung als Mehraufwand oder gar Stressor?

Gleichwohl der Umfang des MRK - wie bereits erwähnt - als zunächst eher abschreckend eingeschätzt wurde, wirkte die Auseinandersetzung mit dem MRK für die Erprobungseinrichtung nicht als Stressor. So wurde insbesondere der bei einer initialen Bearbeitung des MRK nicht unerhebliche Arbeits- und Abstimmungsaufwand aus Sicht der Anwender*innen durch die dem Teilhabethema zugeschriebene Relevanz relativiert. Erwähnt wurde darüber hinaus, dass die Implementationsanalyse den Aufwand einer initialen MRK-Bewertung und -Implementation untersucht hat, nicht jedoch den Aufwand, den die Bearbeitung für spätere Selbsteinschätzungen anderer Einrichtungen bedarf, der nach Einschätzung der Erprobungseinrichtung deutlich geringer ausfallen dürfte: „Ich finde, wir haben uns ja jetzt sehr intensiv damit beschäftigt, weil wir ja auch Verbesserungsvorschläge machen 
sollen, das macht man ja dann nicht. Wir haben ja noch jede Frage zwei-, dreimal gelesen. Das wird ja in Zukunft so nicht sein."

Eine wesentliche zusätzliche Aufgabe und positive Folge, die sich über die konsequente MRK-Bearbeitung ergab, war die Rückkopplung zu und Anpassung von bestehenden Einrichtungskonzepten. So wurden MRK-Kriterien und deren Formulierung zusätzlich mit Passagen aus Konzepten der Einrichtung verglichen, insbesondere dann, wenn im Leitungsteam Unsicherheit hierzu Bestand, inwieweit bzw. wie die jeweiligen Aspekte in den Einrichtungskonzepten festgehalten und formuliert sind. Dieser Aufwand wurde aber von den betroffenen Personen der Lenkungsgruppe durchgängig als notwendig und förderlich eingeschätzt: „Weil, es reicht ja nicht, [...] wenn ich ein Konzept [...] Wenn ich jetzt sage, ich überführe das Konzept in die Wohnhäuser und das QM wird nicht mitgenommen, das geht eigentlich nicht. Es muss ja auch irgendwo, finde ich, verschriftlicht werden, dass es da 'ne Richtlinie gibt oder 'ne Verfahrensanleitung und dann muss ich auch gucken, dass das System darauf ausgerichtet ist. [...] Aber der Nutzen, der muss ja sein, sag ich immer, [...], würd' ich schon sagen, dass es sinnvoll ist. Ja, wenn man es dann noch ein bisschen besser strukturieren kann, denk ich mal, klar, also für mich war es schon gut, so 'nen Leitfaden zu haben“.

\subsubsection{Machbarkeit}

Den Rahmen zur Beurteilung der Machbarkeit modelliert in der Erprobungseinrichtung einerseits die Entscheidung, das Leitungsteam einrichtungsbereichsübergreifend im Lenkungsteam einzubeziehen und darüber hinaus auch die Mitarbeiter*innen in einer Mitarbeiter"innenversammlung für das Thema zu sensibilisieren. Andererseits hat sich als hilfreich erwiesen, dass im Rahmen der Lenkungsgruppe und der Bearbeitung des MRK ein besonderer Blick auf die Konzepte der Einrichtung und des Trägers über die Einbeziehung der Leitung des zentralen Qualitätsmanagements des Trägers erfolgte. Dadurch wurde die Machbarkeit in Bezug auf die Überführung von Anforderungen des MRK sowohl auf konzeptioneller als auch auf praktischer Ebene wesentlich unterstützt. Als Umsetzungsfaktoren und -barrieren wurden hier vor allem die Aspekte benannt, die auch bereits in den vorangegangenen Analysedimensionen der Akzeptanz und Angemessenheit als förderlich oder hemmend festgehalten wurden. 
a) Aufwand: Können wesentliche Anforderungen des MRK in eigene Konzepte, Verfahren, Instrumente etc. überführt werden?

Die Überführung von Anforderungen des MRK in eigene Konzepte, Verfahren, Instrumente etc. wurde als gut möglich eingeschätzt. So konnten schon vorhandene Konzepte unmittelbar mit dem Musterrahmenkonzept abgeglichen und im Bedarfsfall angepasst und schließlich sogar um ein neues Konzept erweitert werden. Auch wenn hiermit keine Erwartung oder Anregung seitens des STAP-Teams verbunden war, wurden also bereits in der Erprobungsphase entsprechende Konzeptanpassungen vorgenommen. Konkret wurde das „Leben-Sterben-Begleiten“-Konzept, das Betreuungsund Beschäftigungskonzept sowie das Pflege- und Betreuungskonzept überarbeitet. Darüber hinaus wurde ein eigenständiges, ausführlicheres Betreuungskonzept entwickelt, das sich teils ebenfalls an Anforderungen des MRK orientiert.

Des Weiteren wurde in der Erprobungseinrichtung im Rahmen der Projektlaufzeit von STAP nicht nur eine unmittelbar aus dem MRK abgeleitete Maßnahme implementiert, sondern auch weitere Veränderungen angeregt und eingeleitet, da während der Mitarbeiter"innenversammlung alle Mitarbeitenden noch einmal für das Thema sensibilisiert wurden. Konkret ergab sich während der Mitarbeiter"innenversammlung die Fragestellung, wie auch die Mahlzeitensituation noch teilhabeorientierter gestaltet werden könnte. In der Folge wurde vereinbart, dass nun der Koch an jedem Mittag in den Speiseraum kommen solle, um die Bewohner*innen nach dem Essen einzeln zu befragen, wie es ihnen geschmeckt habe, so dass für alle Bewohner*innen an jedem Mittag die Möglichkeit zu einer unmittelbaren Rückmeldung zu ihrer Zufriedenheit mit den Mahlzeiten besteht. Es zeigte sich also, dass die Beschäftigung mit dem Thema Teilhabe allein unmittelbar weitere teilhabefördernde Maßnahmen nach sich zog.

Auch der Aufwand für die konkret entwickelte teilhabefördernde Maßnahme - die Entwicklung und Implementation eines zusätzlichen Wochenplans - wurde auf Leitungsebene als überschaubar eingeschätzt. Wobei der Aufwand hier wesentlich von den ausgewäblten Kriterien und davon abgeleiteten Maßnahmen abhängig ist. Entscheidender ist, dass von den in den Kriterien formulierten Anforderungen für die Lenkungsgruppe naheliegend eine Maßnabme abzuleiten war, die den bestehenden Voraussetzungen entsprach und dort integrierbar war. 
b) Umsetzungsfaktoren und -barrieren: Welches sind die wichtigsten fördernden Faktoren und die stärksten Barrieren für die Implementation unter Routinebedingungen? Welche spezifischen Strategien reduzieren die identifizierten Barrieren und stärken die wichtigsten fördernden Faktoren?

\section{Fördernde Faktoren:}

- Als besonders bedeutsam für die Bearbeitung des MRK für die Selbsteinschätzung wurde von Seiten der Erprobungseinrichtung betont, dass das MRK nicht von einer Person allein (z. B. der Leitung) bearbeitet werden sollte. Da der Inhalt des Konzeptes „Teilhabe“ einrichtungsbereichsübergreifend von Bedeutung sei, sei auch eine Bearbeitungsstrategie erforderlich, die breit angelegt ist und alle Fachbereiche einbindet. So wurde die in der Einrichtung durchgeführte Bearbeitung des Konzeptes mittels eines Lenkungsteams, das die meisten Bereiche der Einrichtung erfasste, realisiert. Die Bereiche, die nicht einbezogen wurden (im aktuellen Fall Küche und Haustechnik), müssen aber in dem Prozess mitgedacht werden, weil sie unter Umständen entscheidende Rollen für die selbstbestimmte Teilhabe der Bewohner*innen spielen können. So wurden mehrfach Rücksprachen mit dem Küchenchef gehalten, um die Teilhabekriterien und später -anliegen und -maßnahmen zu diskutieren. Die Strategie zur Bearbeitung des MRK lässt sich auf die „Kurzformel“ bringen, so viele Bereiche wie nötig, so unaufwändig wie möglich einzubeziehen. Als ideale Bearbeitungsteamgröße haben sich in dieser Einrichtung Teams von 2 bis 3 Mitarbeiter*innen bewährt, ergänzt durch Besprechungen des gesamten Lenkungsteams.

- Die Strukturierung des Vorgehens und die gewählte Bearbeitungs- und Kommunikationsstrategie stellte sich als entscheidend und sehr förderlich für die weitere Arbeit mit dem Konzept und dem Ergebnis der Selbsteinschätzung heraus, denn damit gelang es, dass alle betroffenen Mitarbeiter*innen für das Thema sensibilisiert und so nicht nur die einzelne teilhabefördernde Maßnahme, sondern auch die diesen zugrunde liegenden Ideen für alle Betroffenen transparent gemacht werden konnten. Dieses wurde in der Erprobungseinrichtung dadurch sichergestellt, dass in einer eigens dazu durchgeführten Mitarbeiter*innenversammlung alle Mitarbeiter*innen über das Thema Teilhabe informiert und in Bezug auf ihre eigene Arbeit sensibilisiert sowie die geplanten Maßnahmen in diesem Forum diskutiert wurden.

- Über eine initiale Mitarbeiter*innenversammlung hinaus sollte das Thema Teilhabe, um es im Bewusstsein der Mitarbeiter*innen präsent zu 
balten, nach Ansicht der Interviewten in regelmäßig stattfindenden $B e$ sprechungen und Versammlungen immer wieder aufgegriffen werden.

- Als entscheidend für den nachhaltigen Erfolg teilhabefördernder Maßnahmen wird betont, wie wichtig es sei, dass die einzelnen Mitarbeiter"innen eine flexible und offene Arbeitshaltung entwickeln, die sich aus der Orientierung an individuellen Teilhabewünschen der Bewohner*innen ergibt. Dies bedeutet unter Umständen, bewährte Arbeitsroutinen aufgeben zu müssen, um jeweils bedarfs- und situationsgerecht reagieren und terminieren zu können.

- Nachdem Maßnahmen aus einzelnen Konzeptkriterien abgeleitet worden waren, hat es sich als sinnvoll erwiesen, diese nicht nur zu oktroyieren, sondern vorab mit den betroffenen Mitarbeiter"innen gemeinsam zu diskutieren, noch einmal zu hinterfragen und gegebenenfalls zu modifizieren.

- Als zentral für eine nachhaltige Implementation des MRK zeigte sich der Abgleich mit vorhandenen Konzepten der Einrichtung und Trägerorganisation, um zu überprüfen, inwieweit Teilhabeorientierung auch in diesen bereits verankert ist oder an welchen Stellen Präzisierungen und Ergänzungen sinnvoll sind. Um dies zu gewährleisten, hat es sich in der Erprobungsphase bewährt, die Leitung des zentralen Qualitätsmanagements der Trägerorganisation eng in den Bearbeitungsprozess einzubeziehen; denkbar wäre aus Sicht der Diskussionsteilnehmenden aber auch die Einbeziehung einer QM-Beauftragten auf Einrichtungsebene.

\section{Barrieren:}

- Als wesentliche konzeptbezogene Barriere wurde der Umfang des Konzeptes benannt. Als Orientierungsgröße für einen angemessenen Umfang wurde von der Erprobungseinrichtung ein Maximum von 30 Seiten, wie für andere Konzepte der Trägerorganisation, vorgeschlagen.

- Einrichtungsintern zeigte sich dann auch der Faktor Zeit als Barriere der initialen Bearbeitung des Musterrahmenkonzeptes. Die im Rahmen der Erprobung noch intensivere Bearbeitung aller Konzeptkriterien und die damit einhergehende Abstimmungsnotwendigkeit hat sich hier jedoch als wesentliche Barriere offenbart. Wobei sich anhand des Erprobungsbeispiels auch andeutet, dass eine frühzeitige Einbeziehung aller Mitarbeiter*innengruppen und ein Abgleich mit bereits vorhandenen Pflege- oder Betreuungskonzepten dazu beitragen kann, auf Dauer das Thema Teilhabe im Bewusstsein aller Mitarbeiter*innen zu verankern und so die zu investierende Arbeitszeit zu minimieren. Als zeitintensiv 
beschrieben die Anwender*innen auch die Orientierung im Konzept selbst, also häufiges Vor- und Zurückblättern.

- Als Bearbeitungsbarrieren innerhalb des MRK wurde als größtes Problem sein damaliges Format, als Dokument auf Basis eines Textverarbeitungsprogramms, kritisiert. Seitens der Erprobungseinrichtung wurde angemerkt, dass etwa eine verschlagwortete digitale Fassung eine raschere Orientierung in der Vielzahl der Kriterien ermöglichen würde. Darüber hinaus wurde vorgeschlagen, das Konzept noch konsequenter zu strukturieren und zu modularisieren, so dass in der Bearbeitung einzelne inhaltliche Schwerpunkte noch gezielter angesteuert werden können.

- Aus Sicht der Erprobungseinrichtung wurde in Zweifel gezogen, dass eine Altenpflegeeinrichtung allein mittels des Konzeptes und einer kurzen schriftlichen Einführung das MRK bearbeiten könne. Es wurde eine (eintägige) Einführungsschulung für Multiplikator*innen in Altenpflegeeinrichtungen nahegelegt.

\subsubsection{Ergebnisse der Erhebung auf der Mitarbeiter*innenebene (Kurzinterviews)}

Nach der Fokusgruppendiskussion konnten im Rahmen von Kurzinterviews acht Mitarbeiter"innen zu ihren Erfahrungen mit der im Erprobungszusammenhang entwickelten teilhabefördernden Maßnahme - der Entwicklung und Bereitstellung eines bewohner*innenbezogenen Wochenplans - befragt werden. Die nachfolgend referierten Ergebnisse beziehen sich somit ausschließlich auf die Bewertung der aus dem MRK abgeleiteten und schließlich implementierten teilhabefördernden Maßnahme und nicht auf die Bearbeitung des MRK an sich. Die Daten wurden, wie oben beschrieben, mittels eines Kurzinterviews erhoben, bei dem die einleitenden qualitativen Leitfadensequenzen für alle Befragten identisch gestaltet waren. Für den quantitativen Interviewabschnitt unterschieden sich die angelegten Itembatterien nur in den ersten beiden Fragen, die weiteren Fragen waren identisch. Die Einschätzungen der befragten Mitarbeiter"innen bezüglich des neuen Wochenplanes sind in folgender Abbildung 17 angeführt. ${ }^{81}$

81 Die Tabelle umfasst die Angaben aller interviewten Personen $(\mathrm{n}=8)$. Die abgebildeten Items entsprechen der Itembatterie für die Mitarbeiter*innen der Wohnbereichsleitung, Pflege und die zusätzlichen Betreuungskräfte. Hier lautete die erste Frage „1. Ich habe mit dem neuen Plan einen besseren Überblick darüber, wel- 


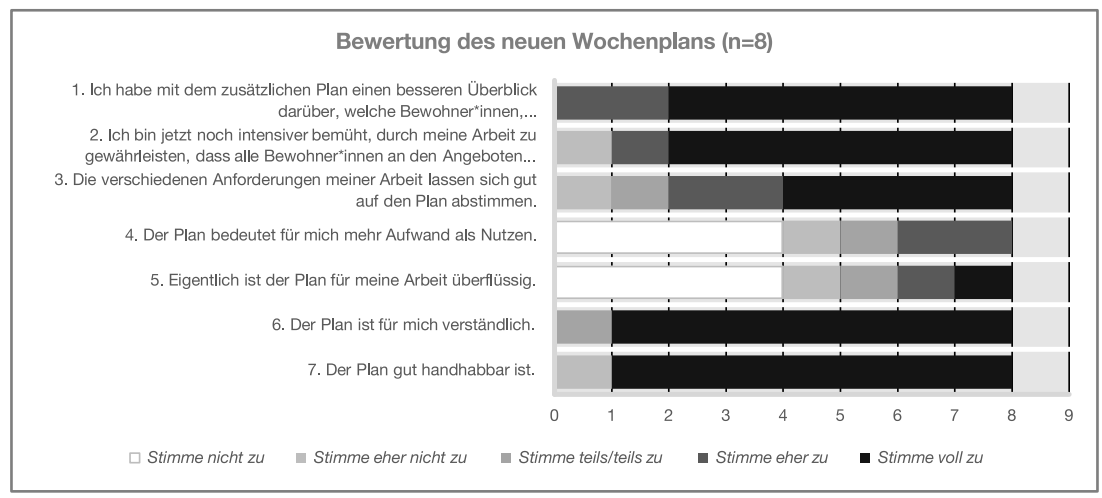

\section{Abbildung 17: Bewertung des neuen Wochenplans}

Es wird demnach deutlich, dass die Mitarbeiter*innen die implementierte Maßnahme für verständlich und sinnvoll hielten sowie in hohem Maße befürworteten. Bei den Mitarbeiter"innen des Sozialen Dienstes war insbesondere der Aspekt interesseleitend, inwiefern sie den Eindruck hätten, dass der neue Wochenplan dazu beitrüge, dass die Bewohner*innen, die dieses wünschen, nun regelmäßiger an den Angeboten des Sozialen Dienstes teilnähmen, was diese bestätigten. So zeigte sich, dass der intendierte Nutzen der Maßnahme tatsächlich eingetreten war.

Die qualitativen Leitfadensequenzen der Kurzinterviews wurden analog zum Fokusgruppeninterview anhand der Kategorien Akzeptanz, Angemessenheit und Machbarkeit ausgewertet. Dabei ergaben sich nachstehende Ergebnisse.

\subsubsection{Akzeptanz}

Insgesamt ließ sich auf Seiten aller befragten Mitarbeiter"innen eine ausgeprägte Einstellungs- wie Verhaltensakzeptanz in Bezug auf die implementierte

che Bewohner*innen, wann, an welchen Angeboten des Sozialen Dienstes teilnehmen möchten." Die Itembatterie für den Sozialen Dienst unterschied sich davon, indem hier zwei Items mit der umgekehrten Perspektive wie folgt aufgenommen wurden: 1. „Ich habe den Eindruck, dass die Mitarbeiter*innen der Pflege jetzt besser über unsere Angebote informiert sind“ und 2. „Ich habe den Eindruck, dass die zusätzlichen Betreuungskräfte jetzt besser über unsere Angebote informiert sind“. 
teilhabefördernde Maßnahme nachweisen. Dabei wurde deutlich, dass die Akzeptanz in diesem Erprobungsfall über das Kriterium „Informationen über Teilhabe" moderiert wurde. Die hohe Akzeptanz der Maßnahme ist nach Einschätzung der Forschenden darauf zurückzuführen, dass alle Mitarbeiter*innen über das Thema Teilhabe informiert wurden und die Mitarbeiter*innen somit den neuen Wochenplan als sinnvoll für die Teilhabe der Bewohner*innen und ihrer Förderung einschätzten. Auf Nachfrage konnten zum Beispiel alle Befragten Bezüge ihrer eigenen spezifischen Arbeitsaufgabe zum Thema Teilhabe herstellen. Sie schrieben in den Leitfadeninterviews dem Thema Teilhabe eine hohe Relevanz zu, ordneten die neue Maßnahme entsprechend thematisch zu und erachteten sie als sinnvoll. So etwa eine Pflegemitarbeiterin: „Es gibt Bewohner, die nicht zu vielen Aktivitäten gehen, aber die viel Wert darauf legen, immer auf die gleichen Aktivitäten zu gehen. Und dann weiß ich, dass die dahin gehen möchten oder wo die sich befinden [...] Der Plan ist eine gute Übersicht für alle". Die Verständlichkeit des Plans wurde im Interviewteil auch positiv hervorgehoben. So beschrieben die Befragten die Orientierung durch den Plan als sehr gut und die Informationen als auf einen Blick erfassbar und somit gut in ihre Arbeitsroutinen integrierbar. Dabei wurde deutlich, dass den Mitarbeiter"innen ebenfalls bewusst war, dass Teilhabe nach dem Prinzip der Selbstbestimmung auch eine Entscheidung zur Nichtteilnahme umfassen kann: „Ich schaue dann auf den Plan und frage dann zum Beispiel die Bewohner: ,Möchten Sie heute in die Kirche gehen, oder nicht?"“ (Pflege).

Die Motivation der Mitarbeiter*innen aus der Pflege und der Bewohner*innenassistenz ließ sich deutlich ausmachen. Sie formulierten den Eindruck, dass der Plan ihnen einerseits die Arbeit erleichtere und andererseits die Zufriedenheit und Teilhabechance der Bewohner*innen verbessere. Nur einzelne Mitarbeiter*innen berichteten, die Teilnahmewünsche der Bewohner*innen an den Angeboten des Sozialen Dienstes auch vor der Implementierung des neuen Wochenplanes stets für ihre Arbeitsplanung berücksichtigt zu haben, weshalb sie diesem eine niedrigere Relevanz zuschrieben. Die Befragten des Sozialen Dienstes interpretierten die Maßnahme als unmittelbare Unterstützung ihrer eigenen Arbeit, weshalb sie ihn entsprechend motiviert einsetzen und aktualisieren. 


\subsubsection{Angemessenheit}

Das Teilhabezugangsproblem, das mit dem zusätzlichen Wochenplan gelöst werden sollte, nämlich zu gewährleisten, dass alle Bewohner*innen, die dies wünschen, an den Angeboten des Sozialen Dienstes teilnehmen können, wurde aus Sicht der betroffenen Mitarbeiter*innen effizient und einfach verständlich gelöst. Die Passung der Maßnahme ist entsprechend den Interviewaussagen offenbar gegeben, weil sich der Wochenplan angemessen in die Arbeitsroutinen einfügt, sich mit einem Blick erfassen lässt und von allen Interviewten als übersichtlich und gut handhabbar eingeschätzt wird. Keine der befragten Personen hat den Plan als zusätzliche Belastung beschrieben, sondern vielmehr als, ,hilfreiche Übersicht und Gedächtnisstütze“. Eine interviewte Person aus dem Bereich der Pflege fasste ihre Einschätzung wie folgt zusammen: „Eigentlich ist der Plan sehr gut aufgenommen worden, weil der auch farblich gekennzeichnet ist, mit den Gruppen, und eigentlich kommt jeder damit super zurecht."

Aus der Perspektive der Mitarbeiter*innen des Sozialen Dienstes zeigte sich, dass für sie, obwohl sie für die Aktualisierung und das wöchentliche Ausdrucken des Planes verantwortlich sind, der Nutzen wesentlich höher als der Aufwand ist. Als Nutzen und in gewisser Weise auch entlastend empfinden sie an der Maßnahme, dass Bewohner*innen nun tatsächlich regelmäßiger an den Angeboten teilnehmen: „Ich kann feststellen, dass manche Bewohner, die gerne teilgenommen hätten, damals [in der Zeit vor der Planeinführung, Anm. d. Verf.] so ein bisschen vernachlässigt worden sind, d. h. dass der Wunsch, obwohl bekannt, nicht immer erfüllt worden ist $[. . .$,$] wo ich dann doch gemerkt habe, jetzt mit diesem Plan, jetzt$ klappt's bei denen, bei denen es nicht immer klappte, jetzt besser."

\subsubsection{Machbarkeit}

Die Berücksichtigung des Wochenplans hat sich für die betroffenen Mitarbeiter*innen bereits verstetigt, sie beziehen ihn selbstverständlich in ihre Arbeitsplanung und zur Orientierung über den aktuellen Aufenthaltsort der Bewohner"innen ein, wie eine Person aus der Pflege anmerkt: „Morgens guckt man auf den Plan drauf, um dann halt zu gucken, wer zu wem geht. Und dann muss man halt danach abwägen.“ 
Fördernde Faktoren:

- Als ausschlaggebender Faktor bei der erfolgreichen Einführung der Maßnahme hat sich die Sensibilisierung der Mitarbeiter*innen für das Thema Teilhabe über die Mitarbeiter*innenversammlung herausgestellt, denn dadurch wurde die Intention einer solchen Maßnahme für die Mitarbeiter*innen nachvollziehbar und dessen Akzeptanz erhöht. Für die Zukunft ist geplant, in regelmäßig stattfindenden Besprechungen das Thema und die Entwicklung der Einrichtung in dieser Hinsicht immer wieder aufzugreifen und zu hinterfragen.

- Die Akzeptanz der implementierten Maßnahme hängt im Falle der erprobten Maßnahme zudem unmittelbar mit ihrer niedrigschwelligen Passung zusammen, d. h. die betroffenen Mitarbeiter*innen können diese gut - ohne Reibungsverluste - in ihr Arbeitshandeln integrieren und fühlen sich durch die Maßnahme nicht von anderen Aufgaben abgehalten.

- Seitens aller Mitarbeitenden ist eine offene Haltung im Hinblick auf die Teilhabewünsche der Bewohner*innen und ihrer Förderung ausschlaggebend für den Erfolg oder Misserfolg teilhabefördernder Maßnahmen. Diese bedingt eine entsprechende Flexibilität im Arbeitshandeln.

\section{Barrieren:}

- Von einzelnen Befragten wurde geäußert, dass das Befolgen der Angaben im neuen Wochenplan kein Problem sei, dass diese jedoch durchaus auch mit anderen, die jeweiligen Bewohner*innen betreffenden Anforderungen konkurrieren könnten, wie etwa Arztbesuche, Angehörigenbesuche o. Ä., die dann aber nicht in dem Plan erfasst bzw. auch gar nicht in der Form erfassbar wären.

\subsection{Zwischenfazit zur Erprobung}

Die Erprobungsphase hat dazu beigetragen, das MRK auf seine Alltagstauglichkeit in Einrichtungen der stationären Altenhilfe zu hinterfragen. Als noch einmal zu überdenkende Faktoren traten der Umfang und das (Medien-)Format des MRK hervor. Seitens der Erprobungseinrichtung wurde deshalb vorgeschlagen, manche Kriterien jeweils zu einem Kriterium zusammenzufassen. Zudem wurde gewünscht, das Konzept zu digitalisieren, um es mit Schlagworten und mit einer Suchfunktion ausstatten zu können, damit eine raschere, gezielte Orientierung im Konzept möglich wäre. Darüber hinaus wurde eine noch stärker inhaltlich strukturierte, mo- 
dularisierte Form vorgeschlagen, so dass im Einrichtungsalltag nicht immer mit dem Gesamtkonzept umgegangen werden müsste. Auf Grundlage dieser Hinweise aus der Implementationsanalyse in der Erprobungseinrichtung ebenso wie durch die Anregungen aus den Projektgremien erfolgten sukzessive mehrere Konzeptmodifizierungen im letzten Projekthalbjahr (siehe Kapitel 7.2 und Anhang).

Bewährt hat sich das MRK in der Erprobungsphase insbesondere in folgenden Bereichen:

1. Es gewährt einen umfassenden Einblick in verschiedenste Aspekte von Teilhabeorientierung in Altenpflegeeinrichtungen. Durch seine zahlreichen Beispiele bietet es Anregungen für einfach umsetzbare, teilhabefördernde Maßnahmen.

2. Der Ist-Stand einer Einrichtung in Bezug auf die Teilhabeorientierung lässt sich mit dem MRK gut diagnostizieren. Die Möglichkeit, die Einschätzung im Konzept mit eigenen Kommentaren zu versehen wurde, als besonders hilfreich für den nachhaltigen Einsatz angesehen.

3. Die Ableitung von teilhabefördernden Maßnahmen ist mittels des Konzeptes leicht möglich. Darüber hinaus kann die mit der Bearbeitung einhergehende Sensibilisierung für das Thema zahlreiche weitere, oft mit geringem Aufwand realisierbare Maßnahmen nach sich ziehen.

4. Bei vorheriger Information der betreffenden Mitarbeiter*innen lässt sich eine sehr hohe Einstellungs- wie Verhaltensakzeptanz teilhabeorientierter Maßnahmen, die aus dem MRK abgeleitet werden, erzielen.

Für die erfolgreiche Arbeit mit dem MRK hat sich als ganz entscheidend dessen Bearbeitung in einem Lenkungsteam, in dem alle entscheidenden Fachbereiche der Einrichtung beteiligt waren, herausgestellt. Darüber hinaus war die Sensibilisierung aller Mitarbeiter*innen für das Thema selbstbestimmte Teilhabe und ein transparentes Vorgehen bei der Maßnahmenumsetzung entscheidend. Zudem wurde deutlich, dass aus dem MRK abgeleitete teilhabefördernde Maßnahmen mitunter verhältnismäßig unaufwändig entwickelt und gut in den Arbeitsalltag integriert werden können. 


\section{Schlussbetrachtung und Ausblick}

In diesem abschließenden Kapitel werden die zentralen empirischen Ergebnissen der STAP-Studie mit Fokus auf die fördernden Faktoren selbstbestimmter Teilhabe resümiert, ein Ausblick zu dem darauf aufbauenden Musterrahmenkonzept vermittelt sowie Empfehlungen an die Politik und weiterer Forschungs- und Entwicklungsbedarf festgehalten.

\subsection{Fördernde Faktoren selbstbestimmter Teilhabe in Altenpflegeeinrichtungen}

Die leitenden Fragstellungen im Rahmen des Projektes STAP lauteten: (1.) Wie können Wünsche und Bedürfnisse von Bewohner*innen stationärer Pflegeeinrichtungen in Bezug auf gesellschaftliche Teilhabe innerhalb und außerhalb der Einrichtung festgestellt und berücksichtigt werden? (2.) Wie kann auf dieser Basis das Recht auf selbstbestimmte Teilhabe dieser Bewohner*innen praktisch verwirklicht und dessen Umsetzung überprüft werden? An diesen beiden Fragen hat sich STAP im Rahmen der empirischen Analysen übergeordnet orientiert, wie in den jeweiligen Untersuchungsbausteinen angeführt - von der Literaturrecherche zum Forschungsstand, über die Wahl der Forschungsmethoden, der Gestaltung der Erhebungsinstrumente und Auswertungskategorien bis zur Einordnung der Forschungsergebnisse.

Im Rahmen der STAP-Studie konnte auf Grundlage eines explorativ ausgerichteten und sequenziell aufgebauten Mixed-Methods-Designs - auch in Anschluss an bislang vorhandene theoretisch-konzeptionelle Überlegungen (Garms-Homolová \& Theiss, 2009b, S. 190) - empirisch fundiert und ausdifferenzierend beleuchtet werden, dass die Förderung der selbstbestimmten Teilhabe von Bewohner*innen in Altenpflegeeinrichtungen potenziell von einer Vielzahl von Faktoren auf unterschiedlichen Ebenen abhängig ist, die sich gegenseitig beeinflussen und deren konkrete Bedeutung personen- und kontextgebunden variieren kann. Diese Vielschichtigkeit ist insofern wenig verwunderlich, weil eine Verwirklichung von Teilhabe nach dem Prinzip der Selbstbestimmung an und für sich mit Bedingungen und Voraussetzungen verbunden ist, die sich individuell - bei den Bewohner*innen, den Mitarbeiter"innen und in der jeweiligen Einrichtung sowie deren Umfeld - unterscheiden (können) und in Wechselbezie- 
hung zueinander stehen. Prozesse der Teilhabeförderung sind demnach prinzipiell von Komplexität, Individualität und Offenheit gekennzeichnet, die sich der Logik verallgemeinerbarer linearer Kausalzusammenhänge entziehen und somit typischerweise auch mit dem Begriff des „Technologiedefizits" (Luhmann \& Schorr, 1982; Spiegel, 2013) verbunden werden können. In diesem Sinne ist das auf die Verwirklichung von selbstbestimmter Teilhabe bezogene professionelle Handeln in Altenpflegeeinrichtungen also potenziell so vielschichtig, dass sich die finalen Zusammenhänge zwischen Vorgehensweisen und Ergebnissen nicht durchgängig planmäßig herstellen oder wiederholen lassen (Spiegel, 2013, S. 31).

Vor diesem Hintergrund lässt sich nachvollziehen, dass die Ergebnisse von STAP nicht auf einem theoretischen Modell basieren, das kausale Verbindungen zwischen einzelnen, wenigen Faktoren herstellt. Demgegenüber markieren die empirischen Ergebnisse von STAP einerseits - mit dem Blick auf die Äußerung, Erfassung, Berücksichtigung und Verwirklichung von Bewohner*innenwünschen zur Teilhabe - einen zentralen Ausgangspunkt und Kernprozess der Teilhabeförderung sowie andererseits - mit Bezug auf die Ebenen der Bewohner*innen, Mitarbeitenden sowie Einrichtungsstrukturen und -prozesse - ein elaboriertes Set an Erklärungsfaktoren für eine gelingende Umsetzung selbstbestimmter Teilhabe von Bewohner*innen in Altenpflegeeinrichtungen.

Mit einer anwendungsorientierten Zielsetzung erfolgte die Forschung einerseits mittels des qualitativen Zugangs primär aussagenorientiert und inhaltsanalytisch in entsprechender Breite der identifizierten Inhalte, ohne Anspruch auf Vollständigkeit. Andererseits wurden der Kernprozess und die identifizieren Faktoren aber in ihrer Relevanz - und ihrer Breite - sowohl durch den quantitativen Zugang als auch durch ergänzende Formate der Validierung (z. B. über Lernworkshops mit den Projekteinrichtungen, Projektgremien, trägerübergreifende Fokusgruppen, eine Jahrestagung von Bewohner"innenbeiräten sowie Fachtagungen mit Praktiker*innen und Wissenschaftler*innen) multimethodisch und mehrperspektivisch ausdrücklich bestätigt. Eine Übersicht zu den über diese Zugänge als relevant identifizierten Ergebnisbereichen bietet nun die folgende Abbildung. 


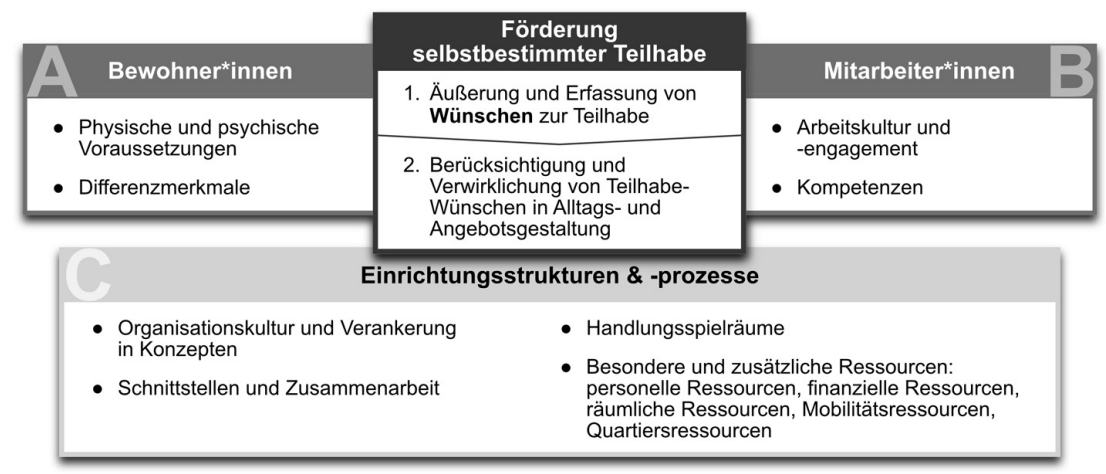

\section{Abbildung 18: Kernprozess sowie Faktoren zur Förderung selbstbestimmter Teilhabe}

Im Zentrum der Abbildung stehen die beiden wesentlichen Phasen im Prozess zur Förderung selbstbestimmter Teilhabe, die bei aller Komplexität, Individualität und Offenheit des Fördergegenstands festgehalten werden können: (1) Die Äußerung und Erfassung von Wünschen zur Teilhabe der Bewohner*innen. ${ }^{82}$ (2) Die Berücksichtigung und Verwirklichung der Teilhabewünsche in der Alltags- und Angebotsgestaltung. Auch wenn diese Unterscheidung im Praxishandeln nicht immer chronologisch trennscharf ist (etwa wenn geäußerte Teilhabewünsche situativ unmittelbar umgesetzt werden), kann sie schematisch als prototypische Abfolge der zentralen Prozessphasen in der Teilhabeförderung von Bewohner*innen in stationären Altenpflegeeinrichtungen betrachtet werden, die sich empirisch als relevant herausgestellt haben.

\section{1. Äußerung und Erfassung von Wünschen zur Teilhabe}

Die individuellen Wünsche der Bewohner*innen zur Teilhabe sind der Ausgangspunkt zur Verwirklichung ihrer selbstbestimmten Teilhabe, da eine Förderung von Teilhabe nach dem Prinzip der Selbstbestimmung vor-

82 In dieser abschließend fokussierten Darstellung der fördernden Voraussetzungen zur Verwirklichung selbstbestimmter Teilhabe werden die Wunschäußerung und -erfassung als zusammenhängende Schritte in einer Prozessphase betrachtet, die bestenfalls aufeinander aufbauen, gleichwohl sie in der Ergebnisdarstellung in Kapitel 4.1.2 als zwei getrennte Prozessphasen näher beleuchtet wurden. 
aussetzt, dass individuelle Wünsche berücksichtigt werden, die zuvor von den Bewohner*innen verbal geäußert oder situativ nonverbal (z. B. durch Mimik, Gestik) angezeigt wurden. Zu berücksichtigen ist, dass die Motivationen und Möglichkeiten zur Äußerung von Teilhabewünschen bei den Bewohner*innen durch verschiedene Voraussetzungen eingeschränkt sein können (z. B. gesundheitliche Einschränkungen, Bescheidenheit, wahrgenommenes Abhängigkeitsverhältnis). Daher sollten - entsprechend den Voraussetzungen der Bewohner"innen - verschiedene Formen der Einbeziehung genutzt werden, indem entweder Anlässe zur Wunschäußerung gegeben oder Angebote zur Teilhabe sensibel offeriert werden und deren Akzeptanz gedeutet wird. Letztlich kann nur sichergestellt werden, dass Menschen mit Pflegebedarf auch im Kontext von Altenpflegeeinrichtungen einen gleichberechtigten Zugang zu allen Teilhabeleistungen haben, wenn sie vom Tag ihres Einzugs an systematisch und kontinuierlich nach ihren Wünschen gefragt werden bzw. auf ihre Wunschäußerungen geachtet wird. Sie bewusst zu motivieren, ihre Wünsche zu äußern, ist sinnvoll, um einem möglichen Anpassungsverhalten von Bewohner"innen an empfundene oder existierende institutionelle Bedingungen und Abläufe von Seiten der Mitarbeitenden entgegenzuwirken. Zudem können eine sensible Unterstützung der Wunschäußerung oder eine Weckung von Wünschen bei Bewohner*innen förderlich sein, die von sich aus keine Wünsche äußern (können). Allerdings ist auch der selbstbestimmte Wunsch der Nichtteilnahme an Teilhabeangeboten ausdrücklich zu achten. Außerdem kommt dem Bewohner*innenbeirat als Selbstvertretungsorgan der Bewohner*innen bei der Äußerung und auch späteren Erfassung von Wünschen $\mathrm{zu}$ teilhabeorientierten Angeboten und Leistungen der Einrichtung eine besondere Rolle zu. Da sich aber auch gezeigt hat, dass die Partizipation von Bewohner*innen im Rahmen des Bewohner*innenbeirates und dessen Angebote (z. B. Sprechstunde für Bewohner"innen) in der Praxis aufgrund der veränderten Bewohner*innenstruktur tendenziell abnimmt oder diffiziler wird, sind hier mitunter kreativere Lösungen gefragt. Folgende Faktoren können die Wunschäußerung ${ }^{83}$ von Bewohner*innen fördern:

83 Hier und im Folgenden wird eine Auswahl der in der Studie zentral identifizierten - in Bezug auf die selbstbestimmte Teilhabe von Bewohner*innen - fördernden Faktoren aufgelistet. Auf eine Darstellung der gleichermaßen identifizierten hemmenden Faktoren wird verzichtet (siehe hierzu v. a. die Kapitel 4 und 5). 
Wunschäußerung - fördernd, zum Beispiel: Bewohner*innen- und Biografieorientierung in Alltagsgestaltung und Angebotsplanung / fortlaufende partizipative Gespräche in Bezug auf Teilhabewünsche / Schaffung konkreter Anlässe zur Wunschäußerung wie etwa partizipative Angebotsplanungen / Vorschläge von konkreten Angeboten und deren Abstimmung / bei eingeschränkter Kommunikationsfähigkeit sensibles Anbieten und Erproben von Teilhabeangeboten.

Äußerungen zu Teilhabewünschen von Bewohner*innen können in verschiedenen Situationen stattfinden, in denen die Initiative von den Bewohner*innen, Angehörigen oder Mitarbeitenden erfolgt. Geäußerte Wünsche von Bewohner*innen zur Teilhabe sollten aber in der Einrichtung regulär erfasst werden, um eine Teilhabeförderung dementsprechend planen, koordinieren und umsetzen zu können. Dass für die Erfassung von Bewohner*innenwünschen grundsätzlich genügend Kontexte und Verfahren in den Einrichtungen existieren, soll mit der folgenden Abbildung nochmal zum Ausdruck gebracht werden. Dort sind zentrale formelle und informelle Erfassungsformen in Altenpflegeeinrichtungen im "Zeitverlauf“ aufgeführt, die mit unterschiedlichen Zielsetzungen Voraussetzungen, Wünsche und Bedürfnisse von Bewohner*innen erfassen und mit denen prinzipiell auch Wünsche zur Teilhabe erfasst werden können. 
7.1. Fördernde Faktoren selbstbestimmter Teilhabe in Altenpflegeeinrichtungen

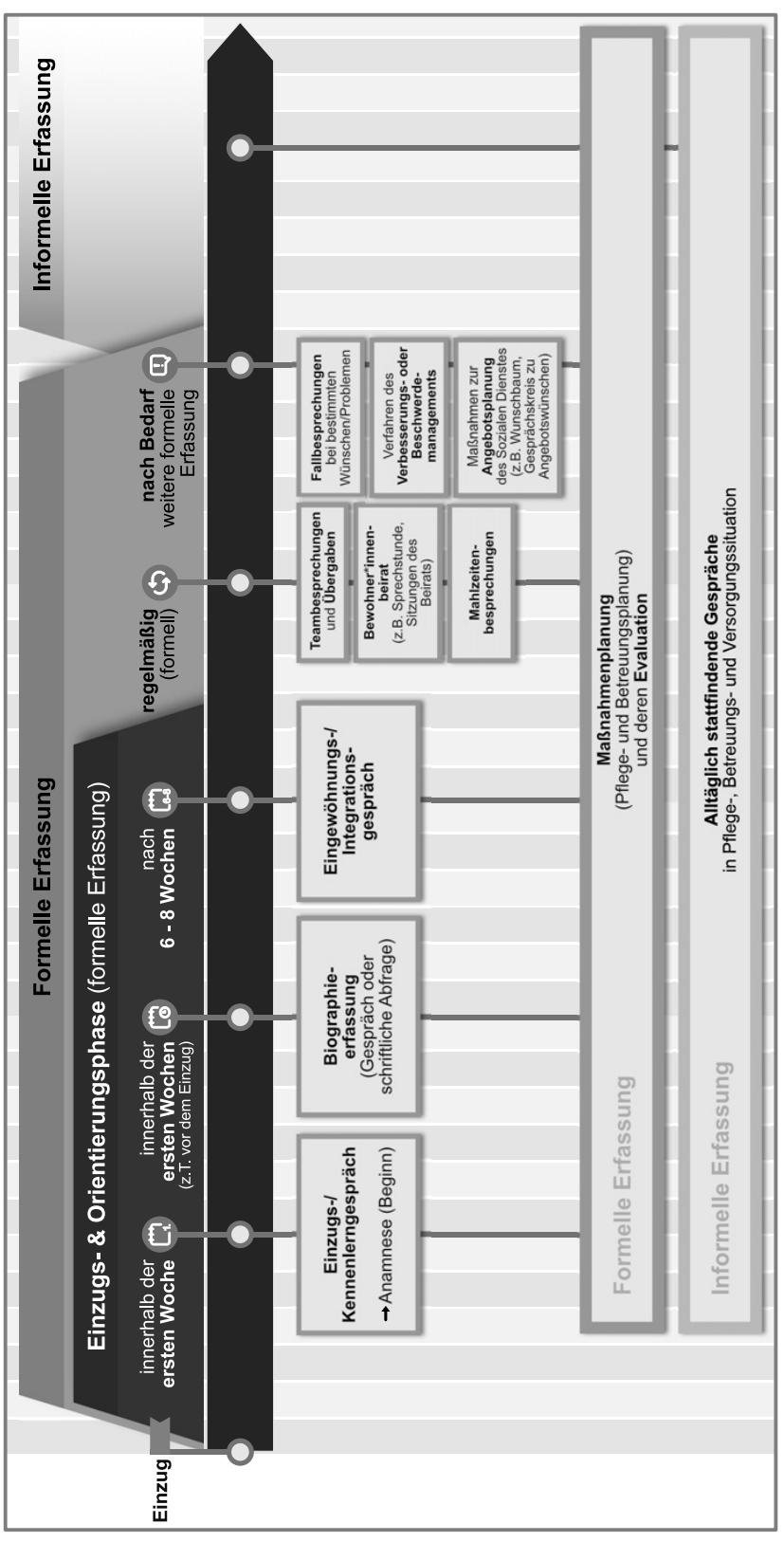

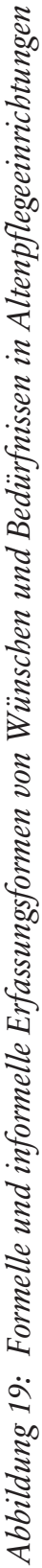


Somit können Wünsche von Bewohner*innen in der Einzugs- und Orientierungsphase (z. B. über Einzugs-, Biografie- und Eingewöhnungsgespräche) prinzipiell sehr ausgeprägt erfasst sowie potenziell auch fortlaufend in der Maßnahmenplanung (z. B. Pflege- und Betreuungsplanung) berücksichtigt werden. Außerdem können Bewohner*innenwünsche im Rahmen des Besprechungswesens (z. B. bei der Übergabe) sowie in speziellen Angeboten des Bewohner"innenbeirats (z. B. Sprechstunde) und der Hauswirtschaft (z. B. Mahlzeitenbesprechung) von Bewohner*innen regelmäßig angesprochen werden. Darüber hinaus existieren verschiedene Kontexte der formellen Erfassung, die eher nach Bedarf und unregelmäßig erfolgen (z. B. Fallbesprechungen, Verfahren des Verbesserungs- oder Beschwerdemanagements und Angebote zur Maßnahmenplanung des Sozialen Dienstes).

Vor diesem Hintergrund ist nach den Ergebnissen der vorliegenden Studie festzuhalten, dass kein Bedarf besteht, neue Instrumente der Wunscherfassung zu entwickeln. Entscheidend ist jedoch, dass individuelle Wünsche zur Teilhabe in den vorhandenen Instrumenten auch ausdrücklich berücksichtigt und routinemäßig dokumentiert werden. Bislang werden aber Aspekte der Pflege und Versorgung regulärer erfasst (z. B. in Instrumenten der Pflegeprozessplanung) und thematisiert (z. B. in Übergaben) als Teilhabethemen. Auch wenn eine Feststellung von Teilhabewünschen informell erfolgt und eine informelle Weitergabe dieser Information an aktuell zuständige Mitarbeitende sinnvoll ist, sollten die festgestellten Wünsche danach dennoch dokumentiert werden, damit die Informationen langfristig und für alle Mitarbeitenden festgehalten sind. Grundlegend ist, den Umgang mit Wünschen als einen andauernden und offenen Prozess zu verstehen, der methodisch über Biografiearbeit und bei Bedarf über Angehörigengespräche unterstützt werden kann, aber stets die Aktualität von Wünschen der Bewohner*innen zur Teilhabe in der Wunscherfassung berücksichtigt. Teilhabefördernd ist folglich auch, wenn die Erfassung von Teilhabewünschen von den Mitarbeitenden bereichsübergreifend als Teil der wahrgenommenen Aufgaben verstanden wird.

Wunscherfassung - fördernd, zum Beispiel: Sensibilisierung der Mitarbeiter*innen durch Leistungskräfte / Wunscherfassung als Teil der wahrgenommenen Aufgaben aller Mitarbeiter*innen / prinzipielle Dokumentation von wahrgenommenen Wünschen / Abstimmung zwischen Mitarbeitenden verschiedener Schichten und Einrichtungsbereiche / Biografiearbeit / fortlaufende bzw. aktualisierte Wunscherfassungen / Kontaktpflege zu Angehörigen, wenn Bewohner*innen sich nicht mehr selbst äußern können. 
2. Berücksichtigung und Verwirklichung von Teilhabewünschen in Alltags- und Angebotsgestaltung

Die zweite Prozessphase in der Teilhabeförderung beinhaltet, dass die erfassten Wünsche zur Teilhabe von Bewohner*innen im Kontext der Alltags- und Angebotsgestaltung - je nach Teilhabewunsch wiederholend oder einmalig - tatsächlich berücksichtigt und verwirklicht werden. Die Berücksichtigung der Wünsche meint hier, dass die erfassten Wünsche in der Planung und Vorbereitung von Teilhabeangeboten beachtet werden, während sich die Verwirklichung dann auf die eigentliche Realisierung des gewünschten Teilhabeangebotes in der Alltags- und Angebotsgestaltung bezieht.

Die Alltags- und Angebotsgestaltung beinhaltet hier alle Kontexte und Prozesse der Versorgung, Betreuung sowie Begleitung der Bewohner*innen in Altenpflegeeinrichtungen, in denen Teilhabeförderung unmittelbar stattfindet oder in denen mittelbar dazu beigetragen wird. Daher gehören etwa Angebote, Leistungen und Handlungen der sozialen Betreuung ebenso dazu wie jene der Pflege und Mahlzeitengestaltung. Teilhabewünsche sind hierbei nicht an die bestehende Alltags- und Angebotsgestaltung anzupassen, sondern die Alltags- und Angebotsgestaltung orientiert sich an den Wünschen der Bewohner*innen oder versucht ihre Teilhabewünsche $\mathrm{zu}$ integrieren. So sollte in der Organisation und Gestaltung der Pflege und Versorgung auf die besonderen Wünsche, Bedürfnisse und Voraussetzungen der Bewohner*innen eingegangen werden, auch wenn fehlende personelle Ressourcen hierbei oft hemmende Rahmenbedingungen darstellen. Unmittelbar auf die Förderung von Teilhabe ausgerichtet sind in der Regel die Angebote der sozialen Betreuung. So existieren Einzel- und Gruppenangebote innerhalb und außerhalb der Einrichtungen, die von regelmäßigen Einzelbetreuungen und Gruppenangeboten über besondere Veranstaltungen innerhalb der Einrichtung bis zu Einzelaktivitäten, Gruppenausflügen und Urlaubsfahrten außerhalb der Einrichtung reichen. Auch hier lässt sich festhalten, dass im Prinzip genügend Kontexte zur Verwirklichung von Teilhabe existieren, fraglich ist aber, inwieweit die Inhalte und Gestaltung dieser Angebote den Wünschen der einzelnen Bewohner*innen entsprechen und inwiefern davon unabhängig individuelle Teilhabewünsche berücksichtigt und verwirklicht werden (können). So sollte bei der Alltags- und Angebotsgestaltung ein durchgängiger Maßstab sein, dass diese auf Basis der individuellen Wünsche zur Teilhabe erfolgt. Gleichermaßen sollte (selbst)kritisch hinterfragt werden, inwieweit Angebote des Sozialen Dienstes über die Zwecke der allgemeinen Beschäftigung und 
Tagesstrukturierung hinausgehend auch das Ziel der Verwirklichung von persönlich bedeutsamer Teilhabe verfolgen.

Neben der Orientierung an der Selbstbestimmung ist in stationären Einrichtungen daher vor allem das Prinzip der Normalität ausschlaggebend, um die Teilhabe individuell nach den aktuellen Wünschen der Bewohnerinnen sowie nach ihren biografisch gewohnten Normen und Routinen zu unterstützen. Dass die Bewohner*innen in ihrem Alltag in ihrer selbstbestimmten Teilhabe durch Mitarbeitende begleitet und unterstützt werden, ist aber nicht als besondere Dienstleistung, sondern als reguläre Aufgabe einer Altenpflegeeinrichtung und als Rechtsanspruch der Bewohner*innen zu verstehen, denn aufbauend auf einem menschenrechtsbasierten Verständnis von Teilhabe ist der "gleichberechtigte Zugang zu sozialen Umweltbeziehungen $[\ldots]$ Bedingung für die Verwirklichung von Grundrechten" (Fuchs, 2009, S. 20).

Alltags- und Angebotsgestaltung - fördernd, zum Beispiel: Teilhabewünsche
werden in Alltags- und Angebotsgestaltung in Kontexten der Pflege, Ver-
sorgung und Betreuung prinzipiell berücksichtigt / erforderliche Res-
sourcen werden entsprechend ein- oder umgeplant / Angebotsplanungen
sind nicht von der Anzahl der Teilnehmenden, sondern von Wünschen
und Voraussetzungen der Bewohner*innen abhängig / Gruppenangebote
mit inhaltlicher Breite und zu verschiedenen Zeiten sind vorhanden / In-
formation der Bewohner*innen und Mitarbeitenden über Angebote /
barrierefreie, für verschiedene Bewohner*innengruppen zugängliche An-
gebote; / Angebote mit größeren Gruppen oder Bewohner*innen mit he-
terogenen Voraussetzungen werden von mehreren Mitarbeitenden be-
gleitet.

Die oben erläuterten Phasen der Äußerung und Erfassung von Teilhabewünschen sowie deren Berücksichtigung und Verwirklichung in der Alltags- und Angebotsgestaltung sind beeinflusst von einer Vielzahl von Faktoren, die hier auf der Ebene der Bewohner*innen, der Mitarbeitenden sowie der Einrichtungsstrukturen und -prozesse unterschieden werden. An diesen Ebenen wird nun konkret deutlich, dass die damit verbundenen Faktoren vor Ort sowohl zwischen als auch innerhalb der einzelnen Altenpflegeeinrichtungen variieren (können) und diese Ebenen bzw. deren Faktoren in Wechselwirkung zueinanderstehen, denn die Voraussetzungen von Bewohner*innen sind individuell ebenso wie sich die Kompetenzen von Leitungskräften und Mitarbeitenden sowie Einrichtungsstrukturen und -prozesse unterscheiden. Gleichermaßen ist zu berücksichtigen, dass 
mit den oben benannten Anforderungen zur Teilhabeförderung auch Ressourcen verbunden sind, die daher eine besondere Bedeutung auf Ebene der Einrichtung aufweisen.

\section{a) Bewohner*innen}

Die Voraussetzungen zur Äußerung von Teilhabewünschen und deren Verwirklichung sind bei den Bewohner*innen äußerst heterogen und dementsprechend individuell zu berücksichtigen. Dennoch können schematisch verschiedene Teilgruppen von Bewohner*innen unterschieden werden, die differierende Voraussetzungen zur Teilhabeförderung "mitbringen“. Für die Teilhabeförderung bedeutsam scheinen zunächst Differenzen zwischen Bewohner*innen in Bezug auf physische und psychische Voraussetzungen („orientierte“ Bewohner“innen, kognitiv eingeschränkte bzw. psychisch veränderte Bewohner*innen, physisch stark eingeschränkte Bewohner*innen und Bewohner*innen in der Sterbephase). Hier ist eine Fortsetzung der Entwicklung zu erwarten, da die Bewohner*innen, die in stationäre Altenpflegeeinrichtungen einziehen, zunehmend von Hochaltrigkeit, Multimorbidität und schwerer Pflegebedürftigkeit geprägt sein werden. Das darf aber keineswegs zu der gelegentlich zu hörenden, pauschalen Aussage führen, die Bewohner*innen wollten oder könnten überhaupt nicht mehr teilhaben. Vielmehr ist von zentraler Bedeutung, Prozesse der Wunscherfassung und Teilhabeförderung differenziert nach den jeweiligen Voraussetzungen vorzunehmen und auch etwa bei Bewohner*innen mit psychischen Veränderungen und kognitiven Einschränkungen Teilhabewünsche sensibel zu identifizieren oder zu wecken. Zudem gilt es, dies auch bei der Bereitstellung und Planung notwendiger Ressourcen in der Altenhilfe zu berücksichtigen.

Quer liegend zu den Differenzierungen in Bezug auf physische und psychische Voraussetzungen sind weitere Differenzmerkmale von Bedeutung, die sich in den vorliegenden Ergebnissen vor allem auf das Geschlecht, die finanzielle Lage und die Herkunft der Bewohner*innen beziehen. Hier ist absehbar, dass die Pluralisierung und Individualisierung von Lebensformen und -stilen im hohen Alter und damit auch in der stationären Altenhilfe als bedeutsames Thema zunehmen wird. Somit gilt es also wiederum, die Wünsche und Voraussetzungen zur Verwirklichung von Teilhabe individuell zu erfassen und zu berücksichtigen.

Auch wenn die hier vorgenommenen Unterscheidungen für unterschiedliche Voraussetzungen in der Teilhabeförderung sensibilisieren kön- 
nen, dürfen diese umgekehrt nicht zu Stigmatisierungen und Defizitzuschreibungen sowie - entgegen gleichberechtigter Teilhabezugänge - zu fixen Zuteilungen in spezifische Teilhabeoptionen und Angebotsformen führen.

Bewohner*innen - fördernd, zum Beispiel: individuelle Differenzierungen in Bezug auf die Teilnahme an Einzel- und Gruppenangeboten / keine fixen Zuordnungen von Teilgruppen zu Angebotstypen / Ressourcenorientierung / Beobachtung des Verhaltens von Bewohner*innen im Teilhabeangebot

\section{b) Mitarbeitende}

Der Umfang und die Qualität der Teilhabeförderung steht nach den vorliegenden Ergebnissen auch erheblich im Zusammenhang mit den individuellen Kompetenzen und persönlichen Eigenschaften der jeweiligen Mitarbeiter*innen, denn die Berücksichtigung und Verwirklichung der selbstbestimmten Teilhabe der Bewohner*innen findet im Alltag unmittelbar in der Wechselbeziehung zwischen Bewohner*innen und Mitarbeitenden statt. Doch auch wenn hierbei die Individualität der Mitarbeitenden ebenso zu berücksichtigen ist, wie jene der Bewohner*innen, darf es auf Ebene der Mitarbeitenden nicht primär von der einzelnen Person abhängig bleiben, inwieweit und wie das Recht der Bewohner*innen auf Teilhabe tatsächlich verwirklicht wird. Vielmehr sollten Mitarbeitende über gemeinsame Ziele und Wissensgrundlagen sowie wesentliche Fähigkeiten für das Handeln mit Bewohner*innen in Altenpflegeeinrichtungen verfügen. Hierzu ist von Bedeutung, dass die - für eine Förderung von selbstbestimmter Teilhabe - relevanten Ziele, Werte und Prinzipien in der Einrichtung auch vermittelt sowie wesentliche Wissensgrundlagen und Fähigkeiten bei den Mitarbeitenden gefördert werden.

Relevant auf dieser Ebene ist zunächst die individuelle Arbeitskultur der Mitarbeitenden, die in enger Verbindung mit der auf Einrichtungsebene verorteten Organisationskultur steht und hier primär auf die individuellen Annahmen, Werte und Überzeugungen sowie das jeweilige Arbeitshandeln der Mitarbeitenden fokussiert. Diese oft auch als „Haltung“ oder „Selbstverständnis“ der Mitarbeitenden umschriebene Arbeitskultur prägt maßgeblich, ob überhaupt und mit welchen Zielen und wie die Teilhabe von Bewohner"innen gefördert wird. Und sie kann etwa über das Thematisieren der für die Einrichtung relevanten Ziele, Werte und Prinzipien in 
Besprechungen oder Schulungen begünstigt werden. Ein Einflussfaktor ist hierbei auch das jeweilige Arbeitsengagement, das an die Arbeitskultur anschließt und insbesondere die jeweilige Einstellung zum Arbeiten selbst betrifft, die etwa in Mitarbeiter"innengesprächen thematisiert werden kann.

Arbeitskultur und -engagement - fördernd, zum Beispiel: Orientierung an den Bewohner*innen im professionellen Handeln / Zugewandtheit gegenüber Bewohner*innen / kooperatives Denken und Handeln unter Mitarbeitenden / positive Einstellung zur Arbeit und kein „Dienst nach Vorschrift" / Förderung etwa im Kontext von Mitarbeiter*innenversammlungen, Teamgesprächen, Übergaben, Fallbesprechungen, Mitarbeiter*innengesprächen, Hospitationen in anderen Einrichtungsbereichen.

Ferner sind spezifische Wissensgrundlagen und Fähigkeiten bei den Mitarbeitenden bedeutsam. Sie können in Fach-, Methoden-, Sozial- und Selbstkompetenzen unterschieden und mit verschiedenen für Prozesse der Teilhabeförderung inhaltlichen Aspekten verbunden und etwa über Schulungen, Fort- und Weiterbildungen gefördert werden.

Fachkompetenz-fördernd, zum Beispiel: Wissen über Handlungsprinzipien (z. B. Normalitätsprinzip) / Handlungsmethoden (z. B. Biografiearbeit) / zentrale Krankheitsbilder (z. B. Demenz) und Voraussetzungen (z. B. Mobilisation) zur Teilhabeförderung / Grundlagen der Kommunikation und Gesprächsführung (z.B. aktives Zuhören) / gesetzliche Vorgaben bzw. Vorschriften, die sich auf selbstbestimmte Teilhabe auswirken (z. B. Hygienevorschriften, Brandschutz) / Förderung der Fachkompetenz etwa über Schulungen, Fort- und Weiterbildungen, interdisziplinäre Fallbesprechungen.

Methodenkompetenz - fördernd zum Beispiel: Fähigkeiten in der Anwendung von Methoden und Techniken (z. B. Biografiearbeit, soziale Netzwerkarbeit, soziale Gruppenarbeit, Sozialraumorientierung, motivierende Gesprächsführung, Beobachtungsmethoden) / Förderung der Methodenkompetenz etwa über Schulungen, Fort- und Weiterbildungen, Hospitationen. 
Sozialkompetenz - fördernd zum Beispiel: Fähigkeiten zur Gestaltung von Interaktionen und Beziehungen (z. B. Empathiefähigkeit, Kommunikationsfähigkeit, Teamfähigkeit) / Förderung der Sozialkompetenz etwa über Schulungen, Fort- und Weiterbildungen, Teamsitzungen.

Subjektkompetenz - fördernd zum Beispiel: Fähigkeiten der Selbstregulation in Wahrnehmung und Handlung (z. B. in Bezug auf Flexibilität im professionellen Handeln, Reflexionsfähigkeit, Einsatzbereitschaft) / Förderung der Subjektkompetenz etwa über Fort- und Weiterbildungen, Mitarbeiter*innengespräche.

\section{c) Einrichtungsstrukturen und -prozesse}

Für die Erfassung und Berücksichtigung von Teilhabewünschen sowie die Verwirklichung der selbstbestimmten Teilhabe von Bewohner*innen spielen die Rahmenbedingungen sowie organisatorischen Abläufe in der jeweiligen Altenpflegeeinrichtung eine wesentliche Rolle. Auf dieser komplexen Ebene der Einrichtungsstrukturen und -prozesse wurden die meisten fördernden und hemmenden Faktoren identifiziert, die hier zusammenfassend in den Bereichen "Organisationskultur und Verankerung in Konzepten und Instrumenten“, „Schnittstellen und Handlungsspielräume“ sowie „Besondere und zusätzliche Ressourcen“ wiedergegeben und diskutiert werden.

\section{Organisationskultur und Verankerung in Konzepten}

Wesentlich als Rahmenbedingung ist eine in der Einrichtung entwickelte Organisationskultur, die eine gemeinsame Orientierung der Mitarbeitenden an der selbstbestimmten Teilhabe der Bewohner*innen fördert und die individuelle Arbeitskultur prägt. Die Organisationskultur bezieht sich auf die Einrichtungsziele sowie organisationalen bzw. einrichtungsbezogenen Werte und Prinzipien, die von den Mitarbeitenden der Einrichtung bereichsübergreifend - hier in Bezug auf die selbstbestimmte Teilhabe der Bewohner*innen - anerkannt werden. In der Entwicklung und Förderung der Organisationskultur haben die Leitungskräfte eine zentrale Rolle. Diese sollten im Alltag wiederholend das Ziel der selbstbestimmten Teilhabe einbringen und an Mitarbeitende transportieren. Dabei unterstützt eine Verankerung des Themas in Konzepten der Einrichtung, damit dort für 
die Mitarbeitenden gemeinsame Orientierungsgrundlagen konkret festgehalten, präsent und nachvollziehbar sind. Förderlich sind zudem Fortbildungen zu Aspekten selbstbestimmter Teilhabe, um die Mitarbeitenden entsprechend informieren, sensibilisieren und motivieren zu können - wie auf der Ebene der Mitarbeitenden angesprochen. Allerdings reichen singuläre Fortbildungen für die langfristige Entwicklung einer teilhabeförderlichen Organisationskultur nicht aus, vielmehr ist hierfür auch das stete Reflektieren in Bewohner*innensituationen und Besprechungen - unter Beteiligung von Leitungsverantwortlichen - wichtig und nützlich. Beachtenswert ist hierbei einerseits, dass die Entwicklung einer Organisationskultur (z. B. über bereichsübergreifende Schulungen und Mitarbeiter"innenversammlungen) auf der Ebene der Einrichtung einen Ausgangspunkt für den Weg zu einem teilhabeförderlichen Arbeiten darstellen kann. Andererseits ist somit aber auch zu beachten, dass die Vermittlung einer teilhabeförderlichen Organisationskultur mit einem andauernden Prozess verbunden ist, in dem die Leitungskräfte immer wieder für das Thema der selbstbestimmten Teilhabe im Arbeitsalltag sensibilisieren und motivieren.

Organisationskultur und Verankerung in Konzepten - fördernd, zum Beispiel: geteiltes Verständnis zu Einrichtungszielen, Werten und Prinzipien bezogen auf selbstbestimmte Teilhabe im Leitungsteam und bei Mitarbeitenden / Initiierung und Verantwortungsübernahme in der Organisationskulturförderung durch das Leitungsteam / Coaching oder Beratung des Leitungsteams / Aufnahme von Zielen, Werten und Prinzipien in Konzepten (z. B. Leitbild, Einrichtungs-, Pflege-, Sozialdienst-, Hauswirtschafts- und Verpflegungskonzept) / Schulungen, Fort- und Weiterbildung der Mitarbeitenden / fortlaufend bedarfsorientierte Thematisierung im Arbeitsalltag durch Leitungskräfte (z. B. in Teamsitzungen, Übergaben, Mitarbeiter*innengesprächen).

Schnittstellen und Kooperation

Für die Versorgung, Betreuung und Begleitung der Bewohner*innen in einer Altenpflegeeinrichtung im Allgemeinen sowie die Förderung selbstbestimmter Teilhabe im Speziellen ist es unabdingbar, dass verschiedene Funktionsbereiche mit spezifischen Qualifikationen und Kompetenzen vorhanden sind und die jeweiligen Mitarbeitenden miteinander kooperieren. Die für die Teilhabeförderung relevante Kooperation findet einerseits im Rahmen von direkt bewohner*innenbezogenen Aktivitäten ( $z$. B. 
Gruppen- und Einzelangeboten, Ausflügen, Festen) und andererseits von indirekt bewohner*innenbezogenen Aktivitäten (z. B. Übergaben, Fallbesprechungen, Dokumentationen) statt. Hierbei wurde als relevant identifiziert, dass Zeitpunkte der Schnittstelle und Aufgaben der Kooperation von den jeweiligen Einrichtungsbereichen eingeplant werden und die Einrichtungsbereiche wechselseitig informiert sind. Ferner verbessert ein gegenseitiges Verständnis für die jeweiligen Funktionen und Aufgaben sowie ein geteiltes Verständnis von Zielen, Werten und Prinzipien der Einrichtung die Kooperation. Auch im Kontext der vorhandenen Besprechungs- und Dokumentationsverfahren sollten zwischen den Einrichtungsbereichen insbesondere zwischen Pflege und Sozialem Dienst - regelmäßige Abstimmungen erfolgen, damit teilhaberelevante Informationen über Bewohner*innen weitergegeben werden. Das heißt nicht, dass etwa der Soziale Dienst täglich an der Dienstübergabe in einem Wohnbereich teilnehmen muss, dennoch sollte dessen Teilnahme regelmäßig, zumindest aber bedarfsorientiert selbstverständlich sein. Darüber hinaus ist als wichtige Rahmenbedingung festzuhalten, wie die Stellen des Sozialen Dienstes und die der zusätzlichen Betreuungskräfte in der Einrichtung integriert sind und ihre Kooperation systematisch organisiert ist. Hier sollte der Soziale Dienst auf „Augenhöhe“ mit der Pflege agieren (können) und auch im Leitungsteam der Einrichtung vertreten sein. Ferner hat sich als bedeutsam herausgestellt, dass die zusätzlichen Betreuungskräfte (nach $₫ 43 \mathrm{~b}$ SGB XI) eine besondere personelle Ressource für die - insbesondere auf einzelne Bewohner*innen bezogene - Teilhabeförderung darstellen und sie daher dementsprechend eingesetzt werden und nicht Aufgaben außerhalb ihres Aufgabenbereichs übernehmen sollten. Die enge Kooperation mit den Fachkräften des Sozialen Dienstes ist hierbei besonders relevant. Innerhalb derer ist es dann durchaus sinnvoll, wenn Aufgaben von Vorgesetzten bezüglich der zusätzlichen Betreuungskräfte bzw. Anteile von ihnen über die Leitung bzw. koordinierende Kraft des Sozialen Dienstes erfolgen, auch wenn die Einhaltung der Vorgaben der Betreuungskräfterichtlinie formal der Pflegedienstleitung obliegt (BetrKrRL $\$ 2$, Absatz 4).

Schnittstellen und Kooperation - fördernd, zum Beispiel: gemeinsame Orientierungsgrundlagen im Sinne der Organisationskultur / gegenseitiges Verständnis von den Funktionen und Aufgaben der Einrichtungsbereiche / Kooperation der Professionen auf „Augenhöhe“ / Kenntnis über und Einplanung der Schnittstellenaufgaben / aufgaben- und bewohner*innenbezogene Abstimmung über das Besprechungs- und Dokumentationswesen / Hospitation in anderen Einrichtungsbereichen. 
Handlungsspielräume

Um eine Teilhabeförderung zu verwirklichen, die sich an den individuellen Wünschen der Bewohner*innen orientiert, müssen Mitarbeitende innerhalb der gegebenen Arbeitsprozesse und Organisationsstrukturen flexibel agieren (können). Die Nutzung von Handlungsspielräumen wird auf Ebene der Mitarbeitenden über die individuelle Flexibilität als Teil der Subjektkompetenz begünstigt. Auf der Ebene der Einrichtung sind es aber die Routinen und Abläufe der Einrichtung sowie vorhandene Vorgaben sowie Werte der Vorgesetzten und Kolleg*innen, die Handlungsspielräume beschränken oder tolerieren. Auch wenn diesem Faktor - zumindest im Vergleich mit den anderen Faktoren - in der quantitativen Analyse eine geringere Relevanz zugeordnet wurde, bleibt mit den Ergebnissen der qualitativen Analyse dennoch festzuhalten, dass die Offenheit für und Wahrnehmung von Handlungsspielräumen für situativ erforderliche Unterstützungen und Begleitungen von Bewohner*innen selbstverständlich sein sollte - zumindest dann, wenn das Normalitätsprinzip und nicht institutionelle Reglementierungen im Vordergrund stehen.

Handlungsspielräume, zum Beispiel: Legitimation von Handlungsspielräumen zwischen Wochentagen und Dienstschichten durch Vorgesetzte (z. B. Verschiebung von Aufgaben auf andere Schichten oder Wochentage) / bei Bedarf Abweichungen von Routinen und Rahmenplänen / Personalplanung entsprechend arbeitsintensiver Zeiten / Kenntnisse zu Handlungsspielräumen innerhalb rechtlicher Vorgaben und Vorschriften (z. B. Brandschutz, Hygienevorschriften)

\section{Besondere und zusätzliche Ressourcen}

Für eine Teilhabeförderung nach dem Prinzip der Selbstbestimmung bedarf es spezifischer Ressourcen in der Einrichtung, die es zu identifizieren, beschaffen und nutzen gilt. Besondere und zusätzliche Ressourcen beziehen sich hier auf (fördernde Faktoren der) Rahmenbedingungen, die Zugänge für die Teilhabe der Bewohner*innen eröffnen und die Prozesse der Teilhabeförderung innerhalb und außerhalb der Einrichtung unterstützen bzw. ermöglichen. So werden die Möglichkeiten zur Teilhabe der einzelnen Bewohner*innen etwa über die Vermittlung von finanziellen Mitteln, externen Dienstleistungen oder Hilfsmitteln sowie die Beteiligung ihrer Angehörigen, Betreuer*innen und Bevollmächtigten gefördert. Dabei ist 
die Erschließung zusätzlicher personeller und finanzieller Ressourcen hilfreich, um regulär vorhandene Strukturen (und Mittel) in der Einrichtung für die Teilhabeförderung erweitern zu können. Auch die vorhandenen Raumressourcen und die damit verbundene Materialausstattung sind wichtige Ressourcen, da sie Aneignungsmöglichkeiten durch die Bewohner*innen und das Setting der Teilhabeförderung innerhalb der Einrichtung beeinflussen. Für eine Teilhabe am gesellschaftlichen Leben außerhalb der Einrichtung sind ferner Unterstützungen und Hilfen der Mobilität der Bewohner*innen relevante Ressourcen. Darüber hinaus sind die Ressourcen im Quartier von Bedeutung, die in Kooperationen mit Akteur*innen und Nutzung der Quartierstrukturen liegen und über Öffnungen der Einrichtung für das und zum Quartier die Teilhabe der Bewohner*innen innerhalb und außerhalb der Einrichtung fördern. Von Interesse wäre zudem, welche Rolle in Zukunft soziale Medien, technische Hilfen und Assistenzsysteme in der Förderung selbstbestimmter Teilhabe spielen werden, die in dieser Zusammenstellung (noch) nicht als relevanter Faktorenbereich aufgenommen wurden, weil dazu offenbar die Erfahrungen noch zu gering sind und daher in der vorliegenden Studie die empirischen Hinweise fehlten.

Grundlegend ist im Themenbereich der Ressourcen aber, dass die Ressourcen, die zur Teilhabeförderung (potenziell) zur Verfügung stehen, für die jeweilige Einrichtung eruiert und ihre Mitarbeitenden dafür sensibilisiert werden, diese zu nutzen. Hervorzuheben ist auch, dass viele dieser Ressourcen geprägt sind durch politische Rahmenbedingungen auf Ebene des Bundes, des Landes und der Kommune, so dass fehlende Ressourcen oder Rahmensetzungen durchaus auch von den Einrichtungen thematisiert und auf politischer Ebene eingebracht werden sollten (hierzu Kapitel 7.3).

Personelle Ressourcen - fördernd, zum Beispiel: zusätzliche Betreuungskräfte nicht als „zusätzliche“, sondern als besondere Ressource für teilhabebezogene Einzelangebote / Ehrenamtler*innen zur individuellen Begleitung einzelner Bewohner*innen / Angehörige, die etwa bei der Gewinnung biografischer Informationen, individuellen Begleitungen von Bewohner*innen oder im Rahmen von Gruppenangeboten und Ausflügen unterstützen.

Finanzielle Ressourcen - fördernd, zum Beispiel: Spenden über Stiftungen, (eigene) Fördervereine oder Sponsoring / Projektmittel / Personalstelle beim Träger, die für Akquise von Spenden und Projektmitteln zuständig ist. 
Räumliche Ressourcen - fördernd, zum Beispiel: Aufenthaltsbereiche, die selbstbestimmten Aufenthalt und selbstbestimmte Beschäftigungen ermöglichen / Existenz geeigneter Räume hinsichtlich Größe und Ausstattung für Gruppenangebote / bewohner*innenorientierte Raumgestaltung / keine Zweckentfremdung von Aufenthaltsräumen.

Mobilitätsressourcen - fördernd, zum Beispiel: Koordination der Kräfte (z. B. zusätzliche Betreuungskräfte, Ehrenamtler*innen) zur Begleitung von Bewohner*innen durch den Sozialen Dienst / Flexibilität in personellen Ressourcen für Begleitungen von Bewohner*innen / kommunale Unterstützung von Taxifahrten / einrichtungseigenes, geräumiges Fahrzeug.

Quartiersressourcen - fördernd, zum Beispiel: Kooperationen und Vernetzungen mit Institutionen und Schlüsselpersonen des Quartiers / Öffnung von Veranstaltungen im Haus für Quartiersbewohner*innen / Nutzung von Veranstaltungen und Angeboten im Quartier / eine im Quartier wahrnehmbare Öffentlichkeitsarbeit / Beteiligung an politischen Gremien im Quartier.

Mit Blick auf den Kernprozess von Teilhabeförderung als auch auf die drei Ebenen der Bewohner*innen, Mitarbeitenden sowie Einrichtungsstrukturen und -prozesse konnten also nochmals resümierend die als relevant identifizierten Faktoren zur Förderung selbstbestimmter Teilhabe in Altenpflegeeinrichtungen dargestellt werden. Entsprechend den Untersuchungsfragen wurde damit sowohl differenziert beleuchtet, wie Wünsche von Bewohner*innen festgestellt und berücksichtigt werden können, als auch wie auf dieser Basis ihr Recht auf selbstbestimmte Teilhabe praktisch verwirklicht werden kann.

Überprüfung der Umsetzung selbstbestimmter Teilhabe

Nicht näher eingegangen wurde bislang auf den zweiten Teil der zweiten Untersuchungsfrage, wie die Umsetzung selbstbestimmter Teilhabe überprüft werden kann. Dieser Aspekt wurde deswegen ausgelassen, weil die empirischen Erhebungen keine expliziten Antworten dazu geliefert haben. So wurden in den Interviews keine Instrumente genannt, mit denen ausdrücklich die Umsetzung selbstbestimmter Teilhabe der Bewohner"innen überprüft wird. Als vorhandene Instrumente, mit denen generell die Qualität der Einrichtung, ihrer Angebote und Leistungen erfasst sowie Verände- 
rungsprozesse angestoßen werden, wurden demgegenüber aber vor allem Bewertungsinstrumente wie Zufriedenheitsbefragungen von Bewohner*innen und Angehörigen, das Beschwerde- bzw. Verbesserungsmanagement sowie die individuelle Evaluation im Rahmen der Pflege- und Betreuungsplanung genannt. Damit stehen wiederum Instrumente im Fokus, die auch im Kontext der Wunscherfassung eine Rolle spielen. Allerdings wurde dazu auch - und noch expliziter als im Kontext der Wunscherfassung festgehalten, dass die Perspektive der selbstbestimmten Teilhabe im Rahmen dieser Instrumente bislang kaum bzw. nur eingeschränkt eine Rolle spiele. Demnach ist auf die Frage nach Möglichkeiten zur Überprüfung der Umsetzung der selbstbestimmten Teilhabe von Bewohner*innen in Altenpflegeeinrichtungen zunächst $\mathrm{zu}$ antworten, dass hier wiederum eine stärkere Berücksichtigung des Themas in den vorhandenen Instrumenten sinnvoll erscheint. Konkret ist es einerseits unerlässlich, auf der Ebene der einzelnen Bewohner*innen die Verwirklichung der individuellen Teilhabewünsche zu überprüfen, was dann vor allem über vorhandene Instrumente der Pflege- und Betreuungsplanung - mit Abstimmungs- oder $\mathrm{Zu}-$ griffsmöglichkeiten zwischen den relevanten Einrichtungsbereichen - erfolgen sollte und über Pflegevisiten sowie Fallbesprechungen flankiert werden kann. Andererseits wäre eine Bewertung auf Einrichtungsebene möglich, indem etwa in Zufriedenheitserhebungen (z. B. im Kontext von Bewohner*innen- und Angehörigenbefragungen, aber auch von Wunschund Evaluationsrunden zu Angeboten des Sozialen Dienstes, Bewohner*innenbeirats- und Angehörigentreffen) teilhaberelevante Aspekte konkret aufgenommen werden. Damit könnten Daten und Aussagen zur Umsetzung der selbstbestimmten Teilhabe von Bewohner*innen in der Einrichtung aggregiert gewonnen werden - im Sinne einer Selbstevaluation im Kontext der Ergebnisqualität des Qualitätsmanagements und mit entsprechenden Zugängen, die nicht den Anspruch einer Wirkungsevaluation haben können. Sinnvoll ist es außerdem, die Teilhabeangebote selbst bzw. die Angebotsplanung regelmäßig zu reflektieren und gegebenenfalls anzupassen (z. B. bezüglich Zielgruppenspezifik bzw. Inhalt des Angebots, angewandten Methoden und eingesetzten Materialien sowie bei Gruppenangeboten die Anpassung der Gruppengröße und -zusammensetzung im Verhältnis zum eingesetzten Personal). Für Erweiterungen der bestehenden Instrumente auf Einrichtungsebene kann sowohl auf bereits vorhandene Indikatoren zur Teilhabe, die im Rahmen von (Lebens-)Qualitätsmessungen vorliegen (z. B. Weidekamp-Maicher, 2016, S. 81ff), als auch auf Kriterien, die im Rahmen von STAP für das Musterrahmenkonzept festgehalten wurden, zurückgegriffen werden. Zudem kann das Musterrahmenkonzept 
selbst als Instrument betrachtet werden, das Hinweise zur Umsetzung der selbstbestimmten Teilhabe auf Einrichtungsebene gibt, da damit zumindest im Rahmen einer Selbsteinschätzung eine kriteriengeleitete Überprüfung des Ist-Standes in der eigenen Einrichtung möglich ist.

\subsection{Ausblick zum Musterrabmenkonzept}

Basierend auf den empirischen Ergebnissen der STAP-Studie und ergänzt durch gute Praxisbeispiele sowie Hinweise aus der Forschungsliteratur, im Rahmen von Projektveranstaltungen und der Erprobung wurden im Musterrahmenkonzept wesentliche Kriterien, Erläuterungen und Beispiele für die Umsetzung von selbstbestimmter Teilhabe in stationären Altenpflegeeinrichtungen festgehalten, auch wenn damit immer noch kein Anspruch auf Vollständigkeit besteht. Mit dem Musterrahmenkonzept soll ermöglicht werden, den Ist-Zustand zu den einzelnen Kriterien für die eigene Einrichtung in Bezug auf den Handlungsbedarf einschätzen und auf dieser Basis Vorschläge für Verbesserungsmaßnahmen festhalten zu können.

Inhaltlich wurden die Kriterien im Musterrahmenkonzept den in Altenpflegeeinrichtungen bekannten Ebenen von Struktur, Prozess und Ergebnis zugeordnet. Innerhalb dessen sind jedoch bereits in den Kapitelüberschriften des Musterrahmenkonzeptes die Ergebnisse der STAP-Studie wiederkennbar (siehe dazu auch den Auszug im Anhang): ${ }^{4}$

- Strukturkriterien: 1. Organisationskultur, 2. Arbeitskultur, Kompetenzen und Handlungsspielräume der Mitarbeitenden, 3. Organisationsstruktur, 4. Zusätzliche Ressourcen

- Prozesskriterien: 5. Umgang mit Wünschen, 6. Selbstbestimmung, Alltags- und Angebotsgestaltung

- Ergebniskriterien: 7. Evaluation der Konzepte und Maßnahmen, 8. Zufriedenheitserhebungen und Verbesserungsmanagement

Im Rahmen der Implementationsanalyse des Musterrahmenkonzeptes in der Erprobungseinrichtung sowie durch die Rückmeldungen von den vier Projekteinrichtungen konnte festgehalten werden, dass das Musterrahmenkonzept einen umfassenden Überblick und Einblick in verschiedenste Aspekte zur Förderung der selbstbestimmten Teilhabe von Bewohner*innen in Altenpflegeeinrichtungen vermittelt. Auch wurde bestätigt, dass sich der Ist-Stand einer Einrichtung in Bezug auf die Teilhabeorientierung gut mit dem MRK einschätzen lässt und auf dieser Basis eine Ableitung teilha-

84 Vollständig verfügbar ist das Musterrahmenkonzept unter www.stap-teilhabe.de. 
befördernder Maßnahmen möglich ist. Kritisch betrachtet wurden demgegenüber das Format und der Umfang des Musterrahmenkonzeptes. Diese Kritik wurde berücksichtigt, indem einerseits das Musterrahmenkonzept, das ursprünglich in einem Textverarbeitungsprogramm als fortlaufende Tabelle vorlag, in ein Dateiformat exportiert wurde, das zum Beispiel über Seitenregister die Navigation innerhalb der Kapitel des Musterrahmenkonzeptes erleichtert und Erläuterungen nur bei Bedarf erscheinen lässt. Andererseits wurde der Umfang des Musterrahmenkonzeptes auch inhaltlich angepasst, indem einige ursprünglich getrennt aufgeführte Kriterien durch Zusammenlegungen oder Subsumtionen zwar nicht gestrichen, aber strukturell reduziert wurden.

Für die Zukunft wird die entscheidende Frage sein, ob und wie das Musterrahmenkonzept von anderen Einrichtungen genutzt und angenommen wird. Im Rahmen der weiteren Transferaktivitäten werden die Untersuchungsergebnisse und das Musterrahmenkonzept in Gremien und Veranstaltungen von Politik, Praxis und Wissenschaft eingebracht. Zudem ist zu prüfen, inwieweit und in welchem Rahmen einführende Schulungen zur Nutzung des Musterrahmenkonzeptes angeboten werden können, um die Bedingungen für dessen Implementation zu fördern.

Im Sinne der Akzeptanz des Musterrahmenkonzeptes ist abschließend darauf hinzuweisen, dass die Vielzahl der aufgenommenen Kriterien nicht den Eindruck vermitteln darf, dass eine Förderung selbstbestimmter Teilhabe im Sinne der STAP-Ergebnisse zu anspruchsvoll und komplex ist, um sie in stationären Altenpflegeeinrichtungen umsetzen zu können. Vielmehr bietet das Musterrahmenkonzept eine Übersicht relevanter Kriterien, deren nähere Betrachtung in den meisten Einrichtungen auch deutlich machen wird, dass zu vielen Kriterien bereits Voraussetzungen bestehen. Von Interesse ist dann aber, in welchem Umfang und in welcher Intensität diese Voraussetzungen in den einzelnen Bereichen bereits gegeben sind. Bislang fehlte den Einrichtungen eine entsprechende Orientierung, die Anregungen zu relevanten Kriterien bietet und ermöglicht, für sich einzuschätzen und festzuhalten, wo und inwieweit Handlungsbedarf besteht. Für diesen Zweck wurde das Musterrahmenkonzept entwickelt.

\subsection{Empfehlungen an die Politik}

Abschließend werden aus Sicht der STAP-Studie relevante strukturelle Rahmenbedingungen zur Förderung der selbstbestimmten Teilhabe von Bewohner*innen in Altenpflegeeinrichtungen in den Blick genommen 
und in Anschluss an die Untersuchungsergebnisse Möglichkeiten der gesetzlichen und untergesetzlichen Weiterentwicklung als Empfehlungen an die Politik festgehalten. Allgemein ist aber zunächst festzuhalten, dass die Diskussion um eine qualitativ und quantitativ bedarfsgerechte Personalausstattung in Pflegeheimen auch für die Verwirklichung selbstbestimmter Teilhabe von hoher Bedeutung ist. Die Personalbemessung für Altenpflegeeinrichtungen steht derzeit auf Bundesebene auf dem Prüfstand. Die weiteren Entwicklungen werden auch von den am STAP-Projekt Beteiligten beobachtet. Wichtig wird grundlegend sein, dass in den Einrichtungen ein Organisationsentwicklungsprozess beginnt. Es ist davon auszugehen, dass eine Anpassung des MRK an gegebenenfalls neue Erfordernisse in dieser Hinsicht leicht möglich ist. Neben diesem Punkt der allgemeinen personellen Ressourcenausstattung in der Altenpflege erscheinen darüber hinaus die folgenden teilhabespezifischen Ansätze der gesetzlichen und untergesetzlichen Weiterentwicklung von Belang:

a) Anpassung des SGB XI an die Anforderungen der UN-

Behindertenrechtskonvention

Leistungsanbieter*innen von Altenpflegeeinrichtungen sind - wie in Kapitel 2.3.1 angeführt - nach den Bestimmungen der UN-Behindertenrechtskonvention (UN-BRK), dem Alten- und Pflegesetz Nordrhein-Westfalen (APG NRW), dem Wohn- und Teilhabegesetz Nordrhein-Westfalen (WTG NRW) sowie der Durchführungsverordnung dazu aufgefordert und verpflichtet, die Teilhabe der Bewohner"innen von Altenpflegeeinrichtungen am Leben in der Gesellschaft zu fördern.

Allerdings ist die konkrete Verpflichtung zur Förderung der selbstbestimmten Teilhabe von Menschen mit pflegerischem Versorgungsbedarf bisher lediglich in einigen Heimgesetzen der Länder, wie eben u. a. im Wohn- und Teilhabegesetz NRW, d. h. im Ordnungsrecht, nicht aber im Leistungsrecht der Pflegeversicherung verankert, denn der Teilhabebegriff ist im SGB XI bislang nicht expliziert.

Die Bundesrepublik Deutschland hat sich mit ihrem Beitritt zur UN-Behindertenrechtskonvention (UN-BRK) aber verpflichtet, nach Art. 19 Abs. 1 i. V. m. Art. 25, 26 Abs. UN-BRK wirksame und geeignete Maßnahmen zu treffen, um Menschen mit Behinderungen in die Lage zu versetzen, ein Höchstmaß an Unabhängigkeit, umfassende körperliche, geistige, soziale und berufliche Fähigkeiten sowie die volle Einbeziehung in alle As- 
pekte des Lebens und die volle Teilhabe an allen Aspekten des Lebens zu erreichen und zu bewahren.

Nach Art. 4 Abs. 1 Buchst. a besteht die Verpflichtung zur Gewährleistung und Förderung der vollen Verwirklichung aller Menschenrechte und Grundfreiheiten für alle Menschen mit Behinderungen und ohne jede Diskriminierung aufgrund von Behinderung. Es sind alle geeigneten Gesetzgebungs-, Verwaltungs- und sonstigen Maßnahmen zur Umsetzung der in diesem Übereinkommen anerkannten Rechte zu treffen.

Die hier im Fokus stehenden Menschen mit pflegerischem Versorgungsbedarf gehören zweifelsfrei zu den Menschen mit Behinderungen im Sinne von Art. 1 Abs. 2 UN-BRK, da sie langfristig seelische, geistige oder Sinnesbeeinträchtigungen haben, welche sie in Wechselwirkung mit verschiedenen Barrieren an der vollen, wirksamen und gleichberechtigten Teilhabe an der Gesellschaft hindern können.

Das Elfte Sozialgesetzbuch (SGB XI) ist mithin ein geeignetes Gesetz zur Umsetzung der UN-BRK. Dennoch hat der Gesetzgeber, trotz verschiedener Novellierungen des SGB XI in den letzten Jahren, dieses Gesetz nicht an die Anforderungen der UN-BRK angepasst. Ziel des SGB XI ist unverändert nicht die Förderung der selbstbestimmten Teilhabe pflegebedürftiger Menschen. Stattdessen sollen die Leistungen des SGB XI Pflegebedürftigen lediglich „helfen, trotz ihres Hilfebedarfs ein möglichst selbstständiges und selbstbestimmtes Leben zu führen, das der Würde des Menschen entspricht. Die Hilfen sind darauf auszurichten, die körperlichen, geistigen und seelischen Kräfte der Pflegebedürftigen, auch in Form der aktivierenden Pflege, wiederzugewinnen oder zu erhalten“.

Statt der Förderung der selbstbestimmten Teilhabe am Leben in der Gesellschaft beschränken sich die Leistungen der Pflegeversicherung nach dem Wortlaut des $\$ 2$ SGB XI auf die Verbesserung des individuellen geistigen und körperlichen Zustandes der Berechtigten durch Erhaltung bzw. Wiedergewinnung der körperlichen, geistigen und seelischen Kräfte der Pflegebedürftigen. Folgerichtig ist das Leistungsrecht der Pflegeversicherung auf die medizinisch-pflegerische Versorgung und die soziale Betreuung der Berechtigten orientiert. Die gesetzliche Definition von Gegenstand, Umfang, Ausführung und Qualität der Leistungen der Pflegeversicherung kennt keine Inhalte zur Förderung der Teilhabe. Im Rahmen der STAP-Studie hat sich jedoch gezeigt, dass die Orientierung an der selbstbestimmten Teilhabe der Bewohner*innen mit einer entsprechenden Verankerung des Themas in Konzepten und Instrumenten der Altenpflegeeinrichtungen grundlegend gestärkt werden sollte. 
Aus Sicht der STAP-Studie wird daher vorgeschlagen, zur Umsetzung der UN-BRK die Zielsetzung der Leistungen der Pflegeversicherung auch auf die Förderung der selbstbestimmten Teilhabe pflegebedürftiger Menschen auszurichten und entsprechende Leistungstatbestände in die Definition von Gegenstand, Umfang, Ausführung und Qualität der Leistungen zur Pflege aufzunehmen.

\section{b) Aufwand der Teilhabeförderung im Vergütungsrecht des SGB XI verankern}

Die Einbeziehung der selbstbestimmten Teilhabe in das Leistungsrecht der Pflegeversicherung erfordert dann auch eine Anpassung der Bemessungsgrundsätze für die Pflegesätze ( $\mathbb{\$} 84$ SGB XI) in der Weise, dass der entsprechende Leistungsaufwand als leistungsgerecht anerkannt und vergütet wird.

c) Förderung selbstbestimmter Teilhabe als Qualitätskriterium im SGB XI

Nach $₫ 115$ Abs. 1 SGB XI stellen die Landesverbände der Pflegekassen sicher, dass die von den Pflegekassen erbrachten Leistungen und deren Qualität hinsichtlich der Ergebnis- und Lebensqualität veröffentlicht werden. Der Qualitätsausschuss soll nach $\mathbb{1 1 3}$ Abs. 4 Nr. 4 SGB XI wissenschaftliche Einrichtungen oder Sachverständige beauftragen, ,ergänzende Instrumente für die Ermittlung und Bewertung von Lebensqualität zu entwickeln." Derartige Instrumente stehen bisher aber nicht zur Verfügung.

Die Qualitätsprüfungsrichtlinien nach $\mathbb{1} 114$ SGB XI enthalten in verschiedenen Abschnitten zum Beispiel der Wohnqualität oder der Speiseversorgung Fragestellungen, die Teilaspekte sowohl von Lebensqualität wie auch von Teilhabe sein könnten. Die Fragestellungen sind aber nicht auf die Beurteilung von Lebensqualität ausgerichtet.

In der Ausfüllanleitung für die Prüfer (Ziffer 35bb) ist die Frage, ob Selbstbestimmung bei der Pflege und Betreuung berücksichtigt wird, mit Ja zu beantworten, wenn aus der Dokumentation zu ersehen ist, dass Selbstbestimmung berücksichtigt wird. Die Dokumentation enthält jedoch keine spezifisch auf die selbstbestimmte Teilhabe orientierten, für die Beurteilung der Selbstbestimmung im Sinne der UN-BRK geeigneten Kriterien. Im Übrigen umfasst die selbstbestimmte Teilhabe alle Aspekte des 
Lebens (Art. 26 UN-BRK) und ist nicht auf den Aspekt der Lebensqualität beschränkt.

Es wird vorgeschlagen, entsprechend Art. 26 UN-BRK die Förderung der selbstbestimmten Teilhabe sowohl als Ziel der Pflegeversicherung wie auch als nachzuweisendes und zu überprüfendes Qualitätskriterium im SGB XI zu verankern.

\section{d) Weiterentwicklung des WTG NRW}

Das WTG NRW enthält - im Gegensatz zu einigen anderen Bundesländern - insbesondere in den $\mathbb{S} 1$ und 3 grundsätzliche, auf die selbstbestimmte Teilhabe orientierende Bestimmungen. Dabei konkretisiert $₫ 5$ Abs. 2 die Unterstützung- und Förderverpflichtung der Leistungserbringer ( $\$ 5$ Abs. 1), allerdings beschränkt auf vier sehr allgemein gehaltene Aspekte, von denen drei auf Aktivitäten außerhalb der Einrichtung ausgerichtet sind.

Die Ergebnisse der STAP-Studie zeigen jedoch auch, dass eine wirksame Förderung der selbstbestimmten Teilhabe innerhalb und außerhalb von Pflegeeinrichtungen erreichbar ist. Voraussetzung ist allerdings, dass das Selbstverständnis der Einrichtung und die Rahmenbedingung in der Einrichtung neben der pflegerischen Versorgung die Förderung der selbstbestimmten Teilhabe als völlig gleichwertiges Ziel und Aufgabe adaptiert und konsequent auch auf die Förderung der selbstbestimmten Teilhabe ausgerichtet werden.

Der Landesgesetzgeber kann diese Entwicklung dadurch fördern, dass er die selbstbestimmte Teilhabe

- als verbindliches Konzept in das Qualitätsmanagement des $₫ 4$ Abs. 3 sowie

- in die Aufzählung der Leistungstatbestände des $₫ 19$ Abs. 1 aufnimmt, die durch eine fachliche Konzeption hinterlegt sein müssen.

Der Handlungskatalog des $\ 5$ Abs. 2 sollte um die „Unterstützung der Bewohner bei der Geltendmachung von Leistungen zur Förderung der Teilhabe" ergänzt werden. Neben den Leistungen der Pflegeversicherung bestehen zur Förderung der Teilhabe auch nach anderen Sozialgesetzen Leistungen, für deren Erschließung die Bewohner Unterstützung benötigen.

Das Ergebnis von STAP, insbesondere des Musterrahmenkonzeptes, enthält Kriterien, die auch im Rahmen des ordnungsrechtlichen Prüfauftrages der Aufsichtsbehörden in den Rahmenprüfkatalog einfließen könnten. 
e) Anpassung der Betreuungskräfte-Richtlinien nach $₫ 53$ c SGB XI

Aus Sicht der Studie stellen zusätzliche Betreuungskräfte eine besondere personale Ressource zur individuellen Unterstützung der selbstbestimmten Teilhabe von Bewohner*innen innerhalb und außerhalb von Altenpflegeeinrichtungen dar. Allerdings wurden hier auch Verbesserungsbedarfe in Bezug auf die Aufgabenzuschnitte und Qualifikation der zusätzlichen Betreuungskräfte festgehalten.

Die Richtlinien nach $\$ 53$ c SGB XI zur Qualifikation und zu den Aufgaben von zusätzlichen Betreuungskräften in stationären Pflegeeinrichtungen sollten daher weiterentwickelt werden. Die Weiterentwicklung sollte insbesondere die teilhabeorientierte Aufgabenstellung und die entsprechende Qualifizierung der Betreuungskräfte umfassen.

Nach $\$ 1$ Satz 2 letzter Halbsatz der Richtlinien soll Pflegebedürftigen in Pflegeeinrichtungen mehr Austausch mit anderen Menschen und mehr Teilhabe am Leben in der Gemeinschaft ermöglicht werden. Die nachfolgenden Bestimmungen der Richtlinie sind jedoch weniger auf die Förderung der Teilhabe am Leben in der Gesellschaft ausgerichtet, sondern eher auf Tätigkeiten und Angebote der „klassischen Beschäftigung und Tagesstrukturierung" beschränkt.

Es wird vorgeschlagen, die Richtlinie auf die Förderung der selbstbestimmten Teilhabe im Sinne der UN-BRK bei den Inhalten der Aufgaben und der Qualifikation weiter zu entwickeln und damit die konkrete Teilhabeförderung innerhalb und außerhalb von Pflegeeinrichtungen stärker hervorzuheben und zu ermöglichen. Wie zusätzliche Betreuungskräfte organisatorisch zugeordnet werden, obliegt der Organisationsverantwortung der Einrichtungen. Die Richtlinie sollte allerdings konkrete Regelungen zu den Aufgabenstellungen der Vorgesetzten enthalten. Da es sich um eine untergesetzliche Regelung handelt, wäre hier auch eine zeitnahe Weiterentwicklung im Rahmen der Selbstverwaltung der Pflegeversicherung möglich.

\section{f) Pflegeausbildung}

Bei der Ausbildung der Pflegefachkräfte sollten in den Curricula Inhalte zur Bedeutung und Förderung von selbstbestimmter Teilhabe sowie zur diesbezüglichen Wunscherfassung berücksichtigt werden. Bezogen auf die neue generalistische Pflegeausbildung gibt der Rahmenlehrplan der Fachkommission nach $\$ 53$ Pflegeberufsgesetz eine hilfreiche Orientierung zur 
Integration von teilhaberelevanten Inhalten in die Ausbildung (vor allem in der curricularen Einheit 09). Diese Hinweise gilt es jetzt bei der Erstellung der pflegeschulinternen Curricula zu nutzen. Und es ist wichtig, den Transfer der theoretischen Kenntnisse in die Praxis zu gestalten. Eine gute Grundlage dafür bildet der Rahmenausbildungsplan der Fachkommission und vor allem auch die von der Fachkommission bewusst angelegten Verknüpfungen zwischen Rahmenlehr- und Rahmenausbildungsplan. Eine gelingende Umsetzung in der Praxis setzt die konstruktive Zusammenarbeit zwischen Pflegeschulen und Praxiseinrichtungen voraus.

\section{g) Anwendung des Musterrahmenkonzepts bei der Durchführung des $₫ 5$ WTG NRW}

Ein Ziel des Forschungsprojekts war die Entwicklung eines praxisnahen Musterrahmenkonzeptes, mit dem die Verwirklichung selbstbestimmter Teilhabe in Altenpflegeeinrichtungen gefördert werden kann. Die Praxiserprobung des entwickelten Musterrahmenkonzepts hat gezeigt, dass auf dieser Grundlage eine Selbsteinschätzung des Ist-Standes und die Identifizierung von Handlungsbedarfen sowie die Entwicklung von Maßnahmen zur Organisation selbstbestimmter Teilhabe in Altenpflegeeinrichtungen möglich ist.

Zur Umsetzung des $₫ 5$ WTG NRW bietet es sich an, den Altenpflegeeinrichtungen in Nordrhein-Westfalen zu empfehlen, der einrichtungsspezifischen Förderung der selbstbestimmten Teilhabe dieses Musterrahmenkonzept zugrunde zu legen. Um eine möglichst flächendeckende Entwicklung für Nordrhein-Westfalen zu erreichen, wird angeregt, dass sich der Landesausschuss Alter und Pflege mit dem Ergebnis des Forschungsprojekts und der Wirkungsweise des Musterrahmenkonzepts befasst. Die Sicherstellung der selbstbestimmten Teilhabe in Altenpflegeeinrichtungen in Nordrhein-Westfalen würde eine signifikante Dynamik erreichen, wenn der Landesausschuss Alter und Pflege eine Empfehlung zur Anwendung des Musterrahmenkonzeptes abgibt.

\subsection{Weiterer Forschungs- und Entwicklungsbedarf}

Die praktische Erprobung des Musterrahmenkonzepts hat gezeigt, dass die Organisation, Koordination und Steuerung der vielfältigen Aktivitäten in der Lebenssituation pflegebedürftiger Menschen im Allgemeinen sowie 
die Förderung und Verwirklichung ihrer selbstbestimmten Teilhabe im Speziellen hohe Anforderungen an Altenpflegeeinrichtungen und deren Mitarbeitenden stellt. Die derzeitigen, zum Teil historisch gewachsenen und auf die Ausführung der Anforderungen der medizinische-pflegerischen Versorgung orientierten Abläufe in den Einrichtungen stoßen zunehmend an ihre Grenzen. Im Rahmen der STAP-Studie wurden nicht nur besondere, sondern auch zusätzliche Ressourcen identifiziert und benannt, die es aus Sicht der Studie sowohl von den einzelnen Einrichtungen zu berücksichtigen als auch auf politischer Ebene in den Blick zu nehmen gilt. Darüber hinaus könnte die Organisation, Koordination und Steuerung der Teilhabeförderung auch durch digitale Innovationen maßgeblich unterstützt werden, etwa in Form von digitalen, einrichtungsbereichsübergreifend zugänglichen Planungs- und Dokumentationssystemen. Digitale Dokumentationssysteme werden zwar bereits vielfach in den Altenpflegeeinrichtungen eingesetzt, die Möglichkeiten solcher Systeme zur Steuerung der Teilhabeaktivitäten sind aber häufig noch nicht oder nur begrenzt vorhanden. Es bietet sich an, Art und Umfang solcher Innovationen im Rahmen eines Forschungsprojekts zu erheben und entsprechende Lösungsvorschläge zu entwickeln.

In Bezug auf das im Projekt entwickelte Musterrahmenkonzept wäre es empfehlenswert, wenn dessen Transfer in die Praxis einerseits über Schulungen in Altenpflegeeinrichtungen unterstützt und andererseits mit Blick auf dessen Akzeptanz, Angemessenheit und Machbarkeit sowie auf bewirkte Veränderungen in den geschulten Einrichtungen auf Basis einer größeren trägerübergreifenden Stichprobe näher untersucht werden könnte. Aus Sicht der STAP-Studie kann das Musterrahmenkonzept ausdrücklich zur Weiterentwicklung des Praxishandelns zur Förderung und Verwirklichung der selbstbestimmten Teilhabe in Altenpflegeeinrichtungen beitragen - im Sinne der Bewohner*innen wäre daher ein erfolgreicher Praxistransfer wünschenswert. 


\section{Literatur}

Ackermann, A., \& Oswald, W. D. (2006). Erhalt und Förderung der Selbstständigkeit bei Pflegeheimbewohnern. Zeitschrift für Gerontopsychologie \& -psychiatrie, 19(2), 59-71.

Andresen, M., Hoff, M., \& Puggaard, L. (o. J.). Decisional autonomy among nursing home residents: A meta-synthesis. Unveröffentlichtes Manuskript.

Andresen, M., Runge, U., Hoff, M., \& Puggaard, L. (2009). Perceived Autonomy and Activity Choices Among Physically Disabled Older People in Nursing Home Settings: A Randomized Trial. Journal of Aging and Health, 21(8), 11331158.

Arbeitskreis Teilhabeorientierte Pflege. (o. J.). Empfehlungen für eine teilhabeorientierte Pflege. www.caritas.de/stellungnahmen/empfehlungen-fuer-eine-teilhabeori entierte-pflege-/150158/.

Aronson, P., \& Mahler, C. (2016). Menschenrechte in der Pflegepraxis: Herausforderungen und Lösungsansätze in Pflegeheimen. Deutsches Institut für Menschenrechte.

Baas, S., Hedtke-Becker, A., \& Wolfinger, M. (2017). Beeinflussung von Woblbefinden und Lebensqualität älterer Menschen im Pflegeheim - Wie kann das in der Praxis geschehen? (Abschlussbericht zum „Modellprojekt zur Messung der Lebensqualität und subjektiven Wohlbefindens im Pflegeheim“ MoMeL). Institut für Sozialpädagogische Forschung Mainz gGmbH (ism).

Bartholomeyczik, S., Halek, M., Sowinski, C., Besselmann, K., Dürrmann, P., Haupt, M., Kuhn, C., Müller-Hergl, C., Perrar, K. M., Riesner, C., Rüsing, D., Schwerdt, Ruth, Kooij, C. von der, \& Zegelin, A. (2006). Rahmenempfehlungen zum Umgang mit herausforderndem Verhalten bei Menschen mit Demenz in der stationären Altenpflege (Abschlussbericht). Bundesministerium für Gesundheit. www.bundesgesundheitsministerium.de/fileadmin/Dateien/Publikationen/Pfleg e/Berichte/Bericht_Rahmenempfehlungen_zum_Umgang_mit_herausfordernde m_Verhalten_bei_Menschen_mit_Demenz_in_der_stationaeren_Altenhilfe.pdf.

Bartoszek, G., Fischer, U., Clarenau, S. C. von, Grill, E., Mau, W., Meyer, G., Strobl, R., Thiesemann, R., Nadolny, S., \& Müller, M. (2015). Development of an International Classification of Functioning, Disability and Health (ICF)-based standard set to describe the impact of joint contractures on participation of older individuals in geriatric care settings. Archives of Gerontology and Geriatrics, 61(1), 61-66.

Beelmann, A., \& Karing, C. (2014). Implementationsfaktoren und -prozesse in der Präventions- forschung: Strategien, Probleme, Ergebnisse, Perspektiven. Psychologische Rundschau, 65(3), 129-139.

Behrens, J., \& Zimmermann, M. (2006). Das Bedürfnis nach Selbstbestimmung bei Pflegebedürftigkeit: Konzept und Forschungsperspektiven. Zeitschrift für Gerontologie und Geriatrie, 39(3), 165-172. 
Benninghoven, C. (2013). Kurze Wege. Ein Altenheim wird zum Nachbarschaftszentrum. Und rückt ins Zentrum des Stadtlebens. Diakonie magazin, 1(2013), 42-44.

Bernsteiner, M., \& Boggatz, T. (2016). Wohlbefinden im Alter. Pflege, 29(3), 137149.

Bleck, C., Rießen, A. van, Knopp, R., \& Schlee, T. (2018). Sozialräumliche Perspektiven in der stationären Altenhilfe. Eine empirische Studie im städtischen Raum. Springer.

Bleck, C., Rießen, A. van, \& Schlee, T. (2018). Sozialraumorientierung in der stationären Altenhilfe. Aktuelle Bezüge und zukünftige Potenziale. In C. Bleck, A. van Rießen \& R. Knopp (Hrsg.), Alter und Pflege im Sozialraum. Theoretische Erwartungen und empirische Bewertungen (S. 225-247). Springer VS.

Blumer, H. (1954). What is Wrong with Social Theory. American Sociological Review, 1954(18), 3-10.

Bobbert, M. (2002). Patientenautonomie und Pflege. Begründung und Anwendung eines moralischen Rechts. Campus.

Bode, I. (2014). Akteure in der stationären Altenhilfe. In H. Brandenburg, I. Bode, B. Werner \& B. Berger (Hrsg.), Soziales Management in der stationären Altenhilfe: Kontexte und Gestaltungsspielräume (S. 87-112). Verlag Hans Huber.

Bogert, B. (2013). Vernetzt im Quartier: Projekt. Altenheim, 52(12), 40-43.

Böhm, E. (1999). Psychobiographisches Pflegemodell nach Böhm - Band I: Grundlagen. facultas Universitätsverlag.

Böhmer, A. (2015). Verfahren und Handlungsfelder der Sozialplanung: Grundwissen für die Soziale Arbeit. Springer VS.

Bohnsack, R., Nentwig-Gesemann, I., \& Nohl, A.-M. (Hrsg.). (2007). Die dokumentarische Methode und ibre Forschungspraxis: Grundlagen qualitativer Sozialforschung (2., erw. aktualis. Aufl.). Springer VS.

Bräutigam, C. (2008). Messen - Bewerten - Besser werden Meten - Boordelen - Beter worden (Projektbericht). Institut für Arbeit und Technik (IAT). www.sozial-hold ing.de/upload/download/endbericht_mbb_2008_deutsch.pdf.

Brütt, A. L., Buschmann-Steinhage, R., Kirschning, S., \& Wegscheider, K. (2016). Teilhabeforschung. Bundesgesundheitsblatt - Gesundheitsforschung - Gesundheitsschutz, 59(9), 1068-1074.

Bundesministerium Familie, Soziales, Frauen und Jugend (2002). Vierter Altenbericht zur Lage der älteren Generation in der Bundesrepublik Deutschland: Risiken, Lebensqualität und Versorgung Hochaltriger unter besonderer Berücksichtigung demenzieller Erkrankungen. Bundesministerium Familie, Soziales, Frauen und Jugend. www.bmfsfj.de/bmfsfj/service/publikationen/4--altenbericht-/95594.

Bundesministerium Familie, Soziales, Frauen und Jugend (2005). Fünfter Bericht zur Lage der älteren Generationen in der Bundesrepublik Deutschland. Potenziale des Alters in Wirtschaft und Gesellschaft. Der Betrag der älteren Menschen zum Zusammenhalt der Generationen. (Bericht der Sachverständigenkommission). Bundesministerium Familie, Soziales, Frauen und Jugend. www.bmfsfj.de/blob/79080/ 8a95842e52ba43556f9ebfa600f02483/fuenfter-altenbericht-data.pdf. 
Bundesministerium für Arbeit und Soziales (2018). Sozialhilfe und Grundsicherung im Alter und bei Erwerbsminderung. Bundesministerium für Arbeit und Soziales. www.bmas.de/SharedDocs/Downloads/DE/PDF-Publikationen/a207-sozialhilfeund-grundsicherung.pdf.

Collopy, B. J. (1988). Autonomy in Long Term Care: Some Crucial Distinctions. The Gerontologist, 28(Suppl), 10-17.

Cook, C., Heath, F., \& Thompson, R. L. (2000). A Meta-Analysis of Response Rates in Web- or Internet-Based Surveys. Educational and Psychological Measurement, 60(6), 821-836.

Davies, S., Laker, S., \& Ellis, L. (1997). Promoting autonomy and independence for older people within nursing practice: A literature review. Journal of Advanced Nursing, 26(2), 408-417.

Degener, T. (2009). Die UN-Behindertenrechtskonvention als Inklusionsmotor. $R d$ JB Recht der Jugend und des Bildungswesens, 2/2009, 200-219.

Degener, T., \& Diehl, E. (Hrsg.) (2015). Handbuch Behindertenrechtskonvention: Teilhabe als Menschenrecht - Inklusion als gesellschaftliche Aufgabe. BpB.

Denzin, N. (1970). The research act. A theoretical introduction to sociological methods. Aldine Pub. Co.

Deutscher Evangelischer Verband für Altenarbeit und Pflege e.V. (2008). Den Sozialraum mitgestalten. DEVAP.

Dewing, J. (2007). Participatory research A method for process consent with persons who have dementia. Dementia, 6(1), 11-25.

Dittrich, C. (2009). Lebensqualität für Menschen mit Demenz - Wie Teilhabe von Menschen mit Demenz und ihren Angehörigen gefördert werden kann. Theorie und Praxis der Sozialen Arbeit, 60(3), 174-180.

Döring, N., \& Bortz, J. (2016). Forschungsmethoden und Evaluation in den Sozial- und Humanwissenschaften (5., vollst. überarb., aktualis., erw. Aufl.). Springer.

Dräger, D., Kölzsch, M., Kalinowski, S., Ellert, S., Kopke, K., Fischer, T., \& Kreutz, R. (2013). Autonomie trotz Schmerz? Ressourcen und Barrieren in der Lebenswelt von Pflegeheimbewohnern und -bewohnerinnen. In A. Kuhlmey \& C. Tesch-Römer (Hrsg.), Autonomie trotz Multimorbidität Ressourcen für Selbstständigkeit und Selbstbestimmung im Alter (S. 165-202). Hogrefe.

Eid, M., Gollwitzer, M., \& Schmitt, M. (2017). Statistik und Forschungsmethoden: Mit Online-Materialien (5., korr. Auflage). Beltz.

Elander, G., Drechsler, K., \& Persson, K. W. (1993). Ethical dilemmas in long-term care settings; interviews with nurses in Sweden and England. International Journal of Nursing Studies, 30(1), 91-97.

Enggruber, R., \& Bleck, C. (2005). Modelle der Kompetenzfeststellung im beschäftigungs- und bildungstheoretischen Diskurs - Unter besonderer Berücksichtigung von Gender Mainstreaming. Entwicklungspartnerschaft „Arbeitsplätze für junge Menschen in der Sozialwirtschaft". www.bildungsserver.de/onlineressource.html?onlineress ourcen_id=40624.

Euler, D., \& Sloane, P. F. E. (1998). Implementation als Problem der Modellversuchsforschung. Unterrichtswissenschaft, 26(4), 312-326. 
Falk, K., \& Heusinger, J. (2011). Selbstbestimmt trotz Armut, Alter, Krankheit?. Ein Projektbericht. Dr. med. Mabuse, 32(192), 48-50.

Falk, K., \& Wolter, B. (2018). Sozialräumliche Voraussetzungen für Teilhabe und Selbstbestimmung sozial benachteiligter älterer Menschen mit Pflegebedarf. In C. Bleck, A. van Rießen \& R. Knopp (Hrsg.), Alter und Pflege im Sozialraum (S. 143-160). Springer VS.

Fan, W., \& Yan, Z. (2010). Factors affecting response rates of the web survey: A systematic review. Computers in Human Behavior, 26(2), 132-139.

Farin, E. (2012). Konzeptionelle und methodische Herausforderungen der Teilhabeforschung. Die Rehabilitation, 51(S 01), 3-11.

Farrokhzad, S., \& Mäder, S. (2014). Nutzenorientierte Evaluation: Ein Leitfaden für die Arbeitsfelder Integration, Vielfalt und Toleranz. Waxmann.

Faulbaum, F., Prüfer, P., \& Rexroth, M. (2009). Was ist eine gute Frage? Die systematische Evaluation der Fragenqualität. GWV Fachverlage.

Fischbach, C., \& Veer, T. (2008). Bürgerschaftliches Engagement in der Altenhilfe. In M. Erlinghagen \& K. Hank (Hrsg.), Produktives Altern und informelle Arbeit in modernen Gesellschaften. Theoretische Perspektiven und empirische Befunde (S. 235256). Springer VS.

Fix, E., \& Kurzke-Maasmeier, S. (Hrsg.). (2009). Das Menschenrecht auf gute Pflege: Selbstbestimmung und Teilhabe verwirklichen. Lambertus.

Fixsen, D. L., \& Blase, K. A. (2009). Implementation: The Missing Link Between Research and Practice. NIRN Implementation Brief, 1. https://files.eric.ed.gov/full text/ED507422.pdf.

Fixsen, D. L., Blase, K. A., Naoom, S. F., \& Wallace, F. (2009). Core Implementation Components. Research on Social Work Practice, 19(5), 531-540.

Fixsen, D. L., Naoom, S. F., Blase, K. A., \& Wallace, F. (2007). Implementation: The Missing Link Between Research and Practice. The ASPAC Advisor, $19(1$ \& 2), 4-11.

Flick, U. (Hrsg.). (2006). Qualitative Evaluationsforschung. Konzepte, Methoden, Umsetzung. Rowohlt Taschenbuch Verlag.

Flick, U. (2014). Qualitative Sozialforschung. Eine Einführung (6., vollst. überarb., erw. Aufl.). Rowohlt Taschenbuch Verlag.

Flick, U. (2016). Qualitative Sozialforschung. Eine Einführung (7., vollst. überarb., erw. Aufl.). Rowohlt Taschenbuch Verlag.

Fossey, J., Lee, L., \& Ballard, C. (2002). Dementia Care Mapping as a research tool for measuring quality of life in care settings: Psychometric properties. International Journal of Geriatric Psychiatry, 17(11), 1064-1070.

Franzen, A. (2014). 51. Antwortskalen in standardisierten Befragungen. In N. Baur \& J. Blasius (Hrsg.), Handbuch Methoden der empirischen Sozialforschung (S. 701712). Springer VS.

Fuchs, H. (2009). Zur Reichweite sozialrechtlicher Rahmenbedingungen zur Selbstbestimmung und Teilhabe. In V. Garms-Homolová, E. von Kardorff, K. Theiss, A. Meschnig \& H. Fuchs (Hrsg.), Teilhabe und Selbstbestimmung von Menschen mit Pflegebedarf. Konzepte und Methoden (S. 19-35). Mabuse. 
Fuchs, H. (2016). UN-Behindertenrechtskonvention, SGB IX und Lebensqualität. In S. V. Müller \& C. Gärtner (Hrsg.), Lebensqualität im Alter: Perspektiven für Menschen mit geistiger Behinderung und psychischen Erkrankungen (S. 403-422). Springer VS.

Garms-Homolová, V., \& Theiss, K. (2009a). Professionelle Standards für die „gute Praxis": Kriterien und Konzepte der Evidenzbasierung. In V. Garms-Homolová, K. Theiss, E. von Kardorff, A. Meschnig \& H. Fuchs (Hrsg.), Teilhabe und Selbstbestimmung von Menschen mit Pflegebedarf. Konzepte und Methoden (S. 189-215). Mabuse.

Garms-Homolová, V., \& Theiss, K. (2009b). Selbstbestimmung und Teilhabe von Menschen mit Pflegebedarf - Therapeutische Konzepte und Voraussetzungen der Realisierung. In V. Garms-Homolová, E. von Kardorff, K. Theiss, A. Meschnig \& H. Fuchs (Hrsg.), Teilhabe und Selbstbestimmung von Menschen mit Pflegebedarf. Konzepte und Methoden (S. 105-188). Mabuse.

Garms-Homolová, V., \& Theiss, K. (2009c). Teilhabe und Selbstbestimmung in den Pflegeeinrichtungen - realisierbare Maßnahmen. In V. Garms-Homolová, E. von Kardorff, K. Theiss, A. Meschnig \& H. Fuchs (Hrsg.), Teilhabe und Selbstbestimmung von Menschen mit Pflegebedarf. Konzepte und Methoden (S. 215-252). Mabuse.

Garms-Homolová, V., Kardorff, E. von, Theiss, K., Meschnig, A., \& Fuchs, H. (2009). Teilhabe und Selbstbestimmung von Menschen mit Pflegebedarf. Konzepte und Methoden. Mabuse.

Geisler, L. S. (2004). Patientenautonomie - Eine kritische Begriffsbestimmung. DMW - Deutsche Medizinische Wochenschrift, 129(9), 453-456.

Giesbers, M. (2016). Zwischen Autonomie und Fürsorge. Pflege und Pflegepolitik aus der Sicht der Praxis institutioneller Pflege. Jabrbuch für Christliche Sozialwissenschaften, 57(0), 21-30.

Greenberg, M. T., Domitrovic, C. E., Graczyk, P. A., \& Zins, J. E. (2005). The Study of Implementation in School-Based Preventive Interventions: Theory, Research, and Practice (Volume 3). Center for Mental Health Services, Substance Abuse and Mental Health Services Administration.

Hahn, W. (2011). Ein neues Zuhause? Eine ethnographische Studie in einem Altenpflegeheim. Peter Lang.

Hämel, K. (2012). Öffnung und Engagement: Altenpflegeheime zwischen staatlicher Regulierung, Wettbewerb und zivilgesellschaftlicher Einbettung. Springer VS.

Hasseler, M. (2016). Entwicklung eines wissenschaftlich basierten Qualitätsverständnisses für die Pflege- und Lebensqualität (Abschlussbericht). GKV Spitzenverband. www.gkv-spitzenverband.de/media/dokumente/pflegeversicherung/qualitaet_in _der_pflege/wiss_qualitaetsverstaendnis/2016-08-25_Abschlussbericht_wiss_Qua litaetsverstaendnis.pdf.

Heeg, F. J. (1991). Moderne Arbeitsorganisation: Grundlagen der organisatorischen Gestaltung von Arbeitssystemen bei Einsatz neuer Technologien (2., überarb. Aufl). Hanser.

Heimverzeichnis gGmbH (2017). Altenheime, Pflegeeinrichtungen und Seniorenresidenzen mit Lebensqualität. www.heimverzeichnis.de/index.php?id=126. 
Heusinger, J., \& Knoch, T. (2007). Fallstudien zur Qualität von Pflege und Versorgung in stationären Pflegeeinrichtungen. In U. Schneekloth \& H.-W. Wahl (Hrsg.), Pflegebedarf und Versorgungssituation bei älteren Menschen in Heimen. Demenz, Angehörige und Freiwillige, Beispiele für Good Practice (S. 288-336). Kohlhammer.

Hoben, M. (2016). Stand der pflegerischen Implementierungsforschung im deutschsprachigen Raum. In M. Hoben, M. Bär \& H.-W. Wahl (Hrsg.), Implementierungswissenschaft für Pflege und Gerontologie: Grundlagen, Forschung und Anwendung; ein Handbuch (S. 115-132). Kohlhammer.

Huber, M., Siegel, S. A., Wächter, C., \& Brandenburg, A. (2005). Autonomie im Alter: Leben und Altwerden im Pflegeheim; wie Pflegende die Autonomie von alten und pflegebedürftigen Menschen fördern. Schlütersche.

Institut für Qualifikation und Arbeit (2017). Empfänger von Hilfe zur Pflege 19922016 innerhalb und außerhalb von Einrichtungen und Gesamtzahl in 1.000. Institut für Qualifikation und Arbeit der Universität Duisburg-Essen. www.sozialpolitikaktuell.de/tl_files/sozialpolitik-aktuell/_Politikfelder/Sozialstaat/Datensammlun g/PDF-Dateien/abbIII55.pdf.

IT.NRW (2017). Kommunalprofile. Landesbetrieb IT.NRW Statistik und IT-Dienstleistungen. https://www.it.nrw/kommunalprofile-82197.

Jenull-Schiefer, B. (2005). Aktivität und Selbstbestimmung im Pflegeheimalltag (Ergebnisse aus dem Projekt „Heri-Aktiv“). In T. Klie, A. Buhl, H. Entzian \& A. Hedtke-Becker (Hrsg.), Die Zukunft der gesundheitlichen, sozialen und pflegerischen Versorgung älterer Menschen. Mabuse.

Kammerer, K., Falk, K., Heusinger, J., \& Kümpers, S. (2012). Selbstbestimmung bei Pflegebedürftigkeit. Zeitschrift für Gerontologie und Geriatrie, 45(7), 624-629.

Kane, R. A., Pratt, M., \& Schoeneman, K. (2004). Measures, indicators, and improvement of quality of life in nursing homes: Final Report. School of Public Health, University of Minnesota.

Kardorff, E. von (2014). Partizipation im aktuellen gesellschaftlichen Diskurs. Anmerkungen zur Vielfalt eines Konzepts und seiner Rolle in der Sozialarbeit. Archiv für Wissenschaft und Praxis der Sozialen Arbeit, 2(2014), 4-15.

Kardoff, E. von und Meschnig, A. (2009a). Pflege und Pflegepolitik im gesellschaftlichen Wandel. In V. Garms-Homolová, E. von Kardorff, K. Theiss, A. Meschnig \& H. Fuchs (Hrsg.), Teilhabe und Selbstbestimmung von Menschen mit Pflegebedarf. Konzepte und Methoden (S. 35-59). Mabuse.

Kardoff, E. von, \& Meschnig, A. (2009b). Selbstbestimmung, Teilhabe und selbstständige Lebensführung: Konzeptionelle Überlegungen. In V. Garms-Homolová, E. von Kardorff, K. Theiss, A. Meschnig \& H. Fuchs (Hrsg.), Teilhabe und Selbstbestimmung von Menschen mit Pflegebedarf. Konzepte und Methoden (S.6188). Mabuse.

Kardorff, E. von, \& Meschnig, A. (2009c). Die Perspektive der Betroffenen. Ergebnisse einer Fokusgruppen-Studie. In V. Garms-Homolová, E. von Kardorff, K. Theiss, A. Meschnig \& H. Fuchs (Hrsg.)Teilhabe und Selbstbestimmung von Menschen mit Pflegebedarf. Konzepte und Methoden (S. 253-278). Mabuse.

Kempchen, U. (2017). Der Heimbeirat als Partner. Altenheim, 4/2017, 78-80. 
Klie, T., Vollmann, J., \& Pantel, J. (2014). Autonomie und Einwilligungsfähigkeit bei Demenz als interdisziplinäre Herausforderung für Forschung, Politik und klinische Praxis. informationsdienst altersfragen, 4(41), 5-15.

Kohls, M. (2012). Pflegebedürftigkeit und Nachfrage nach Pflegeleistungen von Migrantinnen und Migranten im demographischen Wandel, Kurzfassung (Forschungsbericht). Bundesamt für Migration und Flüchtlinge. www.bamf.de/SharedDocs/A nlagen/DE/Forschung/Forschungsberichte/Kurzberichte/fb12-pflegebeduerftigk eit-kurzfassung.pdf?_blob=publicationFile\&v=10.

Kotsch, L., \& Hitzler, R. (2013). Selbstbestimmung trotz Demenz? Ein Gebot und seine praktische Relevanz im Pflegealltag. Beltz Juventa.

Krahmer, U., \& Klie, T. (Hrsg.). (2009). Sozialgesetzbuch XI. Soziale Pflegeversicherung (3. Aufl.). Nomos.

Krems, B. (2011). Best Practice. Beitrag im Online-Verwaltungslexikon olev.de, Version 1.3. https://olev.de/b/best-practice.htm.

Kruse, A., \& Schröder. (2006). H.I.L.DE. Abschlussbericht Erste Förderphase (Abschlussbericht). Institut für Gerontologie und Sektion Gerontopsychiatrie an der Psychiatrischen Universitätsklinik Heidelberg. www.gero.uni-heidelberg.de/ imperia/md/content/fakultaeten/vekw/ifg/forschung/hildekongress/hilde_abschl ussbericht_erste_foerderphase.pdf.

Kruse, J., \& Schmieder, C. (2014). Qualitative Interviewforschung: Ein integrativer Ansatz. Beltz Juventa.

Kuckartz, U. (2016). Qualitative Inhaltsanalyse: Methoden, Praxis, Computerunterstützung (3., überarb. Aufl.). Beltz Juventa.

Kuckartz, U. (2018). Qualitative Inhaltsanalyse. Methoden, Praxis, Computerunterstützung (4. Aufl.). Beltz Juventa.

Kuhlmey, A., \& Tesch-Römer, C. (2013). Autonomie trotz Multimorbidität Ressourcen für Selbstständigkeit und Selbstbestimmung im Alter. Hogrefe.

Kühn, T., \& Koschel, K.-V. (2011). Gruppendiskussionen: Ein Praxis-Handbuch (1. Aufl.). Springer VS.

Kühn, T., \& Koschel, K.-V. (2018). Gruppendiskussionen: Ein Praxis-Handbuch (2. Aufl.). Springer VS.

Kümpers, S., \& Heusinger, J. (2012). Autonomie trotz Armut und Pflegebedarf? Altern unter Bedingungen von Marginalisierung. Huber.

Kümpers, S., \& Wolter, B. (2015). Soziale Teilhabe pflegebedürftiger älterer Menschen in innovativen stationären Wohnformen. In K. Jacobs, A. Kuhlmey, S. Greß \& A. Schwinger (Hrsg.), Pflege-Report 2015. Pflege zwischen Heim und Häuslichkeit (S. 135-145). Schattauer.

Kümpers, S., \& Zander, M. (2012). Der Autonomiebegriff in Kontext von Hilfeund Pflegebedürftigkeit und sozialer Benachteiligung. In S. Kümpers \& J. Heusinger (Hrsg.), Autonomie trotz Armut und Pflegebedarf? Altern unter Bedingungen von Marginalisierung (S. 21-38). Huber.

Lamnek, S. (2005). Qualitative Sozialforschung: Lehrbuch (4., vollst., überarb. Aufl.). Beltz. 
Lamnek, S., \& Krell, C. (2016). Qualitative Sozialforschung: Mit Online-Material (6., überab. Aufl.). Beltz.

Luhmann, N., \& Schorr, K. E. (1982). Das Technologiedefizit der Erziehung und die Pädagogik. In N. Luhmann \& K. E. Schorr (Hrsg.), Zwischen Technologie und Selbstreferenz. Fragen an die Pädagogik (S. 11-41). Suhrkamp.

Marshall, M. (1997). State of the art in dementia care. Centre for Policy on Aging.

Mattiasson, A. C., \& Andersson, L. (1995). Nursing home staff attitudes to ethical conflicts with respect to patient autonomy and paternalism. Nursing Ethics, 2(2), 115-130.

Mayerhofer, W. (2009). Das Fokusgruppeninterview. In R. Buber \& H. H. Holzmüller (Hrsg.), Qualitative Marktforschung. Konzepte - Methoden - Analysen (2., überarb. Aufl., S. 477-490). Gabler.

Mayring, P. (2001). Kombination und Integration qualitativer und quantitativer Analyse. Forum Qualitative Sozialforschung / Forum: Qualitative Social Research, 2(1). http://nbn-resolving.de/urn:nbn:de:0114-fqs010162.

Mayring, P. (2010). Qualitative Inhaltsanalyse: Grundlagen und Techniken (11., aktualis., überarb. Aufl.). Beltz.

Mayring, P. (2015). Qualitative Inhaltsanalyse: Grundlagen und Techniken (12., überarb. Aufl.). Beltz.

Meeks, S., Young, C. M., \& Looney, S. W. (2007). Activity participation and affect among nursing home residents: Support for a behavioural model of depression. Aging \& Mental Health, 11(6), 751-760.

Meeks, Suzanne, \& Looney, S. W. (2011). Depressed Nursing Home Residents' Activity Participation and Affect as a Function of Staff Engagement. Behavior Therapy, 42(1), 22-29.

Mehnert, T., \& Kremer-Preiß, U. (2014). Ist-Analyse im Quartier. Handreichung im Rabmen des Förderbausteins 3.1.1 "Projekte im Ansatz zur Quartiersentwicklung" des Deutschen Hilfswerks, Stand 02/14 (S.34). Kuratorium Deutsche Altershilfe. www.fernsehlotterie.de/content/uploads/2016/06/Quartiersentwicklung_Handre ichung-1_Ist-Analyse_02-14_1.pdf.

Merkens, H. (1992). Teilnehmende Beobachtung: Analyse von Protokollen teilnehmender Beobachter. In Analyse verbaler Daten. Über den Umgang mit qualitativen Daten (S. 216-247). Westdeutscher Verlag.

Meuser, M., \& Nagel, U. (1991). ExpertInneninterviews - Vielfach erprobt, wenig bedacht. Ein Beitrag zur qualitativen Methodendiskussion. In Qualitativ-empirische Sozialforschung. Konzepte, Methoden, Analysen (S. 441-471). Springer VS.

Meuser, M., \& Nagel, U. (2009). Das Experteninterview - Konzeptionelle Grundlagen und methodische Anlage. In S. Pickel, G. Pickel, H.-J. Lauth \& D. Jahn (Hrsg.), Methoden der vergleichenden Politik- und Sozialwissenschaft. Neue Entwicklungen und Anwendungen (S. 465-479). Springer VS.

Michell-Auli, P., \& Sowinski, C. (2012). Die 5. Generation: KDA-Quartiershäuser: Ansätze zur Neuausrichtung von Alten- und Pflegeheimen. Kuratorium Deutsche Altershilfe. 
Michie, S., Johnston, M., Abraham, C., Lawton, R., Parker, D., \& Walker, A. (2005). Making psychological theory useful for implementing evidence based practice: A consensus approach. Qual Saf Health Care, 26-33.

Ministerium für Gesundheit, Emanzipation, Pflege und Alter des Landes Nordrhein-Westfalen (Hrsg.) (2017). Selbstbestimmt Leben, gemeinsam Teilhabe ermöglichen (Landesförderplan „Alter und Pflege“ des Landes Nordrhein-Westfahlen 2017-2018 gemäß $\ 19$ APG NRW). Ministerium für Gesundheit, Emanzipation, Pflege und Alter des Landes Nordrhein-Westfalen. www.mags.nrw/sites/defa ult/files/asset/document/pflege_landesfoerderplan_2017.pdf.

Netten, A., Beadle-Brown, J., Trukeschitz, B., Towers, A.-M., Welch, E., Forder, J., Smith, J., \& Alden, E. (2010). Measuring Outcomes for Public Service Users (Discussion Paper 2696/2). PSSRU, University of Kent. www.pssru.ac.uk/pub/dp2696_2 .pdf.

Nullmeier, F. (2015). Inklusive Sozialpolitik und die Entwicklung des Teilhabegedankens. In Heinrich-Böll-Stiftung (Hrsg.), Inklusion. Wege in die Teilhabegesellschaf (S. 92-104). Campus.

Olbrecht, T. (2010). Akzeptanz von E-Learning. Eine Auseinandersetzung mit dem Technologieakzeptanzmodell zur Analyse individueller und sozialer Einflussfaktoren. Friedrich-Schiller-Universität Jena.

Oswald, F., Wahl, H.-W., Antfang, P., Heusel, C., Maurer, A., \& Schmidt, H. (2014). Lebensqualität in der stationären Altenpflege mit INSEL: Konzeption, praxisnahe Erfassung, Befunde und sozialpolitische Implikationen. Lit Verlag.

Patton, M. Q. (2002). Qualitative research and evaluation methods (3. Aufl.). Sage Publications.

Petermann, F. (2014). Implementationsforschung: Grundbegriffe und Konzepte. Psychologische Rundschau, 65(3), 122-128.

Pleschberger, S. (2005). Nur nicht zur Last fallen: Sterben in Würde aus der Sicht alter Menschen in Pflegeheimen. Lambertus.

Porst, R. (2014). Fragebogen. Ein Arbeitsbuch. (4. Aufl.). Springer VS.

Rädiker, S., \& Kuckartz, U. (2019). Analyse qualitativer Daten mit MAXQDA: Text, Audio und Video. Springer VS.

Rasch, B., Friese, M., Hofmann, W., \& Naumann, E. (2014). Quantitative Methoden 2: Einführung in die Statistik für Psychologen und Sozialwissenschaftler (4. Aufl.). Springer.

Reckwitz, A. (2010). Auf dem Weg zu einer kultursoziologischen Analytik zwischen Praxeologie und Poststrukturalismus. In M. Wohlrab-Sahr (Hrsg.), Kultursoziologie (S. 179-205). Springer VS.

Reinmann-Rothmeier, G., \& Mandl, H. (1998). Wenn kreative Ansätze versanden: Implementation als verkannte Aufgabe. Unterrichtswissenschaft, 26(4), 292-311.

Ryle, G. (1945). Knowing How and Knowing That: The Presidential Address. Proceedings of the Aristotelian Society, 46, 1-16. 
Saal, S., Meyer, G., Beutner, K., Klingshirn, H., Strobl, R., Grill, E., Mann, E., Köpke, S., Bleijlevens, M. H. C., Bartoszek, G., Stephan, A.-J., Hirt, J., \& Müller, M. (2018). Development of a complex intervention to improve participation of nursing home residents with joint contractures: A mixed-method study. BMC Geriatrics, 18(1), 61.

Schmitt, M. (2015). Innovationskultur - Grundlage einer zukunftsorientierten Arbeitskultur. In W. Widuckel, M. J. Ringlstetter, K. de Molina \& D. Frey (Hrsg.), Arbeitskultur 2020. Herausforderungen und Best Practices der Arbeitswelt der Zukunft (S. 73-88). Springer Fachmedien Wiesbaden.

Schönberg, F. (2011). Quartiersnahe Versorgung und die Charta. In Evangelisches Johanneswerk (Hrsg.), Quartiersnah. Die Zukunft der Altenhilfe (S. 47-55). Vincentz.

Schopp, A., Dassen, T., Välimäki, M., Leino-Kilpi, H., Bansemir, G., Gasull, M., Lemonidou, C., Scott, P., \& Arndt, M. (2001). Autonomie, Privatheit und die Umsetzung des Prinzips der „informierten Zustimmung“ im Zusammenhang mit pflegerischen Interventionen aus der Perspektive des älteren Menschen. Pflege, 14(1), 29-37.

Schreier, M. (2014). Varianten qualitativer Inhaltsanalyse: Ein Wegweiser im Dickicht der Begrifflichkeiten. Forum Qualitative Sozialforschung / Forum: Qualitative Social Research, 15(1). http://nbn-resolving.de/urn:nbn:de:0114-fqs1401185.

Schuhmacher, B. (2018). Inklusion für Menschen mit Demenz: Exklusionsrisiken und Teilhabechancen. Springer VS.

Schulz, M., Mack, B., \& Renn, O. (Hrsg.). (2012). Fokusgruppen in der empirischen Sozialwissenschaft: Von der Konzeption bis zur Auswertung. Springer VS.

Schütz, A. (1972). Studien zur soziologischen Theorie (Brodersen, Arvin, Hrsg.). Nijhoff.

Selinger, Y., Weber, A., \& Behrens, J. (2010). Das Verhältnis von selbstbestimmter Teilhabe und Pflege: Konzepte in anderen europäischen Ländern. Archiv für Wissenschaft und Praxis der Sozialen Arbeit, 2010(3), 72-88.

Spiegel, H. v. (2013). Methodisches Handeln in der sozialen Arbeit (5., vollst. überarb. Aufl.). Reinhardt.

Stamann, C., Janssen, M., \& Schreier, M. (2016). Qualitative Inhaltsanalyse - Versuch einer Begriffsbestimmung und Systematisierung. Forum Qualitative Sozialforschung / Forum: Qualitative Social Research, 17(3). http://nbn-resolving.de/urn: nbn:de:0114-fqs1603166.

Steiner, G. (1999). Die Idee der Selbstbestimmung. INFORUM, 3/99, 44-51.

Streblow, C. (2005). Schulsozialarbeit und Lebenswelten Jugendlicher: Ein Beitrag zur dokumentarischen Evaluationsforschung. Edition Recherche.

Voigt-Radloff, S., Stemmer, R., Behrens, J., Horbach, A., Ayerle, G. M., Schäfers, R., Binnig, M., Mattern, E., Heldmann, P., Wasner, M., Braun, C., Marotzki, U., Kraus, E., George, S., Müller, C., Corsten, S., Lauer, N., Schade, V., \& Kempf, S. (2013). Forschung zu komplexen Interventionen in der Pflege- und Hebammenwissenschaft und in den Wissenschaften der Physio- therapie, Ergotherapie und Logopädie. Deutsches Cochrane Zentrum Universitätsklinikum Freiburg. 
Vries, B. de (2009). Aufbruch ins Quartier: Versorgungssicherheit außerhalb des Heims. Altenheim, 48(4), 22-25.

Wagner, P., \& Hering, L. (2014). Online-Befragung. In N. Baur \& J. Blasius (Hrsg.), Handbuch Methoden der empirischen Sozialforschung (S. 661-673). Springer VS.

Wahl, H.-W., \& Diehl, M. (2016). Implementierung und Implementierungsforschung in der Gerontologie. In M. Hoben, M. Bär \& H.-W. Wahl (Hrsg.), Implementierungswissenschaft für Pflege und Gerontologie (S. 133-149). Kohlhammer.

Walter, T. (Hrsg.). (2005). Das Normalisierungsprinzip. Ein Lesebuch zu Geschichte und Gegenwart eines Reformkonzepts. Lebenshilfe-Verlag.

Weichbold, M. (2014). Pretest. In N. Baur \& J. Blasius (Hrsg.), Handbuch Methoden der empirischen Sozialforschung (S. 299-297). Springer VS.

Weidekamp-Maicher, M. (2016). Lebensqualität in der stationären pflegerischen Versorgung. Rabmenmodell und Merkmale (Abschlussbericht). GKV Spitzenverband. www.gkv-spitzenverband.de/media/dokumente/pflegeversicherung/qualitaet_in _der_pflege/wiss_qualitaetsverstaendnis/2016-08-25_Abschlussbericht_wiss_Qua litaetsverstaendnis.pdf.

Weins, C. (2010). Uni- und bivariate deskriptive Statistik. In C. Wolf \& H. Best (Hrsg.), Handbuch der sozialwissenschaftlichen Datenanalyse (S. 65-89). Springer VS.

Welti, F. (2005). Behinderung und Rehabilitation im sozialen Rechtstaat. Freiheit, Gleichheit und Teilhabe behinderter Menschen. Mohr Siebeck.

Werner, S. (2013). So lange wie möglich selbstbestimmt leben. Was bedeutet Autonomie(verlust) für Menschen mit Demenz? Pflegezeitschrift, 66(10), 594-597.

WHO. (2002). Aktiv Altern. Rahmenbedingungen und Vorschläge für politisches Handeln. World Health Organization. https://apps.who.int/iris/bitstream/handle/106 65/67215/WHO_NMH_NPH_02.8_ger.pdf.

Widuckel, W. (2015). Arbeitskultur 2020 - Herausforderungen für die Zukunft der Arbeit. In W. Widuckel, M. J. Ringlstetter, K. de Molina \& D. Frey (Hrsg.), Arbeitskultur 2020. Herausforderungen und Best Practice der Arbeitswelt der Zukunft (S. 27-43). Springer Fachmedien.

Wingenfeld, K., Büscher, A., \& Gansweid, B. (2011). Das neue Begutachtungsinstrument zur Feststellung von Pflegebedürftigkeit (Schriftenreihe Modellprogramm zur Weiterentwicklung der Pflegeversicherung Band 2). GKV Spitzenverband. www.gkv-spitzenverband.de/media/dokumente/presse/publikationen/schriftenre ihe/GKV-Schriftenreihe_Pflege_Band_2_18962.pdf.

Wirth, K., \& Husmann, A.-K. (2013). Akzeptanz konsekutiver Ausbildungsgänge aus Sicht teilnehmender Betriebe - Ergebnisse des Schulversuchs EARA.bwp@ Spezial 7 - Weiterentwicklung dualer Berufsausbildung: Konsekutiv, kompetenzorientiert, konnektiv. Erfahrungen und Impulse aus dem Schulversuch EARA. www.bwpat .de/spezial7/wirth_husmann_eara2013.pdf.

Witzel, A. (2000). Das problemzentrierte Interview. Forum Qualitative Sozialforschung / Forum: Qualitative Social Research, 1(1). http://dx.doi.org/10.17169/fqs-1. 1.1132 . 
Wohn- und Teilhabegesetz NRW. Abgerufen 23. Januar 2017, von https://recht.nr w.de/lmi/owa/br_text_anzeigen?v_id=10000000000000000678\#det321090.

Wulff, I., Budnick, A., Kölzsch, M., Kreutz, R., \& Dräger, D. (2012). Deutschsprachige Testkurzversion der Hertz Perceived Enactment of Autonomy Scale (HPEASKD) für ältere Menschen in Pflegeheimen. Zeitschrift für Gesundheitspsychologie, 20(4), 157-165.

Wulff, I., Kalinowski, S., \& Dräger, D. (2010). Autonomie im Pflegeheim - Konzeptionelle Überlegungen zu Selbstbestimmung und Handlungsfähigkeit anhand eines Modells. Pflege, 23(4), 240-248.

Wunder, M. (2008). Demenz und Selbstbestimmung. Ethik in der Medizin, 20(1), 17-25.

Zündel, M. (2012). Nichtsprachliche Interaktion und das Entstehen von Bedeutung in der Pflege. In A. Hanses \& K. Sander (Hrsg.), Interaktionsordnungen: Gesundheit als soziale Praxis. Springer VS. 
Anhang: Auszüge aus dem Musterrahmenkonzept

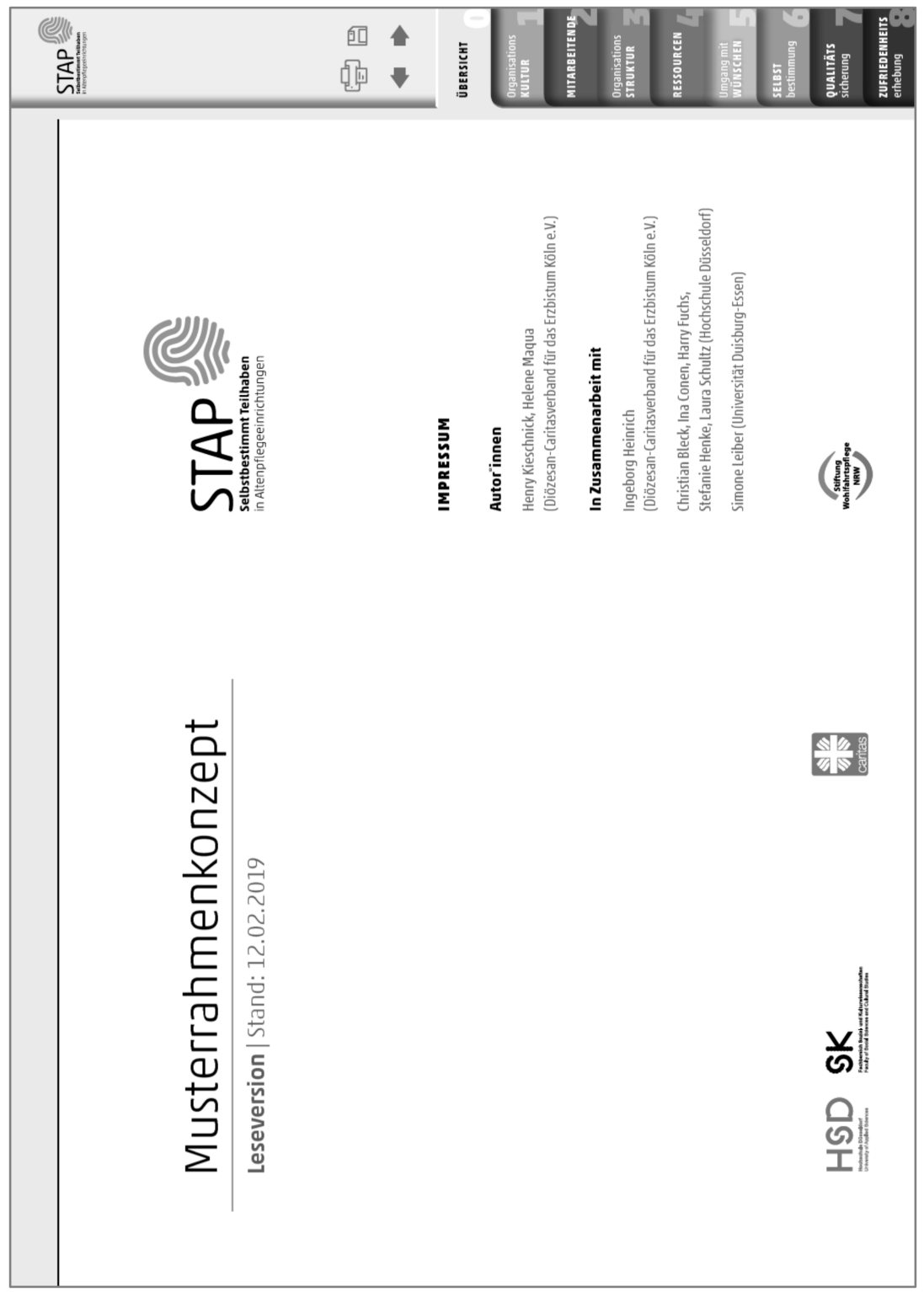


Anhang: Auszüge aus dem Musterrahmenkonzept

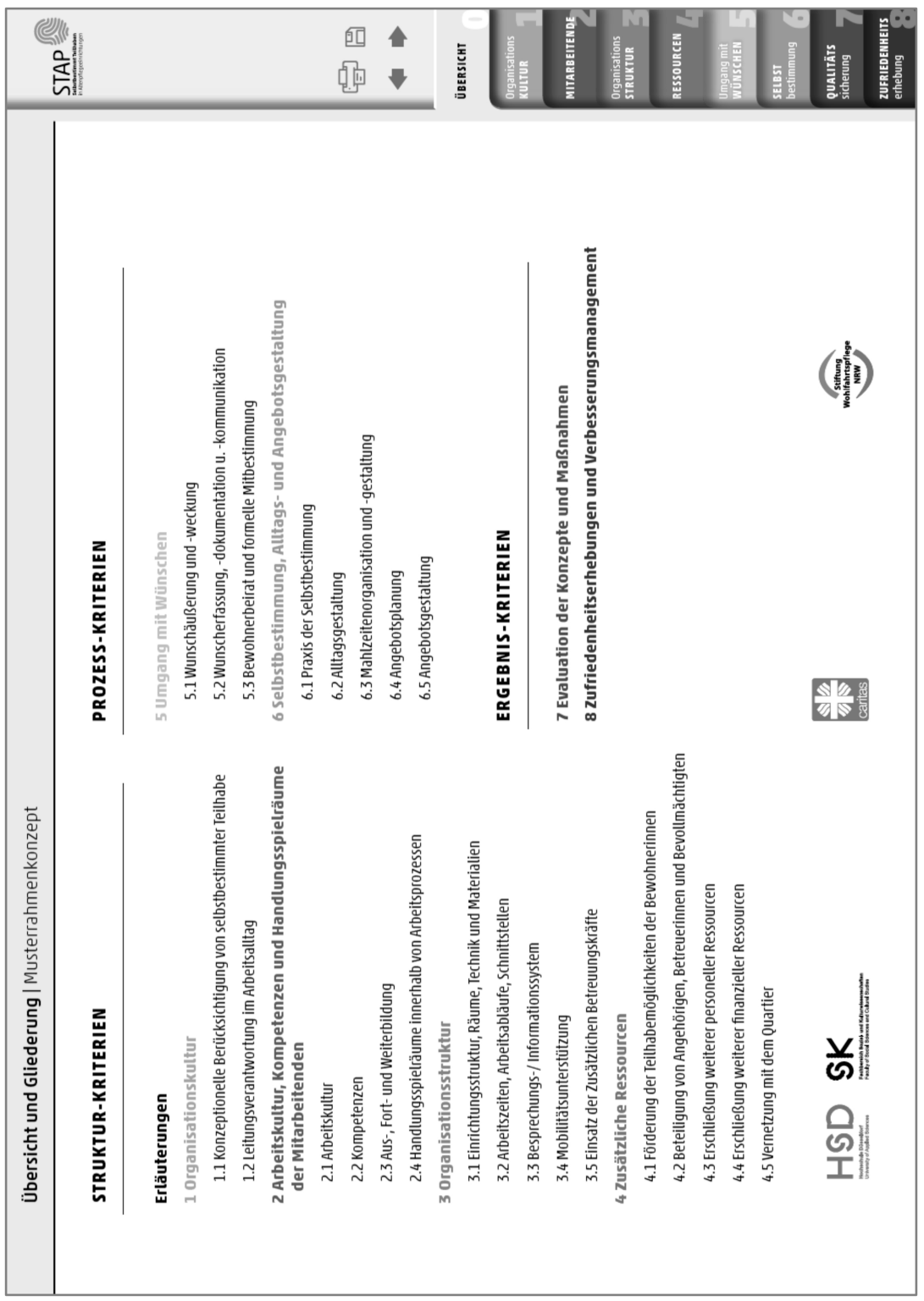









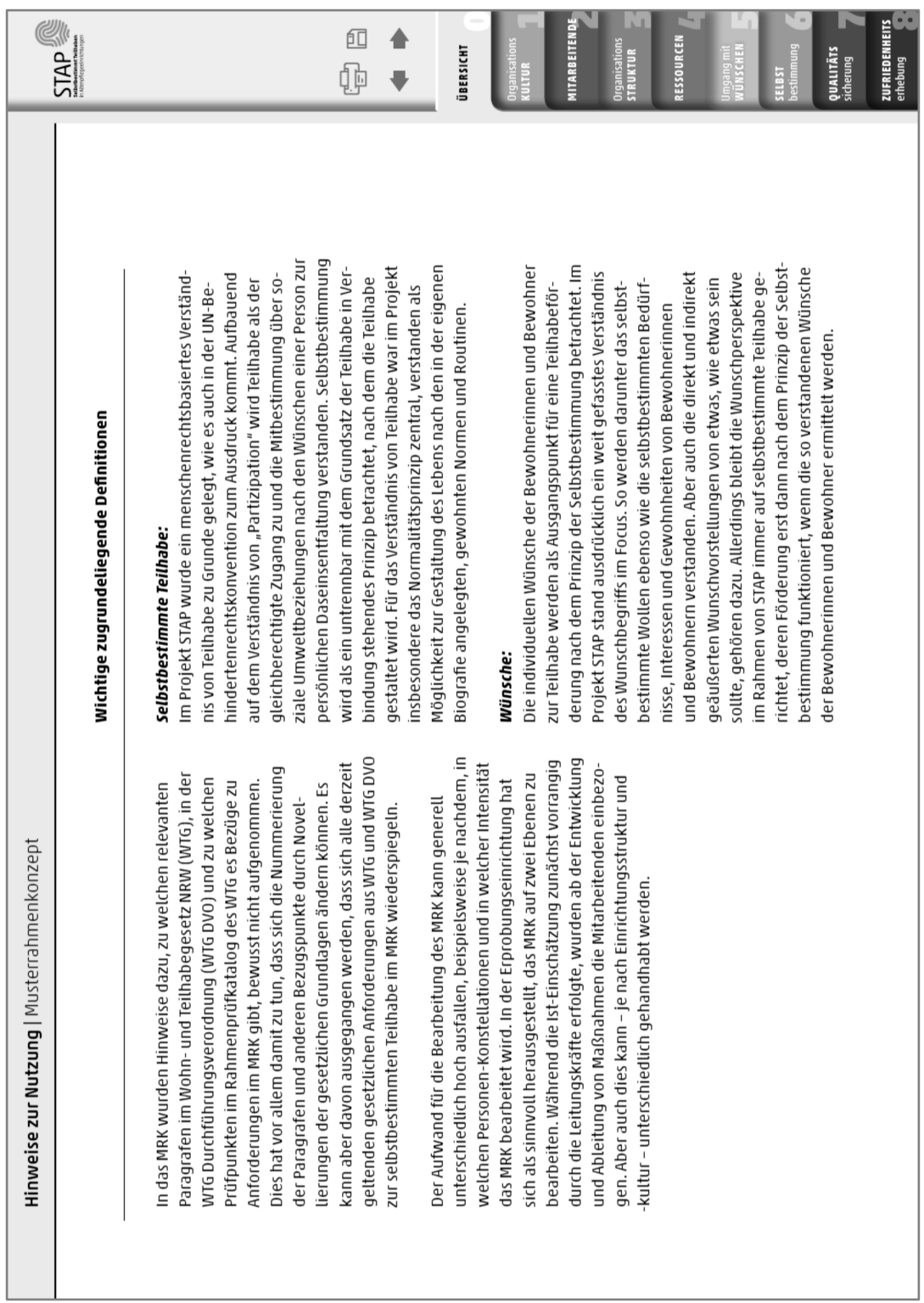




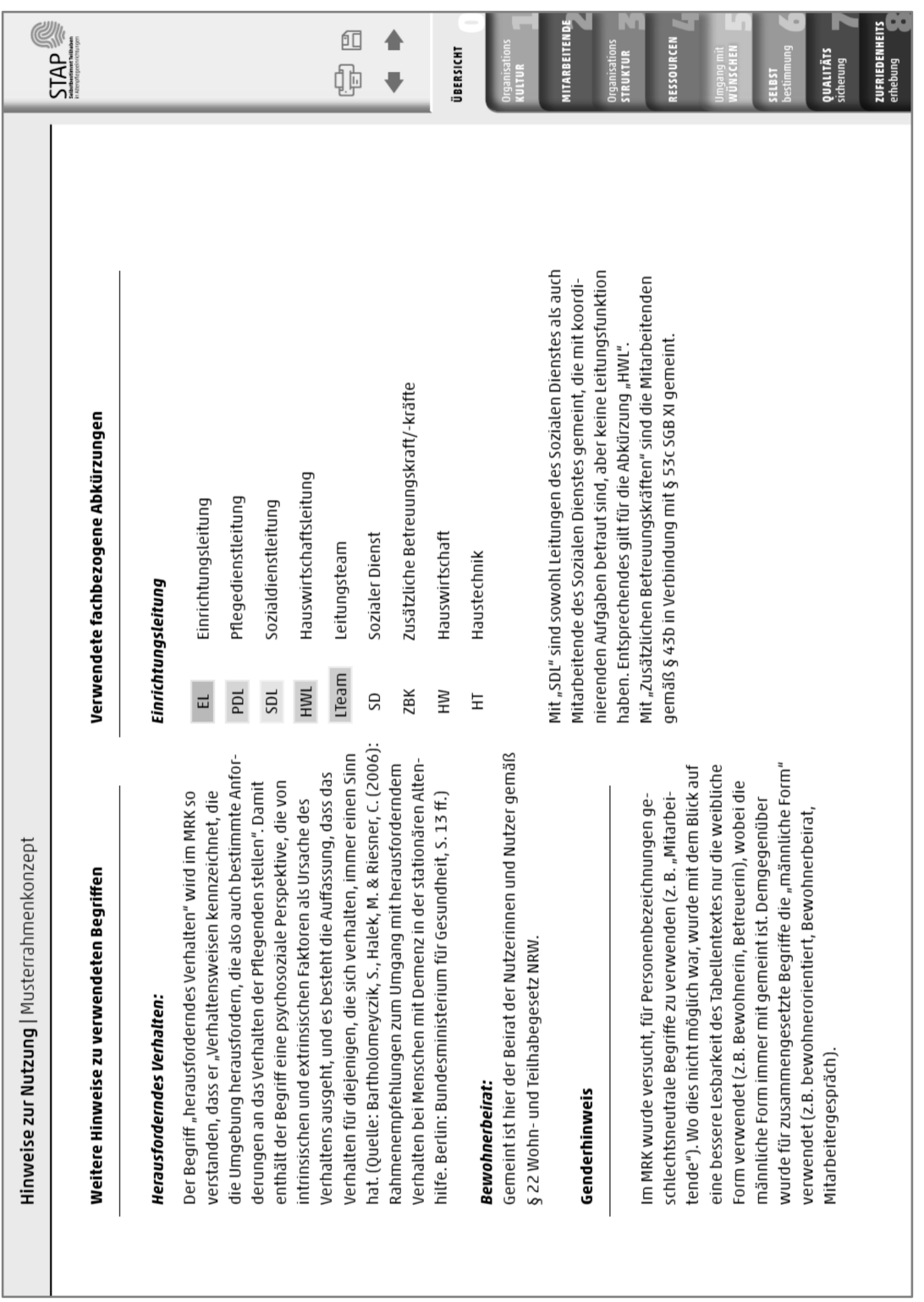




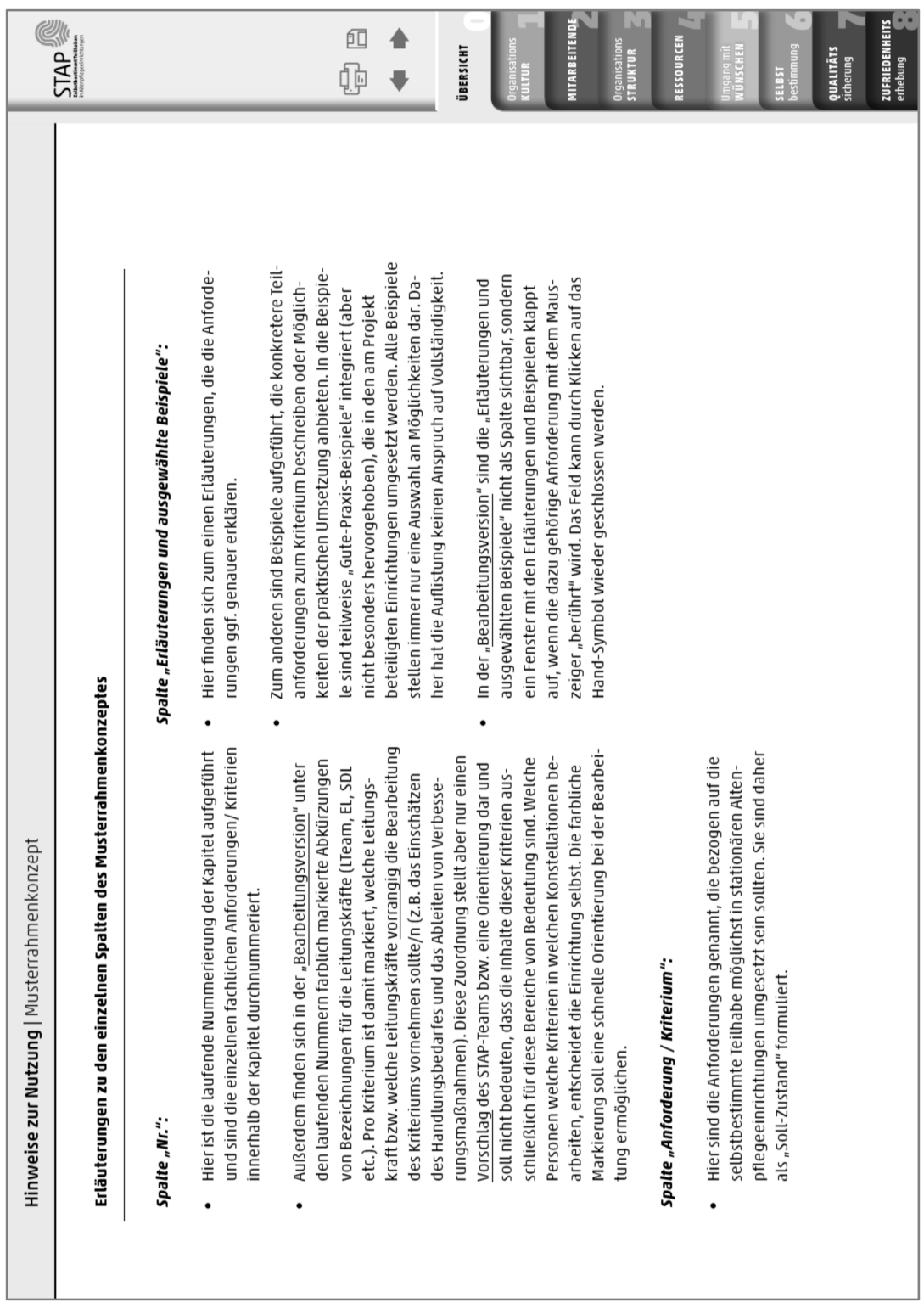




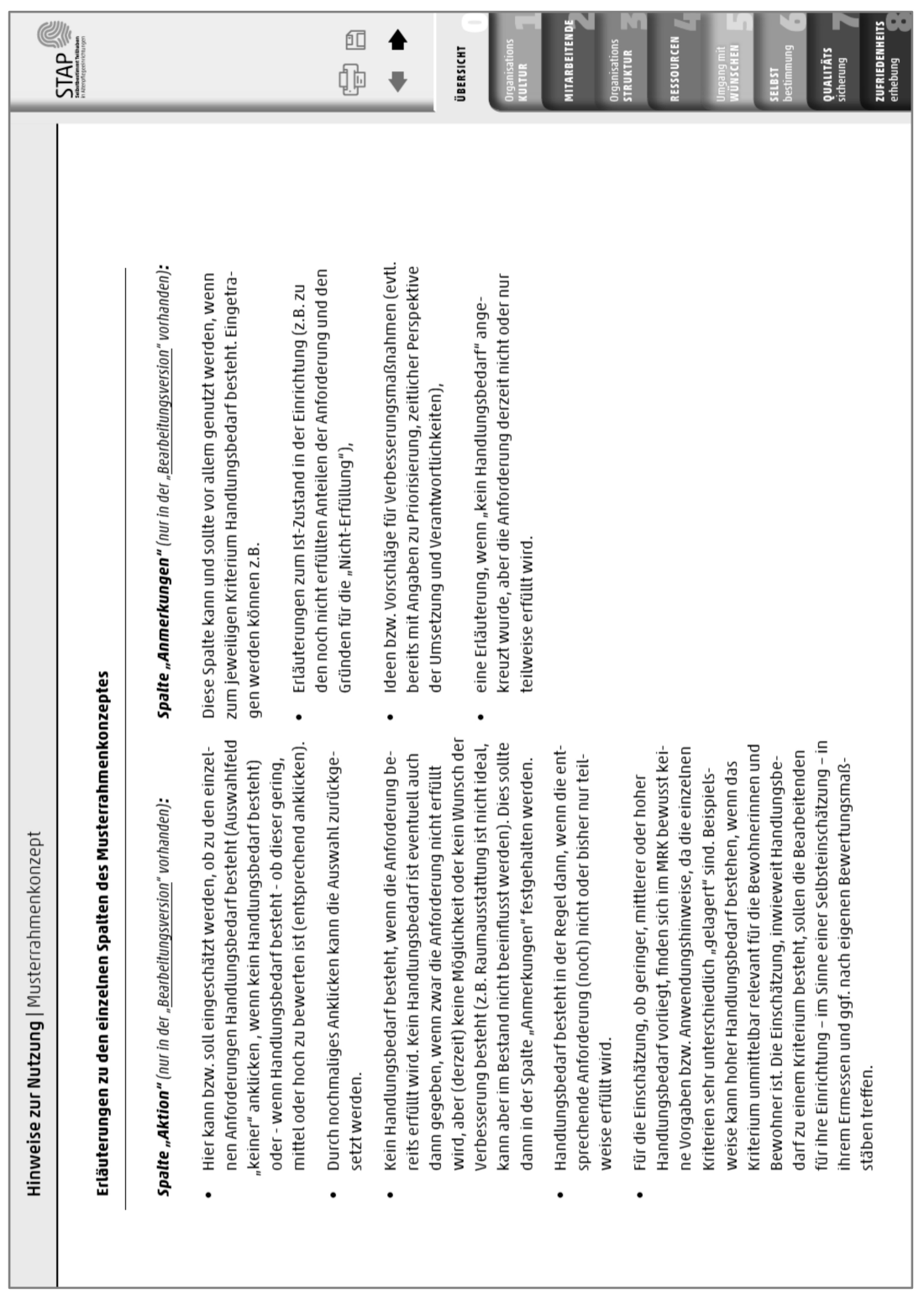




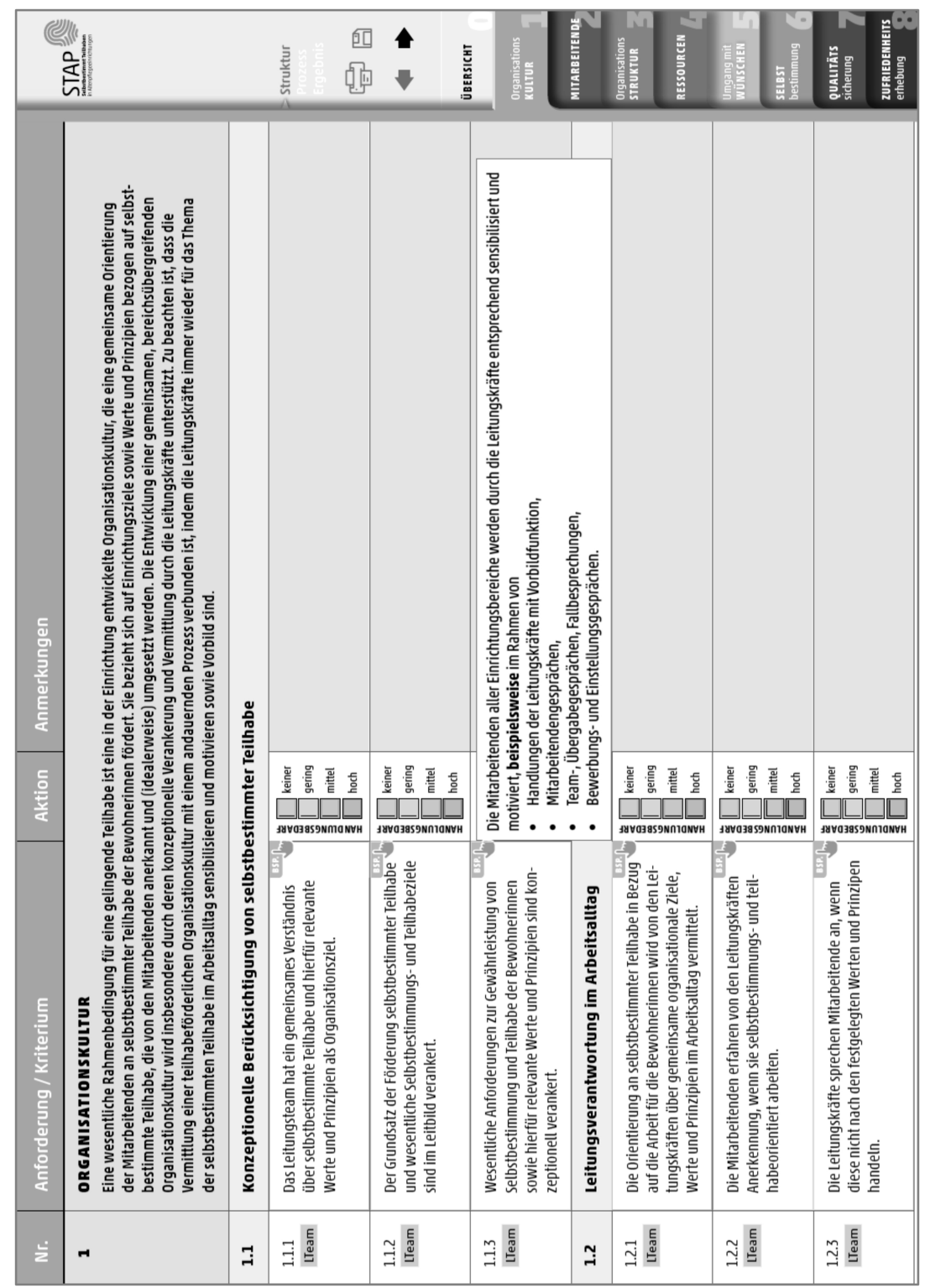




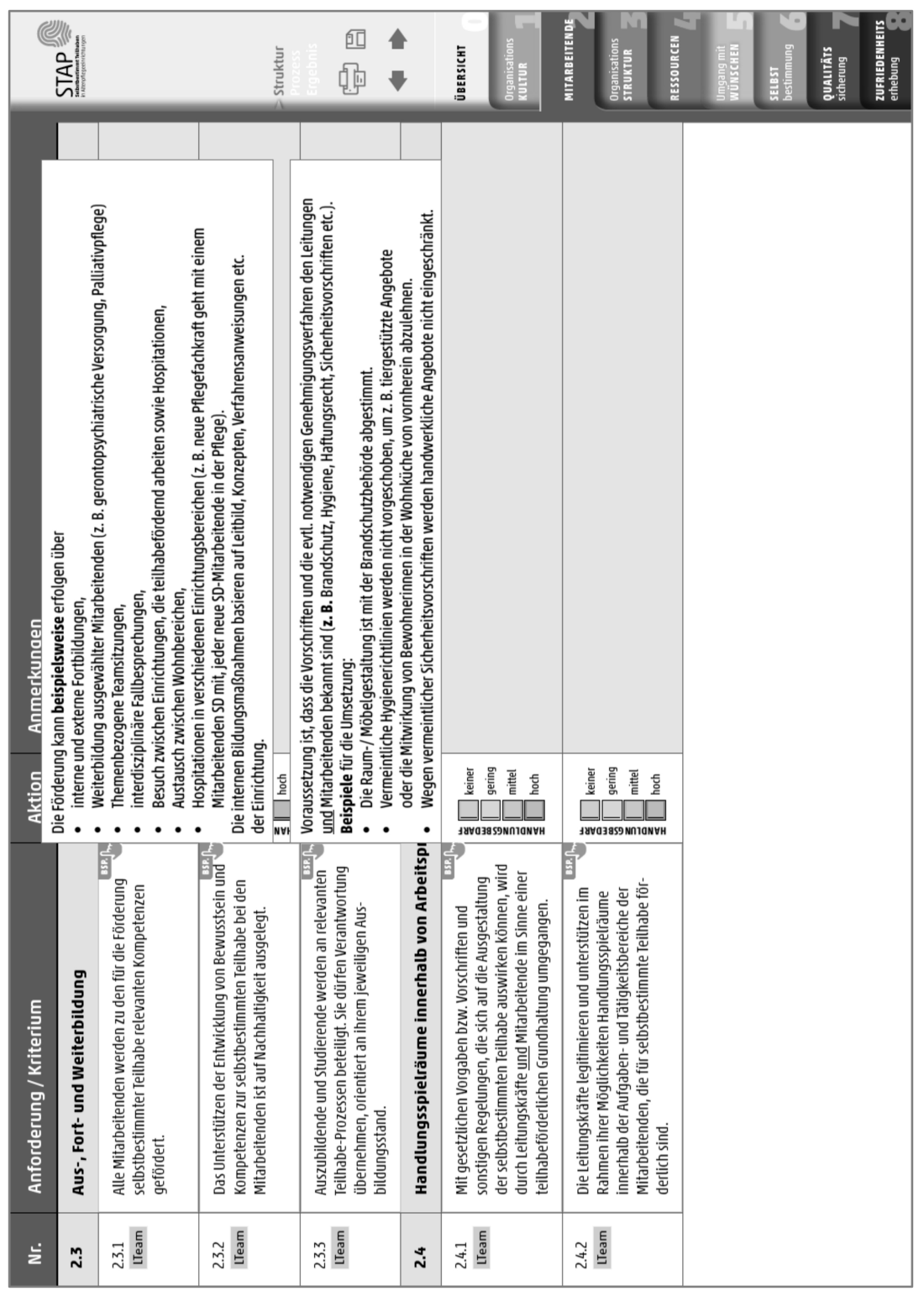




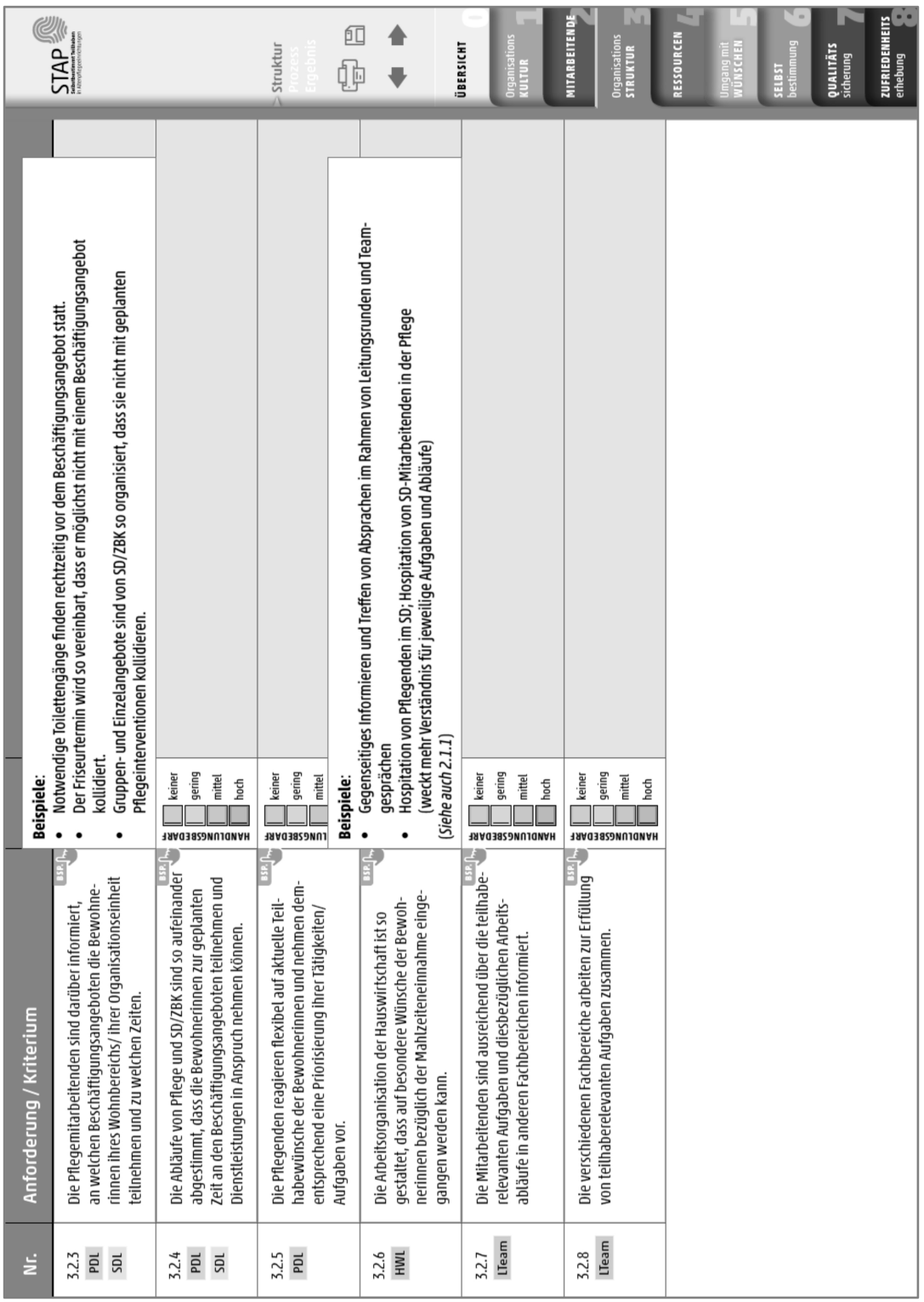




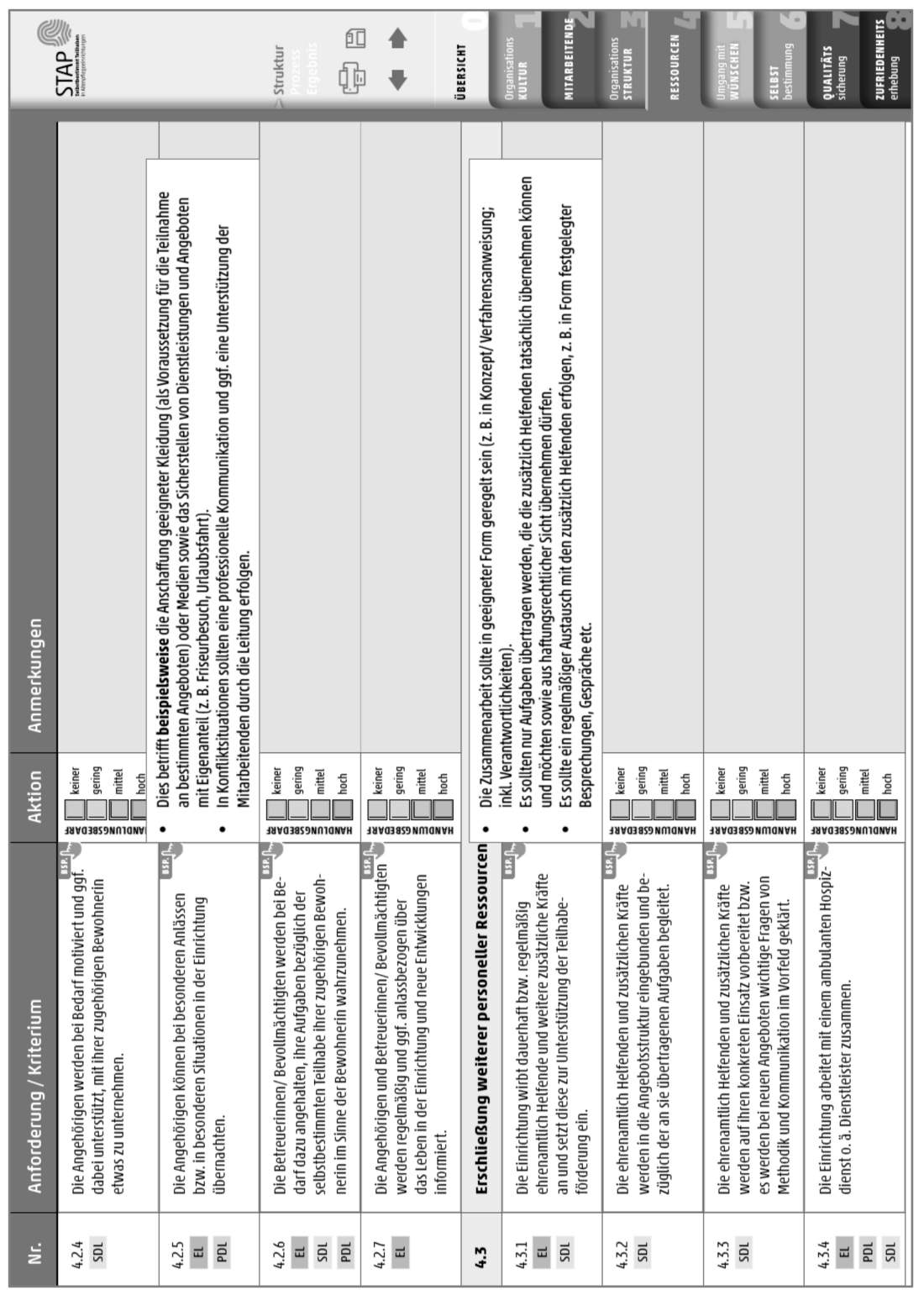




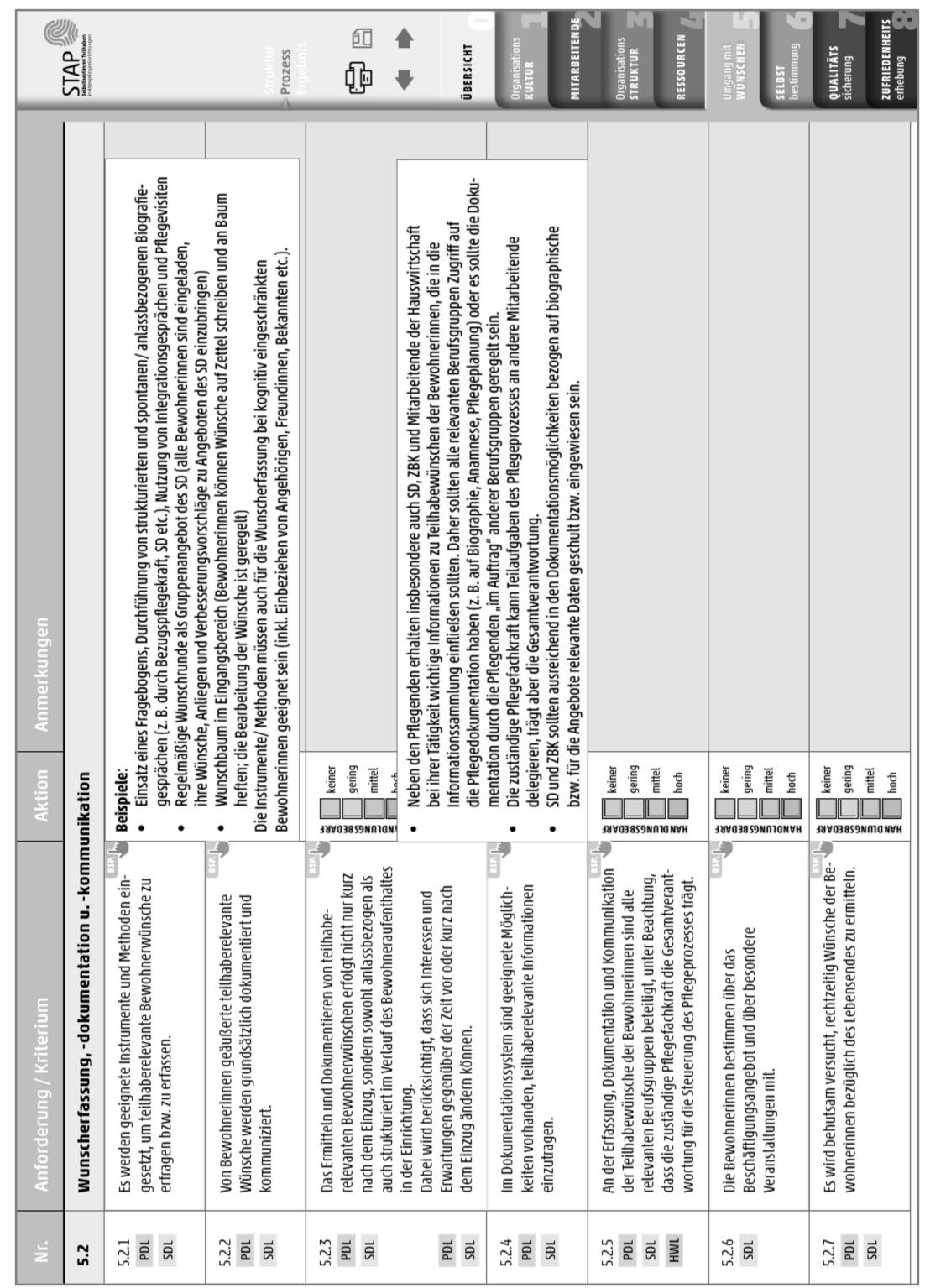




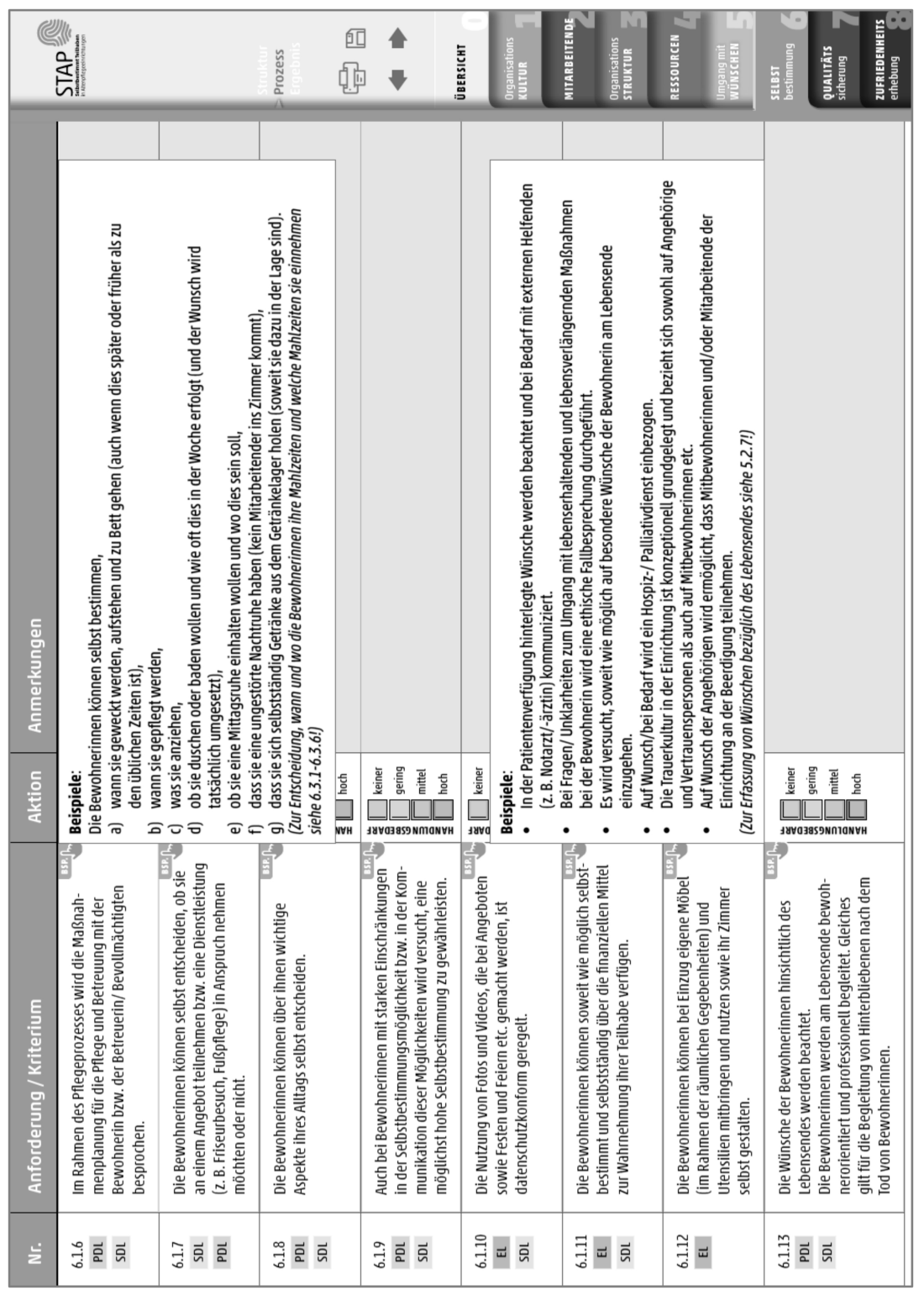




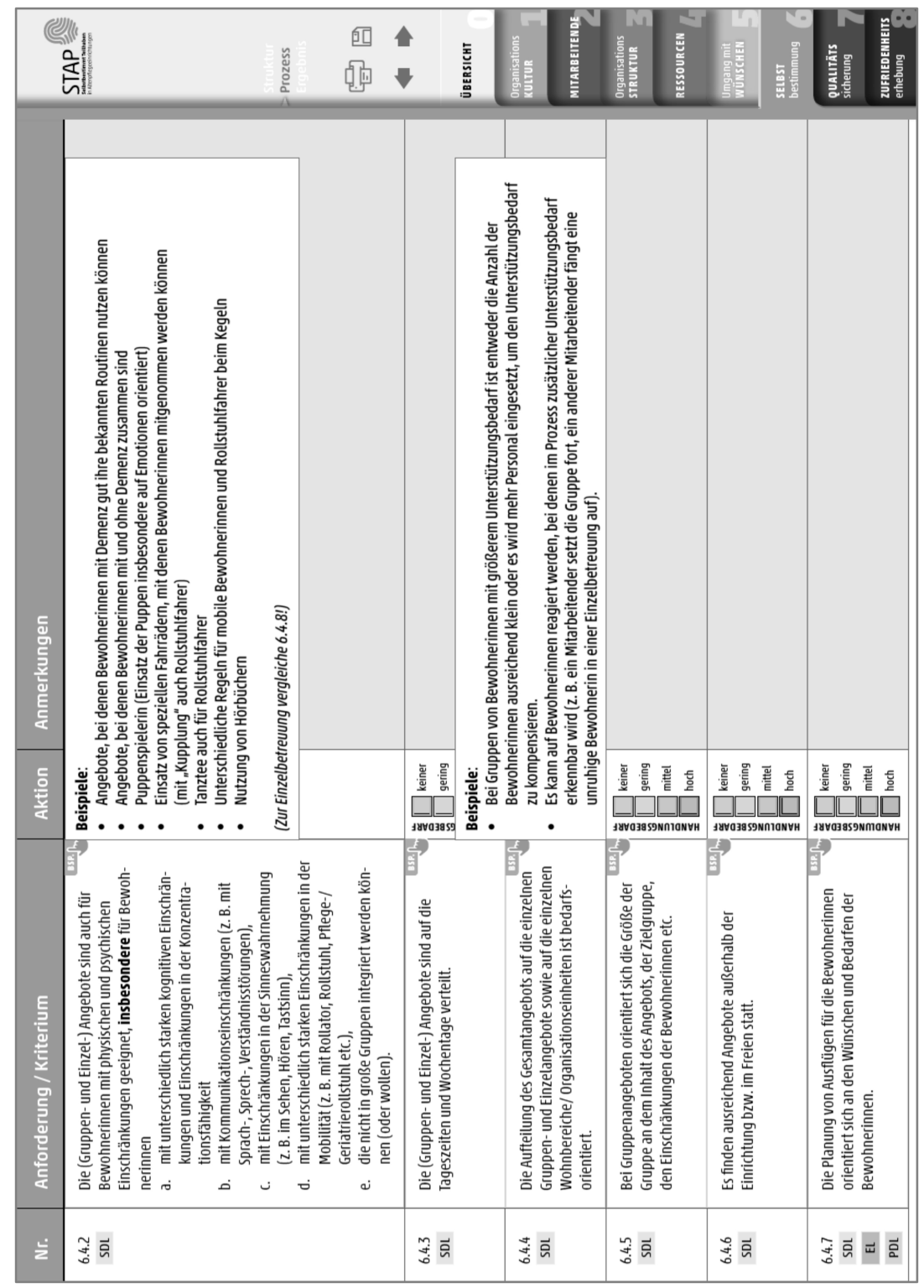









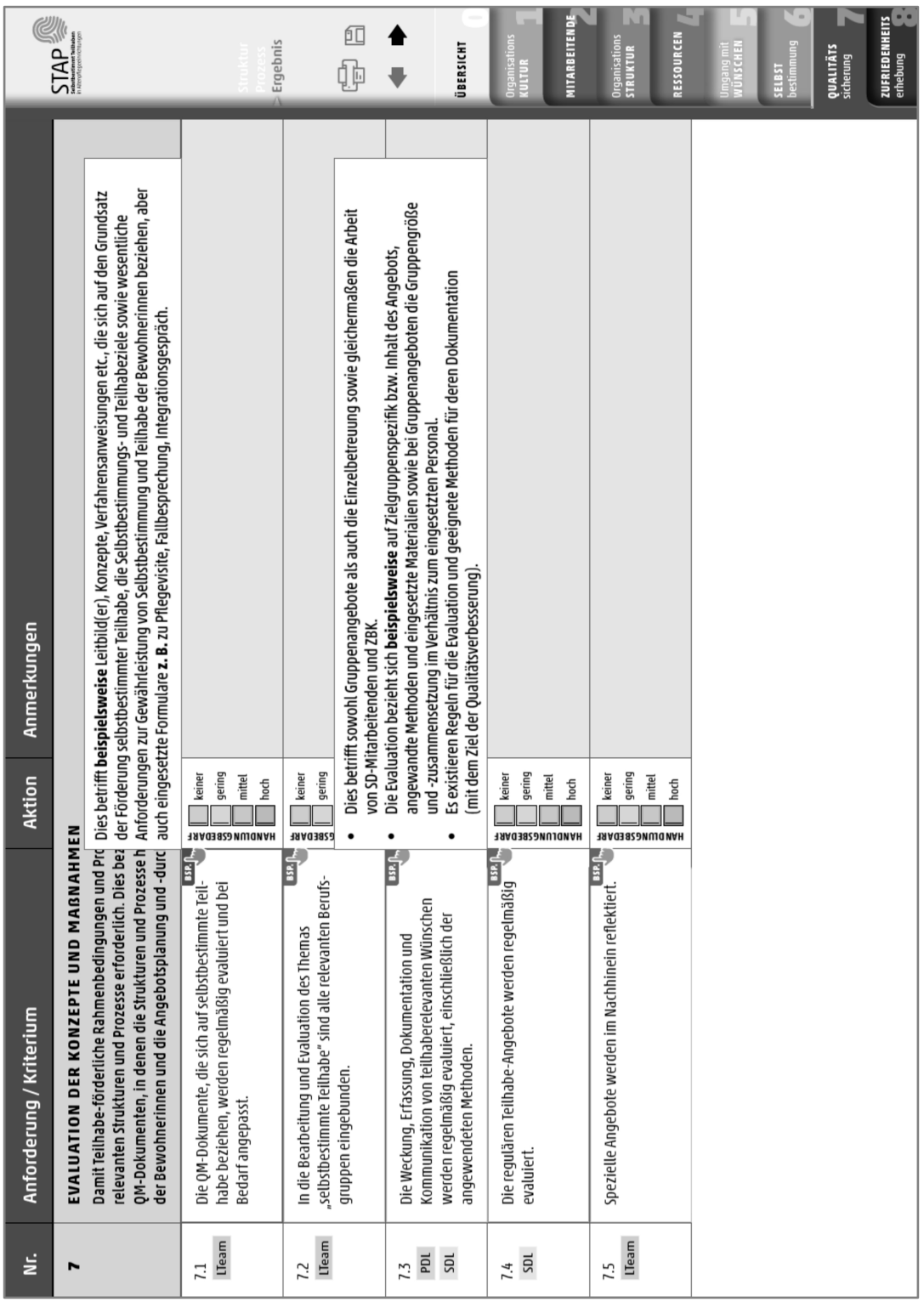

